\title{
Automatic hydraulic fracturing design for low permeability reservoirs using artificial intelligence
}

Andrei Sergiu Popa

West Virginia University

Follow this and additional works at: https://researchrepository.wvu.edu/etd

\section{Recommended Citation}

Popa, Andrei Sergiu, "Automatic hydraulic fracturing design for low permeability reservoirs using artificial intelligence" (2004). Graduate Theses, Dissertations, and Problem Reports. 2638.

https://researchrepository.wvu.edu/etd/2638

This Dissertation is protected by copyright and/or related rights. It has been brought to you by the The Research Repository @ WVU with permission from the rights-holder(s). You are free to use this Dissertation in any way that is permitted by the copyright and related rights legislation that applies to your use. For other uses you must obtain permission from the rights-holder(s) directly, unless additional rights are indicated by a Creative Commons license in the record and/ or on the work itself. This Dissertation has been accepted for inclusion in WVU Graduate Theses, Dissertations, and Problem Reports collection by an authorized administrator of The Research Repository @ WVU.

For more information, please contact researchrepository@mail.wvu.edu. 


\title{
Automatic Hydraulic Fracturing Design for Low Permeability Reservoirs Using Artificial Intelligence
}

\author{
Andrei Sergiu Popa \\ Dissertation submitted to the \\ College of Engineering and Mineral Resources \\ at West Virginia University \\ in partial fulfillment of the requirements \\ for the degree of \\ Doctor of Philosophy \\ in \\ Petroleum and Natural Gas Engineering \\ Shahab Mohaghegh, Ph.D., Chair \\ Samuel Ameri, M.S., Department Chair \\ Khashayar Aminian, Ph.D. \\ H. Ilkin Bilgesu, Ph.D. \\ Sinisha Jikich, Ph.D. \\ John Loth, Ph.D. \\ Morgantown, West Virginia \\ 2004
}

Keywords: Hydraulic Fracturing, Artificial Neural Networks, Genetic Algorithms, Fuzzy Logic, Vector Quantization, Lithology Identification

Copyright 2004 Andrei Sergiu Popa 


\title{
ABSTRACT \\ Automatic Hydraulic Fracturing Design for Low Permeability Reservoirs Using Artificial Intelligence
}

\author{
Andrei Sergiu Popa
}

The hydraulic fracturing technique is one of the major developments in petroleum engineering in the last two decades. Today, nearly all the wells completed in low permeability gas reservoirs require a hydraulic fracturing treatment in order to produce at an economical level. This study presents a new methodology, applicable to tight gas reservoirs, for designing hydraulic fractures.

This study is intended to develop an automatic hydraulic fracture design tool to help users design fracture jobs without being an expert in the art and science of hydraulic fracturing. This process is composed entirely of an integration of several artificial intelligence techniques.

The methodology consists of three modules: formation stress determination, optimum treatment design and net treatment pressure prediction. The first module combines the classic approach of stress calculations with a fuzzy lithology identification system to better characterize the reservoir and estimate the stress profile. The result of this module is essential for the fracture treatment design. The second module incorporates an optimization system composed of neural networks and a genetic algorithm to search for the optimum treatment design. The third, and final, module is designed to predict the net treating pressure expected during fracturing. A onedimensional vector quantization technique samples and extracts the main characteristic of the pressure profile. The net treatment pressure neural network generates the main features of the pressure profile and then reconstructs the entire signal.

The methodology was integrated in a computer program aimed to help petroleum engineers design optimum treatment schedules and predict net treatment pressure for hydraulic fracturing. This tool is designed to reduce the engineering time for designing optimum treatment schedules. 


\section{DEDICATION}

To my wife Carrie, the best thing that ever happened in my life. 


\section{ACKNOWLEDGEMENTS}

I express my sincere appreciation to Dr. Shahab Mohaghegh, my graduate advisor and committee Chair. His vision, passion, and continuous encouragement are a big part of my accomplishments.

I am thankful to Dr. and Mrs. Sinisha Jikich, who mentored my steps since my first days in West Virginia and for assistance and guidance with my thesis. They were like family to me.

Special thanks to my professors in the Department of Petroleum and Natural Gas Engineering for providing me the knowledge to complete my degree and serving on my committee: Sam Ameri, Professor and Chair, for his support and encouragement; Dr. Khashayar Aminian, Dr. Ilkin Bilgesu, and Dr. John Loth for their positive feedback.

I would like to mention Sami, my irreplaceable cat, who slept on my lap or keyboard during the long nights of research and was always around in times of stress and loneliness. $\mathrm{He}$ was my first "American Friend."

I am so grateful to have such wonderful parents, Sergiu and Stela Popa, who always believed in me and provided me with the best advice. Their love and encouragement gave me the strength to continue my education at West Virginia University. 


\section{TABLE OF CONTENTS}

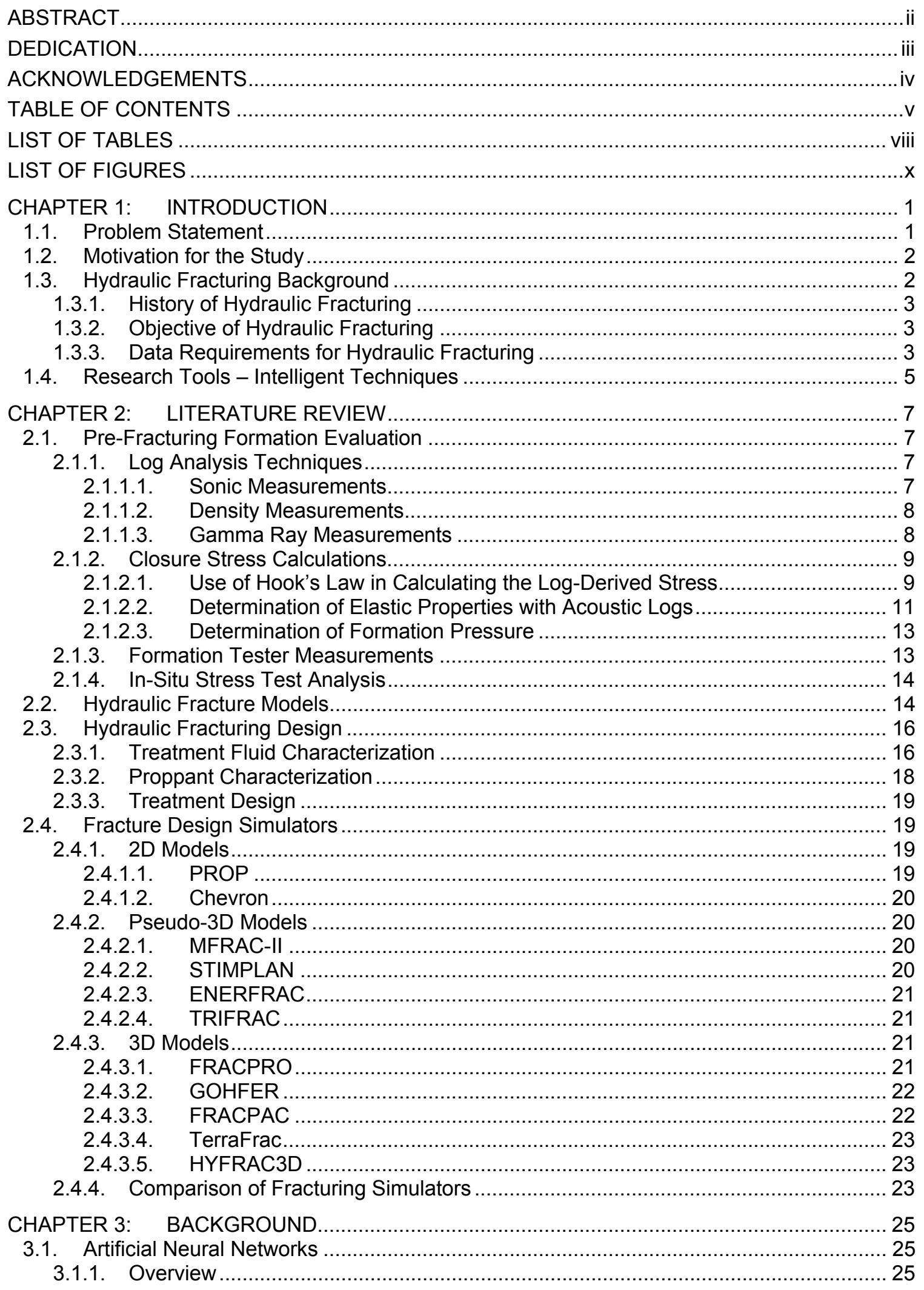




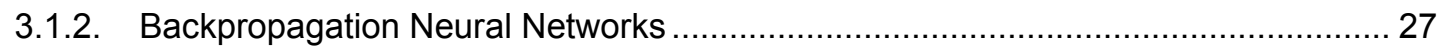

3.1.3. Neural Nets versus Conventional Computing ................................................... 28

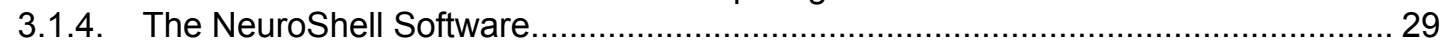

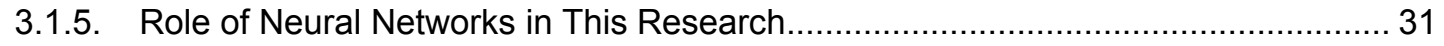

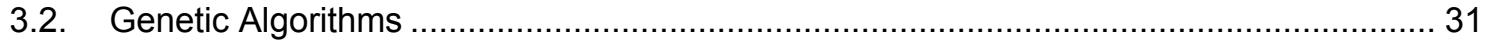

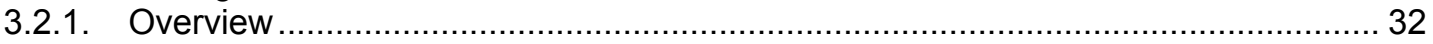

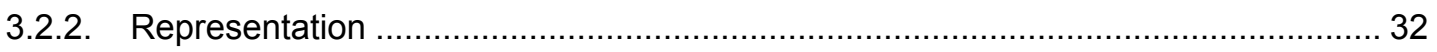

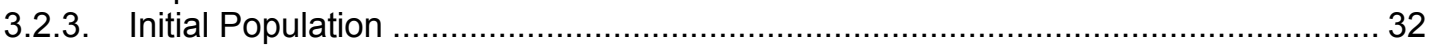

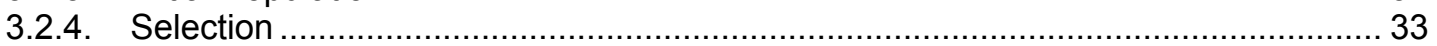

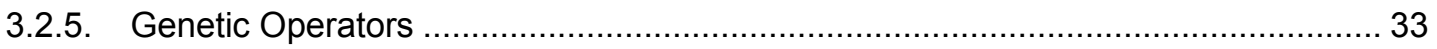

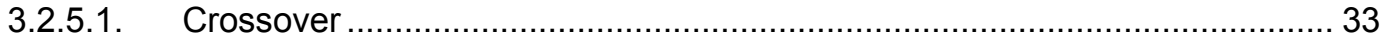

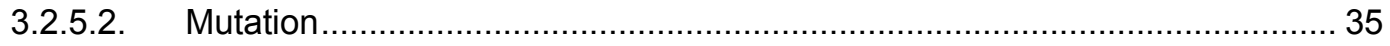

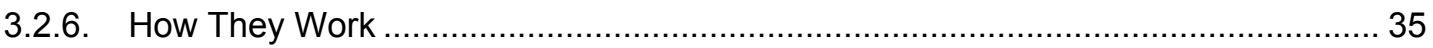

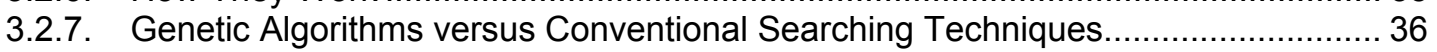

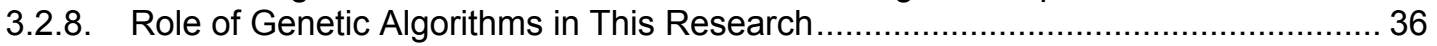

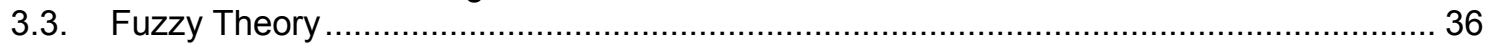

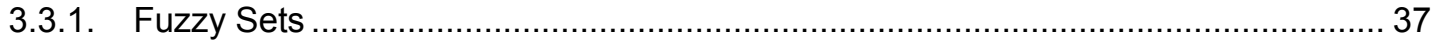

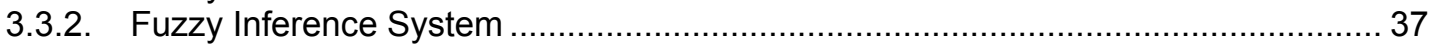

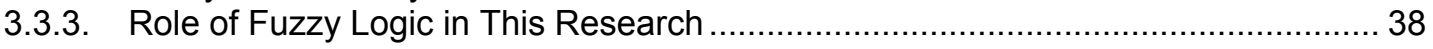

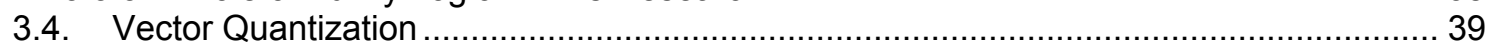

3.4.1. Role of Vector Quantization in This Research.................................................. 40

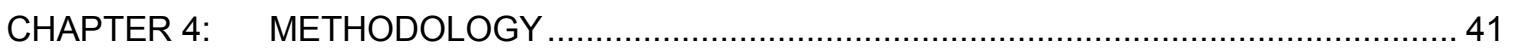

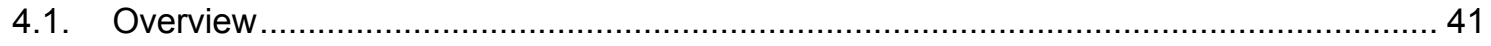

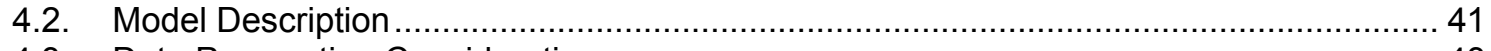

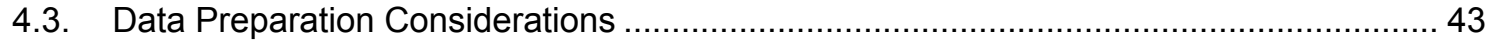

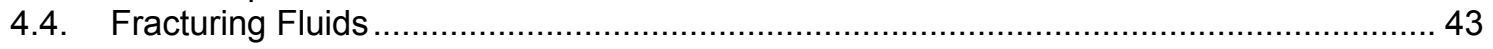

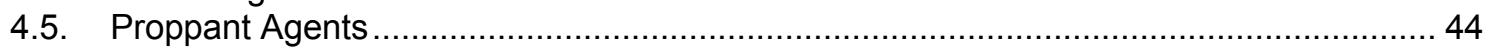

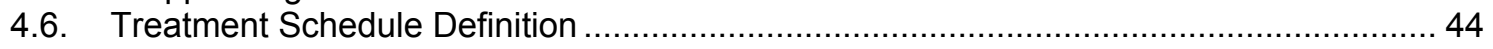

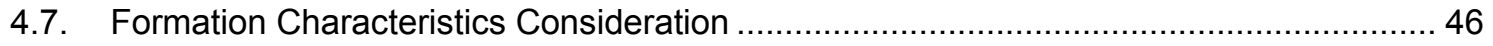

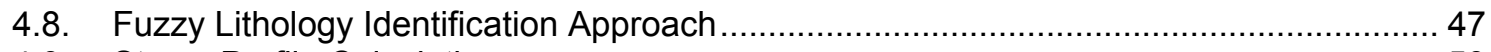

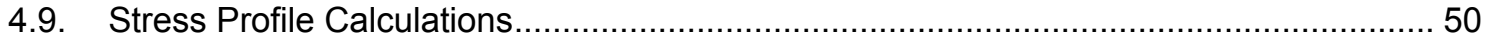

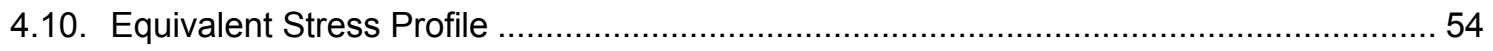

4.10.1. Methodology for Generating Equivalent Stress Profile ...................................... 55

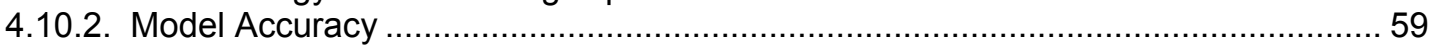

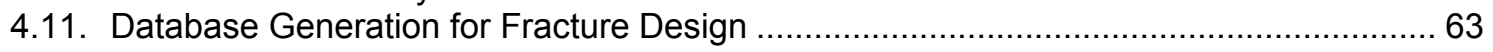

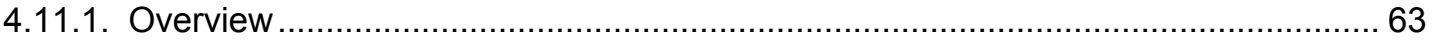

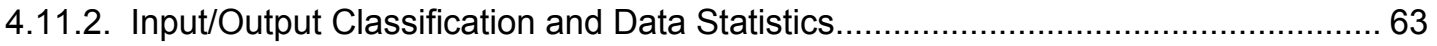

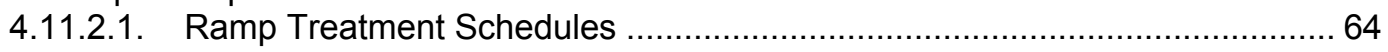

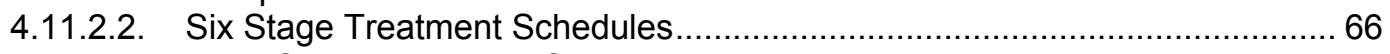

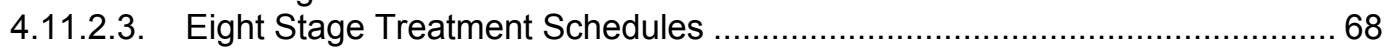

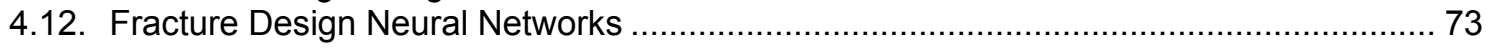

4.12.1. Developing Neural Networks for Ramp Treatments ............................................ 74

4.12.2. Developing Neural Networks for Stage Treatments ........................................... 77

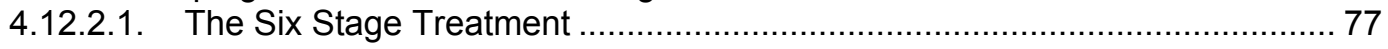

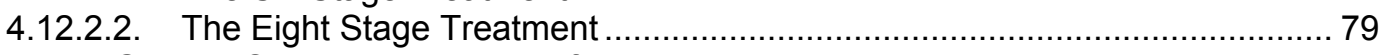

4.13. Neuro-Genetic Optimization Module for Fracture Treatment Design.............................. 82

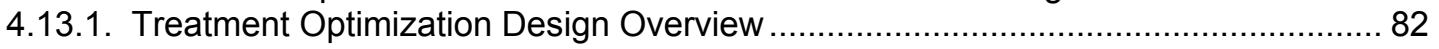

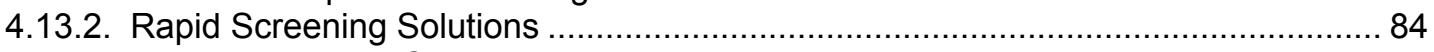

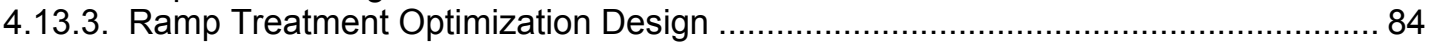

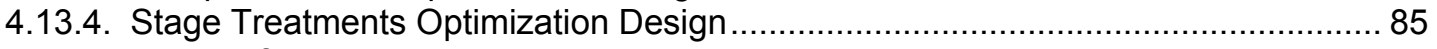

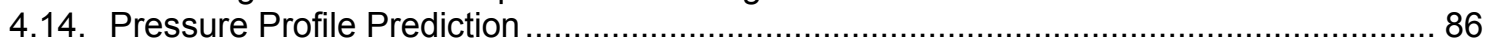

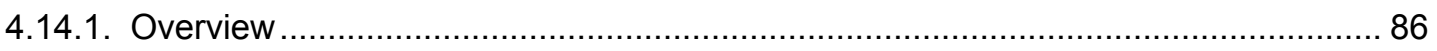

4.14.2. Defining Pressure Components Used For Pressure Matching.............................. 87

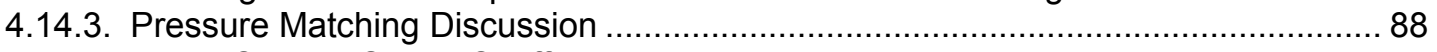

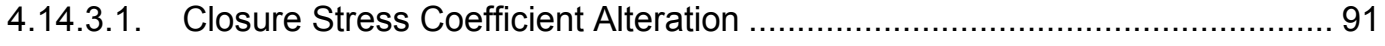


4.14.3.1.1. Top, Overburden, and Bottom Layers 92

4.14.3.1.2. Pay Zone Layer $\quad 96$

4.14.3.1.3. Closure Stress Coefficient Alteration Summary 98

4.14.3.2. Correction Percentage Value Determination................................................ 99

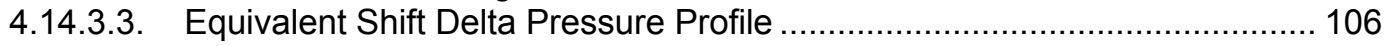

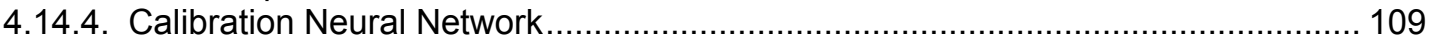

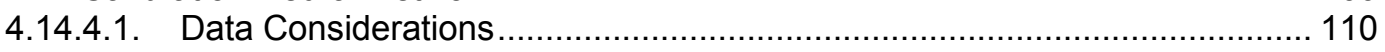

4.14.4.2. White Noise Data Generation ............................................................ 112

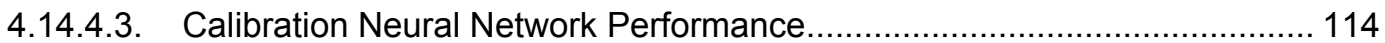

4.14.5. Neural Network for Pressure Profile Prediction ................................................ 116

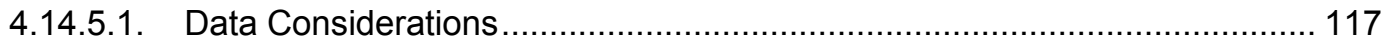

4.14.5.2. Data Sampling using Vector Quantization ............................................ 118

4.14.5.3. Neural Network Design ................................................................... 120

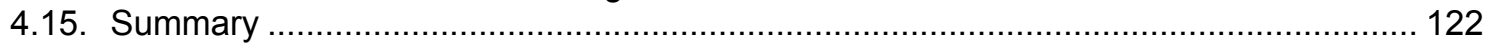

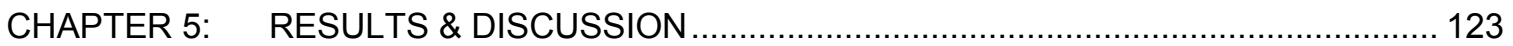

5.1. Fuzzy Logic Lithology Technique for Stress Profile Calculations............................... 123

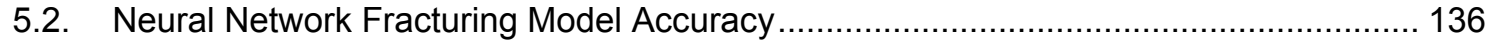

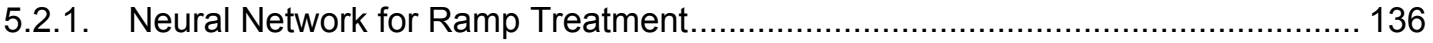

5.2.2. Neural Network for Six Stage Treatment....................................................... 146

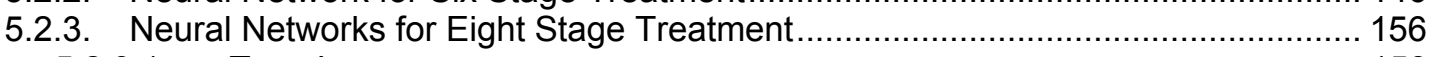

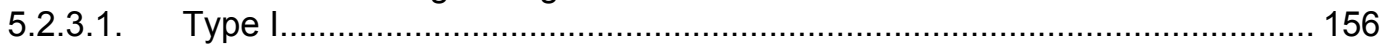

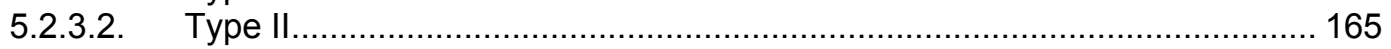

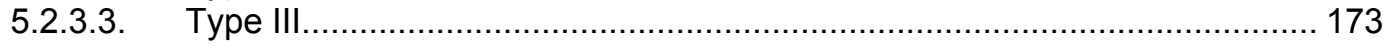

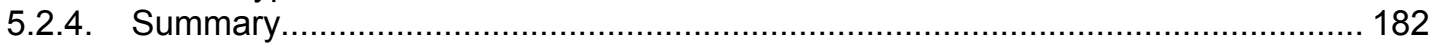

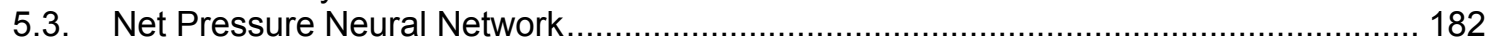

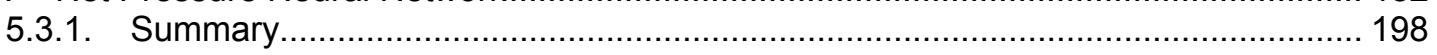

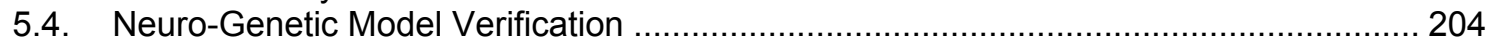

5.4.1. Tool Design vs FRACPRO vs Actual for Treatment Schedule ............................... 204

5.4.2. Design versus FRACPRO for Net Pressure Prediction ........................................ 206

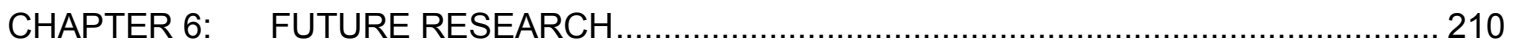

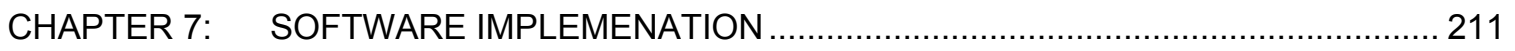

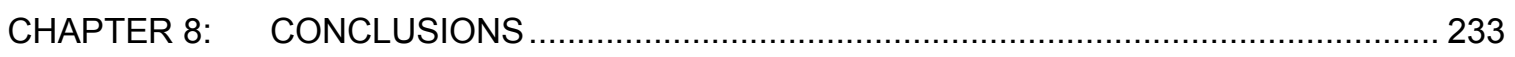

BIBLIOGRAPHY

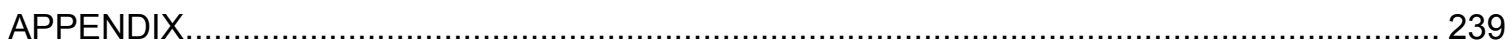

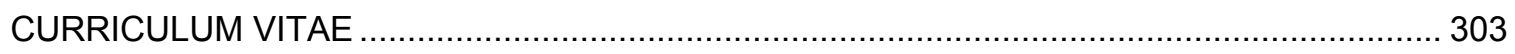




\section{LIST OF TABLES}

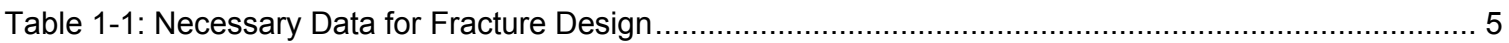

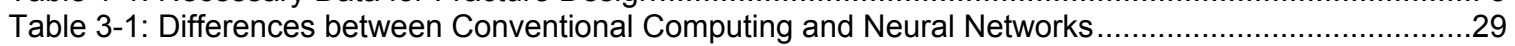

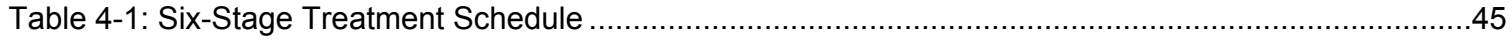

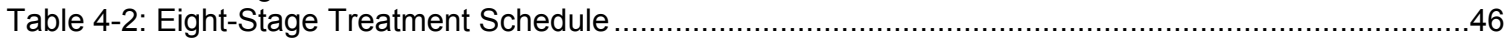

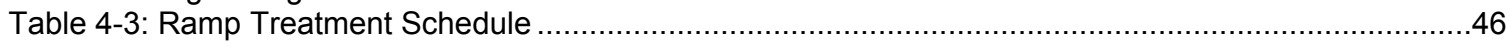

Table 4-4: Fuzzy Rules for the Fuzzy Lithology Identification System ...........................................49

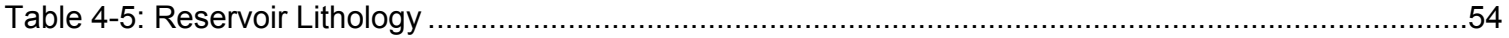

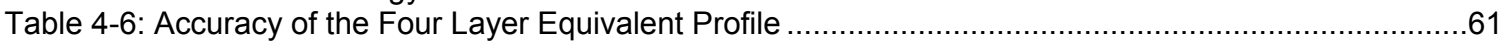

Table 4-7: Percentage of Total Fluid Volume per Stage for Six-Stage Treatment Schedule ......................67

Table 4-8: Stage Fluid Volume Structure of Six-Stage Treatment Schedule..........................................67

Table 4-9: Proppant Concentration for Eight-Stage Treatment Schedule ............................................69

Table 4-10: Percentage of Total Fluid Volume per Stage for Eight-Stage Treatment Schedule ...................69

Table 4-11: Type I Stage Fluid Volume Structure of Eight-Stage Treatment Schedule .............................70

Table 4-12: Types II and III Stage Fluid Volume Structure of Eight-Stage Treatment Schedule ...................71

Table 4-13: Inputs/Outputs for Ramp Treatment Neural Network ......................................................75

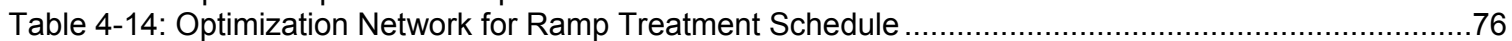

Table 4-15: Inputs/Outputs for Six-Stage Treatment Schedule Neural Network .......................................78

Table 4-16: Optimization Network for Six-Stage Treatment Schedule ...............................................79

Table 4-17: Correlation Coefficients for Eight Stage Treatment Schedule Neural Network ........................80

Table 4-18: Optimization Network for Eight Stage Treatment Schedule ...............................................82

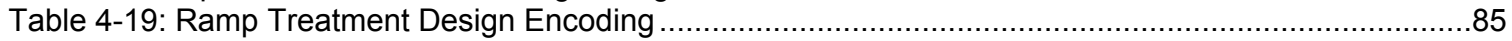

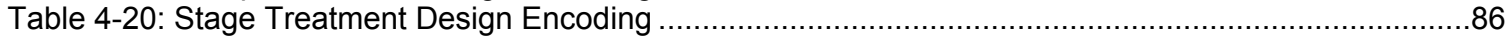

Table 4-21: Closure Stress Gradient Values for Equivalent Altered Profiles from Scenario A Case 1..........92

Table 4-22: Closure Stress Gradient Values for Equivalent Altered Profiles from Scenario B Case $1 \ldots . . . . . . .93$

Table 4-23: Closure Stress Gradient Values for Equivalent Altered Profiles from Scenario A Case 3 ...........95

Table 4-24: Closure Stress Gradient Values for Equivalent Altered Profiles from Scenario B Case 3..........95

Table 4-25: Closure Stress Gradient Values for Pay Zone Equivalent Altered Profiles from Case 1 ............97

Table 4-26: Closure Stress Gradient Values for Pay Zone Equivalent Altered Profiles from Case 1 ...........97

Table 4-27: Generated Reservoir Lithology Case .................................................................... 100

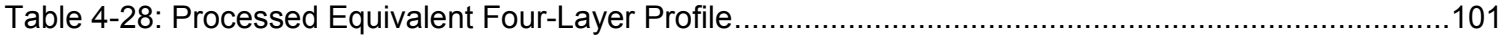

Table 4-29: Correction Percentage Coefficients for 50 Cases ......................................................104

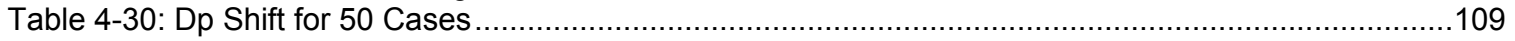

Table 4-31: Parameters for Delta Pressure Shift Neural Network ..................................................110

Table 4-32: Data for Training the Delta Pressure Shift Neural Network ...........................................111

Table 4-33: White Noise Data for Training the Calibration Neural Network .........................................113

Table 4-34: Calibration Neural Network, $1^{\text {st }}$ Noise Data Set Included ...................................................114

Table 4-35: Calibration Neural Network, 1 st and $2^{\text {nd }}$ Noise Data Sets Included ....................................114

Table 4-36: Calibration Neural Network, $1^{\text {st }}, 2^{\text {nd }}$, and $3^{\text {rd }}$ Noise Data Sets Included ..................................114

Table 4-37: Calibration Neural Network, $1^{\text {st }}, 2^{\text {nd }}, 3^{\text {rd }}$, and $4^{\text {th }}$ Noise Data Sets Included .................................115

Table 4-38: Calibration Neural Network, All 5 Noise Data Sets Included .............................................115

Table 4-39: Inputs and Outputs for the Net Pressure Neural Network ..........................................121

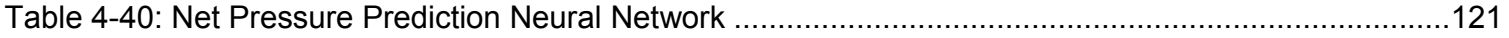

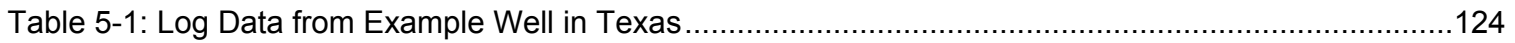

Table 5-2: Fuzzy Rules for the Fuzzy Lithology Identification System .............................................125

Table 5-3: Lithology Identification using Fuzzy Lithology System .................................................127

Table 5-4: Example of Stress Calculation using ABC Methodology ..............................................130

Table 5-5: Example of Stress Profile Processing for Four Layers ...................................................132

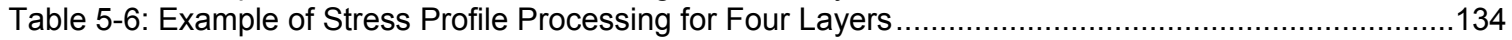

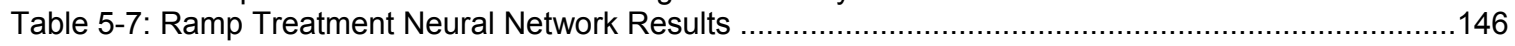

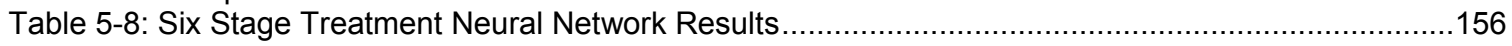

Table 5-9: Eight Stage Type I Treatment Neural Network Results.................................................164

Table 5-10: Eight Stage Type II Treatment Neural Network Results.................................................173

Table 5-11: Eight Stage Type III Treatment Neural Network Results.............................................181

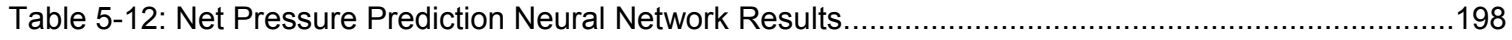

Table 5-13: Original Ramp Treatment with Optimum Suggested Treatments........................................204

Table 5-14: Original Halliburton Treatment with Eight Stage Optimum Suggested Treatments ..................205

Table 5-15:Original Halliburton Treatment with Six Stage Optimum Suggested Treatment......................206

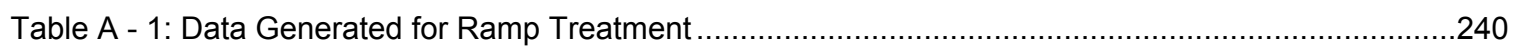




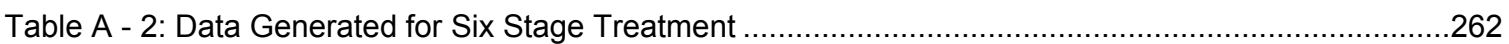

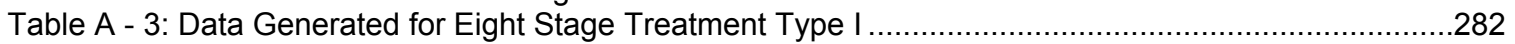

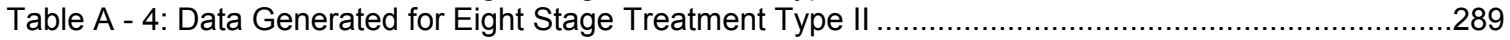

Table A - 5: Data Generated for Eight Stage Treatment Type III .....................................................296 


\section{LIST OF FIGURES}

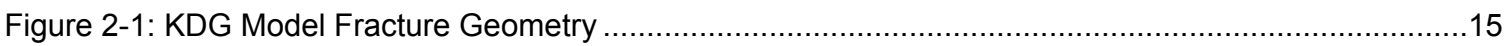

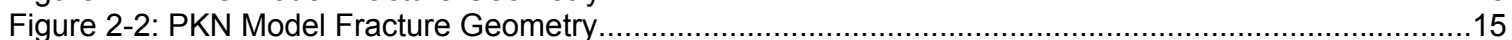

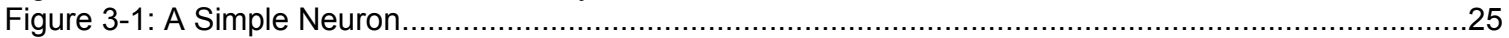

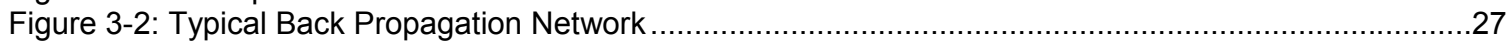

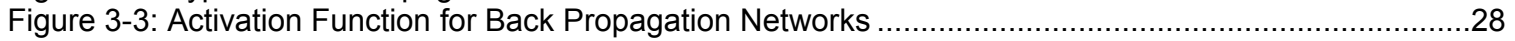

Figure 3-4: Multiple Slabs in an Artificial Neural Network ...................................................................

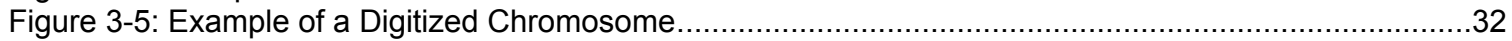

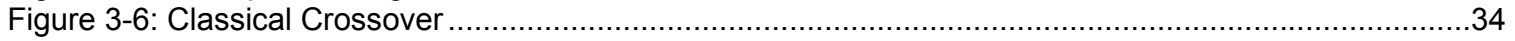

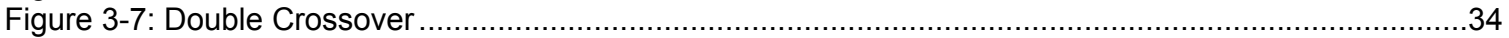

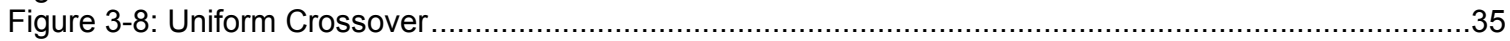

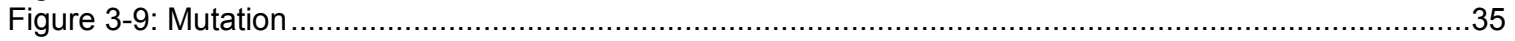

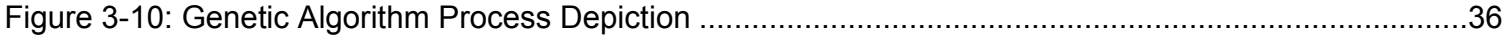

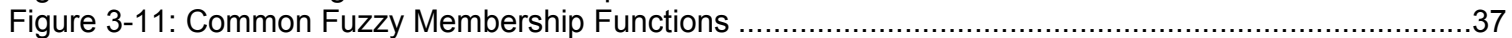

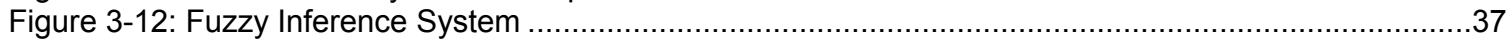

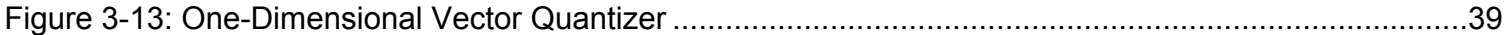

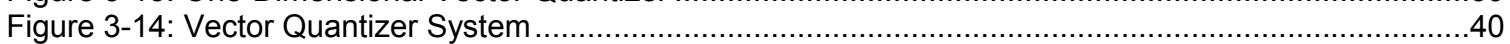

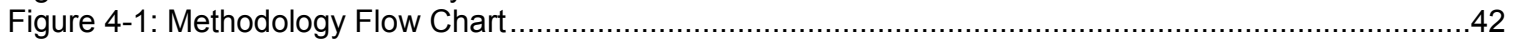

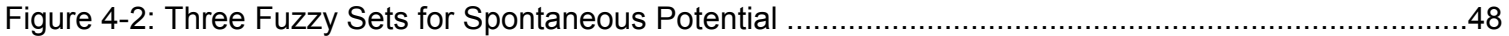

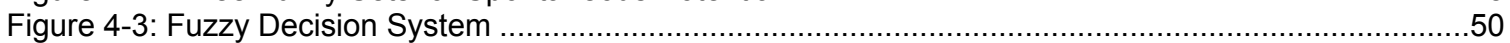

Figure 4-4: Log-Derived Stress Profile ..................................................................................52

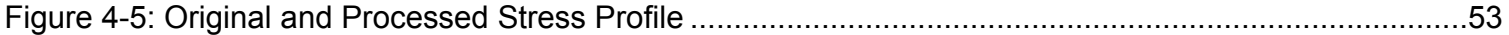

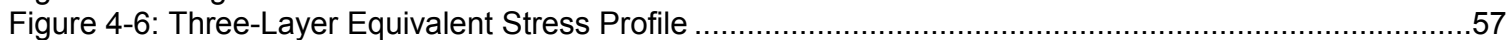

Figure 4-7: Four-Layer Equivalent Stress Profile ………................................................................62

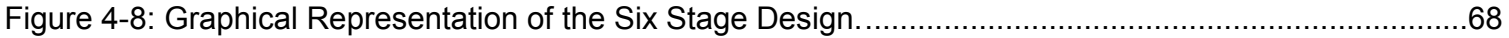

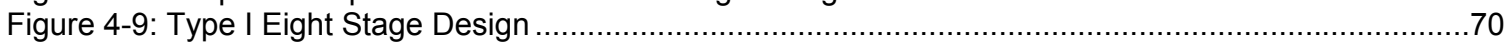

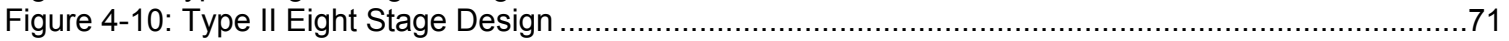

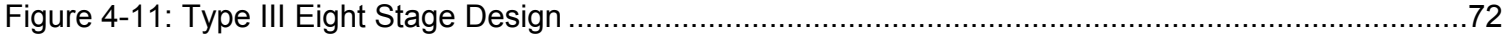

Figure 4-12: Comparison of Eight Stage Treatment Schedules ........................................................

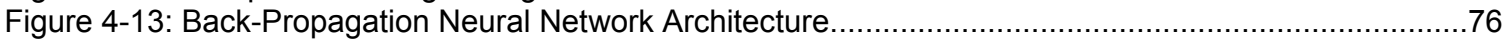

Figure 4-14: Correlation Coefficient Variation with Number of Cases .................................................... 81

Figure 4-15: Pressure Matching Example between Observed Net Pressure and Predicted Net Pressure ....89

Figure 4-16: Net Pressures for Original Lithology and Equivalent Profile from Case 1 ...............................90

Figure 4-17: Net Pressures for Original Lithology and Equivalent Profile from Case 3 ..............................90

Figure 4-18: Net Pressures for Original Lithology and Equivalent Profile from Case 8 …..........................91

Figure 4-19: Net Pressure Profile Comparisons for Equivalent Altered Profiles from Case 1 ......................94

Figure 4-20: Net Pressure Profile Comparisons for Equivalent Altered Profiles from Case 3 .......................95

Figure 4-21: Net Pressure Profile Comparisons for Pay Zone Equivalent Altered Profiles from Case 1 1........97

Figure 4-22: Net Pressure Profile Comparisons for Pay Zone Equivalent Altered Profiles from Case 3........98

Figure 4-23: Original, Equivalent, and Altered Equivalent Stress Profiles............................................101

Figure 4-24: Net Pressure Profile Matching Process for Case 10 ………..........................................102

Figure 4-25: Net Pressure Profile Matching Process for Case 23 ......................................................102

Figure 4-26: Net Pressure Profile Matching Process for Case 37 …..................................................103

Figure 4-27: Net Pressure Profile Matching Process for Case 50 .......................................................103

Figure 4-28: Frequency Distribution Analysis for Percentage Coefficient ...........................................105

Figure 4-29: Regression Plot of Pay Zone Depth vs. Correction Percentage Coefficient ...........................105

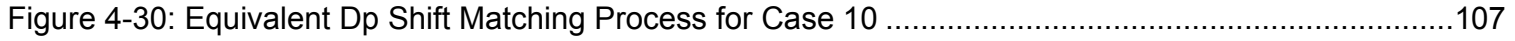

Figure 4-31: Equivalent Dp Shift Matching Process for Case 23 .......................................................107

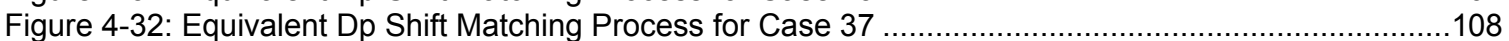

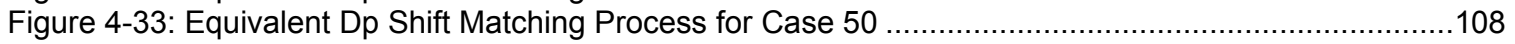

Figure 4-34: Calibration Neural Network Training Set Accuracy .........................................................115

Figure 4-35: Calibration Neural Network Calibration Set Accuracy ....................................................116

Figure 4-36: Calibration Neural Network Verification Set Accuracy ……...........................................116

Figure 4-37: Net Pressure Profiles with Various Duration Times ....................................................117

Figure 4-38: Net Pressure Profiles for Original Signal and Compressed Signal Example 2 ..........................119

Figure 4-39: Net Pressure Profiles for Original Signal and Compressed Signal Example 2 …..................120

Figure 5-1: Fuzzy Sets for Deep Induction Log ............................................................................125

Figure 5-2: Fired Rules in the Process of Lithology Identification ......................................................125

Figure 5-3: Outcome of the Fuzzy Lithology System ……..............................................................126 
Figure 5-4: Spontaneous Potential, Gamma Ray, Deep Induction, and Lithology .................................128

Figure 5-5: Processing Methodology for Original Stress Profile ....................................................... 131

Figure 5-6: Original Stress Profile and Processed Stress Profile ..................................................133

Figure 5-7: Processed Stress Profile and Equivalent Four-Layer Stress Profile ....................................135

Figure 5-8: Ramp Treatment Training Set Results for Fracture Efficiency .......................................137

Figure 5-9: Ramp Treatment Calibration Set Results for Fracture Efficiency......................................137

Figure 5-10: Ramp Treatment Verification Set Results for Fracture Efficiency .....................................138

Figure 5-11: Ramp Treatment Training Set Results for Propped Fracture Length .................................138

Figure 5-12: Ramp Treatment Calibration Set Results for Propped Fracture Length...............................139

Figure 5-13: Ramp Treatment Verification Set Results for Propped Fracture Length ............................139

Figure 5-14: Ramp Treatment Training Set Results for Proppant Concentration ....................................140

Figure 5-15: Ramp Treatment Calibration Set Results for Proppant Concentration................................140

Figure 5-16: Ramp Treatment Verification Set Results for Proppant Concentration ................................141

Figure 5-17: Ramp Treatment Training Set Results for Dimensionless Conductivity Ratio .......................141

Figure 5-18: Ramp Treatment Calibration Set Results for Dimensionless Conductivity Ratio ....................142

Figure 5-19: Ramp Treatment Verification Set Results for Dimensionless Conductivity Ratio.......................142

Figure 5-20: Ramp Treatment Training Set Results for Maximum Fracture Width..................................143

Figure 5-21: Ramp Treatment Calibration Set Results for Maximum Fracture Width ..............................143

Figure 5-22: Ramp Treatment Verification Set Results for Maximum Fracture Width................................144

Figure 5-23: Ramp Treatment Training Set Results for Fracture Height.............................................144

Figure 5-24: Ramp Treatment Calibration Set Results for Fracture Height.......................................145

Figure 5-25: Ramp Treatment Verification Set Results for Fracture Height ........................................ 145

Figure 5-26: Six Stage Treatment Training Set Results for Fracture Efficiency ...................................147

Figure 5-27: Six Stage Treatment Calibration Set Results for Fracture Efficiency ................................147

Figure 5-28: Six Stage Treatment Verification Set Results for Fracture Efficiency .................................148

Figure 5-29: Six Stage Treatment Training Set Results for Propped Fracture Length .............................148

Figure 5-30: Six Stage Treatment Calibration Set Results for Propped Fracture Length ..........................149

Figure 5-31: Six Stage Treatment Verification Set Results for Propped Fracture Length .........................149

Figure 5-32: Six Stage Treatment Training Set Results for Proppant Concentration ................................150

Figure 5-33: Six Stage Treatment Calibration Set Results for Proppant Concentration ............................150

Figure 5-34: Six Stage Treatment Verification Set Results for Proppant Concentration ............................151

Figure 5-35: Six Stage Treatment Training Set Results for Dimensionless Conductivity Ratio...................151

Figure 5-36: Six Stage Treatment Calibration Set Results for Dimensionless Conductivity Ratio...............152

Figure 5-37: Six Stage Treatment Verification Set Results for Dimensionless Conductivity Ratio ...............152

Figure 5-38: Six Stage Treatment Training Set Results for Maximum Fracture Width ..............................153

Figure 5-39: Six Stage Treatment Calibration Set Results for Maximum Fracture Width..........................153

Figure 5-40: Six Stage Treatment Verification Set Results for Maximum Fracture Width ..........................154

Figure 5-41: Six Stage Treatment Training Set Results for Fracture Height .......................................154

Figure 5-42: Six Stage Treatment Calibration Set Results for Fracture Height .....................................155

Figure 5-43: Six Stage Treatment Verification Set Results for Fracture Height ....................................155

Figure 5-44: Eight Stage Type I Treatment Training Set Results for Fracture Efficiency ..........................157

Figure 5-45: Eight Stage Type I Treatment Calibration Set Results for Fracture Efficiency......................157

Figure 5-46: Eight Stage Type I Treatment Verification Set Results for Fracture Efficiency ......................158

Figure 5-47: Eight Stage Type I Treatment Training Set Results for Propped Fracture Length ..................158

Figure 5-48: Eight Stage Type I Treatment Calibration Set Results for Propped Fracture Length ..............159

Figure 5-49: Eight Stage Type I Treatment Verification Set Results for Propped Fracture Length ..............159

Figure 5-50: Eight Stage Type I Treatment Training Set Results for Proppant Concentration .....................160

Figure 5-51: Eight Stage Type I Treatment Calibration Set Results for Proppant Concentration.................160

Figure 5-52: Eight Stage Type I Treatment Verification Set Results for Proppant Concentration ...............161

Figure 5-53: Eight Stage Type I Treatment Training Set Results for Maximum Fracture Width...................161

Figure 5-54: Eight Stage Type I Treatment Calibration Set Results for Maximum Fracture Width...............162

Figure 5-55: Eight Stage Type I Treatment Verification Set Results for Maximum Fracture Width .............162

Figure 5-56: Eight Stage Type I Treatment Training Set Results for Fracture Height ..............................163

Figure 5-57: Eight Stage Type I Treatment Calibration Set Results for Fracture Height ...........................163

Figure 5-58: Eight Stage Type I Treatment Verification Set Results for Fracture Height ........................164

Figure 5-59: Eight Stage Type II Treatment Training Set Results for Fracture Efficiency .........................165

Figure 5-60: Eight Stage Type II Treatment Calibration Set Results for Fracture Efficiency.......................166

Figure 5-61: Eight Stage Type II Treatment Verification Set Results for Fracture Efficiency ......................166

Figure 5-62: Eight Stage Type II Treatment Training Set Results for Propped Fracture Length .................167

Figure 5-63: Eight Stage Type II Treatment Calibration Set Results for Propped Fracture Length .............167

Figure 5-64: Eight Stage Type II Treatment Verification Set Results for Propped Fracture Length .............168

Figure 5-65: Eight Stage Type II Treatment Training Set Results for Proppant Concentration ...................168 
Figure 5-66: Eight Stage Type II Treatment Calibration Set Results for Proppant Concentration...... 169

Figure 5-67: Eight Stage Type II Treatment Verification Set Results for Proppant Concentration ..............169

Figure 5-68: Eight Stage Type II Treatment Training Set Results for Maximum Fracture Width................170

Figure 5-69: Eight Stage Type II Treatment Calibration Set Results for Maximum Fracture Width..............170

Figure 5-70: Eight Stage Type II Treatment Verification Set Results for Maximum Fracture Width .............171

Figure 5-71: Eight Stage Type II Treatment Training Set Results for Fracture Height .............................171

Figure 5-72: Eight Stage Type II Treatment Calibration Set Results for Fracture Height.........................172

Figure 5-73: Eight Stage Type II Treatment Verification Set Results for Fracture Height .........................172

Figure 5-74: Eight Stage Type III Treatment Training Set Results for Fracture Efficiency ......................174

Figure 5-75: Eight Stage Type III Treatment Calibration Set Results for Fracture Efficiency ....................174

Figure 5-76: Eight Stage Type III Treatment Verification Set Results for Fracture Efficiency ....................175

Figure 5-77: Eight Stage Type III Treatment Training Set Results for Propped Fracture Length ...............175

Figure 5-78: Eight Stage Type III Treatment Calibration Set Results for Propped Fracture Length............176

Figure 5-79: Eight Stage Type III Treatment Verification Set Results for Propped Fracture Length ............176

Figure 5-80: Eight Stage Type III Treatment Training Set Results for Proppant Concentration ..................177

Figure 5-81: Eight Stage Type III Treatment Calibration Set Results for Proppant Concentration................177

Figure 5-82: Eight Stage Type III Treatment Verification Set Results for Proppant Concentration ..............178

Figure 5-83: Eight Stage Type III Treatment Training Set Results for Maximum Fracture Width................178

Figure 5-84: Eight Stage Type III Treatment Calibration Set Results for Maximum Fracture Width..............179

Figure 5-85: Eight Stage Type III Treatment Verification Set Results for Maximum Fracture Width ............179

Figure 5-86: Eight Stage Type III Treatment Training Set Results for Fracture Height ............................180

Figure 5-87: Eight Stage Type III Treatment Calibration Set Results for Fracture Height ..............................180

Figure 5-88: Eight Stage Type III Treatment Verification Set Results for Fracture Height .........................181

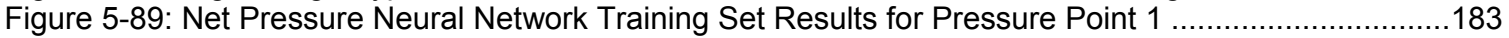

Figure 5-90: Net Pressure Neural Network Calibration Set Results for Pressure Point 1 ........................183

Figure 5-91: Net Pressure Neural Network Verification Set Results for Pressure Point 1........................184

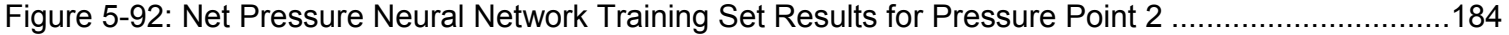

Figure 5-93: Net Pressure Neural Network Calibration Set Results for Pressure Point 2 ........................185

Figure 5-94: Net Pressure Neural Network Verification Set Results for Pressure Point 2 ........................185

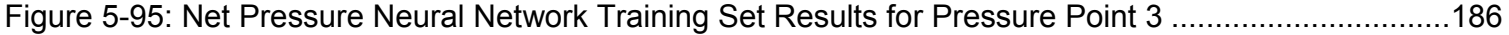

Figure 5-96: Net Pressure Neural Network Calibration Set Results for Pressure Point 3 .........................186

Figure 5-97: Net Pressure Neural Network Verification Set Results for Pressure Point 3.......................187

Figure 5-98: Net Pressure Neural Network Training Set Results for Pressure Point $4 \ldots \ldots \ldots \ldots \ldots \ldots \ldots \ldots \ldots . . \ldots 187$

Figure 5-99: Net Pressure Neural Network Calibration Set Results for Pressure Point 4 ........................188

Figure 5-100: Net Pressure Neural Network Verification Set Results for Pressure Point $4 \ldots \ldots \ldots \ldots \ldots \ldots \ldots \ldots \ldots . . .188$

Figure 5-101: Net Pressure Neural Network Training Set Results for Pressure Point 5 .........................189

Figure 5-102: Net Pressure Neural Network Calibration Set Results for Pressure Point 5 ........................189

Figure 5-103: Net Pressure Neural Network Verification Set Results for Pressure Point 5.......................190

Figure 5-104: Net Pressure Neural Network Training Set Results for Pressure Point 6 ..........................190

Figure 5-105: Net Pressure Neural Network Calibration Set Results for Pressure Point 6 ........................191

Figure 5-106: Net Pressure Neural Network Verification Set Results for Pressure Point $6 \ldots \ldots \ldots \ldots \ldots \ldots \ldots \ldots \ldots . . .191$

Figure 5-107: Net Pressure Neural Network Training Set Results for Pressure Point 7 ..........................192

Figure 5-108: Net Pressure Neural Network Calibration Set Results for Pressure Point 7 .......................192

Figure 5-109: Net Pressure Neural Network Verification Set Results for Pressure Point $7 \ldots \ldots \ldots \ldots \ldots \ldots \ldots \ldots \ldots . .193$

Figure 5-110: Net Pressure Neural Network Training Set Results for Pressure Point 8 .........................193

Figure 5-111: Net Pressure Neural Network Calibration Set Results for Pressure Point 8 .......................194

Figure 5-112: Net Pressure Neural Network Verification Set Results for Pressure Point 8.......................194

Figure 5-113: Net Pressure Neural Network Training Set Results for Pressure Point 9 ..........................195

Figure 5-114: Net Pressure Neural Network Calibration Set Results for Pressure Point 9 .......................195

Figure 5-115: Net Pressure Neural Network Verification Set Results for Pressure Point 9.......................196

Figure 5-116: Net Pressure Neural Network Training Set Results for Pressure Point 10 .........................196

Figure 5-117: Net Pressure Neural Network Calibration Set Results for Pressure Point $10 \ldots \ldots \ldots \ldots \ldots \ldots \ldots . . . \ldots 197$

Figure 5-118: Net Pressure Neural Network Verification Set Results for Pressure Point 10.....................197

Figure 5-119: Case 1 FRACPRO and Neural Network Pressure Profiles .............................................199

Figure 5-120: Case 2 FRACPRO and Neural Network Pressure Profiles ............................................200

Figure 5-121: Case 3 FRACPRO and Neural Network Pressure Profiles ............................................200

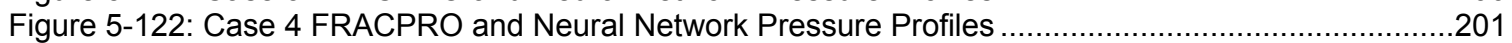

Figure 5-123: Case 5 FRACPRO and Neural Network Pressure Profiles .............................................201

Figure 5-124: Case 6 FRACPRO and Neural Network Pressure Profiles ............................................202

Figure 5-125: Case 7 FRACPRO and Neural Network Pressure Profiles ............................................202

Figure 5-126: Case 8 FRACPRO and Neural Network Pressure Profiles .............................................203

Figure 5-127: FRACPRO and System Generated Pressure Profile for Ramp Case 1 ............................207 
Figure 5-128: FRACPRO and System Generated Pressure Profile for Ramp Case 2 .............................208

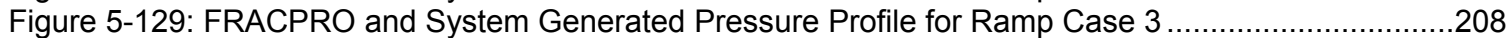

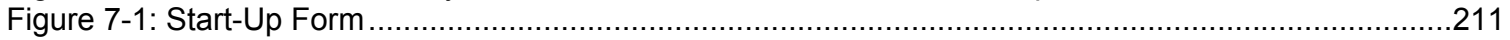

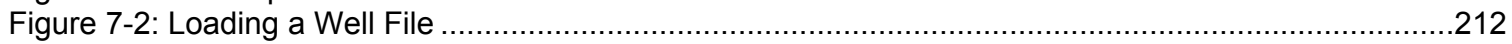

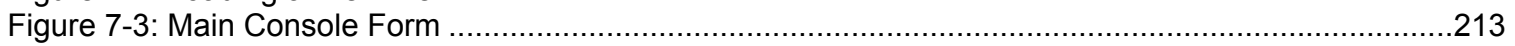

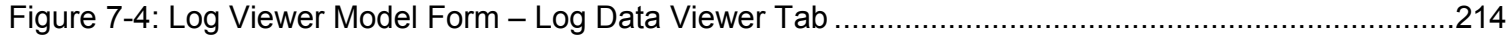

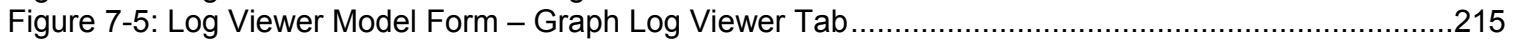

Figure 7-6: Lithology Model Design Form - Input/Output Definition Tab ..........................................216

Figure 7-7: Lithology Model Design Form - Fuzzy Sets Interval Definition Tab...............................217

Figure 7-8: Lithology Model Main Form - Fuzzy Rules Table Tab ................................................218

Figure 7-9: Lithology Model Main Form - Decision Frame Tab ...................................................219

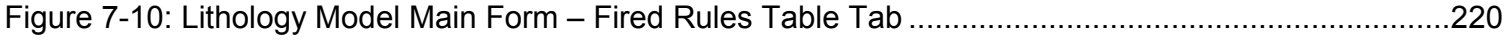

Figure 7-11: Lithology Model Main Form - Tabled Results Tab................................................221

Figure 7-12: Stress Calculation Module Form - ABC Default Method Tab ......................................222

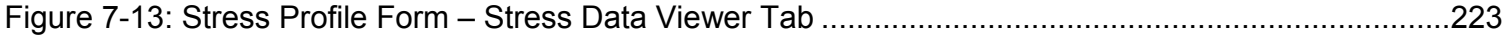

Figure 7-14: Stress Profile Form - Stress Data Viewer Tab ..........................................................224

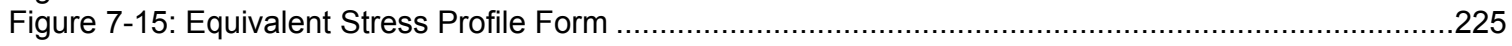

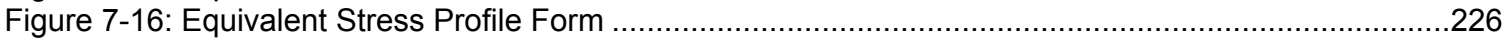

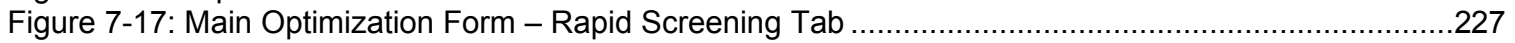

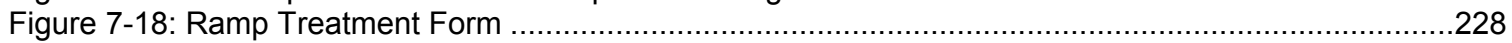

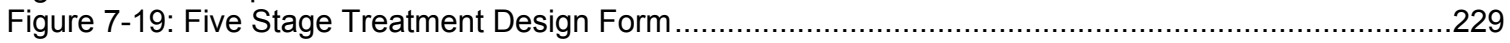

Figure 7-20: Multiple Screens Comparison - Ramp Treatment \& Five Stage Treatment ........................230

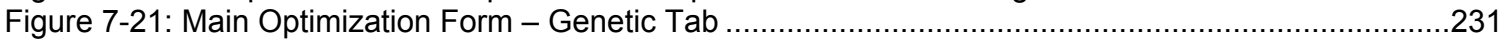

Figure 7-22: Main Optimization Form - Ramp Treatment Display Tab ............................................232 


\section{CHAPTER 1: INTRODUCTION}

\subsection{Problem Statement}

Hydraulic fracturing is a widely used technology to economically increase the productivity of oil and gas reservoirs. Over the last two decades, this technology has been heavily studied, and many sophisticated models have been created in an attempt to simulate the propagation and geometry of induced fractures. However, there are still shortcomings in that the actual results of the job rarely reflect the designed intentions. This is due to the fact that there are many external influences that can affect the results, but are unknown, not incorporated into the model, or not accurately modeled. Some of these influences include (but are not limited to) localized stresses around the wellbore, tortuosity of the reservoir, and the existence of natural minifracs that can alter the direction of propagation.

The current study presents an alternative to classical methods used in fracture design simulators. This work is intended to develop a fracture design simulator that helps users to design hydraulic frac jobs without being an expert in the art and science of hydraulic fracturing. This process is composed of an integration of several artificial intelligence techniques. The advantage of artificial intelligence is that it does not require a mathematical modeling of the problem.

The first step consists of a fuzzy logic decision system that determines the lithology that would assist in the determination of the stress profile. The results of this step are used in the second step, which involves a search and optimization using an integrated system of neural networks and genetic algorithms. The output of this system is the optimum fracturing treatment design. The final step uses vector quantization and neural networks to predict the net treating pressure.

The study verifies the capabilities of this approach in tight gas reservoirs (natural gas reservoirs with an average permeability of less than $0.1 \mathrm{mD}$ ). The outputs of this system (the fracturing treatment design and the net treating pressure) are verified against a commonly used 3-D fracture simulator, FRACPRO. The final outcome of this work is an integrated program able to design optimum fracturing treatment schedules in a matter of seconds once the stress profile has been completed. 


\subsection{Motivation for the Study}

Over the years, many hydraulic fracture simulators have been developed using analytical and empirical equations to solve fracture modeling. However, no attempt has been made thus far to build an intelligent fracture simulator that uses only knowledge and intelligent techniques to design and implement optimum fracture treatments. This study intends to develop a tool, which learns from data, and designs optimum fracture treatments based on historical practices and results.

Due to the unavailability of actual fracturing data (geometry and net pressures), the data used in this study has been generated using a commercial fracture simulator. The intent is that this study should be repeated using the actual results from hydraulic fractures performed in a specific field or basin, which may or may not reflect the analytical fracture simulator.

The goal of the system is to learn from real fracturing data and optimize future treatments based on best practices. Accordingly, this intelligent tool will not only be able to design the optimum frac treatment, but will also be capable of reproducing the net pressure signature for that specific formation or reservoir. If successful, this methodology can be implemented in any field where significant hydraulic fracturing is performed.

The originality of the work is the development of a methodology that will design and monitor hydraulic fracture treatments based on historical results from previous jobs for a specific reservoir, rather than generalized analytical approximations.

\subsection{Hydraulic Fracturing Background}

Well stimulation methods have become very popular in the petroleum industry for maintaining or increasing well productivity. All stimulation practices adjust the skin, which is not due exclusively to damage, but is a multi-component variable for which stimulation may not affect all of its components. Therefore, identification of the individual components of the skin is important in choosing and designing the proper type of stimulation treatment. There are two main types of well stimulation methods employed presently: matrix acidizing and hydraulic fracturing. This study focuses on the practices of hydraulic fracturing.

Hydraulic fracturing is the process of pumping fluid into a wellbore at an injection rate that is high enough to cause the formation to break down. As the resistance to flow in the formation increases, the pressure in the wellbore increases until the breakdown pressure of the formation is exceeded. Once this occurs, a fracture is formed, and the injected fluid begins to move down the fracture. In most formations, a single, vertical fracture is created that propagates in two directions from the wellbore, usually 180 degrees apart and assumed to be identical in shape and size. In naturally fractured reservoirs, it is possible that multiple fractures may occur and each may propagate during a hydraulic fracture treatment. 


\subsubsection{History of Hydraulic Fracturing}

According to a report by the Department of Energy, the first hydraulic fracturing treatment was pumped in 1947 on a Pan American Petroleum Corporation gas well in the Hugoton field ${ }^{1}$. Located in Grant County, Kansas, Kelpper Well No. 1 was a low productivity well, even though it had been acidized. Kelpper Well No. 1 was selected for the first hydraulic fracture stimulation in order to directly compare the results to those of acidizing treatment ${ }^{1}$.

Since that first treatment in 1947, hydraulic fracturing has become a standard treatment for stimulating the productivity of oil and gas wells.

\subsubsection{Objective of Hydraulic Fracturing}

Generally, hydraulic fracture treatments are used to increase the productivity or injectivity index of a producing or injection well, respectively. The productivity index is the volume of oil or gas that can be produced at a given pressure differential between the reservoir and the wellbore, while the injectivity index refers to how much fluid can be injected into the well at a given pressure differential.

There are many applications and uses of hydraulic fracturing; however, the majority of treatments are pumped for the following most common reasons:

- Increase the flow rate of oil and/or gas from low permeability reservoirs.

- Increase the area of drainage or the amount of formation in contact with the wellbore.

- Increase the flow rate of oil and/or gas from wells that have been damaged.

- Connect the natural fractures in a formation to the wellbore.

This study is mainly directed to low permeability reservoirs, which are typically excellent candidates for stimulation by hydraulic fracturing. A low permeability reservoir is one that has a high resistance to fluid flow. In many formations, chemical and/or physical processes alter a reservoir rock over geologic time. Sometimes, these diagenetic processes restrict the openings in the rock and reduce the ability of fluids to flow through the rock.

\subsubsection{Data Requirements for Hydraulic Fracturing}

The data required to run a fracture design simulator can be divided into two groups. The first group includes the data that can be controlled by the engineer such as well completion details, treatment volume, pad volume, injection rate, fracture fluid viscosity, fracture fluid density, fluid loss additives, propping agent type, and propping agent volume. The second group reflects data that must be measured or estimated, but cannot be controlled such as formation depth, formation permeability, in-situ stresses in the pay zone, in-situ stresses in the surrounding layers, 
formation modulus, reservoir pressure, formation porosity, formation compressibility, and the thickness of the reservoir. The most critical data for the design of a fracture treatment are, roughly in order of importance:

- In-situ stress profile

- Formation permeability

- Fluid loss characteristics

- Total fluid volume pumped

- Propping agent type and amount

- Pad volume

- Fracture fluid viscosity

- Injection rate

- Formation modulus

Given a limited amount of time, attention should be focused on the most important parameters. In hydraulic fracture treatment design, the two most important parameters, by far, are the in-situ stress profile and the permeability profile of the zone to be stimulated along with that above and below the target zones.

Typically in new fields, most operating companies are normally willing to spend money to run logs, cut cores and run well tests to determine important factors such as the in-situ stress and the permeability of the major reservoir layers.

By using such data, along with fracture treatment records and production records, accurate data sets can be compiled. These data sets can be used on subsequent wells to optimize the fracture treatment designs. It is normally not practical to cut cores and run well tests on every well. Thus, the data obtained from cores and well tests must be correlated to log parameters so the logs on subsequent wells can be used to compile accurate data sets.

Data necessary for fracture treatment design can be obtained from a number of sources, including drilling records, completion records, well files, open hole geophysical logs, cores and core analyses, well tests, production data, geologic records, and other public records, such as publications. In addition, service companies provide data on their fluids, additives, and propping agents. Table 1-1 illustrates typical data needed to design a fracture treatment and possible sources for the data. 


\begin{tabular}{|l|c|l|}
\hline \multicolumn{1}{|c|}{ Data } & Units & \multicolumn{1}{c|}{ Sources } \\
\hline Formation Permeability & $\mathrm{md}$ & $\begin{array}{l}\text { Cores, Well Tests, } \\
\text { Correlations, } \\
\text { Production Data }\end{array}$ \\
\hline Formation Porosity & $\%$ & Cores, Logs \\
\hline Reservoir Pressure & $\mathrm{psi}$ & $\begin{array}{l}\text { Well Tests, Well Files, } \\
\text { Regional Data }\end{array}$ \\
\hline Formation Modulus & $\mathrm{psi}$ & $\begin{array}{l}\text { Cores, Logs, } \\
\text { Correlations }\end{array}$ \\
\hline $\begin{array}{l}\text { Formation } \\
\text { Compressibility }\end{array}$ & $\mathrm{psi}$ & $\begin{array}{l}\text { Cores, Logs, } \\
\text { Correlations }\end{array}$ \\
\hline Poisson's Ratio & & $\begin{array}{l}\text { Cores, Logs, } \\
\text { Correlations }\end{array}$ \\
\hline Formation Depth & $\mathrm{ft}$ & Logs, Drilling Records \\
\hline In-situ Stress & $\mathrm{psi}$ & $\begin{array}{l}\text { Well Tests, Logs, } \\
\text { Correlations }\end{array}$ \\
\hline Formation Temperature & ${ }^{\circ} \mathrm{F}$ & $\begin{array}{l}\text { Logs, Well Tests, } \\
\text { Correlations }\end{array}$ \\
\hline Fracture Toughness & $\mathrm{psi}-\sqrt{\text { in }}$ & Cores, Correlations \\
\hline Water Saturation & $\%$ & Logs, Cores \\
\hline Net Pay Thickness & Ft & Logs, Cores \\
\hline Gross Pay Thickness & Ft & $\begin{array}{l}\text { Logs, Cores, Drilling } \\
\text { Records }\end{array}$ \\
\hline Formation Lithology & & $\begin{array}{l}\text { Cores, Drilling } \\
\text { Records, Logs, } \\
\text { Geologic Records }\end{array}$ \\
\hline Wellbore Completion & & $\begin{array}{l}\text { Well Files, Completion } \\
\text { Prognosis }\end{array}$ \\
\hline Fracture Fluids & $\begin{array}{l}\text { Service Company } \\
\text { Information }\end{array}$ \\
\hline Fracture Proppants & $\begin{array}{l}\text { Service Company } \\
\text { Information }\end{array}$ \\
\hline
\end{tabular}

Table 1-1: Necessary Data for Fracture Design

\subsection{Research Tools - Intelligent Techniques}

Three main artificial intelligence techniques are employed in the methodology of the Artificial Intelligence Hydraulic Fracturing Simulator: neural networks, genetic algorithms, and fuzzy logic.

Neural Networks are extremely powerful systems for pattern recognition and nonlinear multidimensional interpolation. They use distributive parallel processing to capture existing relationships between input parameters and the output of the system ${ }^{2-4}$. To achieve accurate predictions, neural networks must be trained and exposed to adequate and representative data.

Evolutionary computation it has its root in the biological evolution of the nature. Evolutionary computation is nothing other than searching or optimization algorithms inspired by the biological evolution of nature ${ }^{5}$. Evolutionary computation search and optimization is conducted: (1) based on multiple searching points or solutions candidates (population based search), (2) using operations inspired by biological evolution, such as crossover and mutation, (3) using little information of the searching space $^{6}$. Genetic algorithms are a paradigm of 
evolutionary computation. Genetic algorithms usually represent solutions for chromosomes with bit coding (genotype) and search for the better solution candidates using operations like selection, crossover and mutation.

Fuzzy logic theory provides a means for representing uncertainty ${ }^{7}$. Uncertainty is usually either due to the random nature of events or due to imprecision and ambiguity of information we have about the problem we are trying to solve. Fuzzy logic can use natural language to process information, and it provides efficient use of imprecise information. Fuzzy logic achieves this important task through fuzzy sets. In crisp sets, an object either belongs to a set or it does not. Fuzzy sets are used to determine the degree to which an object belongs to a set (an object may belong to multiple sets in varying degrees). Fuzzy set theory is a capable tool for modeling uncertainty associated with vagueness, imprecision, and/or a lack of information regarding a particular element of the problem at hand ${ }^{7,8}$. 


\section{CHAPTER 2: LITERATURE REVIEW}

\subsection{Pre-Fracturing Formation Evaluation}

Formation parameters used in designing a fracturing treatment can be obtained from wireline logging through direct or indirect measurements. These parameters include stress, shale volume, pressure, and permeability. Usually, the necessary data can be obtained from tools run in open hole on new wells. However, some of the data cannot be acquired in pre-existing cased wells.

\subsubsection{Log Analysis Techniques}

The wireline measurements used in fracturing treatment design are obtained primarily from sonic, gamma ray, density, and formation tester devices ${ }^{9}$. Sonic and density tools provide the measurements needed to calculate formation stresses, while gamma tools are the main instruments for determining the shale volume. Formation testers provide information about the pressure and permeability of the selected formations. However, these last two parameters can be also obtained through other procedures.

The records of sonic, gamma, and density tools are made continuously as the tools move through the wellbore. Typically the measurements are recorded as a function of depth, every 0.5 $\mathrm{ft}$ or $0.1 \mathrm{~m}$. In logging terms, because of this dense sampling of formation properties, these measurements are often considered as being continuous. Continuous data such as this, used for designing fracturing jobs, provides an improved vertical resolution of stress variations.

Rock mechanical properties such as Poisson's ration, Young's modulus, shear modulus, and bulk compressibility are calculated from wireline measurements as an intermediate step in determining formation stress. When rock properties are derived from acoustic measurements, they are referred to as dynamic measurements.

\subsubsection{Sonic Measurements}

Acoustic energy propagates through matter as waves, the most commonly known being compressional and shear waves. By definition, acoustic slowness is the time required for an acoustic wave to travel a specific distance through a material, usually $1 \mathrm{ft}$ or $1 \mathrm{~m}^{9,10}$. Slowness is usually expressed in microseconds per foot or meter $(\mu \mathrm{s} / \mathrm{m})$. The wireline sonic tools use acoustic sources and sensors to measure the formation compressional and shear slowness, $\Delta \mathrm{t}_{\mathrm{c}}$ and $\Delta \mathrm{t}_{\mathrm{s}}$, respectively. These are the two acoustic parameters needed in determining formation stresses. The acoustic velocity is the inverse of the acoustic slowness and is usually expressed in foot or meter per microseconds $(\mathrm{m} / \mu \mathrm{s})$. 
Two types of sonic tools can be used to measure $\Delta \mathrm{t}_{\mathrm{c}}$ and $\Delta \mathrm{t}_{\mathrm{s}}$, depending on whether the formation is slow ${ }^{9}$. A formation is slow when $\Delta t_{s}>\Delta t_{f}$, where $\Delta t_{f}$ is borehole fluid slowness. The normal value of $\Delta \mathrm{t}_{\mathrm{f}}$ for water is $189 \mu \mathrm{s} / \mathrm{ft}$. Full waveform sonic tools use monopole acoustic transmitters and receivers to generate and sense compressional and shear waves that travel along the borehole wall. The tools are thus able to measure $\Delta \mathrm{t}_{\mathrm{c}}$ and $\Delta \mathrm{t}_{\mathrm{s}}$. When the formation is slow, monopole tools cannot give rise to shear waves along the borehole wall and thus cannot measure $\Delta t_{s}$. For this reason, in slow formations, a dipole sonic tool is run. A dipole acoustic transmitter generates flexural waves that travel along the borehole wall and are sensed by dipoles receivers to measure flexural slowness as shear waves. Dipole tools also contain monopole acoustic transmitters and receivers to enable compressional slowness to be measured.

\subsubsection{Density Measurements}

Formation density (also known as formation bulk density, $\rho_{b}$ ) is the combined mass per unit volume of all materials in the formation whether solid, liquid, or gas. Wireline density tools contain a chemical source of gamma rays and two gamma ray detectors ${ }^{9-13}$. The measuring process uses the emission of gamma rays by the source into the formation. Some of the emitted gamma rays are scattered back to the tool and sensed by the detectors. The density measurement is based on the assumption that as formation density increases, the number of gamma rays scattered back to the tool decreases. The measured bulk density is used to determine the overburden pressure. Bulk density and overburden pressure are used with $\Delta \mathrm{t}_{\mathrm{c}}$ and $\Delta \mathrm{t}_{\mathrm{s}}$ in computing formation stresses.

Either traditional or spectral density tools can be used in open holes to measure $\rho_{\mathrm{b}}$. Traditional density tools measure gamma radiation returning to the tool in a single broad energy range. By contrast, spectral density tools measure the amounts of gamma radiation returning to the tool in several specific energy ranges ${ }^{9}$. The spectral tools can provide a more accurate $\rho_{b}$ measurement and also furnish a measurement of formation photoelectric factor used in identifying formation lithology.

\subsubsection{Gamma Ray Measurements}

The shale volume of the formation is determined through the use of gamma ray measurements. Wireline gamma ray tools contain a gamma ray detector but no gamma ray source ${ }^{9,12,13}$. They measure the amount of gamma radiation present in the subsurface environment. The amount of such radiations emanating from a geologic formation is usually a good indictor of the shale volume. Shale volume is used in determining the formation's sanding potential. 
Gamma ray tools are often run in combination with sonic and density tools, when either a conventional or spectral gamma ray tool can be used. Conventional tools measure gamma radiation in a single broad energy range while spectral tools measure the amount of gamma radiation in a larger number of energy bands. With spectral data, shale volume can be calculated more accurately, and clay types can be identified ${ }^{9}$.

Others tools are sometimes used to determine shale volume. These include spontaneous potential, neuron, neutron-density combination, and resistivity devices.

\subsubsection{Closure Stress Calculations}

In-situ stress has been widely accepted as the most important controlling factor for the vertical growth of hydraulic fractures ${ }^{14-16}$. The ability to determine stress profiles and therefore predict the vertical growth of hydraulic fractures is an essential part of a successful stimulation treatment in tight gas reservoirs.

\subsubsection{Use of Hook's Law in Calculating the Log-Derived Stress}

There are published techniques for computing the in-situ stress profile from log measurements and from core analysis ${ }^{10-13,17}$. Several different methodologies for stress calculations follow.

- Hook's Law ${ }^{10-13}$ modified for stress calculation using log data is given by:

$$
\mathrm{e}_{\mathrm{x}}=\frac{1}{\mathrm{E}}(\mathrm{Sx}-\mathrm{Sp})-\frac{v}{\mathrm{E}}(\mathrm{Sy}-\mathrm{Sp})-\frac{v}{\mathrm{E}}(\mathrm{Sx}-\mathrm{Sp}) \quad \text { Equation 2-1 }
$$

where, $e_{\mathrm{x}}=$ elastic strain in the $\mathrm{x}$ direction

$$
\begin{aligned}
& E=\text { Young's modulus } \\
& S x, S y, S z=\text { stress in direction } x, y, z \\
& S p=\text { stress produced by internal pore pressure } \\
& v=\text { Poisson's ratio }
\end{aligned}
$$

When $e_{x}$ is assumed to be zero, $S x=S y=S z$ (closure stress), and $S z=S v$ (overburden stress), the above equation reduces to the classical equation:

$$
\mathrm{Sh}_{\mathrm{h}}=\frac{v}{1-v}\left(\mathrm{~S}_{\mathrm{v}}-\mathrm{S}_{\mathrm{p}}\right)+\mathrm{S}_{\mathrm{p}} \quad \text { Equation 2-2 }
$$

However, different studies have contributed to the equation in an attempt to match the log derived stress profile with the in-situ stress calculations.

- In 1986, Whitehead at $\mathrm{al}^{11}$, modified the classical equation and proposed a new correlation given by: 


$$
\mathrm{Sh}_{\mathrm{h}}=\frac{v}{1-v}\left(1-\mathrm{P}_{\mathrm{p}}^{\prime}\right)+\mathrm{P}_{\mathrm{p}}^{\prime}
$$

where, $\mathrm{P}_{\mathrm{p}}^{\prime}=$ equivalent formation pore pressure

$$
\begin{aligned}
& P_{f}=1.15\left(2.8 P_{f}^{2}+1\right)^{1 / 2}-1 \\
& P p \text { - formation pore pressure }
\end{aligned}
$$

In this equation it can be noticed that the Sv (overburden stress gradient) is equal to 1.

- In 1991, S.A. Holditch and Associates ${ }^{12}$, using the data from a well analyzed in Texas, introduced a tectonic factor to the equation in an attempt to match the log derived stress profile with the measured in-situ stress of the formations. The new form of the correlation is:

$$
\begin{array}{r}
S_{h}=\frac{v}{1-v}\left(S_{v}-S_{p}\right)+S_{p}+\mathrm{X} \\
\text { where, } \mathrm{X}=\text { empirical stress factor } \\
\mathrm{X}=0.794 \text { pnls }-0.075 \\
\phi n \text { nls }=\text { neutron porosity log }
\end{array}
$$

The empirical stress factor, $\mathrm{X}$, is obtained from the plot of delta stress versus neutron porosity $\log ^{12}$.

- In the same year, S.A. Holditch and Associates ${ }^{13}$, using the data from a well analyzed in Wyoming, determined a new form of the correlation:

$$
S_{h}=\frac{v}{1-v}\left(S_{v}-S_{p}\right)+S_{p}(1+\beta)
$$

where, $\beta=$ a unitless, shaliness or "softness" factor

$$
\beta=\left(C_{b}-C_{b m i n}\right) /\left(C_{b m a x}-C_{b m i n}\right)
$$

$\mathrm{C}_{b}=$ bulk compressibility computed from sonic and density log

Cbmax $=$ max bulk compressibility expected for porous sandstone

$\mathrm{C}_{\text {bmin }}=\min$ bulk compressibility expected for solid quartz

- Bragan \& Associates ${ }^{17}$, in a report prepared for Gas Research Institute, evaluated the $A B C$ Stress Derivations Methodology. The $A B C$ method reduces the classical stress equation by using $A, B$ and $C$ coefficients:

$$
S h=A S v+B S p+C
$$


A coefficient is assigned based on formation lithology. In practice, $B$ is assumed to be 0.5 and $C$ zero. A different version of the $A B C$ Methodology calculates $A$ and $B$ coefficients using Poisson's ratio.

$$
\begin{aligned}
& A=\frac{v}{1-v} \\
& B=\frac{1-2 v}{1-v}
\end{aligned}
$$

\subsubsection{Determination of Elastic Properties with Acoustic Logs}

The elastic properties needed in stress calculations are routinely determined from acoustic logs ${ }^{10-13}$. The main parameters used include the following:

- Ratio of shear and compressional slowness, R:

$$
\mathrm{R}=\frac{\Delta \mathrm{ts}}{\Delta \mathrm{tc}}
$$

$$
\text { where, } \begin{aligned}
\Delta t \mathrm{ts} & =\text { shear slowness } \\
\Delta \mathrm{tc} & =\text { compressional slowness }
\end{aligned}
$$

- Compressional velocity, Vc:

$$
\mathrm{Vc}=\frac{10^{6}}{\Delta \mathrm{tc}}
$$

- Shear velocity, Vs:

$$
\mathrm{Vs}=\frac{10^{6}}{\Delta \mathrm{ts}}
$$


- Poisson's Ratio v:

By definition, Poisson's ratio is the ratio of the transverse expansion to the longitudinal stress when a body is compressed ${ }^{10,11}$. It can be determined from static loading tests or from acoustic (dynamic) measurements. The calculation of Poisson's ratio from acoustic log measurement tends to give more consistent results. For the purpose of this analysis, Poisson's ratio is related to the ratio, R, of compressional velocity, $\mathrm{Vc}$, to shear velocity, $\mathrm{Vs}$, through the following expression ${ }^{10,11}$ :

$$
v=\frac{1}{2}\left[\frac{\left[\frac{\mathrm{Vc}}{\mathrm{Vs}}\right]^{2}-2}{\left[\frac{\mathrm{Vc}}{\mathrm{Vs}}\right]^{2}-1}\right]
$$

Equation 2-12

$$
\text { or } v=\frac{1}{2}\left[\frac{\mathrm{R}^{2}-2}{\mathrm{R}^{2}-1}\right]
$$

- Shear modulus, G:

$$
\mathrm{G}=\frac{\left(1.36 * 10^{4}\right) * \rho \mathrm{b}}{\Delta \mathrm{ts}^{2}}
$$

or $G=c * \rho b * V s^{2}$

Equation 2-15

where, $c=$ unit constant

$$
\rho b=\text { bulk density }
$$

- Young's modulus, E:

$$
E=2 G^{*}(1+v)
$$

or $E=\frac{9 K b * G}{(3 K b+G)}$

Equation 2-17

- Bulk modulus, $\mathrm{Kb}$ :

$$
\begin{aligned}
\mathrm{Kb} & =\left(1.36^{*} 10^{-8}\right)^{*} \rho b^{*}\left(\mathrm{Vc}^{2}-\frac{4}{3} V s^{2}\right) \\
\text { or } \mathrm{Kb} & =\frac{\mathrm{GE}}{9 \mathrm{G}-3 E}
\end{aligned}
$$

- Bulk compressibility, $\mathrm{Cb}$ :

$$
\mathrm{Cb}=\frac{1}{\mathrm{~Kb}}
$$




\subsubsection{Determination of Formation Pressure}

Formation pressure is a major component in the stress profile calculation expression. It is known that in normally pressured zones the formation pressure contributes well over one half

of the closure stress. For accurate calculations of the stress profile, the formation pressure must be known or closely estimated. In permeable zones, pressure can be determined from wireline testing; the most common procedure is the Repeat Formation Tester ${ }^{9-13}$.

In the case when well tests are not run, the formation pressure can be estimated using industry accepted correlations. The most common correlation consists of a linear dependency between formation pressure and depth.

\subsubsection{Formation Tester Measurements}

Formation testers provide stationary measurements of formation pressure ${ }^{10,11}$. The tool is positioned at a depth at which pressure is to be measured and is then held stationary while pressure is recorded as a function of time. This is in contrast to most other logging tools, where measurements are usually recorded as a function of depth. Analysis of pressure measurements yields reservoir pressure and permeability. These parameters are used directly in the simulation program.

Pore pressure, $p_{p}$, is one of the most crucial parameters in evaluating the stress field around the borehole. Although the assumption of a $0.46 \mathrm{psia} / \mathrm{ft}$ pore pressure gradient is accurate enough for most analyses, the exceptions will cause erroneous stress profile calculations. For example, over-pressured zones and depleted zones have different pore pressure gradients and therefore drastically affect the final stress profile. Although reservoir engineers generally know the pore pressure gradients in such zones, the precise boundaries at which pressure gradients change must be identified. This can be accomplished using wireline formation tester or microfrac data. If such data are not available, over-pressured or depleted zones may be detected using density, resistivity, or sonic logs ${ }^{9}$.

Pore pressure gradients can vary on a smaller scale within a reservoir or zone of interest, dependent upon the density of the fluid present. Although a slight gradient shift may not be critical in the final stress evaluation, data from a wireline formation tester should be used when available. Density, resistivity, and sonic logs along with reservoir engineering data are usually not able to sufficiently resolve variations in pore pressure gradients within an interval. 


\subsubsection{In-Situ Stress Test Analysis}

The objective of an in-situ stress test is to determine when a fracture opens (or closes) by monitoring pressure data ${ }^{10-13}$. With this information, the minimum principal stress can be estimated.

Several methods are used to estimate the minimum principal stress (or in-situ stress) for an interval. These methods include injection falloff or injection flowback tests, mini-fracs, and micro-fracs ${ }^{10-12}$. Injection falloff tests are generally more accurate ${ }^{9}$. In these tests, very small fluid volumes (less than 500 gallons) are pumped at very low injection rates (usually less than 20 gpm). A downhole shut-off tool is used to record the pressure. In this way, the effects of afterflow or wellbore storage during shut-in or pressure falloff stages are minimized. Two types of analyses are used for determining the fracture closure pressure: the log-log plot of pressure versus time and the plot of square root of time versus pressure. The half-slope region on the loglog plot represents the linear flow and the end of the half slope line should agree with the straight line on the square root of time plot $^{11}$. The deviation of the data from the straight line represents the fracture closure pressure.

In-situ stress tests are performed in both open-hole and cased-hole. Even though openhole tests are preferable, it is very difficult to get the packer to seal on the formation making the measurements untrustworthy ${ }^{11}$. For this reason, cased-hole tests are run to determine the fracture closure pressure with a high rate of success. A good set of the packer in the cased hole allows for better control of the point of injection. However, a poor sealing of the packer in the casing allows communication with the annulus and the measurements become unclear.

\subsection{Hydraulic Fracture Models}

From the early years of oil and gas production, hydraulic fracturing seemed to be a very promising technology to increase the well flow rate and thus produce the well at higher economic levels. However, not much was known about the propagation and geometry of the fracture.

In 1955, Khristianovic and Zlethov $^{18}$ proposed the first model to simulate fracture propagation. It was a simple two-dimensional model that considered the formation as a single layer bounded by two impermeable layers. Since this first model was introduced, many authors have proposed different hydraulic fracturing models. Throughout the years, the models have become more and more complex in an attempt to integrate the mathematical equations describing the fundamental and constitutive laws ${ }^{19,20}$.

In 1969, Geertsma and de Klerk ${ }^{21}$ improved Khristianovic's ${ }^{18}$ model and proposed a new 2D-model known as the KGD Model. This model was a breakthrough at the time and is still incorporated in many commercial simulators. The particularities of this model are: uniform height of the fracture, rectangular cross section of the fracture, and the crack opening is solved in the 
horizontal plane. A schematic description of the fracture geometry considered in the KGD model is presented in Figure 2-1.

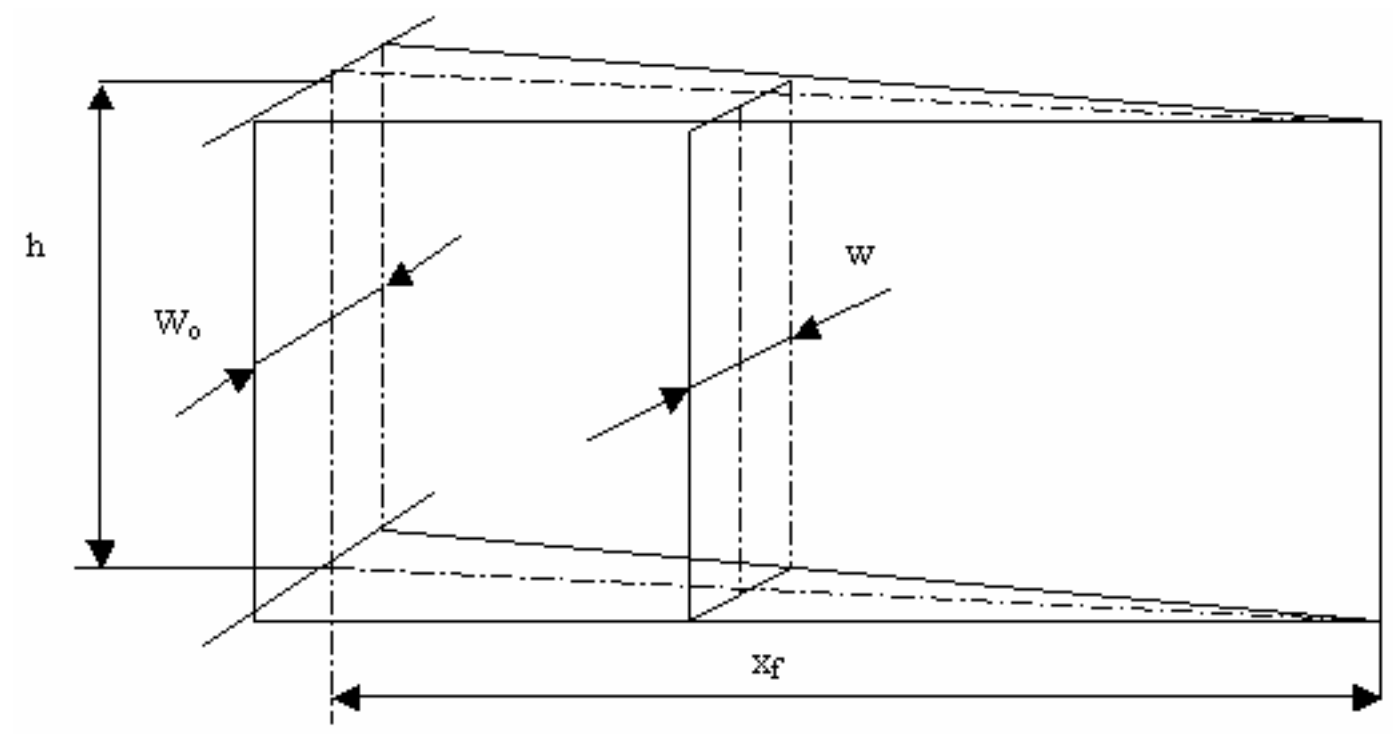

Figure 2-1: KDG Model Fracture Geometry

In 1961 Perkins and Kern ${ }^{22}$, and Nordgen ${ }^{15}$ in 1972, proposed a new 2D-model known as the PKN Model. They considered a new approach in solving the crack opening equations based on the assumption that the shape of the fracture in cross section, is not rectangular, but elliptical. The model considered the following assumptions: uniform height of the fracture, elliptical cross section of the fracture, and the crack opening is solved in the vertical plane. A schematic description of the fracture geometry considered by the PKN model is presented in Figure 2-2.

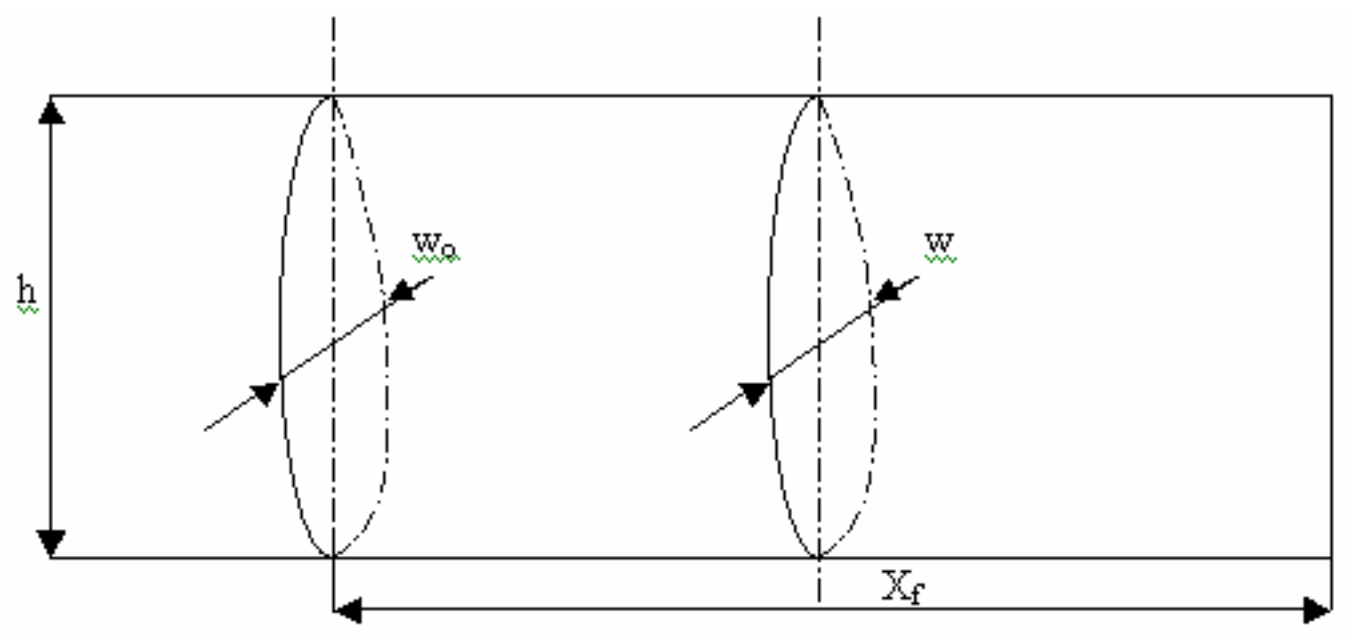

Figure 2-2: PKN Model Fracture Geometry 
In 1982, Nolte and Smith ${ }^{14}$ developed a pseudo-three-dimensional model ( $\left.p-3 D\right)$, which was based mainly on the PKN system. The primary difference from the classical 2D-model is the estimation of the vertical fluid-flow term through the fracture, which provides a non-uniform height of the fracture.

In 1991, Mike Cleary ${ }^{23-25}$ developed one of the most complex 3-D fracturing models in existence today. The model was implemented in complex software called FRACPRO ${ }^{26}$, by Resource Engineering Systems, Inc. (RES) with support from the Gas Research Institute, (GRI).

\subsection{Hydraulic Fracturing Design}

Hydraulic fracturing removes the skin around the wellbore, and also improves the well productivity by creating a large contact surface between the well and the reservoir ${ }^{19}$. Experience has demonstrated that hydraulic fracturing, in appropriate reservoirs, has increased and maintained well productivity to values by far greater than achieved by matrix acidizing.

Hydraulic fracturing is a complex treatment that deals with four main domains: formation characteristics, treatment fluid characteristics, proppant characteristics, and treatment type. Hydraulic fracture modeling uses fundamental laws (mass, momentum, and energy conservation), constitutive laws, and the previously mentioned domains to simulate the propagation and geometry of the fracture ${ }^{19}$.

\subsubsection{Treatment Fluid Characterization}

The fracturing fluid is a critical component of the hydraulic fracturing treatment due to its important functions: to open the fracture and transport the proppant into the fracture ${ }^{9,19,26}$. For this reason, the fracturing fluid must not only have good viscous properties, but also many other properties that can be summarized as follows:

- Low friction pressure in tubing/casing and in the fracture

- Good fluid-loss control and low damage on the productive formation

- Rapidly break at the end of the treatment

- Low cost

Three types of fracturing fluids are currently available in the industry: oil-based fluids, water-based fluids, and multiphase fluids.

Oil-based fluids were the first fluids used in fracturing treatments because they were perceived to be less damaging to the production formation than water-based fluids. Also, their viscosity made them more attractive. However, oil-based fluids are expensive and operationally difficult to handle. Therefore, this type of fluid is now only used for extremely water-sensitive formations ${ }^{19}$. 
Water-based fluids are the most widely used fracturing fluids in the industry ${ }^{19}$. After the discovery of polymers and crosslinkers, water-based fluids replaced most oil-based fluids due to their high performance and low cost. By adding polymers to a water-based fluid, the viscosity of the solution increases considerably as well as the proppant transport property of the fluid. All polymers (basically high molecular weight molecules) increase the viscosity of the solution at ambient temperatures. Because reservoir temperature is much higher, some solutions thin significantly. In these conditions, the polymer concentration can be increased to maintain the solution viscosity and offset the thermal conditions, but this approach is expensive. For these cases, the use of crosslinker agents has proved to be more efficient. Crosslinker agents are used to increase the effective molecular weight of the polymer and, as a result, increase the viscosity of the solution. The most used ions to crosslink water-soluble polymers are borate, $\mathrm{Ti}$ (IV), and $\mathrm{Zr}$ (IV).

Multiphase fluids are basically foams and emulsions. Foam is a stable mixture of liquid and gas. In the case of hydraulic fracturing, foams are created by adding gas to water-based or oil-based fluids. Oftentimes, surface-active agents are added to increase the stability of the foams. These agents, also known as surfactants, act at the gas-liquid interface to reduce the surface tension. The most common foams used in the industry are nitrogen $\left(\mathrm{N}_{2}\right)$ and carbon dioxide $\left(\mathrm{CO}_{2}\right)$ foam fracturing fluids.

Foams are not only good fluid-loss control fluids in low permeability formations, but are also non-damaging ${ }^{19}$. Foam fracturing fluids are described by a specific parameter called "foam quality" which is defined by the following relationship:

$$
\text { Foam Quality }=\frac{\text { GasVolume }}{\text { FoamVolume }} \times 100 \quad \text { Equation 2-21 }
$$

It should be mentioned that foam fracturing fluids are becoming more commonly used in the industry.

The other type of multiphase fluids is the emulsion. An emulsion is a dispersion of two immiscible phases, usually stabilized with a surfactant. In the case of fracturing fluids, emulsions are created by mixing oil in water or water in oil and stabilized with a surfactant. The primary advantage of the emulsion-based fracturing fluids is its high viscosity, which confers a very good transport property. Disadvantages include high friction pressure and the high cost of the fluid (hydrocarbon phase).

There are a series of additives that are added to fracturing fluids to control their properties and increase their functionality and usability. Among these additives are buffers (to adjust the $\mathrm{pH}$ of the fluid), bactericides (to prevent viscosity loss due to bacterial degradation of the polymer), stabilizers (to prevent degradation of the polymer at high temperatures), breakers (to break the gel polymer at the end of the treatment), surfactants (to reduce the interface tension 
between two immiscible substances), and fluid-loss additives (to control fluid-loss in the productive formation).

\subsubsection{Proppant Characterization}

Proppant is the material transported with the fracturing fluid and deposited in the fracture to keep the fracture open at the end of the treatment ${ }^{9,19,26}$. Since the beginning of hydraulic fracturing, several materials have been used as proppants. Some proppants used in the industry today include sands, resin-coated sands, intermediate-strength proppants (ISP), and high-stress proppants.

Sand is by far the most widely used proppant due to its good performance and low cost. There are different types of sand, named for the region where they are found such as "Northern sand", "Ottawa sand", "Brady sand", "Jordan sand", etc.

Resin-coated sand is sand treated with resin coatings to improve the proppant strength. It has been proven that resin-coated sand provides higher conductivity and withstands higher confining pressure than conventional sands ${ }^{19}$. The cost of this proppant is higher than conventional sands.

Intermediate-strength proppants are basically fused ceramics. These proppants are used only in cases where the formation yields higher closure stress.

High-strength proppants are sintered bauxite and zirconium oxide. They yield a very high strength and are very seldom used due to high cost.

The main characteristics of propping agents that have an impact on fracture conductivity are:

- Proppant strength

- Grain size and grain size distribution

- Quality of the proppant

- Roundness and sphericity

- Proppant density

Proppant strength is the property of the proppant to resist the closure stress of the fracture without breaking or crushing. If the proppant is not strong enough and crushes, the permeability of the fracture is significantly reduced.

The conductivity of the fracture is related to the grain size and grain size distribution - the higher the grain size, the higher the conductivity. However, larger grain sizes cannot be used in deep wells due to the low strength to crushing.

The quality of the proppant is related to the purity (the amount of fines and impurities) of the proppant. Fines or impurities can drastically reduce the proppant-packed permeability. 
Roundness and sphericity of the grains have a dramatic effect on the fracture conductivity. Proppant grain roundness is a measure of the relative sharpness of the grain corners, while grain sphericity is a measure of how close the proppant particle approaches the shape of the sphere. Higher roundness and sphericity of grains provide higher conductivity of the fracture and higher load to support before crushing.

\subsubsection{Treatment Design}

A classical hydraulic fracture treatment is designed to provide optimum length and conductivity to effectively stimulate the well. The treatment design consists of the total volume of fracturing fluid, total quantity of proppant used, and injection rate. The total volume of fracturing fluid is divided into pad volume and slurry volume. The pad is the clean volume of the fluid pumped at the beginning of the treatment to fracture the formation, while the slurry is the mixture of fluid and proppant (pumped after the pad) that transports and deposits the proppant in the fracture. Treatment schedules can be designed using different number of stages, proppant concentration, and pumping rate. In order to design an effective treatment job, the appropriate quantities of fluid and proppant must be pumped at a specific flow rate in order to achieve a desired propped length.

\subsection{Fracture Design Simulators}

The purpose of a fracture design simulator is to use a computer to simulate, as closely as possible, the actual downhole events that occur while performing a fracturing treatment. A number of reliable fracture design simulators are currently available on the market ${ }^{20}$. The following discussion focuses on some of major programs that perform fracturing design using 2D, pseudo-3D, and 3D modeling.

\subsubsection{D Models}

2D models simulate the length and width of the fracture, assuming constant height. The most popular 2D models used the PKN or GDK fracture geometries.

\subsubsection{PROP}

PROP is Halliburton's 2D fracture model ${ }^{27}$. It is based on Daneshy's numerical solution and is thus more flexible than other analytical models. It also uses the power-law model for gel fluids and Herschel-Bulkley for nitrogen or carbon dioxide phase fluids. PROP has capabilities to provide both GDK and PKN fracture geometries. 


\subsubsection{Chevron}

Chevron's 2D fracture simulator is used for constant-height, hydraulically induced, vertical fractures using a power-law fluid ${ }^{27}$. It can predict both PKN and GDK fracture geometries. It is most applicable for fracture design where geological conditions prevent height growth. It also has a proppant transport model that uses proppant settling and production, which calculates the final propped concentration, width, and bank height, given the settling velocity. Chevron's 2D simulator can predict possible problems caused by proppant bridging or screenout.

\subsubsection{Pseudo-3D Models}

Pseudo-3D models were developed based on the PKN model by removing the requirement of constant fracture height. However, they assume one-dimensional flow along the fracture length.

\subsubsection{MFRAC-II}

MFRAC-II is a pseudo-3D hydraulic fracturing simulator that models penny, GDK and PNK geometries and accounts for the parameters affecting fracture propagation and proppant transport $^{27}$. It also includes multilayer, asymmetrical confining stress contrast; fracture toughness and tip/overpressure effects; rock deformation; variable injection rate and time-dependent fluid rheology properties; multilayer leakoff with spurt loss; and 2D proppant transport. The fracture propagation model calculates fracture length, upper and lower heights, net pressure, efficiency, geometry parameters as function of time, and width variation as a function of height and confining stress. MRAC-II also presents other options to allow customization and applicability over a broad range of conditions.

\subsubsection{STIMPLAN}

The STIMPLAN program, developed by NSI Inc., is a fracture design simulator with special modifications that allows for tip-screenout designs ${ }^{27}$. At tip-screenout initiation, fracture extension is stopped and the program calculates a width increase based on the increase in the net treating pressure. This program will analyze complex formations composed of multiple productive layers with varying fluid-loss coefficients to calculate height growth including proppant settling and simulate fracture closure. It includes a history-matching module for net treating pressure to provide fracture geometry and behavior. STIMPLAN is well designed, easy to use, and is a popular choice among professionals who need an effective tool for designing fracturing treatments for high permeability formations ${ }^{27}$. 


\subsubsection{ENERFRAC}

ENERFRAC is Shell's pseudo-3D hydraulic fracture model that predicts fracture dimensions for uncontained and contained fractures ${ }^{27}$. It accounts for fracture-tip effects through direct input of apparent fracture toughness or the instantaneous shut-in pressure minus the closure pressure, which can be determined in the field from mini-frac tests. ENERFRAC also incorporates other interacting processes such as viscous fluid flow, elastic rock deformations, and fluid loss ${ }^{27}$.

\subsubsection{TRIFRAC}

S. A. Holditch and Associates uses TRIFRAC ${ }^{27}$, a pseudo-3D model, which utilizes a finite-difference numerical approach to determine created and propped fracture dimensions from fracture propagation and proppant transport. It can use up to 22 multiple nonsymmetrical stress layers, with each layer having unique values for Young's modulus, Poisson's coefficient, and fracture toughness, permeability, porosity, and fluid-leak-off coefficients. The apparent viscosity of the fracturing fluid is calculated from the shear rate inside the fracture and owes its variations of temperature (a temperature calculation model is provided) and time to changes in flowbehavior index and consistency index. Initiating the hydraulic fracture from up to 10 different layers is possible. Special options can be used for nitrogen foam ${ }^{27}$.

Proppant distribution, transport, and settling, along with fracture growth are simultaneously solved using the geometry computations module coupled with the finite-difference proppant transport simulator. It can compute the proppant profile for each time step for certain fluid velocities and proppant settling rates. TRIFRAC also has the ability to model PKN and GDK (including horizontal fracture) geometries. All models use the proppant-transport calculations module.

\subsubsection{D Models}

Fully $3 \mathrm{D}$ fracture models calculate the fracture geometry with no restrictions on fracture height. They consider 3D fluid flow into the fracture.

\subsubsection{FRACPRO}

Resource Engineering Systems, Inc. (RES) developed the FRACPRO ${ }^{26,27}$ program with support from the Gas Research Institute (GRI). Currently, FRACPRO belongs to Pinnacle Technologies.

The FRACPRO ${ }^{26}$ system is designed to provide engineers with comprehensive tools for hydraulic fracturing design and analysis. The fracture design model has the capability of 
acquiring real-time fracturing data during treatment. The program can be used to design fracturing treatments and can acquire downhole data during field operations from a treatment database to confirm the design estimates or perform detailed post-treatment analysis. Changes to the design and treatment can be made to better match the job data to design criteria. The capabilities of designing, monitoring, and analyzing the fracturing treatment make FRACPRO ${ }^{26} a$ versatile model for both minifrac and fracture design.

The FRACPRO model can analyze several layers of formation with varying rock properties and fluid-loss coefficients. Also, the model allows the user to select either wall-building or non-wall-building fracturing fluids to be used during the treatment. The fluid selection feature is important, since both of these fluid types (HEC and borate-crosslinked fluids) are used in high permeability formations. HEC does not build a filter cake and is controlled by viscous invasion of the formation, whereas borate-crosslinked fluids simulate a wall-building fluid with high spurt volumes. Multiple fluid designs can be handled with changing fluid-loss coefficients and different fluid-loss characteristics of fracturing fluids ${ }^{26,27}$.

\subsubsection{GOHFER}

Grid Oriented Hydraulic Fracture Extension Replicator (GOHFER) ${ }^{27}$ is a planar 3D model provided by Marathon Oil Company. For elastic rock displacement, it uses a regular grid structure, and a planar 2D finite-difference grid for fluid flow. The fluid flow equations provide the areal pressure distribution and proppant transport, which are used iteratively with the solution for elastic deformation. This allows for the representation of perforations at various locations. Each grid node can be assigned the following values: net stress, pore pressure, permeability, porosity, wall-building coefficient, rock strength, Young's modulus, Poisson's ration, and other variable associated with fracture-wall roughness and tortuosity. Boussinesq is used as the fracture width equation. GOHFER can model multiple fracture initiation and can be used for any planar 3D geometry.

\subsubsection{FRACPAC}

The FRACPAC program, developed by Halliburton, is aimed to assist in design of tipscreenout fracturing treatments ${ }^{9,27}$. The 3-D fracture geometry predictions from the XTENT program are incorporated with modifications to FRACPAC to allow pumping to continue after the tip-screenout initiates. Upon tip-screenout initiation, fracture length extension and fracture height extension stop and injection of additional slurry causes fracture width to grow. Calculations for fluid-loss, fracture width, proppant concentration, and net treating pressure during pumping continue after tip-screenout initiation. Treatment modeling ends when the user-specified increase in net treating pressure is reached. 
The FRACPAC ${ }^{27}$ design simulator offers very good input options such as capability of reading dynamic in-situ rock stress measurements directly from wireline logging files. The program also has limitations, especially in complex reservoirs. FRACPAC allows only one pay zone to be analyzed, and stress values across the zone are averaged. Values for fluid-loss are also limited; only two values are allowed for fluid-loss, one value for the pay zone and the other for outside the pay zone. In wells where several high permeability intervals are separated by small layers of shale, FRACPAC requires the user to make assumptions, which can cause the software to be difficult to use.

\subsubsection{TerraFrac}

TerraFrac is a commercially available, true 3D hydraulic fracture simulator provided by Terra $\mathrm{Tek}^{27}$. TerraFrac uses discrete elements to partition the fracture in order to solve the equations. It uses 3D elasticity, 2D fluid flow, and fracture criteria for intensity of fracture growth25. Proppant and temperature distribution are provided from 2D fluid flow. Multiple stages and multiple layers are available each having different fluids, proppants, injection rates; and insitu stresses, Young's moduli, fracture toughness, Poisson's ratio, and leakoffs, respectively. TerraFrac also has special features for waterfloods, a quasi-Newton method for fluid pressures, and post-shut-in capabilities.

\subsubsection{HYFRAC3D}

HYFRAC3D ${ }^{27}$ is a finite-element based code developed by S. H. Advani of Lehigh University that uses a set of coupled mass conservation, fluid momentum, constitutive elasticity, and fracture mechanics equations governing planar hydraulic fracture propagation in a multilayer reservoir. HYFRAC3D tracks the moving fracture front in a numerical scheme using a mapping technique of the baseline mesh in a unit circle to arbitrarily shaped fracture geometrics. It can also provide PKN results based on a 2D finite-element model simulator with standard PKN model equations, including vertical stiffness and one-dimensional fluid flow.

\subsubsection{Comparison of Fracturing Simulators}

In 1994, the Gas Research Institute sponsored the Stage Field Experiment No. 3 fracture experiment ${ }^{12}$ in which fracture design results were submitted from several different simulators and compared; these results are documented in SPE paper $25890^{27}$. Input data, including rock and reservoir data (depth, zone thickness, in-situ stress, Poisson's ratio, Young's modulus, and fracture toughness) along with treatment data (temperature, reservoir pressure, spurt loss, fluidleakoff, viscosity, fluid volume, injection rate, and proppant), was provided. 
Fracture design results were submitted for GOHFER, PROP, TRIFRAC, FRACPRO, ENERFRAC, MFRAC-II, Chevron, HYFRAC3D, STIMPLAN, and TerraFrac. The results from these models, given the same input parameters, had widely different fracture geometries. For example, in the five-layer case: FRACPRO calculated very short fractures, high net pressures, and large heights; GOHFER predicted short fractures and high net pressures; TRIFRAC, STIMPLAN, TERRAFRAC, and MFRAC-Il all had longer fractures, less height, and somewhat lower net pressures; HYFRAC3D predicted somewhere in the middle.

The differences in results between simulators are partly attributed to the differences in the assumptions for each model. Furthermore, these simulators have many options available to provide better simulation of actual fracture behavior. The use of these options is arbitrary, depending on the reservoir experience of the engineer running the simulation. The authors of SPE paper $25890^{27}$ also recognize that other formations, with different stress and lithology data could provide a considerably different comparison.

It is extremely difficult, if not impossible, to know exactly how the fracture propagates in reality. As such, it cannot be determined which fracture simulator is the best. Each has its advantages and disadvantages. Therefore, due to wide use throughout the industry and availability, the $3 \mathrm{D}$ model FRACPRO ${ }^{26}$ was used for this study. However, any one of these fracture simulators can be used. 


\section{CHAPTER 3: BACKGROUND}

Three main artificial intelligence techniques are employed in the methodology of the Artificial Intelligence Hydraulic Fracturing Simulator: neural networks, genetic algorithms, and fuzzy logic. Each is described in detail throughout the following sections.

\subsection{Artificial Neural Networks}

Artificial neural networks are information-processing systems developed as generalizations of mathematical models of human cognition and neural biology ${ }^{28}$. They were born from the desire to produce systems capable of intelligent and sophisticated computations similar to those the human brain performs.

\subsubsection{Overview}

Biological neural networks are composed of neurons and dendritic connections that form the human brain ${ }^{2}$. The basic processing element in this system is the neuron, while nerve fiber called dendrites form a tree-like network of connections to the cell body. The axon is a single fiber that extends from the cell body and branches into strands and substrands, which are then connected to other neurons through synapses. Figure 3-1 represents a diagram of a simple neuron.

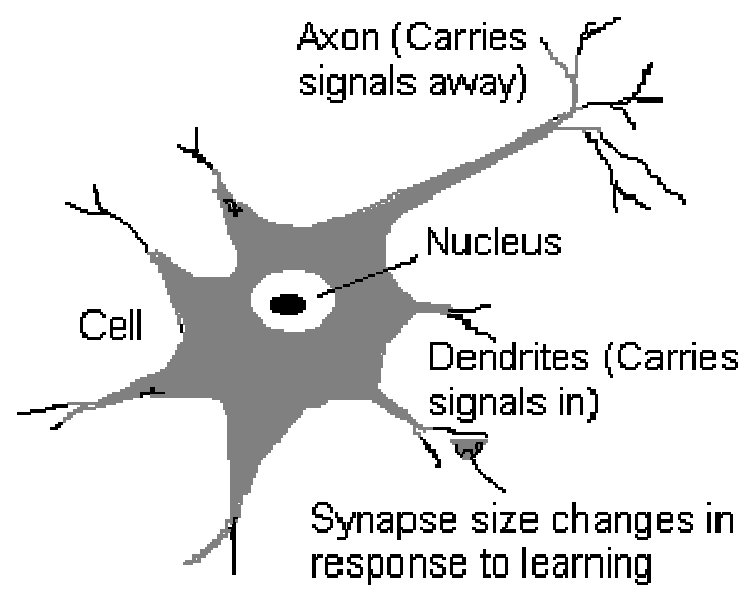

Figure 3-1: A Simple Neuron

Signals are transmitted from one neuron to another at the synapses and involve a complex chemical process in which the sender neuron releases specific transmitter substances to be absorbed by the receiver neuron. The effect of this reaction is the increase of electrical 
potential of the receiver neuron. If the electrical potential reaches a certain threshold, the neuron will fire, sending a pulse down its axon. It is through this process and architecture that complex human learning, reaction, and behavior takes place.

Neural networks are designed to process information in a similar, but simplified, manner as the human brain. Resemblances to the human brain include the following:

- Knowledge acquired through a learning process

- Local processing in artificial neurons (known as nodes or processing elements)

- Storage of experiential knowledge available for future use in interneuron connections (synaptic weights)

- Massive parallel processing implemented by profuse connection pattern

Neural networks present some additional fundamental features providing them with the capability of solving a variety of complex scientific and engineering problems. These features include:

- Adaptability - the ability to deal with small and large data sets with minimal memory requirements; allows for the possibility of developing real-time online solutions where all data is not available at the beginning.

- Massive parallelism and high connectivity - large number of nodes share the burden of computation and often can operate independent of information made available by other nodes; the speed of computation is significantly increased (the output is generated instantaneously).

- Universal function estimation - ability to perform pattern recognition, detect trends, and solve highly non-linear problems through deriving meaning from complicated or imprecise data.

Since the birth of artificial neural networks in the 1980s, neural systems were built in many different forms. However, there are certain characteristics that are common between all paradigms.

- Weight parameters, adjusted to produce learning.

- Interconnected units (nodes).

- Learning rules that dictate information flow in the network.

- Optimization by aiming to minimize a cost function, energy function, or a complex combination of functions.

In neural computing, the artificial neuron is called the processing element or node. Although modeled after biological neurons, their artificial counterparts bear only a modest resemblance. Whereas neurons in the brain can perform at least 150 different processes, processing elements can only perform three:

- Evaluate input signals and determine the strength of each. 
- Calculate the total for the combined input signals and compare this to a certain threshold level.

- Determine the output: to fire or not fire.

As biological neurons can be affected by mechanisms other than inputs, most neural networks attempt to simulate this feature through the use of synaptic weights. Much like the varying of synaptic strengths in biological neurons, weights are adaptive coefficients within the network that determine the intensity of the input signal, thus allowing for some inputs to be more important than others in their combination to form an output or impulse. As mentioned above, neural networks acquire information through the process of learning, which is completed through showing the network examples of input data and the expected output. The synaptic weights adjust themselves until the error between the actual expected output and the output generated by the neural network reaches a desired level. The weights are changed by an amount proportional to the error at that unit times the output of the unit feeding into the weight. This, in effect, allows the processing elements to modify their behavior in response to its inputs ${ }^{3,28}$.

Several processing elements are combined to form a layer of nodes. Inputs are connected to many nodes using various weights, resulting in a series of outputs. The layer receiving the inputs is termed the input layer and generally only buffers the input signal, while the network outputs are generated from the output layer. All other layers are called hidden layers. The network is fully connected if every output from one layer is passed along to every node in the next layer.

\subsubsection{Backpropagation Neural Networks}

Back propagation networks (the most common in use today) allow for weight adjustments to be made in preceding layers of feed forward networks by "backing up" from outputs. Figure 3-2 presents a back-propagation neural network with two hidden layers. The first layer (input layer) reads the inputs and passes them to the next layer, (the first hidden layer). Next, the processed information is passed to the next layer (the second hidden layer) and so on.

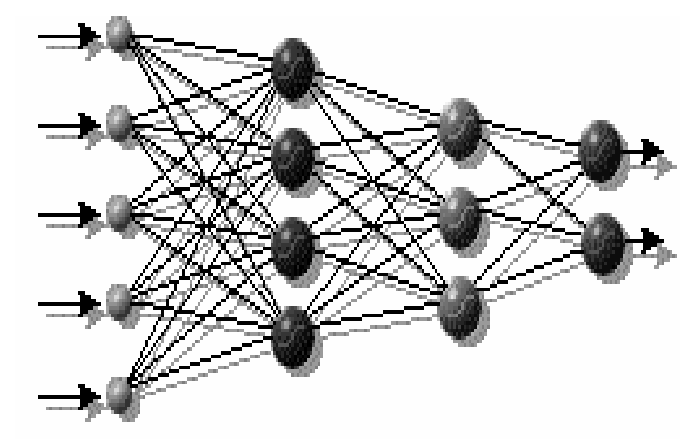

Figure 3-2: Typical Back Propagation Network 
The weights associated to each interneuron connection affect the information so that the network can control the flow of information. The processing units in the hidden and output layers are characterized by the following activation function ${ }^{2}$ :

$$
Y=1 /\left(1+e^{-k\left(\Sigma W_{i n}^{*} X i n\right)}\right)
$$

Equation 3-1

The graphical representation of this function can be seen in Figure 3-3.

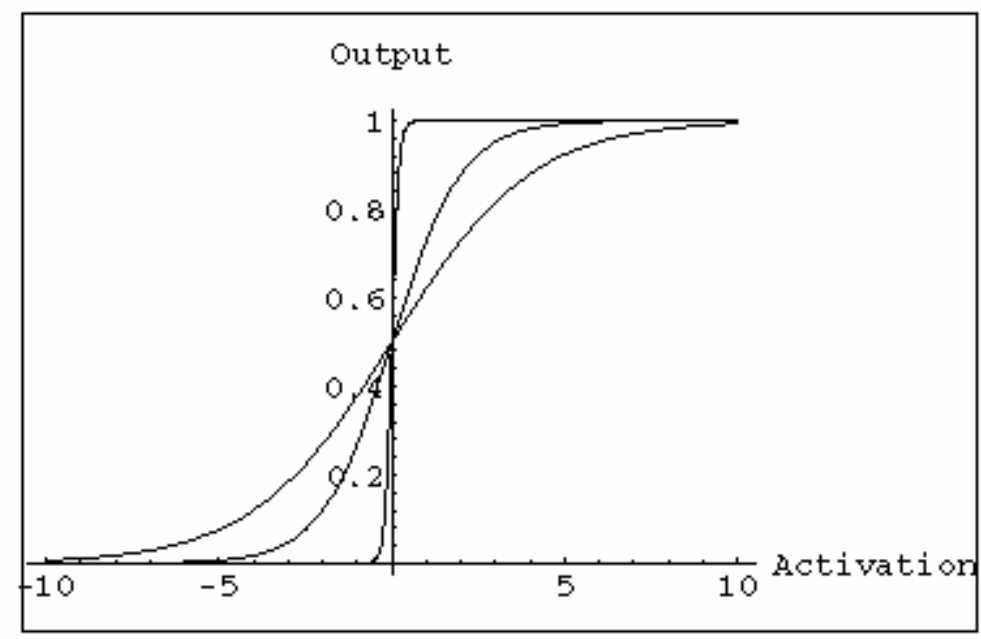

Figure 3-3: Activation Function for Back Propagation Networks

In a typical neural network data processing procedure, the data is divided into three separate sets:

- Training - set of examples used for learning to fit the parameters (weights) of the classifier.

- Testing (or calibration) - set of examples used to tune the parameters of a classifier, such as choosing the number of hidden units in a neural network.

- Production (or verification) - set of examples (not previously seen by the neural network to ensure its integrity and robustness) used only to assess the performance or generalization of a fully specified classifier.

\subsubsection{Neural Nets versus Conventional Computing}

Artificial neural networks are an alternative to conventional computing, but will not replace

it. Neural networks are simply a different way of solving problems that typically cannot be done by conventional computing. Conventional computing (which must follow a specific set of 
instructions) is restricted to problems that can already be solved, while neural networks are able to handle non-linearity, noise in the data, and a large number of parameters. Because neural networks learn by themselves using the examples provided to it, their operation can be unpredictable if not tested thoroughly. A summary of the differences between conventional computing and neural networking follows in Table 3-1:

\begin{tabular}{|c|c|}
\hline Conventional Computing & Neural Networks \\
\hline $\begin{array}{l}\text { Deductive reasoning - known rules are applied to } \\
\text { input data to produce an output. }\end{array}$ & $\begin{array}{l}\text { Inductive reasoning - rules are constructed given } \\
\text { training and testing data. }\end{array}$ \\
\hline $\begin{array}{l}\text { Computation is centralized, synchronous, and } \\
\text { serial. }\end{array}$ & $\begin{array}{l}\text { Computation is collective, asynchronous, and } \\
\text { parallel. }\end{array}$ \\
\hline $\begin{array}{l}\text { Memory is packeted, literally stored, and location } \\
\text { addressable. }\end{array}$ & $\begin{array}{l}\text { Memory is distributed, internalized, and content } \\
\text { addressable. }\end{array}$ \\
\hline Not fault tolerant. & $\begin{array}{l}\text { Fault tolerant, redundant and shared } \\
\text { responsibilities. }\end{array}$ \\
\hline Fast, measured in millionths of a second. & Slow, measured in thousandths of a second. \\
\hline Exact. & Inexact. \\
\hline Static connectivity. & Dynamic connectivity. \\
\hline $\begin{array}{l}\text { Applicable if rules are well defined with precise } \\
\text { input data. }\end{array}$ & $\begin{array}{l}\text { Applicable if rules are unknown or complicated, or } \\
\text { if data is noisy or partial. }\end{array}$ \\
\hline
\end{tabular}

Table 3-1: Differences between Conventional Computing and Neural Networks

Thus, neural networking and conventional algorithmic computing are not in competition, but rather are complementary of each other. Many tasks and engineering problems require systems that use a combination of the two approaches in order for the best solution to be found.

\subsubsection{The NeuroShell Software}

The intelligent fracturing model proposed in this research relies heavily on the prediction capabilities of neural networks. Artificial neural networks are used in the fracturing treatment design (either alone or coupled with genetic algorithms) and for the formation pressure prediction response.

There are numerous artificial neural networks commercial software packages available off the shelf. Examples include: Neurogon, NeuroForcaster/Genetica, Matlab Neural Network Toolbox, NeuroShell, VBBackProp, N-Net, STATISTICA Neural Networks, NeuralWorks, NeuroClassifier, NeuroWindows, MacBrain, PathFinder, etc.

For this research, NeuroShell 2 software, a product of the Ward Systems Group, was used. NeuroShell 2 combines robust neural network architectures, a Microsoft $\circledast$ Windows user interface, and options to provide users with a powerful neural network environment. Previous 
experience with the software together with the preliminary results from training neural network systems concluded that the Ward Architecture Net proved to be the best suited for the types of problems addressed in this research.

NeuroShell 2 includes twelve neural networks architectures for backpropagation training including the following: standard backpropagation type, recurrent networks; Wards nets; TurboProp; Kohonen Self Organizing Map networks; Probabilistic Neural Networks (PNN); General Regression Neural Networks (GRNN); and Group Method of Data Handling polynomial nets (GHMDH). The Ward nets type of backpropagation is able to detect different features in the data through the use of multiple slabs of neurons in the hidden layer, each with a different activation function. Activation functions are functions used internally in neurons to "fire" the neurons. A different activation function is applied to each slab in the hidden layer, which gives the network the possibility to discover novel features in a single pattern processed through the network.

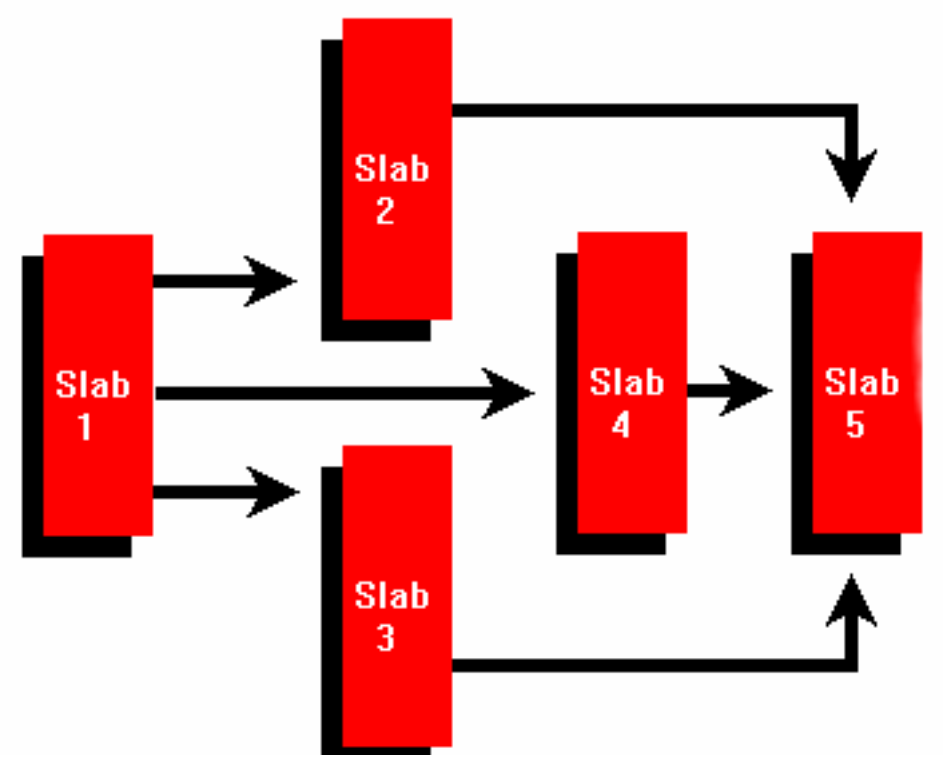

Figure 3-4: Multiple Slabs in an Artificial Neural Network

All backpropagation networks include calibration features, which prevent overtraining (thereby greatly reducing training time), and increase the network's ability to generalize new data well.

The NeuroShell program offers a set of statistical tools to assist in model building and analysis. They include linear and multiple correlation tools.

The performance of the neural network is measured by two main parameters: the linear correlation coefficient $(r)$ and the coefficient of multiple determination $\left(R^{2}\right)$. 
The linear correlation coefficient $(r)$ is the statistical measure of the strength of the relationship between the actual versus the predicted outputs. The correlation coefficient ranges from -1 to +1 , with values approaching 1 denoting a stronger positive linear relationship, values approaching -1 denoting a stronger negative linear relationship, and 0 meaning there is no linear relationship.

The coefficient of multiple determination $\left(R^{2}\right)$ is a much better predictor for the performance of neural networks. It is a statistical indictor usually applied to multiple regression analysis and is a measure of how well the multiple regression equation fits the data. Values approaching +1 indicate very good fit, while values approaching 0 indicate very poor fit. The following is the equation used by NeuroShell 2 for the coefficient of multiple determination:

$$
R^{2}=1-\frac{\sum(y-\hat{y})^{2}}{\sum(y-\bar{y})^{2}}
$$

Equation 3-2

\subsubsection{Role of Neural Networks in This Research}

Neural networks have been used to develop a data driven model that, once trained, can mimic a classic fracturing simulator software. Due to their high response speed, neural networks provide the ideal platform for a genetic algorithm search to find the optimum solution for fracturing treatments.

The model consists of several neural networks, specifically trained to produce certain outputs of a fracture simulation. Five neural networks, representing different types of fracturing treatments, have been trained to mimic the fracture simulation while providing the fracture geometry as output. In addition, two more neural networks have been trained to predict the pressure profile developed during fracturing. The development of each neural network is presented in detailed in the methodology.

\subsection{Genetic Algorithms}

A genetic algorithm is a model of Machine Learning, which derives its behavior from a metaphor of the process of evolution in the nature. Genetic algorithms are a type of evolutionary computation, which attempts to apply the biological principle of natural evolution to artificial systems ${ }^{5,6}$. Evolutionary computation is used to describe all computer-based problem solving systems that use computational models based on the known mechanisms of evolution as key elements in their design and implementation. Evolutionary computing includes genetic algorithms, evolutionary programming, evolution strategies, classifier systems, and genetic programming $^{28}$. All types of evolutionary computation are conceptually similar - they simulate evolution through selection, mutation, and reproduction. 


\subsubsection{Overview}

A genetic algorithm is an iterative procedure that provides a powerful and adaptive search method, practical in complex optimization problems and valuable for application to spaces that are too large to be exhaustively searched. It can cover very large search spaces in a small amount of time without requiring an exact mathematical model of the problem. Genetic algorithms have the ability to simultaneously search several solutions and combine the best of these solutions to come up with the optimum combination from all these possibilities. The genetic algorithm maintains a constant-size population of individuals that evolve according to the rules of selection and other search operators. Each individual in the population, which is a possible solution in the given problem space, is represented by a chromosome (a finite string of symbols, often binary), and has a measure of fitness according to the particular environment. Using this information, reproduction occurs with attention focused on high fitness individuals (those that are better are more likely to survive and propagate their genetic materials). Thus, the offspring have a greater probability of a high measure of fitness.

\subsubsection{Representation}

A chromosome is a finite string of symbols that represents an individual in the population. The chromosome may be digitized (a binary string), analog (a series of real numbers), characterbased, or a tree representation. Digitized (binary) chromosomes are most often used and will be used for explanatory purposes here. Figure 3-5 shows an example of a digitized chromosome.

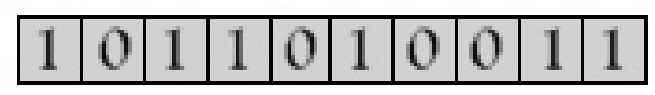

Figure 3-5: Example of a Digitized Chromosome

\subsubsection{Initial Population}

Many random solutions to the given problem are generated in order to create the initial population. The fitness of each member of the initial population is evaluated according to a determined fitness function. The fitness is a numerical value, which is representative of the ability of the individual. The fitness value eval $\left(v_{j}\right)$ is calculated for each chromosome $v_{j}(j=1$ ...pop_size), then the total population fitness is determined: 


$$
F=\sum_{j=1}^{\text {Pop_size }} \operatorname{eval}\left(v_{j}\right)
$$

Equation 3-3

\subsubsection{Selection}

In order to generate new and better populations, chromosome selection is extremely important so that the best chromosomes survive to produce the next generation. The most apt parents are selected from the population using the fitness values. More fit individuals will likely be selected several times in a generation, while less fit ones may not be selected at all.

The most widely used method is the classical selection (or roulette wheel) method, which is based on a probability distribution with respect to fitness evaluation. After the total population fitness is determined, the following steps are completed:

- Calculate the probability of selection $P_{j}$ for each chromosome $v_{j}(j=1 \ldots$ pop_size $)$ :

$$
P_{j}=\frac{\operatorname{eval}\left(v_{j}\right)}{F}
$$

Equation 3-4

- Calculate the cumulative probability $q_{j}$ for each chromosome $v_{j}(j=1 \ldots$ pop_size $)$ :

$$
F=\sum_{k=1}^{j}\left(P_{k}\right)
$$

Equation 3-5

This selection process is based on spinning the roulette wheel pop_size times, where a single chromosome is selected each time for the new population.

\subsubsection{Genetic Operators}

After the parent chromosomes have been selected, the offspring must be created through implementation of genetic operations. There are several types of genetic operators such as crossover, fitness-proportionate reproduction, and mutation. In implementation, simple bit manipulation operations allow for the creation of new populations. The most common operators are crossover and mutation.

\subsubsection{Crossover}

Crossover, or recombination, involves the exchange of chunks of genetic information. It is reflective of sexual reproduction in nature, which allows for the creation of genetically different offspring of the same species. Crossover is quite important in genetic algorithms for it allows portions of chromosomes to be combined with portions of other chromosomes. A corresponding 
point (or points) along the parent chromosomes is selected at random. The parent chromosomes are swapped at each crossover point to produce two offspring chromosome. Each offspring inherits genes from each parent. Three types of crossover are most common: classical crossover, double crossover, and uniform crossover.

In classical crossover, one random number (between one and the total number of bits in the chromosome) is generated to determine where the two chromosomes will exchange parts, as seen in Figure 3-6 below:

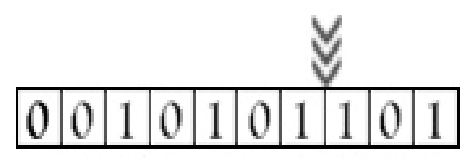
Chromosome 1

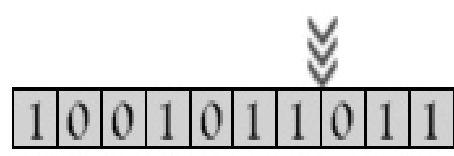

Chromosome 2

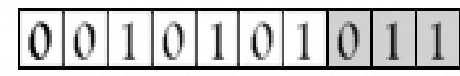

New Chromosome 1

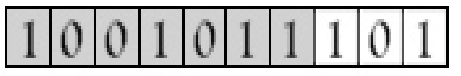

New Chromosome 2

Figure 3-6: Classical Crossover

In double crossover, two random numbers (between one and the total number of bits in the chromosome) are generated to determine where the two chromosomes will be broken into three parts and the middle exchanged, as seen in Figure 3-7:

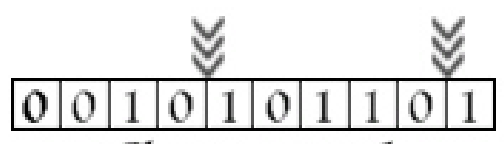

Chromosome 1

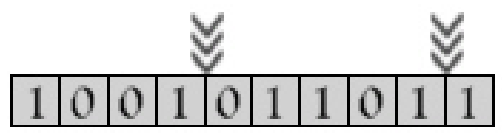

Chromosome 2

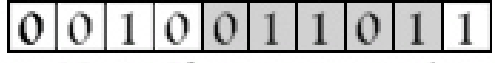

New Chromosome 1

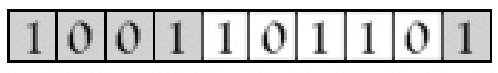

New Chromosome 2

Figure 3-7: Double Crossover

In uniform crossover, an indefinite number of random numbers (between one and the total number of bits in the chromosome) are generated that will represent the bits exchanged between the two chromosomes, as seen in Figure 3-8: 


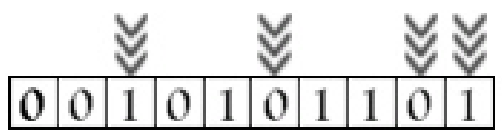

Chromosome 1

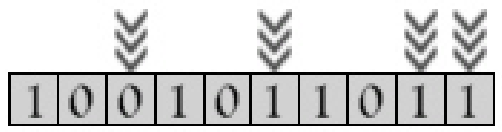

Chromosome 2 \begin{tabular}{|l|l|l|l|l|l|l|l|l|l|}
\hline 0 & 0 & 0 & 0 & 1 & 1 & 1 & 1 & 1 & 1 \\
\hline
\end{tabular}

New Chromosome 1

\begin{tabular}{|l|l|l|l|l|l|l|l|l|l|}
\hline 1 & 0 & 1 & 1 & 0 & 0 & 1 & 0 & 0 & 1 \\
\hline
\end{tabular}

New Chromosome 2

Figure 3-8: Uniform Crossover

\subsubsection{Mutation}

Mutation is another major operator in genetic algorithms, although its relative importance continues to be a matter of debate. In mutation, a bit is randomly selected and its value is altered to produce the new individual. For example as seen below in Figure 3-9, the fourth bit in the chromosome is randomly selected and its value is flipped from zero to one to form the offspring. In other types of chromosomes, such as analog, the new value is selected from amongst a parameter range. Mutation typically occurs with a small frequency.

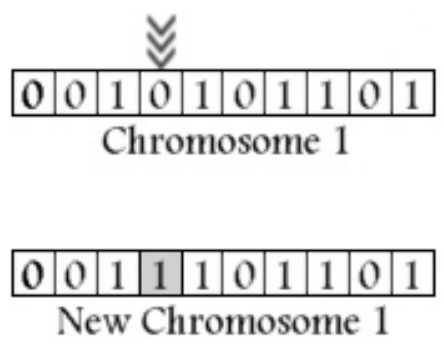

Figure 3-9: Mutation

\subsubsection{How They Work}

The following steps represent the implementation of the genetic algorithm process:

- Generate the initial population.

- Evaluate the fitness (a numerical value, representative of the ability of the individual) for all individuals in the population.

- Select the best individuals from those whose fitness has just been measured.

- Perform operations such as crossover, fitness-proportionate reproduction, and mutation on the best individuals.

- Generate a new population (offspring) via the operations just performed.

- Discard the old population and iterate using the new population. 
Each iteration of this loop is referred to as a generation. During each iteration, the genetic operations (using the fitness measure) work to improve the population. This procedure is repeated until no improvement in the fitness value is observed. Figure 3-10 represents the genetic algorithm process:

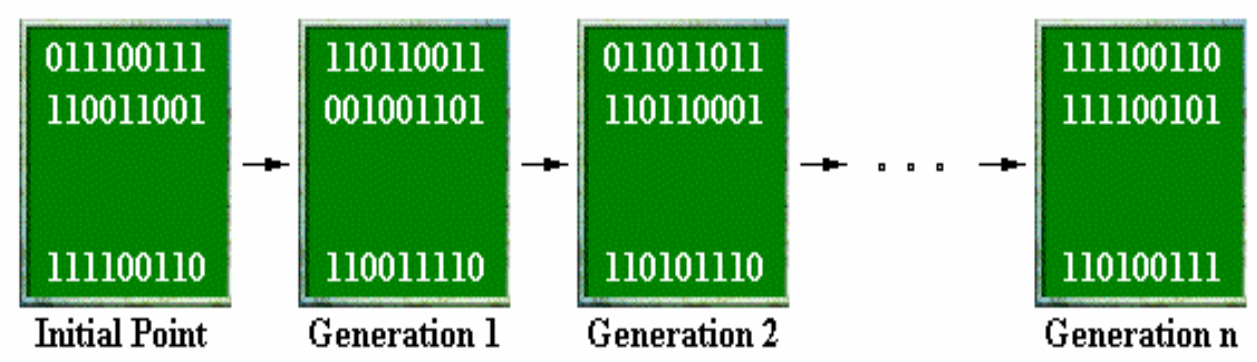

Figure 3-10: Genetic Algorithm Process Depiction

\subsubsection{Genetic Algorithms versus Conventional Searching Techniques}

It cannot be stressed too strongly that the genetic algorithm is not a random search for a solution to a problem. The genetic algorithm uses stochastic processes, but the result is distinctly non-random (better than random). Genetic algorithms are used for a number of different applications, including multidimensional optimization problems in which each bit in the chromosome represents the value for a different optimization parameter.

\subsubsection{Role of Genetic Algorithms in This Research}

In this study, genetic algorithms were combined with neural networks in a hybrid system, aimed at designing optimum fracturing treatments. The complexity of the problem is given by the number of parameters to be optimized. The problem calls for simultaneously finding the best combination of four parameters to match the desired fracture length and conductivity. Due to their unmatchable capabilities of searching large spaces and providing accurate solutions in relative short time, genetic algorithms were the best fit technique for this research. The development of the genetic algorithm for designing optimum fracturing treatments is presented in detail in the methodology.

\subsection{Fuzzy Theory}

Fuzzy theory is a broad field that allows for the use of intermediate values between standard values (such as true/false, yes/no, 0/1, etc.) in an attempt to mimic the human thought process. In other words, it provides an array of grays to be defined between black and white and permits vague answers such as "OK" when asked, "How are you?" Fuzzy logic values are 
continuous ranging from 0 to 1 (where 0 is false and 1 is true), allowing statements to be either false by various degrees or true by various degrees.

\subsubsection{Fuzzy Sets}

In traditional set theory, an element either belongs to the set (completely inside the set) or does not belong to the set (completely outside the set). Fuzzy set theory allows the element to belong partially to the set (both inside and outside the set) or belong to a certain amount, called the degree of membership ${ }^{7}$. Furthermore, the element can belong to multiple sets with varying degrees of membership. In addition, fuzzy sets correspond more closely to human language than tradition sets.
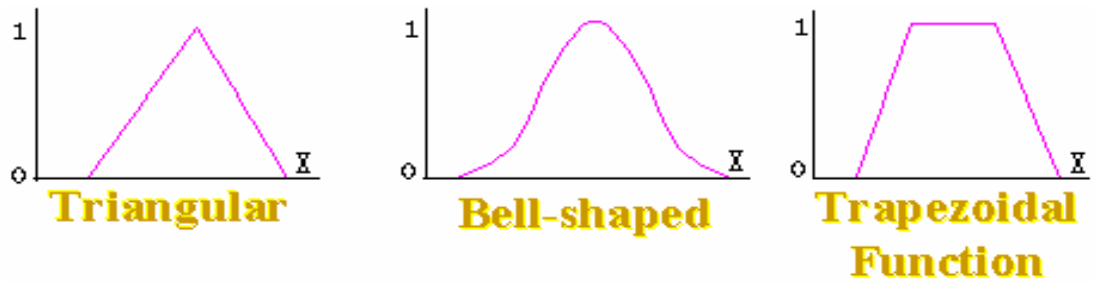

Figure 3-11: Common Fuzzy Membership Functions

All fuzzy sets are specific by their membership functions, provided by human experts. Some commonly used membership functions can be seen above in Figure 3-11. These are used with fuzzy rules that represent associations between input and output fuzzy sets.

\subsubsection{Fuzzy Inference System}

A collection of these rules is called a fuzzy inference system, which provides exceptional representation of expert knowledge and is highly interpretable. In a practical fuzzy inference system, two added steps (fuzzification and defuzzification) are required to combine crisp inputs with expert knowledge to produce a crisp output, as seen below in Figure 3-12.

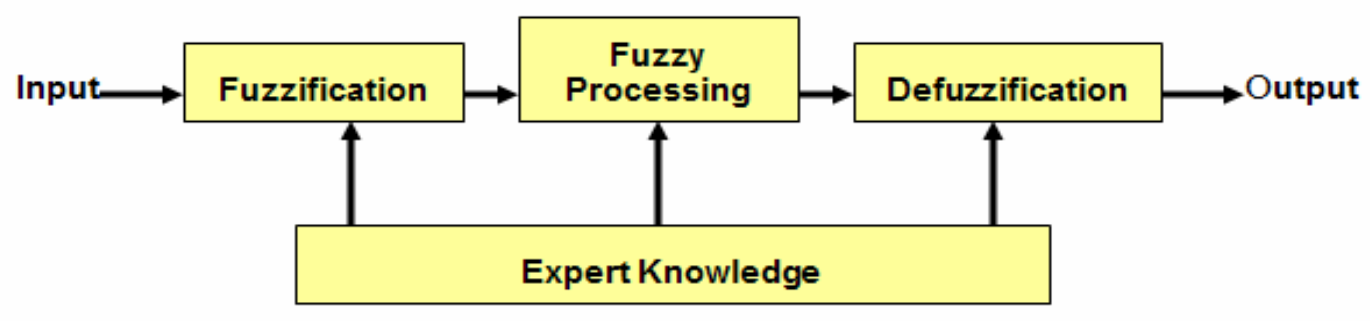

Figure 3-12: Fuzzy Inference System 
Fuzzification, the first step in the inference system, is the process by which a fuzzy representation of a crisp input value is obtained. In other words, during the fuzzification process, the membership function is calculated for each crisp input for each fuzzy rule. The second step is rule output evaluation, which allows for the modification of the output fuzzy set for each rule based on the rule strength. The final step, defuzzification, is the process by which fuzzy output values are converted into the crisp equivalent.

The main benefit of fuzzy logic lies in the fact that it simplifies the process of tapping the knowledge of domain experts ${ }^{7}$. Fuzzy methodologies are most useful for human inference, particularly for applications that involve the following:

- Encompassing expert knowledge about unknown dependencies.

- Using post-processing of outputs for decision-making.

- Interpreting models produced by non-fuzzy methods.

\subsubsection{Role of Fuzzy Logic in This Research}

Fuzzy Logic has been used to develop a lithology identification expert system. This study introduces a new and automatic approach for lithology identification. The lithology is necessary for stress calculations when sonic logs are not recorded.

The methodology uses a combination of three different logs such as spontaneous potential, resistivity, gamma ray, or other available logs. Fuzzy sets are defined for each three selected logs and the expected output. The output of the system is the type of lithology. Expert knowledge is incorporated in the fuzzy rules. The final outcome of the system is the continuous lithology identification for every foot or half-foot of log data. This continuous data lithology is used later in an empiric stress equation to determine the stress profile required for fracturing simulation. The development of each fuzzy lithology system is presented in detail in the methodology. 


\subsection{Vector Quantization}

Vector quantization is one of the most powerful technologies for data compression and dimension reduction ${ }^{29}$. The dictionary definition of quantization is the division of a quantity into a discrete number of small parts, often assumed to be integral multiples of a common quantity. The objective of vector quantization is to minimize a well-defined approximation error when the number of prototypes is fixed beforehand ${ }^{30}$. This is in contrast to clustering methods that implicitly define groupings by use of a computational procedure. In practice, the objective of vector quantization is to compress a particular sample by a pre-specified number of prototypes.

The original purpose of vector quantization was to encode continuous or discrete data vectors so that they could be transmitted over a digital communications channel. It is often used in speech and image processing where compression is necessary and some loss in quality can be endured.

A vector quantizer is a system for mapping a sequence of continuous or discrete vectors into a digital sequence suitable for communication or storage in a digital channel. Vector quantization is nothing more than an approximator. The idea is similar to that of "rounding-off" to the nearest integer. An example of a 1-dimensional VQ is shown in Figure 3-13:

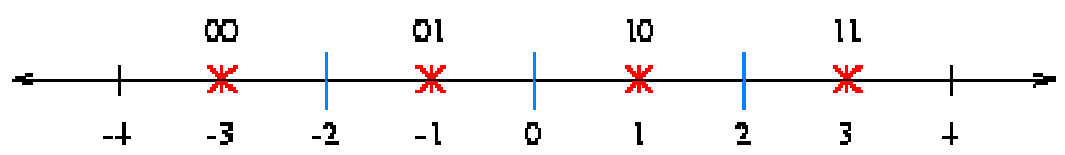

Figure 3-13: One-Dimensional Vector Quantizer

Every number less than -2 is approximated by -3 . Every number between -2 and 0 is approximated by -1 . Every number between 0 and 2 is approximated by +1 . Every number greater than 2 is approximated by +3 . Note that 2 bits uniquely represent the approximate values. This is a 1-dimensional, 2-bit VQ with the rate of 2 bits/dimension.

A vector quantizer system includes both an encoder and a decoder, as seen in Figure 3-14. First, the input vectors are divided into a constant number of separate regions, and an output vector for each region is found. Once this is complete, input vectors can be given to the encoder. The encoder will provide the index (or channel symbol) of the region to which the input vector belongs. The index is then transmitted over the digital channel. Once it reaches the decoder, it is mapped to its corresponding output vector ${ }^{29,30}$. 


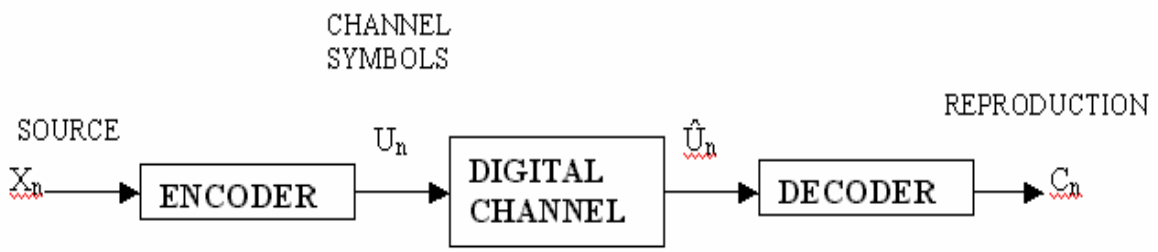

Figure 3-14: Vector Quantizer System

The data or information source $\left\{x_{n} n=0,1, \ldots\right\}$ is a sequence of random vectors. The encoder produces a sequence of channel symbols $\left\{u_{n} n=0,1, \ldots\right\}$. The sequence $\quad\left\{\bar{u}_{n} n=0,1, \ldots\right\}$ is delivered to the receiver by the digital channel. The decoder maps this sequence into the final representation of $\left\{c_{n} n=0,1, \ldots\right\}$.

\subsubsection{Role of Vector Quantization in This Research}

In this study, vector quantization has been used to compress the pressure profile signal into a smaller number of prototypes (called pressure points or pressure quantizers) in order to train a neural network. The data available for a pressure profile can reach up to 1000 pressure points when small time steps are used in recording. However, using generated data with time steps of one minute, the range of data points is between 40 and 100. Since the number of pressure points is very large to be used as output in the neural network, a simple linear vector quantization algorithm was used to reduce the number of points to 8 . Two end points were added to the existing, so that 10 points can represent every pressure profile, no matter its length. The development and implementation of the vector quantization system is presented in detail in the methodology. 


\section{CHAPTER 4: METHODOLOGY}

\subsection{Overview}

The goal of the project is to develop an intelligent application to design optimum fracture treatments and predict the expected net treating pressure. The application is designed for tight gas reservoirs where, due to very low values of permeability, the wells have to be stimulated by hydraulic fracture treatments in order to achieve an economically adequate production rate.

A classical hydraulic fracture treatment is designed to provide optimum length and conductivity to effectively stimulate the well. Similarly, this application uses an intelligent system composed of neural networks and genetic algorithms to search for the optimum fracture treatment that creates the desired fracture length. This is an optimization problem that requires the optimum combination of four treatment parameters: total fluid volume, pad volume, proppant concentration, and injection rate. The methodology consists of three steps: lithology determination and stress profile calculation, optimum fracture treatment design, and net treatment pressure prediction. These steps are outlined in detailed in the following sections.

\subsection{Model Description}

The model described in Figure 4-1 presents the big picture methodology flow chart. Three main modules form the methodology, (1) lithology identification \& stress profile, (2) fracture design treatment, and (3) pressure profile prediction. Each of these modules is described in detail in the following sections. 


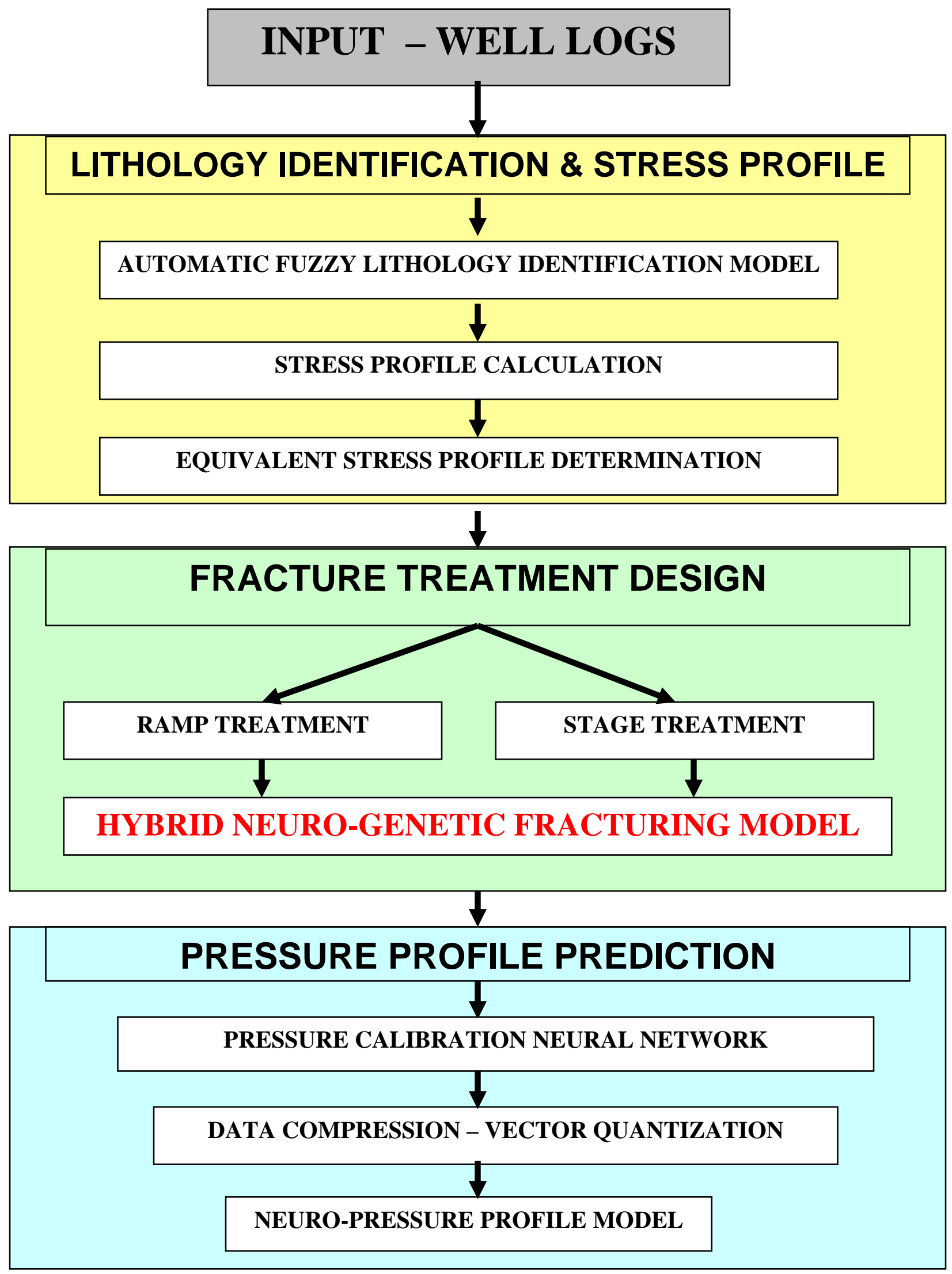

Figure 4-1: Methodology Flow Chart 


\subsection{Data Preparation Considerations}

The methodology proposed in this study is intended to design optimum fracturing treatments and predict the formation pressure response during fracturing. If this methodology proves successful it is intended to be used for actual fracturing data in a reservoir or basin.

In order to test the feasibility of this methodology, sample data was generated using a commercial fracture simulator. FRACPRO software was used for this purpose due to its powerful 3D capabilities and widespread use in the industry.

As presented in a previous chapter, fracture simulation requires quite a significant amount of information. This information can be divided into four categories:

- Fracturing Fluids Characteristics - fracture fluid viscosity, fracture fluid density, fluid loss additives.

- Proppant Characteristics - propping agent type, and propping agent volume.

- Treatment Schedule - number of stages, treatment volume, pad volume, injection rate.

- Formation characteristics - formation depth, formation permeability, in-situ stresses in the pay zone, in-situ stresses in the surrounding layers, formation modulus, reservoir pressure, formation porosity, formation compressibility, and the thickness of the reservoir.

\subsection{Fracturing Fluids}

The main types of the treatment fluids and their characteristics and properties were described in section 2.3.1 Treatment Fluid Characterization.

Typically each service company develops its own fracturing fluids, using proprietary chemicals and compositions. Depending on the base chemical used and treatment scope, the fracturing fluids are grouped in different classes, such as: Boragel, Fracgel, Hybor, Thermagel, Versagel, etc.

FRACPRO's Fluid Library was used to generate the data needed for building the model. FRACPRO includes a Fluid Library, which contains several types of fluids developed by major service companies such as Halliburton, Dowell Schlumberger, BJ Services. As a result, the fluids bear their names. Examples of the Halliburton naming conventions for different types of fluids are presented below. 
- HL_BOR_G25_1

class Boragel

- HL_FG_25_1

class Fracgel

- HL_HYB_25_1

class Hybor

- HL_THG_30

class Thermagel

- HL_VER_30

class Versagel

Since fluids from class Boragel are most often used in tight gas sands stimulation, HL_BOR_G30_1 was used for data generation. Once the methodology is proven, other fluids will be added to the final model.

\subsection{Proppant Agents}

The detail description and role of the propping agents were presented in section 2.3.2 Proppant Characterization.

In the industry there are several well-known types of sands, which typically carry the name of the region where they are produced. Examples are Brady, Jordan, Ottawa, Colorado sand, etc. Moreover, each type of sand comes in different mesh size such as: 20/40, 30/50, $16 / 30,14 / 20,12 / 20$ etc.

Proppants already available in the FRACPRO's Proppant Library were used in generating the data needed to build the model. FRACPRO included a Proppant Library that contains the most popular types of proppants used in fracturing treatments.

Examples of the most common types of proppants presented below.

- Brady $20 / 40$

- Jordan $16 / 30$

- Ottawa 20/40

- Col_It 12/20 (Colorado Sand)

Since 20/40 mesh size is one of the most popular sands used in fracturing tight gas sands, Brady 20/40 sand was used in generating data. Again, once the methodology is proven other mesh sizes and sand brands can be added to the model.

\subsection{Treatment Schedule Definition}

Designing an optimum fracturing treatment is a complex process, which requires both art and science. Typically, the treatment is designed to provide an optimum fracture length and conductivity. To achieve these desired requirements, engineers are able to control a few main parameters such as fluid (pad and slurry), proppant concentration, and injection rate.

In the early times, due to mechanical limitations the fracturing treatments were executed in stages, each stage consisting of different amount of fluid (slurry) pumped at a specific proppant 
concentration. These types of treatment are called "Stage Treatment Schedule" and are discussed in detail in this manuscript.

In this study two examples of Stage Treatment Schedules were considered in order to demonstrate the capability of this methodology in comprehensively addressing all possible variations:

- The Six Stage Treatment

- The Eight Stage Treatment

Table 4-1 presents in detail the structure of the Six Stage Treatment while Table 4-2 displays the structure of the Eight Stage Treatment.

The treatment schedule can be composed of as many stages as the user desires. In practice the total number of stages rarely exceeds ten. The example in Table 4-1 presents a stage treatment schedule containing five slurry stages, each one being characterized by specific fluid volume and proppant concentration. In this case the proppant is pumped in steps being constant during every stage. After the last slurry stage, one more stage is pumped. This last stage is called flush and is equal with the wellbore volume. Usually, during flush, Slickwater is pumped to transport the remaining slurry from the last stage from the tubing or casing into the formation, thus cleaning all the sand from the tubing/casing.

\begin{tabular}{|l|l|l|l|l|l|l|}
\hline No & Stage & $\begin{array}{l}\text { Volume } \\
(\mathrm{gal})\end{array}$ & $\begin{array}{l}\text { Rate } \\
(\mathrm{bbl} / \mathrm{min})\end{array}$ & $\begin{array}{l}\text { Starting Proppant } \\
\text { Conc }(\mathrm{ppg})\end{array}$ & $\begin{array}{l}\text { Final Proppant } \\
\text { Conc (ppg) }\end{array}$ & Fluid Type \\
\hline & & & & & & \\
\hline 1 & Pad & 21598 & 20 & - & - & HL_HYB_30_1 \\
\hline 2 & Slurry & 4773 & 20 & 1.5 & 1.5 & HL_HYB_30_1 \\
\hline 3 & Slurry & 4773 & 20 & 3 & 3 & HL_HYB_30_1 \\
\hline 4 & Slurry & 9546 & 20 & 4.5 & 4.5 & HL_HYB_30_1 \\
\hline 5 & Slurry & 11932 & 20 & 6 & 6 & HL_HYB_30_1 \\
\hline 6 & Slurry & 14318 & 20 & 7.5 & 7.5 & HL_HYB_30_1 \\
\hline 7 & Flush & 2150 & 20 & - & - & SLICKWATER \\
\hline
\end{tabular}

Table 4-1: Six-Stage Treatment Schedule

Table 4-2 presents a stage treatment containing 7 slurry stages, one pad stage, and the flush (Slickwater). Depending upon engineering practices the Stage Treatments may have a smaller or larger number of stages. 


\begin{tabular}{|l|l|l|l|l|l|l|}
\hline No & Stage & $\begin{array}{l}\text { Volume } \\
(\mathrm{gal})\end{array}$ & $\begin{array}{l}\text { Rate } \\
(\mathrm{bbl} / \mathrm{min})\end{array}$ & $\begin{array}{l}\text { Starting Proppant } \\
\text { Conc } \\
(\mathrm{ppg})\end{array}$ & $\begin{array}{l}\text { Final Proppant } \\
\text { Conc (ppg) }\end{array}$ & Fluid Type \\
\hline & & & & & & \\
\hline 1 & Pad & 21598 & 20 & - & - & HL_HYB_30_1 \\
\hline 2 & Slurry & 3573 & 20 & 1 & 1 & HL_HYB_30_1 \\
\hline 3 & Slurry & 4793 & 20 & 2 & 2 & HL_HYB_30_1 \\
\hline 4 & Slurry & 6546 & 20 & 3 & 3 & HL_HYB_30_1 \\
\hline 5 & Slurry & 8732 & 20 & 4 & 4 & HL_HYB_30_1 \\
\hline 6 & Slurry & 11318 & 20 & 5 & 5 & HL_HYB_30_1 \\
\hline 7 & Slurry & 13434 & 20 & 6 & 6 & HL_HYB_30_1 \\
\hline 8 & Slurry & 16890 & 20 & 7 & 7 & HL_HYB_30_1 \\
\hline 9 & Flush & 2150 & 20 & - & - & SLICKWATER \\
\hline
\end{tabular}

Table 4-2: Eight-Stage Treatment Schedule

With the advance of technology the new blenders allow the entire job to be pumped continuously ramping up the proppant concentration during the job. In this case the treatment consists of one pad stage and one slurry stage. This type of treatment is called "Ramp Treatment Stage". The structure of the Ramp Treatment Schedule is described in Table 4-3.

\begin{tabular}{|l|l|l|l|l|l|l|}
\hline No & Stage & $\begin{array}{l}\text { Volume } \\
\text { (gal) }\end{array}$ & $\begin{array}{l}\text { Rate } \\
\text { (bbl/min) }\end{array}$ & $\begin{array}{l}\text { Starting Proppant } \\
\text { Conc }(\mathrm{ppg})\end{array}$ & $\begin{array}{l}\text { Final Proppant } \\
\text { Conc (ppg) }\end{array}$ & Fluid Type \\
\hline & & & & & & \\
\hline 1 & Pad & 21598 & 20 & - & - & HL_HYB_30_1 \\
\hline 2 & Slurry & 39458 & 20 & 1 & 7.5 & HL_HYB_30_1 \\
\hline 3 & Flush & 2150 & 20 & - & - & SLICKWATER \\
\hline
\end{tabular}

Table 4-3: Ramp Treatment Schedule

In comparison with the Stage Treatment Schedule the Ramp Treatment Schedule consists of two main stages, pad and slurry, and ends with the flush. As in the case of Stage Treatment Schedule the flush is equal with the wellbore volume. All the proppant is pumped as ramp typically starting with a concentration of $1 \mathrm{ppg}$ to the final desired concentration.

FRACPRO is a user-friendly flexible tool that allows the possibility of using different types of treatment schedules, including both Stage Treatment Schedules and Ramp Treatment Schedules, each one being unique in its way. In this study the three types of treatments described above are considered.

\subsection{Formation Characteristics Consideration}

An accurate fracture simulation requires a significant amount of data not only related to the formation to be fractured but also considerable information describing the formations above and below. In a previous chapter the amount of information needed, its role in fracturing 
simulation and sources of collection were described in detail. The most important information that controls the fracture geometry is the stress profile, which reflects in-situ stress contrast between the production formation and the barrier formations.

In practice not all desired information is available or easy to collect. Due to high cost some of the required information is uneconomic to collect. However, in most cases well logs are already available at no extra cost. The main information that can be obtained from logs is the depth and thickness of each formation. Moreover, logs provide significant information regarding the lithology of the productive formation and the surrounding layers.

Sonic logs, together with density logs and gamma ray logs are required for closure stress gradient calculation. If sonic logs are not run in the well, the Poisson coefficient cannot be computed and as a result nor the stress gradient.

This study proposes a new methodology to calculate the closure stress gradient without sonic logs. Instead, it uses basic logs such as spontaneous potential (SP), gamma ray (GR) and deep induction logs (or resistivity). The process employs a fuzzy logic approach to automatically determine formation lithology. Once lithology is identified, a model such as the ABC stress model can be used to determine the stress profile. This methodology is presented in detail in the following chapter.

The other information defining the reservoir is related to formation pressure and temperature, porosity, permeability and viscosity of the existing fluid. Some of this information can be either measured or estimated from correlations.

In this study most of these parameters will be calculated using well-accepted correlations.

\subsection{Fuzzy Lithology Identification Approach}

Reservoir lithology is vital, not only in formation characterization ${ }^{31,32}$, but also in providing information used in fracture design and calculations. Several techniques use lithology description to calculate the stress profile. For example, the $A B C$ default model ${ }^{17}$ developed by RES (section 2.1.2.1) involves the use of defined coefficients for different types of lithology. Prior knowledge of the lithology is required in order to assign the appropriate coefficient to the corresponding formation.

The technique proposed in this study presents a flexible approach for automatic lithology identification through the use of a fuzzy logic system. The intelligent procedure will determine the type of lithology and automatically assign the corresponding coefficient, necessary for stress calculation in Equation 2.6. The system allows for flexible, changeable rules to match different reservoirs in different zones. It is designed to use basic logs for lithology identification such as spontaneous potential, gamma ray and deep induction log (or resistivity). These three logs have been selected due to their high availability in most wells. However, other logs may be used with the system, if available. 
Lithology identification usually is done visually using logs. Geologists, geophysicists, and engineers visually inspect the logs and identify the possible lithology based on specific deflections and values. Lithology identification is more accurate when using three or more logs, since at same depths different logs exhibit similar, yet specific characteristics.

In this study, it was identified that such qualitative information reflecting expert knowledge, can contribute to the determination of the in-situ stress. The best-known analytical technique for incorporation of expert knowledge into calculations is fuzzy logic.

Since formations transition from sandstone to shale, or from shale to carbonate, using crisp values in defining the lithology may induce significant errors. These zones of transition are defined by uncertainty, and this is where fuzzy sets are able to provide the best answer.

A three-input, one-output fuzzy expert system to determine the formation lithology from well logs was developed. The inputs to this system are Spontaneous Potential, Gamma Ray, and Deep Induction logs. As previously mentioned, other logs can be used in the system as well.

Three fuzzy sets (low, medium, and high) were defined for each input log. The range interval of the log is defined first and then the type of fuzzy set is considered. In Figure 4-2, the range interval was selected between 0 and 150 . For example, a value of $40 \mathrm{mv}$ of spontaneous potential has membership value of 0.67 in fuzzy set "Low" and a membership value of 0.33 in fuzzy set "Medium". Similar fuzzy sets are defined for gamma ray log and induction log.

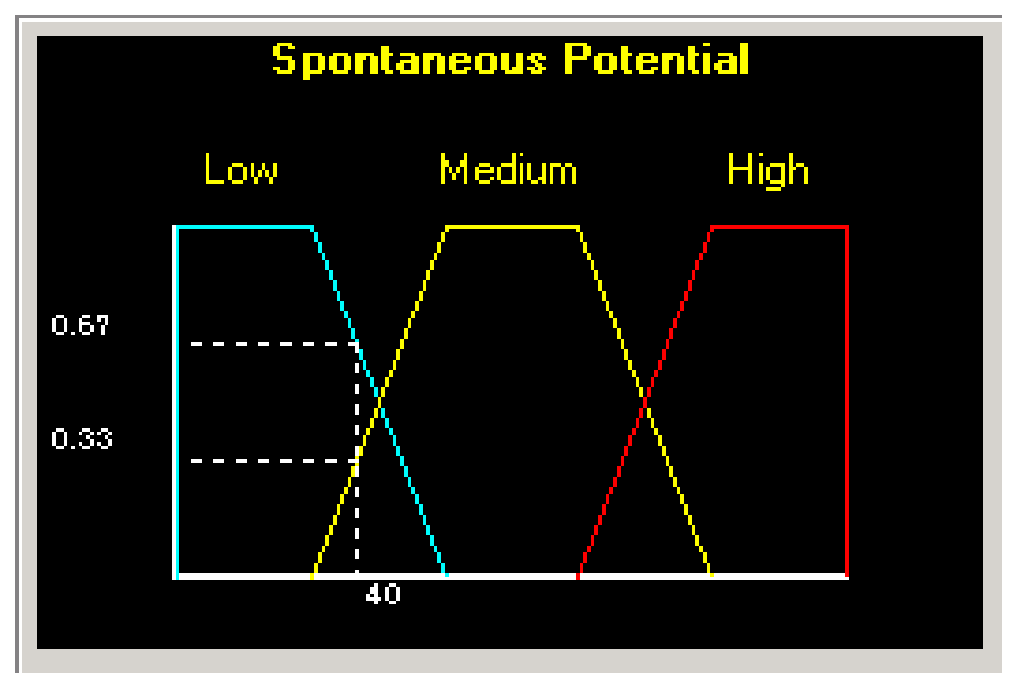

Figure 4-2: Three Fuzzy Sets for Spontaneous Potential

The fuzzy rules were defined based on the fuzzy sets. The three input logs, each one with three fuzzy sets require 27 fuzzy rules as shown in Table 4-4. Each rule was defined using expert knowledge such that the system can function for any reservoir or basin. The output of the system can be sandstone, shaly-sandstone (dirty sandstone), or shale. An example rule follows: 
If Deep Induction is "Low" and Gamma Ray is "High"

and Spontaneous Potential is "Medium", then the formation is "Shale"

\begin{tabular}{|c|c|c|c|c|c|c|c|c|c|c|c|c|}
\hline & & \multicolumn{3}{|c|}{ Deep Induction Log } & & \multicolumn{3}{|c|}{ Deep Induction Log } & & \multicolumn{3}{|c|}{ Deep Induction Log } \\
\hline & & Low & Medium & High & & Low & Medium & High & & Low & Medium & High \\
\hline \multirow{7}{*}{ Gamma Ray } & Low & Shale & SS.Sh & SandStone & Low & SS.Sh & SandStone & SandStone & Low & SS.Sh & SandStone & $\overline{\text { SandStone }}$ \\
\hline & & $T$ & $\mathrm{FT}$ & UT & & FT & $T$ & UT & & FT & $T$ & UT \\
\hline & Medium & Shale & Shale & SS.Sh & Medium & SS.Sh & SS.Sh & SandStone & Medium & SS.Sh & SS.Sh & SandStone \\
\hline & & $T$ & $\mathrm{FT}$ & $T$ & & FT & $T$ & FT & & $T$ & UT & $T$ \\
\hline & High & Shale & Shale & Shale & High & Shale & Shale & Shale & High & Shale & Shale & Shale \\
\hline & & UT & NNT & NNT & & UT & $T$ & NNT & & UT & NNT & NNT \\
\hline & & \multicolumn{3}{|c|}{ Spontaneous Potential - LOW' } & & \multicolumn{3}{|c|}{ Spontaneous Potentia - MEDUUM } & & \multicolumn{3}{|c|}{ Spontaneous Potential $\cdot H I G H$} \\
\hline
\end{tabular}

Table 4-4: Fuzzy Rules for the Fuzzy Lithology Identification System

The previous statement defines a rule of the fuzzy set. In addition, each rule is qualified using an approximate reasoning methodology as being true (T), very true (VT), fairly true (FT), and not necessarily true (NNT). In this context, not necessarily true refers to cases that are nearly impossible to be found in nature.

Using a fuzzy set theory-based approach for defining formation lithology provides the necessary means to identify rock types that cannot distinctly be defined as sandstone, shale or carbonate. Fuzzy terms, used for years in the oil industry such as sandy-shale or shaly-sand, are easily accommodated in this approach and contribute to success of the methodology presented in this study.

As seen in Table 4-4, the rules of log interpretation can be described by fuzzy terms used in fuzzy rules, which are incorporated in the fuzzy decision support system. During a fuzzy inference process, several fuzzy rules will be fired in parallel. The parallel rule firing, unlike sequential evaluation, is much closer to the human reasoning process. The output of the fuzzy inference process is the type of lithology. An example of the lithology determination by the fuzzy system is presented in Figure 4-3. In this figure, both sandstone and shale are represented in the fuzzy sets and the lithology estimated is dirty sandstone or shaly-sand. 


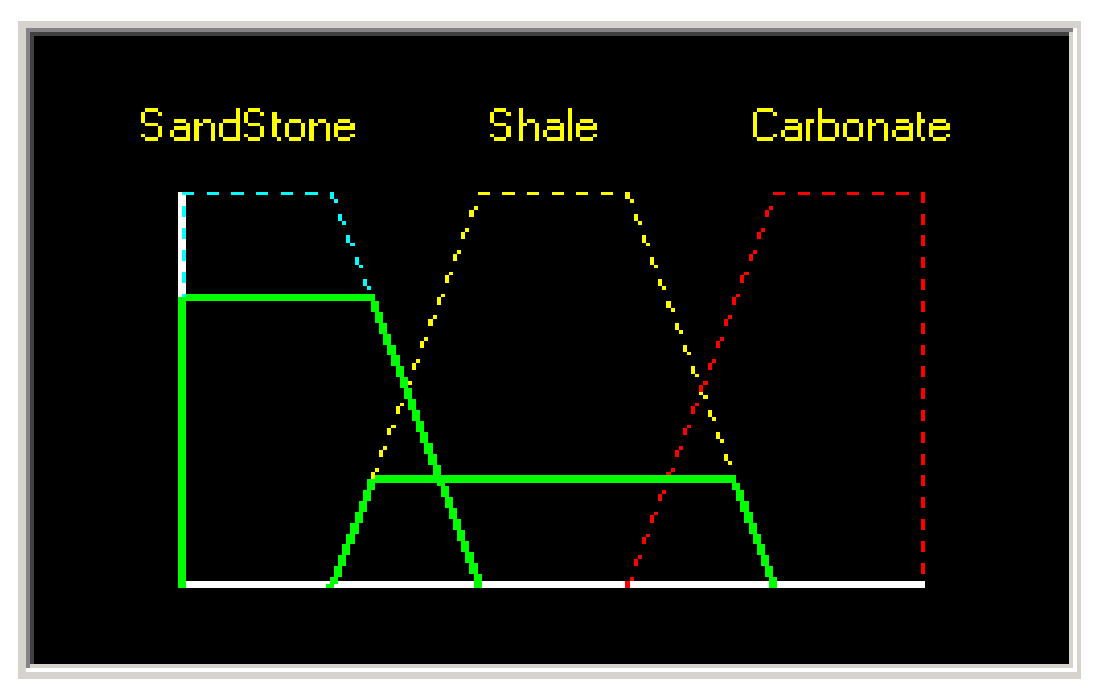

Figure 4-3: Fuzzy Decision System

The estimation is done for each recorded value of spontaneous potential, gamma ray and resistivity. Since logs are recorded each 0.5 or $1 \mathrm{ft}$, the fuzzy system assigns the type of lithology for every recording. With the lithology defined for every 0.5 or $1 \mathrm{ft}$, the system automatically assigns the corresponding lithology coefficients, needed in Equation 2-6 for stress calculation.

\subsection{Stress Profile Calculations}

The stress profile is known to be the most important input in designing a successful fracture treatment. Oftentimes, the formation intended for fracturing is not neatly bound by top and bottom layers. Occasionally, the productive formation itself contains many thin layers of nonproductive rock. It is important to understand the stresses in the surrounding zones as well as the productive zone. As mentioned in previous chapters, the stress profile can be estimated from log measurements. Since these calculations are crucial in the design of a fracture treatment, the calculated stress profile is usually calibrated with the stress values obtained from in-situ stress test analysis, when these are available.

The stress profile can be calculated using one of the models presented in section 2.1.2.1 Use of Hook's Law in Calculating the Log-Derived Stress. However, some of the models can be used only when sonic logs are available. In the absence of the sonic logs, the lithology model ( $A B C$ model) presented in section 2.1.2.1, can be used ${ }^{33}$. The result is a continuous stress profile curve for the considered interval as shown in Figure 4-4. Next, the stress profile is processed to illustrate a clear definition of the stress zones.

Stress profile processing is an early step that engineers take in order to compress data and better define different layers. Similar consecutive stress values are grouped together to define possible shale and sandstone formations by visually analyzing the stress profile curve and 
logs. For example, in Figure 4-4, there is a different stress value for every foot between 11,040 and 11,100 ft. However, these values are very similar and all within a close range. This interval would be considered one formation and represented by one stress value, which is calculated as the arithmetic average of all stress profile values between the two depths. This is shown in Figure 4-5, where the continuous jagged red curve represents the original stress profile and the thick blue step line shows the processed stress profile. In addition, Table 4-5 presents the lithology of the formations resulted after geology and engineering interpretation. In section 4.10 Equivalent Stress Profile, an explicit example of stress profile processing is provided. 


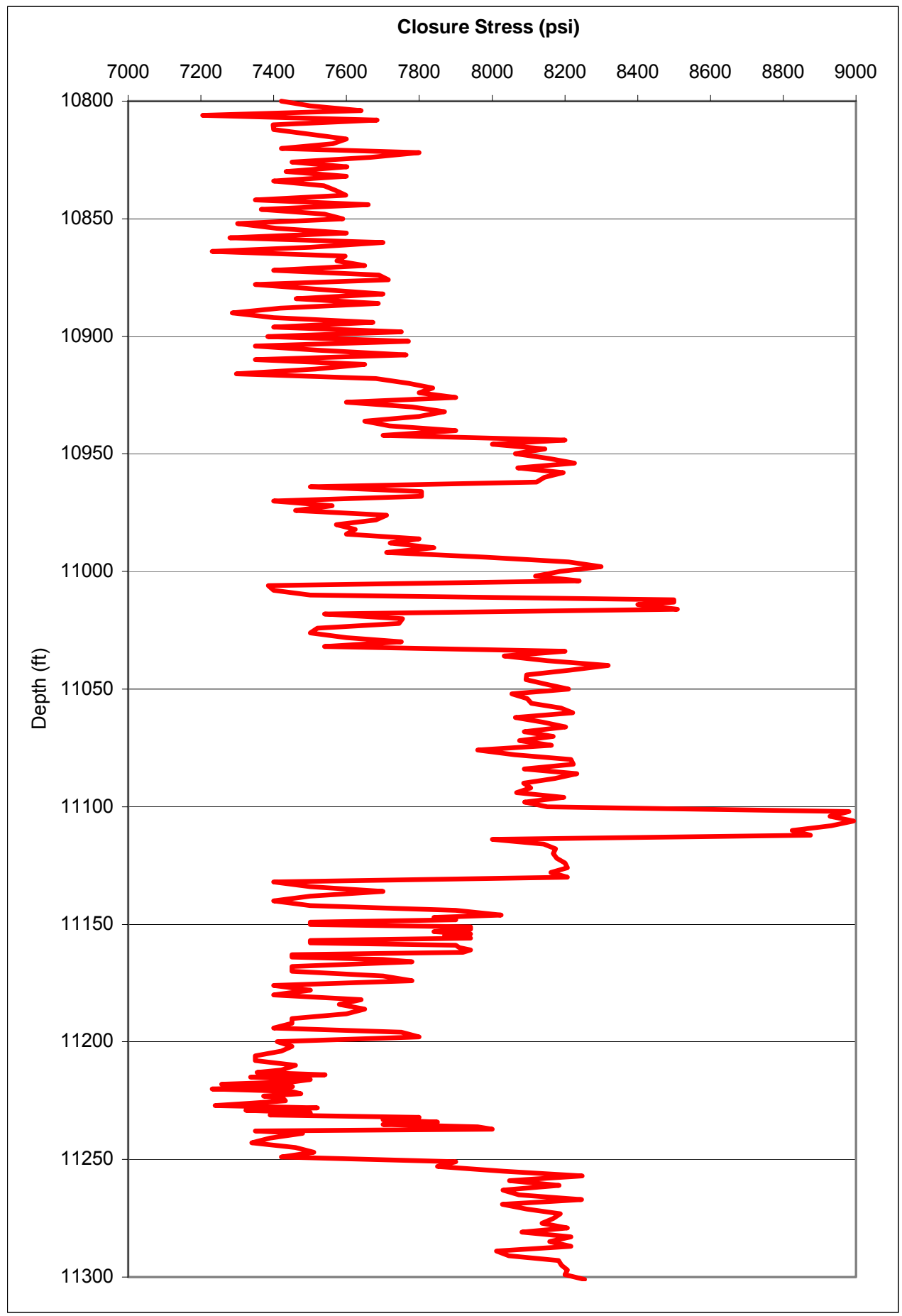

Figure 4-4: Log-Derived Stress Profile 


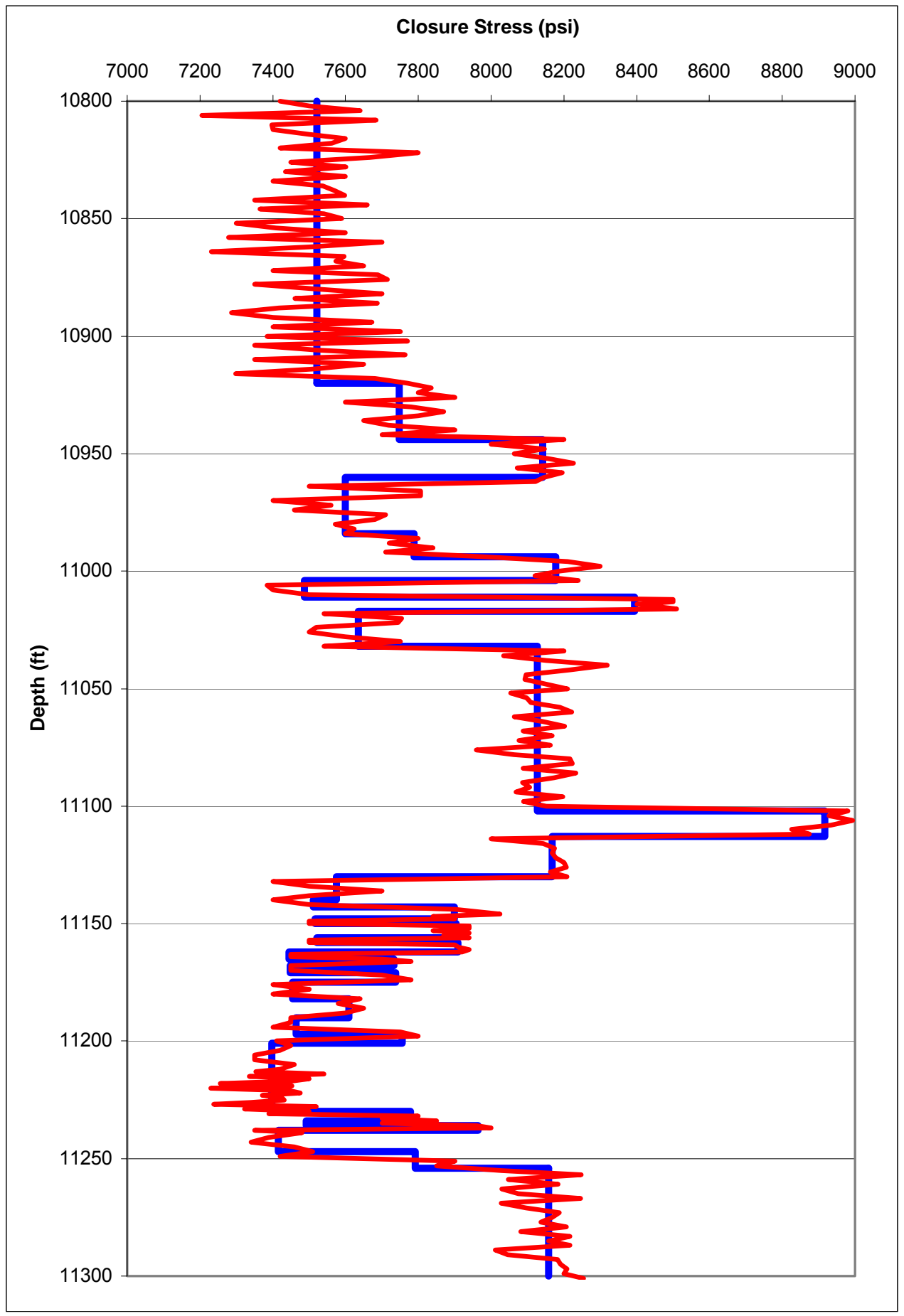

Figure 4-5: Original and Processed Stress Profile 


\begin{tabular}{|c|c|c|c|c|c|c|c|}
\hline Layer & Upper & Lower & Layer & Layer & Poisson's & Closure Str. & Closure \\
\hline Description & Depth & Depth & Thickness & Permeability & Ratio & Gradient & Stress \\
\hline Shale 1 & 10800 & 10920 & 120 & 0 & 0.23 & 0.69 & 7521.07 \\
\hline Shale 2 & 10920 & 10944 & 24 & 0 & 0.25 & 0.71 & 7747.24 \\
\hline Shale 3 & 10944 & 10960 & 16 & 0 & 0.29 & 0.74 & 8141.81 \\
\hline Shale 1 & 10960 & 10984 & 24 & 0 & 0.23 & 0.69 & 7598.63 \\
\hline Shale 2 & 10984 & 10994 & 10 & 0 & 0.25 & 0.71 & 7787.64 \\
\hline Shale 3 & 10994 & 11004 & 10 & 0 & 0.29 & 0.74 & 8176.75 \\
\hline Shale 4 & 11004 & 11011 & 7 & 0 & 0.22 & 0.68 & 7487.15 \\
\hline Shale 5 & 11011 & 11017 & 6 & 0 & 0.30 & 0.76 & 8394.24 \\
\hline Shale 1 & 11017 & 11032 & 15 & 0 & 0.23 & 0.69 & 7634.99 \\
\hline Shale 6 & 11032 & 11102 & 70 & 0 & 0.28 & 0.73 & 8127.56 \\
\hline Shale 7 & 11102 & 11113 & 11 & 0 & 0.34 & 0.80 & 8916.29 \\
\hline Shale 6 & 11113 & 11132 & 19 & 0 & 0.28 & 0.73 & 8168.32 \\
\hline Shale 4 & 11132 & 11140 & 8 & 0 & 0.22 & 0.68 & 7574.56 \\
\hline Redfork 1 & 11140 & 11143 & 3 & 0.01182 & 0.21 & 0.67 & 7511.35 \\
\hline Shale 2 & 11143 & 11148 & 5 & 0 & 0.25 & 0.71 & 7898.55 \\
\hline Redfork 1 & 11148 & 11150 & 2 & 0.01182 & 0.21 & 0.67 & 7516.40 \\
\hline Shale 2 & 11150 & 11156 & 6 & 0 & 0.25 & 0.71 & 7903.86 \\
\hline Redfork 1 & 11156 & 11158 & 2 & 0.01182 & 0.21 & 0.67 & 7521.80 \\
\hline Shale 2 & 11158 & 11162 & 4 & 0 & 0.25 & 0.71 & 7908.82 \\
\hline Redfork 2 & 11162 & 11165 & 3 & 0.01182 & 0.20 & 0.67 & 7444.05 \\
\hline Shale 1 & 11165 & 11168 & 3 & 0 & 0.23 & 0.69 & 7733.33 \\
\hline Redfork 2 & 11168 & 11171 & 3 & 0.01182 & 0.20 & 0.67 & 7448.05 \\
\hline Shale 1 & 11171 & 11175 & 4 & 0 & 0.23 & 0.69 & 7737.83 \\
\hline Redfork 2 & 11175 & 11182 & 7 & 0.01182 & 0.20 & 0.67 & 7454.05 \\
\hline Shale 4 & 11182 & 11190 & 8 & 0 & 0.22 & 0.68 & 7608.57 \\
\hline Redfork 2 & 11190 & 11197 & 7 & 0.01182 & 0.20 & 0.67 & 7464.05 \\
\hline Shale 1 & 11197 & 11201 & 4 & 0 & 0.23 & 0.69 & 7755.84 \\
\hline Redfork 3 & 11201 & 11230 & 29 & 0.01182 & 0.19 & 0.66 & 7398.08 \\
\hline Shale 1 & 11230 & 11234 & 4 & 0 & 0.23 & 0.69 & 7778.69 \\
\hline Redfork 2 & 11234 & 11236 & 2 & 0.01182 & 0.20 & 0.67 & 7491.73 \\
\hline Shale 2 & 11236 & 11238 & 2 & 0 & 0.25 & 0.71 & 7963.39 \\
\hline Redfork 3 & 11238 & 11247 & 9 & 0.01182 & 0.19 & 0.66 & 7415.89 \\
\hline Shale 1 & 11247 & 11254 & 7 & 0 & 0.23 & 0.69 & 7791.51 \\
\hline Shale 8 & 11254 & 11500 & 246 & 0 & 0.26 & 0.72 & 8157.74 \\
\hline
\end{tabular}

Table 4-5: Reservoir Lithology

\subsection{Equivalent Stress Profile}

In Figure 4-5 the continuous scatter line represents the stress profile calculated every 0.5 or $1 \mathrm{ft}$, while the bold step line illustrates a clear definition of the stress zones, which defines each potential layer. Table 4-5 clearly points to the large amount of input data (number of layers and their properties) needed for a proper fracture simulation. Moreover, no two reservoirs are the 
same; reservoirs exist at different depths and have different lithologies. Therefore, based on the number of the inputs, it can be concluded that:

- The number of the layers may vary from 3 to 50 or more.

- Each layer is situated at a specific depth.

- Each layer has a unique thickness.

- Each layer has a unique closure gradient stress (and closure stress).

- Each layer has a unique permeability, if productive.

In the conditions outlined above, it is impractical to train a neural network using all the layers from the lithology. Therefore, it becomes necessary to develop a methodology that reduces the total number of layers to a few representative layers. Meanwhile, the methodology should have a universal applicability for different numbers of layers, different lithology, and different layer thicknesses. In addition, this equivalent lithology should provide not only the same output as the original profile, but also the same shape of the fracture when used in simulation.

This study proposes an algorithm to reduce the stress profile, and consequently the reservoir representation, to a representative model of a few layers.

\subsubsection{Methodology for Generating Equivalent Stress Profile}

A first attempt to develop an equivalent stress profile considered reducing the total number of layers to three. These layers were named as follows:

- Top layer - The formations above the productive formation merged together.

- Pay zone - The production layers and the intercalation merged together.

- Bottom layer - The formations below the pay zone merged together.

A real case of a well in Texas was considered to analyze the robustness of the threelayer profile compared to the original profile. The well has a complete data set and was hydraulic fractured for increased productivity. To determine the characteristics of the proposed three-layer model, an averaging technique was used. This methodology merges the layers into one layer with a thickness equal to the sum of the thicknesses of all layers. The methodology also determines the closure stress gradient of the equivalent layer, defined by the following relationship: 


$$
\begin{aligned}
& \sigma_{\text {eq }}= \frac{\sum \sigma{ }^{*} \mathrm{hi}}{\sum \mathrm{hi}} \\
& \text { where, } \sigma_{\mathrm{eq}}=\text { closure stress gradient of the equivalent layer, psi/ft } \\
& \sigma \mathrm{i}=\text { closure stress of each layer, psi } \\
& \mathrm{hi}=\text { thickness of each layer, } \mathrm{ft}
\end{aligned}
$$

A similar relationship was used to calculate the equivalent permeability of the productive formation.

$$
\begin{aligned}
& \mathrm{K}_{\mathrm{eq}}=\frac{\sum \mathrm{ki} * \mathrm{hi}}{\sum \mathrm{hi}} \\
& \text { where, } \mathrm{K}_{\mathrm{eq}}=\text { equivalent permeability of the productive layer, } \mathrm{mD} \\
& \mathrm{ki}=\text { permeability of each layer, } \mathrm{mD} \\
& \mathrm{hi}=\text { thickness of each layer, } \mathrm{ft}
\end{aligned}
$$

Using Equation 4-1, top layer is defined by an average stress value and thickness equal to sum of all layers thickness above. Similarly, the production layers were merged into one layer, as were the formations below the target formation. The permeability of the pay zone was calculated using Equation 4-2. Figure 4-6 shows the processed stress profile and the three layers equivalent profile.

FRACPRO was run in both cases, using the three layers stress profile and the original profile. Analyzing the results, it was concluded that the major difference between two cases was the geometry of the fracture. 


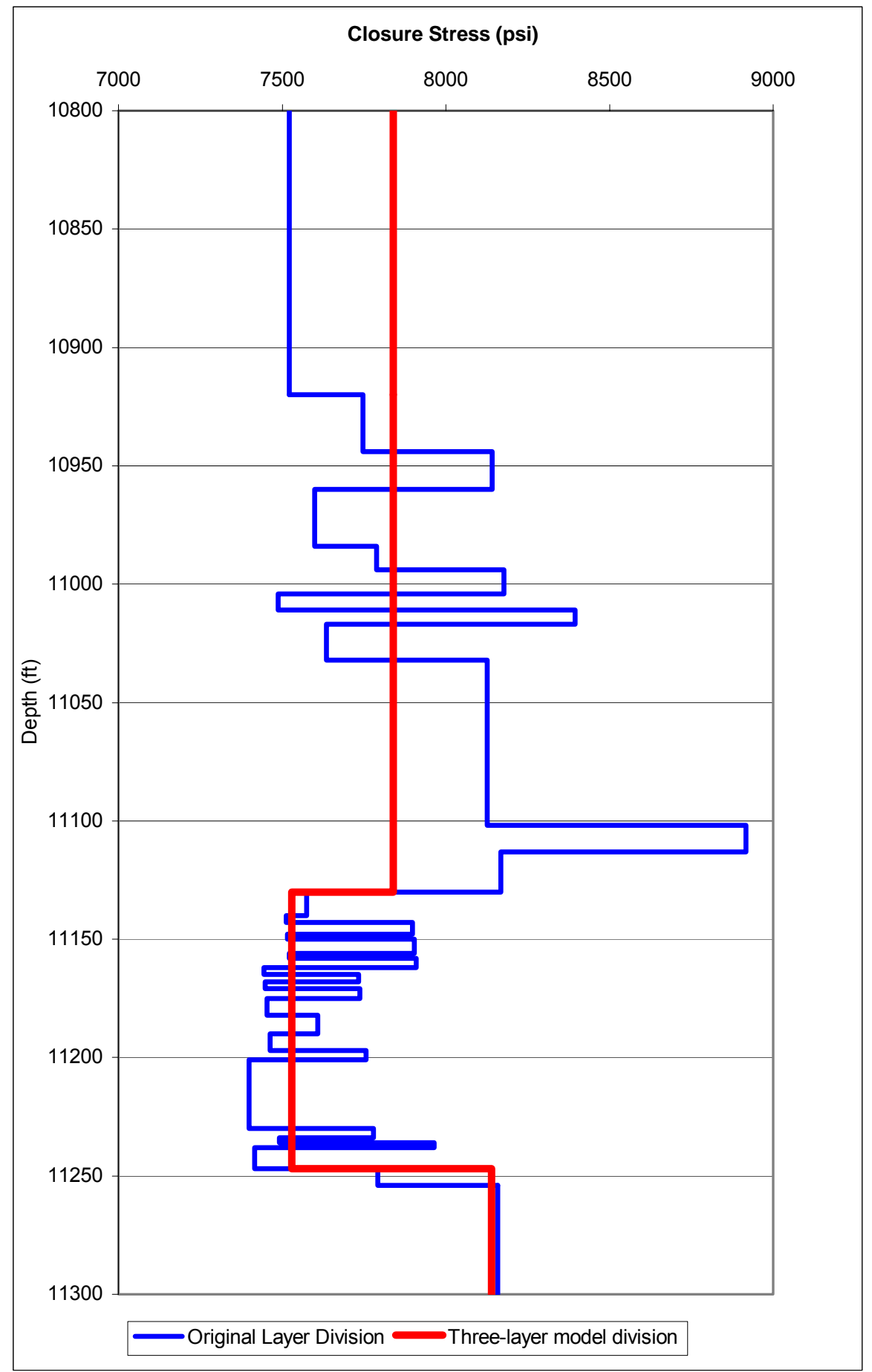

Figure 4-6: Three-Layer Equivalent Stress Profile 
To study the impact of these three layers on the shape of the fracture and the typical outputs (fracture length, total height, width and conductivity), 72 random generated cases were considered. These cases were generated using the following assumptions:

- The number of layers above the productive formation was considered between 6 and 14 layers.

- The number of layers for the productive formation was considered between 6 and 12 layers.

- Poisson coefficient for each layer was randomly generated between 0.215 and 0.34 . This was considered a good range for the coefficient.

- Each formation thickness was considered between 5 and $70 \mathrm{ft}$.

- The sum of layer thicknesses above the productive formation should not be greater then $270 \mathrm{ft}$.

The closure stress gradient was calculated using the relationship:

$$
\begin{aligned}
\operatorname{GradPc} & =\left(\frac{v}{1-v}\right) \operatorname{Pob}+\left(1-\left(\frac{v}{1-v}\right)\right) \mathrm{Pp} \quad \text { Equation 4-3 } \\
\text { where, } & \mathrm{Pob}=\text { overburden pressure }(\mathrm{psi} / \mathrm{ft}) \\
& \mathrm{Pp}=\text { pore pressure }(\mathrm{psi} / \mathrm{ft}) \\
& v=\text { Poison coefficient }
\end{aligned}
$$

The value for the overburden pressure was assumed as $1.1 \mathrm{psi} / \mathrm{ft}$, which represents a typical value for this type of calculation. The pore pressure is usually defined between 0.43 and $0.65 \mathrm{psi} / \mathrm{ft}$. A default average value of $0.54 \mathrm{psi} / \mathrm{ft}$ was used. The closure stress of each layer was calculated based on the gradient closure stress and depth to the middle of the layer.

$$
\begin{aligned}
& \sigma_{\mathrm{j}}=\left(\mathrm{H}_{\mathrm{j}}+\mathrm{H}_{\mathrm{j}+1}\right) / 2 \text { * GradPc } \\
& \text { where, } \sigma_{\mathrm{j}}=\text { closure stress of the layer } \mathrm{j} \text {, psi } \\
& \qquad \begin{array}{l}
\mathrm{Hj}=\text { depth to the top of the layer } \mathrm{j}, \mathrm{ft} \\
\mathrm{H}_{\mathrm{j}+1}=\text { depth to the bottom of the layer } \mathrm{j}, \mathrm{ft} \\
\mathrm{hj}=\text { thickness of the layer } \mathrm{j}\left(\mathrm{hj}=\mathrm{H}_{\mathrm{j}+1}-\mathrm{Hj}\right), \mathrm{ft}
\end{array}
\end{aligned}
$$

A simple Visual Basic code was developed to generate the cases used in the study. The program generates a random number of layers, random values for Poisson Ratio and thickness, all within the specified ranges. Using these values, the gradient closure stress and formation closure stress is calculated for each generated layer. 


\subsubsection{Model Accuracy}

Using the methodology presented above, all 72 generated cases were reduced to a three-layer model. For each of the 72 cases FRACPRO was run using both methodologies; first using all the layers (original profile) and then the three equivalent layers. The error value between the outputs of the original stress profile and the equivalent stress profile was calculated in order to measure the fit between the fracture geometries. For most of the 72 cases, the error for the fracture propped length between the two models was greater than $12 \%$. This was a significantly high value that was not accepted for the equivalent model.

Analyzing the shape of the fracture for the two scenarios, it was concluded that the highest closure stress layer plays a very important role in the geometry of the fracture. This layer was named the significant overburden layer. Consequently, the significant overburden layer was placed at the bottom of the top layer, right above the target zone. Figure 4-7 shows the new stress profile arrangement with the overburden layer placed above pay zone. With this assumption FRACPRO was run again for the 72 cases. In this scenario, the error for the fracture propped length was diminished to a range between 0 and $8 \%$, with an average of less than $4 \%$. These results were considered satisfactory and this new four-layer model was adopted. Table 4-6 shows the error for the fracture propped length between the original lithology and the equivalent profile.

In addition, the influence of the position of the significant overburden layer within the sequence of layers on fracture propped length was studied. The entire top layer was divided in four equally regions as follows:

- Region A - topmost region of the top layer,

- Region B - right below region A,

- Region $\mathrm{C}$ - right below region $\mathrm{B}$,

- Region $\mathrm{D}$ - the region right above the productive formation.

The study showed that higher error values are present when the overburden layer belongs to regions $A$ and $B$. As the position of the overburden layer gets closer to the top of the productive formation, the error values diminish as seen in Table 4-6.

The newly added layer, placed right on top of the target formation, was named the immediate overburden layer in the model. It is defined as the highest stress layer in the top sequence of the lithology and proved to be the main component in controlling the fracture geometry. As a result, the equivalent stress profile adopted in this study consists of four-layer equivalent model. In this new representation, the number of inputs required for neural network training decreases to eight, as listed below: 
1. Thickness of the top layer.

2. Closure gradient stress of the top layer.

3. Thickness of the overburden layer.

4. Closure gradient stress of the overburden layer.

5. Thickness of the pay zone.

6. Closure gradient stress of the pay zone.

7. Permeability of the pay zone.

8. Closure gradient stress of the bottom layer. 


\begin{tabular}{|c|c|c|c|c|c|}
\hline \multirow[t]{2}{*}{ No } & \multirow[t]{2}{*}{ Region } & \multirow{2}{*}{$\begin{array}{l}\text { Overburdon Layer } \\
\text { Thickness }\end{array}$} & \multicolumn{2}{|c|}{ Fracture Propped Length } & \multirow[t]{2}{*}{ Error } \\
\hline & & & All Layers & 4 Layers Model & \\
\hline & & $\mathrm{ft}$ & ft & $\mathrm{ft}$ & \\
\hline & & & & & \\
\hline 1 & $\mathrm{C}$ & 13.72 & 290 & 299 & 3.10 \\
\hline 2 & $\mathrm{C}$ & 19 & 302.57 & 289.3 & 4.39 \\
\hline 3 & A & 22.58 & 292.08 & 290.57 & 0.52 \\
\hline 5 & A & 27 & 361.37 & 340.47 & 5.78 \\
\hline 7 & B & 16.5 & 363.94 & 349.25 & 4.04 \\
\hline 1 & $\mathrm{D}$ & 42.5 & 351.45 & 331.22 & 5.76 \\
\hline 2 & $\mathrm{C}$ & 12.54 & 338.96 & 353.89 & -4.40 \\
\hline 5 & B & 12.8 & 356.41 & 337.22 & 5.38 \\
\hline 6 & $\mathrm{D}$ & 7.77 & 347.41 & 337.73 & 2.79 \\
\hline 7 & $\bar{B}$ & 19.5 & 350.63 & 340.75 & 2.82 \\
\hline 9 & $\mathrm{C}$ & 15.7 & 347.49 & 337.88 & 2.77 \\
\hline 11 & $\mathrm{C}$ & 28.3 & 326.85 & 330.09 & -0.99 \\
\hline 14 & $\mathrm{D}$ & 10.5 & 343.26 & 342.23 & 0.30 \\
\hline 15 & $\mathrm{D}$ & 19.5 & 335.8 & 344.36 & -2.55 \\
\hline 16 & $\mathrm{C}$ & 19.63 & 329.25 & 317.23 & 3.65 \\
\hline 18 & $\bar{\theta}$ & 8 & 355.8 & 334.34 & 6.03 \\
\hline 19 & $\mathrm{D}$ & 10.45 & 360.54 & 337.54 & 6.38 \\
\hline 20 & $\mathrm{D}$ & 24.54 & 346.52 & 329.86 & 4.81 \\
\hline 19 & $\mathrm{D}$ & 11.49 & 353.33 & 333.93 & 5.49 \\
\hline 20 & $\bar{B}$ & 11.68 & 332.37 & 335.63 & -0.98 \\
\hline 21 & $\mathrm{AB}$ & 24 & 358.36 & 338.76 & 5.47 \\
\hline 22 & $\mathrm{C}$ & 8.93 & 344.42 & 332.08 & 3.58 \\
\hline 23 & $\bar{B}$ & 10.68 & 301.51 & 329.95 & -9.43 \\
\hline 24 & $\bar{B}$ & 15.62 & 352.68 & 335.66 & 4.83 \\
\hline 25 & B & 43.61 & 350.39 & 346.23 & 1.19 \\
\hline 26 & $\mathrm{C}$ & 44.4 & 334.35 & 347.78 & -4.02 \\
\hline 27 & $\mathrm{~B}$ & 21.54 & 351.93 & 334.74 & 4.88 \\
\hline 28 & $\bar{\theta}$ & 24.8 & 340.23 & 341.06 & -0.24 \\
\hline 29 & $\mathrm{D}$ & 13.77 & 354.33 & 335.53 & 5.31 \\
\hline 30 & $\mathrm{C}$ & 8.78 & 324.3 & 290.72 & 10.35 \\
\hline 31 & $\mathrm{D}$ & 21.45 & 359.65 & 342.48 & 4.77 \\
\hline 32 & $\mathrm{D}$ & 23.7 & 361.88 & 342.83 & 5.26 \\
\hline 33 & $\mathrm{C}$ & 49.88 & 335.17 & 348.24 & -3.90 \\
\hline 34 & $\mathrm{~A}$ & 36.18 & 311.59 & 346.13 & -11.09 \\
\hline 35 & $\bar{B}$ & 59.67 & 348.71 & 350.37 & -0.48 \\
\hline 36 & $\bar{D}$ & 30.3 & 357.04 & 350.55 & 1.82 \\
\hline 37 & $\bar{D}$ & 25.95 & 358.4 & 343.56 & 4.14 \\
\hline 38 & $\mathrm{D}$ & 20.22 & 355.3 & 341.61 & 3.85 \\
\hline 39 & $\mathrm{D}$ & 11.76 & 355.66 & 338.94 & 4.70 \\
\hline 40 & A & 40.05 & 346.47 & 347.55 & -0.31 \\
\hline 41 & $\mathrm{~B}$ & 25.27 & 338.78 & 337.49 & 0.38 \\
\hline 42 & $\mathrm{C}$ & 31.48 & 325.02 & 341.19 & -4.98 \\
\hline
\end{tabular}

Table 4-6: Accuracy of the Four Layer Equivalent Profile 
A graphical representation of the original stress profile and the representative four-layer profile are seen in Figure 4-7.

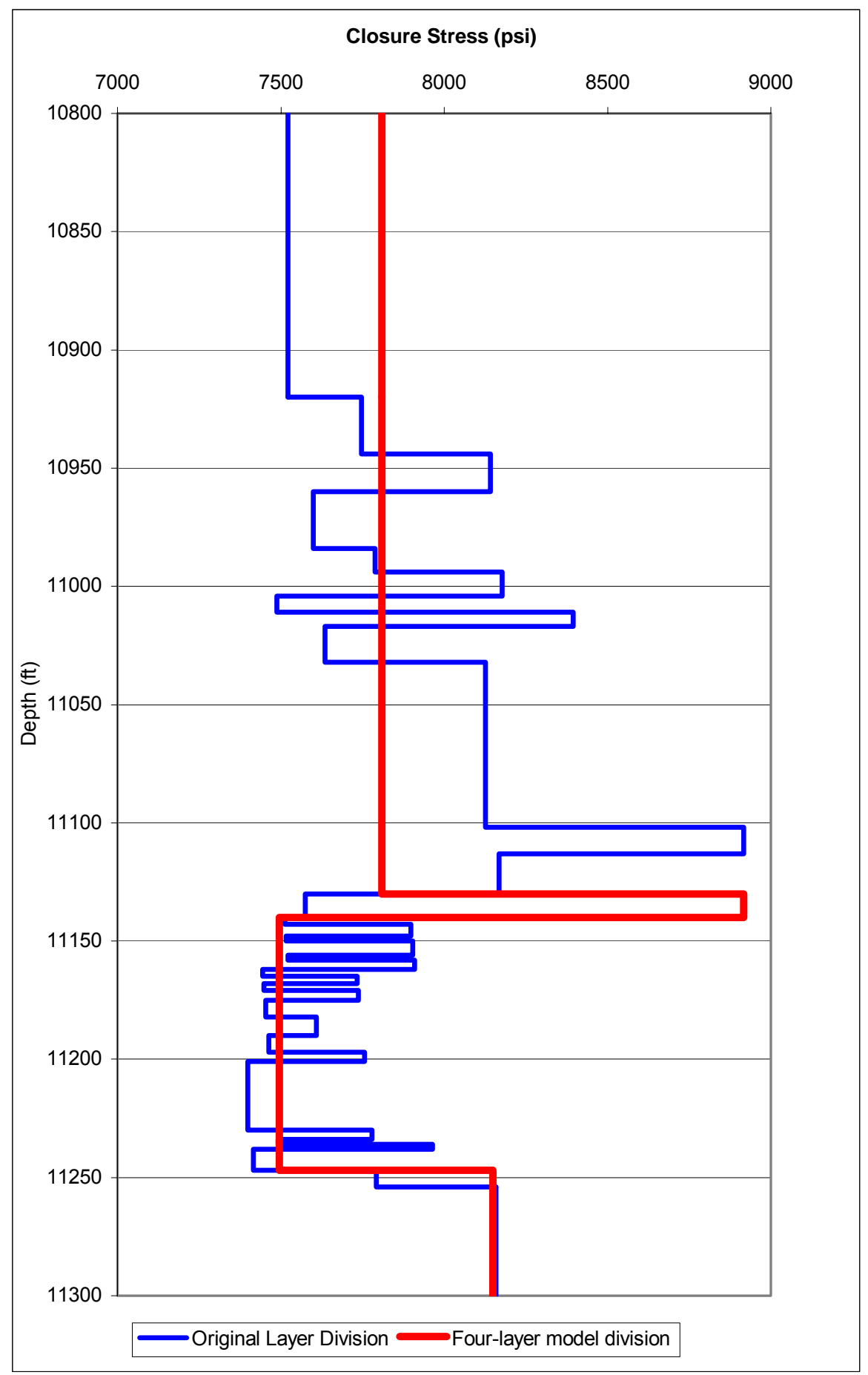

Figure 4-7: Four-Layer Equivalent Stress Profile 


\subsection{Database Generation for Fracture Design}

In this study a combination of neural networks and genetic algorithms, both regarded as artificial intelligence tools, are used to search for the optimum fracture treatment to create the desired fracture length. Typically, the neural network is trained on historic data and captures the relationship between a certain data set of input parameters and the corresponding output parameters.

In order to train a neural network model with reasonable accuracy, one must have access to a significant amount of data. This study uses simulated data to prove its intended methodology.

\subsubsection{Overview}

Since fracture treatments are performed in fields or basins that typically contain hundreds of wells, large databases containing fracturing information are continuously generated. Moreover, these databases grow with every new treatment executed. The information recorded in these databases can be of a great value to engineers attempting to optimize fracturing treatments.

Since the information used in this work is simulated, the data must contain all required parameters needed for a complex fracturing treatment. Furthermore, is it required that the data would show significant variation in order to capture the relationships between the input and output $^{34}$. FRACPRO was used to generate the data for training the neural networks. The model was designed for tight gas sands where long fractures are desired.

\subsubsection{Input/Output Classification and Data Statistics}

Input data required for modeling a fracture simulation typically consists of treatment details and formation data as was stated in detail in section 1.3.3 Data Requirements for Hydraulic Fracturing. The output of the simulation contains several parameters. Since most fractures are designed to achieve a specific fracture length and conductivity, these two parameters are the most important outcomes of the system. In addition to fracture length and conductivity, a simulation run also calculates total fracture height and width. These two parameters, along with the fracture length, define the geometry of the fracture. FRACPRO provides a final important parameter: fracture efficiency, which is related to the effectiveness of fluid use during fracturing. If no leak-off occurs, the value of the fracture efficiency is 1.

The following sections will focus on the input and output parameters as defined for each of the treatment designs considered in the study. Statistics for each of the input/output data sets are included for each type of treatment. 


\subsubsection{Ramp Treatment Schedules}

As described in section 4.6 Treatment Schedule Definition, ramp treatment schedules are pumped while continuously ramping up the proppant concentration during the job. The input parameters listed below are used for running FRACPRO to generate the output. A total number of 675 cases (listed in the Appendix Table A - 1) were generated for training and testing the neural network. Each case is unique and contains 14 different parameters as follows:

1. Total fluid volume - the range of values considered for this parameter was based on recommendations provided by Halliburton engineers. Typical treatment jobs performed by Halliburton use between 10,000 to 200,000 gallons.

2. Pad volume - typically is considered between $35-45 \%$ of total fluid volume.

3. Final proppant concentration - the range for this parameter was considered between 6 and 16 pounds per gallon (ppg). Typical ramp jobs are 6 to $10 \mathrm{ppg}$. The proppant is pumped as a ramp function, from a starting value or $1 \mathrm{ppg}$ (default) to the final concentration.

4. Average injection rate - controls the time of the treatment. In the case of jobs where large amounts of fluid are pumped, the injection rate is higher, thus reducing the job time. The range for the injection rate was considered between 15 and $40 \mathrm{bbl} / \mathrm{min}$.

5. Depth to the top of the "productive formation" - the range for the depth was considered to be $5000-15,000 \mathrm{ft}$. The depth controls the stress profile and pore pressure.

6. Reservoir pressure - considered as an input although calculated as a function of depth. In the case of tight gas sands, fracture jobs are executed in new wells or when the formation pressure has not dropped more than 2000 psia. Therefore, the reservoir pressure was calculated as the initial reservoir pressure (depth * pressure gradient) minus a value of 2000 multiplied by a random number. This assumption provides a good range in reservoir pressure values that can occur in reservoirs.

7. Permeability - controls the leakoff of the fluid in the formation. Since the study addresses the case of the tight gas sands, the values for this parameter were considered within a range of $0.001-0.1 \mathrm{mD}$. This permeability is actually the permeability of the "equivalent pay zone" which in a real case will be calculated using an averaging technique.

8. Top layer thickness - was considered in a range of $170-250 \mathrm{ft}$. Two facts contribute to this range selection:

- It is time consuming to define lithology above the productive formation for more than $300 \mathrm{ft}$, assuming that logs are available

- The upper height of the fracture does not develop higher than 200-250 ft.

9. Closure stress gradient for the equivalent top layer - considered between 0.72-0.85 psi/ft. This was concluded from the previous analysis when the layers above the productive formation were merged to form the equivalent top layer. 
10. Overburden layer thickness - restricted to values between 10 and $50 \mathrm{ft}$. It has been observed from logs that a high stress layer does not usually present a high value of thickness.

11. Closure stress gradient of the overburden layer - considered within a range of $0.74-0.85$ psi/ft. This parameter is designed to always be greater than the closure stress of the equivalent top layer.

12. Pay zone thickness - values between 50 and $130 \mathrm{ft}$. The pay zone thickness is an average of the productive sands, and the thin shale intercalations, when the later exists.

13. Closure stress gradient for the pay zone - considered within a range of 0.64 to $0.72 \mathrm{psi} / \mathrm{ft}$. This value is, in a real case, obtained from the merging algorithm developed for the production layer.

14. Closure stress gradient of the bottom layer - considered within a range of 0.715 and $0.810 \mathrm{psi} / \mathrm{ft}$. Most of the time, little information is known about the layers below the production formation or little lithology change exists. For these reasons, simulations consider only one layer below the production formation and take into account only its closure stress and no thickness. This is also the case of FRACPRO when the thickness of the bottom layer is not required as input.

Since porosity is an important reservoir characteristic, its influence on the simulation outputs was studied. Keeping all the inputs for the FRACPRO simulator constant, while altering porosity values from 7 to $13 \%$, it was concluded that porosity does not induce significant difference in the geometry (height, width, length) of the fracture. Accordingly, the average value of $9 \%$ was considered satisfactory for all cases.

The leakoff fluid permeability ratio, $\mathrm{Kp} / \mathrm{KI}$ (ratio of formation pore-fluid permeability to formation leakoff-fluid permeability), was determined by expert engineers to have a range of 2530.

The average pressure along the fracture was calculated as a function of the depth and the fracture closure gradient using the following formula:

$$
\text { Average pressure in the fracture }=\text { Depth * } \text { Grad }_{\text {closure_stress }} \text { Equation 4-5 }
$$

Since the slurry has a linear relationship with total volume and pad volume, it was not considered as an input in training the neural network.

All 14 inputs presented above were used in the FRACPRO simulator for generating the data. A simple Visual Basic computer program, using a random number generation function and the constraints defined above, generated 660 cases for the parameters within the specified ranges. 
According to Halliburton experts, most ramp treatments in tight rock country $(k<1 \mathrm{md})$ start with $1 \mathrm{lb} / \mathrm{gal}$ and are increased to around $6 \mathrm{lb} / \mathrm{gal}$. In highly permeable sands, the ramping may grow in $2 \mathrm{lb} /$ gal increments. It is intended to get $60 \%$ or better of the total sand volume in the last one or two stages.

\subsubsection{Six Stage Treatment Schedules}

The main difference between a stage treatment and a ramp treatment consists of the number of stages when pumping the slurry. Also, in the case of stage treatment the proppant concentration remains constant during each stage, which mathematically can be described as a step function. The six stages treatment as defined in this study consists of five steps and the pad.

All 14 inputs described in the ramp treatment section remain the same. In addition, three more parameters are added to describe volume and proppant concentration by stage for this type of treatment. These three new inputs are:

1. Initial proppant concentration in the first slurry stage. For this parameter three values were considered: 1, 1.5, and 2 ppg. Halliburton engineers suggested these values, which are typically used when designing treatments.

2. Proppant concentration increment - used to define the proppant concentration for the intermediate stages, which are the stages between the first and the last. The range values for the final proppant concentration were defined in the previous section. The proppant concentration increment is calculated using the following relationship:

$$
\mathrm{PCl}=\frac{\mathrm{FPC}-\mathrm{IPC}}{\mathrm{S}-1} \quad \text { Equation 4-6 }
$$

where, $\mathrm{PCl}=$ proppant concentration increment. ppg

$\mathrm{FPC}=$ final proppant concentration, ppg

IPC = initial proppant concentration, ppg

$S=$ number of stages excluding pad

3. Fluid volume increment - defines the fluid volume for the intermediate stages. The fluid volumes by stage are not generated randomly, as each stage must respect a specified range. The fluid volumes for each stage are defined as a function of the total fluid volume. Table 4-7 shows the stage volume as percentage of the total treatment volume. 


\begin{tabular}{|c|c|}
\hline Stage Type & $\begin{array}{c}\text { Percentage from Total } \\
\text { Fluid Volume }\end{array}$ \\
\hline Pad (Stage 1) & $35-45 \%$ \\
\hline Stage 2 & $5-10 \%$ \\
\hline Stage 3 & $5-10 \%$ \\
\hline Stage 4 & $10-15 \%$ \\
\hline Stage 5 & $15-20 \%$ \\
\hline Stage 6 & $15-25 \%$ \\
\hline
\end{tabular}

Table 4-7: Percentage of Total Fluid Volume per Stage for Six-Stage Treatment Schedule

In order to generate stage treatments, which respects the intervals defined in Table 4-7, the value of the fluid increment was thoroughly studied. Different types of combinations were considered and analyzed. However the one meeting the most requirements is structured as shown in Table 4-8.

\begin{tabular}{|c|c|}
\hline Stage Type & $\begin{array}{c}\text { Stage Volume Structure } \\
(35-45) \% \text { from Total Fluid } \\
\text { Volume }\end{array}$ \\
\hline Pad (Stage 1) & $2{ }^{*}$ Increment \\
\hline Stage 2 & $2{ }^{*}$ Increment \\
\hline Stage 3 & $3{ }^{*}$ Increment \\
\hline Stage 4 & $4{ }^{*}$ Increment \\
\hline Stage 5 & $5{ }^{*}$ Increment \\
\hline Stage 6 &
\end{tabular}

Table 4-8: Stage Fluid Volume Structure of Six-Stage Treatment Schedule

To achieve this structure, a new relationship was introduced to calculate the fluid increment. This equation defines the fluid increment volume as:

$$
\mathrm{FI}=\frac{T F V-P F V}{N}
$$

where, $\mathrm{FI}=$ fluid volume increment, gal

TFV = total fluid volume, gal

$\mathrm{PFV}=$ pad fluid volume, gal

$\mathrm{N}=$ parameter calculated function of number of stages in the treatment

(In the present case of 5 slurry stages, $\mathrm{N}$ is equal to 16. ) 
The graphical representation of the six-stage treatment is shown in Figure 4-8.

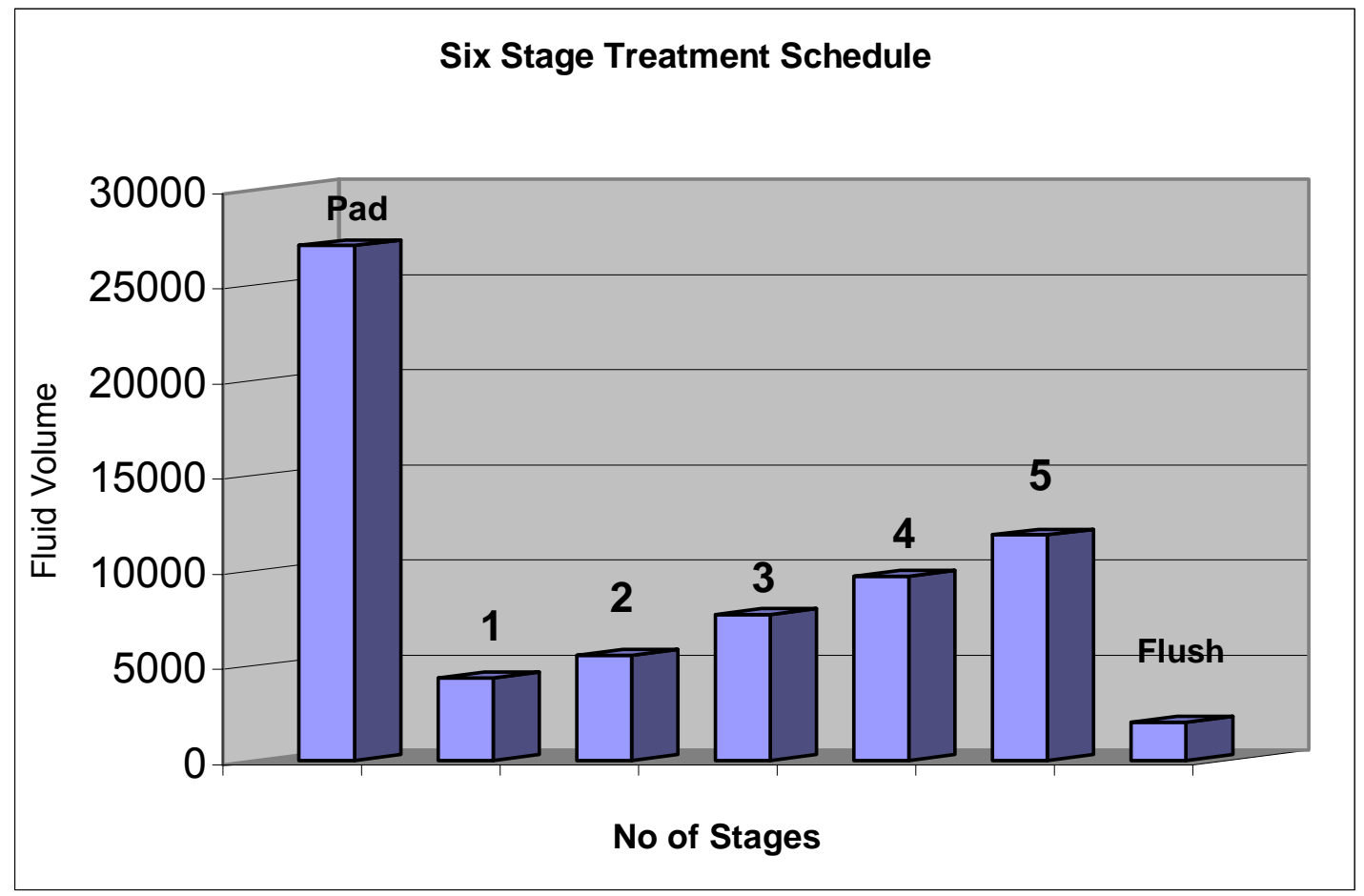

Figure 4-8: Graphical Representation of the Six Stage Design.

The 17 inputs defined above were used in FRACPRO simulator for generating the data, which can be viewed in the Appendix Table A - 2. The final system consists of a total number of 17 inputs and 5 outputs.

\subsubsection{Eight Stage Treatment Schedules}

A treatment with large numbers of stages (eight or more) is designed when job placement problems are anticipated. For example, if the desired conductivity would be achieved from 5 and $6 \mathrm{lb} / g a l$ stages, but there might be problems placing 5 and $6 \mathrm{lb} / g a l$ into the formation, then smaller incremental steps of 1,2 , and $3 \mathrm{lb} /$ gal stages are designed so they can be analyzed as each stage enters the formation. In this case, if problems occur, the job design can be altered to overcome any formation problems that might exist (i.e. increase rate, increase volume etc.). As a rule of thumb, if the reservoir is prone to job placement problems a higher number of stages are recommended.

The design of the eight-stage treatment runs basically on the same platform as the sixstage treatment. The only difference consists in the percentage of fluid volume injected for each stage. As in the case of six stages treatment, the system consists of 17 parameters as defined 
above. The proppant concentration increment and the fluid volume increment are calculated with equations 4-6 and 4-7.

The initial proppant concentration in the first slurry stage was considered as having three values: 1, 1.1, $1.2 \mathrm{ppg}$. These values were suggested by Halliburton engineers, which are typically used when designing treatments. The proppant concentration increment, used to define the proppant concentration for the intermediate stages, is calculated using the same equation (4.6). Table 4-9 shows how proppant increment concentration is applied in defining each of the treatment stages.

\begin{tabular}{|c|c|}
\hline Stage & Proppant Conc \\
\hline Pad (Stage 1) & - \\
\hline Stage 2 & Xi \\
\hline Stage 3 & $x i+1^{*}$ p_inc \\
\hline Stage 4 & $x i+2^{*} \mathrm{p}$ inc \\
\hline Stage 5 & $\mathrm{xi}+3^{*} \mathrm{p}$ _inc \\
\hline Stage 6 & $\mathrm{xi}+4^{*} \mathrm{p}$ _inc \\
\hline Stage 7 & $\mathrm{xi}+5^{*} \mathrm{p}$ _inc \\
\hline Stage 8 & $\mathrm{xf}$ \\
\hline
\end{tabular}

Table 4-9: Proppant Concentration for Eight-Stage Treatment Schedule

The fluid volume increment is used to define the fluid volume for the intermediate stages. Again the fluid volumes by stage are not generated randomly; each stage must respect a specified range. The fluid volumes for each stage are defined as a function of the total fluid volume. Table 4-10 shows the stage volume as percentage of the total treatment volume.

\begin{tabular}{|c|c|}
\hline Stage Type & $\begin{array}{c}\text { Percentage from } \\
\text { Total Fluid Volume }\end{array}$ \\
\hline Pad (Stage 1) & $25-43 \%$ \\
\hline Stage 2 & $5-8 \%$ \\
\hline Stage 3 & $5-8 \%$ \\
\hline Stage 4 & $8-10 \%$ \\
\hline Stage 5 & $8-10 \%$ \\
\hline Stage 6 & $12-20 \%$ \\
\hline Stage 7 & $15-20 \%$ \\
\hline Stage 8 & $12-15 \%$ \\
\hline
\end{tabular}

Table 4-10: Percentage of Total Fluid Volume per Stage for Eight-Stage Treatment Schedule

The ranges provided in Table 4-10 are typical ranges for an eight-stage treatment design. In order to generate stage treatments that respect the ranges defined in Table 4-10, a thorough 
analysis of the fluid increment value was carried out. One equation that respects the structure of stages is a derivation of equation (4.7), where $\mathrm{N}$ is equal to 23 . The number $\mathrm{N}$ is the sum of all fluid increments as can be seen in Table 4-11.

\begin{tabular}{|c|c|}
\hline & Type I \\
\hline Stage & Fluid Volume \\
\hline Pad (Stage 1) & $30-40 \%$ \\
\hline Stage 2 & $2^{*}$ inc \\
\hline Stage 3 & $2^{*}$ inc \\
\hline Stage 4 & $3^{*}$ inc \\
\hline Stage 5 & $3^{*}$ inc \\
\hline Stage 6 & $4^{*}$ inc \\
\hline Stage 7 & $4^{*}$ inc \\
\hline Stage 8 & $5^{*}$ inc \\
\hline
\end{tabular}

Table 4-11: Type I Stage Fluid Volume Structure of Eight-Stage Treatment Schedule

The treatment with the structure presented in Table 4-11 column two, was named "8 Stages Design Type I". The graphical representation of this type of treatment is shown in Figure 4-9.

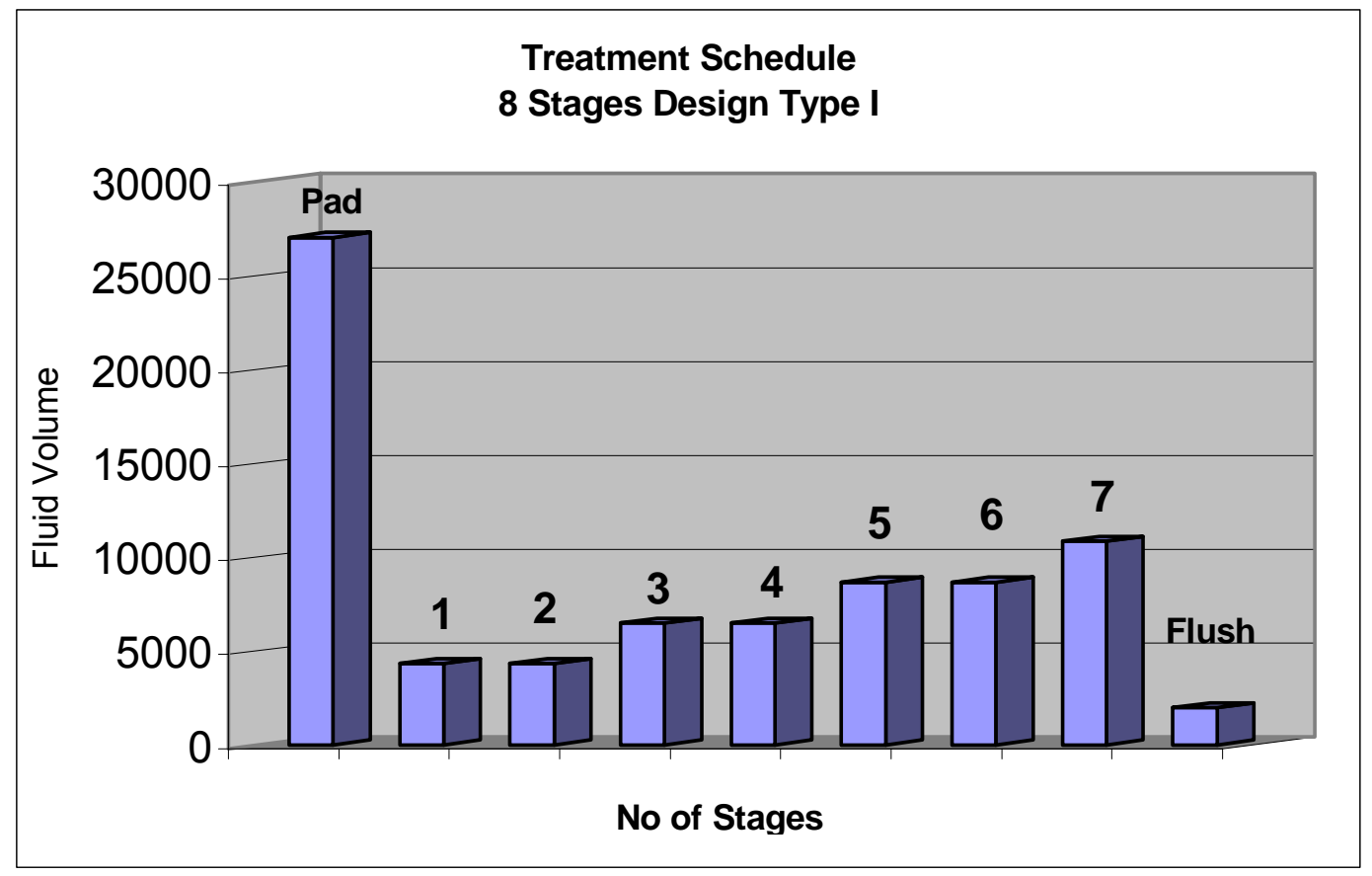

Figure 4-9: Type I Eight Stage Design

In designing fracture treatments, the first proppant stages are basically an extension of the pad portion of the job. Increasing proppant concentrations build fracture width and serve as an indicator for any near wellbore or fracture growth problems. The last proppant stage is 
designed to provide maximum conductivity near well bore, and therefore is not a significant percentage of the job.

With these considerations, the volume for each stage was altered to accommodate for designs that provide higher conductivity near the well bore. Two more structures for the eightstage treatment were defined as shown in Table 4-12.

\begin{tabular}{|c|c|c|}
\hline & Type II & Type III \\
\hline Stage & Fluid Volume & Fluid Volume \\
\hline Pad & $30-40 \%$ & $30-40 \%$ \\
\hline Stage 1 & $2^{*}$ inc & $2^{*}$ inc \\
\hline Stage 2 & $2.5^{*}$ inc & $2.5^{*}$ inc \\
\hline Stage 3 & $3^{*}$ inc & $3^{*}$ inc \\
\hline Stage 4 & $4.5^{*}$ inc & $4^{*}$ inc \\
\hline Stage 5 & $4^{*}$ inc & $4.5^{*}$ inc \\
\hline Stage 6 & $5^{*}$ inc & $5^{*}$ inc \\
\hline Stage 7 & $3^{*}$ inc & $2^{*}$ inc \\
\hline
\end{tabular}

Table 4-12: Types II and III Stage Fluid Volume Structure of Eight-Stage Treatment Schedule

The treatment with the structure presented in Table 4-12 column two was named "8 Stages Design Type II". The graphical representation of this type of treatment is shown in Figure 4-10.

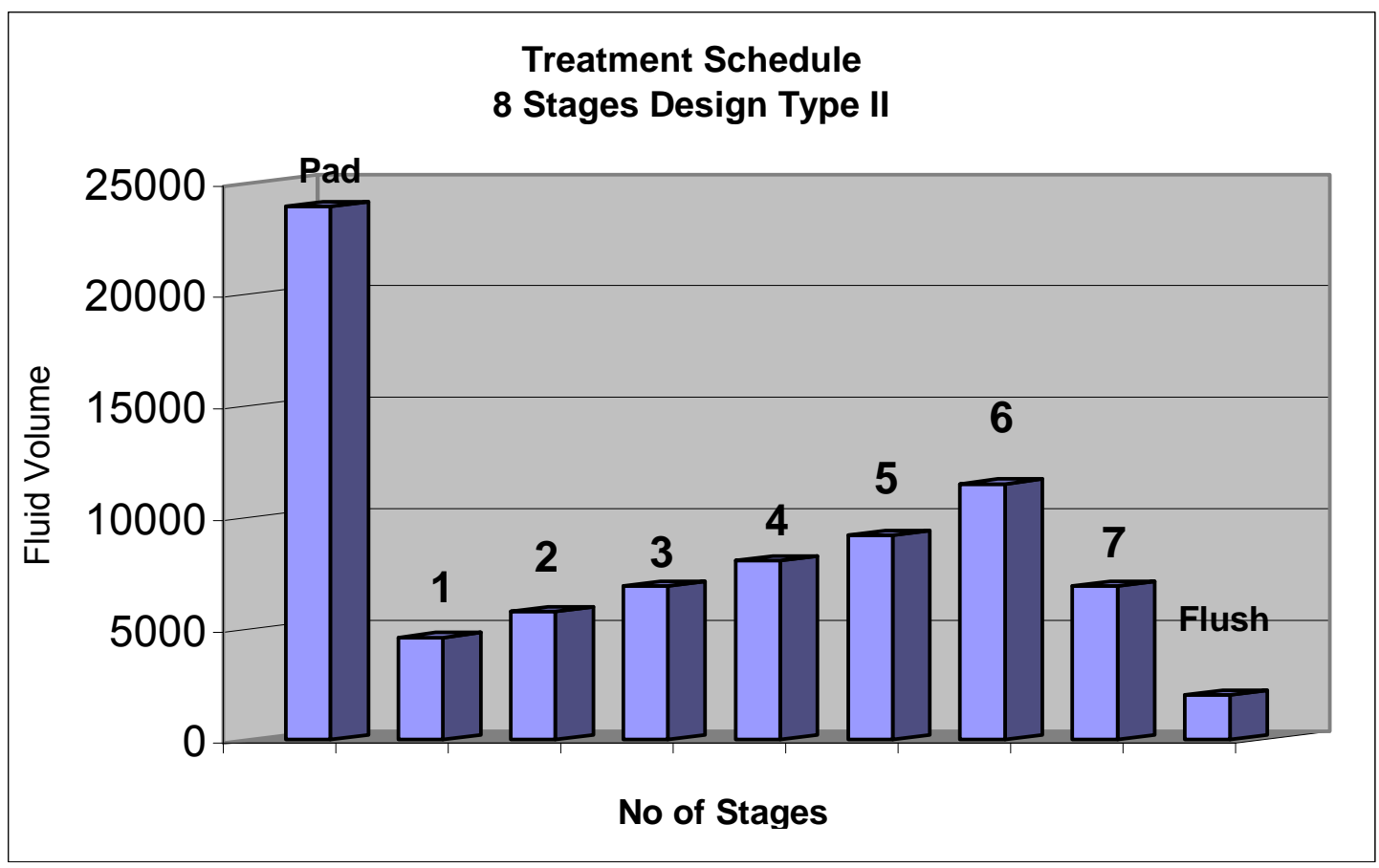

Figure 4-10: Type II Eight Stage Design 
The treatment with the structure presented in Table 4-12 column three, was named "8 Stages Design Type III". The graphical representation of this type of treatment is shown in Figure 4-11.

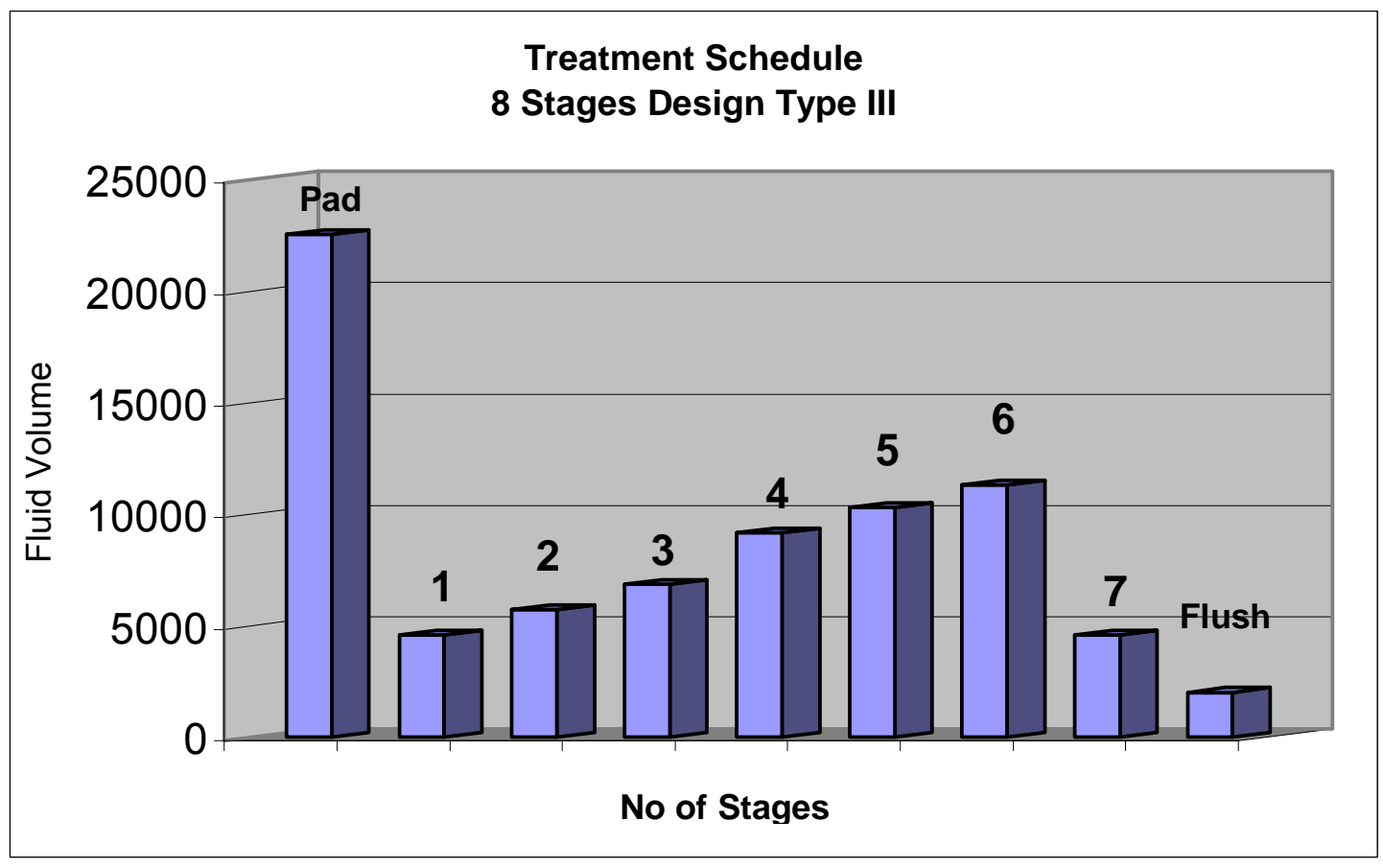

Figure 4-11: Type III Eight Stage Design

The comparison between the three types of treatments is shown in Figure 4-12. As can be seen from the graph, Types II and III are designed so that higher proppant concentrations are carried with low amount of fluid, thus creating high conductivity around the well bore. 


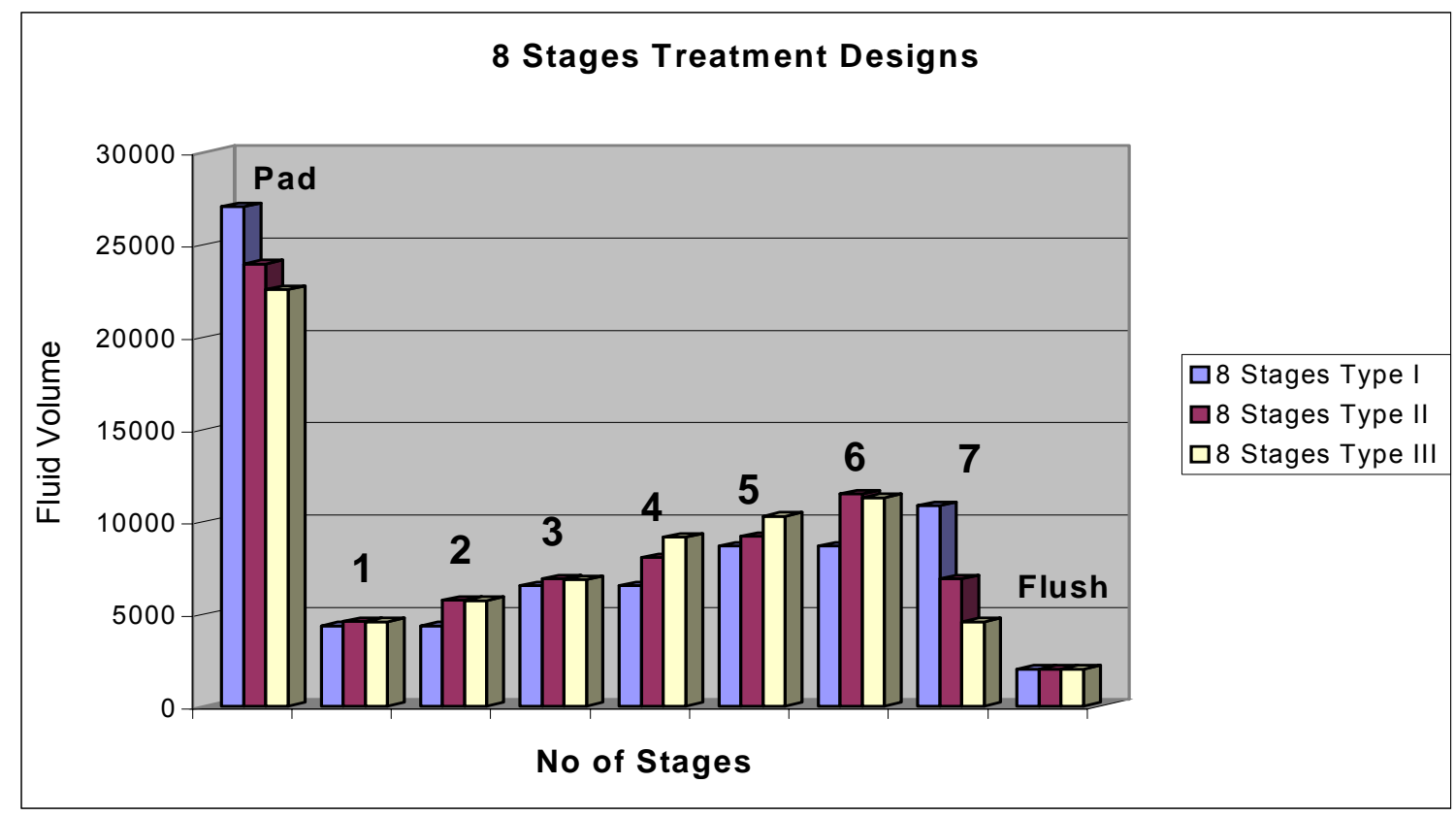

Figure 4-12: Comparison of Eight Stage Treatment Schedules

All 17 inputs were used in the FRACPRO simulator for data generation. For each of the three types of treatments, 200 cases were generated and can be viewed in the Appendix Table $A$ - 3, Table A - 4, and Table A - 5.

\subsection{Fracture Design Neural Networks}

The next step in the development of an intelligent tool that designs optimum fracture treatments is to build a module that replaces the hydraulic fracture simulator. Since neural networks have a natural propensity to store knowledge and make it available for later use, they can be trained to mimic the fracture simulation and provide the same output as the computer model.

It is impractical, if not impossible, to couple vendor software such as FRACPRO with a search engine, like genetic algorithms, particularly because FRACPRO requires human data entry for each new run. Furthermore, a simulation run may take up to a minute depending upon the complexity of the reservoir lithology and treatment design. In addition, no source code of the software was available or easy to acquire.

Since neural networks can learn, they can be trained to perform a particular task, such as the output of a fracturing simulation treatment. Neural networks learn from presented data and can generalize to a certain extent, but they cannot predict something that is beyond the range of the training data. Therefore, it is important for the training data to be representative and cover a wide range of possibilities. The module that replaces the fracture simulator is composed of a few 
neural networks with specific functionality. These networks are the heart of the optimization methodology presented in this study.

The geometry of the fracture is defined by fracture length, fracture height, and fracture width. Three additional parameters, fracture efficiency and proppant concentration and dimensionless fracture conductivity, are also carefully considered when placing a treatment job. All six parameters represent the output of this system.

In training the neural networks that will replace FRACPRO, a commercial product named "NeuroShell 3.0" developed by Ward System Groups was used. "NeuroShell 3.0" provides a relatively wide variety of net architectures and training algorithms, with a friendly user interface.

\subsubsection{Developing Neural Networks for Ramp Treatments}

The first neural network developed was the ramp treatment network. It was designed to mimic a hydraulic fracture simulator for ramp treatments schedules. Table 4-13 summarizes the inputs and outputs used for training the neural network. The final system consisted of a total number of 14 inputs and 6 outputs. Column two defines the names of each parameter, column three identifies whether it is an input or output, and column four provides the range of each parameter. 


\begin{tabular}{|c|c|c|c|c|}
\hline Index & Parameter Name & Type & Range Interval & Units \\
\hline & & & & \\
\hline 1 & Total fluid volume & input & $10,000-200,000$ & gal \\
\hline 2 & Pad volume & input & $3,500-90,000$ & gal \\
\hline 3 & Final proppant Concentration & input & $6-15$ & $\mathrm{ppg}$ \\
\hline 4 & Flow pumping rate & input & $15-40$ & $\mathrm{bbl} / \mathrm{min}$ \\
\hline 5 & Depth to the "pay zone" & input & $5,000-15,000$ & $\mathrm{ft}$ \\
\hline 6 & Reservoir pressure & input & $1,500-7,000$ & psia \\
\hline 7 & Permeability & input & $0.001-0.1$ & $\mathrm{mD}$ \\
\hline 8 & Top layer thickness & input & $170-250$ & $\mathrm{ft}$ \\
\hline 9 & Top layer closure stress gradient & input & $0.72-0.85$ & $\mathrm{psi} / \mathrm{ft}$ \\
\hline 10 & Overburden layer thickness & input & Oct-50 & $\mathrm{ft}$ \\
\hline 11 & Overburden layer closure stress gradient & input & $0.74-0.85$ & $\mathrm{psi} / \mathrm{ft}$ \\
\hline 12 & Pay zone thickness & input & $50-130$ & $\mathrm{ft}$ \\
\hline 13 & Pay zone closure stress gradient & input & $0.64-0.72$ & $\mathrm{psi} / \mathrm{ft}$ \\
\hline 14 & Bottom layer closure stress gradient & input & $0.71-0.81$ & $\mathrm{psi} / \mathrm{ft}$ \\
\hline 1 & Fracture Total Height & output & $220-600$ & $\mathrm{ft}$ \\
\hline 2 & Fracture Propped Length & output & $170-900$ & $\mathrm{ft}$ \\
\hline 3 & Fracture Maximum Width & output & $0.14-1.5$ & in \\
\hline 4 & Dimensionless Conductivity Ratio & output & $1-500$ & \\
\hline 5 & Fracture Proppant Concentration & output & $0.4-2.0$ & $\mathrm{Ib} / \mathrm{ft} 2$ \\
\hline 6 & Fracture Efficiency & output & $0.2-0.97$ & \\
\hline
\end{tabular}

Table 4-13: Inputs/Outputs for Ramp Treatment Neural Network

FRACPRO was used for generating a representative set of examples, namely pairs of input and output samples, necessary for training the neural network as described in section 4.11.2.1. The results are displayed in the Appendix Table A - 1.

Due to the complexity of the problem and the wide range of parameters, several neural network types and corresponding architectures were considered. Because of its outstanding performance, a back-propagation neural network with an architecture consisting of three hidden layers with different activation functions was used in training. The graphical representation of the selected neural network architecture is shown in Figure 4-13. 


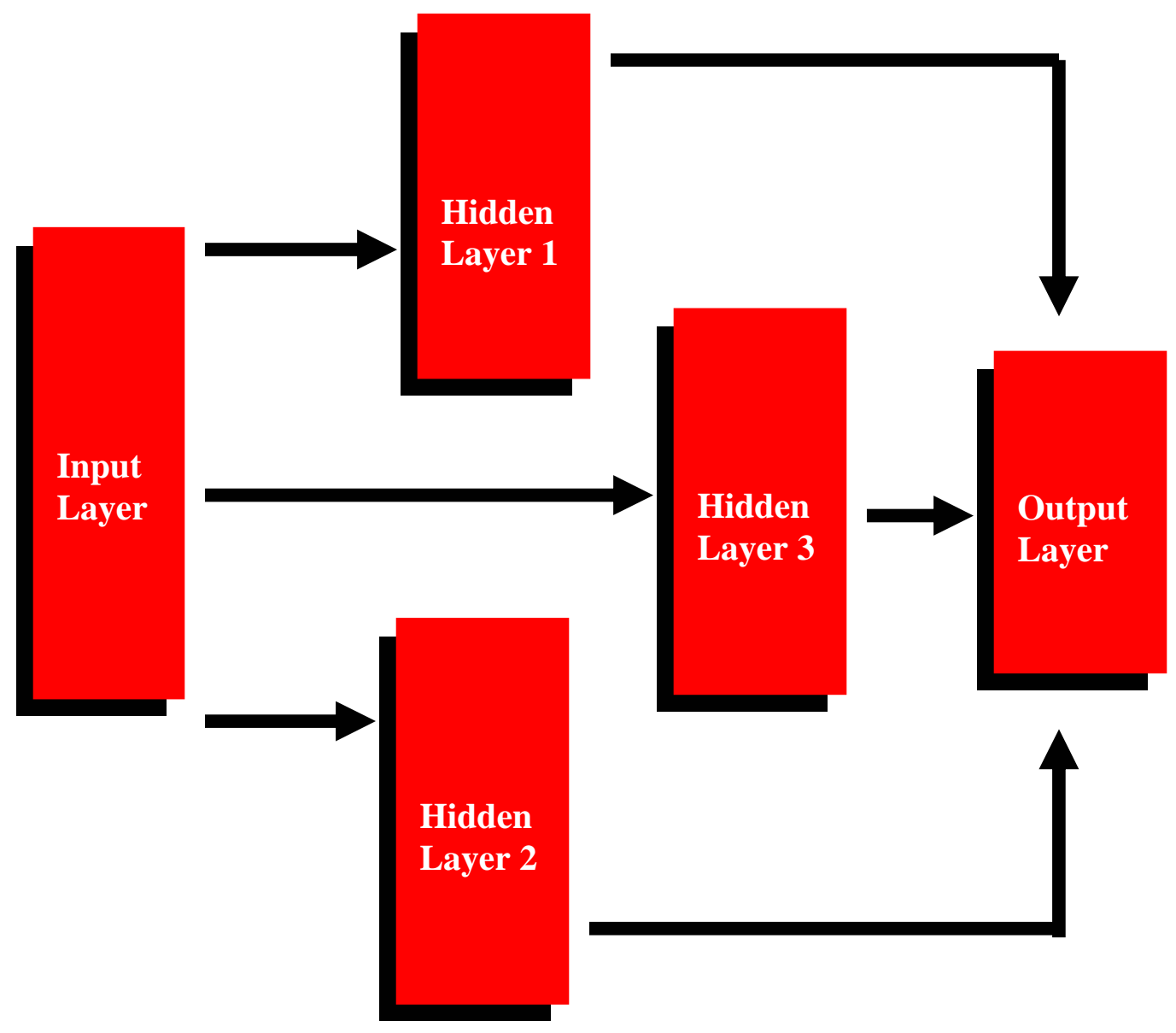

Figure 4-13: Back-Propagation Neural Network Architecture

A summary architecture description of the Neural Networks presented in Figure 4-13 is given in Table 4-14.

\begin{tabular}{|c|c|c|c|c|c|c|c|}
\hline $\begin{array}{c}\text { Optimization } \\
\text { Network }\end{array}$ & $\begin{array}{c}\text { Input } \\
\text { Layer }\end{array}$ & $\begin{array}{c}\text { Hidden } \\
\text { Layer 1 }\end{array}$ & $\begin{array}{c}\text { Hidden } \\
\text { Layer 2 }\end{array}$ & $\begin{array}{c}\text { Hidden } \\
\text { Layer 3 }\end{array}$ & Output & $\begin{array}{c}\text { Learning } \\
\text { Rate }\end{array}$ & Momentum \\
\hline $\begin{array}{c}\text { Number of } \\
\text { Neurons }\end{array}$ & 14 & 12 & 12 & 12 & 6 & 0.1 & 0.1 \\
\hline $\begin{array}{c}\text { Type of } \\
\text { activaton } \\
\text { Function }\end{array}$ & Linear & Gaussian & Tanh & Gaussian & Logistic & & \\
\hline
\end{tabular}

Table 4-14: Optimization Network for Ramp Treatment Schedule 
The ramp treatment optimization network has one input layer with 14 input neurons, three hidden layers with 36 hidden neurons (12 in each hidden layer) and 6 output neurons. The learning rate was 0.1 and the momentum 0.1 .

\subsubsection{Developing Neural Networks for Stage Treatments}

Two scenarios were considered in order to demonstrate the capability of the methodology by addressing multiple variations of the stage treatment design schedules. Once the model proved that these two types of stage treatment schedules provide accurate results, the model can be expanded to any number of stages that can be reasonably implemented in the field.

The next two sections provide a detailed explanation of the implementation for the six and eight stage treatments schedules design.

\subsubsection{The Six Stage Treatment}

The second neural network developed was the six-stage treatment network. This network models cases where fracturing treatments are placed in stages with a step in proppant concentration for each stage. Previously, an algorithm was introduced to represent different stages while keeping the lowest possible number of inputs. An example of the structure of a sixstage treatment was defined in Table 4-1.

Table 4-15 summarizes the inputs/outputs neural network system. Column two defines the names of each parameter, column three identifies the type of parameter, and column four provides the range of each parameter. 


\begin{tabular}{|c|c|c|c|c|}
\hline Index & Parameter Name & Type & Range Interval & Units \\
\hline & & & & \\
\hline 1 & Total fluid volume & Input & $10,000-200,000$ & gal \\
\hline 2 & Pad volume & Input & $3,500-90,000$ & gal \\
\hline 3 & Increment volume & Input & Calculated & gal \\
\hline 4 & Initial proppant concentration & input & $1,1.5,2$ & ppg \\
\hline 5 & Proppant concentration increment & input & Calculated & ppg \\
\hline 6 & Final proppant Concentration & input & 10.8 & ppg \\
\hline 7 & Flow pumping rate & input & $15-40$ & $\mathrm{bbl} / \mathrm{min}$ \\
\hline 8 & Depth to the "pay zone" & input & $5,000-15,000$ & $\mathrm{ft}$ \\
\hline 9 & Reservoir pressure & input & $1,500-7,000$ & psia \\
\hline 10 & Permeability & input & $0.001-0.1$ & $\mathrm{mD}$ \\
\hline 11 & Top layer thickness & input & $170-250$ & $\mathrm{ft}$ \\
\hline 12 & Top layer closure stress gradient & input & $0.72-0.85$ & psi/ft \\
\hline 13 & Overburden layer thickness & input & Oct-50 & $\mathrm{ft}$ \\
\hline 14 & Overburden layer closure stress gradient & input & $0.74-0.85$ & $\mathrm{psi} / \mathrm{ft}$ \\
\hline 15 & Pay zone thickness & input & $50-130$ & $\mathrm{ft}$ \\
\hline 16 & Pay zone closure stress gradient & input & $0.64-0.72$ & $\mathrm{psi} / \mathrm{ft}$ \\
\hline 17 & Bottom layer closure stress gradient & input & $0.71-0.81$ & $\mathrm{psi} / \mathrm{ft}$ \\
\hline 1 & Fracture Total Height & output & $220-600$ & $\mathrm{ft}$ \\
\hline 2 & Fracture Propped Length & output & $170-900$ & $\mathrm{ft}$ \\
\hline 3 & Fracture Maximum Width & output & $0.14-1.5$ & in \\
\hline 4 & Dimensionless Conductivity Ratio & output & $1-500$ & \\
\hline 5 & Fracture Proppant Concentration & output & $0.4-2.0$ & $\mathrm{lb} / \mathrm{ft} 2$ \\
\hline 6 & Fracture Efficiency & output & $0.2-0.97$ & \\
\hline
\end{tabular}

Table 4-15: Inputs/Outputs for Six-Stage Treatment Schedule Neural Network

As in the case of the ramp treatment schedule, FRACPRO was used for generating data necessary for training the neural network. The stage treatment neural network used has an identical architecture with the one used for ramp treatment (Figure 4-13). A summary description of the neural networks architecture is presented in Table 4-16. 


\begin{tabular}{|c|c|c|c|c|c|c|c|}
\hline $\begin{array}{c}\text { Optimization } \\
\text { Network }\end{array}$ & $\begin{array}{c}\text { Input } \\
\text { Layer }\end{array}$ & $\begin{array}{c}\text { Hidden } \\
\text { Layer 1 }\end{array}$ & $\begin{array}{c}\text { Hidden } \\
\text { Layer 2 }\end{array}$ & $\begin{array}{c}\text { Hidden } \\
\text { Layer 3 }\end{array}$ & Output & $\begin{array}{c}\text { Learning } \\
\text { Rate }\end{array}$ & Momentum \\
\hline $\begin{array}{c}\text { Number of } \\
\text { Neurons }\end{array}$ & 17 & 11 & 11 & 11 & 6 & 0.1 & 0.1 \\
\hline $\begin{array}{c}\text { Type of } \\
\text { activaton } \\
\text { Function }\end{array}$ & Linear & Gaussian & Tanh & Gaussian & Logistic & & \\
\hline
\end{tabular}

Table 4-16: Optimization Network for Six-Stage Treatment Schedule

The six-stage optimization network has one input layer with 17 input neurons, three hidden layers with 33 hidden neurons (11 in each hidden layer) and 6 output neurons. The learning rate was 0.1 and the momentum 0.1 .

\subsubsection{The Eight Stage Treatment}

The third type of neural network was the eight-stage treatment network. A small study was performed to determine the minimum number of cases needed to be generated in order to train an acceptable accurate neural network to mimic the type of treatment. 660 cases were generated for the ramp treatment and 675 cases for the six-stage treatment. This was based on the runs already available from the six-stage treatment schedule.

The procedure consisted of the following steps:

1. 100 random cases were selected from the 675 total available.

2. The cases were divided into the training set and the testing set, representing $90 \%$ and $10 \%$ respectively of the 100 random cases.

3. A neural network was trained using the training and testing cases. Once optimized the correlation coefficient for the training set is recorded.

4. The process is repeated each time increasing the number of random cases selected using a step of 25 or 50 cases. The correlation coefficient for each step is recorded for five of the outputs.

Table 4-17 presents each step of the process, the structure of the training and testing sets as well as the recorded correlation coefficients for each step. The first column represents the step number, while column two shows the total number of cases selected for the current step. Columns three and four show the structure of the training and testing sets. The last five columns are the values for the correlation coefficients for each of the five outputs considered in the neural network. The last row in the table corresponds to the correlation coefficients for the optimized six-stage treatment neural network. 


\begin{tabular}{|l|c|c|c|c|c|c|c|c|c|}
\hline & \multicolumn{3}{|c|}{ Number of Cases } & \multicolumn{5}{c|}{ Correlation Coefficient Values } \\
\hline $\begin{array}{c}\text { Total \# } \\
\text { of } \\
\text { cases }\end{array}$ & $\begin{array}{c}\text { Training } \\
\text { Set, \# } \\
\text { cases }\end{array}$ & $\begin{array}{c}\text { Clibration } \\
\text { Set, } \\
\text { cases }\end{array}$ & $\begin{array}{c}\text { Verification } \\
\text { Set, \# cases }\end{array}$ & $\begin{array}{c}\text { Fracture } \\
\text { Efficiency }\end{array}$ & $\begin{array}{c}\text { Propped } \\
\text { Length, } \\
\text { (ft) }\end{array}$ & $\begin{array}{c}\text { Dimmensionless } \\
\text { Conductivity } \\
\text { Ratio }\end{array}$ & $\begin{array}{c}\text { Maximum } \\
\text { Propped } \\
\text { Width (in) }\end{array}$ & $\begin{array}{c}\text { Fracture } \\
\text { Height, } \\
\text { (ft) }\end{array}$ \\
\hline & & & & & & & & & \\
\hline Step 1 & 100 & 90 & 10 & 578 & 0.801 & 0.88 & 0.848 & 0.889 & 0.87 \\
\hline Step 2 & 150 & 135 & 15 & 528 & 0.846 & 0.921 & 0.834 & 0.904 & 0.904 \\
\hline Step 3 & 200 & 180 & 20 & 478 & 0.838 & 0.929 & 0.892 & 0.902 & 0.918 \\
\hline Step 4 & 250 & 225 & 25 & 428 & 0.838 & 0.94 & 0.88 & 0.899 & 0.912 \\
\hline Step 5 & 300 & 270 & 30 & 378 & 0.828 & 0.946 & 0.898 & 0.913 & 0.918 \\
\hline Step 6 & 350 & 315 & 35 & 328 & 0.864 & 0.931 & 0.905 & 0.919 & 0.93 \\
\hline Step 7 & 400 & 360 & 40 & 278 & 0.887 & 0.939 & 0.901 & 0.919 & 0.927 \\
\hline Step 8 & 425 & 382 & 43 & 253 & 0.858 & 0.929 & 0.908 & 0.935 & 0.918 \\
\hline Step 9 & 450 & 405 & 45 & 228 & 0.883 & 0.943 & 0.935 & 0.945 & 0.942 \\
\hline Step 10 & 475 & 427 & 48 & 203 & 0.884 & 0.955 & 0.929 & 0.945 & 0.938 \\
\hline Step 11 & 500 & 450 & 50 & 177 & 0.882 & 0.958 & 0.925 & 0.939 & 0.933 \\
\hline Step 12 & 525 & 472 & 53 & 153 & 0.907 & 0.954 & 0.925 & 0.94 & 0.924 \\
\hline Step 13 & 550 & 495 & 55 & 127 & 0.927 & 0.954 & 0.941 & 0.951 & 0.924 \\
\hline Step 14 & 575 & 517 & 58 & 103 & 0.943 & 0.943 & 0.938 & 0.942 & 0.916 \\
\hline Step 15 & 600 & 540 & 60 & 75 & 0.937 & 0.946 & 0.933 & 0.945 & 0.939 \\
\hline & & & & & & & & & \\
\hline All & 675 & 480 & 120 & 75 & 0.917 & 0.947 & 0.907 & 0.914 & 0.929 \\
\hline
\end{tabular}

Table 4-17: Correlation Coefficients for Eight Stage Treatment Schedule Neural Network

The graphical representation of the correlation coefficients values versus the number of cases considered in training and testing the neural network is seen in Figure 4-14. 


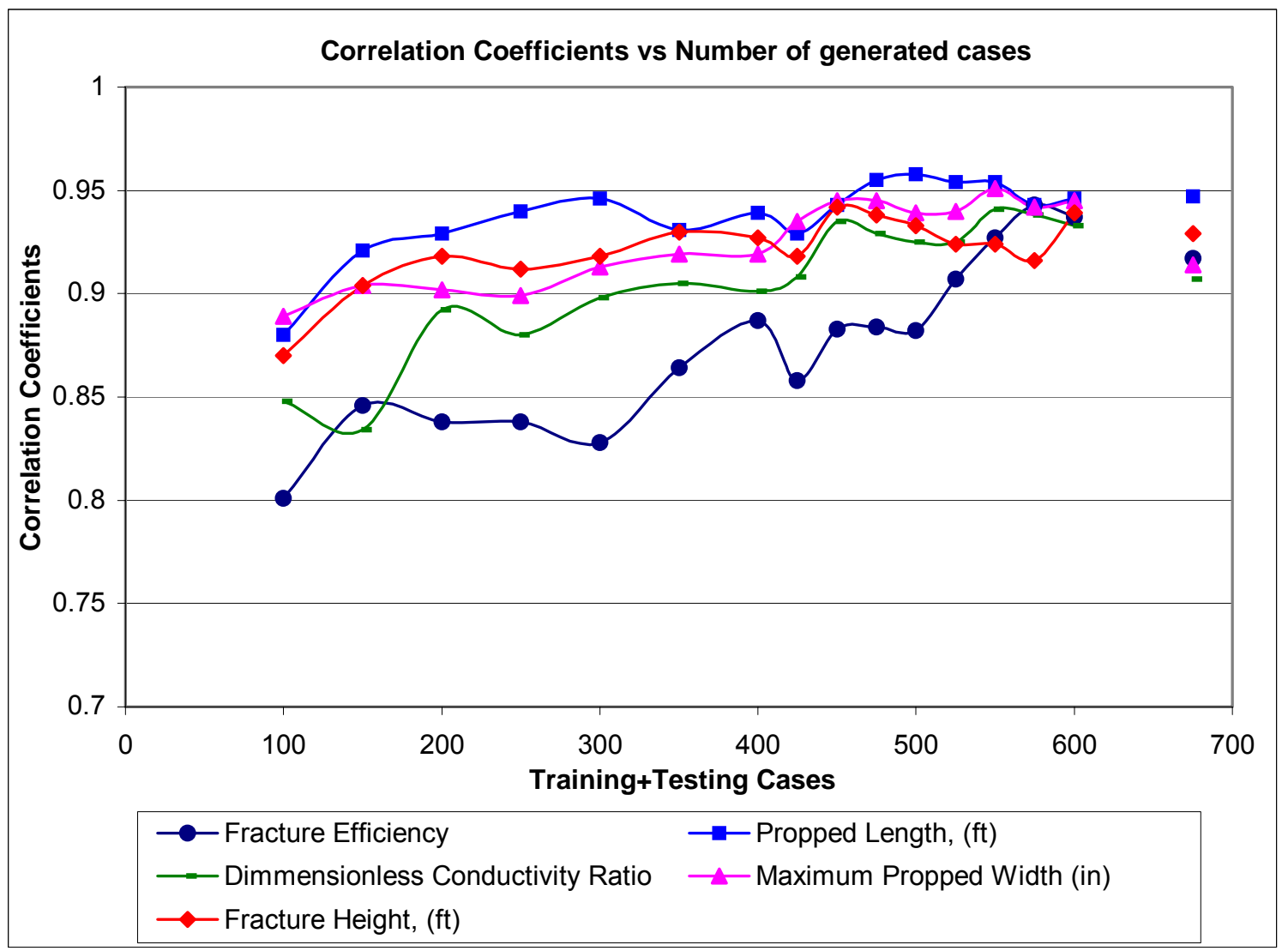

Figure 4-14: Correlation Coefficient Variation with Number of Cases

Table 4-17 and Figure 4-14 show that values for the correlation coefficients are improving with the increase of number of cases. With the exception of fracture efficiency, the values for correlation coefficients are near or above 0.9 for 200 or more cases. Since values above 0.9 are acceptable, 200 cases or more were generated for training the eight stage treatment schedule neural networks.

As with the six stage neural network, three types of treatment structures were also considered for the eight stage neural network. The structures of these three types are defined in Table 4-11 and Table 4-12.

The inputs and outputs for the eight stage neural network are identical to the six stages treatment and are summarized in Table 4-15.

Again, FRACPRO was used to generate data for each type of the three treatments schedules (seen in Appendix Table A - 3, Table A - 4, and Table A - 5). With this data, three neural networks were trained to mimic FRACPRO. All three types neural networks have an identical architecture to that used for ramp and six stage treatments (Figure 4-13).

A summary description of the neural network architectures is presented in Table 4-18. The eight stage optimization networks have one input layer with 17 input neurons, three hidden 
layers with 36 hidden neurons (12 in each hidden layer), and 6 output neurons. The learning rate was 0.1 and the momentum 0.1 .

\begin{tabular}{|c|c|c|c|c|c|c|c|}
\hline $\begin{array}{c}\text { Optimization } \\
\text { Network }\end{array}$ & $\begin{array}{c}\text { Input } \\
\text { Layer }\end{array}$ & $\begin{array}{c}\text { Hidden } \\
\text { Layer 1 }\end{array}$ & $\begin{array}{c}\text { Hidden } \\
\text { Layer 2 }\end{array}$ & $\begin{array}{c}\text { Hidden } \\
\text { Layer 3 }\end{array}$ & Output & $\begin{array}{c}\text { Learning } \\
\text { Rate }\end{array}$ & Momentum \\
\hline $\begin{array}{c}\text { Number of } \\
\text { Neurons }\end{array}$ & 17 & 12 & 12 & 12 & 5 & 0.1 & 0.1 \\
\hline $\begin{array}{c}\text { Type of } \\
\text { activaton } \\
\text { Function }\end{array}$ & Linear & Gaussian & Tanh & Gaussian & Logistic & & \\
\hline
\end{tabular}

Table 4-18: Optimization Network for Eight Stage Treatment Schedule

\subsection{Neuro-Genetic Optimization Module for Fracture Treatment Design}

The objective of this study is to create a front-end optimization routine for hydraulic fracturing models to design the optimum treatment schedule that creates a desired fracture propped length. This is optimization problem requires the optimum combination of four treatment parameters: total fluid volumes, pad volume, proppant concentration and injection rate ${ }^{35}$. The optimum fracture treatment design is found through the use of a two-step method that integrates neural networks with a search engine, in this case a genetic algorithm ${ }^{8,36-38}$.

The first step involves building the neural networks modules to mimic a fracture simulator, as detailed in sections 4.12 .1 and 4.12.2. The second step of the methodology uses the genetic algorithm to search for the optimum fracture design treatment. Genetic algorithms complete the optimization process, which consists of the initialization of a population, fitness evaluation, selection, crossover and mutation to produce the next generation ${ }^{39}$. This process is repeated for each generation until converged to the final solution.

\subsubsection{Treatment Optimization Design Overview}

In designing the optimum hydraulic fracturing treatment an important variable is the dimensionless fracture conductivity. This variable incorporates both fracture conductivity and length. The dimensionless fracture conductivity $\mathrm{C}_{\mathrm{fD}}$, introduced by Cinco-Ley ${ }^{40}$ is defined by the equation: 


$$
\begin{aligned}
& C_{f D}=\frac{k_{f} w}{k x_{f}} \\
& \text { where, } k_{f}=\text { proppant pack fracture permeability, md } \\
& \mathrm{w}=\text { average propped fracture width, in } \\
& \mathrm{k}=\text { formation permeability, } \mathrm{md} \\
& \mathrm{x}_{\mathrm{f}}=\text { fracture half-length, } \mathrm{ft}
\end{aligned}
$$

In high permeability reservoirs, increasing the fracture conductivity is the primary demand, while fracture length is of secondary importance. In low permeability reservoirs, fracture conductivity does not play the primary role, since long fracture lengths are required for the success of the treatment. Accordingly, since this study addresses low permeability reservoirs, fracture length dictates the main optimization followed by the conductivity.

The main outputs of FRACPRO, which were modeled with the neural network, are fracture geometry (height, length, and width), fracture efficiency, proppant concentration, and dimensionless conductivity ratio. FRACPRO does not directly provide the fracture conductivity, but instead the dimensionless conductivity ratio.

According to the FRACPRO manual, the fracture conductivity is derived as the harmonic means of cell conductivities in each region. During simulation, a conductivity value is calculated for each grid block by interpolating the proppant permeability versus laboratory-determined stress. Harmonic mean is the natural way to average conductivities in series and is consistent with the way simulators use permeability to calculate flow between grid blocks. Under harmonic mean averaging, the lowest conductivity tends to dominate.

Since the implementation of genetic algorithms used in this study can only search for one parameter at time, finding the optimum combination of fracture length and conductivity requires a two-step process. The methodology consists of the following steps:

1. Define the desired fracture length.

2. Utilize a genetic algorithm to find the desired optimum fracture length. The result of the genetic optimization is an entire population of solutions evolving around the optimal solution.

3. Select the best $10 \%$ to $20 \%$ of the final population. For each potential solution, calculate the fracture conductivity using equation 4-8.

4. Find the solution with the conductivity value closest to the desired one.

5. If not ideal, minor adjustments in the injection rate or proppant concentrations can achieve a desired solution.

Two genetic algorithms were developed, one for the ramp treatment schedule, the other for the stage treatment schedule. Each of these algorithms uses two neural networks, one as fitness function and the other for optimization. 
The steps for this process, in further detail, are as follows:

1. Generate the initial population of potential treatment designs solutions.

2. Run each one of the potential solutions through the fitness function neural network.

3. Compare the outcome (fracture propped length) to the desired propped length, and rank the treatments based on the error between them.

4. Select the top ranked treatment designs to create the new generation and discard the rest.

5. Generate a new population of treatment designs by performing crossover and mutation processes on the selected treatment designs selected in Step 4.

6. Repeat process from Step 2 until desired error convergence is reached.

\subsubsection{Rapid Screening Solutions}

Rapid screening solutions provide a fast way to estimate potential treatment designs that may fit the desired criteria (fracture length, conductivity, volume of fluid and proppant). It can quickly provide an indication of the size of the treatment (volumes of fluid and proppant) needed to create a fracture with the specified length. The process represents the first generation of genetic optimization.

In this study, a rapid screening solution was developed for each treatment schedule. First, a desired fracture length was set, and then one hundred random treatment designs were generated. These treatments, together with the reservoir data, are sent to the neural network where the corresponding output (the fracture propped length) is created. The treatments are ranked descending according to the fitness function represented by desired propped length so that the top ranked treatment design has the closest calculated fracture length to the desired fracture length. The top five to ten treatments designs were deemed acceptable solutions and were considered as a starting point for the genetic algorithm initial population.

\subsubsection{Ramp Treatment Optimization Design}

Neural networks provide the fitness function for genetic algorithms to solve the highly non-linear optimization problem of finding the optimum treatment schedule in order to create the desired fracture length and conductivity. The data involved in this process is divided in two categories:

- Constant data represented by the formation characteristics

- Treatment design parameters that can be optimized

- Total fluid volume

- Pad fluid volume

- Slurry volume 
- Initial proppant concentration

- Final proppant concentration

- Injection flow rate

Since slurry volume represents the total fluid volume minus the pad volume, it was excluded from the optimization. Furthermore, initial proppant concentration was set to $1 \mathrm{ppg}$ for each case generated, and since it was constant it was not considered for optimization either. With these assumptions, the optimum treatment design represents a combination of the following four parameters:

- Total fluid volume

- Pad fluid volume

- Final proppant concentration

- Injection flow rate

In addition, some constraints were set for the injection rate, such as for small treatment jobs the injection flow rate should not converge towards the high values.

The first step in developing the genetic algorithm module is problem encoding. The binary representation of each parameter is determined. Concatenating the binary representation of the four parameters to be optimized generates one chromosome. The total number of chromosomes forms a population. The number of the bits used for each variable is presented in Table 4-19.

\begin{tabular}{|l|l|c|c|}
\hline \multicolumn{1}{|c|}{ Type } & \multicolumn{1}{|c|}{ Variable } & Range & Number of Bits \\
\hline & \multicolumn{3}{|c|}{} \\
\hline \multirow{3}{*}{ Ramp } & Total Fluid Volume & $10,000-200,000$ gal & 18 \\
\cline { 2 - 4 } & Pad Volume & $3.500-90,000$ gal & 6 \\
\cline { 2 - 4 } & Final Proppant Concentration & 16 & 8 \\
\cline { 2 - 4 } & Injection Rate & $10-20 \mathrm{bpm}$ & 6 \\
\hline
\end{tabular}

Table 4-19: Ramp Treatment Design Encoding

The algorithm creates an initial population of chromosomes by randomly populating the bits, corresponding to each parameter in the chromosomes. This is followed by the selection process, crossover, mutation, and fitness evaluation to form a new population.

\subsubsection{Stage Treatments Optimization Design}

Since both the six stage and the eight stage treatments are designed on the same platform, they can be defined by the same variables as the ramp treatment (total fluid volume, pad volume, slurry volume, initial proppant concentration, final proppant concentration and injection rate). Using the same considerations, the slurry will not be considered in the 
optimization. In the case of stage treatments the initial proppant concentration was set to be equal to the proppant increment for each stage. The same constraints were applied to the injection rate as related to the size of the treatments. Table 4-20 contains the encoding for the stage treatment design, which applies to both six and eight stage treatments.

\begin{tabular}{|c|c|c|c|}
\hline Type & Variable & Range & Number of Bits \\
\hline \multirow{5}{*}{$\begin{array}{l}\text { Stage } \\
\text { Treatment }\end{array}$} & Total fluid volume & $10,000-200,000 \mathrm{gal}$ & 18 \\
\hline & Pad volume & $3,500-90,000 \mathrm{gal}$ & 6 \\
\hline & $\begin{array}{l}\text { Incremental proppant } \\
\text { concentration }\end{array}$ & $1-2 \mathrm{ppg}$ & 5 \\
\hline & Final proppant concentration & $11 \mathrm{ppg}$ & 7 \\
\hline & Injection Rate & $10-40 \mathrm{bpm}$ & 7 \\
\hline
\end{tabular}

Table 4-20: Stage Treatment Design Encoding

\subsection{Pressure Profile Prediction}

Monitoring the pressure in real time is the most precious information that a fracturing engineer can acquire. This provides the real and most accurate response of the formation as it reacts to the fracturing process. The net-pressure matching during fracturing is aimed to support and assist the engineer in two ways. First, a good pressure match while fracturing assures engineer that the treatment is being placed as scheduled. Secondly, good matching between the observed pressure (which is the formation response) and the calculated pressure predicted by the model supports the results of the fracture geometry and fracture conductivity.

This study includes a model to predict the expected net treating pressure for the designed fracture. This model consists of a set of neural networks that mimic the expected net pressure profile during fracturing. This pressure profile can later be used for real time matching.

\subsubsection{Overview}

During a hydraulic fracturing job, the pressure signal can be recorded for any desired time interval. Generally this produces more data points than the neural network can handle as output data. Vector quantization, a powerful technique for data compression, was utilized to resolve this situation.

A simple linear vector quantization technique was used to sample and extract the main characteristics (called quantizers) of the pressure profile. The net treating pressure neural network was trained using these quantizers and, when applied to a new case, reconstructed the entire pressure signal. This technique was used in the development of the pressure profile neural network and was trained in the case of ramp treatments. 


\subsubsection{Defining Pressure Components Used For Pressure Matching}

There are several pressure components that are either calculated or measured during a fracture simulation. These components are defined below:

1. Net Pressure: the pressure in the fracture minus the closure stress that is calculated by the model.

2. Observed Net Pressure: the pressure in the fracture minus the closure stress that is obtained by using one of the three possible measured pressures (bottomhole, surface, or dead string). This pressure is calculated only if a database is available.

3. Tophole Pressure: the pressure at the top of the wellbore, also called treating pressure.

4. Bottomhole Pressure: the calculated bottomhole pressure. This is calculated when it is not an input channel for the simulation.

5. Measured Bottomhole Pressure: the pressure in the wellbore at the fracture initiation depth that is derived from the pressure selected as input channel.

6. Hydrostatic Pressure: the hydrostatic pressure of the fluid and proppant in the wellbore.

7. Wellbore Friction: the calculated wellbore friction pressure. This calculation takes into account different fluids, different proppant concentrations, and different velocities in the wellbore.

8. Perf Friction: the calculated perforation friction pressure.

9. Near Wellbore Friction: the calculated near-wellbore friction.

In pressure matching, FRACPRO uses two specific pressure variables to compare: the observed net pressure and the net pressure. The fracture simulator predicts the net pressure, but there are three choices to calculate the observed net pressure based on the pressure measured at the well site in the database. The observed net pressure calculation options are outlined below:

1. Calculate Observed Net Pressure from Bottomhole Pressure

The recorded bottomhole pressure data is read directly into the simulator as measured bottomhole pressure. Perforation pressure, near wellbore friction, and closure stress in the pay zone are subtracted from the measured bottomhole pressure to give the observed net pressure.

$P_{\text {observed net }}=P_{\text {bottomhole }}-P_{\text {perf }}-P_{\text {near wellbore }}-P_{\text {closure }} \quad$ Equation 4-9

1. Calculate Observed Net Pressure from Dead String Pressure

The pressure measured in the dead string is read into the simulator. The hydrostatic pressure in the dead string, plus the hydrostatic pressure from the end of the dead string down to the center of the fracture, is added to the measured dead string pressure. The wellbore friction present from the bottom of the dead string down to the perforations is subtracted to give the measured bottomhole pressure. The perforation friction, near 
wellbore friction, and closure stress in the pay zone are subtracted from the measured bottomhole pressure to give the observed net pressure.

$P_{\text {observed net }}=P_{\text {dead string }}+P_{\text {dead string hydrostatic }}-P_{\text {perf }}-P_{\text {near wellbore }}-P_{\text {closure }}$ Equation 4-10

2. Calculate Observed Net Pressure from Surface Treating Pressure

The recorded surface treating pressure is read into the simulator directly as tophole pressure. The hydrostatic pressure is added and the wellbore friction is subtracted from the tophole pressure to give the measured bottomhole pressure. The perforation friction, near wellbore friction, and closure stress in the pay zone are subtracted from the measured bottomhole pressure to give the observed net pressure.

$P_{\text {observed net }}=P_{\text {treating }}+P_{\text {hydrostatic }}-P_{\text {perf }}-P_{\text {near wellbore }}-P_{\text {closure }} \quad$ Equation 4-11

\subsubsection{Pressure Matching Discussion}

The pressure profile module is intended to reproduce the net pressure developed during fracturing as predicted by the fracture simulator. This pressure is the observed net pressure during the pressure matching process.

Figure 4-15 shows an example of the pressure matching process, where the jagged red line represents the observed net pressure while the smooth blue line represents the net pressure predicted by the fracture simulator. According to Halliburton engineers this example is considered to be a good match between the two pressures.

The net pressure profile predicted in Figure 4-15 was generated using the original lithology of the reservoir, consisting of a significant number of layers, and the designed treatment schedule. One of the problems with using complex lithologies with a high number of layers is the exponential increase of the number of inputs in designing the neural network. Generating complex lithologies to simulate them using FRACPRO becomes an impractical and timeconsuming task. 


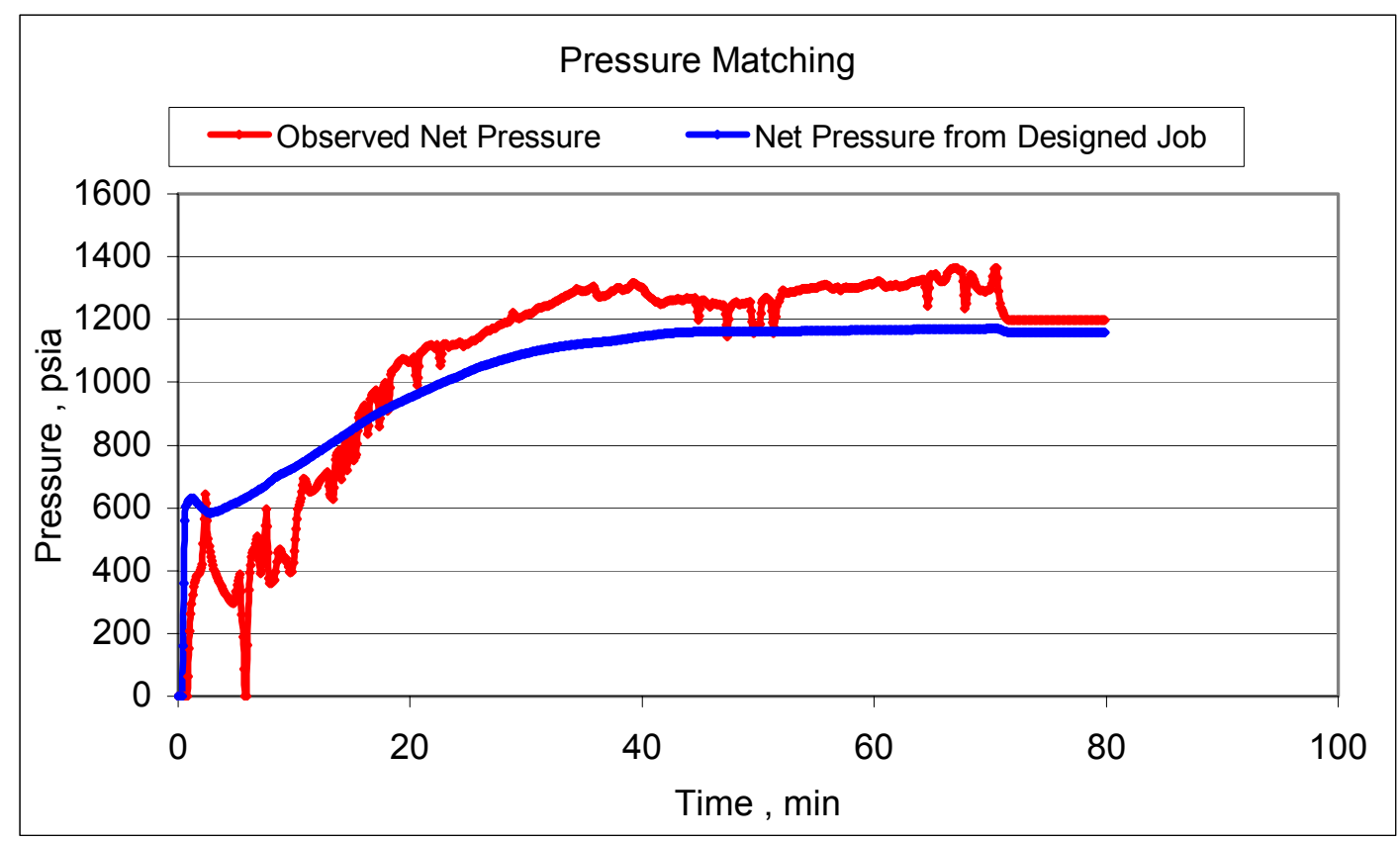

Figure 4-15: Pressure Matching Example between Observed Net Pressure and Predicted Net Pressure

In section 4.10 a new methodology was introduced in order to reduce the number of layers to a four-layer equivalent profile. This equivalent four-layer profile proved to be consistent in providing accurate fracture geometry results compared to the original profile when simulated with FRACPRO.

Since the net pressure profile data is needed to train the neural networks, a first attempt was made to use the four-layer representative profile in the process of data generation. However, before running hundreds of cases, the net pressure profile calculated using original reservoir lithology and the net pressure profile using the equivalent four layers were compared. In order to achieve this, ten random reservoir lithologies were generated, and the corresponding equivalent four-layer profile was calculated. Each case was run through FRACPRO using both scenarios: the entire lithology and the equivalent four-layer profile. The same ramp treatment design was used in all simulations.

The results posed a new problem. In all ten cases, the net pressure profile calculated using the equivalent four-layer algorithm was significantly lower than the net pressure profile calculated using original lithology. Three examples that clearly illustrate this behavior are shown below in Figure 4-16, Figure 4-17, and Figure 4-18. The blue diamond line represents the net pressure profile calculated from the original lithology, and the pink triangular line is the net pressure profile for the four-layer equivalent model. 


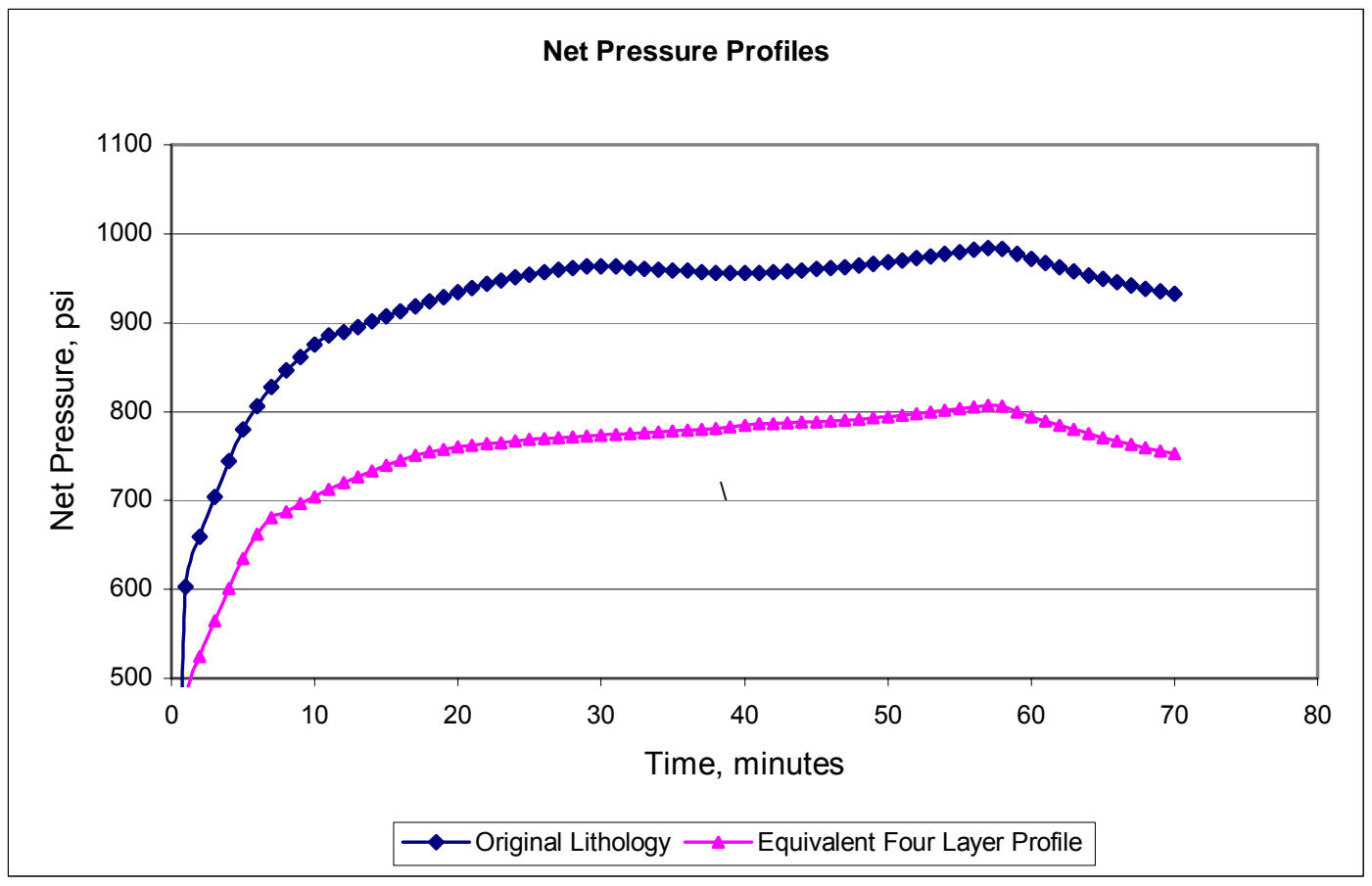

Figure 4-16: Net Pressures for Original Lithology and Equivalent Profile from Case 1

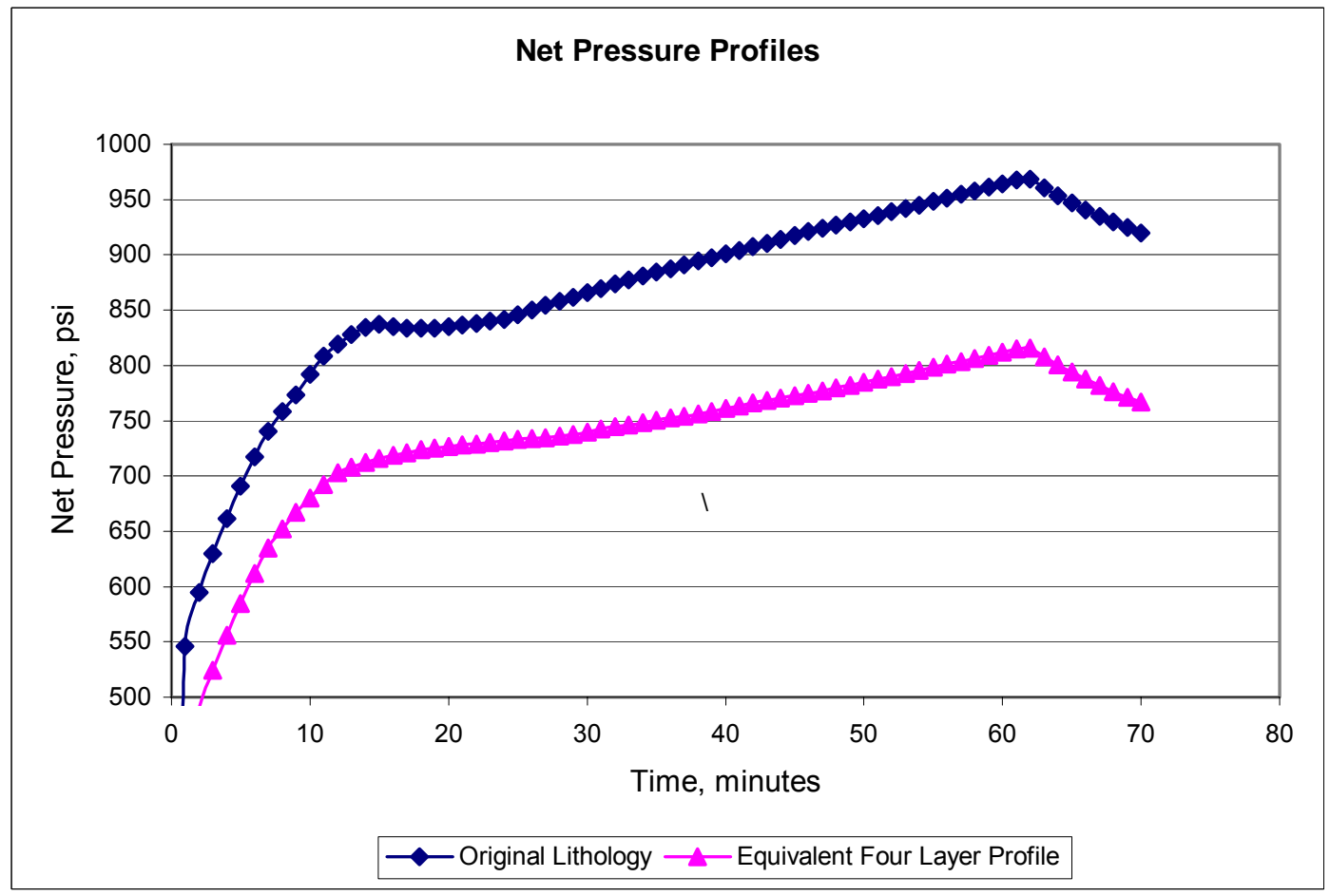

Figure 4-17: Net Pressures for Original Lithology and Equivalent Profile from Case 3 


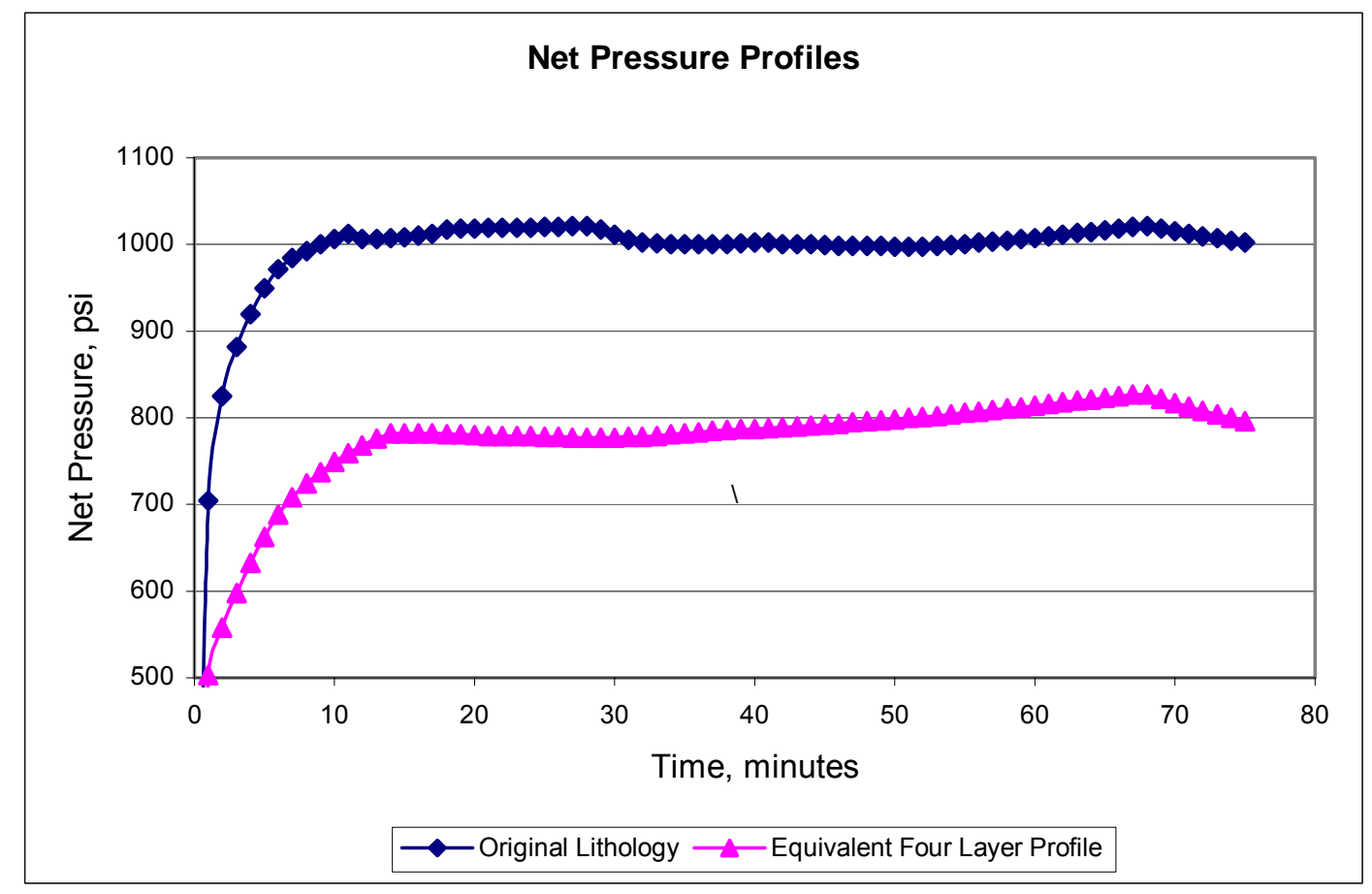

Figure 4-18: Net Pressures for Original Lithology and Equivalent Profile from Case 8

The difference between the original and equivalent profiles was more severe in some cases than others. A credible reason for the net pressure profile to exhibit much lower pressure values for the entire treatment may be related to the stress contrast between the four layer equivalent profiles. This difference and inconsistency was not acceptable for modeling, so a new approach was needed. The goal of this new model was to determine a similar representative stress profile able to provide the same pressure profile as the original lithology stress profile.

\subsubsection{Closure Stress Coefficient Alteration}

The first approach in finding a new representative four-layer stress profile was to alter the closure stress gradients of the four layers in a controlled way. This was done such that the new pressure profile calculated from FRACPRO would provide a closer match with the pressure profile generated by the original stress profile. Only four parameters out of the total eight for the representative four-layer stress model were considered for alteration, namely the closure stress gradients for each of the four layers.

1. Top layer closure stress coefficient

2. Limiting layer closure stress coefficient

3. Pay zone closure stress coefficient

4. Bottom layer closure stress coefficient 
Due to the relatively high number of parameters that can be modified from one run to another the study was conducted in two scenarios. The first scenario considered alterations of the closure stress gradients for top layer, overburden layer and bottom layer. The second scenario altered the closure stress coefficient only for the pay zone layer maintaining the same equivalent permeability.

\subsection{Top, Overburden, and Bottom Layers}

The first scenario (altering the closure stress gradients for top, overburden, and bottom layers) was completed in two sub-steps:

A. Closure stress gradients for the top layer, limiting layer and bottom layer are increased with a percentage between $2-5 \%$.

B. Closure stress gradients for only the top layer and bottom layer are increased with a percentage between $2-5 \%$.

The same ten cases analyzed previously were used in this analysis. FRACPRO was run using the original stress profile and the net pressure profile was recorded. Next, the simulator was run using the altered representative four-layer stress profile. The net pressure profile was again recorded and then compared with the one generated by the original stress profile. The representative four-layer stress profile was then altered four times for scenario $A$ and four times for scenario $B$. This generated eight additional net pressure profiles when run through FRACPRO. The stress data corresponding to the altered four-layer equivalent profiles for scenario $A$ and scenario $B$ are shown in

Table 4-21 and Table 4-22 respectively.

\begin{tabular}{|l|c|c|c|c|c|}
\hline & Equivalent & Equivalent & Equivalent & Equivalent & Equivalent \\
Profile & TOB 2\% & TOB 3\% & TOB 4\% & TOB 5\% \\
\hline & & $2 \%$ & $3 \%$ & $4 \%$ & $5 \%$ \\
\hline Top Layer & & & & & \\
\hline Overburden Layer & 0.729 & 0.751 & 0.758 & 0.765 & 0.773 \\
\hline Pay Zone & 0.646 & 0.815 & 0.823 & 0.831 & 0.611 \\
\hline Bottom Layer & 0.715 & 0.665 & 0.672 & 0.678 & 0.395 \\
\hline
\end{tabular}

Table 4-21: Closure Stress Gradient Values for Equivalent Altered Profiles from Scenario A Case 1 


\begin{tabular}{|l|r|c|r|r|r|}
\hline & $\begin{array}{c}\text { Equivalent } \\
\text { Profile }\end{array}$ & $\begin{array}{c}\text { Equivalent } \\
\text { TB 2\% }\end{array}$ & $\begin{array}{c}\text { Equivalent } \\
\text { TB 3\% }\end{array}$ & $\begin{array}{c}\text { Equivalent } \\
\text { TB 4\% }\end{array}$ & $\begin{array}{c}\text { Equivalent } \\
\text { TB 5\% }\end{array}$ \\
\hline & & $2 \%$ & $3 \%$ & $4 \%$ & $5 \%$ \\
\hline Top Layer & & & & & \\
\hline Overburden Layer & 0.729 & 0.751 & 0.758 & 0.765 & 0.773 \\
\hline Pay Zone & 0.646 & 0.791 & 0.791 & 0.791 & 0.791 \\
\hline Bottom Layer & 0.715 & 0.736 & 0.646 & 0.646 & 0.646 \\
\hline
\end{tabular}

Table 4-22: Closure Stress Gradient Values for Equivalent Altered Profiles from Scenario B Case 1

Figure 4-19 shows eight net pressure profiles, the original stress profile, the equivalent four-layer stress profile, and six altered equivalent four-layer profiles (three from scenario $A$ and three from scenario $B$ ). The following descriptions explain the details for each stress profile:

- Original Lithology: represents the pressure profile for the original stress profile.

- Equivalent Profile: represents the pressure profile corresponding to the representative four layers as defined in section 4.10.

- Equivalent TOB 3\%: represents the case when top layer, overburden layer and bottom layer closure stress coefficients are increased by $3 \%$.

- Equivalent TOB 4\%: represents the case when top layer, overburden layer and bottom layer closure stress coefficients are increased by $4 \%$.

- Equivalent TOB 5\%: represents the case when top layer, overburden layer and bottom layer closure stress coefficients are increased by $5 \%$.

- Equivalent TB 3\%: represents the case when only top layer and bottom layer closure stress coefficients are increased by $3 \%$.

- Equivalent TB 4\%: represents the case when only top layer and bottom layer closure stress coefficients are increased by $3 \%$.

- Equivalent TB 5\%: represents the case when only Top layer and Bottom layer closure stress coefficients are increased by $3 \%$. 


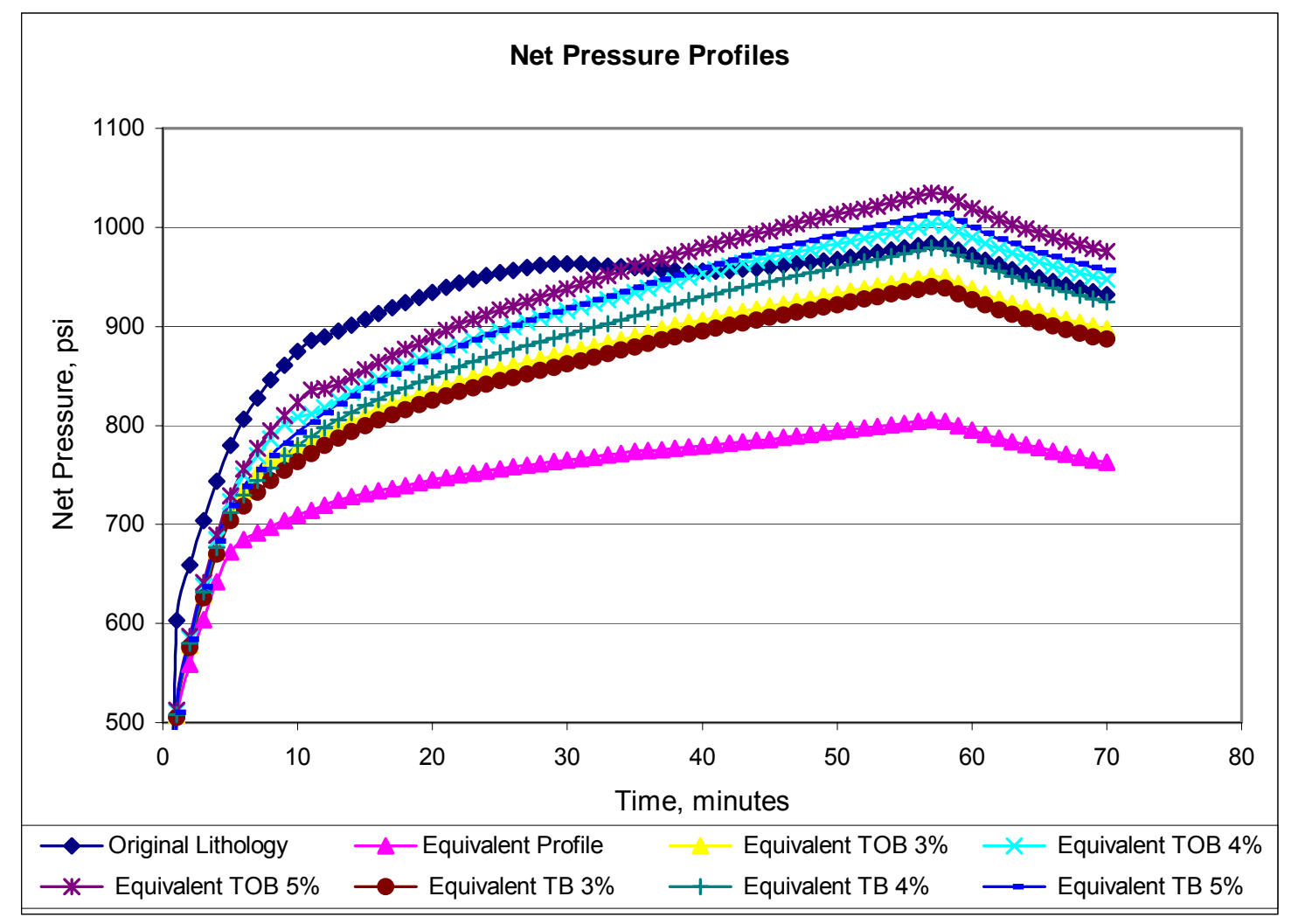

Figure 4-19: Net Pressure Profile Comparisons for Equivalent Altered Profiles from Case 1

The net pressure profiles for Equivalent TOB 2\% and Equivalent TB 2\% are not displayed on the figure since their results were very close to the equivalent four-layer profile, which clutters the graph and makes it difficult to see.

Another example is Case 3 presented below. The stress values corresponding to the altered fourlayer equivalent profiles for scenario $A$ and scenario $B$ are shown in

\begin{tabular}{|l|c|c|c|c|c|}
\hline & $\begin{array}{c}\text { Equivalent } \\
\text { Profile }\end{array}$ & $\begin{array}{c}\text { Equivalent } \\
\text { TOB 2\% }\end{array}$ & $\begin{array}{c}\text { Equivalent } \\
\text { TOB 3\% }\end{array}$ & $\begin{array}{c}\text { Equivalent } \\
\text { TOB 4\% }\end{array}$ & $\begin{array}{c}\text { Equivalent } \\
\text { TOB 5\% }\end{array}$ \\
\hline & & $2 \%$ & $3 \%$ & $4 \%$ & $5 \%$ \\
\hline Top Layer & 0.750 & 0.765 & 0.773 & 0.780 & 0.788 \\
\hline Overburden Layer & 0.809 & 0.825 & 0.833 & 0.841 & 0.849 \\
\hline Pay Zone & 0.665 & 0.665 & 0.665 & 0.665 & 0.665 \\
\hline Bottom Layer & 0.730 & 0.745 & 0.752 & 0.759 & 0.767 \\
\hline
\end{tabular}

Table 4-23 and

Table 4-24. 


\begin{tabular}{|l|r|c|c|c|c|}
\hline & $\begin{array}{c}\text { Equivalent } \\
\text { Profile }\end{array}$ & $\begin{array}{c}\text { Equivalent } \\
\text { LOB 2\% }\end{array}$ & $\begin{array}{c}\text { Equivalent } \\
\text { TOB 3\% }\end{array}$ & $\begin{array}{c}\text { Equivalent } \\
\text { TOB 4\% }\end{array}$ & $\begin{array}{c}\text { Equivalent } \\
\text { TOB 5\% }\end{array}$ \\
\hline & & $2 \%$ & $3 \%$ & $4 \%$ & $5 \%$ \\
\hline Top Layer & & & & & \\
\hline Overburden Layer & 0.750 & 0.765 & 0.773 & 0.780 & 0.788 \\
\hline Pay Zone & 0.609 & 0.825 & 0.833 & 0.841 & 0.849 \\
\hline Bottom Layer & 0.730 & 0.665 & 0.665 & 0.665 & 0.665 \\
\hline
\end{tabular}

Table 4-23: Closure Stress Gradient Values for Equivalent Altered Profiles from Scenario A Case 3

\begin{tabular}{|l|r|r|r|r|r|}
\hline Layer Name & $\begin{array}{c}\text { Equivalent } \\
\text { Profile }\end{array}$ & $\begin{array}{c}\text { Equivalent } \\
\text { TB 2\% }\end{array}$ & $\begin{array}{c}\text { Equivalent } \\
\text { TB 3\% }\end{array}$ & $\begin{array}{c}\text { Equivalent } \\
\text { TB 4\% }\end{array}$ & $\begin{array}{c}\text { Equivalent } \\
\text { TB 5\% }\end{array}$ \\
\hline & & \multicolumn{1}{c|}{$2 \%$} & $3 \%$ & \multicolumn{1}{c|}{$4 \%$} & \multicolumn{1}{c|}{$5 \%$} \\
\hline Top Layer & 0.75 & 0.765 & 0.7725 & 0.78 & 0.7875 \\
\hline Overburden Layer & 0.809 & 0.809 & 0.809 & 0.809 & 0.809 \\
\hline Pay Zone & 0.665 & 0.665 & 0.665 & 0.665 & 0.665 \\
\hline Bottom Layer & 0.73 & 0.7446 & 0.7519 & 0.7592 & 0.7665 \\
\hline
\end{tabular}

Table 4-24: Closure Stress Gradient Values for Equivalent Altered Profiles from Scenario B Case 3

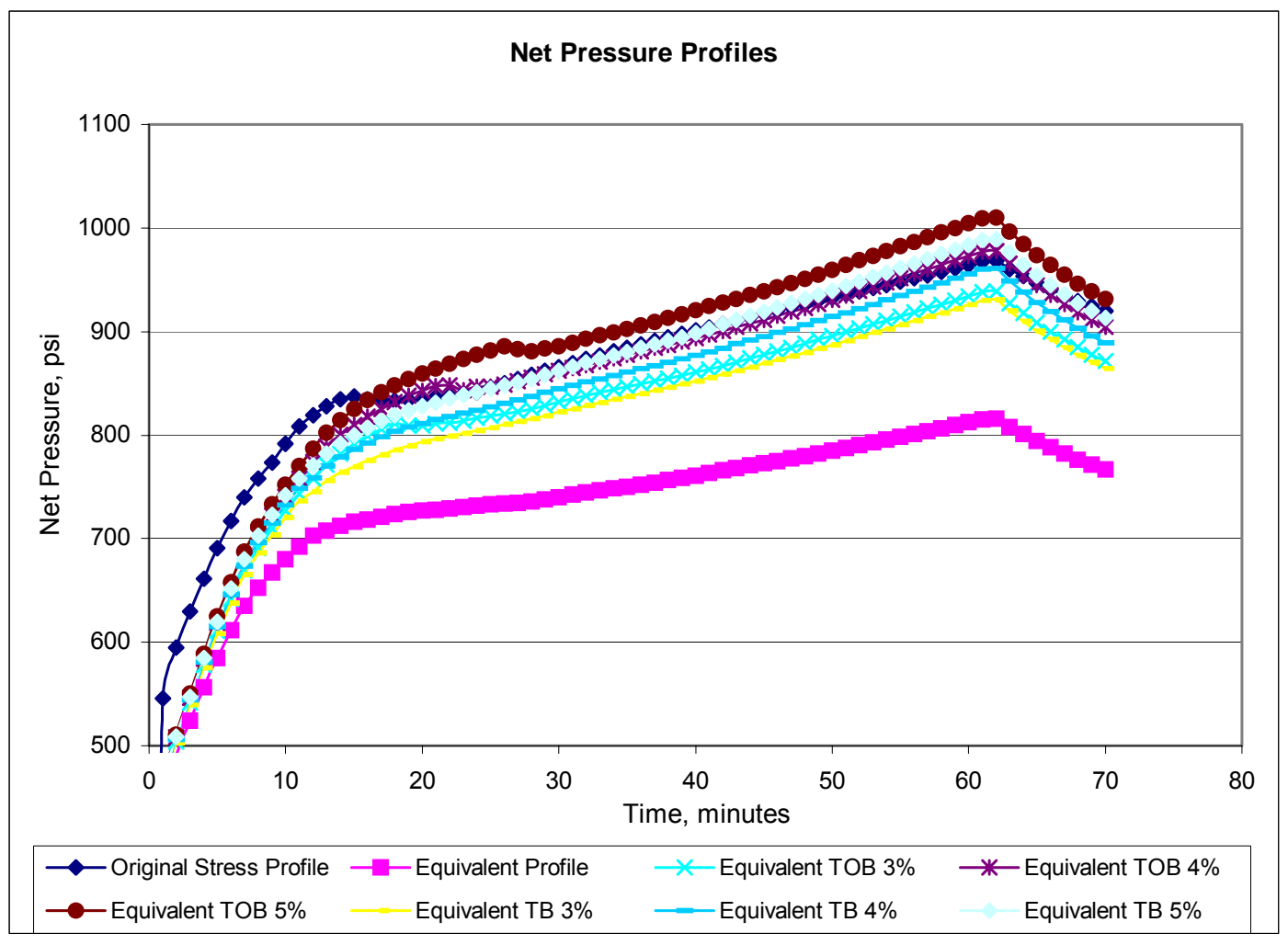

Figure 4-20: Net Pressure Profile Comparisons for Equivalent Altered Profiles from Case 3 
This analysis confirmed that increasing the stress contrast between the non-productive layers and the pay zone layer leads to an increase in the net pressure profile values. Analyzing the shapes of the ten cases showed that some of the altered four-layer profiles almost overlap with original net pressure profile, while others come close in pressure values but not in shape. Case 3 (Figure 4-20) is an example where the net pressure profile generated using Equivalent TOB $4 \%$ provided a similar match to the net pressure profile calculated from original lithology. Furthermore, Case 1 (Figure 4-19) shows an example where pressure values are close using Equivalent TOB $5 \%$ or TOB $4 \%$ but they are not able to reproduce the shape of the pressure profile. The most acceptable pressure match is obtained when the closure stress coefficients of the three non-producing layers of the profile were increased by around $4 \%$.

\subsection{Pay Zone Layer}

The second scenario altered the closure stress coefficient only for the pay zone layer while maintaining the same equivalent permeability. The closure stress gradient for the pay zone layer was decreased by a percentage of $3-7 \%$ psi/ft. Table $4-25$ shows an example of this approach, which was performed each of the previous ten cases in order to provide consistent results.

Table 4-25 shows the altered values of the pay zone closure stress gradient, while Figure 4-21 shows the net pressure profile from Case 1.

- Original Lithology: represents the pressure profile for the original stress profile.

- Equivalent Profile: represents the pressure profile corresponding to the equivalent four-layer profile.

- Equivalent PZ 3\%: represents the case when closure stress gradient of the pay zone layer, is decreased by $3 \%$.

- Equivalent PZ 4.5\%: represents the case when closure stress gradient of the pay zone layer, is decreased $4.5 \%$.

- Equivalent PZ 6\%: represents the case when closure stress gradient of the pay zone layer, is decreased by $6 \%$.

\begin{tabular}{|l|r|r|r|r|}
\hline Layer Name & $\begin{array}{c}\text { Equivalent } \\
\text { Profile }\end{array}$ & $\begin{array}{c}\text { Equivalent } \\
\text { PZ-3\% }\end{array}$ & $\begin{array}{c}\text { Equivalent } \\
\text { PZ-4.5\% }\end{array}$ & $\begin{array}{c}\text { Equivalent } \\
\text { PZ-6\% }\end{array}$ \\
\hline & & & & \\
\hline Top Layer & 0.729 & 0.729 & 0.729 & 0.729 \\
\hline Overburden Layer & 0.791 & 0.791 & 0.791 & 0.791 \\
\hline Pay Zone & 0.646 & 0.626 & 0.616 & 0.607 \\
\hline Bottom Layer & 0.715 & 0.715 & 0.715 & 0.715 \\
\hline
\end{tabular}


Table 4-25: Closure Stress Gradient Values for Pay Zone Equivalent Altered Profiles from Case 1

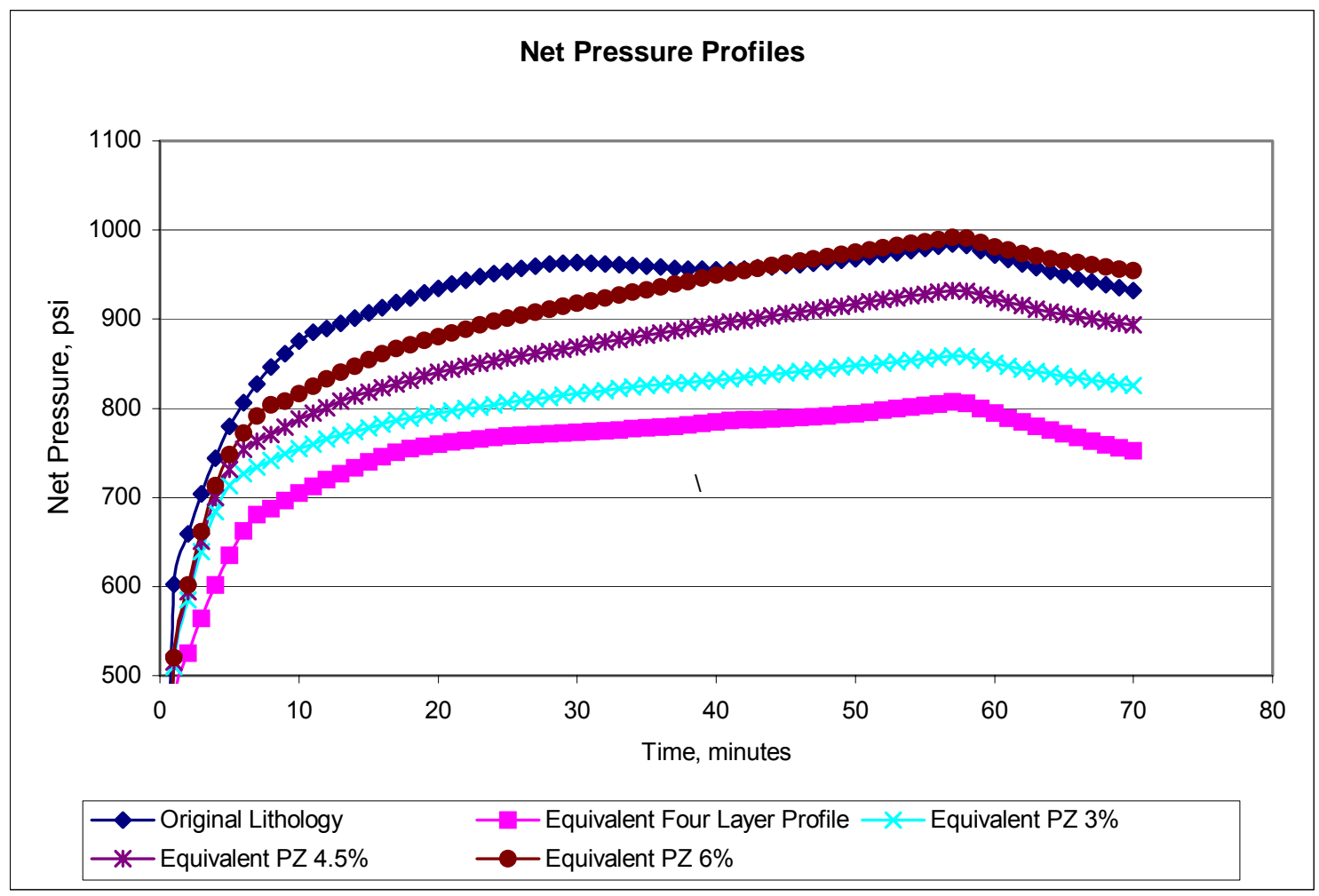

Figure 4-21: Net Pressure Profile Comparisons for Pay Zone Equivalent Altered Profiles from Case 1

Table 4-26 and Figure 4-22 display the analysis from Case 3.

\begin{tabular}{|l|r|r|r|r|}
\hline Layer Name & $\begin{array}{c}\text { Equivalent } \\
\text { Profile }\end{array}$ & $\begin{array}{c}\text { Equivalent } \\
\text { PZ-3\% }\end{array}$ & $\begin{array}{c}\text { Equivalent } \\
\text { PZ-4.5\% }\end{array}$ & $\begin{array}{c}\text { Equivalent } \\
\text { PZ-6\% }\end{array}$ \\
\hline & & & & \\
\hline Top Layer & 0.750 & 0.765 & 0.773 & 0.780 \\
\hline Overburden Layer & 0.809 & 0.825 & 0.833 & 0.841 \\
\hline Pay Zone & 0.665 & 0.645 & 0.635 & 0.625 \\
\hline Bottom Layer & 0.730 & 0.745 & 0.752 & 0.759 \\
\hline
\end{tabular}

Table 4-26: Closure Stress Gradient Values for Pay Zone Equivalent Altered Profiles from Case 1 


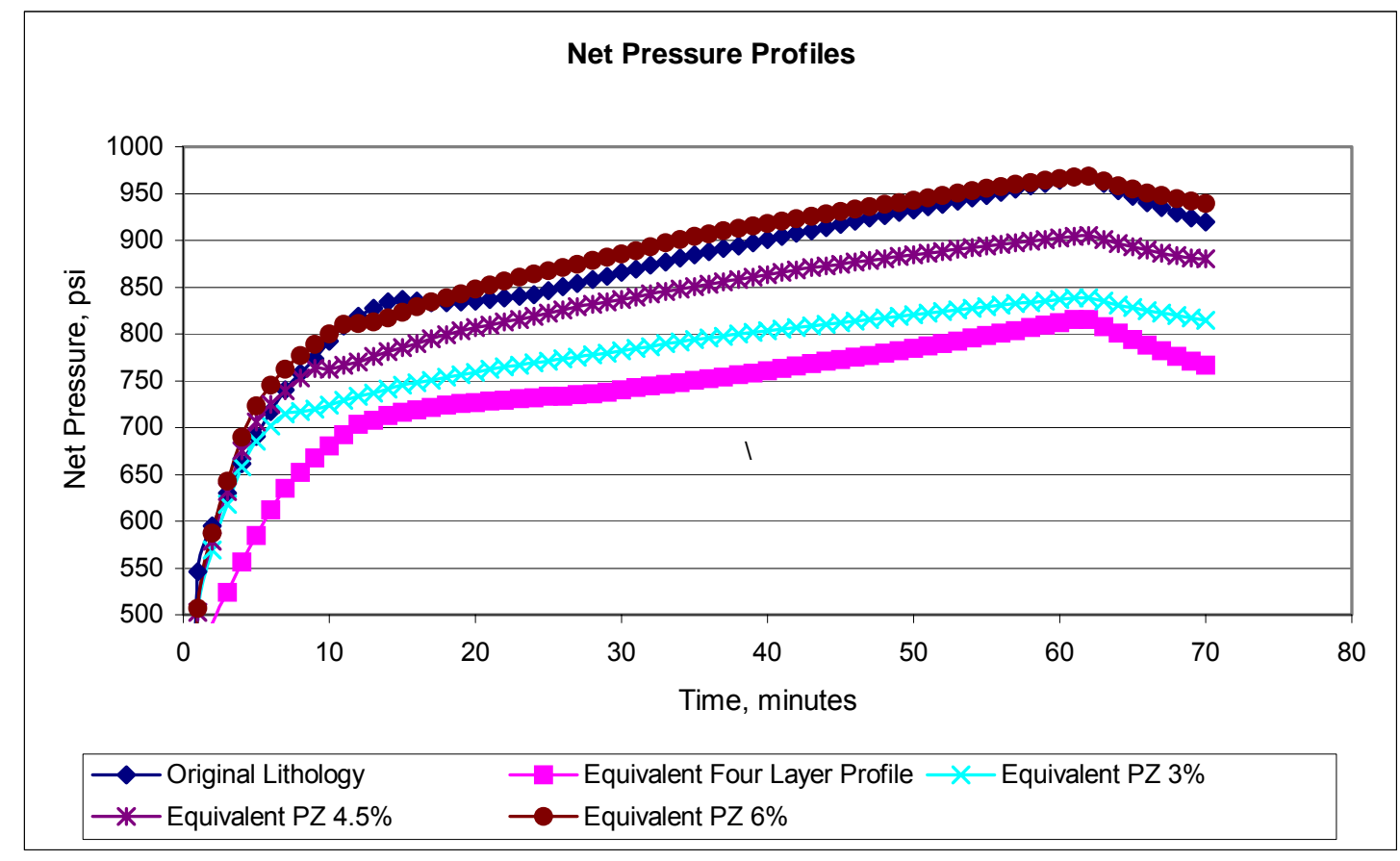

Figure 4-22: Net Pressure Profile Comparisons for Pay Zone Equivalent Altered Profiles from Case 3

Again, much like the altered profiles from top, overburden, and bottom layers previously, altering the closure stress gradient of the pay zone produces a stronger contrast with the nonproductive zones. This leads to increase in the net pressure profile values. In this case, the shapes of the ten cases show that some of the altered four-layer profiles provide a much better overlap, both in values and shapes, with the net pressure profile calculated from the original stress profile. For example, the net pressure profile generated using an Altered Equivalent PZ $6 \%$ provided an excellent match with the original net pressure profile in Case 1 (Figure 4-21) and Case 3 (Figure 4-22). Similar matches were observed for the remaining cases. The most acceptable pressure matches were obtained when the closure stress gradient of the pay zone was decreased by a percentage between 4.5 and $6 \%$.

\subsection{Closure Stress Coefficient Alteration Summary}

The closure stress coefficient alteration process was conducted in two parts until the best pressure match was reached:

- Top, Overburden, and Bottom Layer

This part consisted of two scenarios, the first where the top, overburden, and bottom layers are altered and the second where only the top and bottom layers were altered. Based on the analysis of these two scenarios, it was noted that the increase of the 
closure stress coefficient for the overburden layer does not significantly influence the pressure profile.

- Pay Zone Layer

The closure stress coefficient was decreased by a percentage of $3-7 \% \mathrm{psi} / \mathrm{ft}$ from the equivalent four-layer stress profile. The alteration of the representative layer profile was done empirically based on the rock mechanic and hydraulic fracture behavior.

In conclusion, the alteration of the pay zone closure stress gradient was preferred between the two scenarios presented above. This is for two reasons: first, it provided a better pressure match, both in pressure values and shape; and secondly because of the minimum number of parameters required to be altered (only the closure stress gradient of the pay zone). However, it was still unable to be determined what the critical percentage of the pay zone closure stress gradient should be decreased in order to provide the best net pressure profile match.

\subsubsection{Correction Percentage Value Determination}

The ten previously analyzed cases were not considered sufficient to define a set percentage value that, when applied to the pay zone closure stress gradient, would provide an acceptable match of the net pressure profiles. Further analysis proceeded of which the steps involved are as follows:

1. Generate 50 random cases of reservoir lithology profile where each case contains the following components:

a. Random number of layers above the first permeable productive formation called top layers.

b. Each top layer has a random thickness and a random closures stress gradient.

c. Random number of productive permeable formations called pay zones.

d. Each productive formation has a random thickness and a random closure stress gradient.

e. Random number of thin intercalations between the pay zones.

f. Random number of layers below the deepest permeable productive formation called bottom layers.

g. Each bottom layer has a random thickness and a random closures stress gradient.

This data was generated using a simple computer program. Logic and certain constraints were embedded in the code so that unrealistic cases could not be generated.

2. Apply the equivalent four-layer profile methodology to each of the fifty random cases and calculate the eight parameters defining the four-layer profile.

3. Select first randomly generated case. Design a unique treatment schedule for this case. Run FRACPRO using the reservoir lithology profile, and record the net pressure profile. This net pressure profile was named the original net pressure profile. 
4. Using the same treatment schedule, run FRACPRO using the equivalent four-layer stress profile. Record the net pressure profile. This net pressure profile was named the equivalent net pressure profile.

5. Alter the pay zone closure stress gradient of the equivalent four-layer profile using percentage values between 2 and $8 \%$. Using the same treatment design, run FRACPRO for each altered equivalent four-layer profile. Record net pressures for each run. Typically, start with a value of $3 \%$. Calculate the net pressure profile and plot it on the same graph with the original and equivalent net pressure profiles.

6. Increase the percentage value until a good match in achieved. Record each percentage used, the altered equivalent four-layer profile and the calculated net pressure profile.

7. Repeat the process from step 3.

An example of a generated reservoir lithology is seen in Table 4-27, while Table 4-28 shows the processed equivalent four-layer profile for the same case. Figure 4-23 shows both profiles, original and equivalent, together with two altered equivalent profiles.

\begin{tabular}{|l|c|c|c|c|}
\hline \multicolumn{1}{|c|}{ Layer Index } & $\begin{array}{c}\text { Top } \\
\text { Depth, } \mathrm{ft}\end{array}$ & $\begin{array}{c}\text { Thickness } \\
, \mathrm{ft}\end{array}$ & $\begin{array}{c}\text { Closure Stress } \\
\text { Gradient, psi/ft }\end{array}$ & $\begin{array}{c}\text { Permeability } \\
, \mathrm{md}\end{array}$ \\
\hline & & & & \\
\hline Layer 1 & 7080 & 34 & 0.751 & 0 \\
\hline Layer 2 & 7114 & 22 & 0.772 & 0 \\
\hline Layer 3 & 7136 & 12 & 0.731 & 0 \\
\hline Layer 4 & 7148 & 17 & 0.756 & 0 \\
\hline Layer 5 & 7165 & 12 & 0.755 & 0 \\
\hline Layer 6 & 7177 & 27 & 0.787 & 0 \\
\hline Layer 7 & 7204 & 33 & 0.738 & 0 \\
\hline Layer 8 & 7237 & 8 & 0.706 & 0 \\
\hline Layer 9 & 7245 & 11 & 0.737 & 0 \\
\hline Layer 10 & 7256 & 12 & 0.787 & 0 \\
\hline Layer 11 & 7268 & 29 & 0.8 & 0 \\
\hline Layer 12 & 7297 & 18 & 0.712 & 0 \\
\hline Pay Zone1 & 7315 & 12 & 0.664 & 0.006 \\
\hline Pay Zone2 & 7327 & 6 & 0.664 & 0.006 \\
\hline Layer 13 & 7333 & 10 & 0.719 & 0 \\
\hline Pay Zone 3 & 7343 & 22 & 0.664 & 0.006 \\
\hline Pay Zone 4 & 7365 & 13 & 0.687 & 0.057 \\
\hline Pay Zone 5 & 7378 & 19 & 0.664 & 0.006 \\
\hline Layer 14 & 7397 & 10 & 0.729 & 0 \\
\hline Pay Zone 6 & 7407 & 9 & 0.687 & 0.057 \\
\hline Layer 14 & 7416 & 84 & 0.722 & 0 \\
\hline Layer 15 & 7500 & - & 0.722 & 0 \\
\hline
\end{tabular}

Table 4-27: Generated Reservoir Lithology Case 


\begin{tabular}{|l|r|r|r|r|}
\hline \multicolumn{1}{|c|}{ Layer Index } & $\begin{array}{c}\text { Top } \\
\text { Depth, } \mathrm{ft}\end{array}$ & $\begin{array}{c}\text { Thickness } \\
, \mathrm{ft}\end{array}$ & $\begin{array}{c}\text { Closure Stress } \\
\text { Gradient, psi/ft }\end{array}$ & $\begin{array}{c}\text { Permeability } \\
, \mathrm{md}\end{array}$ \\
\hline & & & & \\
\hline Top Layer & 7080 & 206 & 0.758 & \\
\hline Overburden Layer & 7286 & 29 & 0.8 & \\
\hline Pay Zone & 7315 & 101 & 0.6813 & 0.015 \\
\hline Bottom Layer & 7416 & & 0.722 & \\
\hline
\end{tabular}

Table 4-28: Processed Equivalent Four-Layer Profile

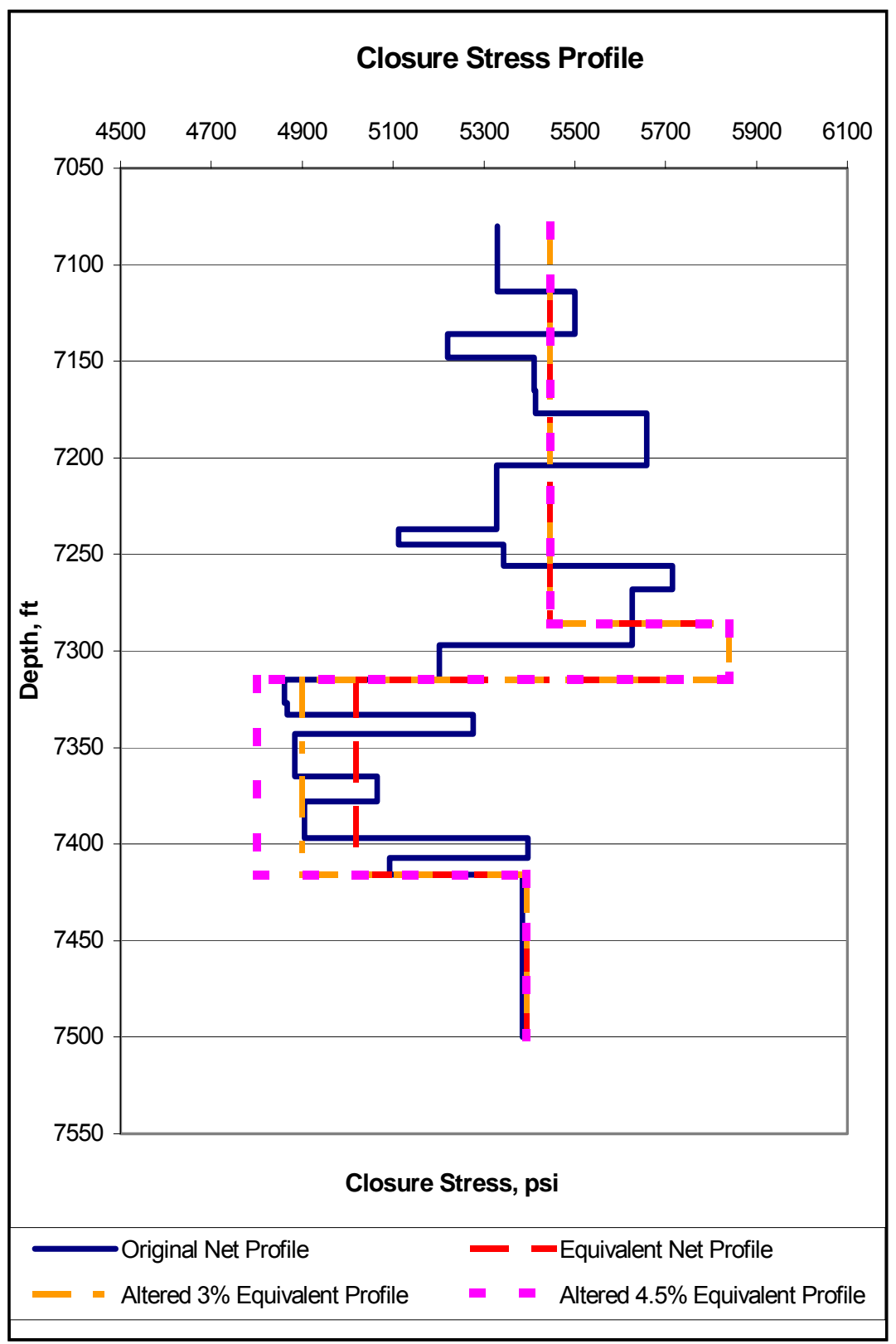

Figure 4-23: Original, Equivalent, and Altered Equivalent Stress Profiles 
The process described above was applied to all fifty generated cases. Several cases of the matching process follow. The remaining examples exhibited similar patterns, the only difference being the value of the net pressure profiles.

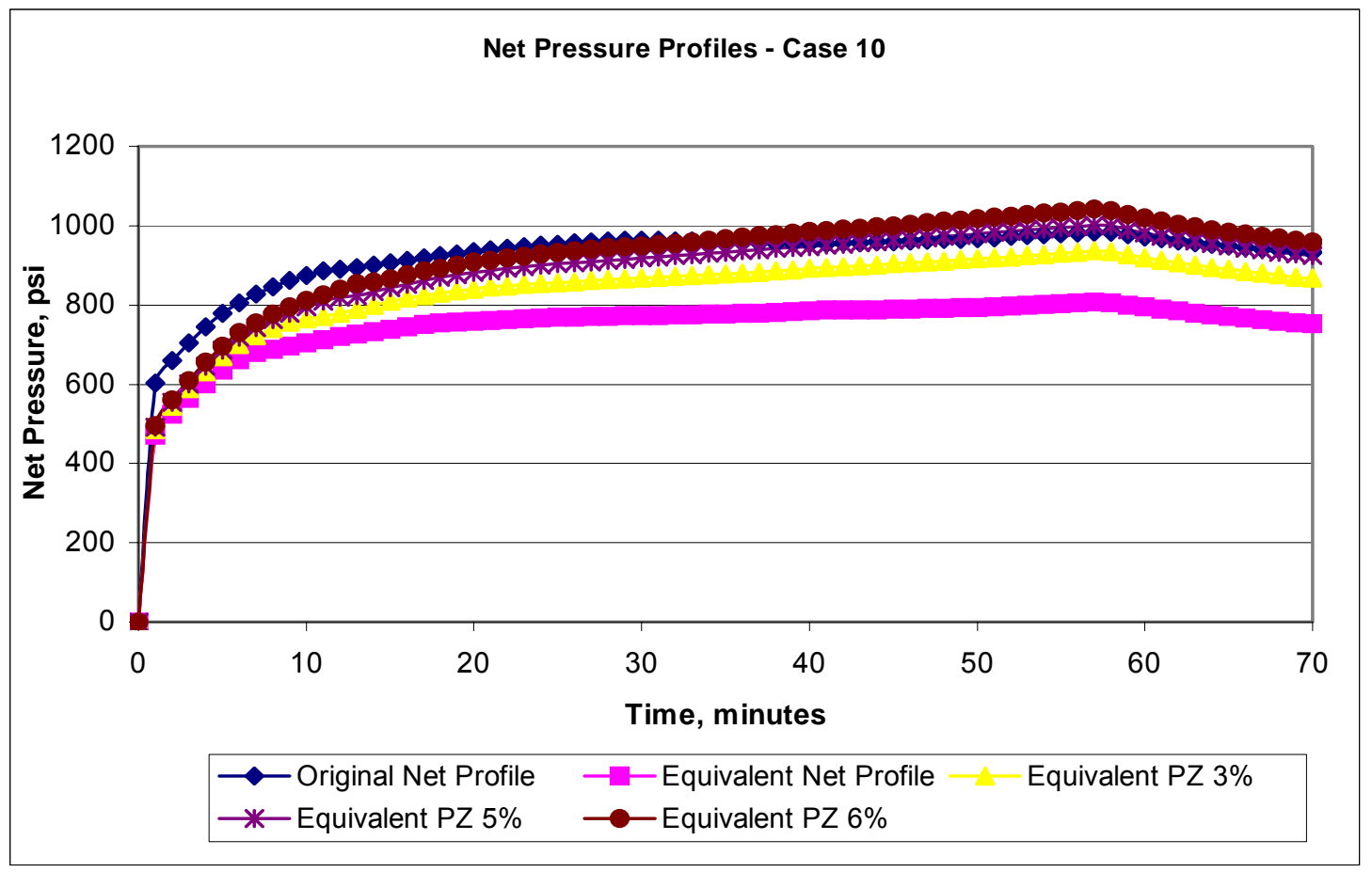

Figure 4-24: Net Pressure Profile Matching Process for Case 10

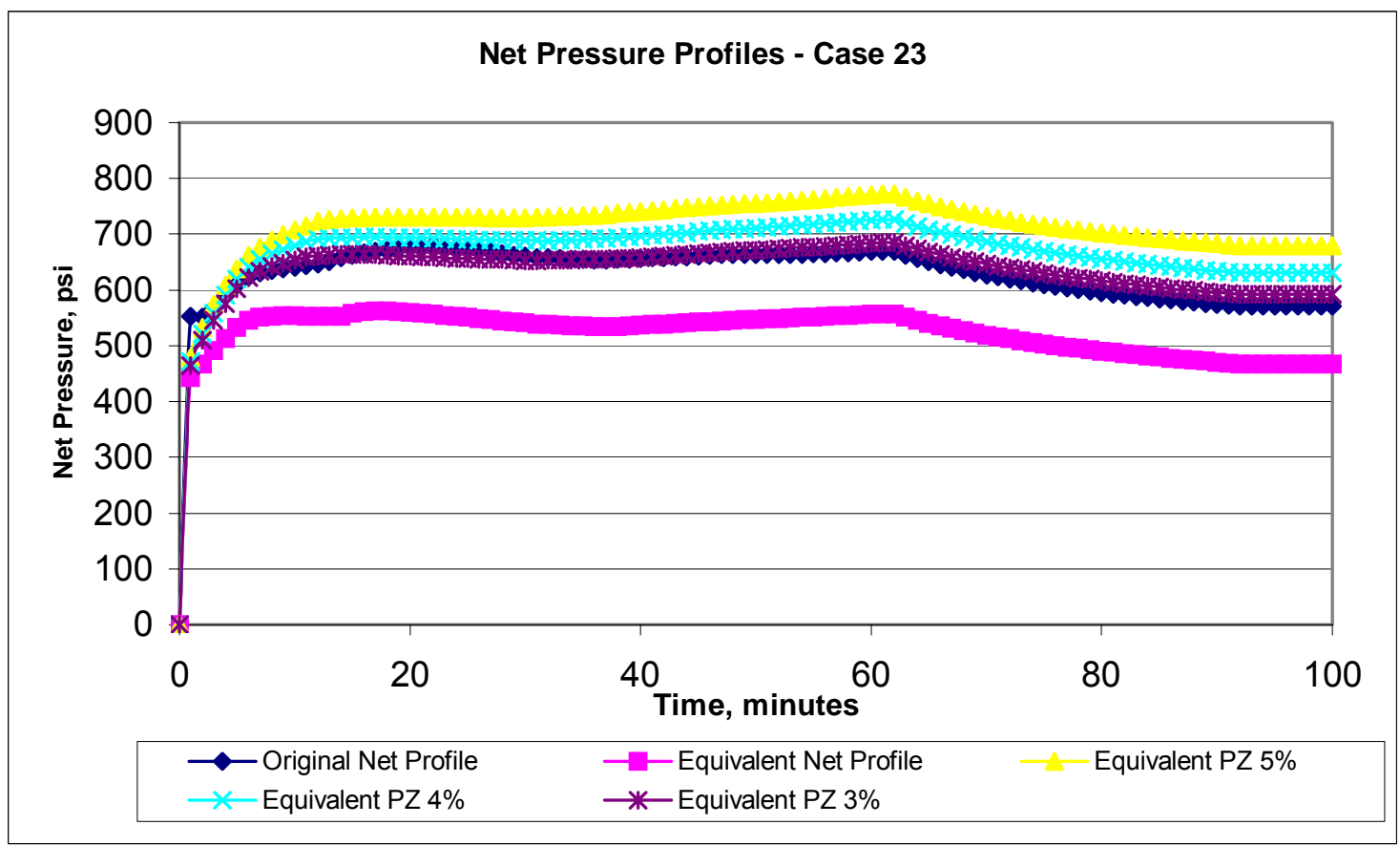

Figure 4-25: Net Pressure Profile Matching Process for Case 23 


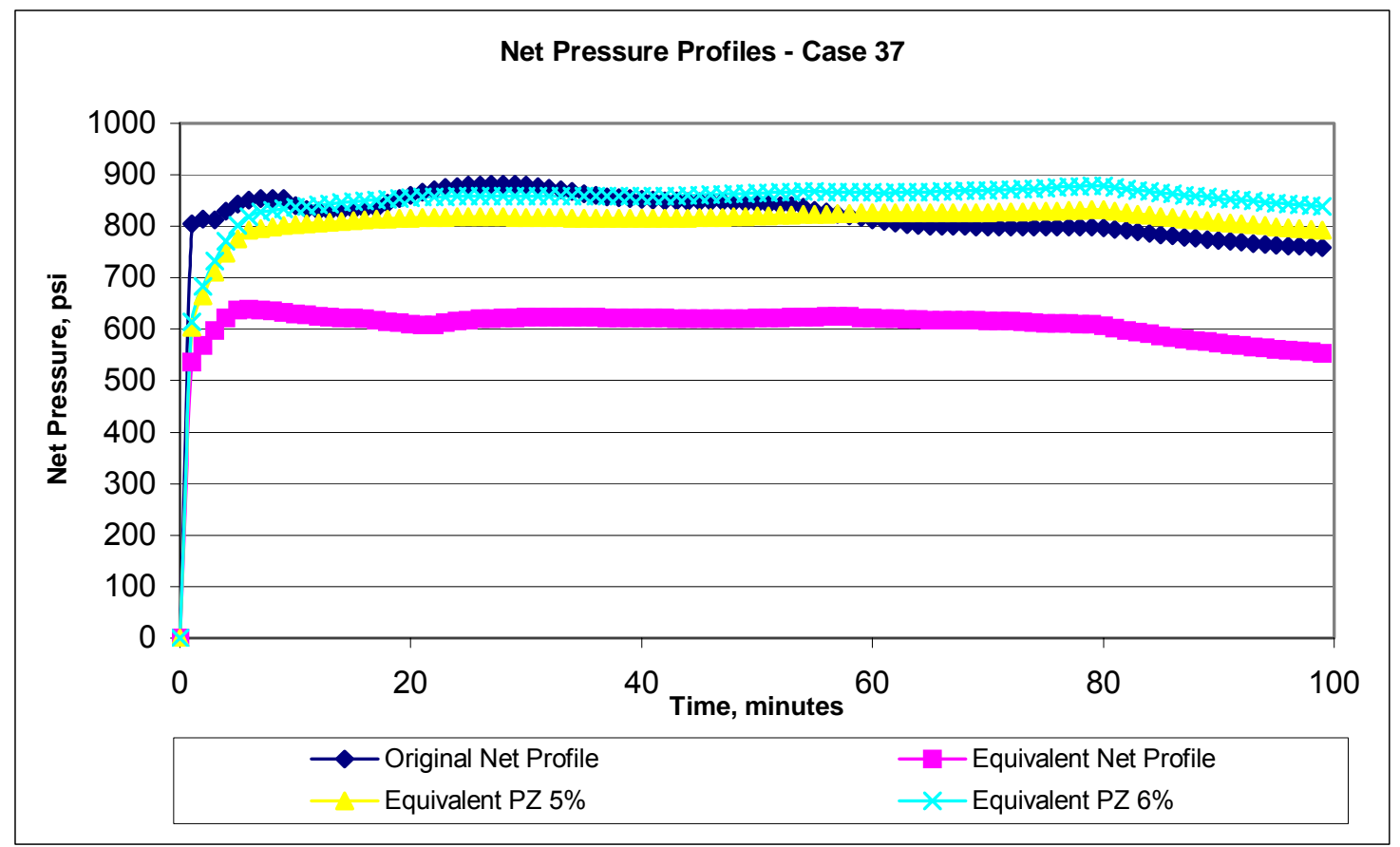

Figure 4-26: Net Pressure Profile Matching Process for Case 37

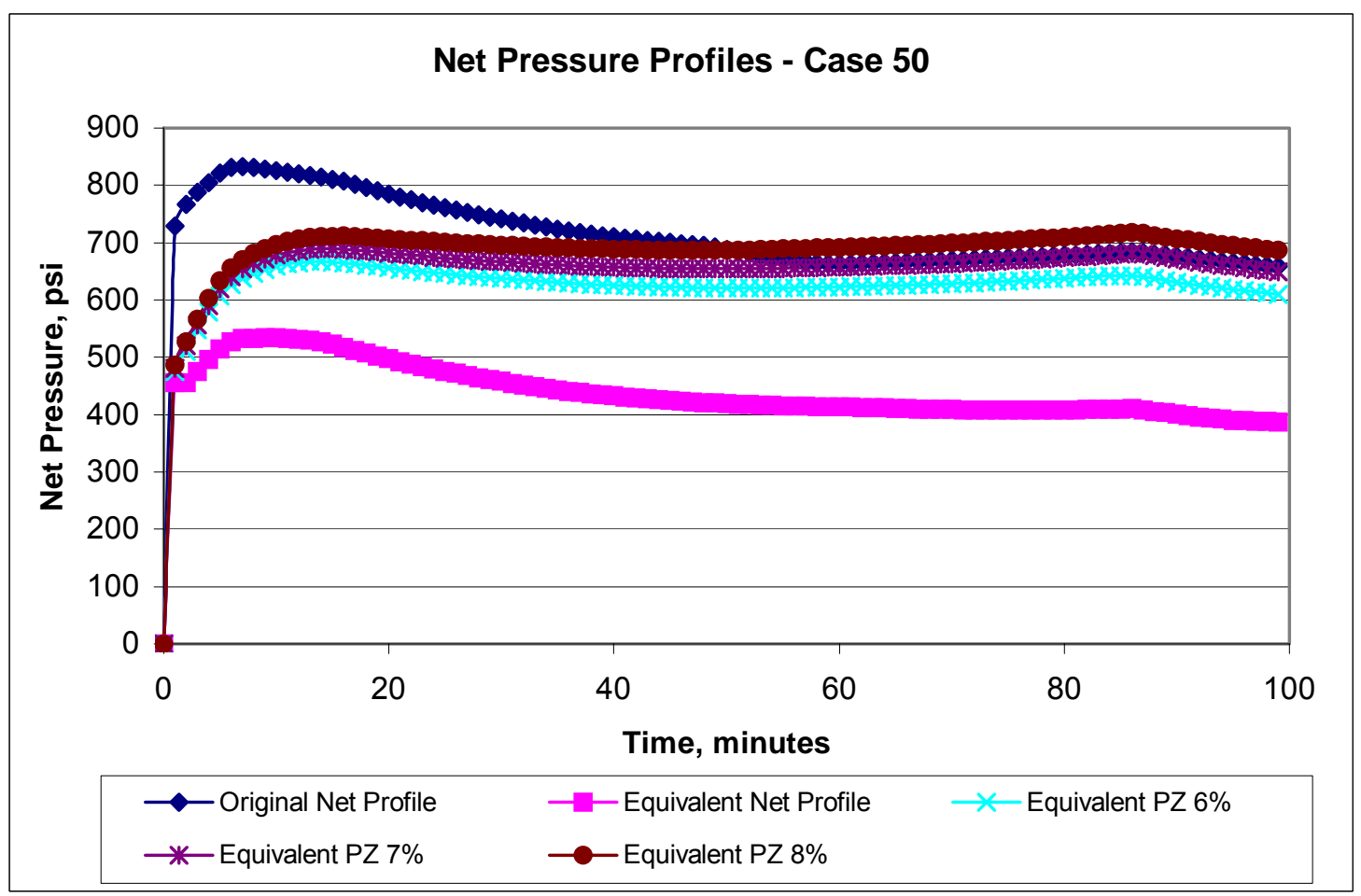

Figure 4-27: Net Pressure Profile Matching Process for Case 50 
Due to the fact that the process of entering data and preparing each simulation is very time consuming, only fifty cases were generated and analyzed. An in-depth visual analysis of all fifty cases allowed better interpretation of each case and matching. It should be noted that some correction percentage coefficients were slightly modified to allow for better matching. Still yet, in some of the cases the matching is not perfect and some pressure domains still do not overlap.

The four examples randomly selected and previously presented show that the correction percentage coefficient appears to have sporadic values. For example, the correction percentage coefficient in Case 10 (Figure 4-24) is 6\%, Case 23 (Figure 4-25) is 3\%, Case 37 (Figure 4-26) is $5 \%$, and Case 50 (Figure 4-27) is around $8 \%$. Table 4-29 shows the correction percentage coefficient for all fifty cases.

\begin{tabular}{|c|c|c|c|}
\hline Case Index & $\begin{array}{c}\text { Correction } \\
\text { percentage } \\
\text { value, } \%\end{array}$ & Case Index & $\begin{array}{c}\text { Correction } \\
\text { percentage } \\
\text { value, } \%\end{array}$ \\
\hline Case 1 & 6 & Case 26 & 9 \\
\hline Case 2 & 9 & Case 27 & 3 \\
\hline Case 3 & 4 & Case 28 & 9 \\
\hline Case 4 & 4.5 & Case 29 & 5 \\
\hline Case 5 & 4.5 & Case 30 & 1.5 \\
\hline Case 6 & 2.5 & Case 31 & 0 \\
\hline Case 7 & 3.5 & Case 32 & 1 \\
\hline Case 8 & 3 & Case 33 & 2.5 \\
\hline Case 9 & 2.5 & Case 34 & 3.5 \\
\hline Case 10 & 6.5 & Case 35 & 6 \\
\hline Case 11 & 3.5 & Case 36 & 1 \\
\hline Case 12 & 9.5 & Case 37 & 5.5 \\
\hline Case 13 & 9.5 & Case 38 & 3 \\
\hline Case 14 & 9.5 & Case 39 & 3.5 \\
\hline Case 15 & 9.5 & Case 40 & 6 \\
\hline Case 16 & 5.5 & Case 41 & 7 \\
\hline Case 17 & 6.5 & Case 42 & 4 \\
\hline Case 18 & 2 & Case 43 & 1.5 \\
\hline Case 19 & 10 & Case 44 & 5.5 \\
\hline Case 20 & 10 & Case 45 & 3 \\
\hline Case 21 & 10 & Case 46 & 9 \\
\hline Case 22 & 3 & Case 47 & 4 \\
\hline Case 23 & 3 & Case 48 & 2.5 \\
\hline Case 24 & 8 & Case 49 & 4.5 \\
\hline Case 25 & 5.5 & Case 50 & 8 \\
\hline
\end{tabular}

Table 4-29: Correction Percentage Coefficients for 50 Cases 
The frequency distribution of the correction percentage coefficient was plotted. Figure 4-28 shows that the distribution is fairly uniform and no clear answer was concluded.

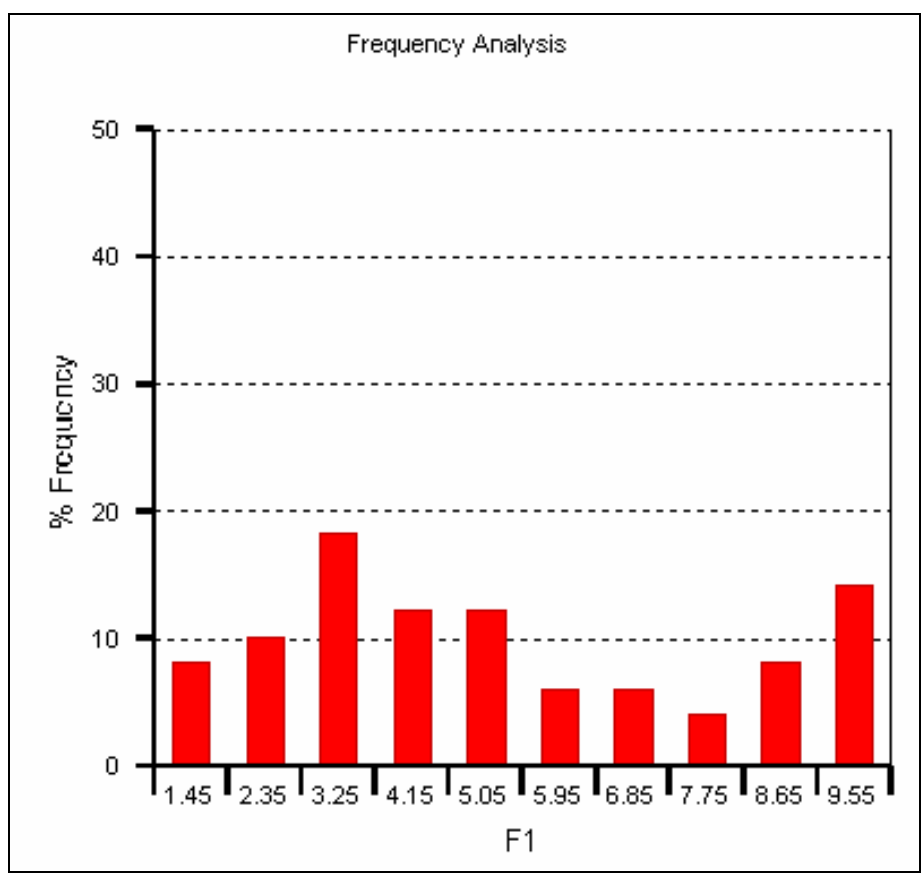

Figure 4-28: Frequency Distribution Analysis for Percentage Coefficient

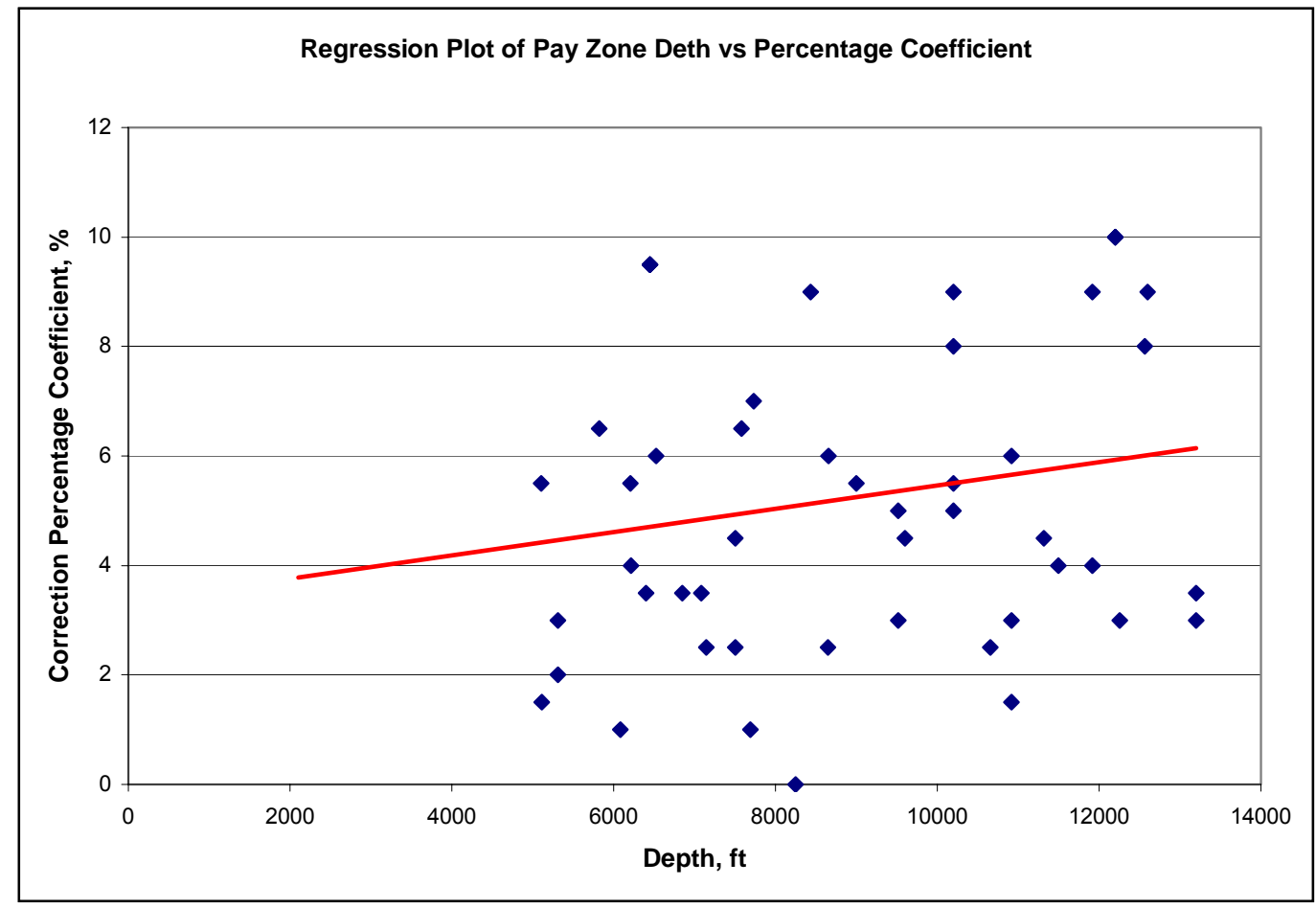

Figure 4-29: Regression Plot of Pay Zone Depth vs. Correction Percentage Coefficient 
Since, the stress profile is directly proportional with the depth, a regression was run comparing the depth of the pay zone with the reservoir lithology versus the correction percentage coefficient. Figure 4-29 clearly shows that the percentage coefficient does not depend on the reservoir depth. Since no default value or significant relationship was found to help estimate the percentage coefficient, a new process was initiated to examine the data.

\subsubsection{Equivalent Shift Delta Pressure Profile}

Careful analysis of the shapes of the pressure profiles indicated that the equivalent net pressure profile shape was much closer to the shape of the original net pressure profile than most of the altered equivalent pressure profiles. In a few cases, the entire equivalent net pressure profile was shifted up, by adding a constant pressure value, until a very close match with the original net profile was achieved. This constant pressure value was called the delta pressure shift $(D p)$, and the new matching profile was called equivalent shift Dp profile. Each case was processed separately. The delta pressure shift was determined for each of the fifty cases such that the new equivalent $\mathrm{Dp}$ shift profile would match as closely as possible the original net pressure profile. Figure 4-30, Figure 4-31, Figure 4-32, and Figure 4-33 show the same cases presented above, with the difference that the matching net pressure is the equivalent $\mathrm{Dp}$ shift. It is clearly visible that equivalent $\mathrm{Dp}$ shift represents a much better match to the original net pressure. However, a different delta pressure shift was determined for each case in order to achieve this match. Table 4-30 summarizes the Dp shift for each of the fifty cases. 


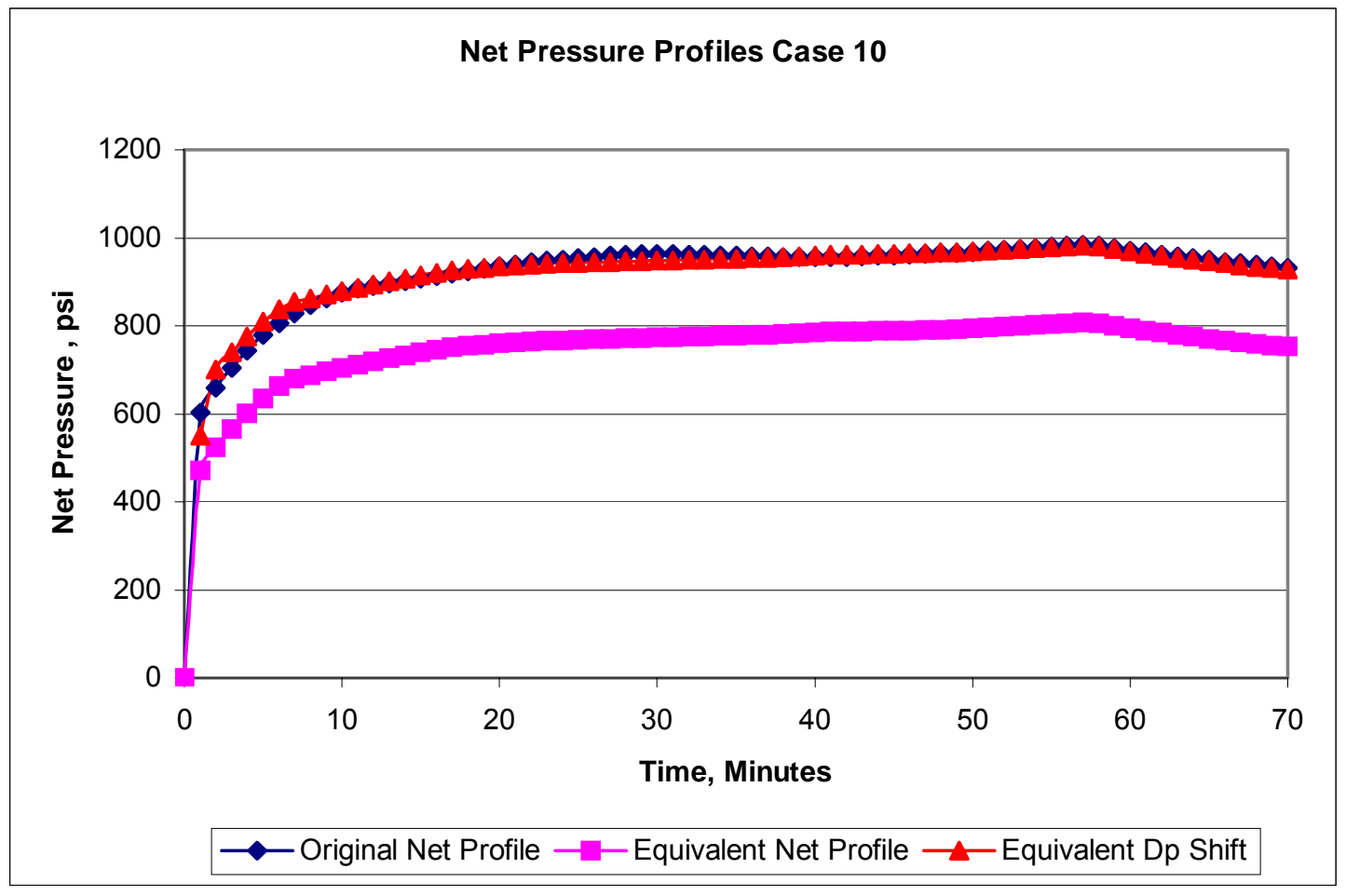

Figure 4-30: Equivalent Dp Shift Matching Process for Case 10

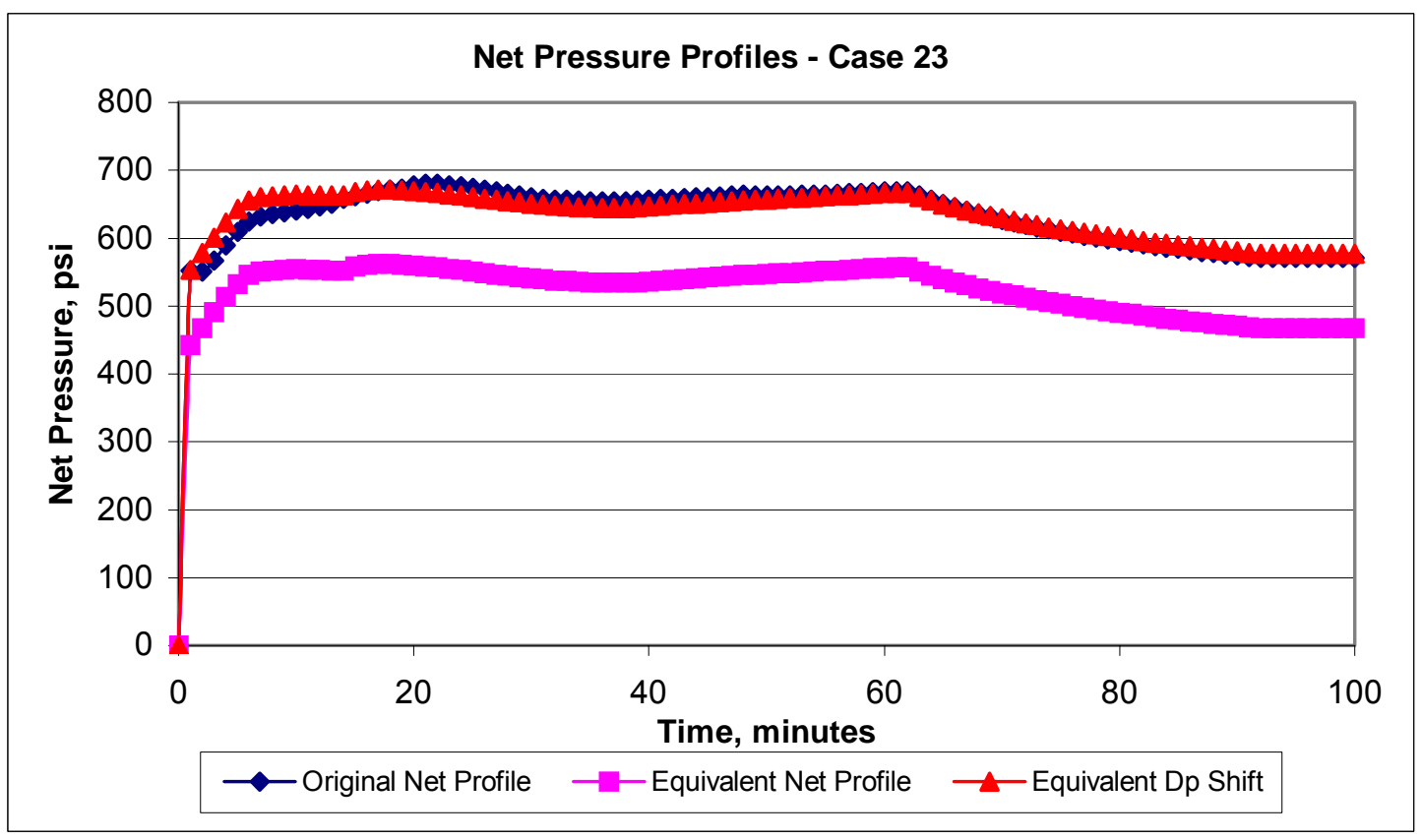

Figure 4-31: Equivalent Dp Shift Matching Process for Case 23 


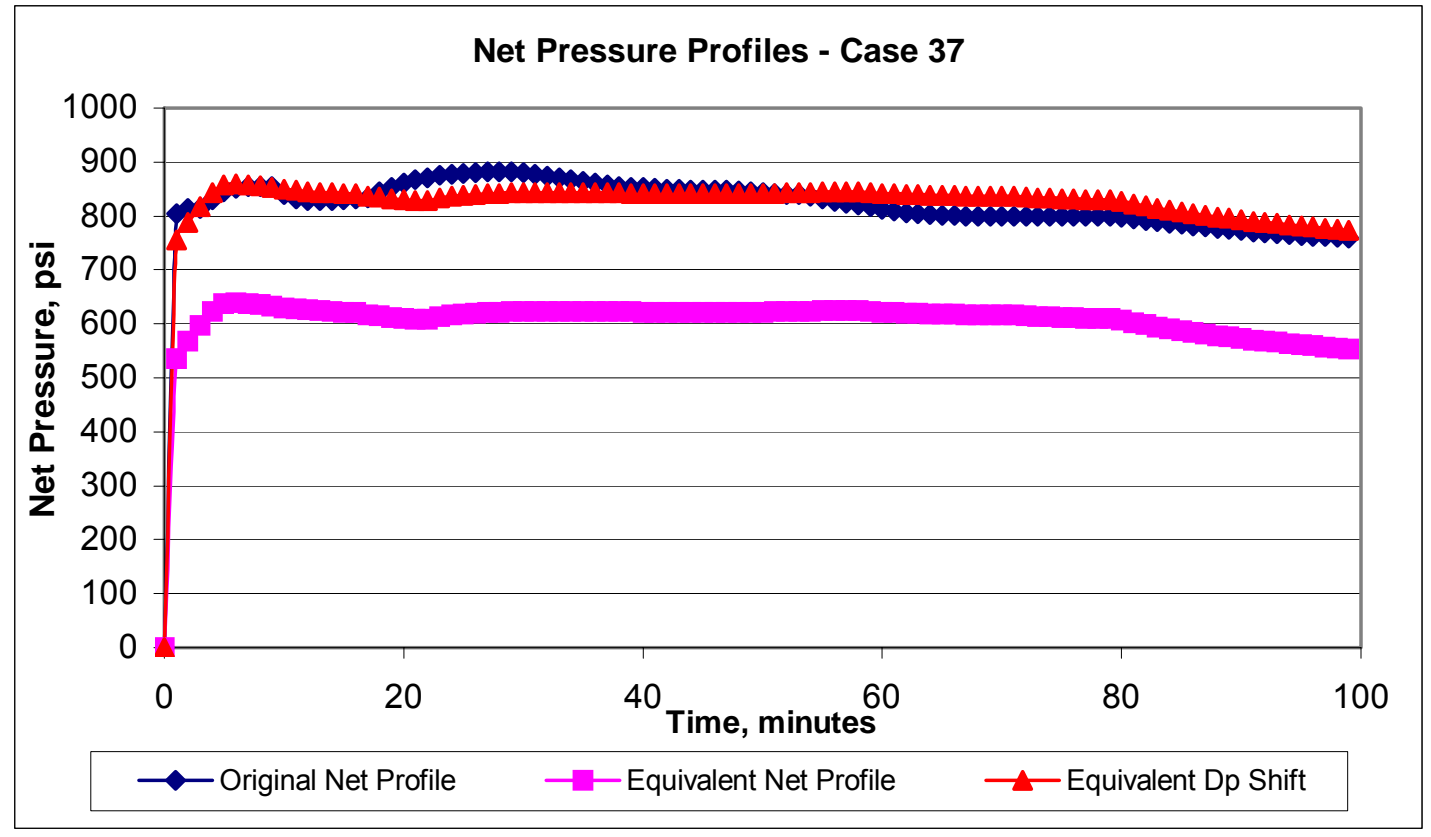

Figure 4-32: Equivalent Dp Shift Matching Process for Case 37

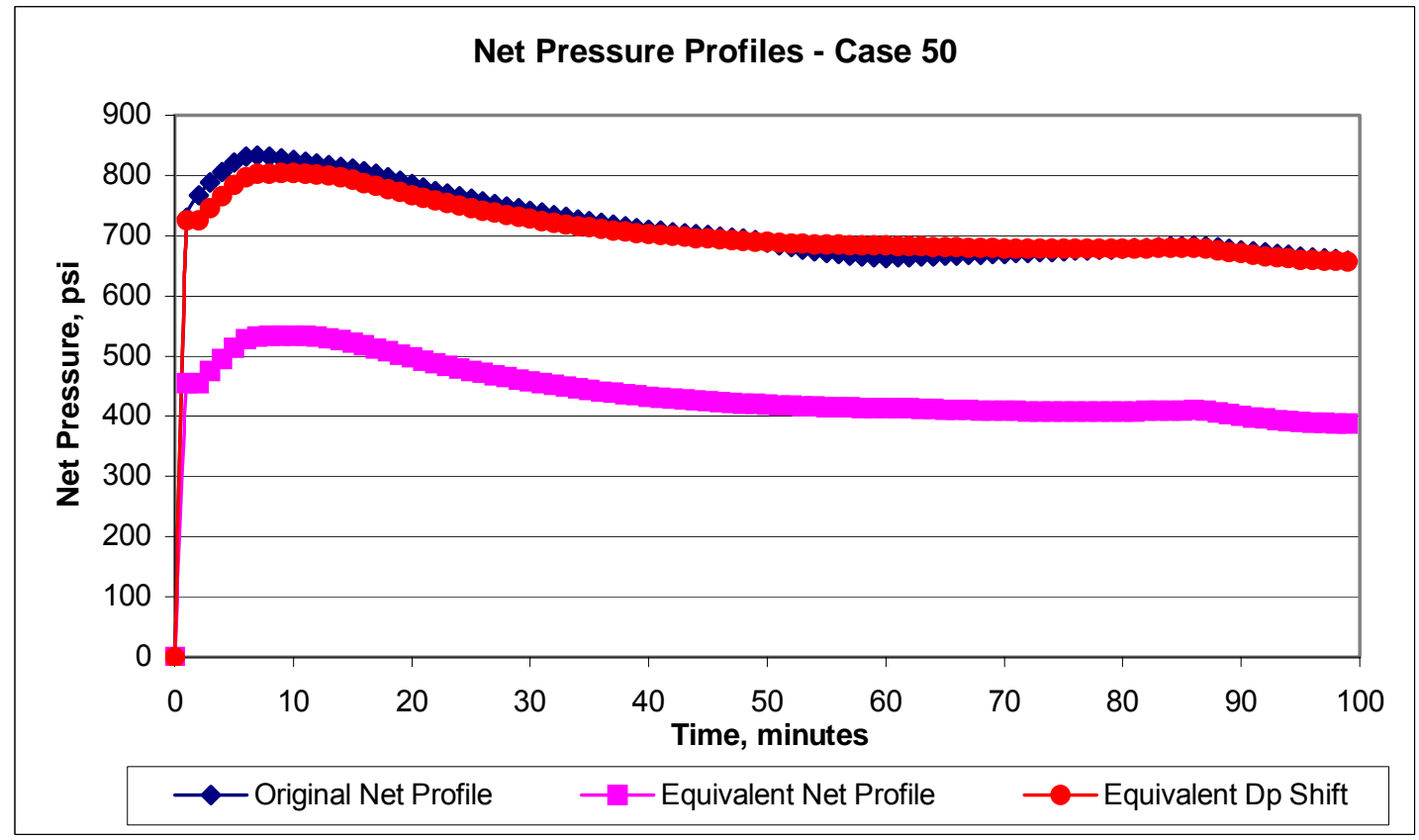

Figure 4-33: Equivalent Dp Shift Matching Process for Case 50 


\begin{tabular}{|c|c|c|c|}
\hline Case Index & $\begin{array}{c}\text { Delta } \\
\text { Pressure } \\
\text { Shift, psi }\end{array}$ & Case Index & $\begin{array}{c}\text { Delta } \\
\text { Pressure } \\
\text { Shift, psi }\end{array}$ \\
\hline Case 1 & 175 & Case 26 & 230 \\
\hline Case 2 & 300 & Case 27 & 50 \\
\hline Case 3 & 125 & Case 28 & 230 \\
\hline Case 4 & 220 & Case 29 & 200 \\
\hline Case 5 & 130 & Case 30 & 40 \\
\hline Case 6 & 40 & Case 31 & 0 \\
\hline Case 7 & 110 & Case 32 & 40 \\
\hline Case 8 & 110 & Case 33 & 80 \\
\hline Case 9 & 55 & Case 34 & 90 \\
\hline Case 10 & 160 & Case 35 & 150 \\
\hline Case 11 & 95 & Case 36 & 20 \\
\hline Case 12 & 260 & Case 37 & 220 \\
\hline Case 13 & 260 & Case 38 & 240 \\
\hline Case 14 & 260 & Case 39 & 175 \\
\hline Case 15 & 260 & Case 40 & 180 \\
\hline Case 16 & 120 & Case 41 & 270 \\
\hline Case 17 & 190 & Case 42 & 120 \\
\hline Case 18 & 50 & Case 43 & 40 \\
\hline Case 19 & 235 & Case 44 & 120 \\
\hline Case 20 & 235 & Case 45 & 140 \\
\hline Case 21 & 235 & Case 46 & 195 \\
\hline Case 22 & 80 & Case 47 & 140 \\
\hline Case 23 & 145 & Case 48 & 280 \\
\hline Case 24 & 160 & Case 49 & 240 \\
\hline Case 25 & 200 & Case 50 & 260 \\
\hline
\end{tabular}

Table 4-30: Dp Shift for 50 Cases

The delta pressure shift has minimum value of 0 (meaning that the equivalent net pressure profile matches the original net pressure profile) and a maximum of $300 \mathrm{psi}$. It has been proven earlier that there is no direct correlation between the percentage coefficient and any other parameter. The same can be said regarding the delta pressure shift. It is still undetermined as to the exact delta pressure shift required in each case to acceptably and accurately match the original pressure profile.

\subsubsection{Calibration Neural Network}

Neural networks have a superior capability of extracting hidden information from complex non-linear data. They are able to learn from patterns in data and predict outcomes when presented with new data. It was believed that a complex relationship or correlation exists 
between the reservoir data (depth, stress, and thickness) and the delta pressure shift; therefore, neural networks were employed to solve this problem.

\subsubsection{Data Considerations}

A neural network was built and trained to predict the delta pressure shift using the available reservoir data. Table 4-31 summarizes the inputs and outputs, while the data used in training the neural network system is shown in Table 4-32.

\begin{tabular}{|c|l|c|c|}
\hline Index & \multicolumn{1}{|c|}{ Parameter Name, Unit } & Type & Range \\
\hline & & & \\
\hline 1 & Depth To Top Layer, $\mathrm{ft}$ & Input & $5102-13200$ \\
\hline 2 & Top Layer Thickness, $\mathrm{ft}$ & Input & $92-249$ \\
\hline 3 & Top Layer Closure Stress, psi/ft & Input & $0.72-0.773$ \\
\hline 4 & Depth to Overburden Layer, ft & Input & $5276-13363$ \\
\hline 5 & Overburden Layer Thickness, ft & Input & $7-81$ \\
\hline 6 & Overburden Layer Stress Gradinent, psi/ft & Input & $0.73-0.83$ \\
\hline 7 & Depth to Pay Zone Layer, ft & Input & $5329-13415$ \\
\hline 8 & Pay Zone Thickness, ft & Input & $63-148$ \\
\hline 9 & Pay Zone Closure Stress Gradient, psi/ft & Input & $0.646-0.69$ \\
\hline 10 & Pay Zone Permeability, md & Input & $0.002-0.554$ \\
\hline 11 & Depth to Bottom Layer, ft & Input & $5419-13497$ \\
\hline 12 & Bottom Layer Closure Stress Gradient, psi/ft & Input & $0.702-0.741$ \\
\hline & & & \\
\hline 1 & Pressure Shift, psi & Output & $0-300$ \\
\hline
\end{tabular}

Table 4-31: Parameters for Delta Pressure Shift Neural Network 


\begin{tabular}{|c|c|c|c|c|c|c|c|c|c|c|c|c|c|}
\hline & \multicolumn{3}{|c|}{ Top Layer } & \multicolumn{3}{|c|}{ Overburden Layer } & \multicolumn{4}{|c|}{ Pay Zone } & \multicolumn{2}{|c|}{ Botom Layer } & \multirow[b]{2}{*}{\begin{tabular}{|c|} 
Output \\
\\
\\
Pressure \\
Shift, psi
\end{tabular}} \\
\hline Index & $\begin{array}{l}\text { Depth } \\
\text { To Top } \\
\text { Layer, } \\
\text { ft }\end{array}$ & $\begin{array}{c}\text { Top Layer } \\
\text { Thicknes } \\
\text { s, ft }\end{array}$ & $\begin{array}{c}\text { Top } \\
\text { Layer } \\
\text { Closure } \\
\text { Stress, } \\
\text { psi/ft }\end{array}$ & $\begin{array}{c}\text { Depth to } \\
\text { Overburden } \\
\text { Layer, } \mathrm{ft}\end{array}$ & $\begin{array}{l}\text { Overburden } \\
\text { Layer } \\
\text { Thickness, } \\
\mathrm{ft}\end{array}$ & $\begin{array}{c}\text { Overburden } \\
\text { Layer } \\
\text { Stress } \\
\text { Gradinent, } \\
\text { psi/ft }\end{array}$ & \begin{tabular}{|c|} 
Depth \\
to Pay \\
Zone \\
Layer, $\mathrm{ft}$
\end{tabular} & $\begin{array}{c}\text { Pay Zone } \\
\text { Thickness } \\
\text {, ft }\end{array}$ & \begin{tabular}{|c|} 
Pay \\
Zone \\
Closure \\
Stress \\
Gradient, \\
psi/ft
\end{tabular} & $\begin{array}{c}\text { Pay Zone } \\
\text { Permeability } \\
\text {, md }\end{array}$ & $\begin{array}{c}\text { Depth } \\
\text { to } \\
\text { Bottom } \\
\text { Layer, } \\
\mathrm{ft}\end{array}$ & \begin{tabular}{|c|} 
Bottom \\
Layer \\
Closure \\
Stress \\
Gradient, \\
psi/ft
\end{tabular} & \\
\hline Case 1 & 10920 & 205 & 0.729 & 11125 & 15 & 0.791 & 11140 & $\overline{100}$ & 0.646 & 0.027 & 11240 & 0.73 & 175 \\
\hline Case 2 & 12600 & 224 & 0.745 & 12824 & 16 & 0.774 & 12840 & 100 & 0.656 & 0.0159 & 12940 & 0.71 & 300 \\
\hline Case 3 & 11500 & 215 & 0.75 & 11715 & 19 & 0.809 & 11734 & 90 & 0.665 & 0.023 & 11824 & 0.73 & 125 \\
\hline Case 4 & 9600 & 200 & 0.772 & 9800 & 30 & 0.82 & 9830 & 80 & 0.665 & 0.039 & 9910 & 0.74 & 220 \\
\hline Case 5 & 7500 & 238 & 0.751 & 7738 & 16 & 0.799 & 7754 & 78 & 0.658 & 0.035 & 7832 & 0.724 & 130 \\
\hline Case 6 & 7500 & 240 & 0.753 & 7740 & 14 & 0.808 & 7754 & 78 & 0.673 & 0.013 & 7832 & 0.715 & 40 \\
\hline Case 7 & 6400 & 232 & 0.745 & 6632 & 8 & 0.8 & 6640 & 76 & 0.67 & 0.0254 & 6716 & 0.718 & 110 \\
\hline Case 8 & 10920 & 197 & 0.734 & 11117 & 23 & 0.787 & 11240 & 100 & 0.677 & 0.05 & 11340 & 0.718 & 110 \\
\hline Case 9 & 8650 & 203 & 0.741 & 8853 & 7 & 0.757 & 8860 & 107 & 0.676 & 0.03 & 8967 & 0.726 & 55 \\
\hline Case 10 & 7580 & 237 & 0.728 & 7817 & 14 & 0.768 & 7831 & 109 & 0.674 & 0.036 & 7940 & 0.71 & 160 \\
\hline Case 11 & 7080 & 206 & 0.758 & 7286 & 29 & 0.8 & 7315 & 101 & 0.6813 & 0.015 & 7416 & 0.722 & 95 \\
\hline Case 12 & 6450 & 198 & 0.741 & 6648 & 29 & 0.803 & 6677 & 103 & 0.672 & 0.0057 & 6780 & 0.728 & 260 \\
\hline Case 13 & 6450 & 198 & 0.741 & 6648 & 29 & 0.803 & 6677 & 103 & 0.672 & 0.0057 & 6780 & 0.728 & 260 \\
\hline Case 14 & 6450 & 198 & 0.741 & 6648 & 29 & 0.803 & 6677 & 103 & 0.672 & 0.0057 & 6780 & 0.728 & 260 \\
\hline Case 15 & 6450 & 198 & 0.741 & 6648 & 29 & 0.803 & 6677 & 103 & \begin{tabular}{l|}
0.672 \\
\end{tabular} & 0.0057 & 6780 & 0.728 & 260 \\
\hline Case 16 & 6210 & 195 & 0.743 & 6405 & 33 & 0.777 & 6438 & 101 & 0.674 & 0.023 & 6539 & 0.73 & 120 \\
\hline Case 17 & 5820 & 192 & 0.726 & 6012 & 13 & 0.771 & 6025 & 92 & 0.675 & 0.022 & 6117 & 0.709 & 190 \\
\hline Case 18 & 5310 & 216 & 0.769 & 5526 & 12 & 0.809 & 5538 & 74 & 0.664 & 0.0072 & 5612 & 0.717 & 50 \\
\hline Case 19 & 12200 & 198 & 0.75 & 12398 & 40 & 0.798 & 12438 & 91 & 0.678 & 0.016 & 12529 & 0.721 & 235 \\
\hline Case 20 & 12200 & 198 & 0.75 & 12398 & 40 & 0.798 & 12438 & 91 & 0.678 & 0.016 & 12529 & 0.721 & 235 \\
\hline Case 21 & 12200 & 198 & 0.75 & 12398 & 40 & 0.798 & 12438 & 91 & 0.678 & 0.016 & 12529 & 0.721 & 235 \\
\hline Case 22 & 9520 & 199 & 0.757 & 9719 & 46 & 0.798 & 9765 & 115 & 0.673 & 0.042 & 9880 & 0.728 & 80 \\
\hline Case 23 & 9520 & 219 & 0.755 & 9739 & 26 & 0.8 & 9765 & 115 & 0.689 & 0.018 & 9880 & 0.72 & 145 \\
\hline Case 24 & 10200 & 236 & 0.739 & 10436 & 16 & 0.783 & 10452 & 148 & 0.667 & 0.01 & 10600 & 0.73 & 160 \\
\hline Case 25 & 10200 & 236 & 0.739 & 10436 & 16 & 0.783 & 10452 & 148 & 0.667 & 0.01 & 10600 & 0.73 & 200 \\
\hline Case 26 & 8435 & 213 & 0.734 & 8648 & 9 & 0.786 & 8657 & 143 & 0.677 & 0.009 & 8800 & 0.717 & 230 \\
\hline Case 27 & 5310 & 216 & 0.767 & 5526 & 12 & 0.795 & 5538 & 74 & 0.663 & 0.007 & 5612 & 0.719 & 50 \\
\hline Case 28 & 10200 & 249 & 0.747 & 10449 & 16 & 0.789 & 10465 & 135 & \begin{tabular}{l|}
0.672 \\
\end{tabular} & 0.011 & 10600 & 0.73 & 230 \\
\hline Case 29 & 10200 & 249 & 0.747 & 10449 & 16 & 0.789 & 10465 & 135 & 0.672 & 0.011 & 10600 & 0.73 & 200 \\
\hline Case 30 & 10920 & 193 & 0.744 & 11113 & 54 & 0.763 & 11167 & 71 & \begin{tabular}{l|}
0.671 \\
\end{tabular} & 0.0033 & 11238 & 0.71 & 40 \\
\hline Case 31 & 8245 & 181 & 0.746 & 8426 & 37 & 0.778 & 8463 & 67 & 0.663 & 0.254 & 8530 & 0.732 & 0 \\
\hline Case 32 & 7687 & 194 & 0.748 & 7881 & 27 & 0.762 & 7908 & 124 & 0.687 & 0.0098 & 8032 & 0.718 & 40 \\
\hline Case 33 & 7145 & 183 & 0.738 & 7328 & 24 & 0.783 & 7352 & 109 & 0.665 & 0.0153 & 7461 & 0.702 & 80 \\
\hline Case 34 & 6851 & 207 & 0.726 & 7058 & 37 & 0.745 & 7095 & 96 & 0.683 & 0.0813 & 7191 & 0.719 & 90 \\
\hline Case 35 & 6521 & 184 & 0.742 & 6705 & 40 & 0.774 & 6745 & 128 & \begin{tabular}{l|}
0.679 \\
\end{tabular} & 0.027 & 6873 & 0.712 & 150 \\
\hline Case 36 & 6080 & 161 & $\begin{array}{l}0.74 \\
\end{array}$ & 6241 & 9 & 0.754 & 6250 & 107 & 0.689 & 0.0425 & 6357 & 0.72 & 20 \\
\hline Case 37 & 8998 & 185 & 0.75 & 9183 & 33 & 0.787 & 9216 & 67 & 0.69 & 0.002 & 9283 & 0.722 & 220 \\
\hline Case 38 & 13200 & 160 & 0.773 & \begin{tabular}{|l|}
13360 \\
\end{tabular} & 55 & 0.795 & 13415 & 63 & 0.6721 & 0.0385 & 13478 & 0.721 & 240 \\
\hline Case 39 & 13200 & 163 & 0.761 & 13363 & 52 & 0.796 & 13415 & 82 & 0.674 & 0.0135 & 13497 & 0.74 & 175 \\
\hline Case 40 & 8657 & 189 & 0.752 & 8846 & 22 & 0.809 & 8868 & 132 & 0.681 & 0.027 & 9000 & 0.722 & 180 \\
\hline Case 41 & 7732 & 217 & $\begin{array}{l}0.72 \\
\end{array}$ & 7949 & 17 & 0.742 & 7966 & 94 & 0.689 & 0.039 & 8060 & 0.715 & 270 \\
\hline Case 42 & 6214 & 198 & 0.731 & 6412 & 23 & 0.779 & 6435 & 81 & 0.685 & 0.11 & 6516 & 0.73 & 120 \\
\hline Case 43 & 5112 & 187 & 0.738 & 5299 & 37 & 0.767 & 5336 & 103 & 0.684 & 0.049 & 5439 & 0.741 & 40 \\
\hline Case 44 & 5102 & 174 & 0.753 & 5276 & 53 & 0.774 & 5329 & 90 & 0.68 & 0.024 & 5419 & 0.721 & 120 \\
\hline Case 45 & 12254 & 217 & 0.754 & 12471 & 17 & 0.83 & 12488 & 90 & 0.676 & 0.0287 & 12578 & 0.715 & 140 \\
\hline Case 46 & 11920 & 139 & 0.724 & 12059 & 81 & 0.73 & 12140 & 100 & 0.682 & 0.007 & 12240 & 0.718 & 195 \\
\hline Case 47 & 11920 & 92 & 0.736 & 12012 & 28 & 0.748 & 12040 & 100 & 0.687 & 0.009 & 12140 & 0.712 & 140 \\
\hline Case 48 & 10658 & 244 & 0.732 & 10902 & 24 & 0.777 & 10926 & 108 & 0.67 & 0.024 & 11034 & 0.726 & 280 \\
\hline Case 49 & 11318 & 244 & 0.727 & 11562 & 24 & 0.75 & 11586 & 108 & 0.668 & 0.03 & 11694 & 0.726 & 240 \\
\hline Case 50 & 12568 & 214 & $\begin{array}{l}0.73 \\
\end{array}$ & \begin{tabular}{|l|}
12782 \\
\end{tabular} & 17 & 0.762 & 12799 & 112 & 0.681 & 0.0132 & 12911 & 0.731 & 260 \\
\hline
\end{tabular}

Table 4-32: Data for Training the Delta Pressure Shift Neural Network 
The first attempt to train a neural network using the data presented above resulted in very poor performance with correlation coefficients around 0.8 and $R^{2}$ values around 0.65 for testing and verification set. Multiple architectures and types of neural networks were tried in the attempt to build the model. It was concluded that the existing data was either insufficient or too sparse since it was randomly generated. Generating additional data would be very time consuming and would not necessarily guarantee the solution of the problem.

From previous experience and literature review ${ }^{41,42}$, utilizing white noise has helped the training process of the neural network while being able to recognize and predict the original data with higher performance. Therefore, white noise was introduced into the system to attempt to overcome the previously described problem.

\subsubsection{White Noise Data Generation}

The process of white noise generation consists of a series of steps. The first of which is data selection. Since the existing dataset has only fifty cases, seven were extracted to form the verification set, representing $14 \%$ of the entire data set. These cases were set aside and were not utilized in noise generation. The remaining 43 cases together with the yet to be generated white noise cases will form the training and calibration data sets.

In this study, a five step process was used for white noise generation:

1. Using a random number generator, one case was extracted from the 43 remaining.

2. For the selected case, a new value for each parameter was generated such that the new value was within $5 \%$ higher or lower than the original value.

3. The result is a white noise case.

4. The process was repeated from step 1 to 3,10 times. The result is one set of 10 white noise cases.

The process outlined above was repeated five times. Table 4-33 shows the five sets of white noise data. 


\begin{tabular}{|c|c|c|c|c|c|c|c|c|c|c|c|c|c|}
\hline & \multicolumn{3}{|c|}{ Top Layer } & \multicolumn{3}{|c|}{ Overburden Layer } & \multicolumn{4}{|c|}{ Pay Zone } & \multicolumn{2}{|c|}{ Botom Layer } & \multirow[b]{2}{*}{\begin{tabular}{|l} 
Output \\
\\
Pressure \\
Shift, ps
\end{tabular}} \\
\hline Index & $\begin{array}{l}\text { Depth } \\
\text { To Top } \\
\text { Layer, } \\
\mathrm{ft}\end{array}$ & $\begin{array}{c}\text { Top Layer } \\
\text { Thicknes } \\
\text { s, ft }\end{array}$ & $\begin{array}{c}\text { Top } \\
\text { Layer } \\
\text { Closure } \\
\text { Stress, } \\
\text { psi/ft }\end{array}$ & $\begin{array}{l}\text { Depth to } \\
\text { Overburden } \\
\text { Layer, } \mathrm{ft}\end{array}$ & $\begin{array}{c}\text { Overburden } \\
\text { Layer } \\
\text { Thickness, } \\
\mathrm{ft}\end{array}$ & \begin{tabular}{|} 
Overburden \\
Layer \\
Stress \\
Gradinent, \\
psi/ft
\end{tabular} & $\begin{array}{l}\text { Depth } \\
\text { to Pay } \\
\text { Zone } \\
\text { Layer, } \mathrm{ft}\end{array}$ & $\begin{array}{c}\text { Pay Zone } \\
\text { Thickness } \\
\text {,ft }\end{array}$ & $\begin{array}{c}\text { Pay } \\
\text { Zone } \\
\text { Closure } \\
\text { Stress } \\
\text { Gradient, } \\
\mathrm{psi} / \mathrm{ft}\end{array}$ & $\begin{array}{c}\text { Pay Zone } \\
\text { Permeability } \\
\text {, md }\end{array}$ & $\begin{array}{l}\text { Depth } \\
\text { to } \\
\text { Bottom } \\
\text { Layer, } \\
\mathrm{ft}\end{array}$ & $\begin{array}{c}\text { Bottom } \\
\text { Layer } \\
\text { Closure } \\
\text { Stress } \\
\text { Gradient, } \\
\text { psi/ft }\end{array}$ & \\
\hline \multicolumn{14}{|c|}{ Noise Set 1} \\
\hline Noise 5 & 7446 & 231 & 0.743 & 7553 & 16 & 0.796 & 7610 & 77 & 0.648 & 0.0344 & 7695 & 0.708 & 127 \\
\hline \begin{tabular}{|l|} 
Noise 28 \\
\end{tabular} & 9908 & 248 & 0.725 & 10237 & 16 & 0.772 & 10418 & 132 & 0.665 & 0.0107 & 10537 & 0.719 & 229 \\
\hline \begin{tabular}{|l|} 
Noise 12 \\
\end{tabular} & 6360 & 195 & 0.736 & 6457 & 28 & 0.801 & 6629 & 102 & 0.662 & 0.0055 & 6697 & 0.719 & 258 \\
\hline \begin{tabular}{|l|} 
Noise 26 \\
\end{tabular} & 8191 & 210 & 0.720 & 8544 & 9 & 0.773 & 8467 & 139 & 0.675 & 0.0087 & 8593 & 0.716 & 228 \\
\hline Noise 10 & 7556 & 236 & 0.722 & 7642 & 14 & 0.764 & 7606 & 106 & 0.670 & 0.0356 & 7886 & 0.707 & 156 \\
\hline \begin{tabular}{|l|} 
Noise 1 \\
\end{tabular} & 10756 & 201 & 0.718 & 10869 & 15 & 0.770 & 10918 & 99 & 0.632 & 0.0266 & 11072 & 0.715 & 175 \\
\hline \begin{tabular}{|l|} 
Noise 15 \\
\end{tabular} & 6269 & 193 & 0.733 & 6554 & 29 & 0.786 & 6550 & 101 & 0.664 & 0.0056 & 6739 & 0.710 & 258 \\
\hline Noise 40 & 8454 & 184 & 0.735 & 8648 & 22 & 0.793 & 8782 & 128 & 0.668 & 0.0267 & 8971 & 0.708 & 180 \\
\hline \begin{tabular}{|l|} 
Noise 34 \\
\end{tabular} & 6838 & 202 & 0.725 & 6890 & 36 & 0.733 & 6947 & 94 & 0.672 & 0.0799 & 7026 & 0.710 & 88 \\
\hline \begin{tabular}{|l|} 
Noise 21 \\
\end{tabular} & 12151 & 196 & 0.749 & 12224 & 39 & 0.786 & 12273 & 91 & 0.666 & 0.0157 & 12418 & 0.700 & 232 \\
\hline \multicolumn{14}{|c|}{ Noise Set 2} \\
\hline Noise 24 & 10084 & 229 & 0.724 & 10355 & 16 & 0.761 & 10255 & 144 & 0.654 & 0.0100 & 10325 & 0.717 & 155 \\
\hline Noise 18 & 5212 & 210 & 0.761 & 5397 & 12 & 0.805 & 5422 & 73 & 0.659 & 0.0072 & 5510 & 0.715 & 50 \\
\hline Noise 35 & 6366 & 182 & 0.720 & 6571 & 40 & 0.758 & 6634 & 125 & 0.662 & 0.0269 & 6684 & 0.704 & 146 \\
\hline \begin{tabular}{|l|} 
Noise 30 \\
\end{tabular} & 10746 & 192 & 0.743 & 11110 & 54 & 0.742 & 10954 & 69 & 0.654 & 0.0032 & 10970 & 0.691 & 39 \\
\hline \begin{tabular}{|l|} 
Noise 2 \\
\end{tabular} & 12421 & 224 & 0.735 & 12804 & 16 & 0.769 & 12555 & 100 & 0.642 & 0.0157 & 12597 & 0.701 & 297 \\
\hline Noise 20 & 11903 & 194 & 0.732 & 12189 & 39 & 0.791 & 12347 & 91 & 0.668 & 0.0159 & 12194 & 0.712 & 233 \\
\hline Noise 38 & 13023 & 158 & 0.751 & 13307 & 53 & 0.795 & 13134 & 63 & 0.660 & 0.0379 & 13170 & 0.717 & 238 \\
\hline \begin{tabular}{|l|} 
Noise 44 \\
\end{tabular} & 5015 & 174 & 0.745 & 5234 & 52 & 0.755 & 5323 & 90 & 0.662 & 0.0240 & 5367 & 0.715 & 119 \\
\hline Noise 49 & 11024 & 243 & 0.718 & 11524 & 24 & 0.735 & 11323 & 105 & 0.657 & 0.0294 & 11616 & 0.708 & 237 \\
\hline \begin{tabular}{|l|} 
Noise 4 \\
\end{tabular} & 9372 & 194 & 0.763 & 9591 & 30 & 0.819 & 9580 & 79 & 0.651 & 0.0384 & 9874 & 0.739 & 216 \\
\hline \multicolumn{14}{|c|}{ Noise Set 3} \\
\hline Noise 32 & 7685 & 191 & 0.738 & 7682 & 27 & 0.743 & 7816 & 121 & 0.669 & 0.0098 & 7850 & 0.710 & 39 \\
\hline \begin{tabular}{|l|} 
Noise 22 \\
\end{tabular} & 9398 & 198 & 0.754 & 9537 & 45 & 0.788 & 9643 & 112 & 0.671 & 0.0410 & 9741 & 0.719 & 78 \\
\hline Noise 48 & 10440 & 238 & 0.722 & 10852 & 23 & 0.754 & 10635 & 105 & 0.655 & 0.0240 & 10824 & 0.724 & 273 \\
\hline \begin{tabular}{|l|} 
Noise 7 \\
\end{tabular} & 6304 & 226 & 0.741 & 6462 & 8 & 0.794 & 6458 & 75 & 0.668 & 0.0253 & 6534 & 0.702 & 109 \\
\hline Noise 46 & 11695 & 136 & 0.715 & 11998 & 80 & 0.715 & 11988 & 100 & 0.673 & 0.0068 & 11891 & 0.717 & 195 \\
\hline Noise 25 & 10020 & 234 & 0.724 & 10127 & 16 & 0.765 & 10371 & 144 & 0.656 & 0.0100 & 10520 & 0.711 & 200 \\
\hline \begin{tabular}{|l|} 
Noise 9 \\
\end{tabular} & 8434 & 197 & 0.730 & 8779 & 7 & 0.745 & 8812 & 105 & 0.674 & 0.0298 & 8714 & 0.704 & 55 \\
\hline \begin{tabular}{|l|} 
Noise 42 \\
\end{tabular} & 6134 & 196 & 0.721 & 6351 & 23 & 0.773 & 6320 & 79 & 0.673 & 0.1083 & 6328 & 0.709 & 117 \\
\hline \begin{tabular}{|l|} 
Noise 14 \\
\end{tabular} & 6443 & 197 & 0.731 & 6577 & 29 & 0.790 & 6483 & 101 & 0.668 & 0.0057 & 6632 & 0.708 & 260 \\
\hline Noise 3 & 11271 & 209 & 0.738 & 11594 & 19 & 0.787 & 11533 & 88 & 0.654 & 0.0225 & 11675 & 0.709 & 125 \\
\hline \multicolumn{14}{|c|}{ Noise Set 4} \\
\hline Noise 11 & 7004 & 205 & 0.745 & 7250 & 28 & 0.777 & 7149 & 101 & 0.673 & 0.0148 & 7315 & 0.718 & 93 \\
\hline Noise 19 & 12171 & 197 & 0.735 & 12043 & 39 & 0.783 & 12147 & 90 & 0.665 & 0.0157 & 12404 & 0.704 & 230 \\
\hline \begin{tabular}{|l|} 
Noise 8 \\
\end{tabular} & 10903 & 196 & 0.720 & 11016 & 23 & 0.775 & 11019 & 99 & 0.665 & 0.0489 & 11334 & 0.705 & 110 \\
\hline \begin{tabular}{|l|} 
Noise 41 \\
\end{tabular} & 7697 & 215 & 0.710 & 7878 & 17 & 0.723 & 7766 & 93 & 0.673 & 0.0387 & 7933 & 0.705 & 268 \\
\hline Noise 39 & 12938 & 162 & 0.739 & 12989 & 51 & 0.788 & 13237 & 80 & 0.667 & 0.0132 & 13144 & 0.732 & 170 \\
\hline Noise 50 & 12469 & 210 & 0.717 & 12542 & 17 & 0.745 & 12483 & 109 & 0.667 & 0.0131 & 12885 & 0.710 & 257 \\
\hline \begin{tabular}{|l|} 
Noise 31 \\
\end{tabular} & 8206 & 181 & 0.741 & 8183 & 37 & 0.767 & 8413 & 66 & 0.646 & 0.2519 & 8285 & 0.721 & 0 \\
\hline \begin{tabular}{|l|} 
Noise 13 \\
\end{tabular} & 6282 & 193 & 0.735 & 6569 & 28 & 0.800 & 6507 & 102 & 0.661 & 0.0056 & 6714 & 0.728 & 259 \\
\hline Noise 36 & 6078 & 161 & 0.737 & 6215 & 9 & 0.733 & 6104 & 105 & 0.672 & 0.0416 & 6245 & 0.715 & 20 \\
\hline \begin{tabular}{|l|} 
Noise 16 \\
\end{tabular} & 6173 & 194 & 0.732 & 6384 & 32 & 0.769 & 6329 & 100 & 0.663 & 0.0223 & 6371 & 0.716 & 118 \\
\hline \multicolumn{14}{|c|}{ Noise Set 5} \\
\hline Noise 6 & 7490 & 234 & 0.732 & 7542 & 14 & 0.797 & 7661 & 77 & 0.658 & 0.0128 & 7805 & 0.715 & 40 \\
\hline \begin{tabular}{|l|} 
Noise 17 \\
\end{tabular} & 5725 & 187 & 0.708 & 5973 & 13 & 0.749 & 5873 & 91 & 0.666 & 0.0216 & 6048 & 0.690 & 186 \\
\hline \begin{tabular}{|l|} 
Noise 23 \\
\end{tabular} & 9400 & 213 & 0.745 & 9636 & 26 & 0.787 & 9682 & 114 & 0.672 & 0.0177 & 9789 & 0.715 & 142 \\
\hline \begin{tabular}{|l|} 
Noise 27 \\
\end{tabular} & 5186 & 213 & 0.756 & 5426 & 12 & 0.782 & 5450 & 74 & 0.662 & 0.0069 & 5571 & 0.719 & 49 \\
\hline Noise 29 & 10015 & 242 & 0.727 & 10250 & 16 & 0.774 & 10344 & 132 & 0.657 & 0.0108 & 10521 & 0.729 & 199 \\
\hline Noise 33 & 7008 & 182 & 0.736 & 7264 & 24 & 0.779 & 7187 & 107 & 0.662 & 0.0149 & 7284 & 0.696 & 79 \\
\hline Noise 37 & 8811 & 181 & 0.737 & 9143 & 33 & 0.780 & 8951 & 66 & 0.682 & 0.0020 & 9053 & 0.715 & 218 \\
\hline \begin{tabular}{|l|} 
Noise 43 \\
\end{tabular} & 5058 & 186 & 0.734 & 5296 & 36 & 0.754 & 5198 & 102 & 0.676 & 0.0482 & 5425 & 0.727 & 39 \\
\hline Noise 45 & 12232 & 213 & 0.733 & 12352 & 17 & 0.811 & 12369 & 90 & 0.667 & 0.0284 & 12245 & 0.711 & 139 \\
\hline Noise 47 & 11674 & 91 & 0.718 & 11995 & 28 & 0.737 & 11793 & 98 & 0.685 & 0.0089 & 12125 & 0.711 & 140 \\
\hline
\end{tabular}

Table 4-33: White Noise Data for Training the Calibration Neural Network 


\subsubsection{Calibration Neural Network Performance}

Five neural networks were trained using the original data set and five sets of white noise data in the following order.

1. The first neural network used the first noise set, which was added to the 43 cases and loaded into NeuroShell. The software defined the training and calibration sets randomly using all 53 cases, and trained the neural network. The performance of the neural network is given in the Table 4-34. The final verification was done using the seven cases set left aside.

2. The second neural network used the first and the second noise data sets together with the original 43 cases. This time the final set consisted of 63 cases. NeuroShell defined the training and calibration sets and trained the second neural network.

3. The process was repeated; each time adding a new white noise data set which increased the total number of cases by ten. The third, fourth, and fifth neural networks were trained this way. The same seven cases, previously extracted from the original fifty cases, were used to measure the performance improvement for each of the neural networks.

\begin{tabular}{|l|c|c|c|}
\hline & \multicolumn{2}{c}{ Training } & \multicolumn{2}{c|}{ Calibration } & Verification \\
& Set & Set & Set \\
\hline Number of Cases & 43 & 10 & 7 \\
\hline $\mathrm{R}^{2}$ & 0.9991 & 0.4345 & 0.8749 \\
\hline $\mathrm{r}^{2}$ & 0.9992 & 0.5351 & 0.9207 \\
\hline Correlation Coefficient & 0.9996 & 0.7315 & 0.9595 \\
\hline
\end{tabular}

Table 4-34: Calibration Neural Network, $1^{\text {st }}$ Noise Data Set Included

\begin{tabular}{|l|c|c|c|}
\hline & \multicolumn{2}{c}{$\begin{array}{c}\text { Training } \\
\text { Set }\end{array}$} & \multicolumn{2}{c|}{ Calibration } & Verification \\
Sumber of Cases & 53 & 10 & Set \\
\hline $\mathrm{R}^{2}$ & 0.9970 & 0.6319 & 0.8535 \\
\hline $\mathrm{r}^{2}$ & 0.9977 & 0.7296 & 0.9080 \\
\hline Correlation Coefficient & 0.9988 & 0.8542 & 0.9529 \\
\hline
\end{tabular}

Table 4-35: Calibration Neural Network, 1 st and $2^{\text {nd }}$ Noise Data Sets Included

\begin{tabular}{|l|c|c|c|}
\hline & \multicolumn{1}{c}{ Training } & \multicolumn{2}{c|}{ Calibration } \\
Set & Verification & Set \\
\hline Number of Cases & 63 & 10 & 7 \\
\hline $\mathrm{R}^{2}$ & 0.6380 & 0.6494 & 0.6802 \\
\hline $\mathrm{r}^{2}$ & 0.6671 & 0.6786 & 0.8217 \\
\hline Correlation Coefficient & 0.8167 & 0.8238 & 0.9065 \\
\hline
\end{tabular}

Table 4-36: Calibration Neural Network, $1^{\text {st }}, 2^{\text {nd }}$, and $3^{\text {rd }}$ Noise Data Sets Included 


\begin{tabular}{|c|c|c|c|}
\hline & $\begin{array}{l}\text { Training } \\
\text { Set }\end{array}$ & $\begin{array}{c}\text { Calibration } \\
\text { Set }\end{array}$ & $\begin{array}{l}\text { Verification } \\
\text { Set }\end{array}$ \\
\hline Number of Cases & 73 & 10 & 7 \\
\hline $\mathrm{R}^{2}$ & 0.9886 & 0.9178 & 0.9194 \\
\hline$r^{2}$ & 0.9912 & 0.9369 & 0.9199 \\
\hline Correlation Coefficient & 0.9956 & 0.9679 & 0.9591 \\
\hline
\end{tabular}

Table 4-37: Calibration Neural Network, $1^{\text {st }}, 2^{\text {nd }}, 3^{\text {rd }}$, and $4^{\text {th }}$ Noise Data Sets Included

\begin{tabular}{|l|c|c|c|}
\hline & \multicolumn{2}{c}{ Training } & \multicolumn{2}{c|}{ Calibration } & Verification \\
Set & Set & Set \\
\hline $\mathrm{R}^{2}$ & 83 & 10 & 7 \\
\hline $\mathrm{r}^{2}$ & 0.9838 & 0.9123 & 0.8804 \\
\hline Correlation Coefficient & 0.9856 & 0.9335 & 0.9077 \\
\hline
\end{tabular}

Table 4-38: Calibration Neural Network, All 5 Noise Data Sets Included

The first neural network (Table 4-34) shows very poor performance in the calibration set; while the second neural network (Table 4-35) shows a slight improvement in the calibration set but the same performance for the verification set. The third neural network (Table 4-36), where three noise data sets were added, shows very poor performance in both calibration and verification sets. The fourth and fifth neural networks (Table 4-37 and Table 4-38, respectively) show very good performance for all three sets - training, calibration, and verification. However, the fourth neural network shows modestly better performance for the verification set $(R 2=0.919)$. The graphical representations of the accuracy of the fourth neural network training, calibration, and verifications sets are shown in Figure 4-34, Figure 4-35, and Figure 4-36 respectively.

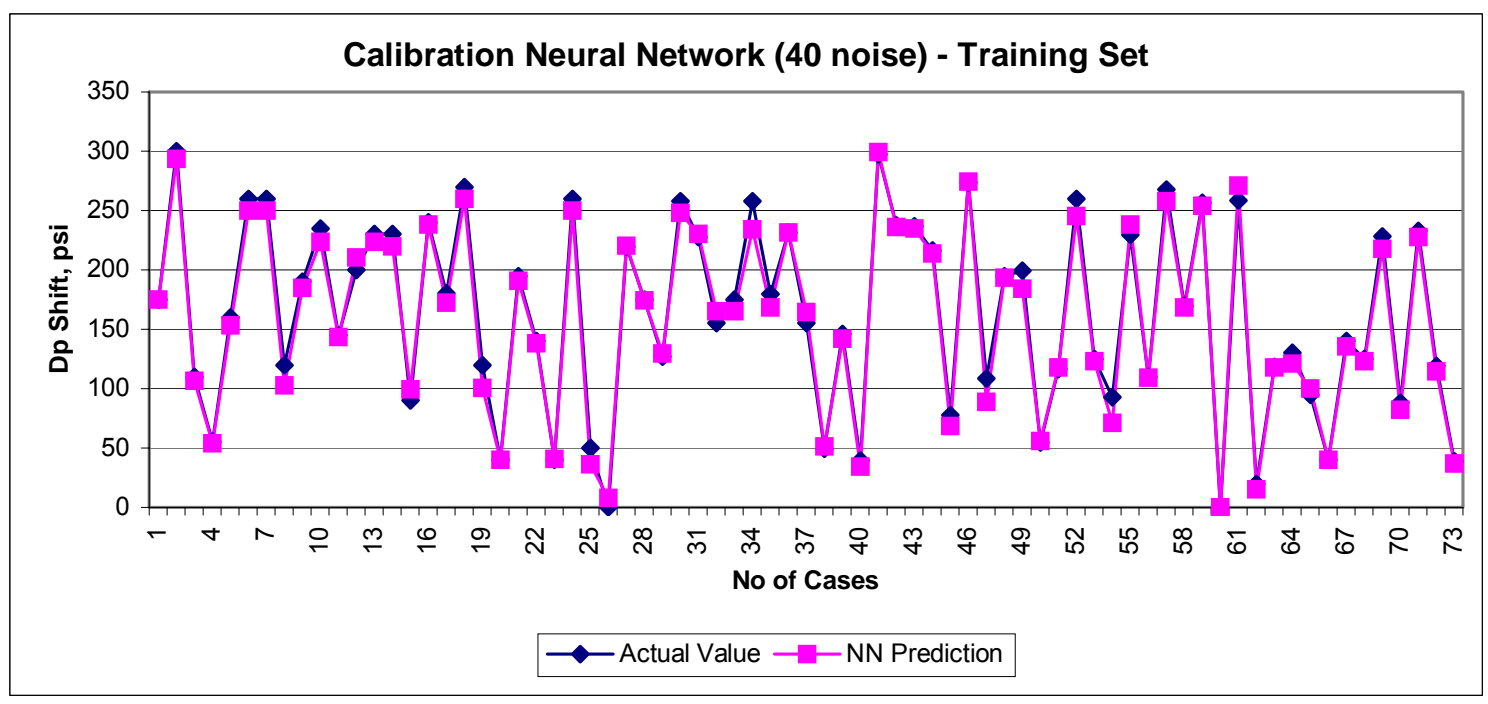

Figure 4-34: Calibration Neural Network Training Set Accuracy 


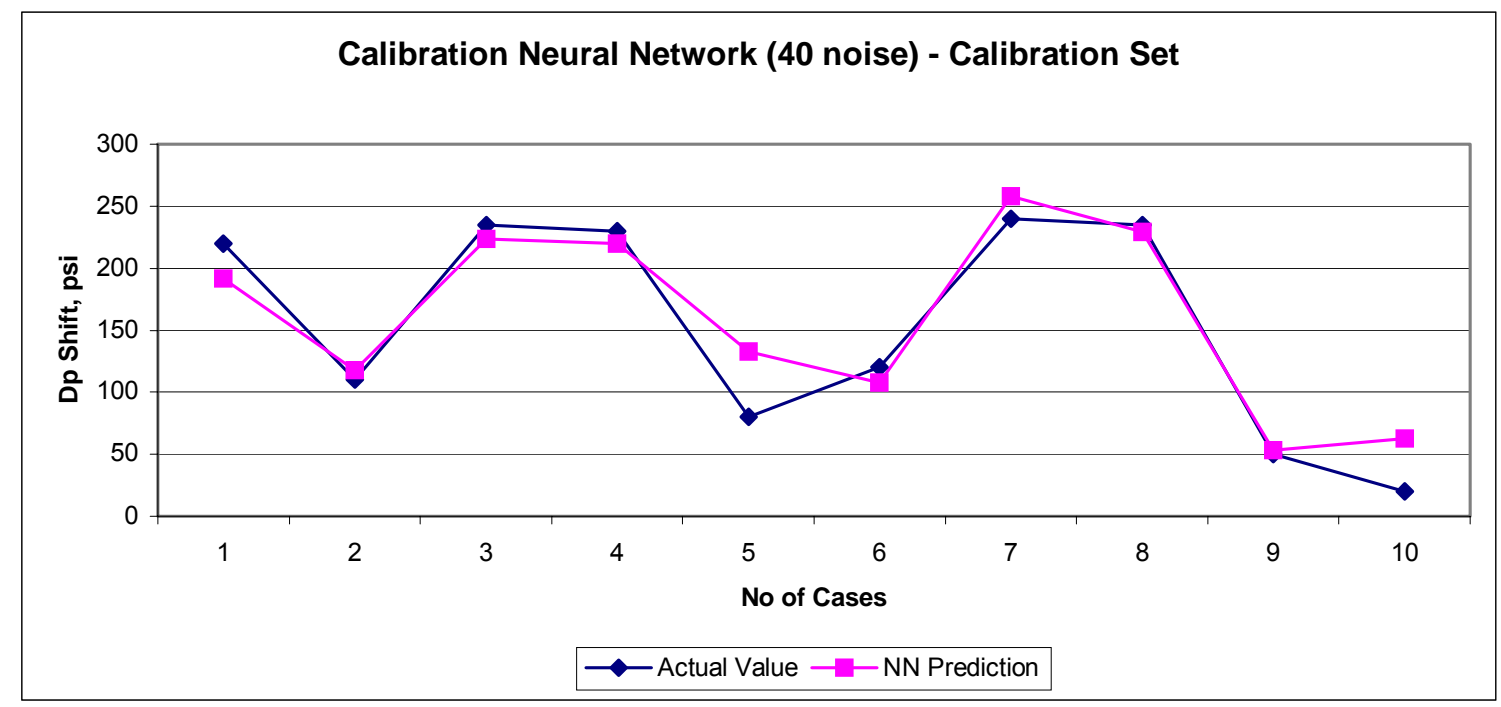

Figure 4-35: Calibration Neural Network Calibration Set Accuracy

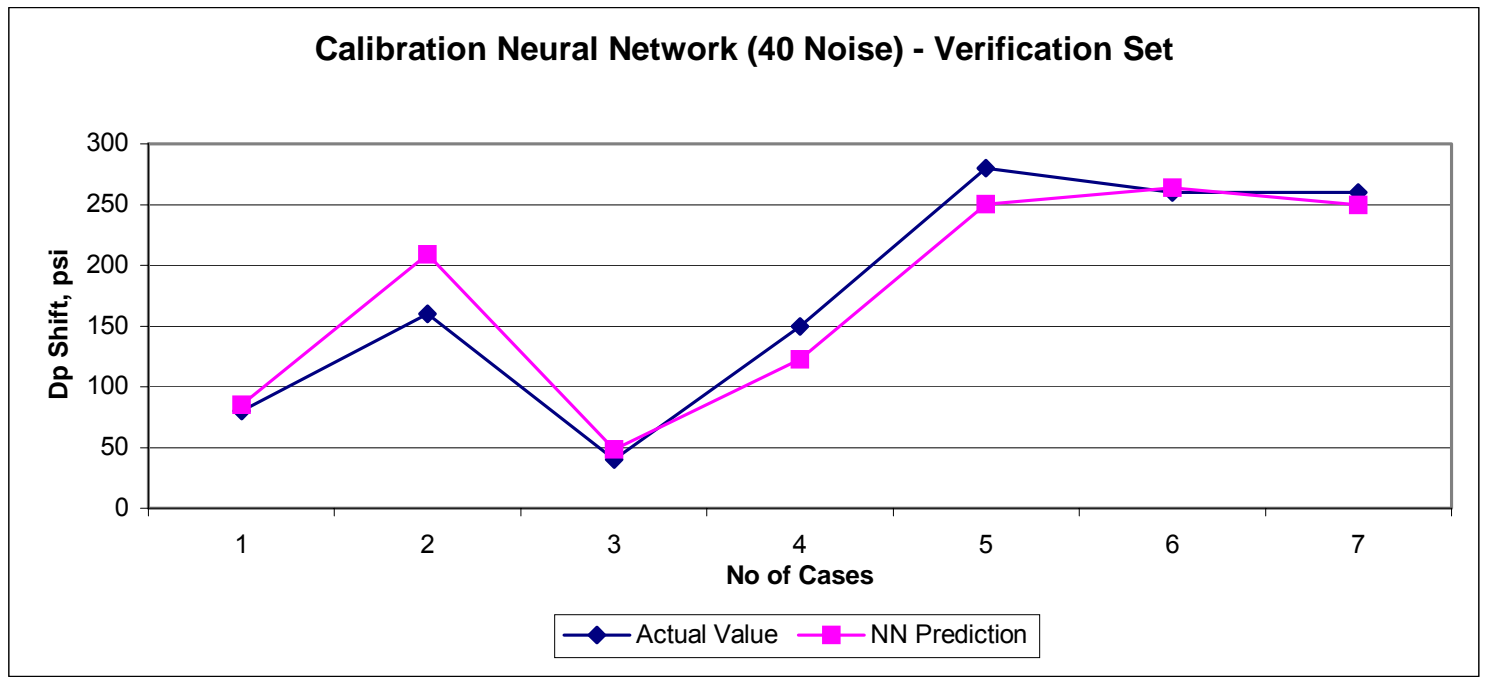

Figure 4-36: Calibration Neural Network Verification Set Accuracy

Due to its improved performance, the fourth neural network was selected to be the calibration neural network. The calibration neural network allows continuing the use of the equivalent four-layer profile for net pressure prediction in the methodology.

\subsubsection{Neural Network for Pressure Profile Prediction}

As previously described, significant work was done to find a methodology able to generate the same net pressure profile when using either the equivalent four-layer profile or original lithology profile with FRACPRO. The result was a calibration neural network trained to add the shift of delta pressure to the net pressure profile generated using the equivalent four- 
layer profile in order to match the net pressure profile from the original lithology. A neural network model was proposed in this study to mimic the fracture simulator and provide the net pressure profile. The development of this system is covered in the following sections.

\subsubsection{Data Considerations}

Since data was needed to train a neural network model, 100 cases were randomly generated using the same algorithm used for ramp treatment schedules as stated in section 4.11.2.1. The inputs are presented in Table 4-3, while the output is the net pressure profile. FRACPRO was run for all 100 cases, and the net pressure profile was recorded for each case.

Each one of the 100 net pressure profiles was generated using a unique treatment design (total fluid, pad, and proppant amount) and specific reservoir conditions (depth, layer stress values, permeability, etc). Since the duration of the job is equal to the ratio of total fluid plus proppant to the injection rate, the treatments have various duration times. The data for each treatment is recorded every minute, resulting in net pressure profile vectors having up to 100 pressure points. In addition, each net pressure profile has a different number of points, based on its duration. Examples of pressure profiles with different duration time for different treatments are presented in Figure 4-37.

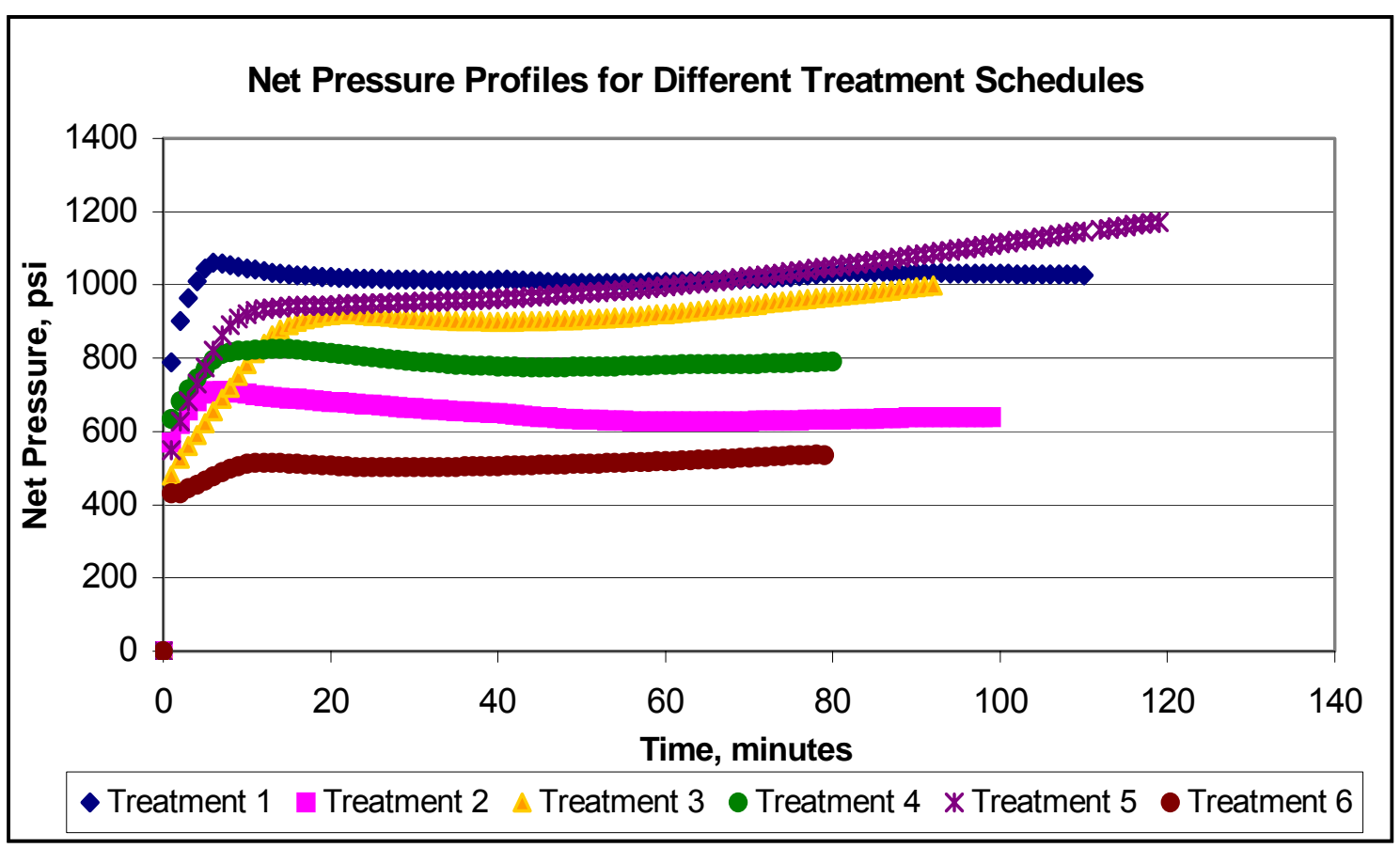

Figure 4-37: Net Pressure Profiles with Various Duration Times 
It is impractical, if not impossible, to use all these points to train a neural network. In these conditions, a methodology to reduce the number of pressure points while maintaining a representative profile is needed. Since vector quantization is one of the most powerful technologies for data compression and dimension reduction, a modified version of the technique was used.

\subsubsection{Data Sampling using Vector Quantization}

The objective of vector quantization is to compress a particular sample by a pre-specified number of prototypes. In this case, the prototypes are the number of pressure points able to reproduce the original pressure profile.

Typically vector quantization is used in communication and data processing, or other high dimensional domains. For this, both an encoder and decoder are needed. However, in this study, the pressure profile is a one-dimensional vector, so a simple extraction of prototypes (pressure points) is sufficient. The net pressure profile will be reproduced using polynomial interpolation.

Vector quantization is simply an approximator. The classic $L B G \vee Q^{30,43}$ design algorithm is an iterative algorithm:

1. Define feature vectors and final number of quantizers. For a number $\mathrm{N}$, of feature vectors $X 1, X 2, \ldots X n$, a number of $k$ quantizers can be found such that $2^{K}<N$.

2. Initialize the first two quantizers, $\mathrm{q} 1$ and $\mathrm{q} 2$, out of $\mathrm{K}$ (usually by assigning a random number to them).

3. Calculate the Euclidian distance from each of the quantizers to all feature vectors. The minimum distance determines Xi's membership to one of the quantizers.

4. Recalculate q1 and q2.

5. Repeat steps 3 and 4 until there is no change in $q 1$ and $q 2$.

6. Split each quantizer such that the new number of quantizers is $K=2 \mathrm{~K}$.

7. Repeat step 3 through 6 until the final number of quantizers is reached.

In this study, each feature vector is one-dimensional. The time vector consists of values for every minute from 0 to final injection time. Similar net pressure vectors consist of values for pressure recorded for each time step, one minute in this case.

The neural network must have a constant number of outputs for each case; therefore, the number of pressure points for all pressure profiles must be consistent in order for the neural network to function. Since not all the pressure profiles have the same duration, sampling at the same time intervals is not possible. Hence, vector quantization was used to extract a fixed number of prototypes (pressure points) from each pressure profile. The algorithm extracts the time and the net pressure for each prototype. By analyzing the shape of each pressure profile, it 
was concluded that 10 points are sufficient to reproduce the pressure profile with acceptable accuracy.

Since vector quantization methodology grows in steps of $2^{\mathrm{K}}$ where $\mathrm{K}$ is the iteration step, the maximum number of prototypes that can be calculated is 8 ( $<10$ points considered). $A$ computer program using the algorithm described below was developed to sample the pressure profile:

1. Use the vector quantization methodology described above to determine the 8 time prototypes (or time quantizers) for the time vector.

2. For this time quantizer, calculate the pressure quantizer (or pressure point) as the average of the pressure values corresponding to the time points represented by the time quantizer.

3. Add two more points to the set of time/pressure quantizers. These points are the time at 1 minute and time of the final injection minute with the corresponding pressures. This ensures that the entire pressure profile is captured, including the start and end.

4. The 10 pressure points (8 quantizers and 2 end points) are the output of the net pressure neural network.

Figure 4-38 and Figure 4-39 show the continuous original net pressure profile generated by FRACPRO (blue diamond points) and the compressed 10-point pressure profile (red square points).

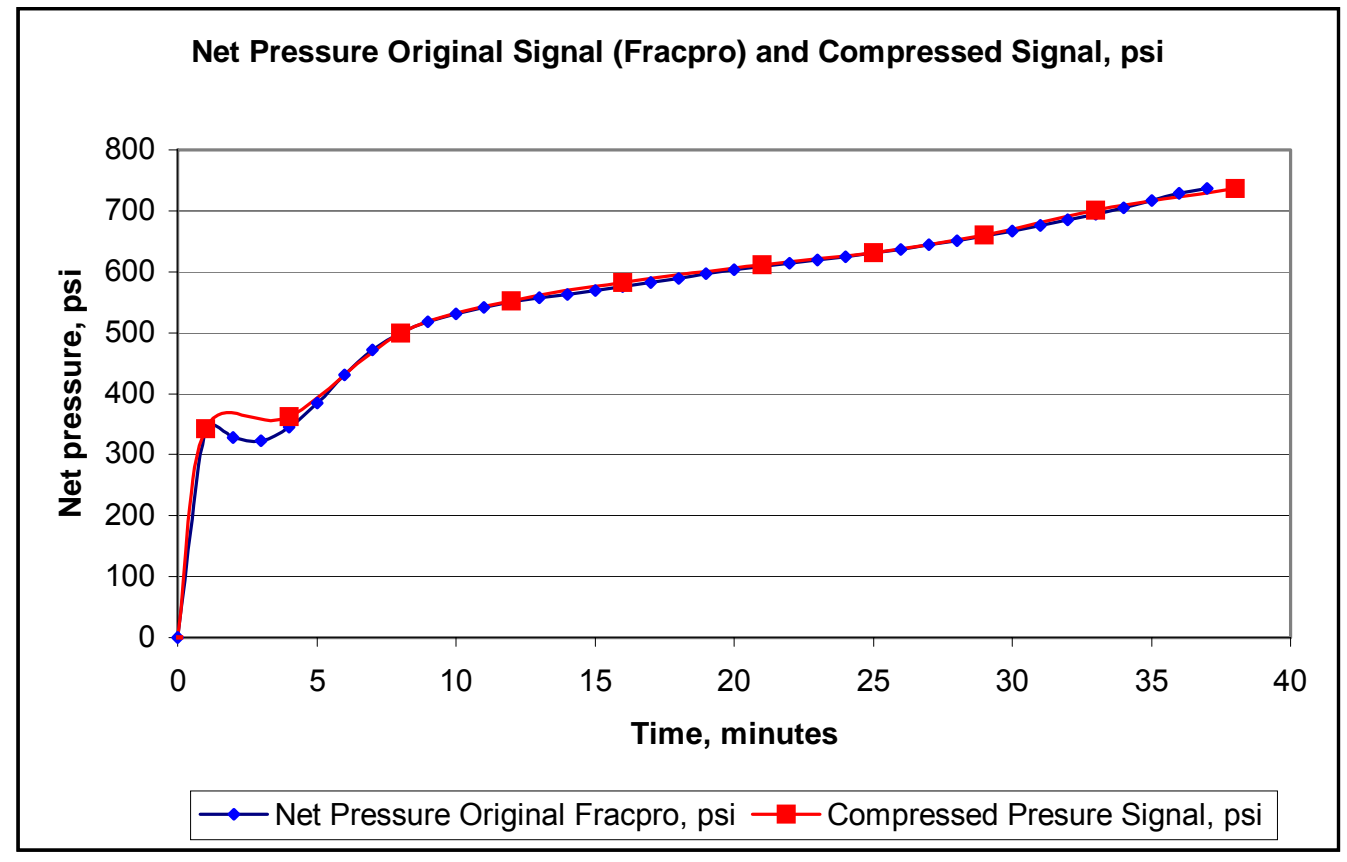

Figure 4-38: Net Pressure Profiles for Original Signal and Compressed Signal Example 2 


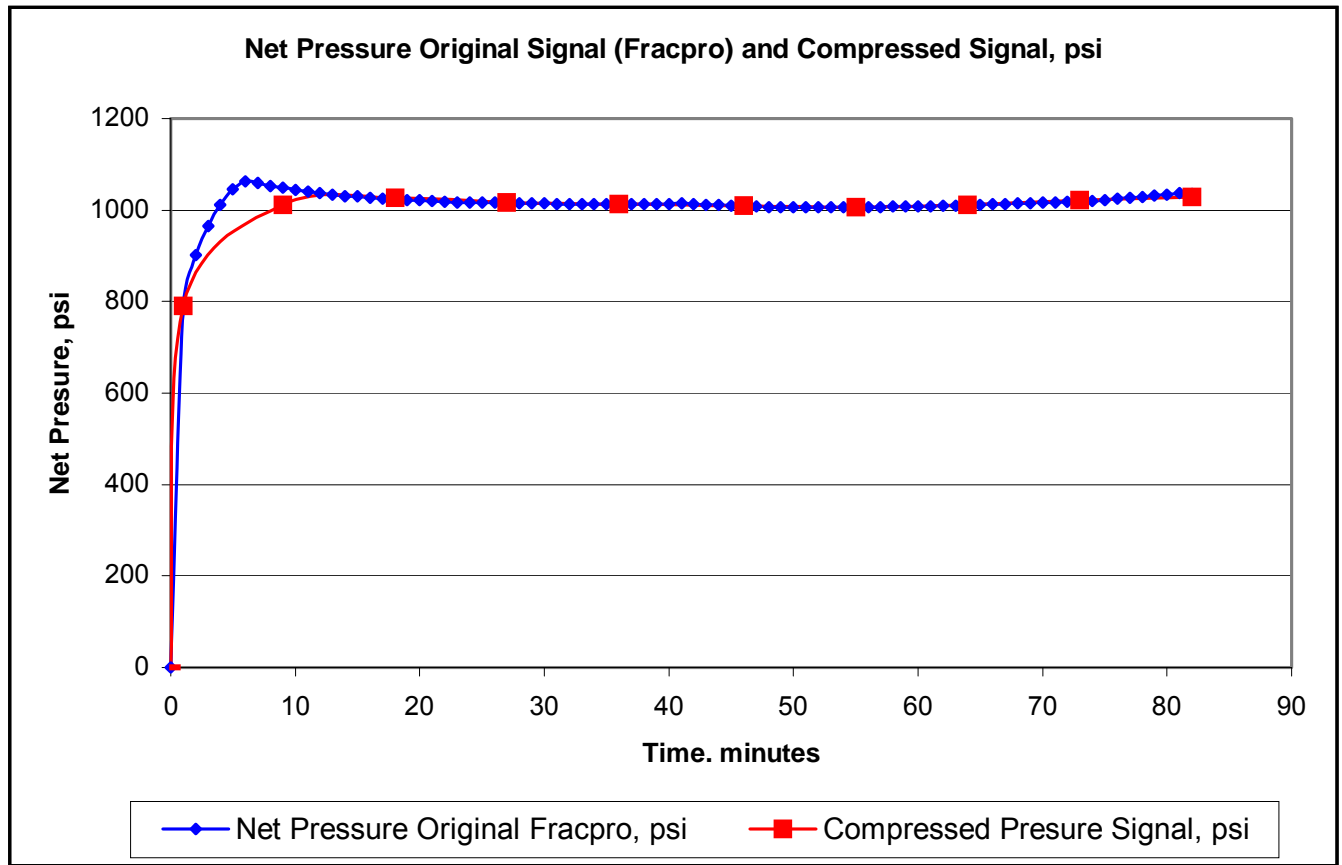

Figure 4-39: Net Pressure Profiles for Original Signal and Compressed Signal Example 2

The algorithm presented above is very convenient when the pressure signal is reproduced back (decoding). Once the size of the treatment and rate are known, the injection time can be easily calculated. Since the time vector is linear, quantizers for any job duration can be calculated by applying the back vector quantization technique.

\subsubsection{Neural Network Design}

The neural network design consists of the inputs and outputs presented in Table 4-39. The net pressure prediction network has one input layer with 14 input neurons, three hidden layers with 21 hidden neurons (7 in each hidden layer) and 10 output neurons. The learning rate was 0.1 and the momentum 0.1 . A summary architecture description of the neural network is given in Table 4-40. 


\begin{tabular}{|c|c|c|c|}
\hline Index & Parameter Name & Type & Units \\
\hline & & & \\
\hline 1 & Total fluid volume & input & gal \\
\hline 2 & Pad volume & input & gal \\
\hline 3 & Final proppant Concentration & input & ppg \\
\hline 4 & Flow pumping rate & input & $\mathrm{bbl} / \mathrm{min}$ \\
\hline 5 & Depth to the "pay zone" & input & $\mathrm{ft}$ \\
\hline 6 & Reservoir pressure & input & psia \\
\hline 7 & Permeability & input & $\mathrm{mD}$ \\
\hline 8 & Top layer thickness & input & $\mathrm{ft}$ \\
\hline 9 & Top layer closure stress gradient & input & $\mathrm{psi} / \mathrm{ft}$ \\
\hline 10 & Overburden layer thickness & input & $\mathrm{ft}$ \\
\hline 11 & Overburden layer closure stress gradient & input & $\mathrm{psi} / \mathrm{ft}$ \\
\hline 12 & Pay zone thickness & input & $\mathrm{ft}$ \\
\hline 13 & Pay zone closure stress gradient & input & $\mathrm{psi} / \mathrm{ft}$ \\
\hline 14 & Bottom layer closure stress gradient & input & $\mathrm{psi} / \mathrm{ft}$ \\
\hline 1 & Pressure Point 1 (at 1 minute) & output & psi \\
\hline 2 & Pressure Point 2 & output & psi \\
\hline 3 & Pressure Point 3 & output & psi \\
\hline 4 & Pressure Point 4 & output & psi \\
\hline 5 & Pressure Point 5 & output & psi \\
\hline 6 & Pressure Point 6 & output & psi \\
\hline 7 & Pressure Point 7 & output & psi \\
\hline 8 & Pressure Point 8 & output & psi \\
\hline 9 & Pressure Point 9 & output & psi \\
\hline 10 & Pressure Point 10 (final minute) & output & psi \\
\hline
\end{tabular}

Table 4-39: Inputs and Outputs for the Net Pressure Neural Network

\begin{tabular}{|c|c|c|c|c|c|c|c|}
\hline $\begin{array}{c}\text { Net } \\
\text { Pressure } \\
\text { Network }\end{array}$ & $\begin{array}{c}\text { Input } \\
\text { Layer }\end{array}$ & $\begin{array}{c}\text { Hidden } \\
\text { Layer 1 }\end{array}$ & $\begin{array}{c}\text { Hidden } \\
\text { Layer 2 }\end{array}$ & $\begin{array}{c}\text { Hidden } \\
\text { Layer 3 }\end{array}$ & Output & $\begin{array}{c}\text { Learning } \\
\text { Rate }\end{array}$ & Momentum \\
\hline $\begin{array}{c}\text { Number of } \\
\text { Neurons }\end{array}$ & 14 & 7 & 7 & 7 & 10 & 0.1 & 0.1 \\
\hline $\begin{array}{c}\text { Type of } \\
\text { activaton } \\
\text { Function }\end{array}$ & Linear & Gaussian & Tanh & Gaussian & Logistic & & \\
\hline
\end{tabular}

Table 4-40: Net Pressure Prediction Neural Network 


\subsection{Summary}

The methodology used in this study combines a series of artificial intelligence tools in a unique manner to build an intelligent tool that designs optimum treatment schedules and predicts net treatment pressure for hydraulic fracturing. Three main modules form the methodology.

In the first module, a new and innovative fuzzy expert system is used for lithology identification. This approach proved to be very effective and provided accurate results. The expert knowledge incorporated in the system makes this tool a great companion for any need of lithology identification. The lithology is then used with a classic approach to calculate the stress profile. Next, the stress profile is processed to illustrate a clear definition of the stress zones. The final part of the module proposes a simple approach to reduce the complex multi-zone lithology stress profile to a four-layer equivalent profile. The equivalent stress profile represents the input to the second module.

The second module incorporates an optimization system composed of neural networks and a genetic algorithm to search for the optimum treatment design. Several types of neural networks were developed to design different types of treatments in this module. This combination of neural networks and genetic algorithms demonstrated fast convergence to optimum solution.

The third, and final, module was designed to predict the fracturing net pressure profile. First, a neural network was developed that calibrates the net pressure profile resulted from the equivalent layer profile with the net pressure profile resulted from original multi-layer profile. Next, a one-dimensional vector quantization technique was used to extract the main characteristic of the pressure profile. A net treatment pressure neural network that uses the pressure quantizers was built to predict the net pressure profile. In the end, the calibration neural network and net pressure neural network predict the pressure profile, and the entire signal is reconstructed. 


\section{CHAPTER 5: RESULTS \& DISCUSSION}

\subsection{Fuzzy Logic Lithology Technique for Stress Profile Calculations}

Over time, several models and equations have been developed to determine the stress profile. The ABC stress profile relationship, developed by Bragan \& Associates ${ }^{17}$, modifies the original Hook's Law equation and introduces the concept of lithology-driven stress calculations. The authors defined a default coefficient for each type of lithology for use in Equation 2-6. However, this approach requires visual and manual coefficient assignment for each formation or zone in addition to the presence of an expert in log interpretation and lithology identification. Moreover, it does not provide any values for transition lithology such as dirty sandstones (shaly sandstone) or shaly carbonates. The fuzzy logic expert system develop in this study identifies lithology without any visual or manual effort and also compensates for transition zones. The system automatically determines the lithology and feeds the corresponding coefficient into Equation 2-6 for stress calculation.

The methodology presented in section 4.8 Fuzzy Lithology Identification Approach was tested on an example well in North Appleby Field, Nacogdoches County, Texas. Three basic logs (spontaneous potential, gamma ray, and deep induction) were obtained from the well. Table 5-1 shows the raw log data for a 30-foot interval (7700-7730 ft) with recordings made every halffoot. 


\begin{tabular}{|c|c|c|c|}
\hline Depth, ft & $\begin{array}{l}\text { Spontaneous } \\
\text { Potential, mV }\end{array}$ & $\begin{array}{c}\text { Gamma Ray, } \\
\text { API }\end{array}$ & $\begin{array}{c}\text { Deep Induction, } \\
\text { ohm-m }\end{array}$ \\
\hline 7700 & -2.6004 & 66.9102 & 5.6821 \\
\hline 7700.5 & -2.7207 & 53.8349 & 5.2905 \\
\hline 7701 & -2.8409 & 41.6089 & 4.5072 \\
\hline 7701.5 & -2.8362 & 39.2341 & 4.2387 \\
\hline 7702 & -2.7065 & 45.2734 & 5.4921 \\
\hline 7702.5 & -2.7017 & 51.4805 & 8.5872 \\
\hline 7703 & -5.1892 & 62.0075 & 11.0407 \\
\hline 7703.5 & -5.1845 & 61.9653 & 12.3901 \\
\hline 7704 & -5.4297 & 52.178 & 10.8485 \\
\hline 7704.5 & -5.425 & 42.1078 & 6.8412 \\
\hline 7705 & -5.2875 & 42.636 & 6.0067 \\
\hline 7705.5 & -5.1577 & 46.3846 & 6.1237 \\
\hline 7706 & -5.028 & 54.2014 & 5.7805 \\
\hline 7706.5 & -5.1404 & 58.5403 & 5.2827 \\
\hline 7707 & -5.167 & 62.2593 & 4.886 \\
\hline 7707.5 & -5.0372 & 64.3599 & 4.3709 \\
\hline 7708 & -4.9075 & 72.039 & 4.0023 \\
\hline 7708.5 & -4.9106 & 80.6483 & 3.7591 \\
\hline 7709 & -5.1558 & 79.1327 & 3.5644 \\
\hline 7709.5 & -5.2761 & 76.7478 & 3.454 \\
\hline 7710 & -5.6464 & 74.3729 & 3.4072 \\
\hline 7710.5 & -5.7745 & 73.2683 & 3.4293 \\
\hline 7711 & -6.2697 & 72.4146 & 3.4614 \\
\hline 7711.5 & -7.015 & 72.3839 & 3.4739 \\
\hline 7712 & -7.3931 & 72.3226 & 3.4716 \\
\hline 7712.5 & -7.8493 & 64.3412 & 6.0416 \\
\hline 7713 & -8.7196 & 54.9708 & 18.8887 \\
\hline 7713.5 & -9.4648 & 48.0022 & 19.487 \\
\hline 7714 & -10.3351 & 42.7926 & 19.8692 \\
\hline 7714.5 & -11.0882 & 40.3232 & 20.3156 \\
\hline 7715 & -11.6928 & 42.9956 & 20.495 \\
\hline 7715.5 & -12.1881 & 46.9197 & 20.6529 \\
\hline 7716 & -12.9646 & 48.5773 & 20.5413 \\
\hline 7716.5 & -13.7255 & 48.6449 & 20.4508 \\
\hline 7717 & -14.2208 & 51.779 & 20.596 \\
\hline 7717.5 & -14.341 & 57.4551 & 21.3248 \\
\hline 7718 & -14.2113 & 57.6 & 22.2165 \\
\hline 7718.5 & -14.2066 & 55.8496 & 31.7005 \\
\hline 7719 & -16.0456 & 49.3581 & 34.0012 \\
\hline 7719.5 & -20.1659 & 47.1824 & 30.3164 \\
\hline 7720 & -21.7861 & 43.1226 & 30.2078 \\
\hline 7720.5 & -22.0314 & 45.2378 & 31.8776 \\
\hline 7721 & -22.0267 & 47.0289 & 34.3436 \\
\hline 7721.5 & -22.0219 & 48.8217 & 37.9456 \\
\hline 7722 & -21.8922 & 43.5953 & 40.7235 \\
\hline 7722.5 & -22.1062 & 41.4603 & 42.3581 \\
\hline 7723 & -21.9609 & 43.2454 & 44.1007 \\
\hline 7723.5 & -22.2061 & 46.3126 & 45.3438 \\
\hline 7724 & -22.5608 & 48.1888 & 46.4139 \\
\hline 7724.5 & -22.7123 & 44.0543 & 46.6483 \\
\hline 7725 & -23.3638 & 45.9305 & 45.1318 \\
\hline 7725.5 & -24.1091 & 43.012 & 46.823 \\
\hline 7726 & -24.6043 & 46.2128 & 44.0916 \\
\hline 7726.5 & -24.7246 & 44.2214 & 43.891 \\
\hline 7727 & -24.8449 & 43.9759 & 42.3834 \\
\hline 7727.5 & -24.8401 & 42.2625 & 36.3123 \\
\hline 7728 & -25.3354 & 37.8586 & 32.1787 \\
\hline 7728.5 & -25.5651 & 35.7222 & 31.4269 \\
\hline 7729 & -25.8103 & 36.2846 & 31.1947 \\
\hline 7729.5 & -26.0556 & 38.8916 & 29.387 \\
\hline 7730 & -26.4415 & 40.8915 & 27.2507 \\
\hline
\end{tabular}

Table 5-1: Log Data from Example Well in Texas 
First, the fuzzy sets were defined for each of the three input parameters. Figure 5-1 shows the data defining the fuzzy sets and the three fuzzy sets for deep induction. Similar sets were defined for spontaneous potential and gamma ray.

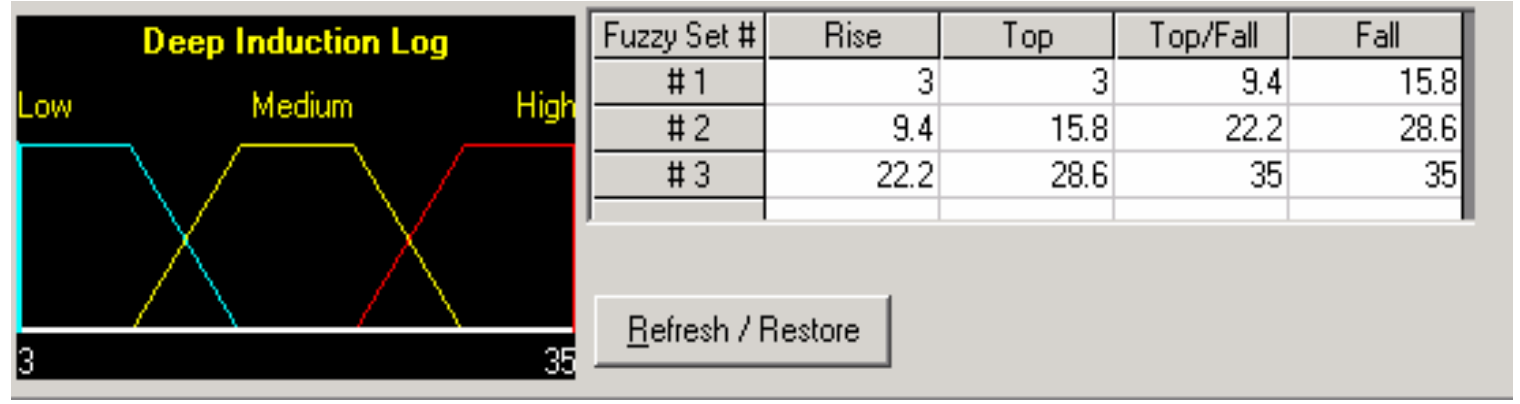

Figure 5-1: Fuzzy Sets for Deep Induction Log

Figure 5-2 shows the parallel processing and decision-making using the fuzzy logic lithology system. In this figure, the system is processing the log values at a depth of 7,845 feet $(22.45 \mathrm{mV}$ for spontaneous potential, 38.2API for gamma ray, and $24.38 \mathrm{ohm}-\mathrm{m}$ for deep induction).
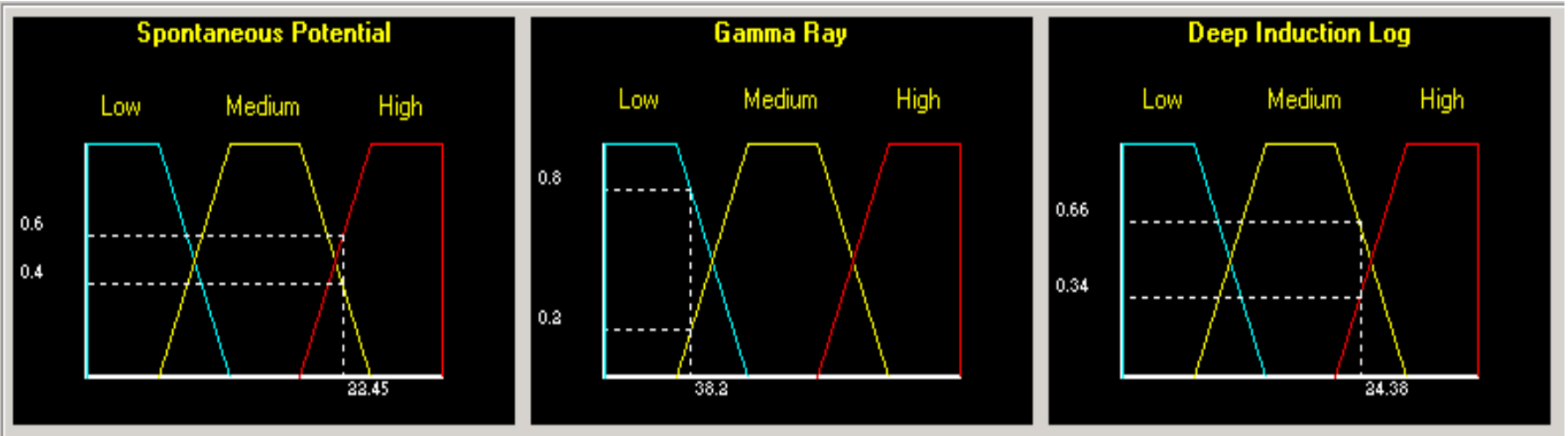

Figure 5-2: Fired Rules in the Process of Lithology Identification

Figure 5-2 shows the fired rules during the decision process for the values mentioned above. Eight rules were fired simultaneously to provide the output of the system. The last column shows the strength of each rule.

\begin{tabular}{|c|c|c|c|c|c|c|c|c|c|}
\hline Rule \# & & Spontaneous Potential & & Gamma Ray & & Deep Induction Log & & Status & $\begin{array}{c}\text { Truth } \\
\text { Qualification }\end{array}$ \\
\hline Rule \#11 & If & Medium & And & Low & And & Medium & Then & SandStone & $T$ \\
\hline Rule \#12 & If & Medium & And & Low & And & High & Then & SandStone & UT \\
\hline Rule \# 14 & If & Medium & And & Medium & And & Medium & Then & SS-Sh & $T$ \\
\hline Rule \# 15 & If & Medium & And & Medium & And & High & Then & SandStone & FT \\
\hline Rule \# 20 & If & High & And & Low & And & Medium & Then & SandStone & $\mathrm{T}$ \\
\hline Rule \# 21 & If & High & And & Low & And & High & Then & SandStone & UT \\
\hline Rule \# 23 & If & High & And & Medium & And & Medium & Then & SS.Sh & UT \\
\hline Rule \# 24 & If & High & And & Medium & And & High & Then & SandStone & $T$ \\
\hline
\end{tabular}

Table 5-2: Fuzzy Rules for the Fuzzy Lithology Identification System 
The outcome of the fuzzy lithology system for the example given above is shown in Figure 5-3. The case belongs to fuzzy set sandstone with a degree of membership equal to 0.6 and to fuzzy set shaly-sandstone with a degree of membership equal to 0.3 .

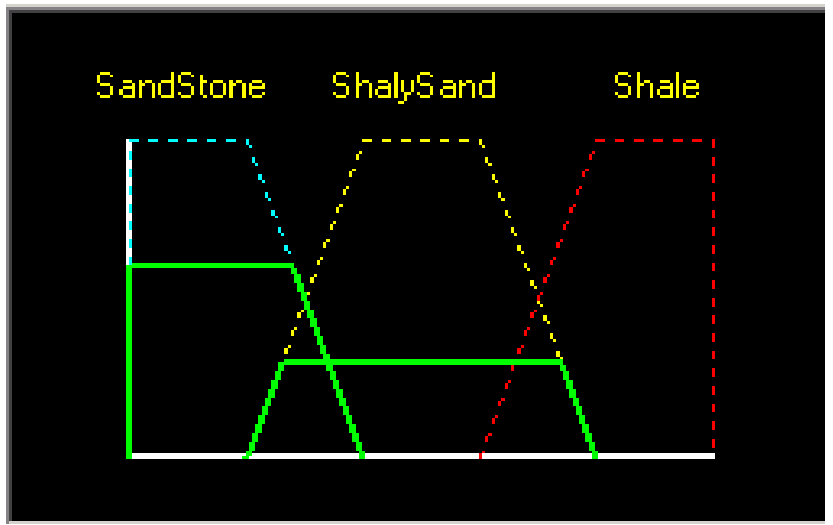

Figure 5-3: Outcome of the Fuzzy Lithology System

Table 5-3 shows the degree of membership value for every half-foot of recorded log data for each of the three lithology fuzzy sets between 7,700 and 7,730 feet. The system identified a shale formation from $7,700-7,714 \mathrm{ft}$, followed by a transition zone $(7,714-7,718 \mathrm{ft})$ identified as shaly-sandstone (dirty sandstone), followed by a cleaner sandstone formation. The logs and lithology output for the interval analyzed in this study is presented in Figure 5-4. The column plotted at the right side of the figure represents the lithology identified using fuzzy lithology system. This lithology was verified by an expert in the domain and proved to be very accurate. 


\begin{tabular}{|c|c|c|c|c|}
\hline \multirow[b]{2}{*}{ Depth, ft } & \multirow[b]{2}{*}{ Lithology } & \multicolumn{3}{|c|}{ Degree of Membership } \\
\hline & & Sandstone & $\begin{array}{c}\text { Shaly- } \\
\text { Sandstone }\end{array}$ & Shale \\
\hline 7700 & Shale & 0 & 0 & 0.85 \\
\hline 7700.5 & Shale & 0 & 0 & 1 \\
\hline 7701 & Shale & 0 & 0 & 0.51 \\
\hline 7701.5 & Shale & 0 & 0 & 0.71 \\
\hline 7702 & Shale & 0 & 0 & 0.84 \\
\hline 7702.5 & Shale & 0 & 0 & 1 \\
\hline 7703 & Shale & 0 & 0 & 0.64 \\
\hline 7703.5 & Shale & 0 & 0 & 0.53 \\
\hline 7704 & Shale & 0 & 0 & 0.77 \\
\hline 7704.5 & Shale & 0 & 0 & 0.56 \\
\hline 7705 & Shale & 0 & 0 & 0.6 \\
\hline 7705.5 & Shale & 0 & 0 & 0.94 \\
\hline 7706 & Shale & 0 & 0 & 1 \\
\hline 7706.5 & Shale & 0 & 0 & 0.95 \\
\hline 7707 & Shale & 0 & 0 & 0.61 \\
\hline 7707.5 & Shale & 0 & 0 & 0.66 \\
\hline 7708 & Shale & 0 & 0 & 1 \\
\hline 7708.5 & Shale & 0 & 0 & 1 \\
\hline 7709 & Shale & 0 & 0 & 1 \\
\hline 7709.5 & Shale & 0 & 0 & 1 \\
\hline 7710 & Shale & 0 & 0 & 1 \\
\hline 7710.5 & Shale & 0 & 0 & 1 \\
\hline 7711 & Shale & 0 & 0 & 1 \\
\hline 7711.5 & Shale & 0 & 0 & 1 \\
\hline 7712 & Shale & 0 & 0 & 1 \\
\hline 7712.5 & Shale & 0 & 0 & 0.66 \\
\hline 7713 & Shale & 0 & 0.06 & 0.91 \\
\hline 7713.5 & Shale & 0 & 0.2 & 0.72 \\
\hline 7714 & Shale & 0.36 & 0.36 & 0.49 \\
\hline 7714.5 & Sandstone & 0.5 & 0.39 & 0.25 \\
\hline 7715 & SS-SH & 0.36 & 0.61 & 0.24 \\
\hline 7715.5 & SS-SH & 0.01 & 0.7 & 0.16 \\
\hline 7716 & SS-SH & 0 & 0.85 & 0.06 \\
\hline 7716.5 & SS-SH & 0 & 0.99 & 0 \\
\hline 7717 & SS-SH & 0 & 1 & 0 \\
\hline 7717.5 & SS-SH & 0 & 1 & 0 \\
\hline 7718 & SS-SH & 0 & 1 & 0 \\
\hline 7718.5 & Sandstone & 1 & 0 & 0 \\
\hline 7719 & Sandstone & 1 & 0 & 0 \\
\hline 7719.5 & Sandstone & 0.74 & 0 & 0 \\
\hline 7720 & Sandstone & 0.48 & 0 & 0 \\
\hline 7720.5 & Sandstone & 0.52 & 0 & 0 \\
\hline 7721 & Sandstone & 0.52 & 0 & 0 \\
\hline 7721.5 & Sandstone & 0.52 & 0 & 0 \\
\hline 7722 & Sandstone & 0.5 & 0 & 0 \\
\hline 7722.5 & Sandstone & 0.6 & 0 & 0 \\
\hline 7723 & Sandstone & 0.51 & 0 & 0 \\
\hline 7723.5 & Sandstone & 0.56 & 0 & 0 \\
\hline 7724 & Sandstone & 0.62 & 0 & 0 \\
\hline 7724.5 & Sandstone & 0.65 & 0 & 0 \\
\hline 7725 & Sandstone & 0.77 & 0 & 0 \\
\hline 7725.5 & Sandstone & 0.64 & 0 & 0 \\
\hline 7726 & Sandstone & 0.93 & 0 & 0 \\
\hline 7726.5 & Sandstone & 0.75 & 0 & 0 \\
\hline 7727 & Sandstone & 0.73 & 0 & 0 \\
\hline 7727.5 & Sandstone & 0.57 & 0 & 0 \\
\hline 7728 & Sandstone & 0.87 & 0 & 0 \\
\hline 7728.5 & Sandstone & 1 & 0 & 0 \\
\hline 7729 & Sandstone & 0.98 & 0 & 0 \\
\hline 7729.5 & Sandstone & 0.8 & 0 & 0 \\
\hline 7730 & Sandstone & 0.64 & 0.31 & 0 \\
\hline
\end{tabular}

Table 5-3: Lithology Identification using Fuzzy Lithology System 


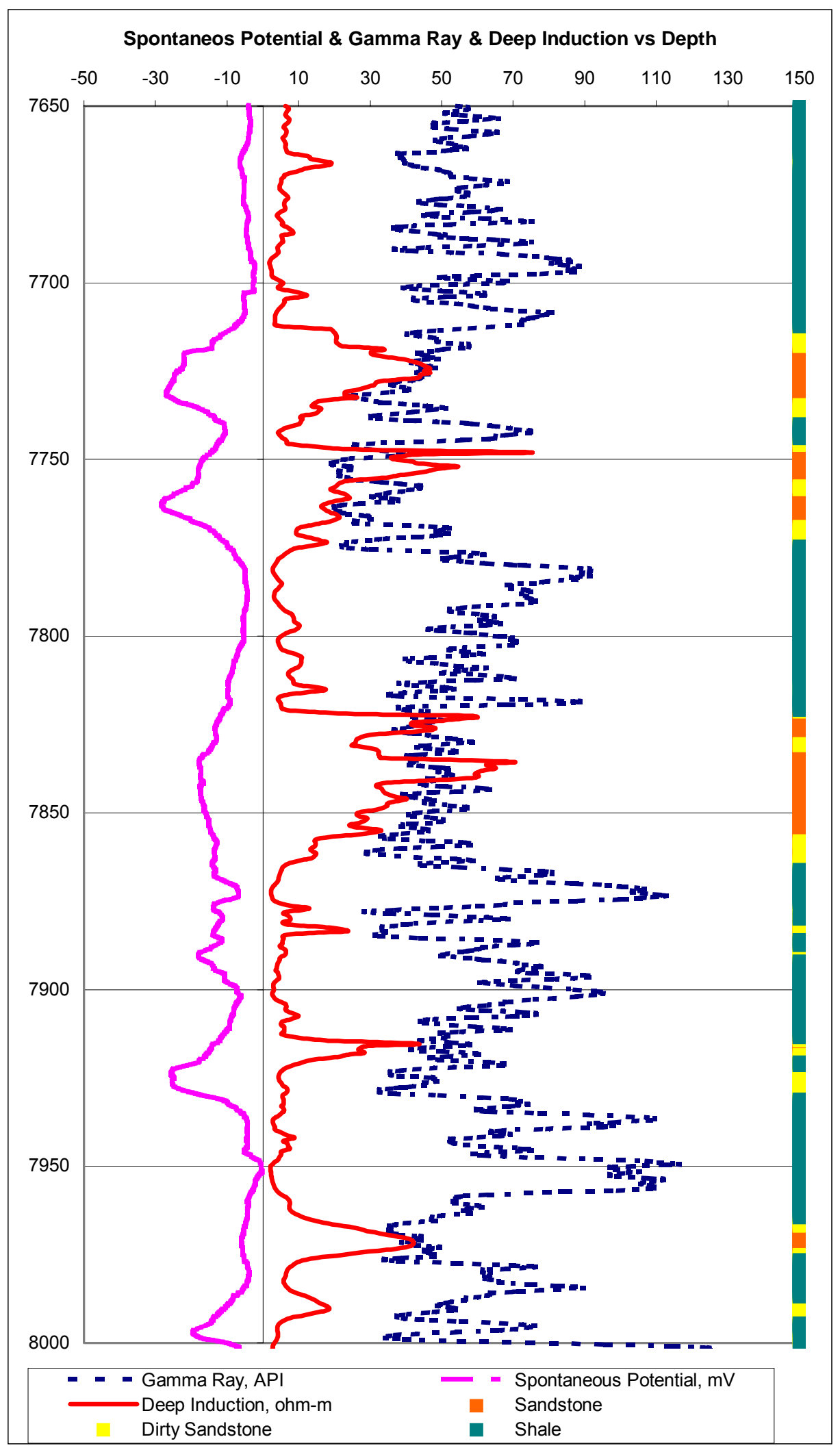

Figure 5-4: Spontaneous Potential, Gamma Ray, Deep Induction, and Lithology 
It is clearly visible that sandstone formations (red in the lithology column) are present where resistivity (deep induction) and spontaneous potential have high values and gamma ray values are low.

After lithology has been established, Lithology Coefficient A was automatically assigned, and Equation 2-6 was used to calculate the stress profile. Table 5-4 shows closure stress calculation for every half-foot of log data, while Figure 5-5 shows the stress profile of the interval.

Next, the stress profile underwent a manual-processing step to clearly illustrate different existing stress layers. This process was done visually, using the stress profile curve and logs, by grouping together similar consecutive stress values to define possible shale and sandstone formations. Figure 5-5 illustrates the separation between different layers with similar characteristics, such as values of closure stress. The horizontal lines and boxes on the right side of the graph show a definition of the four layers between 7,777 and 7,850 feet.

- Layer $1(7,777-7,793.5 \mathrm{ft})$ represents clean shale.

- Layer $2(7,794-7,821 \mathrm{ft})$ is shale with small intercalations of sandstone. These intercalations indicate the presence of sandstone nearby.

- Layer $3(7,821.5-7,832 \mathrm{ft})$ is clean sandstone as indicated from Layer 2 .

- Layer $4(7,832-7,850 \mathrm{ft})$ is poor sandstone (dirty sandstone).

Although every $0.5 \mathrm{ft}$ in these four layers may have a different closure stress value, the final outcome is one closure stress value for each of the four layers. These values are calculated as the arithmetic average of all the stress values between the two depths. Table 5-5 shows the stress profile processing, which is plotted in Figure 5-6. 


\begin{tabular}{|c|c|c|c|c|c|c|c|c|}
\hline & & Degre & of Membe & hip & & & & \\
\hline Depth, ft & Lithology & Sandstone & $\begin{array}{c}\text { Shaly- } \\
\text { Sandstone }\end{array}$ & Shale & $\begin{array}{c}\text { A } \\
\text { Coefficien }\end{array}$ & $\begin{array}{c}\text { B } \\
\text { Coefficien }\end{array}$ & $\begin{array}{c}\text { C } \\
\text { Coefficien }\end{array}$ & $\begin{array}{c}\text { Closure } \\
\text { Stress, psi }\end{array}$ \\
\hline 7700 & Shale & 0 & 0 & 0.85 & 0.524 & 0.5 & 0 & 6162 \\
\hline 7700.5 & Shale & 0 & 0 & 1 & 0.53 & 0.5 & 0 & 6210 \\
\hline 7701 & Shale & 0 & 0 & 0.51 & 0.5104 & 0.5 & 0 & 6052 \\
\hline 7701.5 & Shale & 0 & 0 & 0.71 & 0.5184 & 0.5 & 0 & 6117 \\
\hline 7702 & Shale & 0 & 0 & 0.84 & 0.5236 & 0.5 & 0 & 6160 \\
\hline 7702.5 & Shale & 0 & 0 & 1 & 0.53 & 0.5 & 0 & 6212 \\
\hline 7703 & Shale & 0 & 0 & 0.64 & 0.5156 & 0.5 & 0 & 6096 \\
\hline 7703.5 & Shale & 0 & 0 & 0.53 & 0.5112 & 0.5 & 0 & 6061 \\
\hline 7704 & Shale & 0 & 0 & 0.77 & 0.5208 & 0.5 & 0 & 6139 \\
\hline 7704.5 & Shale & 0 & 0 & 0.56 & 0.5124 & 0.5 & 0 & 6071 \\
\hline 7705 & Shale & 0 & 0 & 0.6 & 0.514 & 0.5 & 0 & 6085 \\
\hline 7705.5 & Shale & 0 & 0 & 0.94 & 0.5276 & 0.5 & 0 & 6195 \\
\hline 7706 & Shale & 0 & 0 & 1 & 0.53 & 0.5 & 0 & 6215 \\
\hline 7706.5 & Shale & 0 & 0 & 0.95 & 0.528 & 0.5 & 0 & 6199 \\
\hline 7707 & Shale & 0 & 0 & 0.61 & 0.5144 & 0.5 & 0 & 6089 \\
\hline 7707.5 & Shale & 0 & 0 & 0.66 & 0.5164 & 0.5 & 0 & 6106 \\
\hline 7708 & Shale & 0 & 0 & 1 & 0.53 & 0.5 & 0 & 6217 \\
\hline 7708.5 & Shale & 0 & 0 & 1 & 0.53 & 0.5 & 0 & 6217 \\
\hline 7709 & Shale & 0 & 0 & 1 & 0.53 & 0.5 & 0 & 6217 \\
\hline 7709.5 & Shale & 0 & 0 & 1 & 0.53 & 0.5 & 0 & 6218 \\
\hline 7710 & Shale & 0 & 0 & 1 & 0.53 & 0.5 & 0 & 6218 \\
\hline 7710.5 & Shale & 0 & 0 & 1 & 0.53 & 0.5 & 0 & 6219 \\
\hline 7711 & Shale & 0 & 0 & 1 & 0.53 & 0.5 & 0 & 6219 \\
\hline 7711.5 & Shale & 0 & 0 & 1 & 0.53 & 0.5 & 0 & 6219 \\
\hline 7712 & Shale & 0 & 0 & 1 & 0.53 & 0.5 & 0 & 6220 \\
\hline 7712.5 & Shale & 0 & 0 & 0.66 & 0.5164 & 0.5 & 0 & 6110 \\
\hline 7713 & Shale & 0 & 0.06 & 0.91 & 0.5264 & 0.5 & 0 & 6191 \\
\hline 7713.5 & Shale & 0 & 0.2 & 0.72 & 0.5188 & 0.5 & 0 & 6130 \\
\hline 7714 & Shale & 0.36 & 0.36 & 0.49 & 0.5044 & 0.5 & 0 & 6014 \\
\hline 7714.5 & Sandstone & 0.5 & 0.39 & 0.25 & 0.445 & 0.5 & 0 & 5533 \\
\hline 7715 & SS-SH & 0.36 & 0.61 & 0.24 & 0.4576 & 0.5 & 0 & 5636 \\
\hline 7715.5 & SS-SH & 0.01 & 0.7 & 0.16 & 0.4964 & 0.5 & 0 & 5950 \\
\hline 7716 & SS-SH & 0 & 0.85 & 0.06 & 0.4924 & 0.5 & 0 & 5918 \\
\hline 7716.5 & SS-SH & 0 & 0.99 & 0 & 0.49 & 0.5 & 0 & 5899 \\
\hline 7717 & SS-SH & 0 & 1 & 0 & 0.49 & 0.5 & 0 & 5900 \\
\hline 7717.5 & SS-SH & 0 & 1 & 0 & 0.49 & 0.5 & 0 & 5900 \\
\hline 7718 & SS-SH & 0 & 1 & 0 & 0.49 & 0.5 & 0 & 5900 \\
\hline 7718.5 & Sandstone & 1 & 0 & 0 & 0.4 & 0.5 & 0 & 5171 \\
\hline 7719 & Sandstone & 1 & 0 & 0 & 0.4 & 0.5 & 0 & 5172 \\
\hline 7719.5 & Sandstone & 0.74 & 0 & 0 & 0.413 & 0.5 & 0 & 5277 \\
\hline 7720 & Sandstone & 0.48 & 0 & 0 & 0.426 & 0.5 & 0 & 5383 \\
\hline 7720.5 & Sandstone & 0.52 & 0 & 0 & 0.424 & 0.5 & 0 & 5367 \\
\hline 7721 & Sandstone & 0.52 & 0 & 0 & 0.424 & 0.5 & 0 & 5368 \\
\hline 7721.5 & Sandstone & 0.52 & 0 & 0 & 0.424 & 0.5 & 0 & 5368 \\
\hline 7722 & Sandstone & 0.5 & 0 & 0 & 0.425 & 0.5 & 0 & 5376 \\
\hline 7722.5 & Sandstone & 0.6 & 0 & 0 & 0.42 & 0.5 & 0 & 5336 \\
\hline 7723 & Sandstone & 0.51 & 0 & 0 & 0.4245 & 0.5 & 0 & 5373 \\
\hline 7723.5 & Sandstone & 0.56 & 0 & 0 & 0.422 & 0.5 & 0 & 5353 \\
\hline 7724 & Sandstone & 0.62 & 0 & 0 & 0.419 & 0.5 & 0 & 5329 \\
\hline 7724.5 & Sandstone & 0.65 & 0 & 0 & 0.4175 & 0.5 & 0 & 5317 \\
\hline 7725 & Sandstone & 0.77 & 0 & 0 & 0.4115 & 0.5 & 0 & 5269 \\
\hline 7725.5 & Sandstone & 0.64 & 0 & 0 & 0.418 & 0.5 & 0 & 5322 \\
\hline 7726 & Sandstone & 0.93 & 0 & 0 & 0.4035 & 0.5 & 0 & 5205 \\
\hline 7726.5 & Sandstone & 0.75 & 0 & 0 & 0.4125 & 0.5 & 0 & 5278 \\
\hline 7727 & Sandstone & 0.73 & 0 & 0 & 0.4135 & 0.5 & 0 & 5287 \\
\hline 7727.5 & Sandstone & 0.57 & 0 & 0 & 0.4215 & 0.5 & 0 & 5352 \\
\hline 7728 & Sandstone & 0.87 & 0 & 0 & 0.4065 & 0.5 & 0 & 5231 \\
\hline 7728.5 & Sandstone & 1 & 0 & 0 & 0.4 & 0.5 & 0 & 5178 \\
\hline 7729 & Sandstone & 0.98 & 0 & 0 & 0.401 & 0.5 & 0 & 5187 \\
\hline 7729.5 & Sandstone & 0.8 & 0 & 0 & 0.41 & 0.5 & 0 & 5260 \\
\hline 7730 & Sandstone & 0.64 & 0.31 & 0 & 0.4324 & 0.5 & 0 & 5442 \\
\hline
\end{tabular}

Table 5-4: Example of Stress Calculation using ABC Methodology 


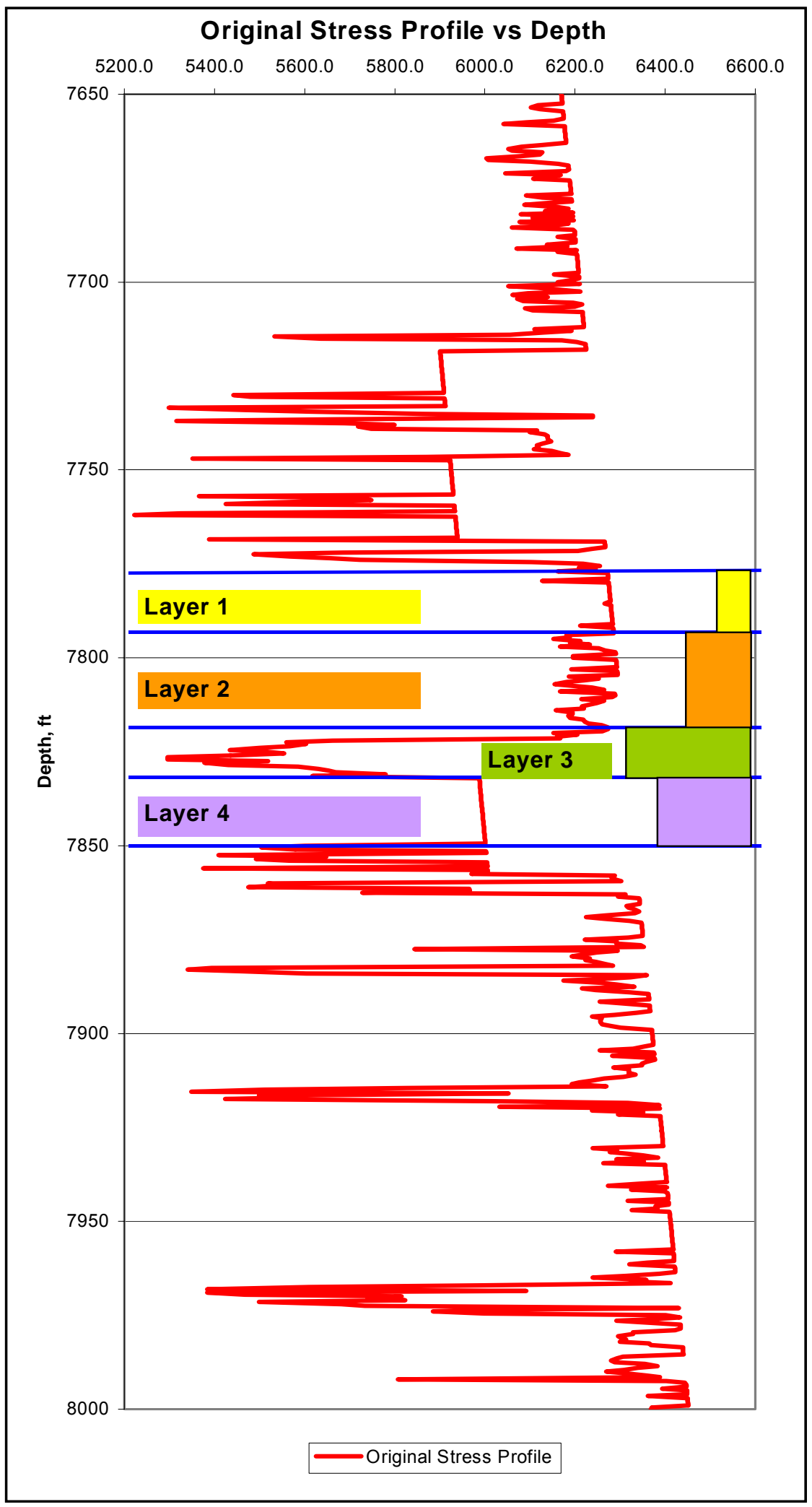

Figure 5-5: Processing Methodology for Original Stress Profile 


\begin{tabular}{|c|c|c|c|c|c|c|c|}
\hline \multicolumn{2}{|c|}{ Layer 1} & \multicolumn{2}{|c|}{ Layer 2} & \multicolumn{2}{|c|}{ Layer 3} & \multicolumn{2}{|c|}{ Layer 4} \\
\hline Depth, ft & $\begin{array}{c}\text { Closure } \\
\text { Stress, psi }\end{array}$ & Depth, ft & $\begin{array}{c}\text { Closure } \\
\text { Stress, psi }\end{array}$ & Depth, ft & $\begin{array}{c}\text { Closure } \\
\text { Stress, psi }\end{array}$ & Depth, ft & $\begin{array}{c}\text { Closure } \\
\text { Stress, psi }\end{array}$ \\
\hline 7777 & 6161 & 7794 & 6181 & 7821.5 & 6167 & 7832.5 & 5988 \\
\hline 7777.5 & 6273 & 7794.5 & 6188 & 7822 & 5662 & 7833 & 5988 \\
\hline 7778 & 6273 & 7795 & 6152 & 7822.5 & 5559 & 7833.5 & 5989 \\
\hline 7778.5 & 6273 & 7795.5 & 6212 & 7823 & 5604 & 7834 & 5989 \\
\hline 7779 & 6274 & 7796 & 6189 & 7823.5 & 5567 & 7834.5 & 5989 \\
\hline 7779.5 & 6127 & 7796.5 & 6232 & 7824 & 5501 & 7835 & 5990 \\
\hline 7780 & 6275 & 7797 & 6167 & 7824.5 & 5435 & 7835.5 & 5990 \\
\hline 7780.5 & 6275 & 7797.5 & 6253 & 7825 & 5516 & 7836 & 5991 \\
\hline 7781 & 6275 & 7798 & 6266 & 7825.5 & 5554 & 7836.5 & 5991 \\
\hline 7781.5 & 6276 & 7798.5 & 6289 & 7826 & 5436 & 7837 & 5991 \\
\hline 7782 & 6276 & 7799 & 6290 & 7826.5 & 5296 & 7837.5 & 5992 \\
\hline 7782.5 & 6277 & 7799.5 & 6195 & 7827 & 5296 & 7838 & 5992 \\
\hline 7783 & 6277 & 7800 & 6196 & 7827.5 & 5518 & 7838.5 & 5993 \\
\hline 7783.5 & 6277 & 7800.5 & 6291 & 7828 & 5378 & 7839 & 5993 \\
\hline 7784 & 6278 & 7801 & 6292 & 7828.5 & 5430 & 7839.5 & 5993 \\
\hline 7784.5 & 6278 & 7801.5 & 6292 & 7829 & 5586 & 7840 & 5994 \\
\hline 7785 & 6279 & 7802 & 6292 & 7829.5 & 5631 & 7840.5 & 5994 \\
\hline 7785.5 & 6266 & 7802.5 & 6293 & 7830 & 5653 & 7841 & 5994 \\
\hline 7786 & 6279 & 7803 & 6192 & 7830.5 & 5668 & 7841.5 & 5995 \\
\hline 7786.5 & 6280 & 7803.5 & 6294 & 7831 & 5780 & 7842 & 5995 \\
\hline 7787 & 6280 & 7804 & 6294 & 7831.5 & 5617 & 7842.5 & 5996 \\
\hline 7787.5 & 6281 & 7804.5 & 6294 & 7832 & 5988 & 7843 & 5996 \\
\hline 7788 & 6281 & 7805 & 6187 & & & 7843.5 & 5996 \\
\hline 7788.5 & 6281 & 7805.5 & 6253 & & & 7844 & 5997 \\
\hline 7789 & 6282 & 7806 & 6220 & & & 7844.5 & 5997 \\
\hline 7789.5 & 6282 & 7806.5 & 6181 & & & 7845 & 5998 \\
\hline 7790 & 6283 & 7807 & 6155 & & & 7845.5 & 5998 \\
\hline 7790.5 & 6283 & 7807.5 & 6195 & & & 7846 & 5998 \\
\hline 7791 & 6283 & 7808 & 6238 & & & 7846.5 & 5999 \\
\hline 7791.5 & 6212 & 7808.5 & 6265 & & & 7847 & 5999 \\
\hline 7792 & 6284 & 7809 & 6167 & & & 7847.5 & 5999 \\
\hline 7792.5 & 6285 & 7809.5 & 6289 & & & 7848 & 6000 \\
\hline 7793 & 6285 & 7810 & 6289 & & & 7848.5 & 6000 \\
\hline 7793.5 & 6285 & 7810.5 & 6283 & & & 7849 & 6001 \\
\hline & & 7811 & 6214 & & & 7849.5 & 6001 \\
\hline & & 7811.5 & 6264 & & & 7850 & 5601 \\
\hline & & 7812 & 6248 & & & & \\
\hline & & 7812.5 & 6232 & & & & \\
\hline & & 7813 & 6216 & & & & \\
\hline & & 7813.5 & 6220 & & & & \\
\hline & & 7814 & 6158 & & & & \\
\hline & & 7814.5 & 6194 & & & & \\
\hline & & 7815 & 6194 & & & & \\
\hline & & 7815.5 & 6185 & & & & \\
\hline & & 7816 & 6189 & & & & \\
\hline & & 7816.5 & 6219 & & & & \\
\hline & & 7817 & 6222 & & & & \\
\hline & & 7817.5 & 6229 & & & & \\
\hline & & 7818 & 6259 & & & & \\
\hline & & 7818.5 & 6273 & & & & \\
\hline & & 7819 & 6273 & & & & \\
\hline & & 7819.5 & 6260 & & & & \\
\hline & & 7820 & 6152 & & & & \\
\hline & & 7820.5 & 6205 & & & & \\
\hline & & 7821 & 6160 & & & & \\
\hline Interval, ft & $\begin{array}{c}\text { Average } \\
\text { Clossure } \\
\text { Stress, psi }\end{array}$ & Interval, ft & $\begin{array}{c}\text { Average } \\
\text { Clossure } \\
\text { Stress, psi }\end{array}$ & Interval, ft & $\begin{array}{l}\text { Average } \\
\text { Clossure } \\
\text { Stress, psi }\end{array}$ & Interval, ft & $\begin{array}{l}\text { Average } \\
\text { Clossure } \\
\text { Stress, psi }\end{array}$ \\
\hline 7777 - 7793.5 & 6269 & $7794-7821$ & 6230 & $7821.5-7832$ & 5584 & $7832.5-7850$ & 5984 \\
\hline
\end{tabular}

Table 5-5: Example of Stress Profile Processing for Four Layers 


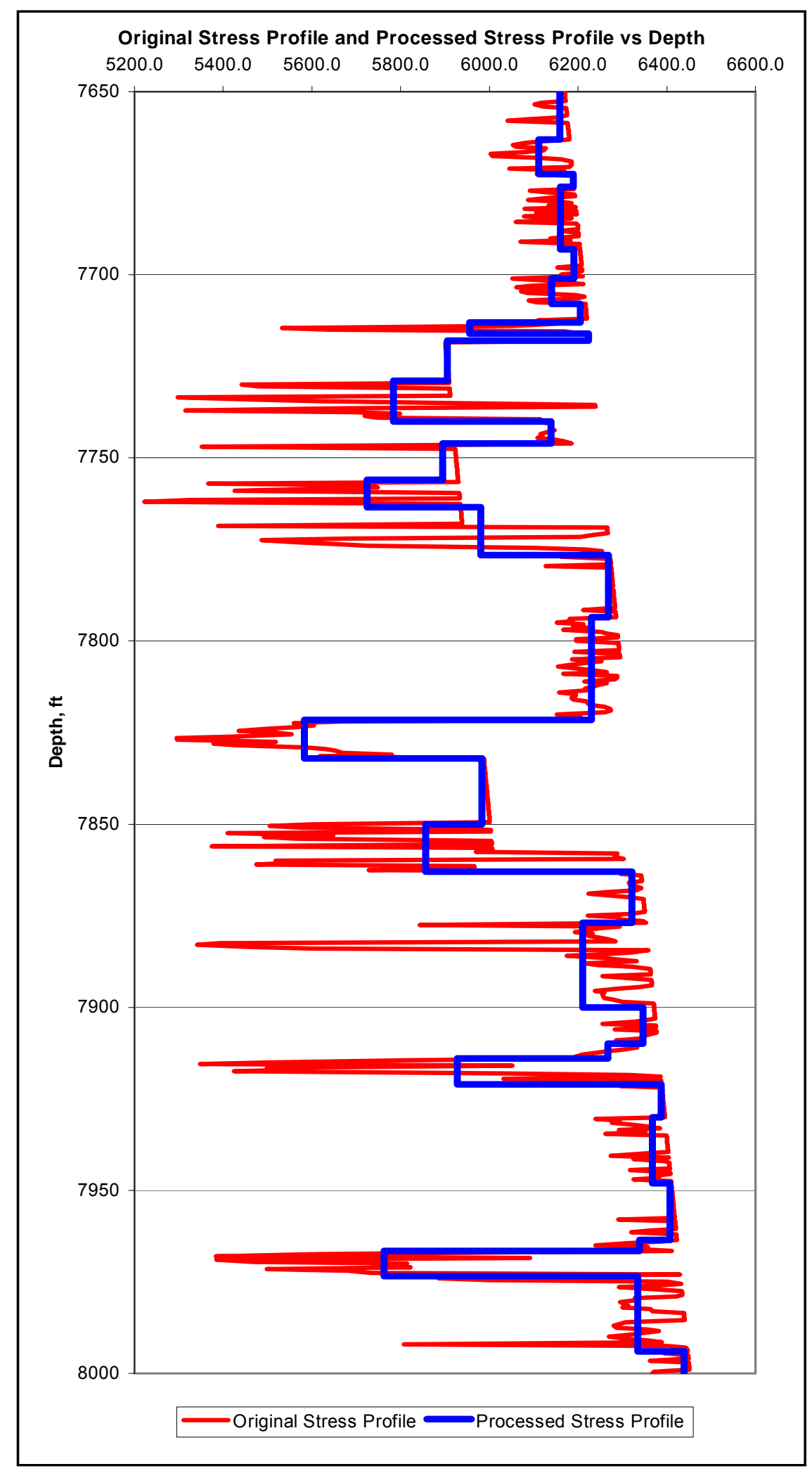

Figure 5-6: Original Stress Profile and Processed Stress Profile 
The final processed stress profile consists of 32 layers and is displayed in Table 5-6. Column two shows the depth interval of each layer while column three gives the average closure stress values of each layer.

\begin{tabular}{|c|c|c|}
\hline Layer Index & $\begin{array}{c}\text { Interval Depth, } \\
\text { ft }\end{array}$ & $\begin{array}{c}\text { Clossure } \\
\text { Stre ss, } \\
\text { psi }\end{array}$ \\
\hline Layer 1 & $7650-7663$ & 6159 \\
\hline Layer 2 & $7663-7672.5$ & 6111 \\
\hline Layer 3 & $7672.5-7676$ & 6189 \\
\hline Layer 4 & $7676-7693$ & 6161 \\
\hline Layer 5 & $7693-7701$ & 6190 \\
\hline Layer 6 & $7701-7708$ & 6140 \\
\hline Layer 7 & $7708-7713$ & 6205 \\
\hline Layer 8 & $7713-7716$ & 5955 \\
\hline Layer 9 & $7716-7718$ & 6224 \\
\hline Layer 10 & $7718-7729$ & 5905 \\
\hline Layer 11 & $7729-7740$ & 5784 \\
\hline Layer 12 & $7740-7746$ & 6139 \\
\hline Layer 13 & $7746-7756$ & 5895 \\
\hline Layer 14 & $7756-7763.5$ & 5724 \\
\hline Layer 15 & $7763.5-7776.5$ & 5980 \\
\hline Layer 16 & $7776.5-7793.5$ & 6269 \\
\hline Layer 17 & $7793.5-7821.5$ & 6230 \\
\hline Layer 18 & $7821.5-7832$ & 5584 \\
\hline Layer 19 & $7832-7850$ & 5984 \\
\hline Layer 20 & $7850-7863$ & 5856 \\
\hline Layer 21 & $7863-7877$ & 6321 \\
\hline Layer 22 & $7877-7900$ & 6211 \\
\hline Layer 23 & $7900-7910$ & 6347 \\
\hline Layer 24 & $7910-7914$ & 6267 \\
\hline Layer 25 & $7914-7921$ & 5928 \\
\hline Layer 26 & $7921-7930$ & 6387 \\
\hline Layer 27 & $7930-7948$ & 6367 \\
\hline Layer 28 & $7948-7963.5$ & 6407 \\
\hline Layer 29 & $7963.5-7966.5$ & 6339 \\
\hline Layer 30 & $7966.5-7973.5$ & 5763 \\
\hline Layer 31 & $7973.5-7994$ & 6334 \\
\hline Layer 32 & $7994-8000$ & 6438 \\
\hline
\end{tabular}

Table 5-6: Example of Stress Profile Processing for Four Layers

In Figure 5-7, the equivalent four-layer stress profile concludes the first module covering reservoir characterization for fracturing simulation. The equivalent four-layer profile is the main input to the optimization module and the net pressure prediction module. 


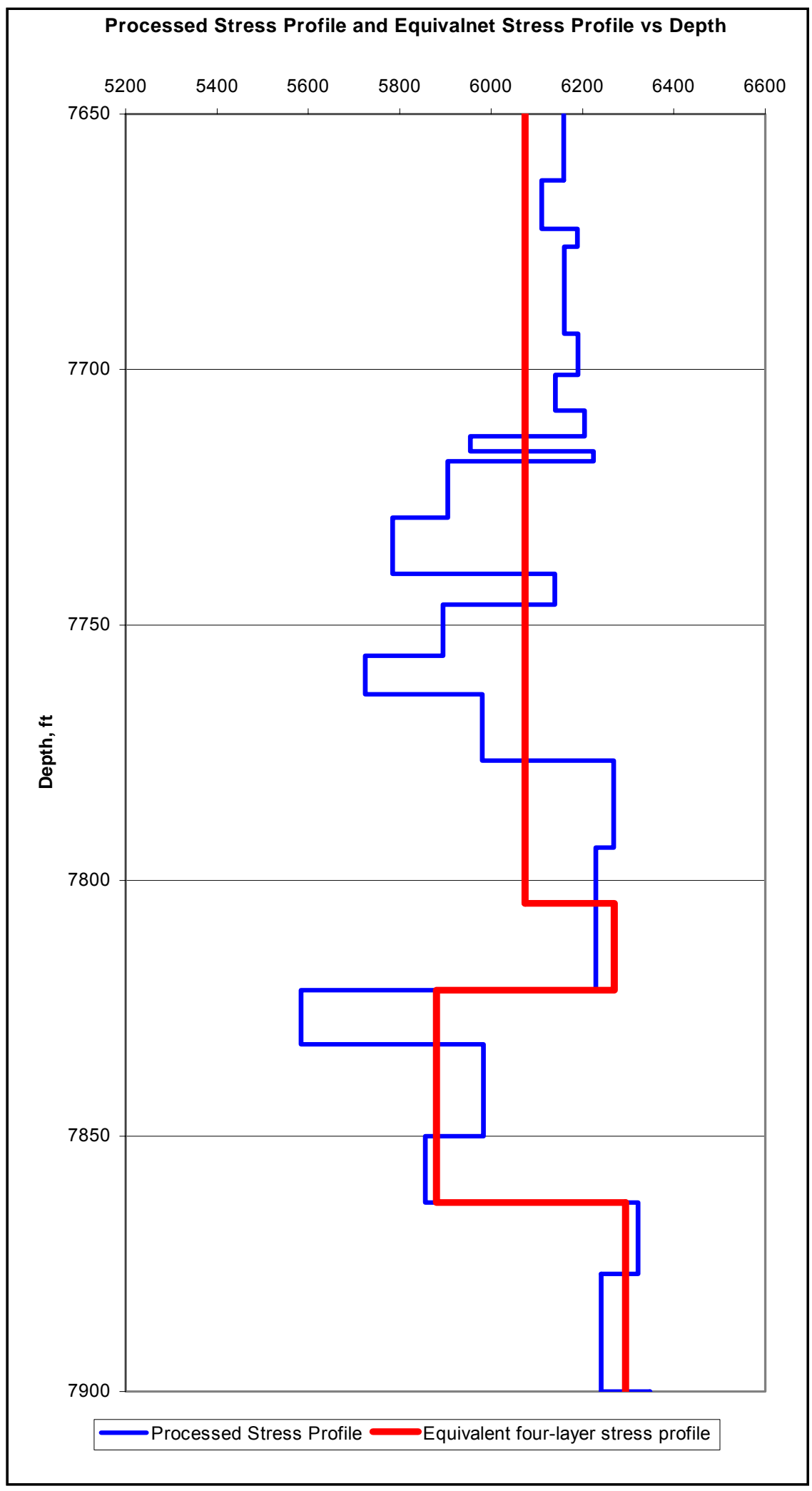

Figure 5-7: Processed Stress Profile and Equivalent Four-Layer Stress Profile 


\subsection{Neural Network Fracturing Model Accuracy}

Two types of fracture treatment design were developed in this study in order to demonstrate the capability of the tool while comprehensively addressing all possible variations. These are the ramp treatment design and stage treatment design. The total number of five neural networks was trained as follows:

- One neural net for ramp treatment design

- One neural network for six stage treatment design

- Three neural networks for three types of eight stage treatment design

The criteria for an acceptable accurate neural network were measured using $\mathrm{R}^{2}$ and the correlation coefficient. Values higher than 0.8 for $R^{2}$ are considered acceptable, while values greater than 0.9 for correlation coefficient show a good agreement in trend between actual values and predicted. In an ideal case, $\mathrm{R}^{2}$ and correlation coefficient are equal to 1 , where the actual values and the predicted values would fall on the 45-degree line. This study illustrates the performance of the neural network, by the closeness of points to the $45^{\circ}$ line.

\subsubsection{Neural Network for Ramp Treatment}

The architecture of the neural network for ramp treatment has three hidden layers with different activation functions, 14 inputs and 6 outputs as described in detail in 4.12.1 Developing Neural Networks for Ramp Treatments. Figure 5-8 through Figure 5-25 show the correlation between the actual FRACPRO values and the neural network predicted values of the training set, calibration set, and verification set for each of the 6 output parameters (fracture efficiency, propped fracture length, proppant concentration, dimensionless conductivity ratio, max fracture width, and fracture height). 
Figure 5-8 shows the training set results for fracture efficiency. $R^{2}$ is $0.89, r^{2}$ is 0.89 , and the correlation coefficient is 0.94 .

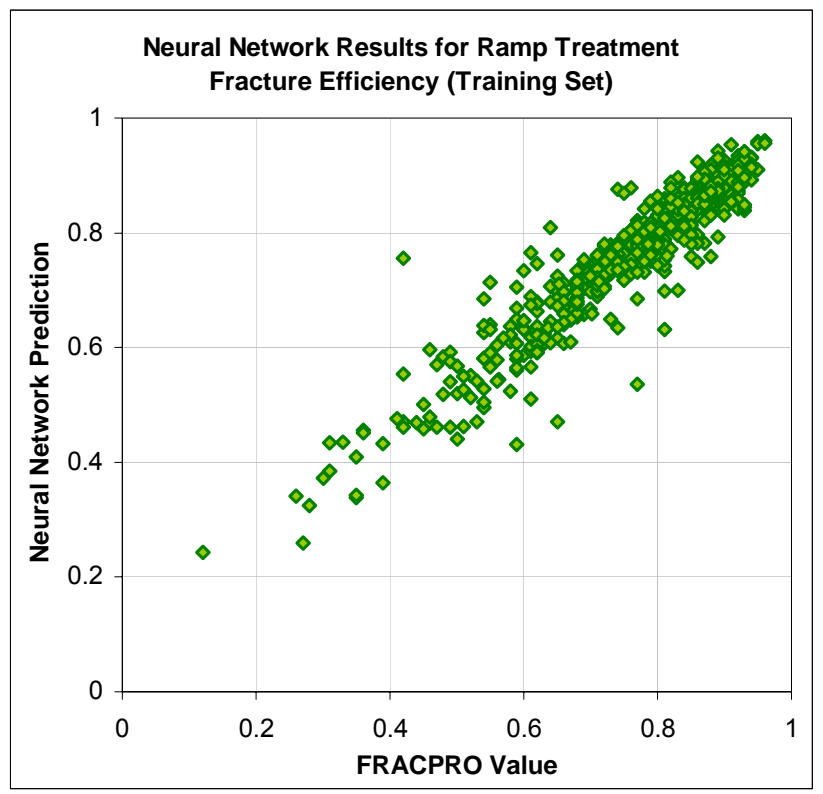

Figure 5-8: Ramp Treatment Training Set Results for Fracture Efficiency

Figure $5-9$ shows the calibration set results for fracture efficiency. $R^{2}$ is $0.83, r^{2}$ is 0.83 , and the correlation coefficient is 0.91 .

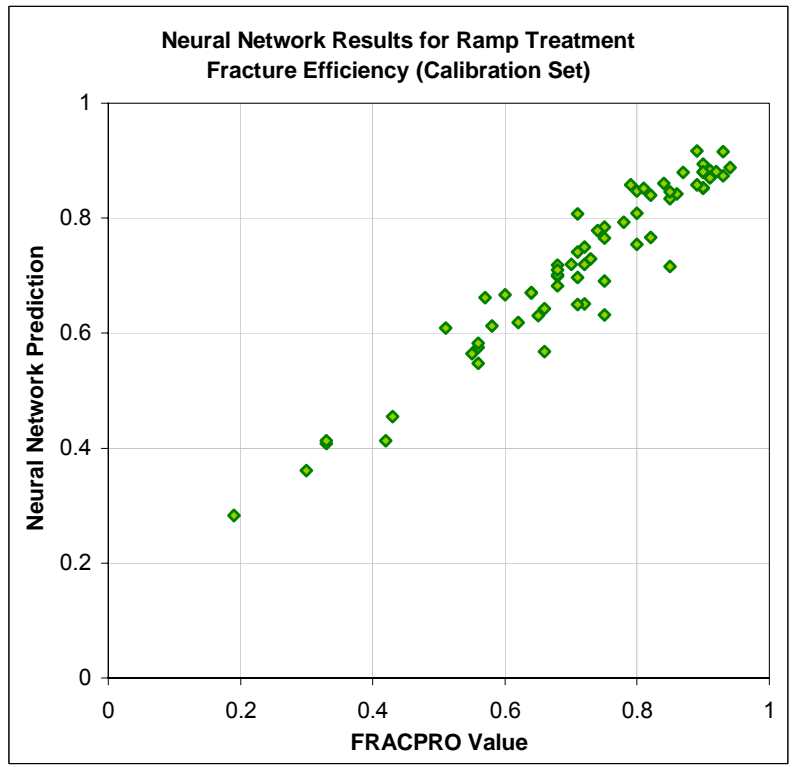

Figure 5-9: Ramp Treatment Calibration Set Results for Fracture Efficiency 
Figure $5-10$ shows the verification set results for fracture efficiency. $R^{2}$ is $0.91, r^{2}$ is 0.92 , and the correlation coefficient is 0.96 .

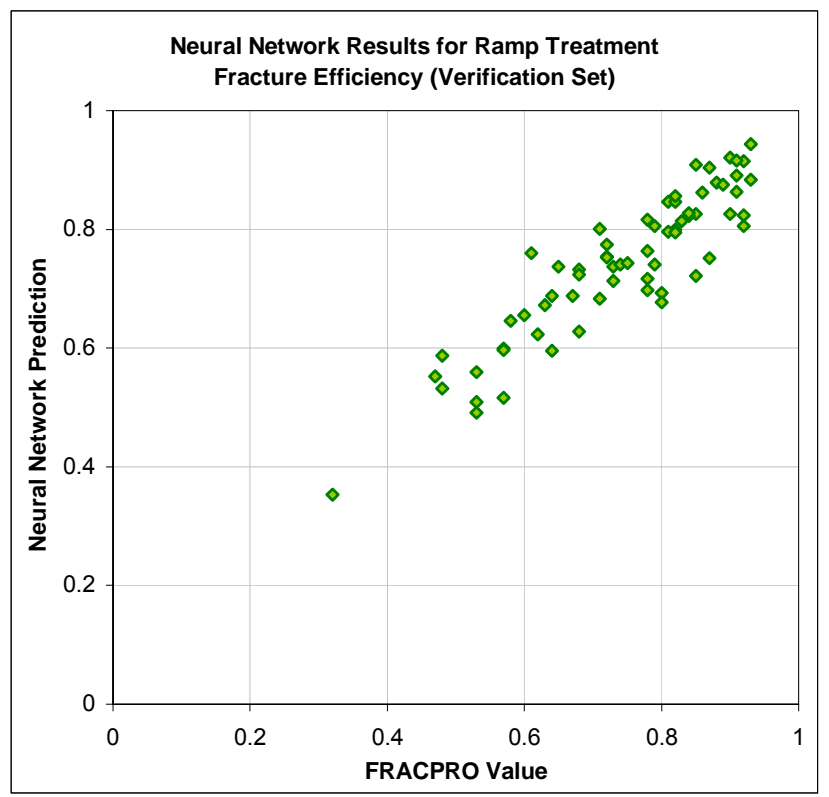

Figure 5-10: Ramp Treatment Verification Set Results for Fracture Efficiency

Figure 5-11 shows the training set results for propped fracture length. $R^{2}$ is $0.92, r^{2}$ is 0.92 , and the correlation coefficient is 0.96 .

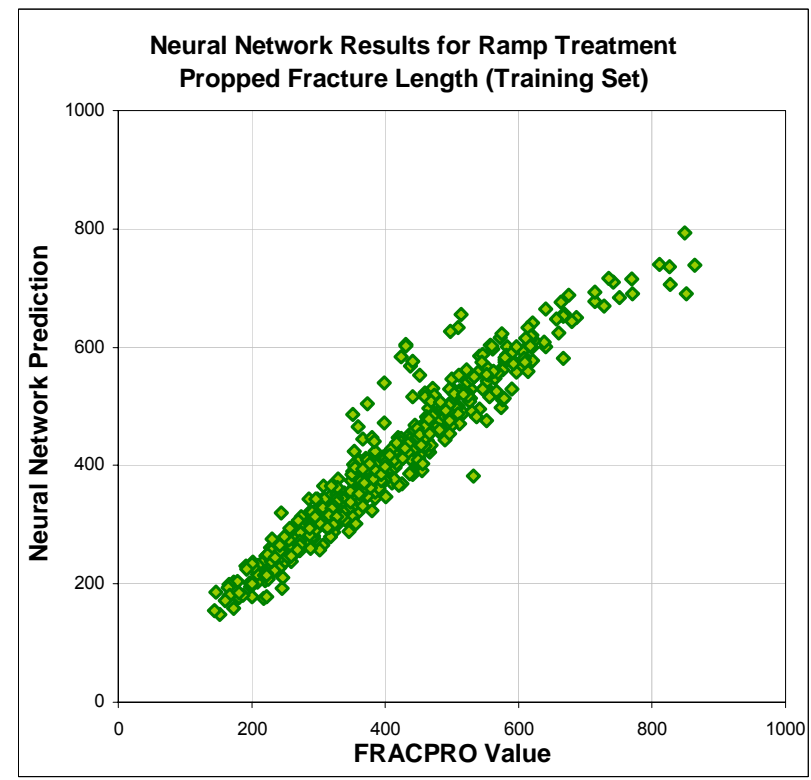

Figure 5-11: Ramp Treatment Training Set Results for Propped Fracture Length 
Figure $5-12$ shows the calibration set results for propped fracture length. $R^{2}$ is $0.90, r^{2}$ is 0.91 , and the correlation coefficient is 0.95 .

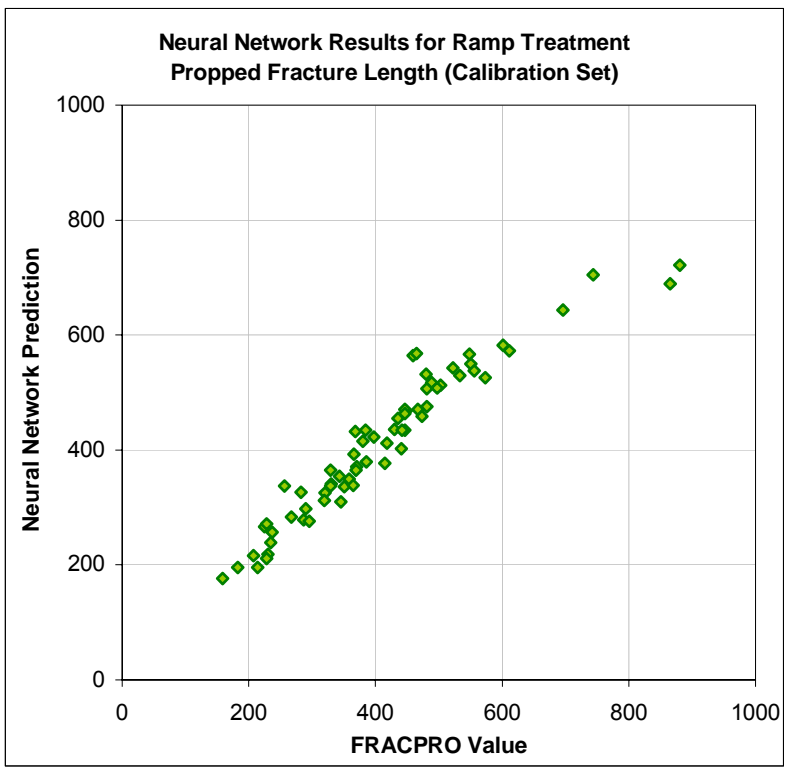

Figure 5-12: Ramp Treatment Calibration Set Results for Propped Fracture Length

Figure $5-13$ shows the verification set results for propped fracture length. $R^{2}$ is $0.90, r^{2}$ is 0.89 , and the correlation coefficient is 0.94 .

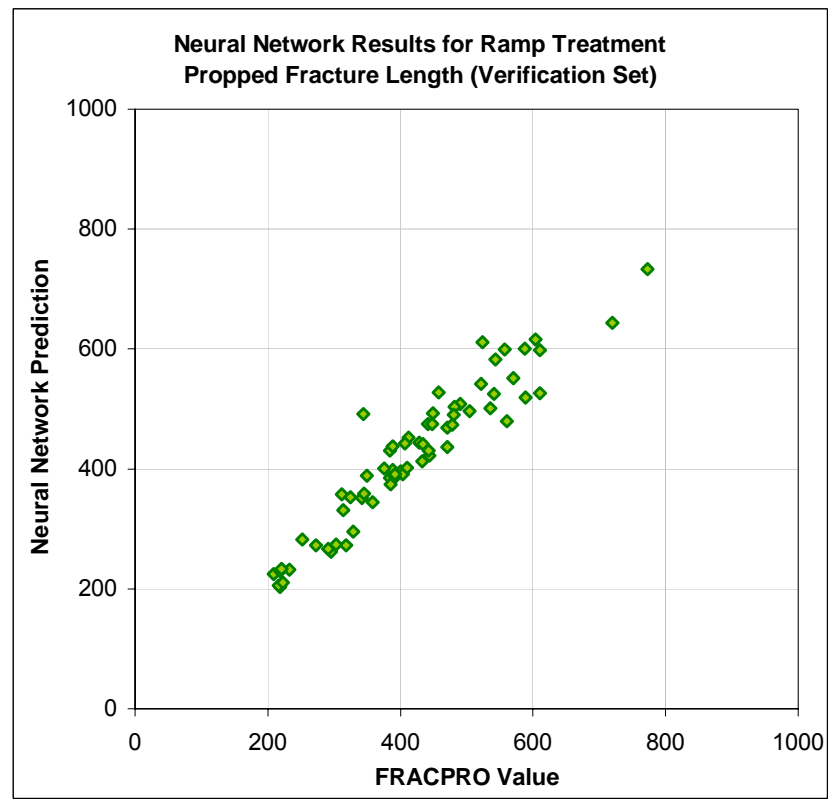

Figure 5-13: Ramp Treatment Verification Set Results for Propped Fracture Length 
Figure 5-14 shows the training set results for proppant concentration. $R^{2}$ is $0.85, r^{2}$ is 0.85 , and the correlation coefficient is 0.92 .

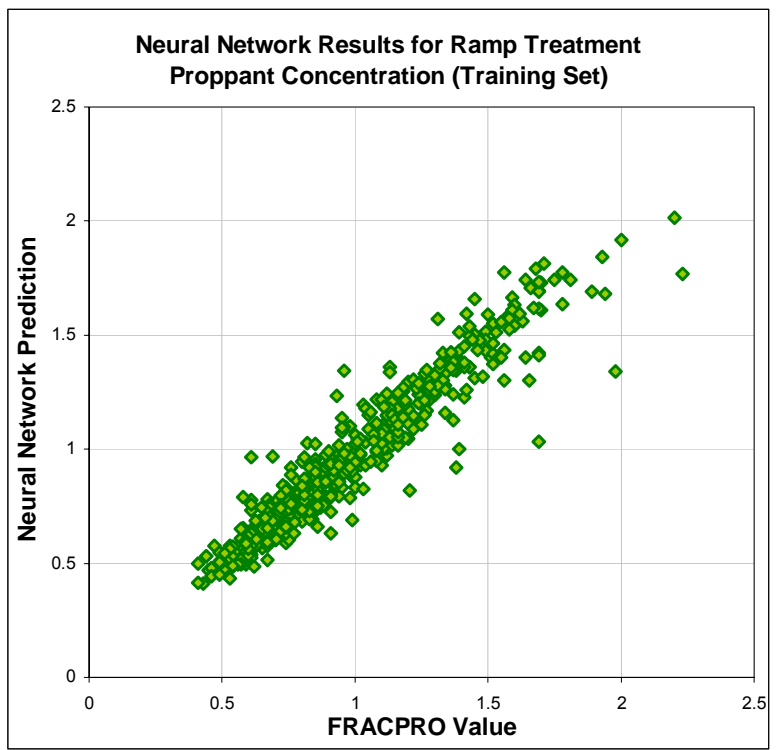

Figure 5-14: Ramp Treatment Training Set Results for Proppant Concentration

Figure $5-15$ shows the calibration set results for proppant concentration. $R^{2}$ is $0.78, r^{2}$ is 0.79 , and the correlation coefficient is 0.89 .

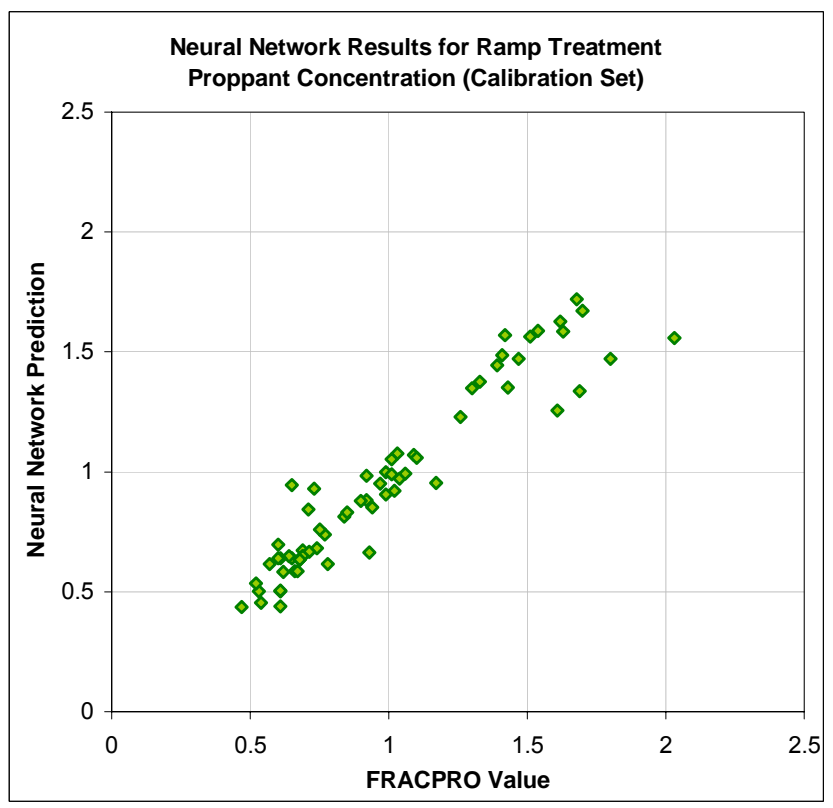

Figure 5-15: Ramp Treatment Calibration Set Results for Proppant Concentration 
Figure 5-16 shows the verification set results for proppant concentration. $R^{2}$ is $0.83, r^{2}$ is 0.84 , and the correlation coefficient is 0.92 .

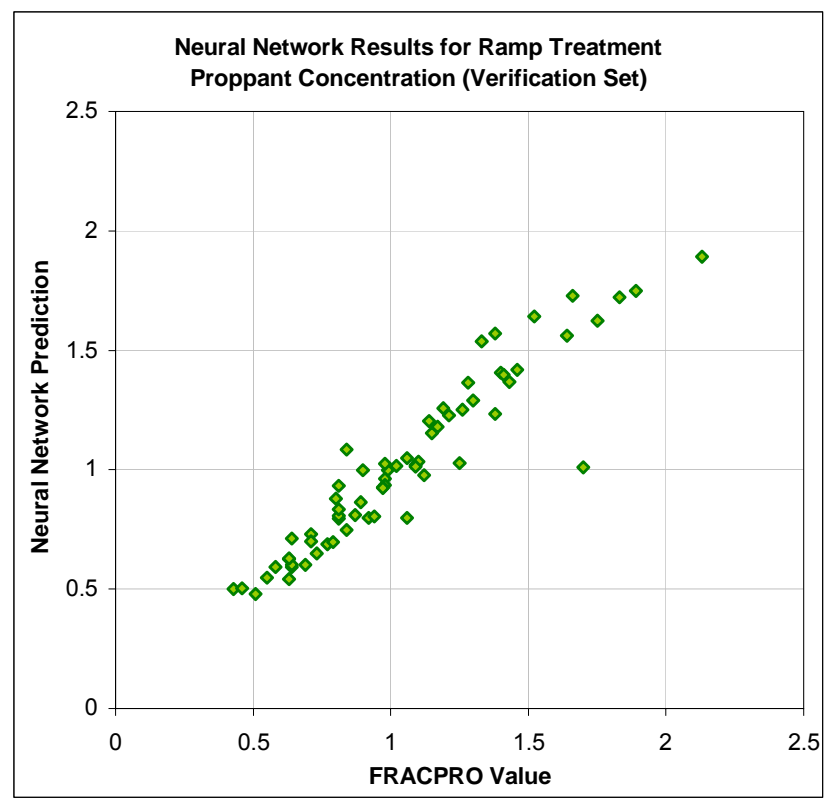

Figure 5-16: Ramp Treatment Verification Set Results for Proppant Concentration

Figure $5-17$ shows the training set results for dimensionless conductivity ratio. $R^{2}$ is 0.87 , $r^{2}$ is 0.88 , and the correlation coefficient is 0.94 .

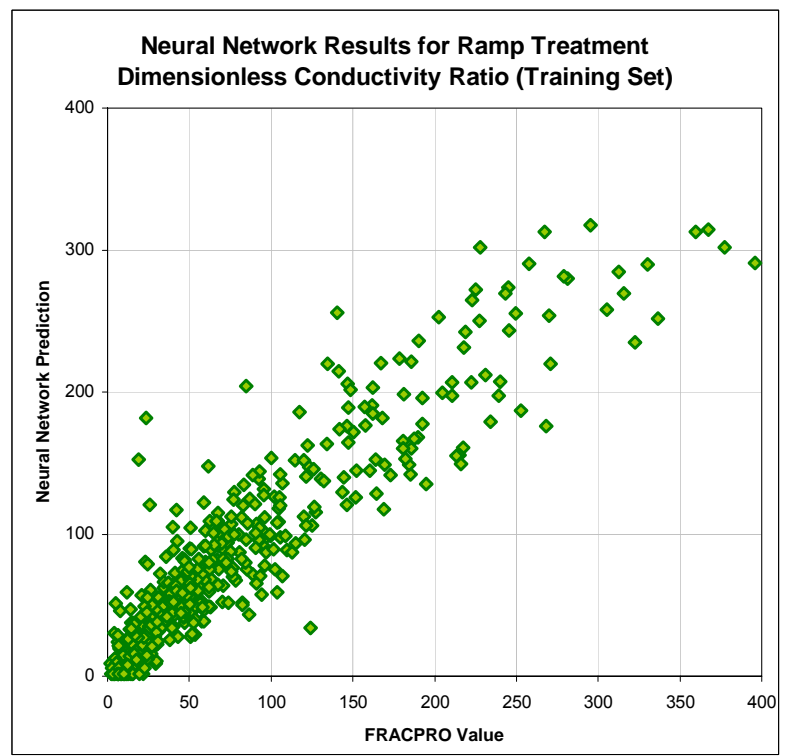

Figure 5-17: Ramp Treatment Training Set Results for Dimensionless Conductivity Ratio 
Figure 5-18 shows the calibration set results for dimensionless conductivity ratio. $R^{2}$ is $0.83, r^{2}$ is 0.83 , and the correlation coefficient is 0.91 .

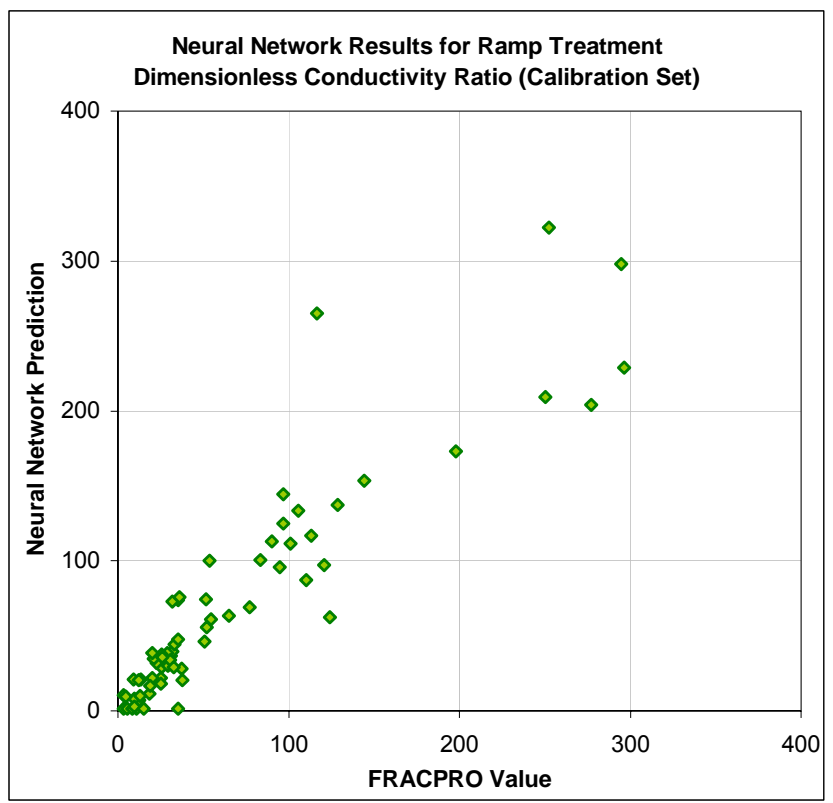

Figure 5-18: Ramp Treatment Calibration Set Results for Dimensionless Conductivity Ratio

Figure $5-19$ shows the verification set results for dimensionless conductivity ratio. $R^{2}$ is $0.87, r^{2}$ is 0.87 , and the correlation coefficient is 0.93 .

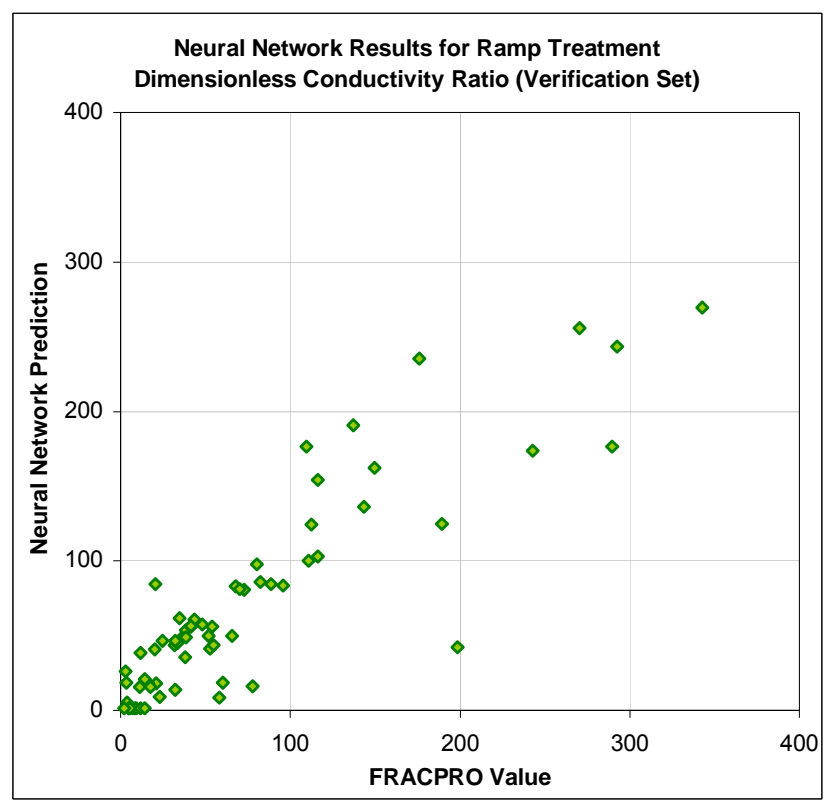

Figure 5-19: Ramp Treatment Verification Set Results for Dimensionless Conductivity Ratio 
Figure $5-20$ shows the training set results for maximum fracture width. $R^{2}$ is $0.95, r^{2}$ is 0.95 , and the correlation coefficient is 0.97 .

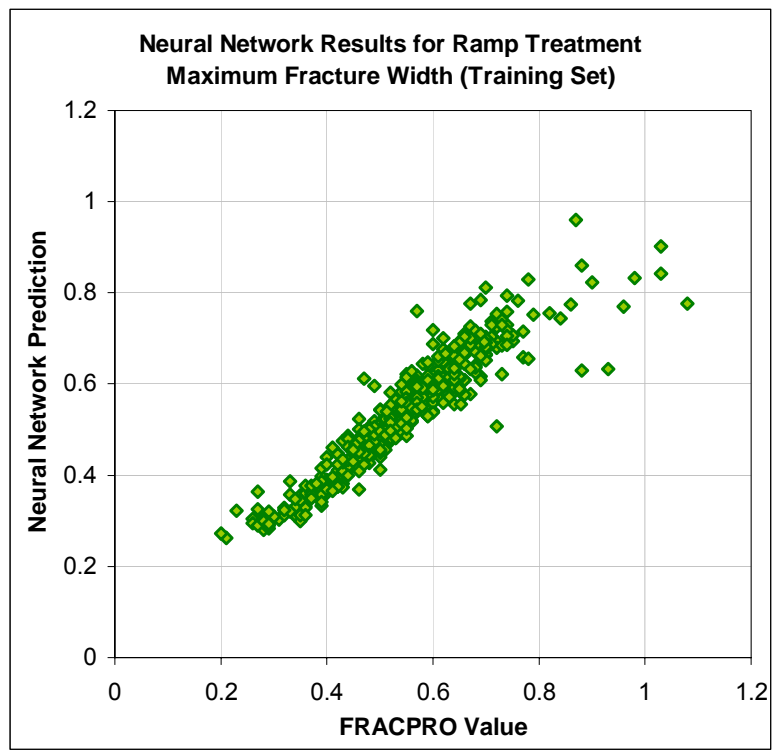

Figure 5-20: Ramp Treatment Training Set Results for Maximum Fracture Width

Figure 5-21 shows the calibration set results for maximum fracture width. $R^{2}$ is $0.95, r^{2}$ is 0.95 , and the correlation coefficient is 0.97 .

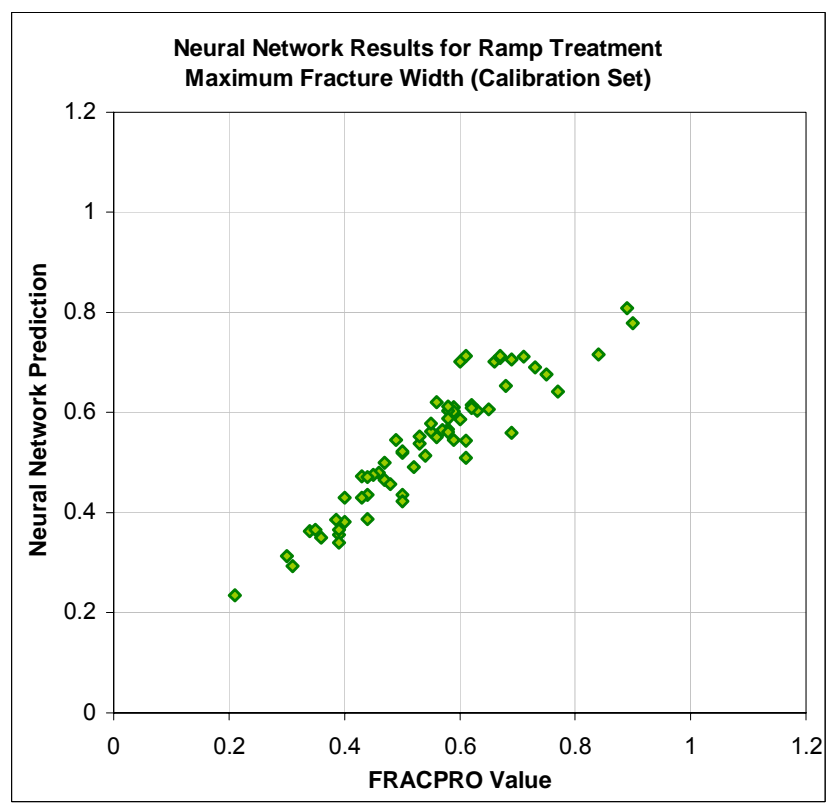

Figure 5-21: Ramp Treatment Calibration Set Results for Maximum Fracture Width 
Figure 5-22 shows the verification set results for maximum fracture length. $R^{2}$ is $0.95, r^{2}$ is 0.95 , and the correlation coefficient is 0.98 .

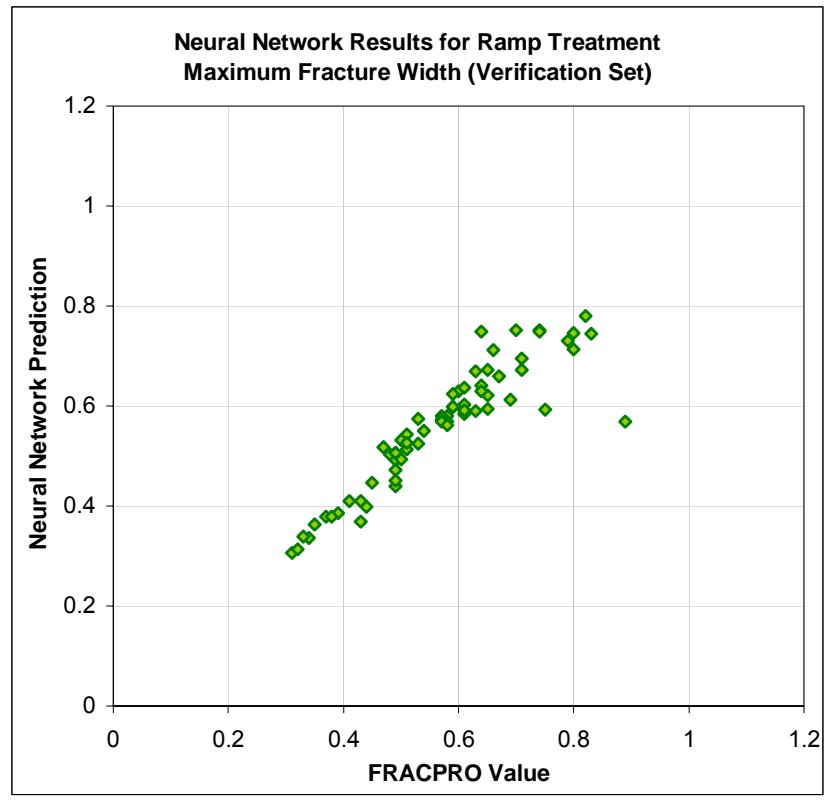

Figure 5-22: Ramp Treatment Verification Set Results for Maximum Fracture Width

Figure $5-23$ shows the training set results for fracture height. $R^{2}$ is $0.91, r^{2}$ is 0.91 , and the correlation coefficient is 0.95 .

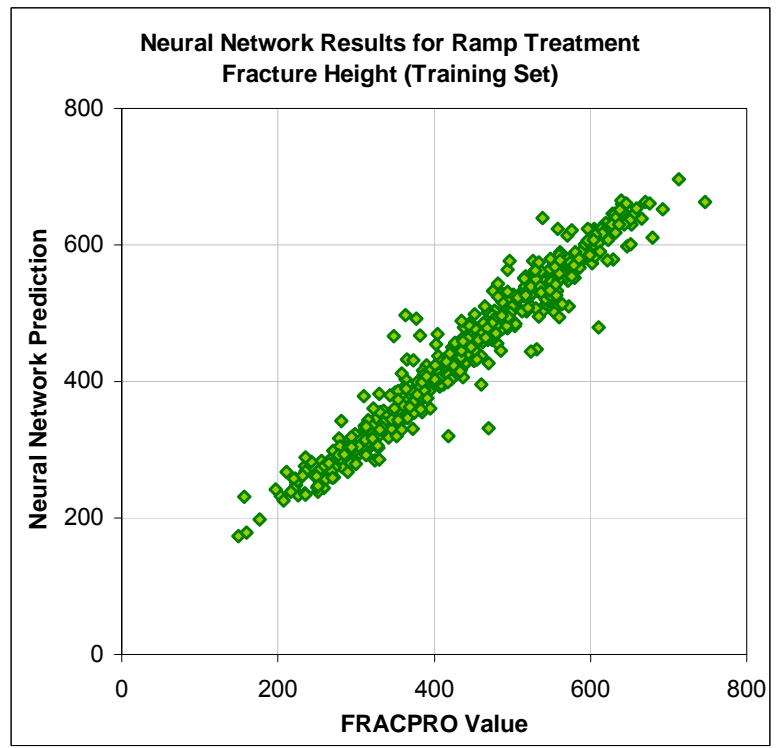

Figure 5-23: Ramp Treatment Training Set Results for Fracture Height 
Figure 5-24 shows the calibration set results for fracture height. $R^{2}$ is $0.88, r^{2}$ is 0.89 , and the correlation coefficient is 0.94 .

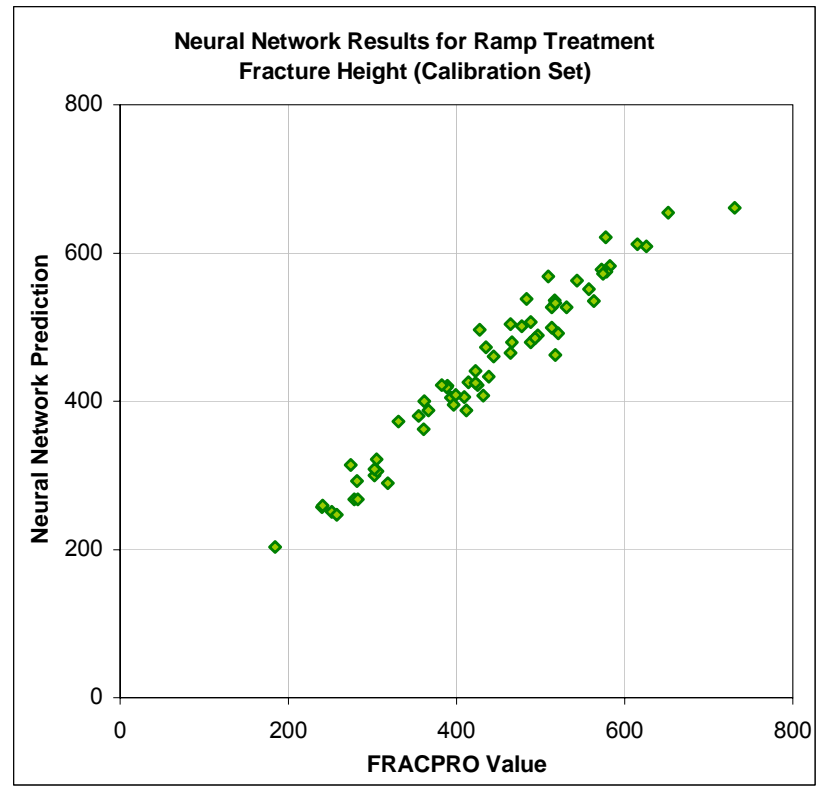

Figure 5-24: Ramp Treatment Calibration Set Results for Fracture Height

Figure 5-25 shows the verification set results for fracture height. $R^{2}$ is $0.89, r^{2}$ is 0.90 , and the correlation coefficient is 0.95 .

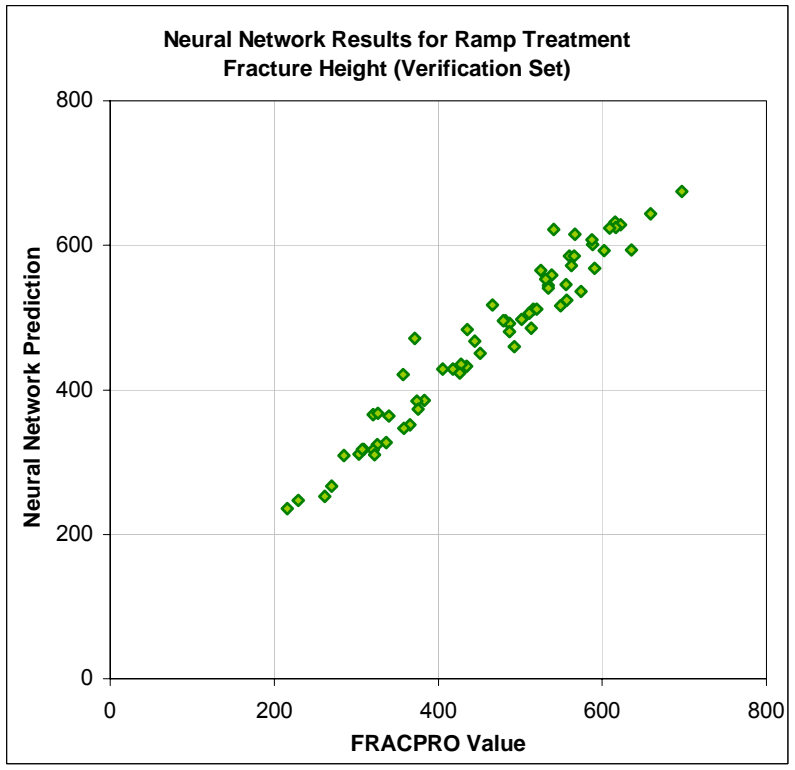

Figure 5-25: Ramp Treatment Verification Set Results for Fracture Height 
The details of the results for each of the six output parameters in the training, calibration, and verification sets can be seen in Table 5-7.

\begin{tabular}{|l|c|c|c|c|c|c|}
\hline \multicolumn{2}{|c|}{$\begin{array}{c}\text { Fracture } \\
\text { Efficiency }\end{array}$} & $\begin{array}{c}\text { Propped } \\
\text { Fracture Length }\end{array}$ & $\begin{array}{c}\text { Proppant } \\
\text { Concentration }\end{array}$ & $\begin{array}{c}\text { Dimensionless } \\
\text { Conductivity } \\
\text { Ratio }\end{array}$ & $\begin{array}{c}\text { Max Fracture } \\
\text { Width }\end{array}$ \\
Fracture Height
\end{tabular}

Table 5-7: Ramp Treatment Neural Network Results

Fracture length and fracture width exhibit the best performance in prediction. This in excellent, particularly since this study addresses tight gas formations where the design optimization criteria is fracture length. Fracture height and fracture efficiency closely follow with regards to performance. Proppant concentration and dimensionless conductivity ratio show a little lower performance than fracture length; however, both have strong correlation coefficients and values above 0.8 for $R^{2}$. As seen in the previous figures for fracture length, fracture height, and fracture width, there is good agreement between the points and the $45^{\circ}$ line.

The training and calibration sets for dimensionless conductivity ratio, Figure 5-17 and Figure 5-18, show sparser values around the $45^{\circ}$ line, compared to other parameters, while the verification set shows poor prediction performance at high values (Figure 5-19). However, current performance was the best achieved and was used in the optimization module.

\subsubsection{Neural Network for Six Stage Treatment}

The architecture of the neural network for six stage treatments has three hidden layers with different activation functions, 17 inputs and 6 outputs as described in detail in 4.12.2.1 The Six Stage Treatment. Figure 5-26 through Figure 5-43 show the correlation between the actual FRACPRO values and the neural network predicted values of the training set, calibration set, and verification set for each of the 6 output parameters (fracture efficiency, propped fracture length, proppant concentration, dimensionless conductivity ratio, max fracture width, and fracture height). 
Figure 5-26 shows the training set results for fracture efficiency. $R^{2}$ is $0.84, r^{2}$ is 0.86 , and the correlation coefficient is 0.93 .

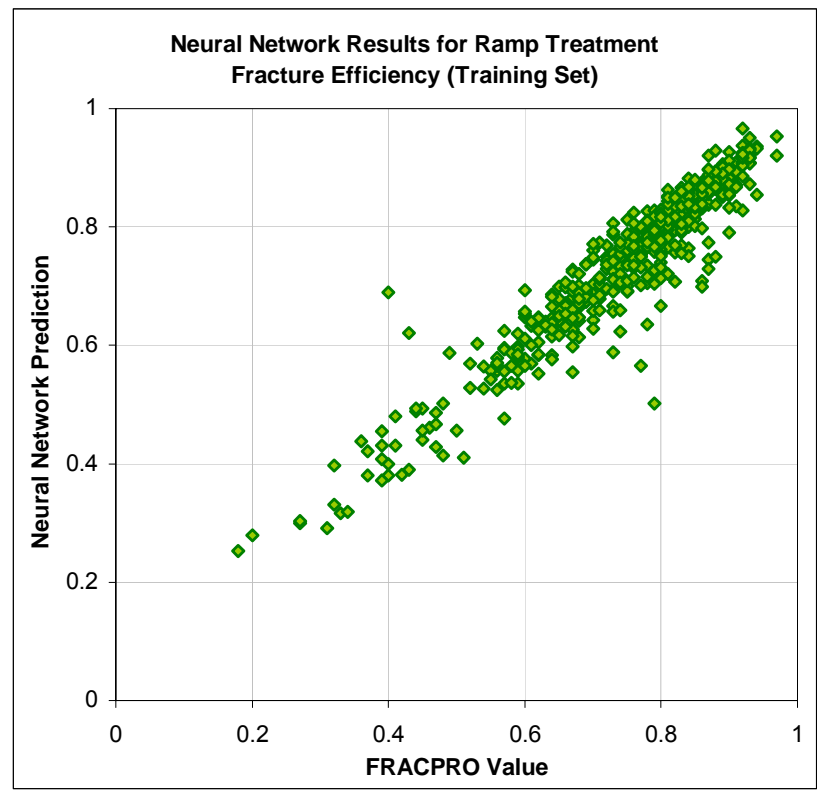

Figure 5-26: Six Stage Treatment Training Set Results for Fracture Efficiency

Figure 5-27 shows the calibration set results for fracture efficiency. $R^{2}$ is $0.81, r^{2}$ is 0.82 , and the correlation coefficient is 0.90 .

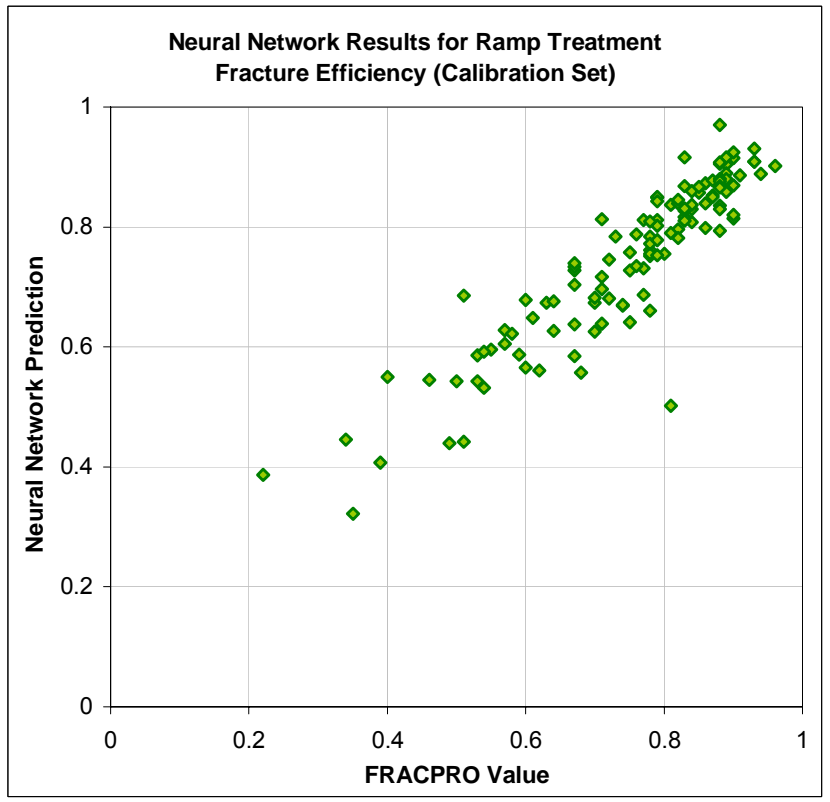

Figure 5-27: Six Stage Treatment Calibration Set Results for Fracture Efficiency 
Figure 5-28 shows the verification set results for fracture efficiency. $R^{2}$ is $0.89, r^{2}$ is 0.90 , and the correlation coefficient is 0.95 .

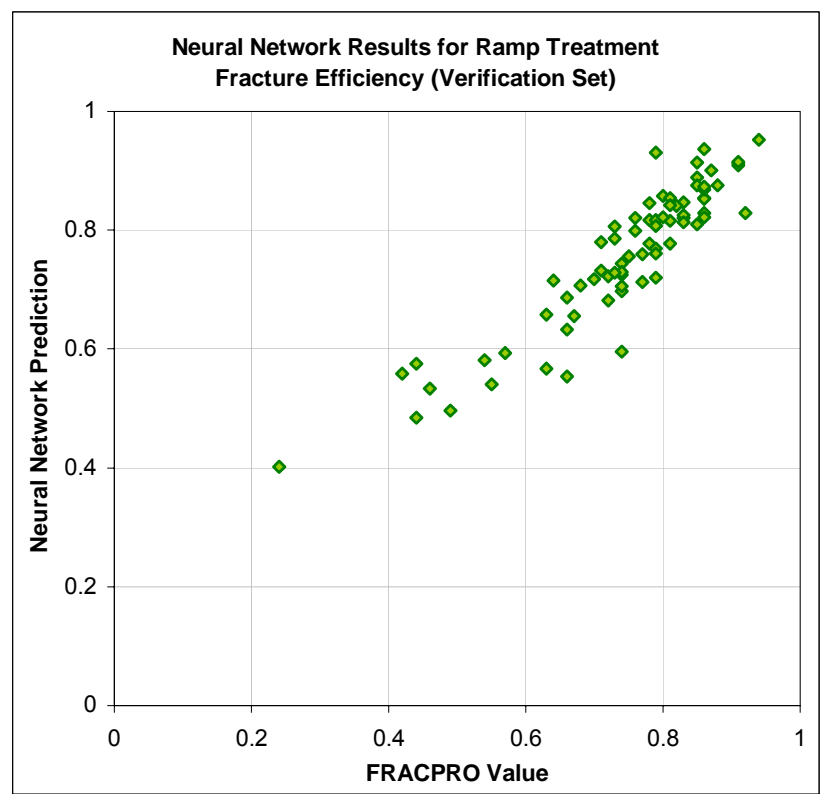

Figure 5-28: Six Stage Treatment Verification Set Results for Fracture Efficiency

Figure $5-29$ shows the training set results for propped fracture length. $R^{2}$ is $0.90, r^{2}$ is 0.89 , and the correlation coefficient is 0.95 .

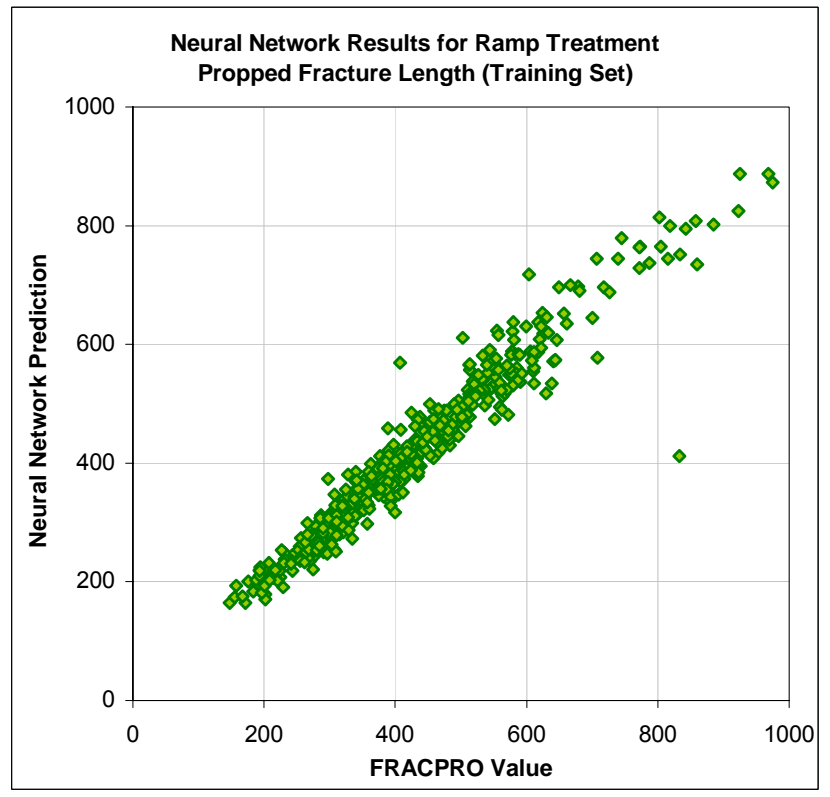

Figure 5-29: Six Stage Treatment Training Set Results for Propped Fracture Length 
Figure 5-30 shows the calibration set results for propped fracture length. $R^{2}$ is $0.87, r^{2}$ is 0.87 , and the correlation coefficient is 0.93 .

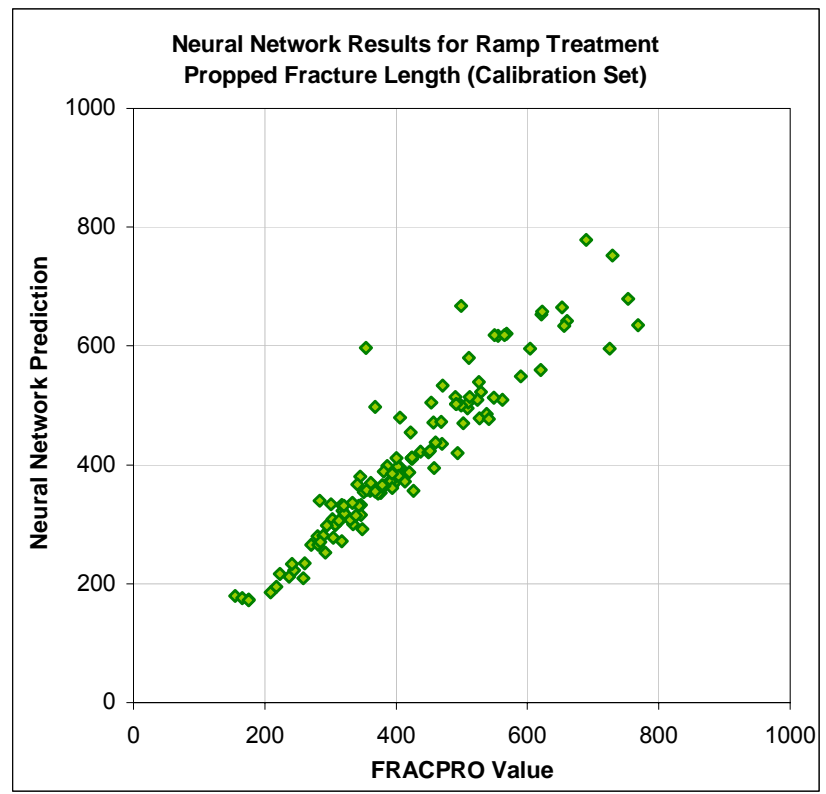

Figure 5-30: Six Stage Treatment Calibration Set Results for Propped Fracture Length

Figure 5-31 shows the verification set results for propped fracture length. $R^{2}$ is $0.93, r^{2}$ is 0.93 , and the correlation coefficient is 0.97 .

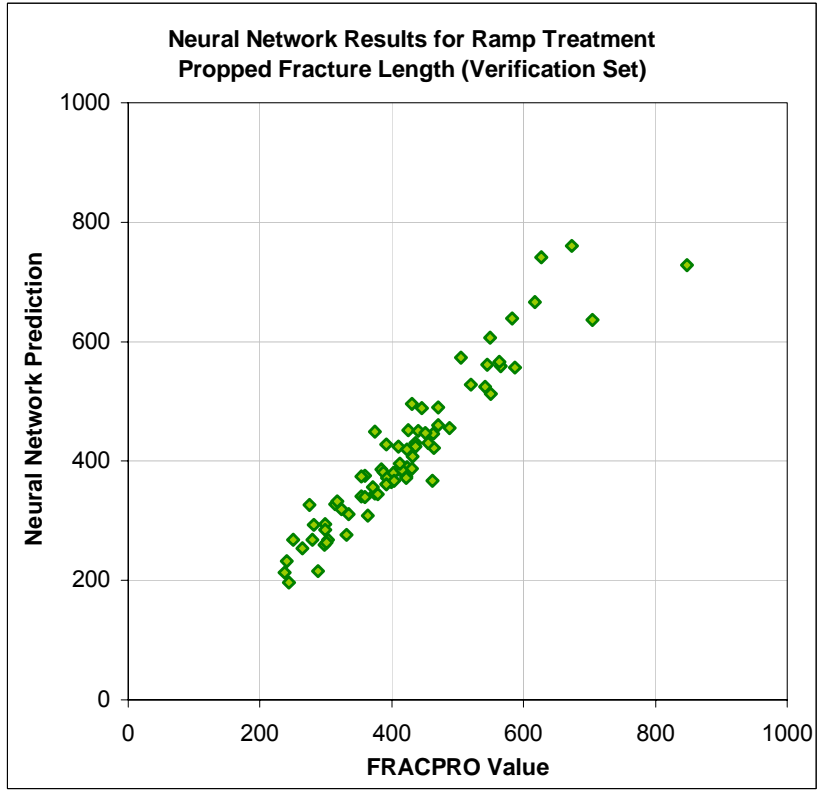

Figure 5-31: Six Stage Treatment Verification Set Results for Propped Fracture Length 
Figure 5-32 shows the training set results for proppant concentration. $R^{2}$ is $0.88, r^{2}$ is 0.89 , and the correlation coefficient is 0.94 .

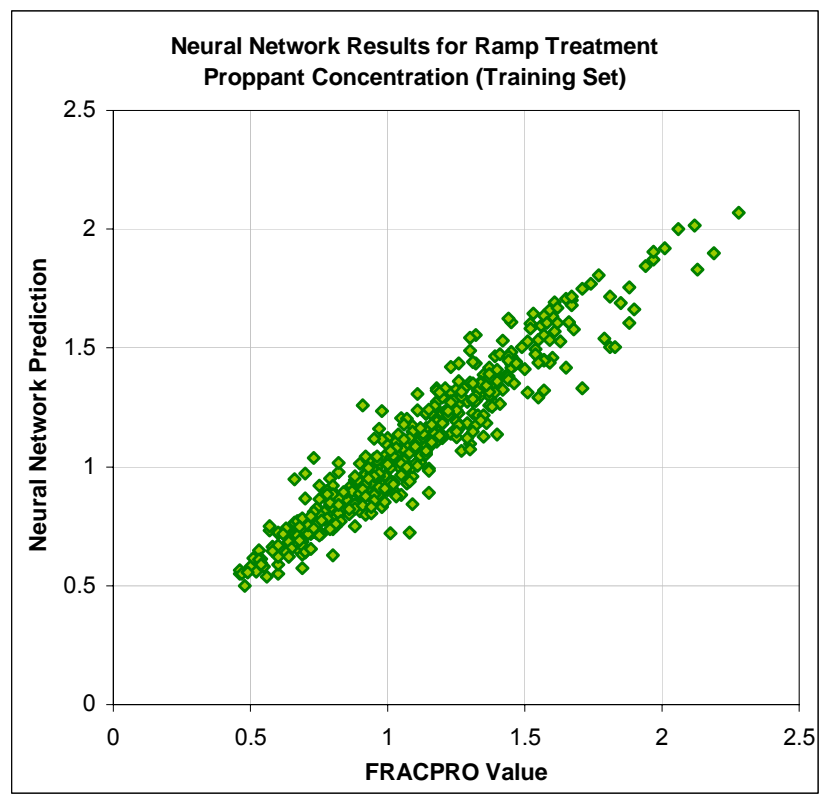

Figure 5-32: Six Stage Treatment Training Set Results for Proppant Concentration

Figure 5-33 shows the calibration set results for proppant concentration. $R^{2}$ is $0.83, r^{2}$ is 0.84 , and the correlation coefficient is 0.92 .

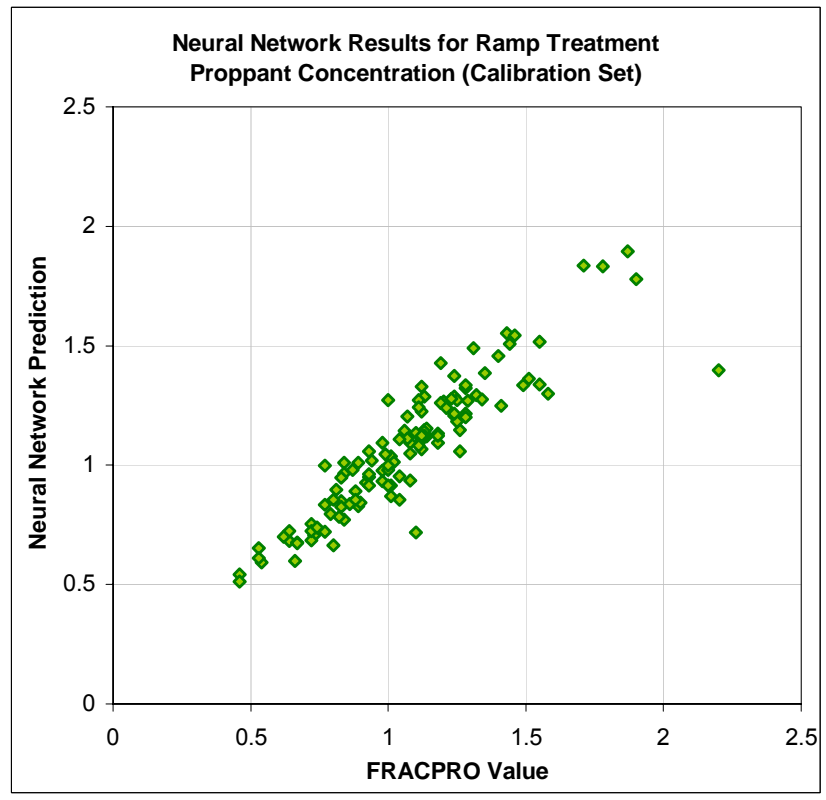

Figure 5-33: Six Stage Treatment Calibration Set Results for Proppant Concentration 
Figure 5-34 shows the verification set results for proppant concentration. $R^{2}$ is $0.89, r^{2}$ is 0.91 , and the correlation coefficient is 0.95 .

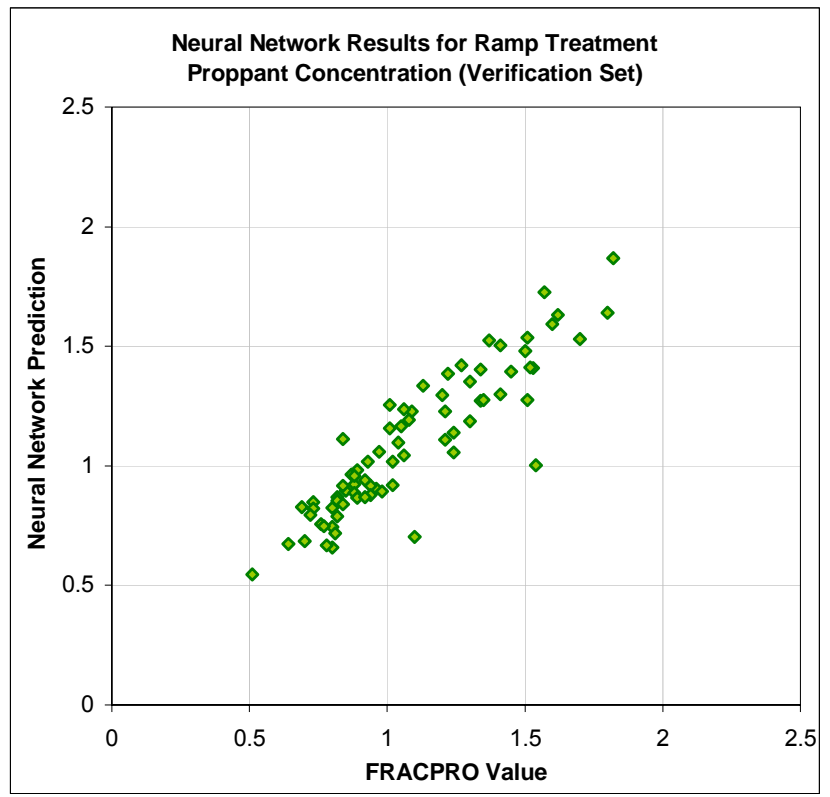

Figure 5-34: Six Stage Treatment Verification Set Results for Proppant Concentration

Figure 5-35 shows the training set results for dimensionless conductivity ratio. $R^{2}$ is 0.80 , $r^{2}$ is 0.81 , and the correlation coefficient is 0.90 .

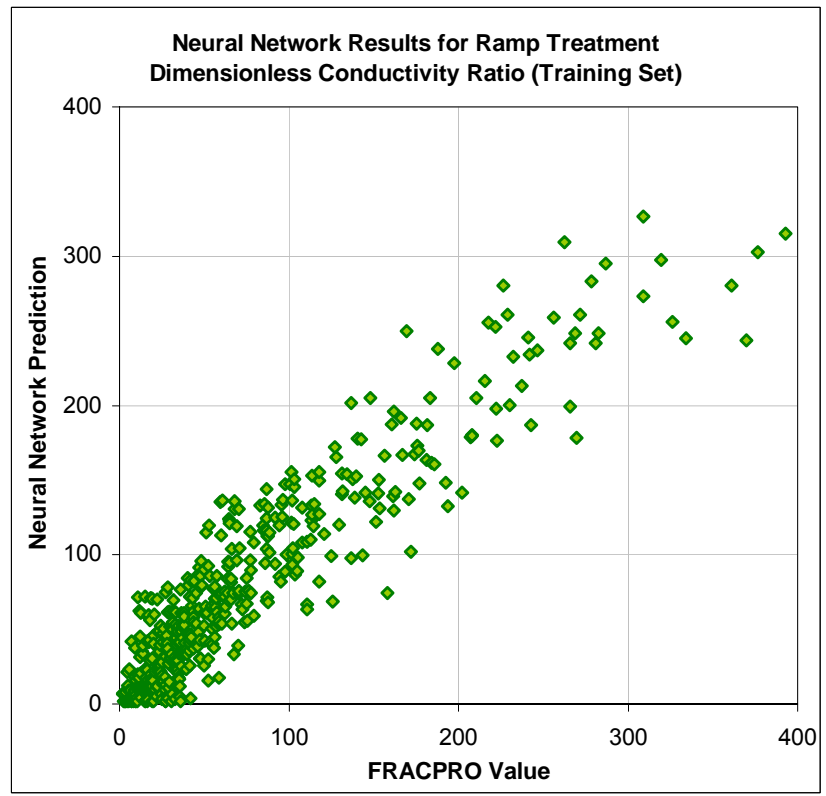

Figure 5-35: Six Stage Treatment Training Set Results for Dimensionless Conductivity Ratio 
Figure 5-36 shows the calibration set results for dimensionless conductivity ratio. $R^{2}$ is $0.83, r^{2}$ is 0.83 , and the correlation coefficient is 0.85 .

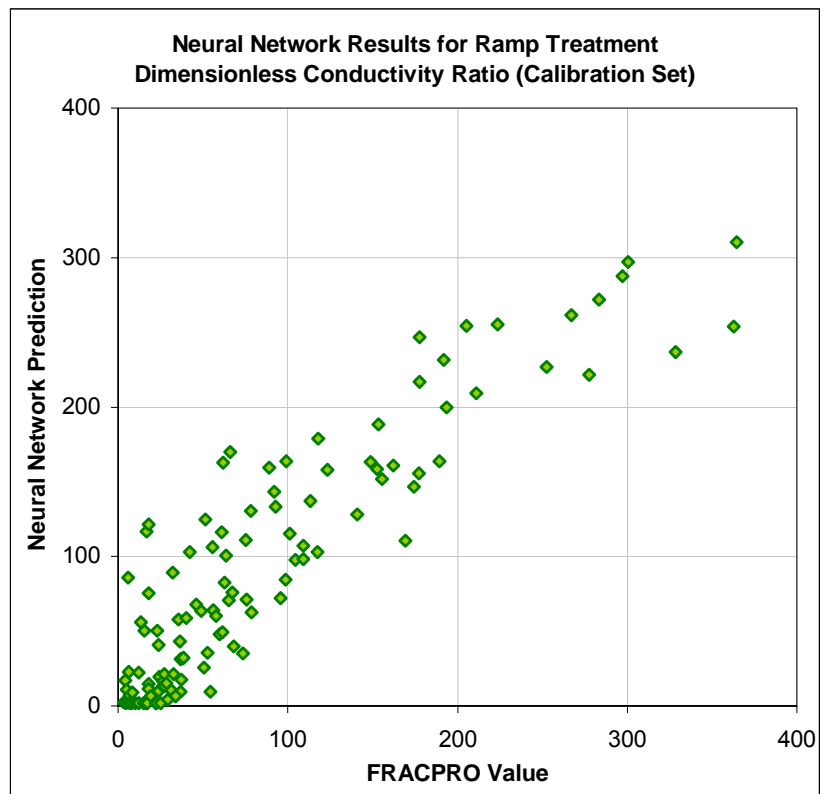

Figure 5-36: Six Stage Treatment Calibration Set Results for Dimensionless Conductivity Ratio

Figure 5-37 shows the verification set results for dimensionless conductivity ratio. $R^{2}$ is $0.80, r^{2}$ is 0.80 , and the correlation coefficient is 0.86 .

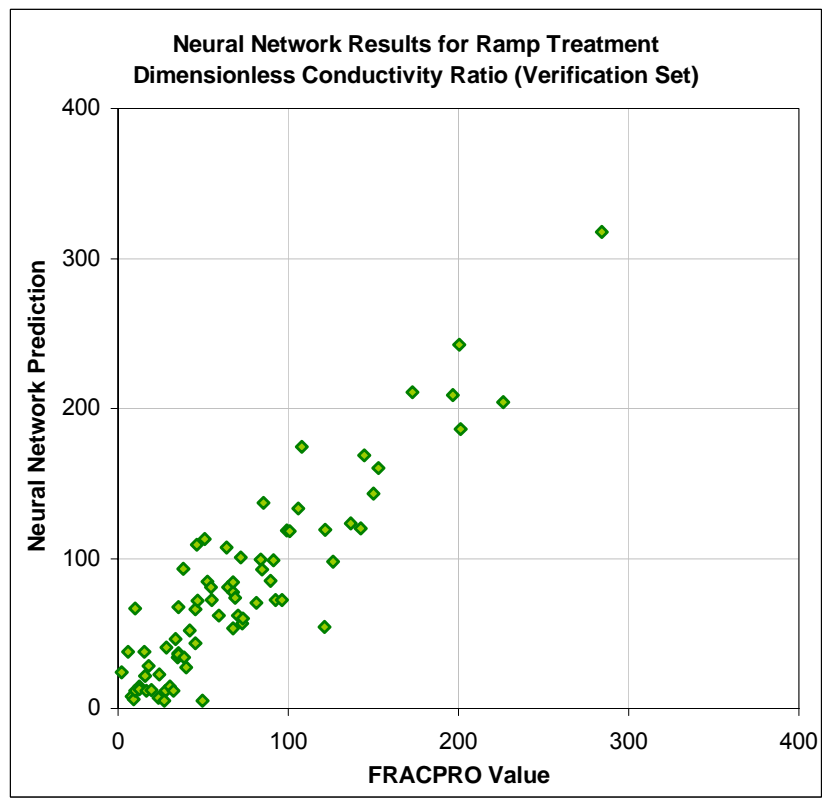

Figure 5-37: Six Stage Treatment Verification Set Results for Dimensionless Conductivity Ratio 
Figure 5-38 shows the training set results for maximum fracture length. $R^{2}$ is $0.84, r^{2}$ is 0.84 , and the correlation coefficient is 0.92 .

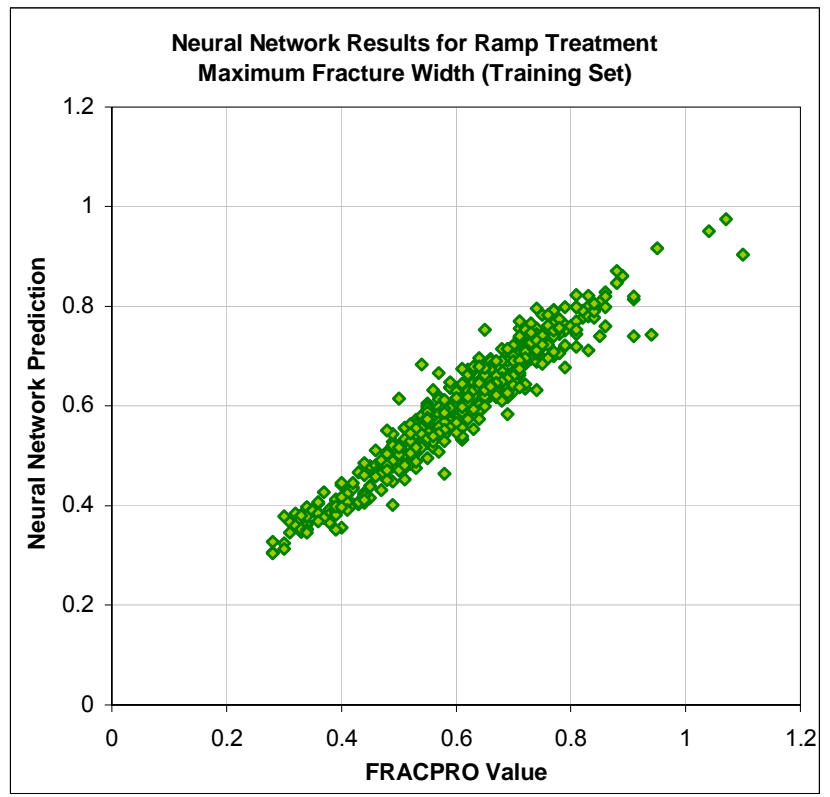

Figure 5-38: Six Stage Treatment Training Set Results for Maximum Fracture Width

Figure 5-39 shows the calibration set results for maximum fracture width. $R^{2}$ is $0.83, r^{2}$ is 0.83 , and the correlation coefficient is 0.91 .

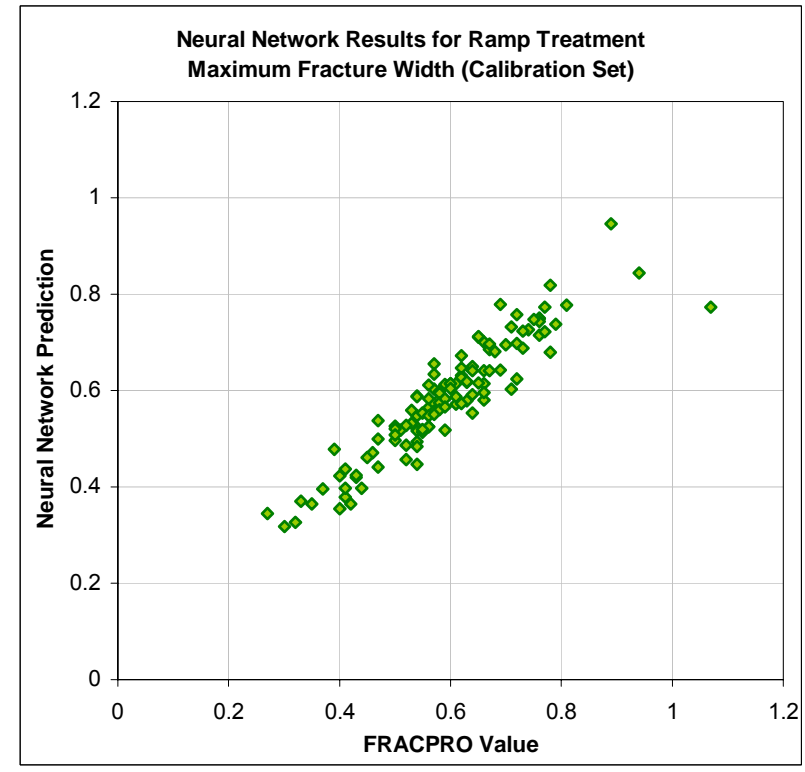

Figure 5-39: Six Stage Treatment Calibration Set Results for Maximum Fracture Width 
Figure 5-40 shows the verification set results for maximum fracture width. $R^{2}$ is $0.85, r^{2}$ is 0.86 , and the correlation coefficient is 0.93 .

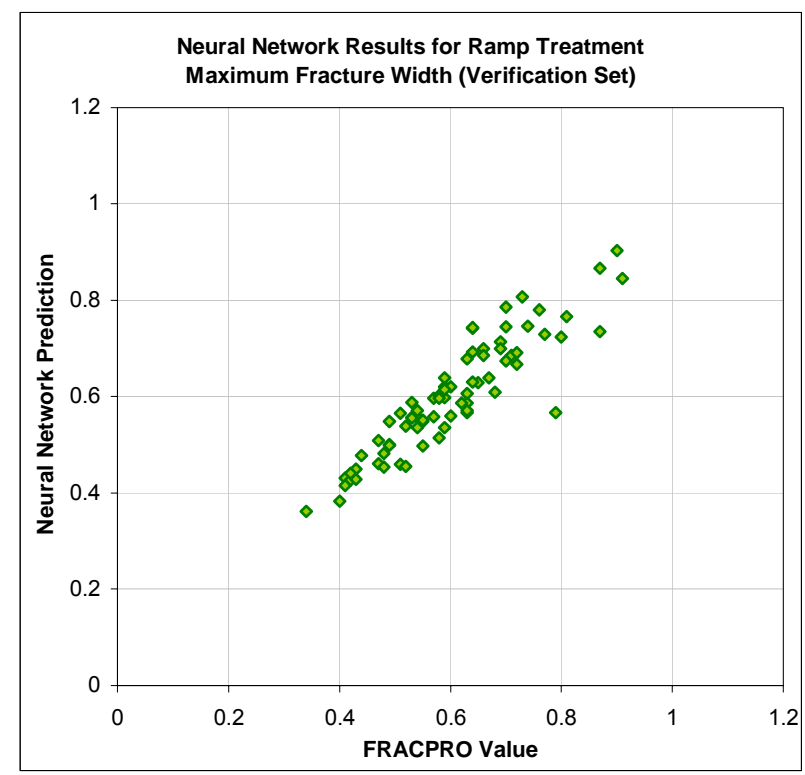

Figure 5-40: Six Stage Treatment Verification Set Results for Maximum Fracture Width

Figure 5-41 shows the training set results for fracture height. $R^{2}$ is $0.94, r^{2}$ is 0.94 , and the correlation coefficient is 0.97 .

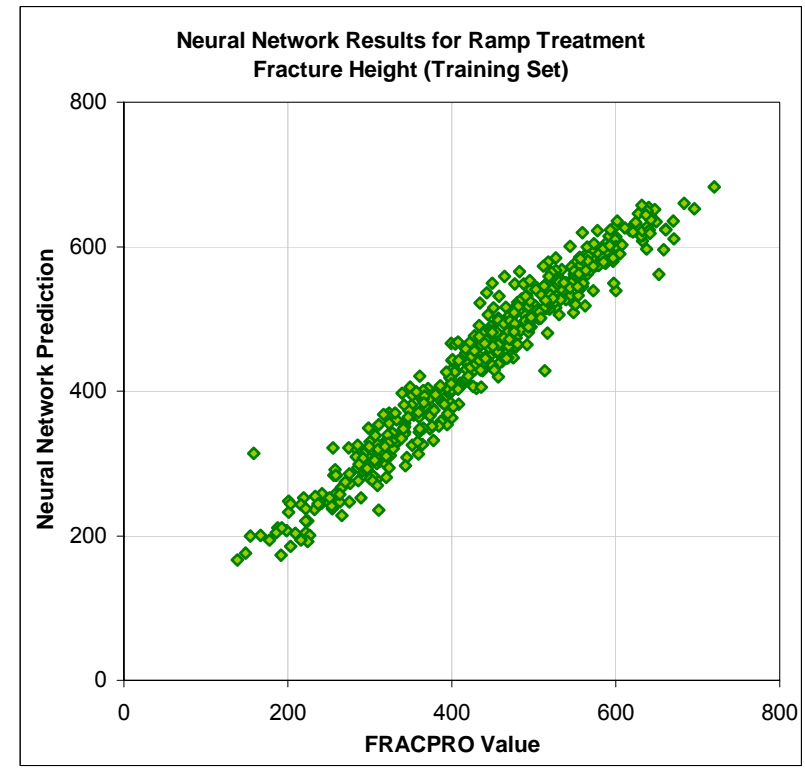

Figure 5-41: Six Stage Treatment Training Set Results for Fracture Height 
Figure 5-42 shows the calibration set results for fracture height. $R^{2}$ is $0.91, r^{2}$ is 0.91 , and the correlation coefficient is 0.95 .

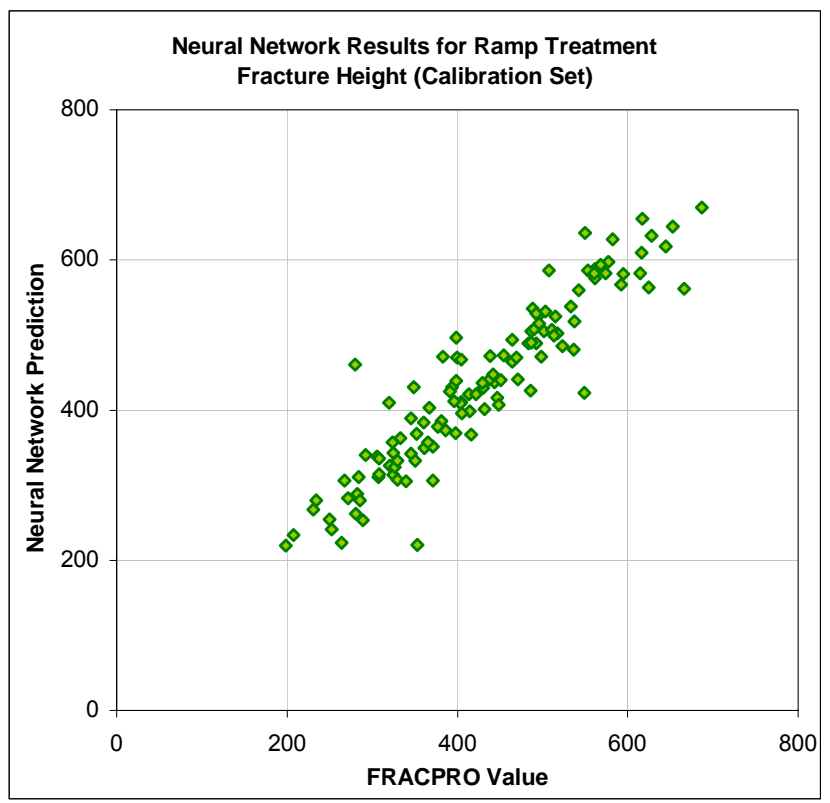

Figure 5-42: Six Stage Treatment Calibration Set Results for Fracture Height

Figure 5-43 shows the verification set results for fracture height. $R^{2}$ is $0.92, r^{2}$ is 0.92 , and the correlation coefficient is 0.96 .

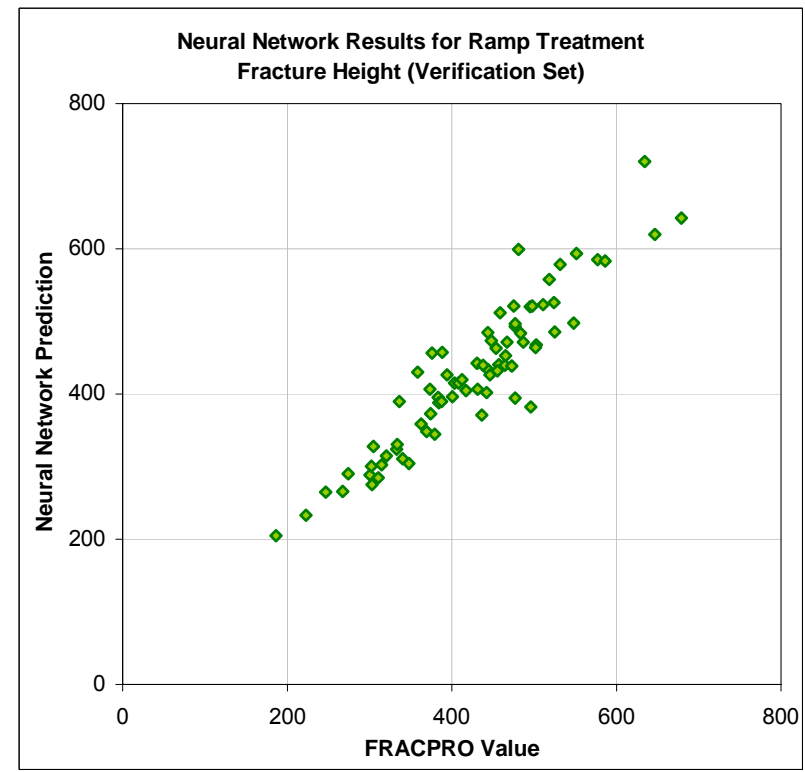

Figure 5-43: Six Stage Treatment Verification Set Results for Fracture Height 
The details of the results for each of the six output parameters in the training, calibration, and verification sets can be seen in Table 5-8.

\begin{tabular}{|c|c|c|c|c|c|c|}
\hline & $\begin{array}{l}\text { Fracture } \\
\text { Efficiency }\end{array}$ & $\begin{array}{c}\text { Propped } \\
\text { Fracture Length }\end{array}$ & $\begin{array}{c}\text { Proppant } \\
\text { Concentration }\end{array}$ & $\begin{array}{c}\text { Dimensionless } \\
\text { Conductivity } \\
\text { Ratio }\end{array}$ & $\begin{array}{l}\text { Max Fracture } \\
\text { Width }\end{array}$ & Fracture Height \\
\hline \multicolumn{7}{|l|}{ Training Set ( 480 cases) } \\
\hline $\mathrm{R}^{2}$ & 0.8440 & 0.8980 & 0.8770 & 0.8030 & 0.8390 & 0.9370 \\
\hline$r^{2}$ & 0.8630 & 0.8900 & 0.8860 & 0.8100 & 0.8410 & 0.9370 \\
\hline Correlation Coefficient, $r$ & 0.9290 & 0.9480 & 0.9410 & 0.9000 & 0.9170 & 0.9680 \\
\hline \multicolumn{7}{|l|}{ Calibration Set (120 cases) } \\
\hline $\mathrm{R}^{2}$ & 0.8060 & 0.8650 & 0.8290 & 0.8260 & 0.8270 & 0.9080 \\
\hline$r^{2}$ & 0.8150 & 0.8660 & 0.8410 & 0.8270 & 0.8280 & 0.9090 \\
\hline Correlation Coefficient, $r$ & 0.9020 & 0.9300 & 0.9190 & 0.8520 & 0.9100 & 0.9520 \\
\hline \multicolumn{7}{|l|}{ Verification Set ( 75 cases) } \\
\hline $\mathrm{R}^{2}$ & 0.8850 & 0.9310 & 0.8880 & 0.8020 & 0.8520 & 0.9200 \\
\hline$r^{2}$ & 0.8960 & 0.9330 & 0.9080 & 0.8010 & 0.8630 & 0.9210 \\
\hline Correlation Coefficient, $r$ & 0.9480 & 0.9660 & 0.9530 & 0.8590 & 0.9290 & 0.9590 \\
\hline
\end{tabular}

Table 5-8: Six Stage Treatment Neural Network Results

Fracture length and fracture height exhibit the best performance in prediction for the six stage neural network. The performance for fracture efficiency and proppant concentration follow closely behind. Fracture width and dimensionless conductivity ratio show lower performance than fracture length; however, both have strong correlation coefficients and values above 0.8 for $R^{2}$. The figures for the training, calibration, and verification sets of fracture length and fracture height show values that fall close the $45^{\circ}$ line.

The training and calibration sets for dimensionless conductivity ratio, Figure 5-35 and Figure 5-36, show poorer alignment with the $45^{\circ}$ line compared to the other parameters. The $\mathrm{R}^{2}$ value is lowest for dimensionless conductivity; however, this performance was the best achieved and was used in the optimization module.

\subsubsection{Neural Networks for Eight Stage Treatment}

The architecture of the neural networks for eight stage treatments has three hidden layers with different activation functions, 17 inputs and 5 outputs as described in detail in 4.12.2.2 The Eight Stage Treatment. Three neural networks were trained for eight stages treatments representing Type I, Type II, and Type III.

\subsubsection{Type I}

Figure 5-44 through Figure 5-58 show the correlation between the actual FRACPRO values and the neural network predicted values of the training set, calibration set, and verification set for each of the 5 output parameters (fracture efficiency, propped fracture length, proppant concentration, max fracture width, and fracture height). 
Figure 5-44 shows the training set results for fracture efficiency. $R^{2}$ is $0.96, r^{2}$ is 0.96 , and the correlation coefficient is 0.98 .

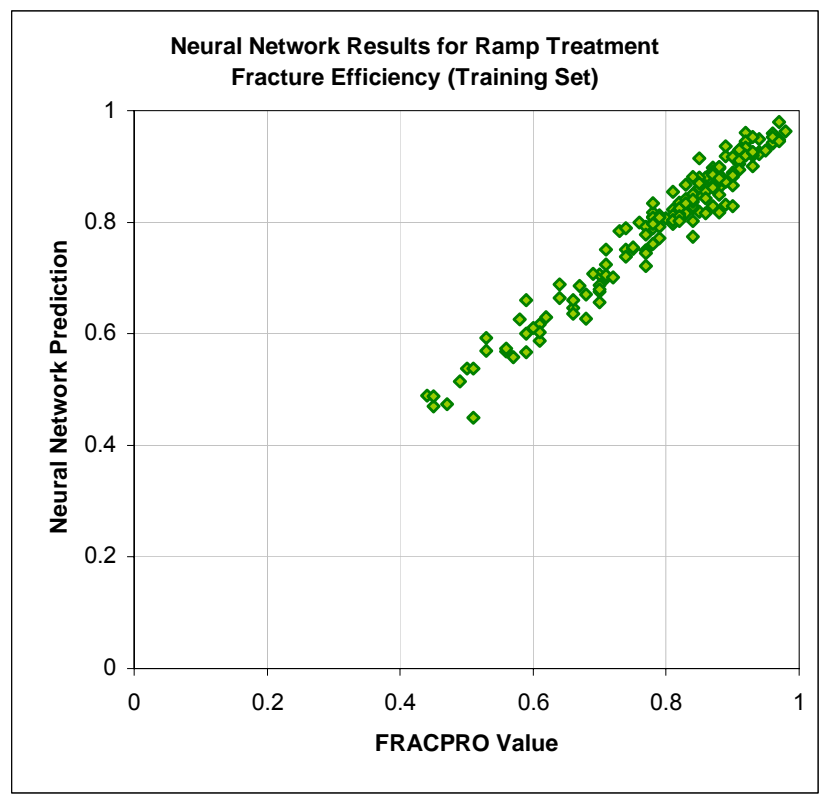

Figure 5-44: Eight Stage Type I Treatment Training Set Results for Fracture Efficiency

Figure $5-45$ shows the calibration set results for fracture efficiency. $R^{2}$ is $0.76, r^{2}$ is 0.79 , and the correlation coefficient is 0.89 .

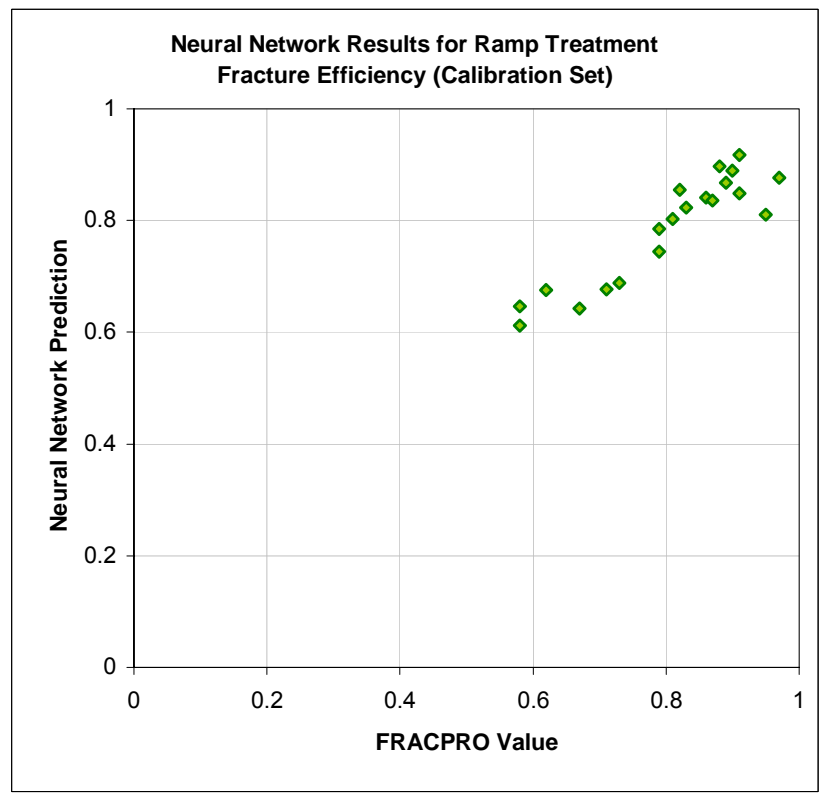

Figure 5-45: Eight Stage Type I Treatment Calibration Set Results for Fracture Efficiency 
Figure 5-46 shows the verification set results for fracture efficiency. $R^{2}$ is $0.72, r^{2}$ is 0.76 , and the correlation coefficient is 0.87 .

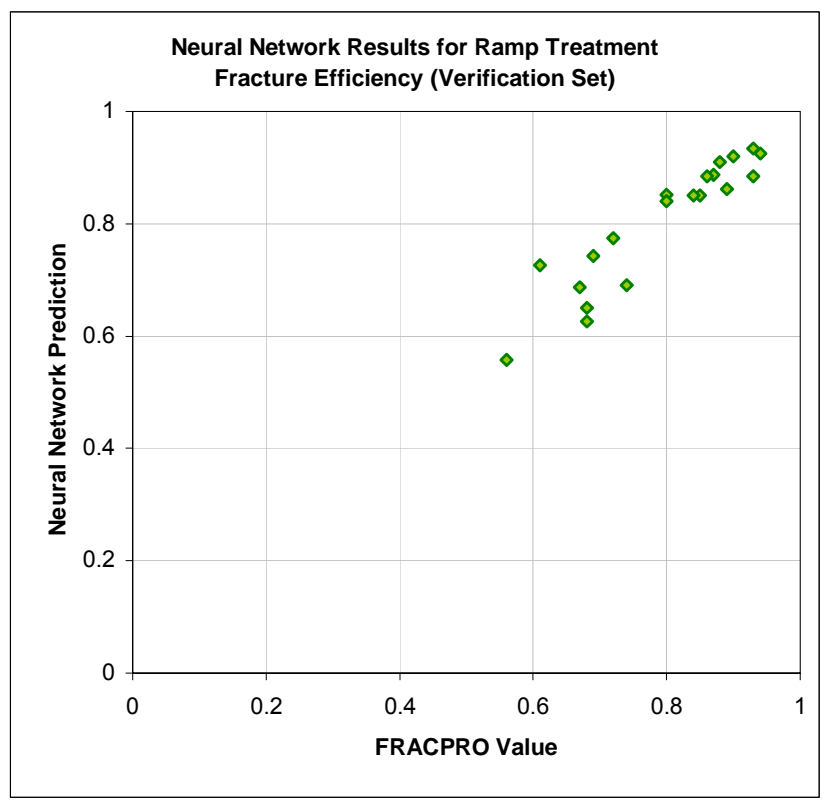

Figure 5-46: Eight Stage Type I Treatment Verification Set Results for Fracture Efficiency

Figure 5-47 shows the training set results for propped fracture length. $R^{2}$ is $0.92, r^{2}$ is 0.93 , and the correlation coefficient is 0.96 .

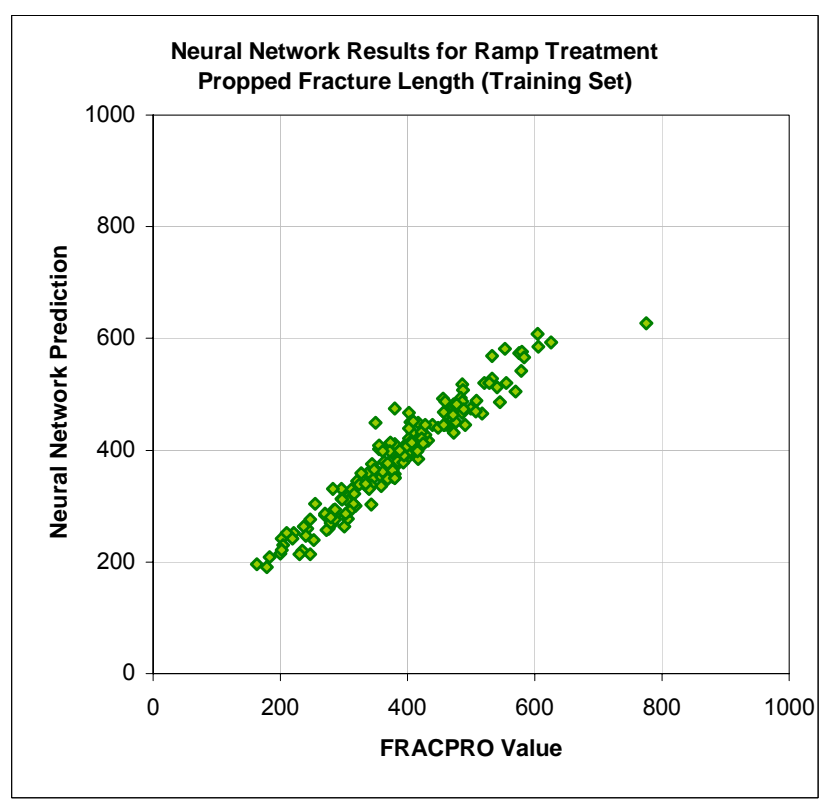

Figure 5-47: Eight Stage Type I Treatment Training Set Results for Propped Fracture Length 
Figure 5-48 shows the calibration set results for propped fracture length. $R^{2}$ is $0.88, r^{2}$ is 0.89 , and the correlation coefficient is 0.95 .

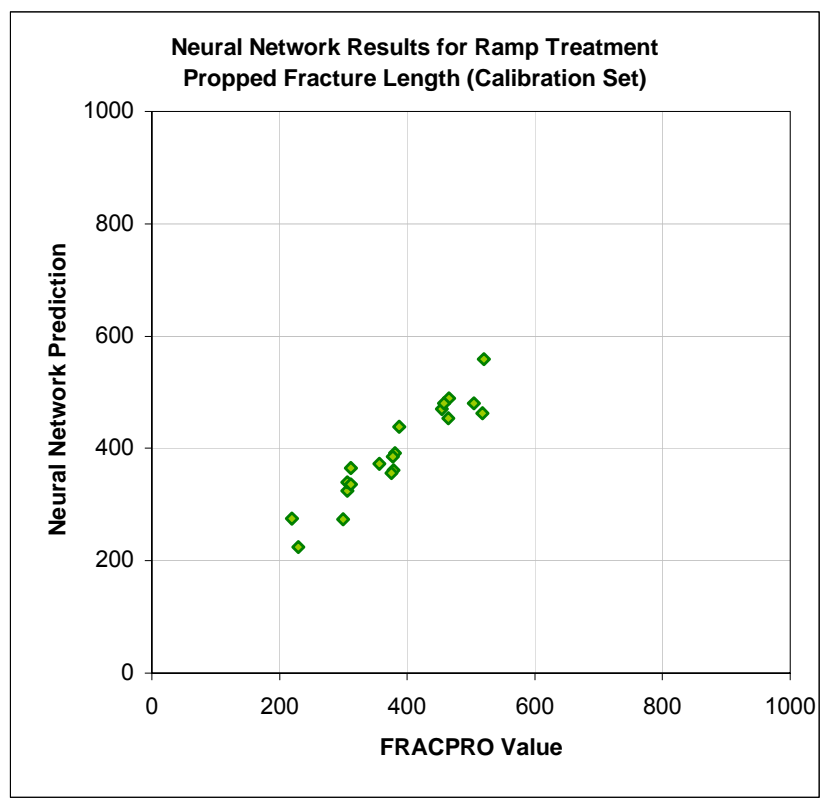

Figure 5-48: Eight Stage Type I Treatment Calibration Set Results for Propped Fracture Length

Figure 5-49 shows the verification set results for propped fracture length. $R^{2}$ is $0.91, r^{2}$ is 0.92 , and the correlation coefficient is 0.96 .

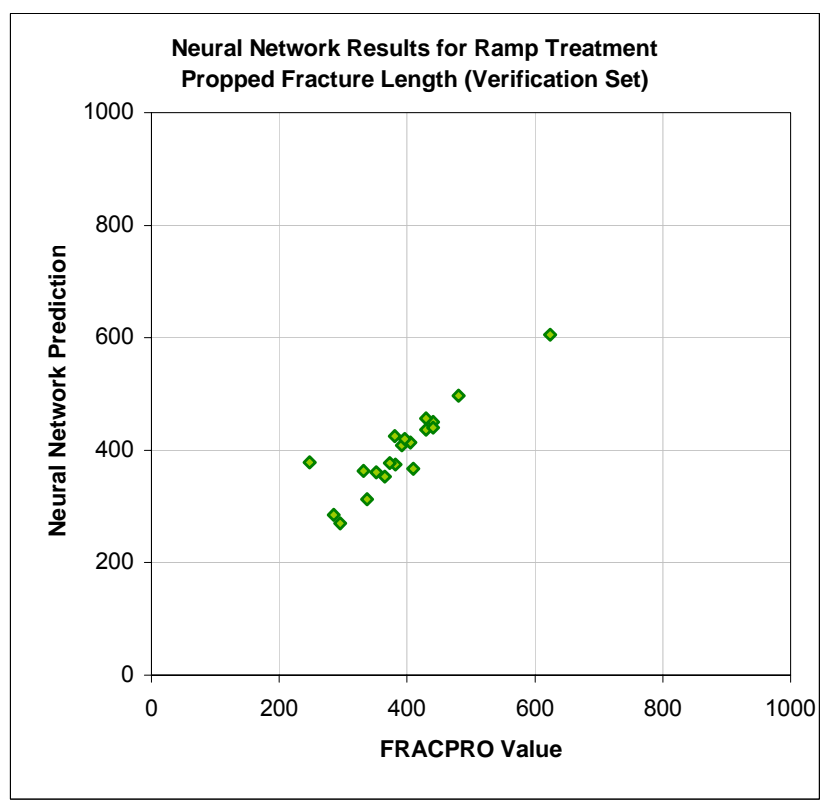

Figure 5-49: Eight Stage Type I Treatment Verification Set Results for Propped Fracture Length 
Figure 5-50 shows the training set results for proppant concentration. $R^{2}$ is $0.80, r^{2}$ is 0.84 , and the correlation coefficient is 0.94 .

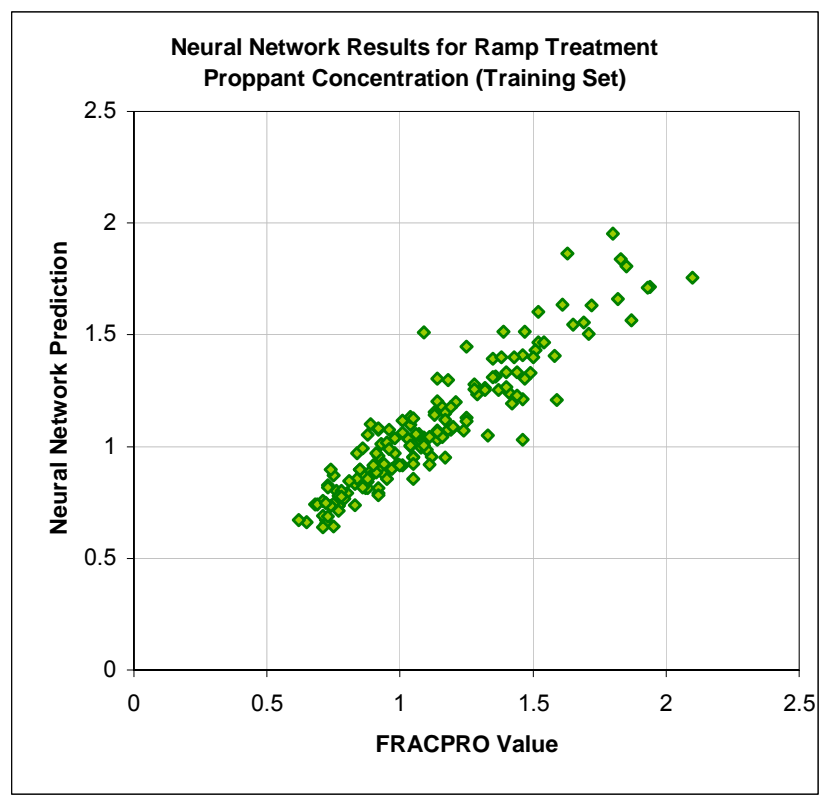

Figure 5-50: Eight Stage Type I Treatment Training Set Results for Proppant Concentration

Figure 5-51 shows the calibration set results for proppant concentration. $R^{2}$ is $0.86, r^{2}$ is 0.82 , and the correlation coefficient is 0.94 .

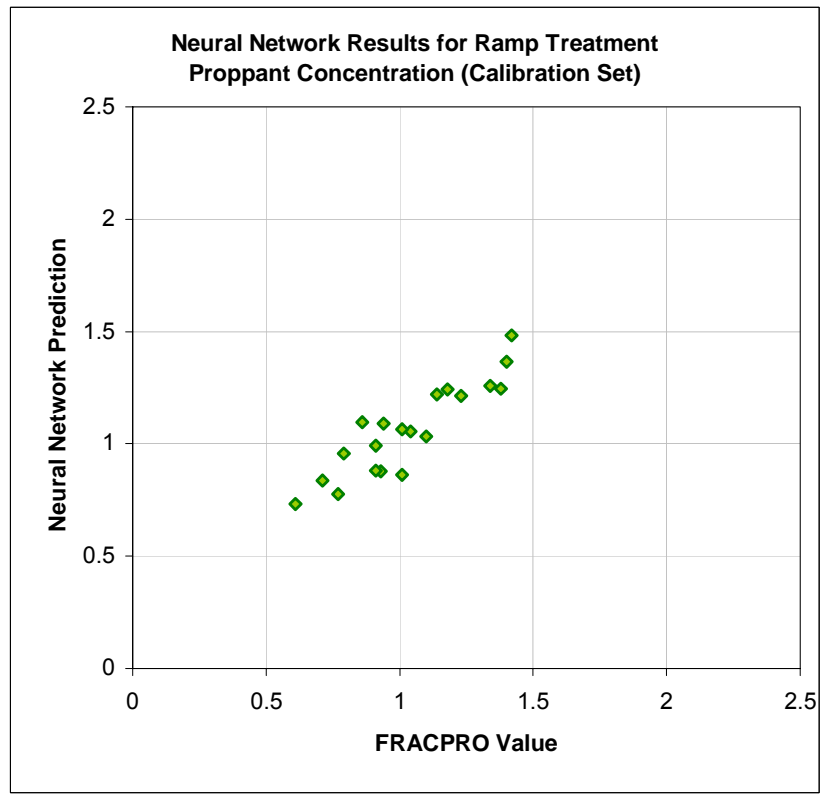

Figure 5-51: Eight Stage Type I Treatment Calibration Set Results for Proppant Concentration 
Figure 5-52 shows the verification set results for proppant concentration. $R^{2}$ is $0.90, r^{2}$ is 0.82 , and the correlation coefficient is 0.90 .

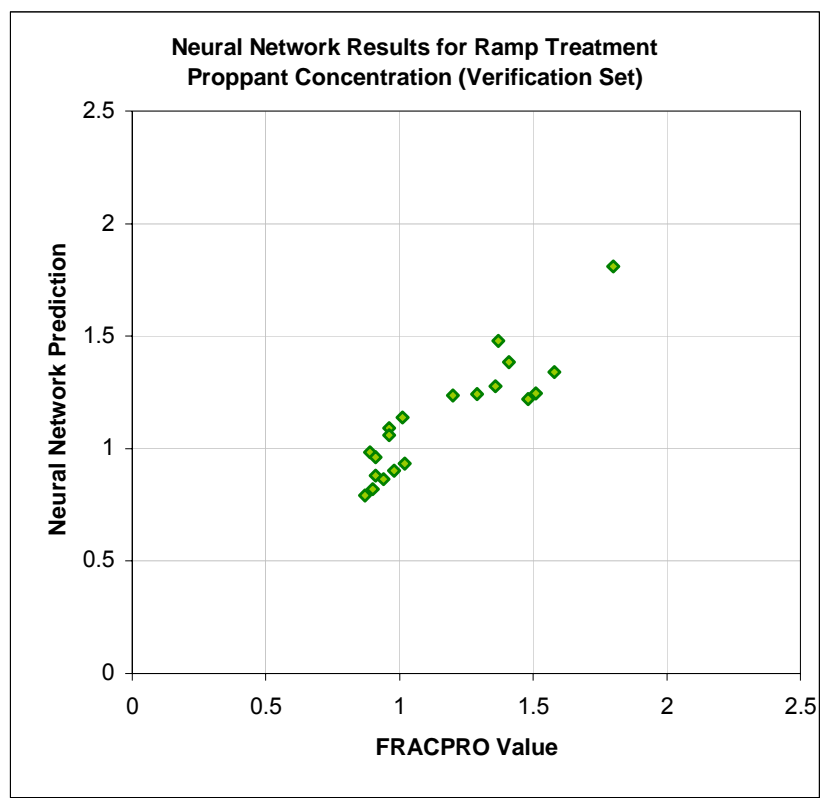

Figure 5-52: Eight Stage Type I Treatment Verification Set Results for Proppant Concentration

Figure $5-53$ shows the training set results for maximum fracture width. $R^{2}$ is $0.82, r^{2}$ is 0.83 , and the correlation coefficient is 0.91 .

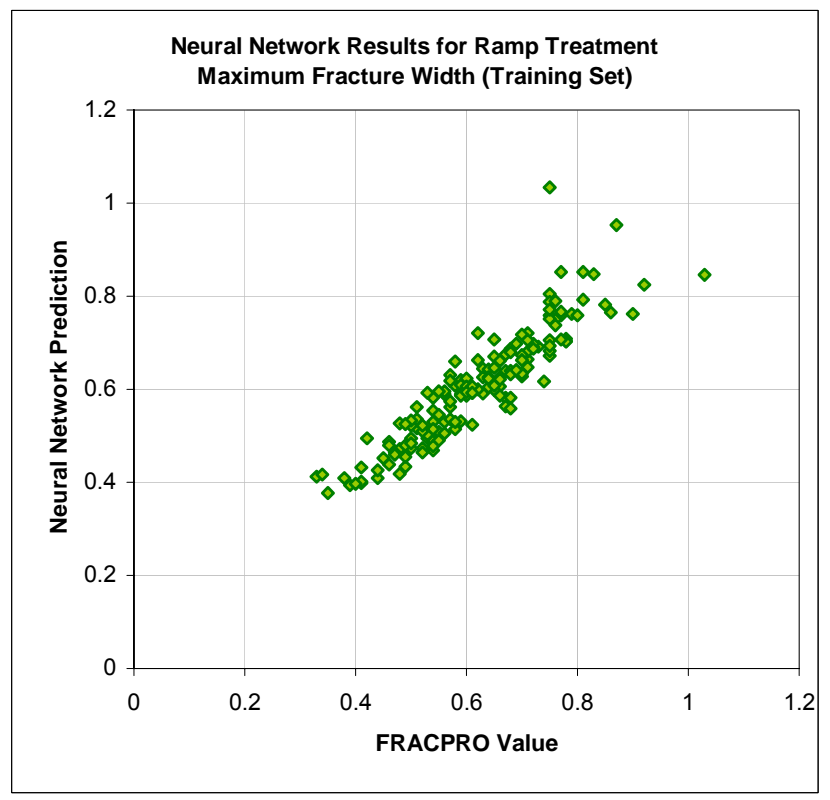

Figure 5-53: Eight Stage Type I Treatment Training Set Results for Maximum Fracture Width 
Figure 5-54 shows the calibration set results for maximum fracture width. $R^{2}$ is $0.87, r^{2}$ is 0.87 , and the correlation coefficient is 0.92 .

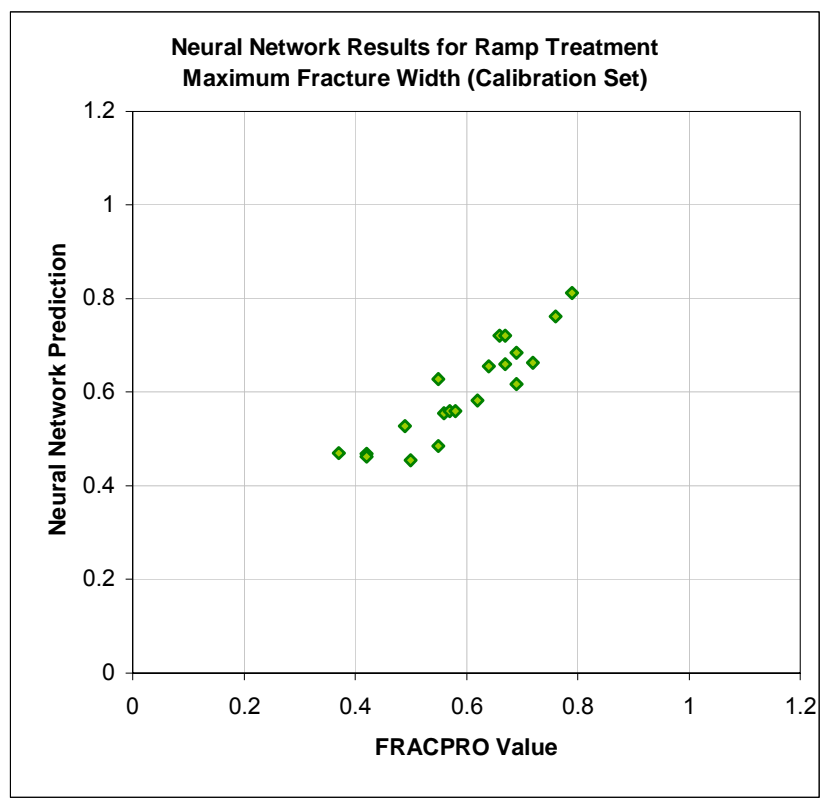

Figure 5-54: Eight Stage Type I Treatment Calibration Set Results for Maximum Fracture Width

Figure 5-55 shows the verification set results for maximum fracture width. $R^{2}$ is $0.84, r^{2}$ is 0.86 , and the correlation coefficient is 0.95 .

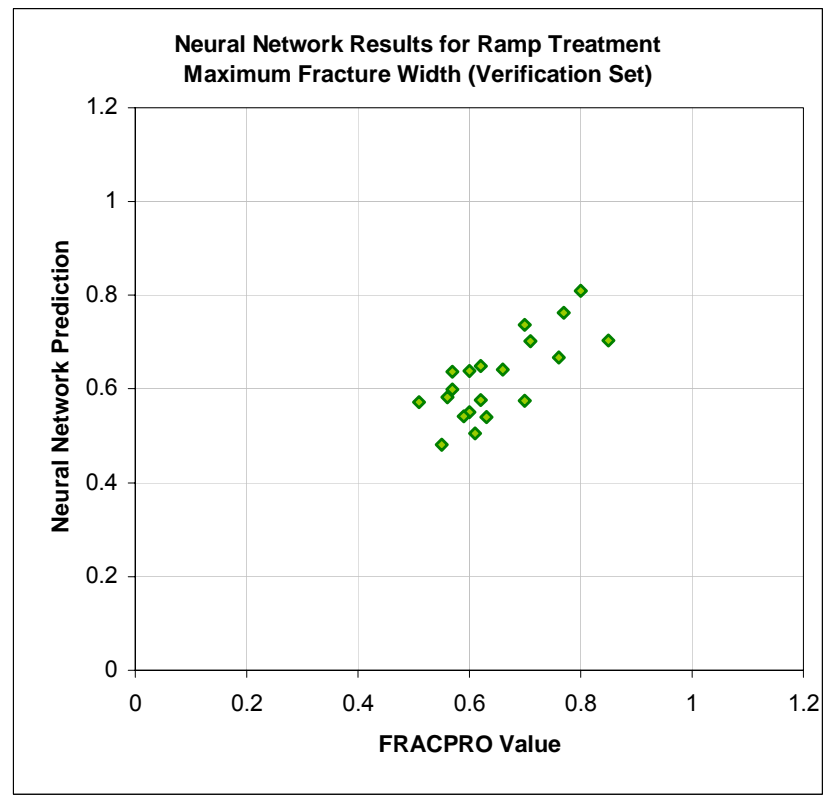

Figure 5-55: Eight Stage Type I Treatment Verification Set Results for Maximum Fracture Width 
Figure 5-56 shows the training set results for fracture height. $R^{2}$ is $0.90, r^{2}$ is 0.90 , and the correlation coefficient is 0.95 .

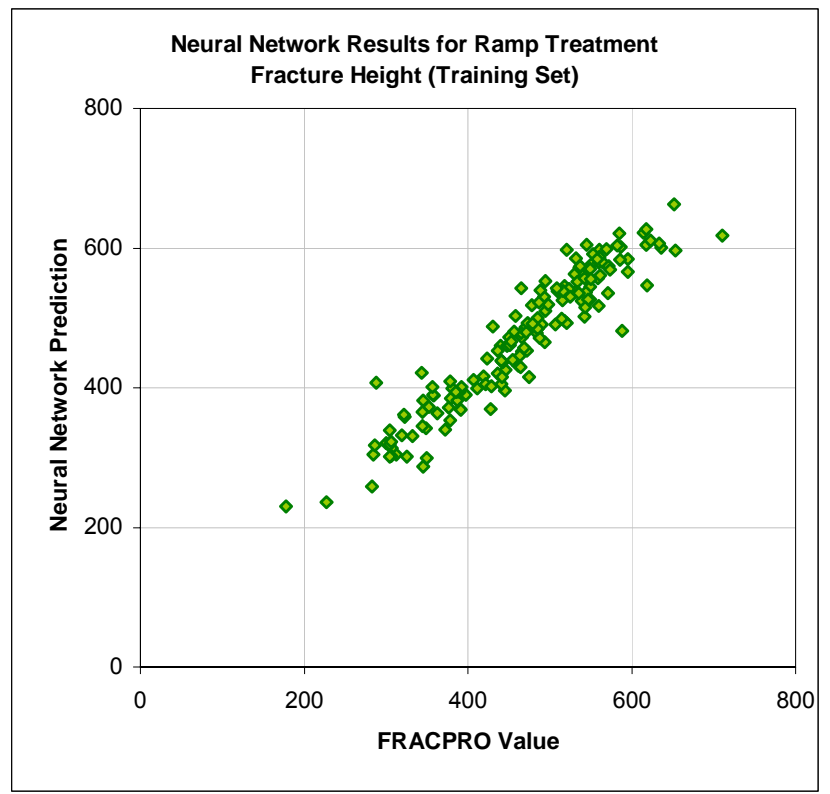

Figure 5-56: Eight Stage Type I Treatment Training Set Results for Fracture Height

Figure 5-57 shows the calibration set results for fracture height. $R^{2}$ is $0.88, r^{2}$ is 0.90 , and the correlation coefficient is 0.95 .

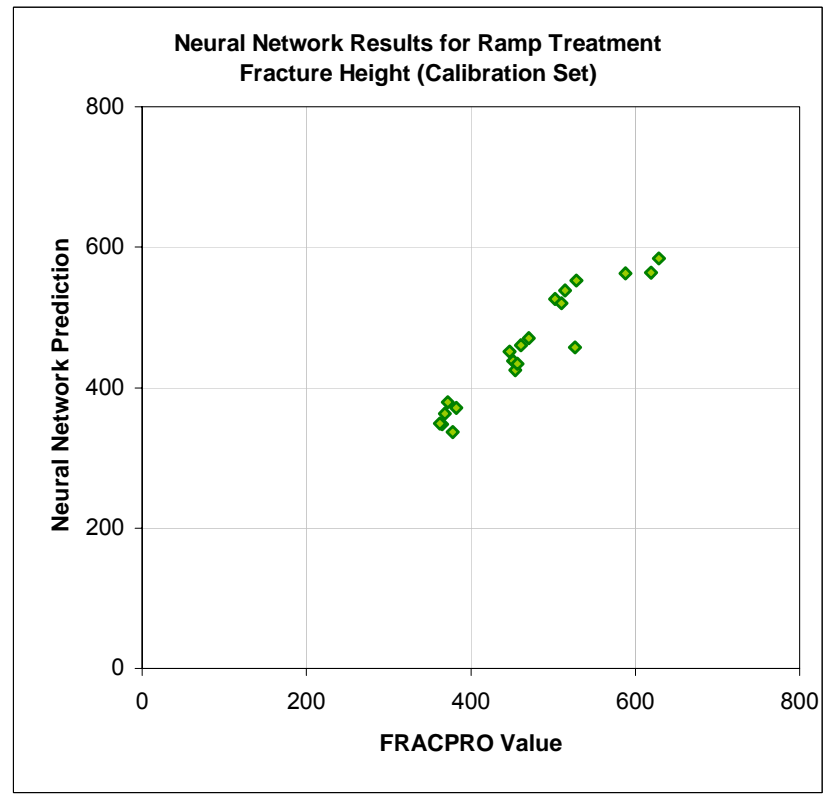

Figure 5-57: Eight Stage Type I Treatment Calibration Set Results for Fracture Height 
Figure 5-58 shows the verification set results for fracture height. $R^{2}$ is $0.90, r^{2}$ is 0.90 , and the correlation coefficient is 0.95 .

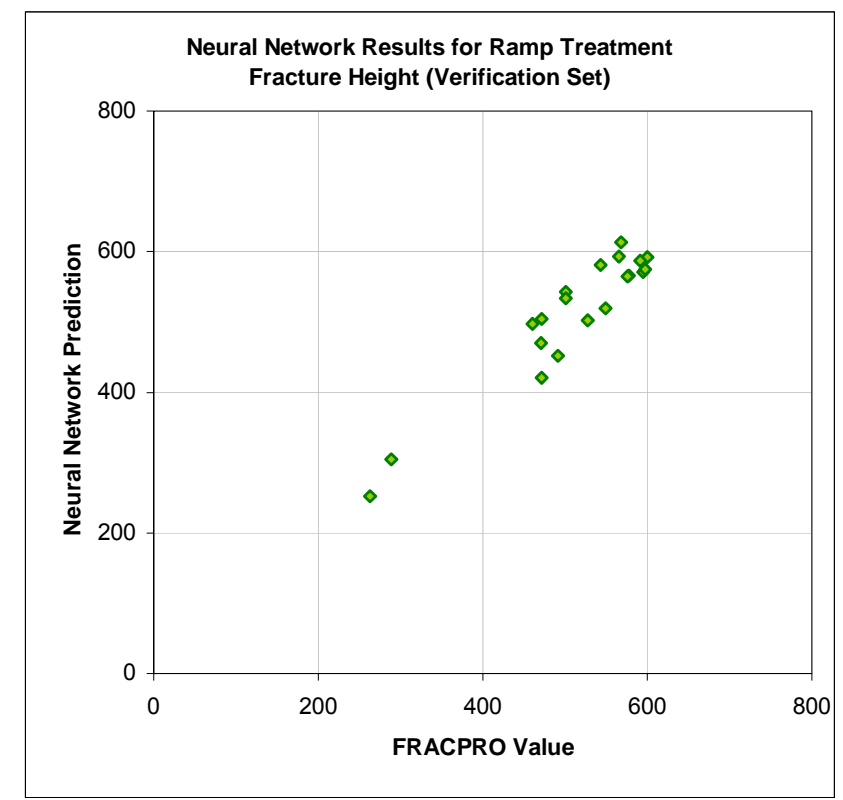

Figure 5-58: Eight Stage Type I Treatment Verification Set Results for Fracture Height

The details of the results for each of the five output parameters in the training, calibration, and verification sets can be seen in Table 5-9.

\begin{tabular}{|c|c|c|c|c|c|}
\hline & $\begin{array}{l}\text { Fracture } \\
\text { Efficiency }\end{array}$ & $\begin{array}{c}\text { Propped } \\
\text { Fracture Length }\end{array}$ & $\begin{array}{c}\text { Proppant } \\
\text { Concentration }\end{array}$ & $\begin{array}{l}\text { Max Fracture } \\
\text { Width }\end{array}$ & Fracture Height \\
\hline \multicolumn{6}{|l|}{ Training Set (162 cases) } \\
\hline $\mathrm{R}^{2}$ & 0.9570 & 0.9241 & 0.8034 & 0.8163 & 0.8953 \\
\hline$r^{2}$ & 0.9575 & 0.9263 & 0.8443 & 0.8287 & 0.8987 \\
\hline Correlation Coefficient, $r$ & 0.9785 & 0.9624 & 0.9413 & 0.9103 & 0.9480 \\
\hline \multicolumn{6}{|l|}{ Calibration Set ( 20 cases) } \\
\hline $\mathrm{R}^{2}$ & 0.7587 & 0.8785 & 0.8611 & 0.8679 & 0.8779 \\
\hline $\mathrm{r}^{2}$ & 0.7900 & 0.8931 & 0.8151 & 0.8710 & 0.9030 \\
\hline Correlation Coefficient, $r$ & 0.8900 & 0.9451 & 0.9443 & 0.9192 & 0.9503 \\
\hline \multicolumn{6}{|l|}{ Verification Set (20 cases) } \\
\hline $\mathrm{R}^{2}$ & 0.7163 & 0.9125 & 0.8998 & 0.8387 & 0.8975 \\
\hline $\mathrm{r}^{2}$ & 0.7618 & 0.9204 & 0.8153 & 0.8594 & 0.9030 \\
\hline Correlation Coefficient, $r$ & 0.8728 & 0.9594 & 0.9829 & 0.9479 & 0.9503 \\
\hline
\end{tabular}

Table 5-9: Eight Stage Type I Treatment Neural Network Results

Note that in training this system only 202 cases were used compared to the two previous neural networks where more than 600 cases were available.

Much like the ramp treatment and six stage neural networks, fracture length and fracture height show the best performance, followed by fracture efficiency and fracture width. Fracture proppant concentration shows lower performance in this neural network. Dimensionless fracture conductivity was not collected for the eight-stage treatment and is not available for prediction. 


\subsubsection{Type II}

Figure 5-59 through Figure 5-73 show the correlation between the actual FRACPRO values and the neural network predicted values of the training set, calibration set, and verification set for each of the 5 output parameters (fracture efficiency, propped fracture length, proppant concentration, max fracture width, and fracture height).

Figure 5-59 shows the training set results for fracture efficiency. $R^{2}$ is $0.81, r^{2}$ is 0.81 , and the correlation coefficient is 0.90 .

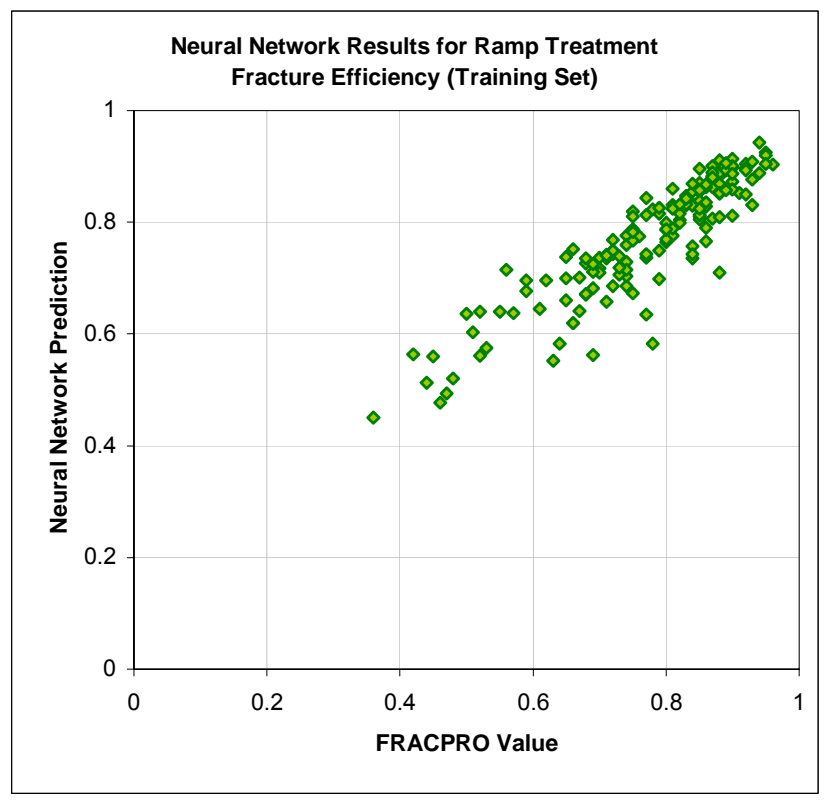

Figure 5-59: Eight Stage Type II Treatment Training Set Results for Fracture Efficiency 
Figure 5-60 shows the calibration set results for fracture efficiency. $R^{2}$ is $0.91, r^{2}$ is 0.91 , and the correlation coefficient is 0.95 .

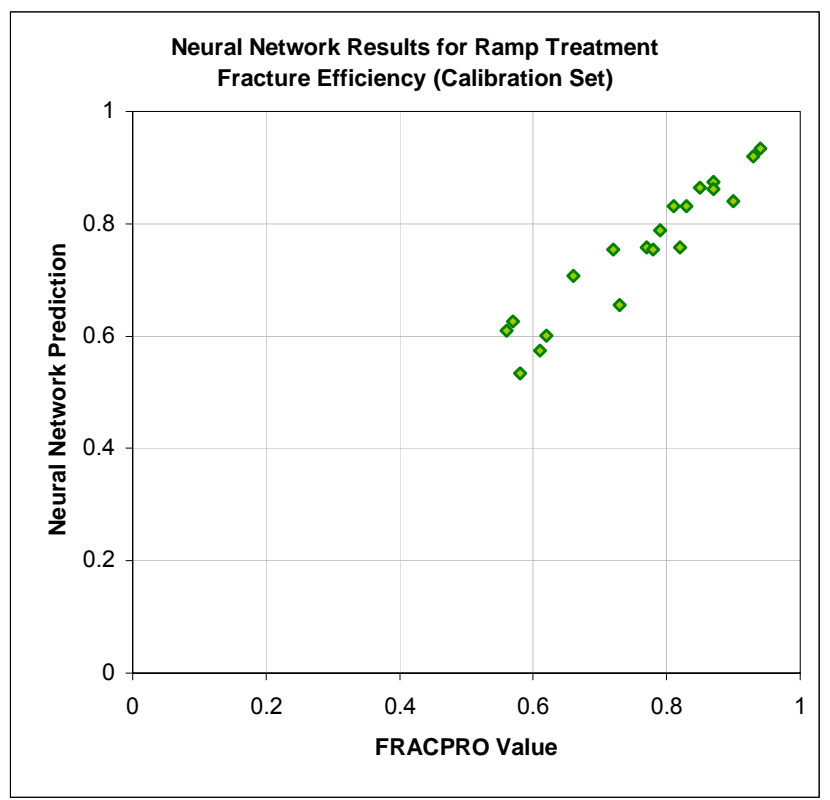

Figure 5-60: Eight Stage Type II Treatment Calibration Set Results for Fracture Efficiency

Figure $5-61$ shows the verification set results for fracture efficiency. $R^{2}$ is $0.83, r^{2}$ is 0.89 , and the correlation coefficient is 0.95 .

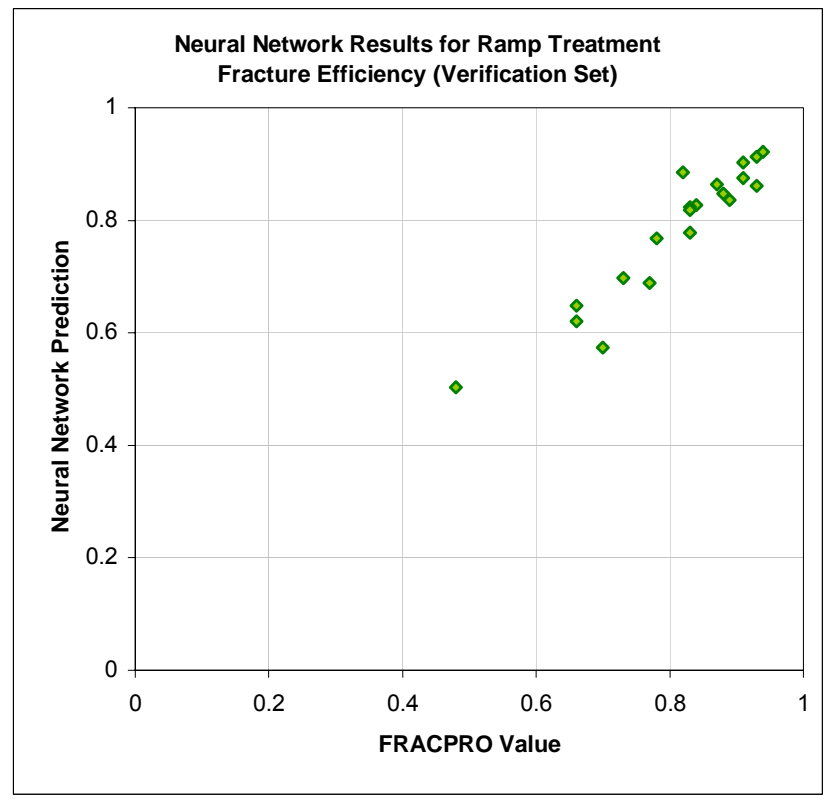

Figure 5-61: Eight Stage Type II Treatment Verification Set Results for Fracture Efficiency 
Figure 5-62 shows the training set results for propped fracture length. $R^{2}$ is $0.89, r^{2}$ is 0.89 , and the correlation coefficient is 0.95 .

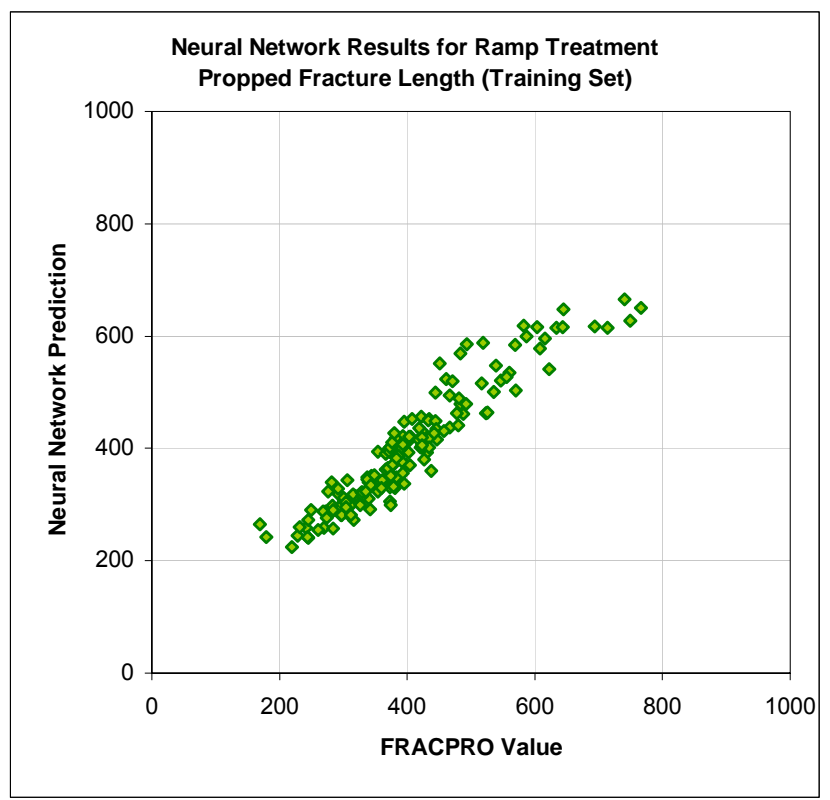

Figure 5-62: Eight Stage Type II Treatment Training Set Results for Propped Fracture Length

Figure 5-63 shows the calibration set results for propped fracture length. $R^{2}$ is $0.84, r^{2}$ is 0.86 , and the correlation coefficient is 0.93 .

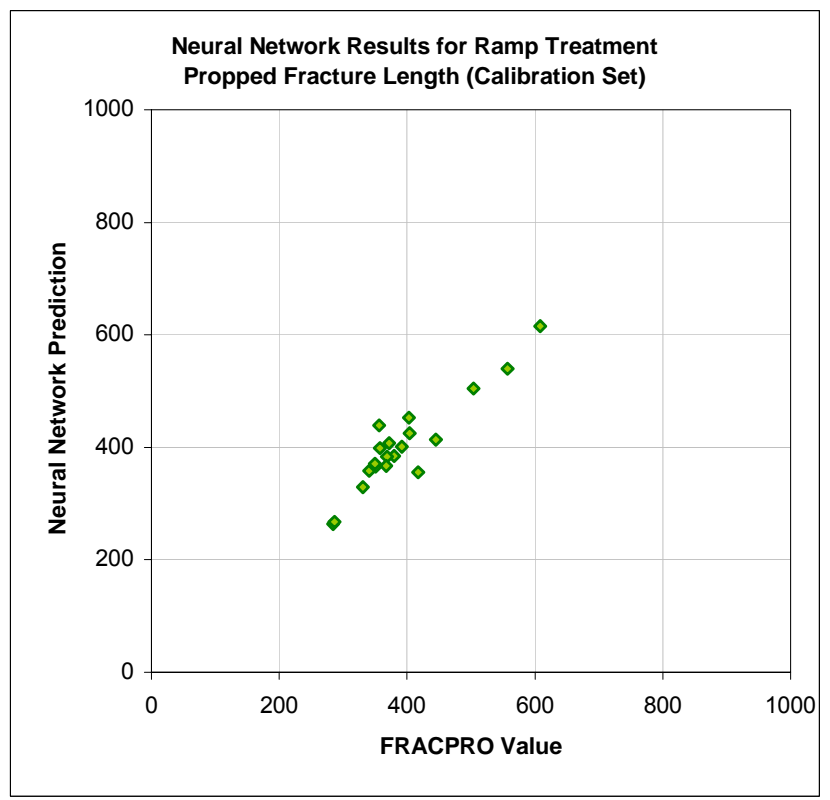

Figure 5-63: Eight Stage Type II Treatment Calibration Set Results for Propped Fracture Length 
Figure 5-64 shows the verification set results for propped fracture length. $R^{2}$ is $0.95, r^{2}$ is 0.95 , and the correlation coefficient is 0.98 .

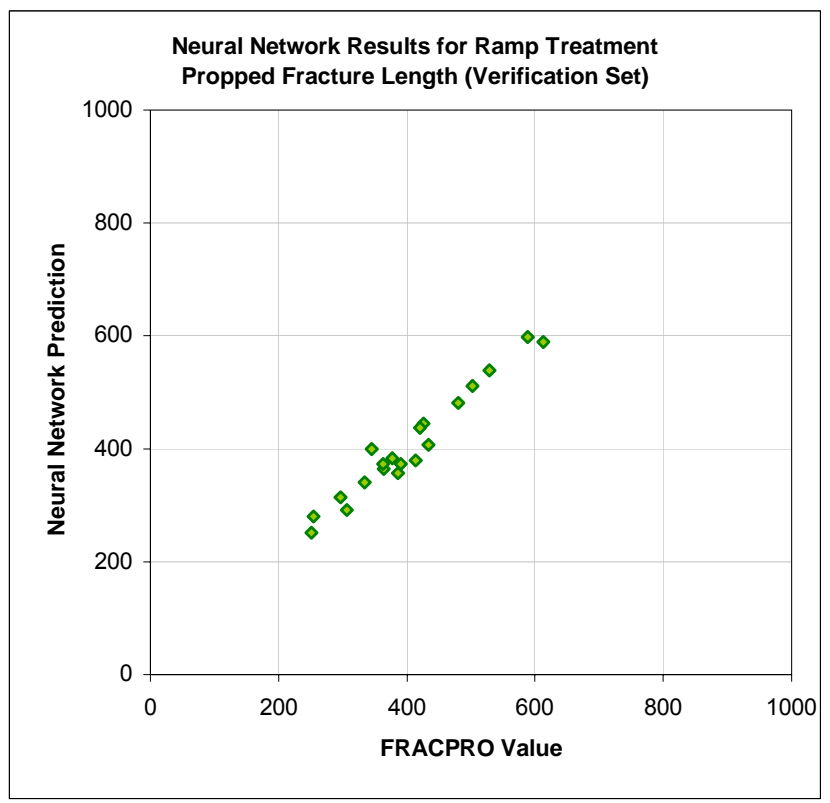

Figure 5-64: Eight Stage Type II Treatment Verification Set Results for Propped Fracture Length

Figure $5-65$ shows the training set results for proppant concentration. $R^{2}$ is $0.74, r^{2}$ is 0.78 , and the correlation coefficient is 0.88 .

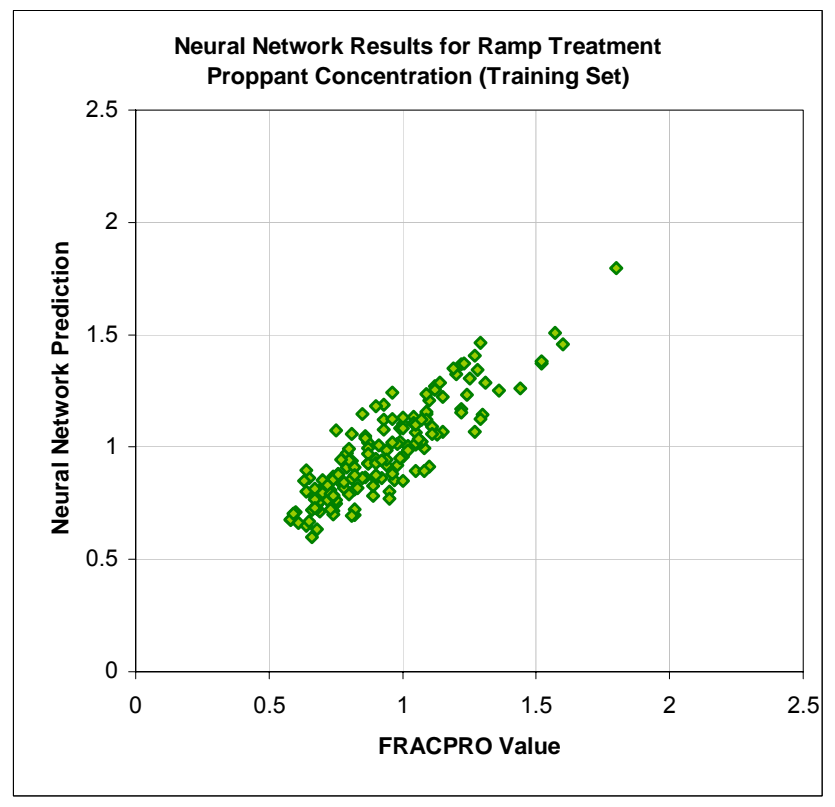

Figure 5-65: Eight Stage Type II Treatment Training Set Results for Proppant Concentration 
Figure 5-66 shows the calibration set results for proppant concentration. $R^{2}$ is $0.84, r^{2}$ is 0.85 , and the correlation coefficient is 0.92 .

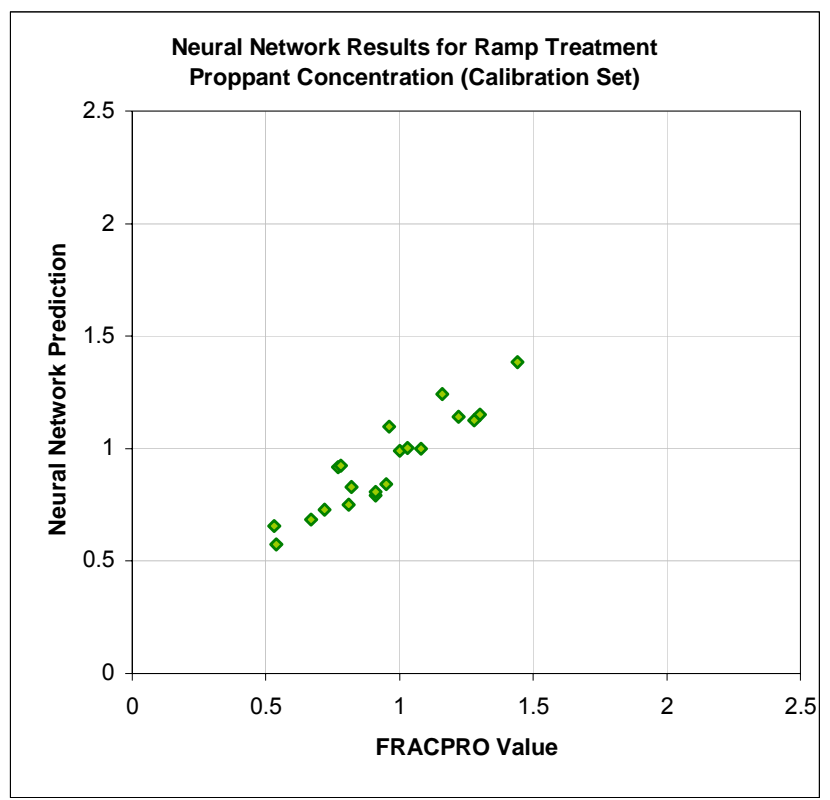

Figure 5-66: Eight Stage Type II Treatment Calibration Set Results for Proppant Concentration

Figure 5-67 shows the verification set results for proppant concentration. $R^{2}$ is $0.91, r^{2}$ is 0.91 , and the correlation coefficient is 0.95 .

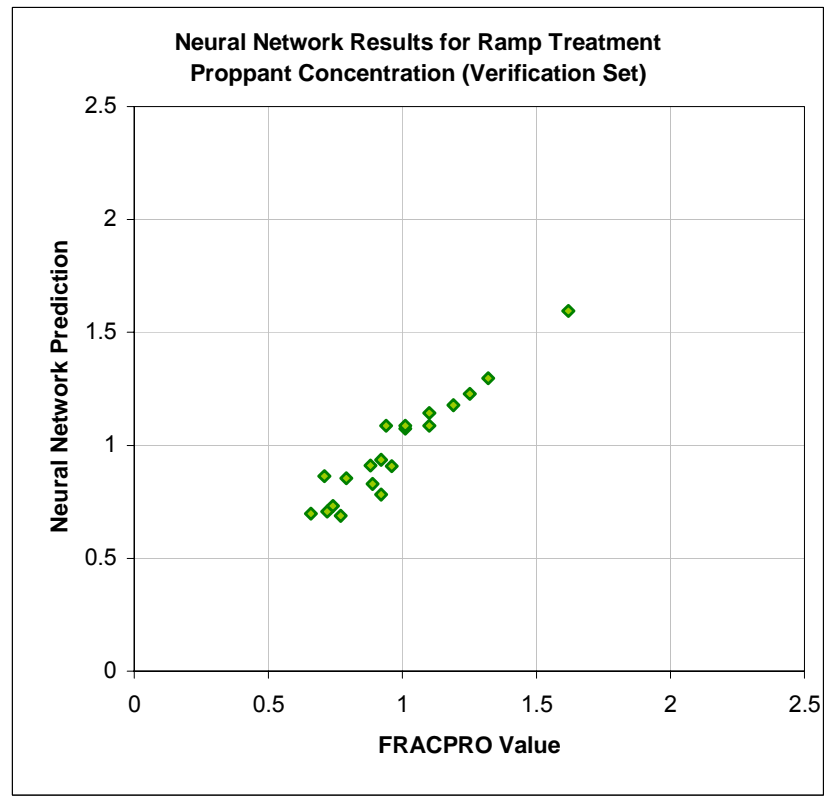

Figure 5-67: Eight Stage Type II Treatment Verification Set Results for Proppant Concentration 
Figure 5-68 shows the training set results for maximum fracture width. $R^{2}$ is $0.81, r^{2}$ is 0.81 , and the correlation coefficient is 0.90 .

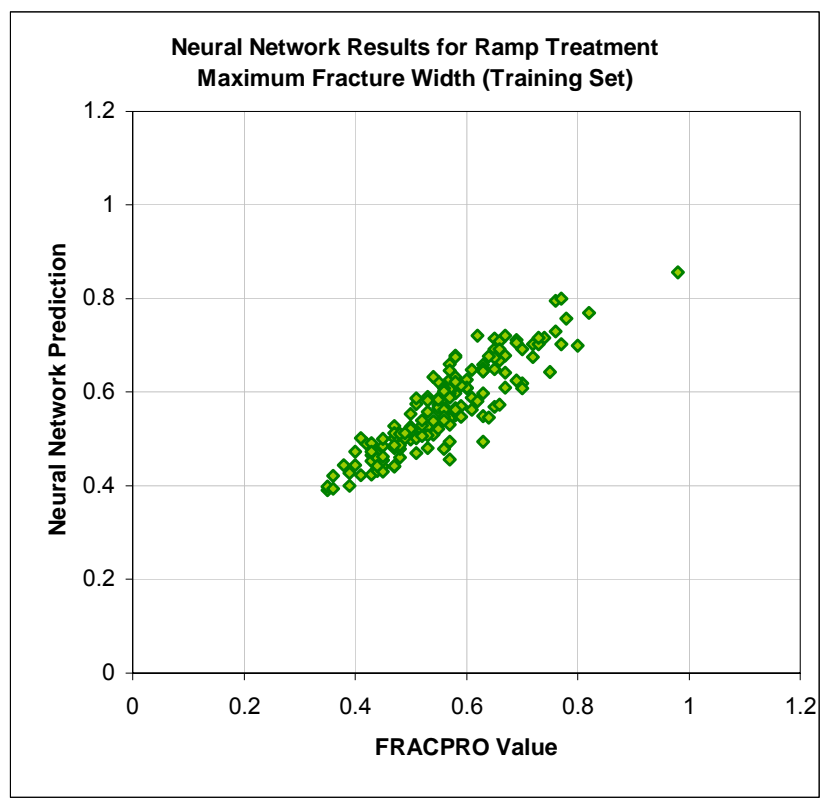

Figure 5-68: Eight Stage Type II Treatment Training Set Results for Maximum Fracture Width

Figure 5-69 shows the calibration set results for maximum fracture width. $R^{2}$ is $0.90, r^{2}$ is 0.90 , and the correlation coefficient is 0.95 .

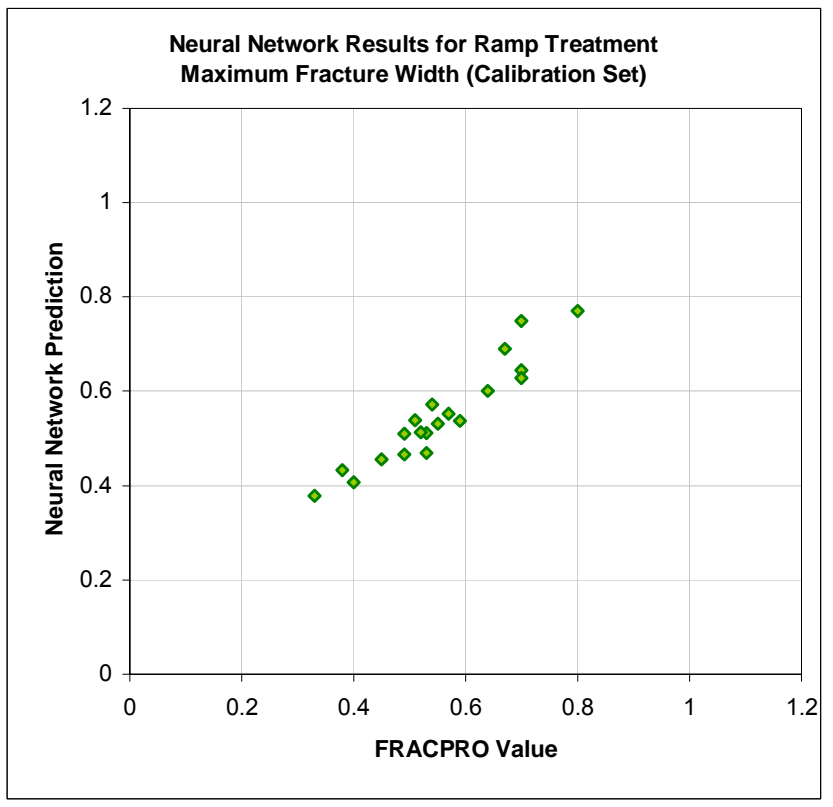

Figure 5-69: Eight Stage Type II Treatment Calibration Set Results for Maximum Fracture Width 
Figure 5-70 shows the verification set results for maximum fracture width. $R^{2}$ is $0.81, r^{2}$ is 0.81 , and the correlation coefficient is 0.89 .

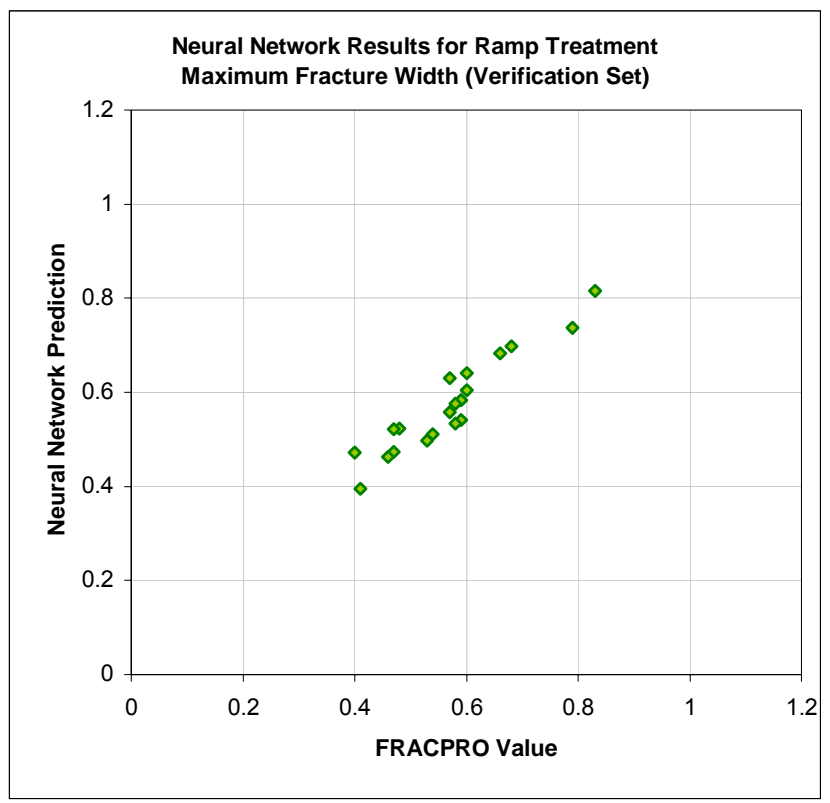

Figure 5-70: Eight Stage Type II Treatment Verification Set Results for Maximum Fracture Width

Figure $5-71$ shows the training set results for fracture height. $R^{2}$ is $0.84, r^{2}$ is 0.86 , and the correlation coefficient is 0.93 .

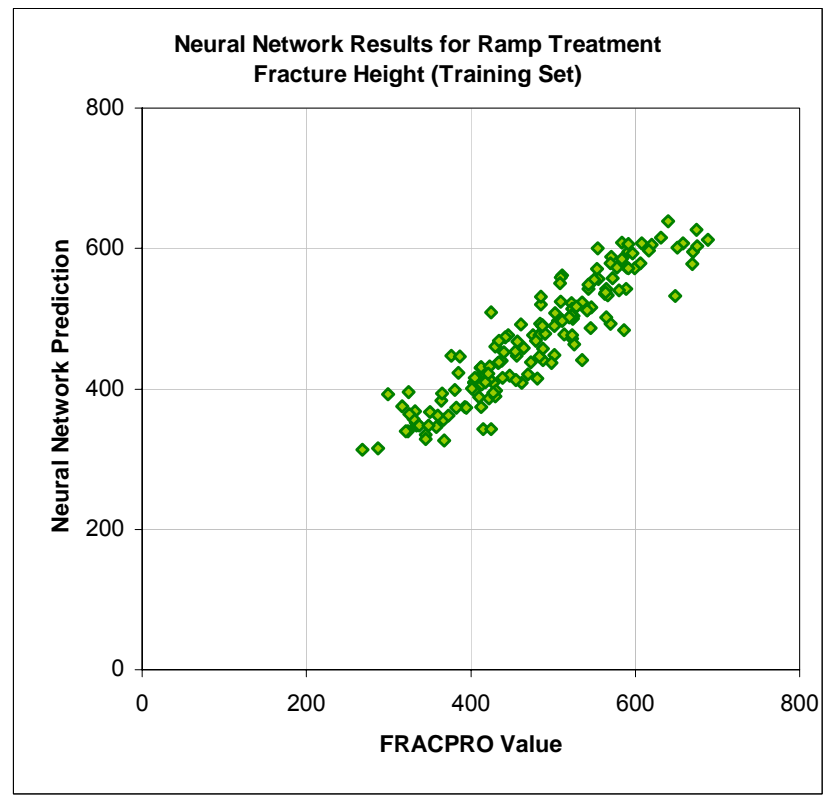

Figure 5-71: Eight Stage Type II Treatment Training Set Results for Fracture Height 
Figure 5-72 shows the calibration set results for fracture height. $R^{2}$ is $0.88, r^{2}$ is 0.88 , and the correlation coefficient is 0.94 .

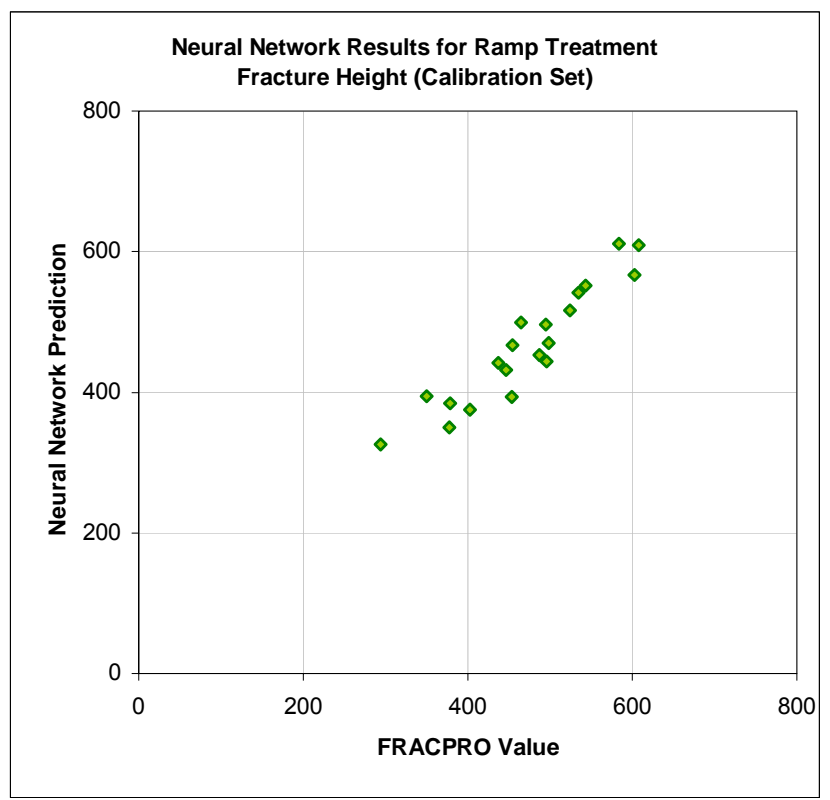

Figure 5-72: Eight Stage Type II Treatment Calibration Set Results for Fracture Height

Figure 5-73 shows the verification set results for fracture height. $R^{2}$ is $0.91, r^{2}$ is 0.92 , and the correlation coefficient is 0.96 .

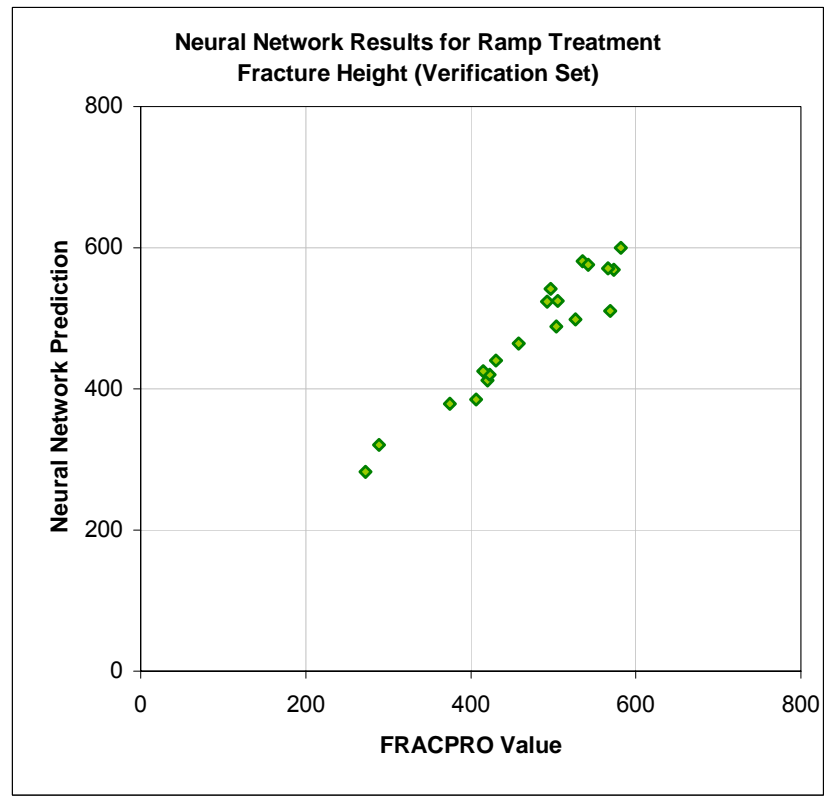

Figure 5-73: Eight Stage Type II Treatment Verification Set Results for Fracture Height 
The details of the results for each of the five output parameters in the training, calibration, and verification sets can be seen in Table 5-10.

\begin{tabular}{|c|c|c|c|c|c|}
\hline & $\begin{array}{l}\text { Fracture } \\
\text { Efficiency }\end{array}$ & $\begin{array}{c}\text { Propped } \\
\text { Fracture Length }\end{array}$ & $\begin{array}{l}\text { Proppant } \\
\text { Concentration }\end{array}$ & $\begin{array}{l}\text { Max Fracture } \\
\text { Width }\end{array}$ & Fracture Height \\
\hline \multicolumn{6}{|l|}{ Training Set (160 cases) } \\
\hline $\mathrm{R}^{2}$ & 0.8100 & 0.8913 & 0.7375 & 0.8056 & 0.8448 \\
\hline$r^{2}$ & 0.8136 & 0.8935 & 0.7754 & 0.8056 & 0.8567 \\
\hline Correlation Coefficient, $r$ & 0.9020 & 0.9452 & 0.8806 & 0.8976 & 0.9256 \\
\hline \multicolumn{6}{|l|}{ Calibration Set (20 cases) } \\
\hline $\mathrm{R}^{2}$ & 0.9054 & 0.8437 & 0.8426 & 0.9021 & 0.8755 \\
\hline $\mathrm{r}^{2}$ & 0.9088 & 0.8618 & 0.8498 & 0.9021 & 0.8823 \\
\hline Correlation Coefficient, $r$ & 0.9533 & 0.9283 & 0.9219 & 0.9498 & 0.9393 \\
\hline \multicolumn{6}{|l|}{ Verification Set (20 cases) } \\
\hline $\mathrm{R}^{2}$ & 0.8298 & 0.9543 & 0.9091 & 0.8114 & 0.9127 \\
\hline$r^{2}$ & 0.8945 & 0.9547 & 0.9118 & 0.8140 & 0.9207 \\
\hline Correlation Coefficient, $r$ & 0.9458 & 0.9771 & 0.9549 & 0.8934 & 0.9595 \\
\hline
\end{tabular}

Table 5-10: Eight Stage Type II Treatment Neural Network Results

The results of the Type II neural network are much like the Type I neural network for eight stage treatments. Fracture length and fracture height show the best performance, followed by fracture efficiency and fracture width. Fracture proppant concentration shows lower performance. Dimensionless fracture conductivity was not collected for eight-stage treatment and is not available for prediction. Again, only 200 cases were used in developing this neural network.

\subsubsection{Type III}

Figure 5-74 through Figure 5-88 show the correlation between the actual FRACPRO values and the neural network predicted values of the training set, calibration set, and verification set for each of the 5 output parameters (fracture efficiency, propped fracture length, proppant concentration, max fracture width, and fracture height). 
Figure 5-74 shows the training set results for fracture efficiency. $R^{2}$ is $0.86, r^{2}$ is 0.87 , and the correlation coefficient is 0.93 .

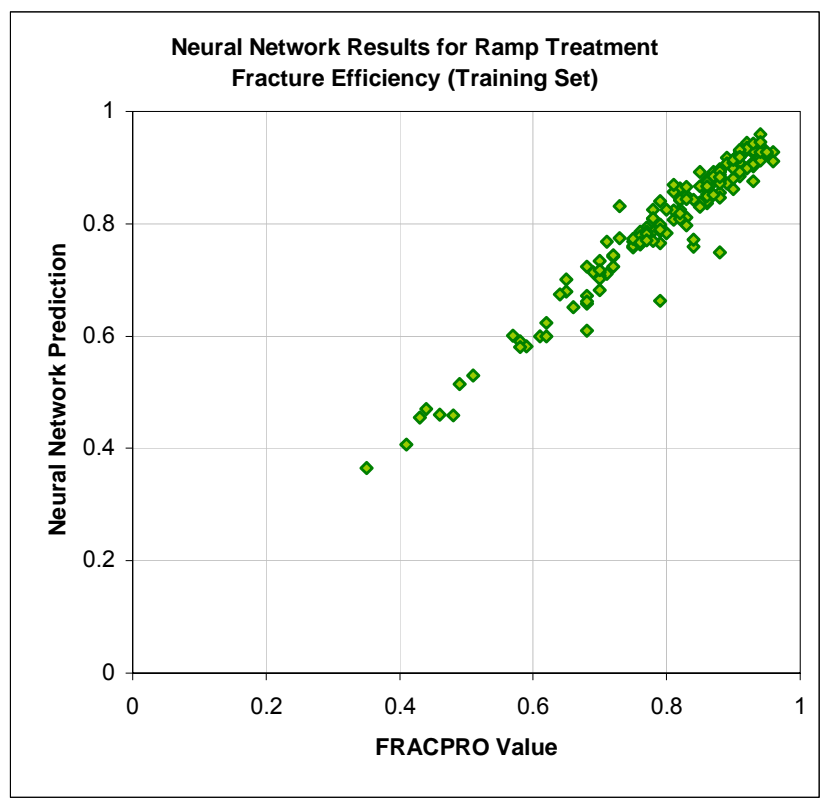

Figure 5-74: Eight Stage Type III Treatment Training Set Results for Fracture Efficiency

Figure 5-75 shows the calibration set results for fracture efficiency. $R^{2}$ is $0.94, r^{2}$ is 0.95 , and the correlation coefficient is 0.97 .

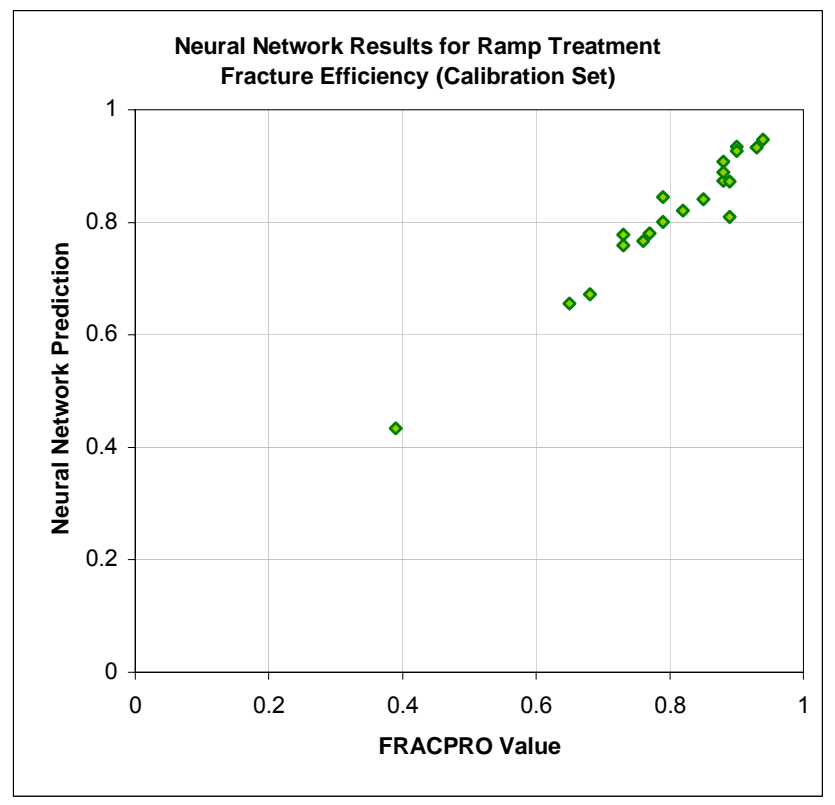

Figure 5-75: Eight Stage Type III Treatment Calibration Set Results for Fracture Efficiency 
Figure 5-76 shows the verification set results for fracture efficiency. $R^{2}$ is $0.91, r^{2}$ is 0.92 , and the correlation coefficient is 0.96 .

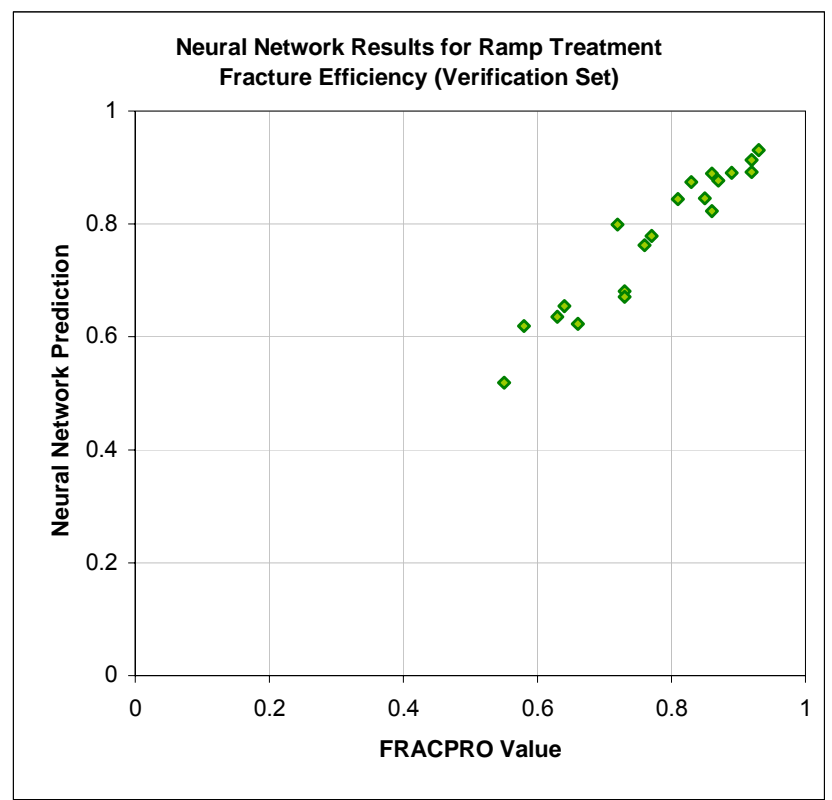

Figure 5-76: Eight Stage Type III Treatment Verification Set Results for Fracture Efficiency

Figure $5-77$ shows the training set results for propped fracture length. $R^{2}$ is $0.92, r^{2}$ is 0.92 , and the correlation coefficient is 0.96 .

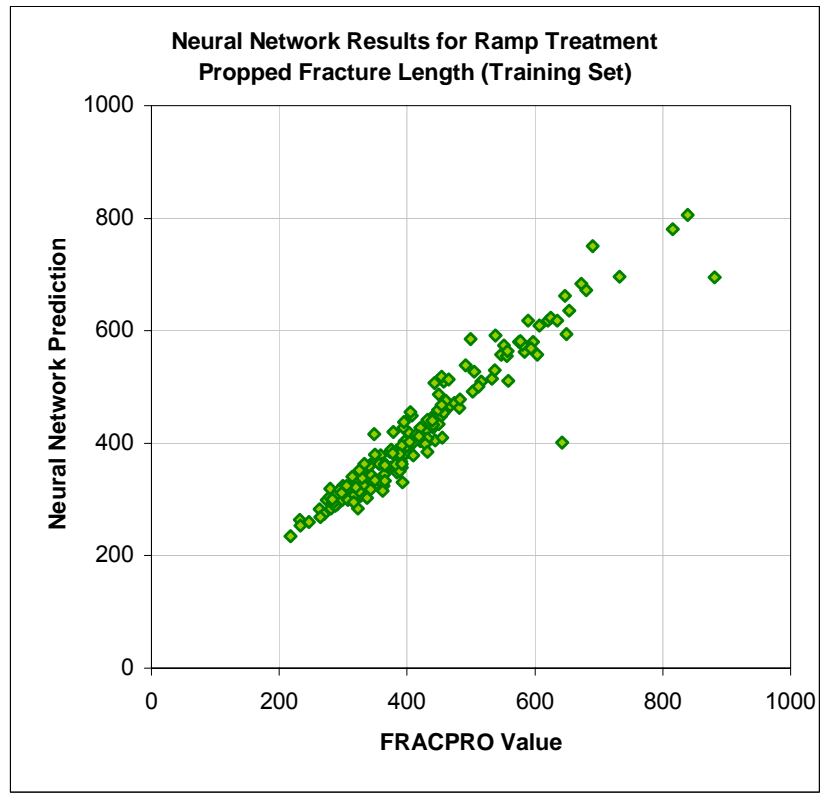

Figure 5-77: Eight Stage Type III Treatment Training Set Results for Propped Fracture Length 
Figure 5-78 shows the calibration set results for propped fracture length. $R^{2}$ is $0.94, r^{2}$ is 0.96 , and the correlation coefficient is 0.98 .

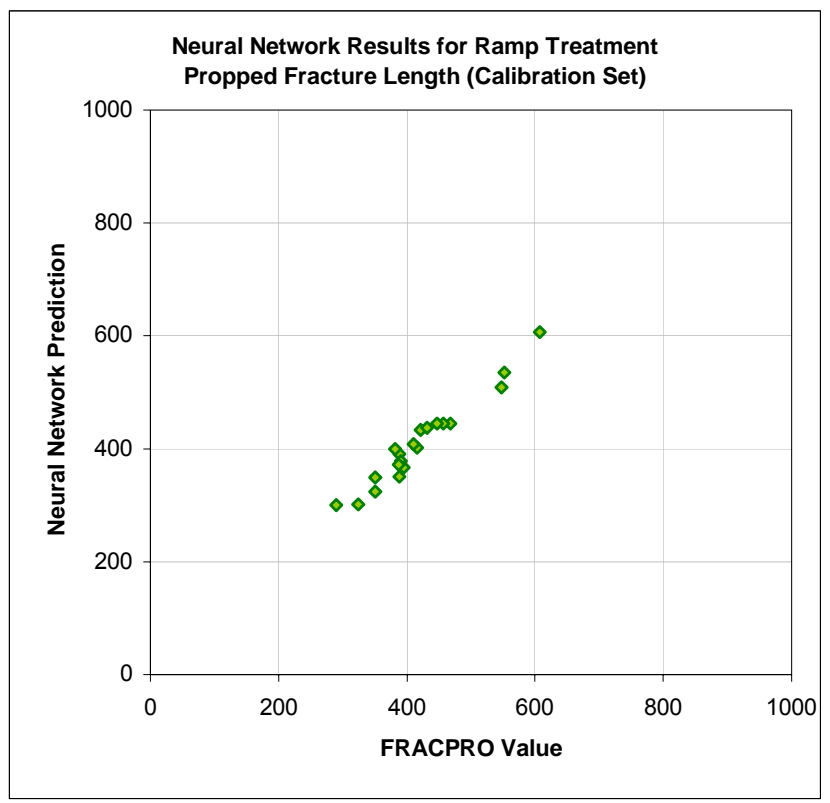

Figure 5-78: Eight Stage Type III Treatment Calibration Set Results for Propped Fracture Length

Figure 5-79 shows the verification set results for propped fracture length. $R^{2}$ is $0.94, r^{2}$ is 0.96 , and the correlation coefficient is 0.98 .

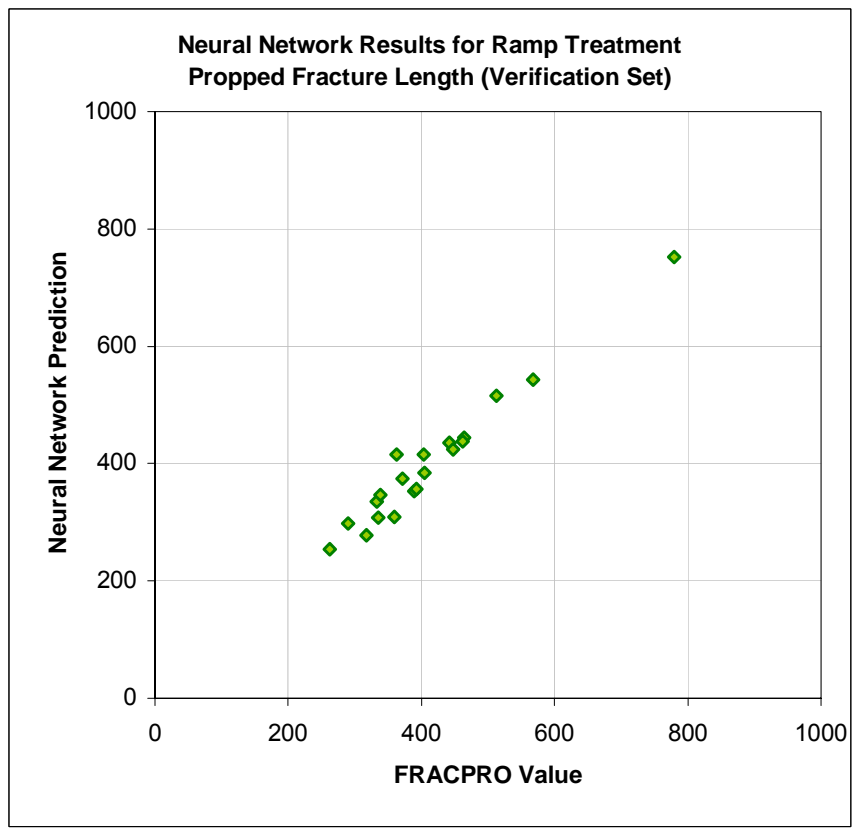

Figure 5-79: Eight Stage Type III Treatment Verification Set Results for Propped Fracture Length 
Figure $5-80$ shows the training set results for proppant concentration. $R^{2}$ is $0.92, r^{2}$ is 0.92 , and the correlation coefficient is 0.96 .

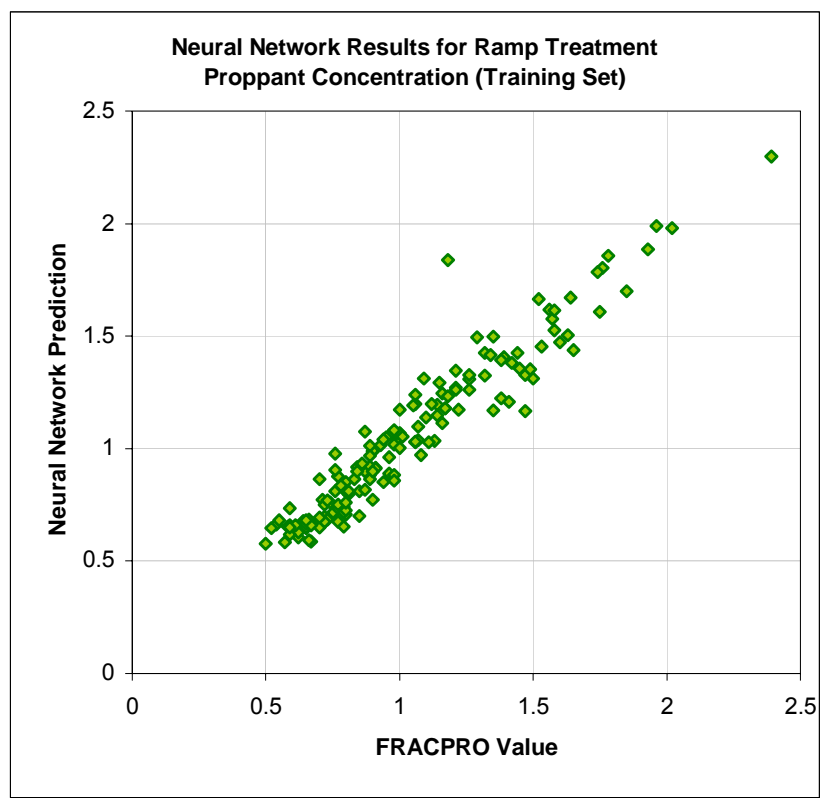

Figure 5-80: Eight Stage Type III Treatment Training Set Results for Proppant Concentration

Figure 5-81 shows the calibration set results for proppant concentration. $R^{2}$ is $0.94, r^{2}$ is 0.94 , and the correlation coefficient is 0.97 .

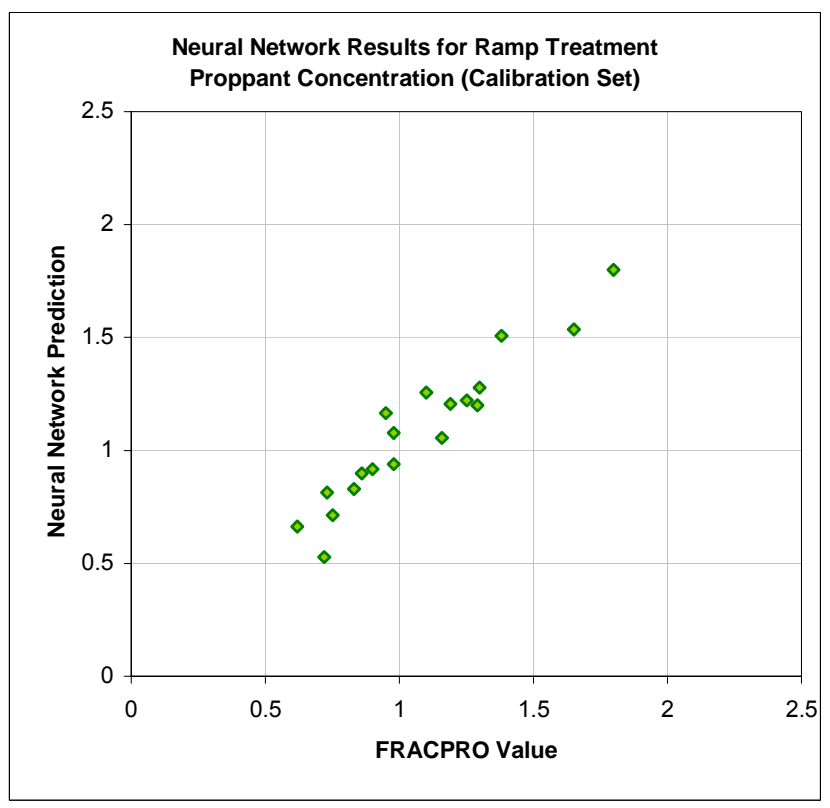

Figure 5-81: Eight Stage Type III Treatment Calibration Set Results for Proppant Concentration 
Figure 5-82 shows the verification set results for proppant concentration. $R^{2}$ is $0.89, r^{2}$ is 0.91 , and the correlation coefficient is 0.95 .

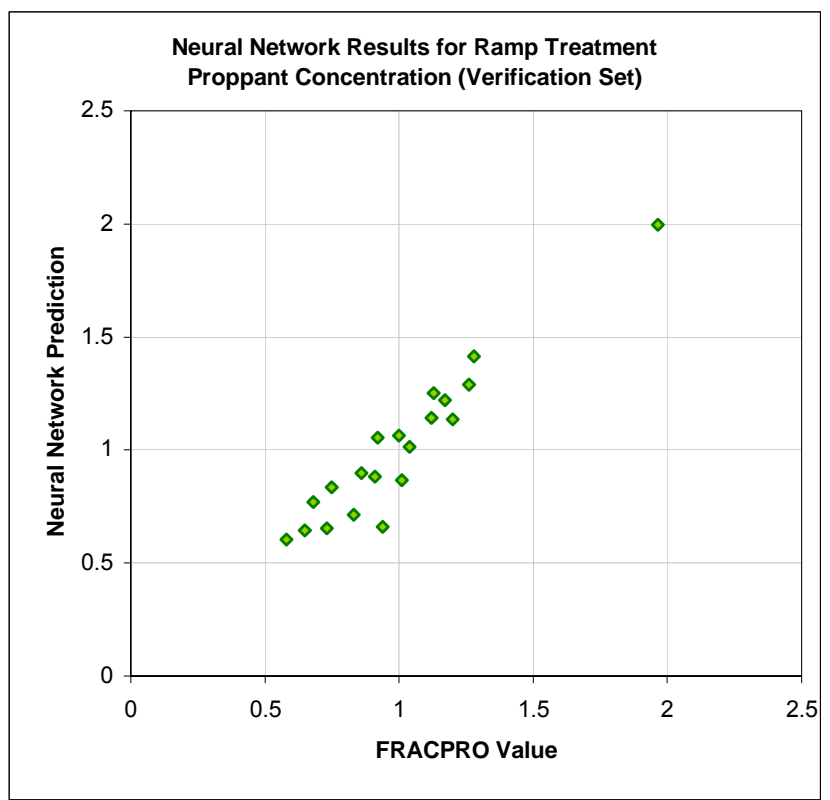

Figure 5-82: Eight Stage Type III Treatment Verification Set Results for Proppant Concentration

Figure 5-83 shows the training set results for maximum fracture width. $R^{2}$ is $0.86, r^{2}$ is 0.87 , and the correlation coefficient is 0.94 .

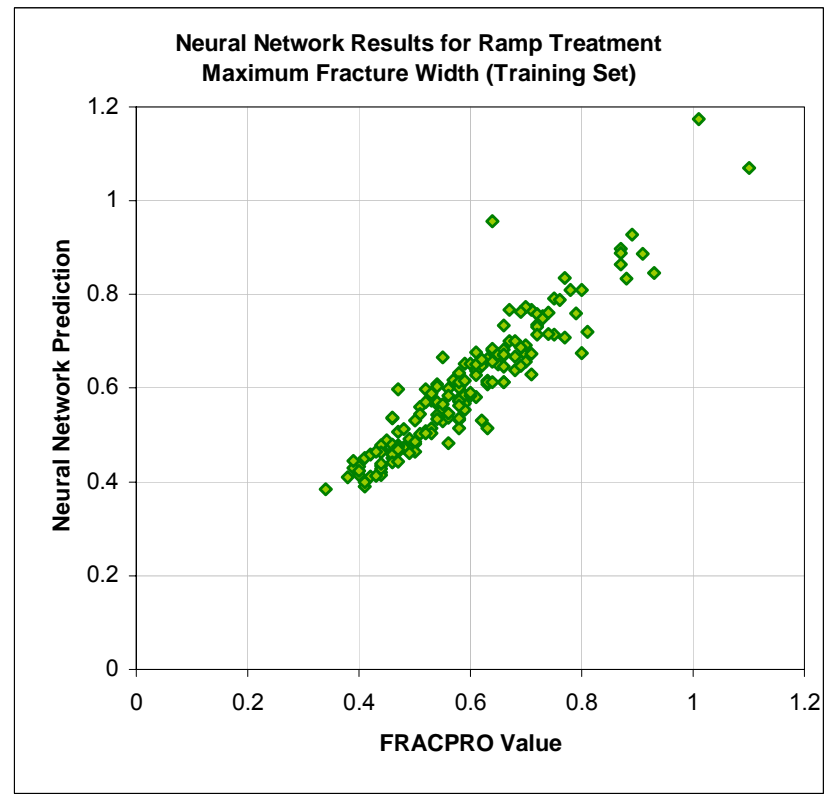

Figure 5-83: Eight Stage Type III Treatment Training Set Results for Maximum Fracture Width 
Figure 5-84 shows the calibration set results for maximum fracture width. $R^{2}$ is $0.92, r^{2}$ is 0.93 , and the correlation coefficient is 0.96 .

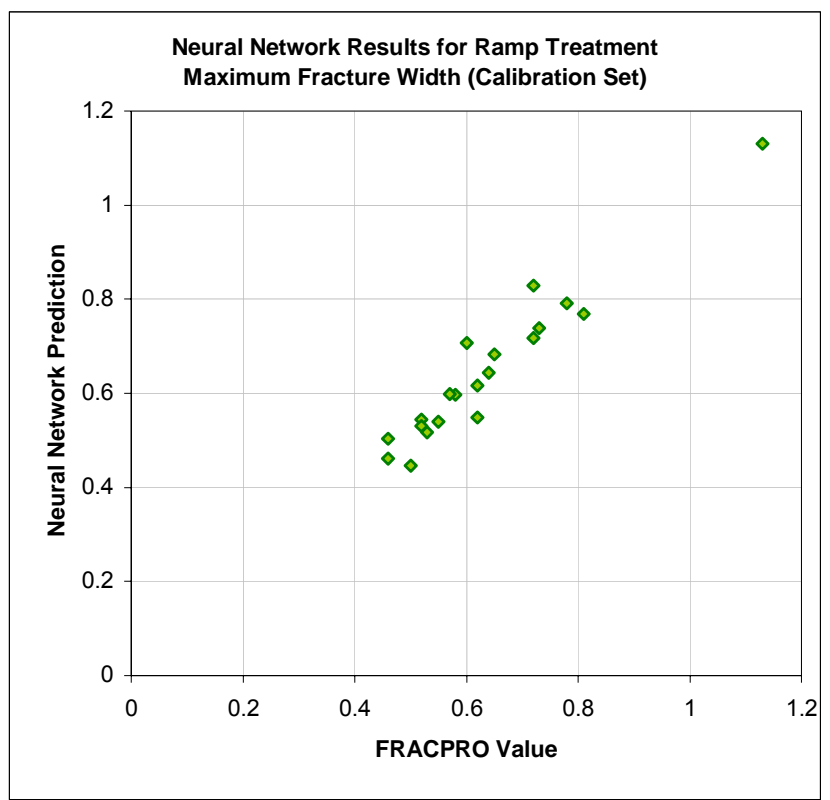

Figure 5-84: Eight Stage Type III Treatment Calibration Set Results for Maximum Fracture Width

Figure 5-85 shows the verification set results for maximum fracture width. $R^{2}$ is $0.93, r^{2}$ is 0.94 , and the correlation coefficient is 0.97 .

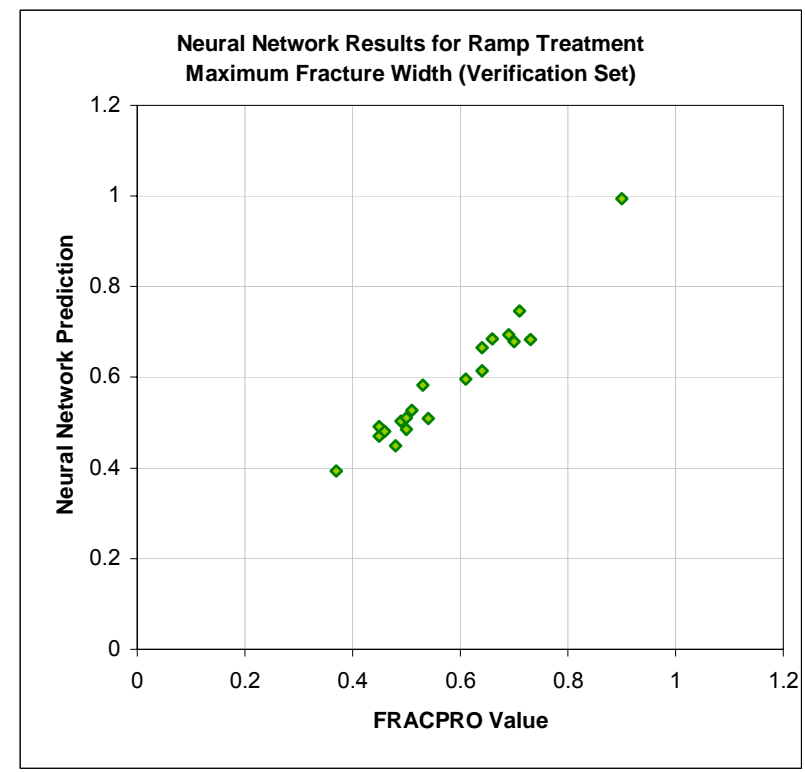

Figure 5-85: Eight Stage Type III Treatment Verification Set Results for Maximum Fracture Width 
Figure 5-86 shows the training set results for fracture height. $R^{2}$ is $0.84, r^{2}$ is 0.85 , and the correlation coefficient is 0.92 .

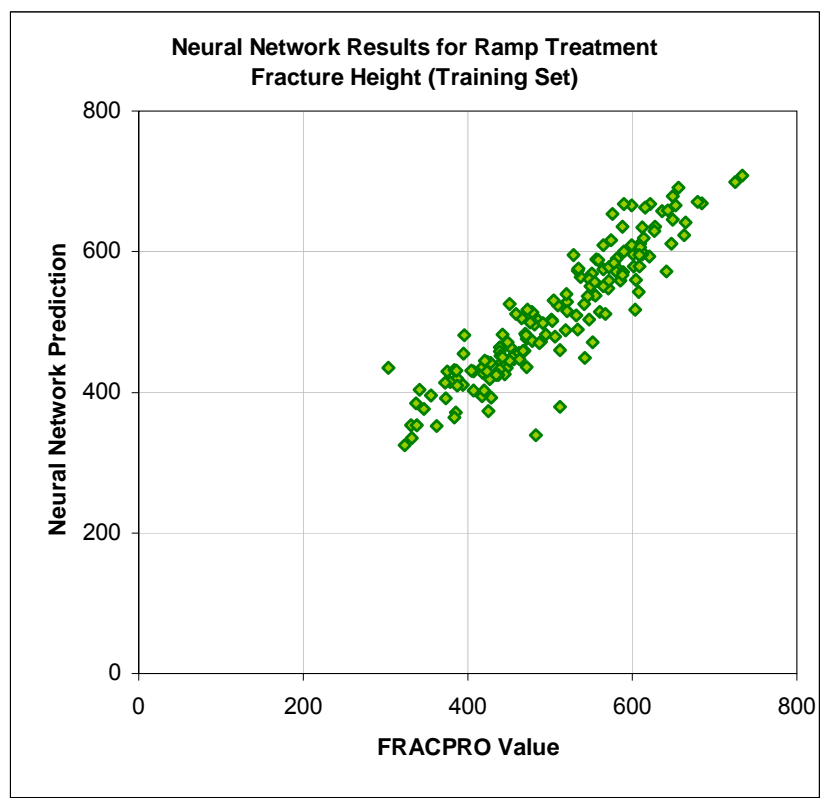

Figure 5-86: Eight Stage Type III Treatment Training Set Results for Fracture Height

Figure $5-87$ shows the calibration set results for fracture height. $R^{2}$ is $0.88, r^{2}$ is 0.88 , and the correlation coefficient is 0.94 .

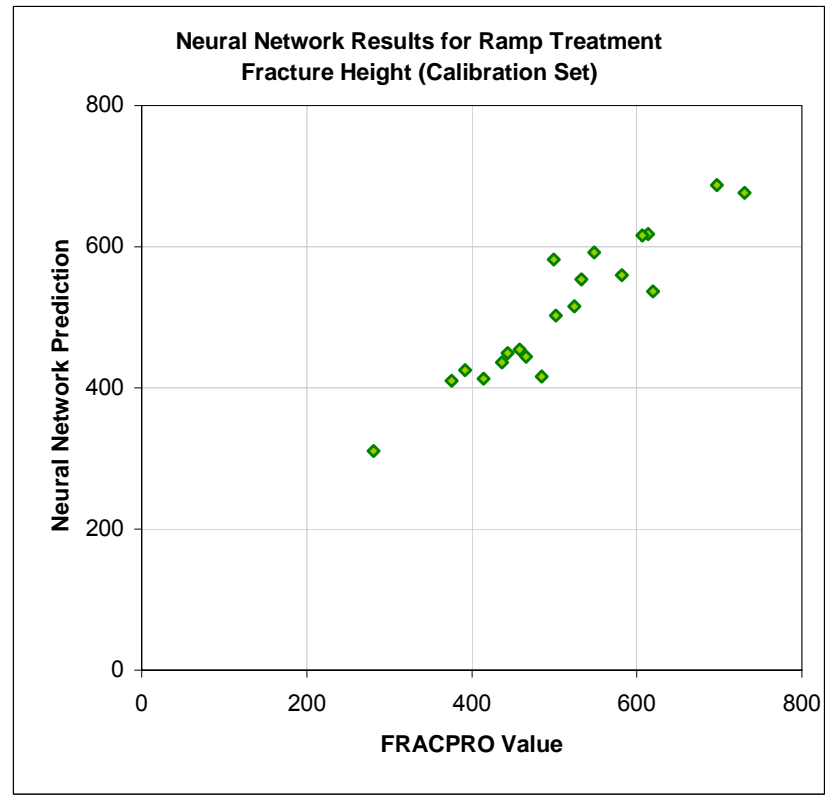

Figure 5-87: Eight Stage Type III Treatment Calibration Set Results for Fracture Height 
Figure 5-88 shows the verification set results for fracture height. $R^{2}$ is $0.90, r^{2}$ is 0.92 , and the correlation coefficient is 0.96 .

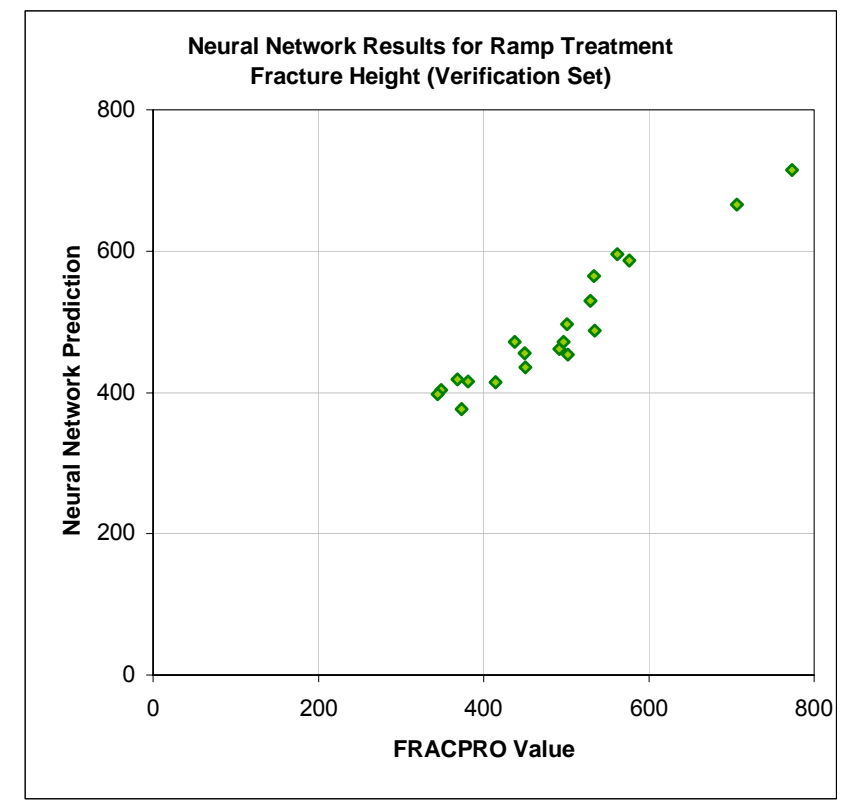

Figure 5-88: Eight Stage Type III Treatment Verification Set Results for Fracture Height

The details of the results for each of the five output parameters in the training, calibration, and verification sets can be seen in Table 5-11.

\begin{tabular}{|c|c|c|c|c|c|}
\hline & $\begin{array}{l}\text { Fracture } \\
\text { Efficiency }\end{array}$ & $\begin{array}{l}\text { Propped } \\
\text { Fracture Length }\end{array}$ & $\begin{array}{c}\text { Proppant } \\
\text { Concentration }\end{array}$ & $\begin{array}{l}\text { Max Fracture } \\
\text { Width }\end{array}$ & Fracture Height \\
\hline \multicolumn{6}{|l|}{ Training Set (162 cases) } \\
\hline $\mathrm{R}^{2}$ & 0.8601 & 0.9184 & 0.9163 & 0.8605 & 0.8426 \\
\hline$r^{2}$ & 0.8653 & 0.9187 & 0.9198 & 0.8742 & 0.8460 \\
\hline Correlation Coefficient, $r$ & 0.9302 & 0.9585 & 0.9591 & 0.9350 & 0.9198 \\
\hline \multicolumn{6}{|l|}{ Calibration Set (20 cases) } \\
\hline $\mathrm{R}^{2}$ & 0.9424 & 0.9377 & 0.9386 & 0.9157 & 0.8787 \\
\hline$r^{2}$ & 0.9493 & 0.9567 & 0.9432 & 0.9255 & 0.8796 \\
\hline Correlation Coefficient, $r$ & 0.9743 & 0.9781 & 0.9712 & 0.9620 & 0.9379 \\
\hline \multicolumn{6}{|l|}{ Verification Set ( 20 cases) } \\
\hline $\mathrm{R}^{2}$ & 0.9129 & 0.9436 & 0.8866 & 0.9251 & 0.8976 \\
\hline$r^{2}$ & 0.9198 & 0.9567 & 0.9068 & 0.9414 & 0.9162 \\
\hline Correlation Coefficient, $r$ & 0.9590 & 0.9781 & 0.9522 & 0.9702 & 0.9572 \\
\hline
\end{tabular}

Table 5-11: Eight Stage Type III Treatment Neural Network Results

As with the other two eight-stage treatment neural networks, 202 cases were used in training. Fracture length and fracture height show the best performance, followed by fracture efficiency and fracture width. Fracture proppant concentration shows lower performance. Dimensionless fracture conductivity was not collected for the eight-stage treatment and is not available for prediction. 


\subsubsection{Summary}

Figure 5-8 through Figure 5-88 show the training, calibration and verification sets for the five neural networks developed for treatment design.

The ramp and the five-stage neural networks exhibit better performance of prediction since more data was used in training the systems. In both cases, the fracture length, fracture height, and fracture width have high $\mathrm{R}^{2}$ and correlation coefficient values. With few exceptions, the cases are very close to the $45^{\circ}$ line in each set. The dimensionless conductivity ratio shows the lowest prediction performance in both neural networks. Even though $\mathrm{R}^{2}$ and the correlation coefficient are good, the training, calibration, and verification plots show sparser distribution along the $45^{\circ}$ line.

The three types of eight-stage neural networks were trained using only a third of the volume of data used for the ramp and six-stage networks. As a result, the expected performance of the neural networks was not as high. However, all three networks show strong performance capabilities for fracture length and fracture height prediction. Fracture width closely follows. The lowest performance was exhibited in predicting the fracture efficiency and fracture proppant concentration. Dimensionless fracture conductivity was not collected for the eight-stage treatment and it is not available for prediction.

Among the many neural networks that were tried for each type of treatment design, the five networks presented above proved to be the best selection for the optimization model.

\subsection{Net Pressure Neural Network}

The final neural network developed in this study is the net pressure prediction network. This neural network generates the pressure profile from the equivalent stress profile. This signal is corrected using the calibration neural network to match the net pressure profile developed when using the original stress profile.

The architecture of the neural network for net pressure has three hidden layers with different activation functions, 14 inputs and 10 outputs as described in detail in section 4.14.5.3 Neural Network Design. Figure 5-89 through Figure 5-118 show the correlation between the actual FRACPRO values and the neural network predicted values of the training set, calibration set, and verification set for each of the 10 pressure point output parameters. 
Figure 5-89 shows the training set results for pressure point $1 . R^{2}$ is $0.83, r^{2}$ is 0.83 , and the correlation coefficient is 0.91 .

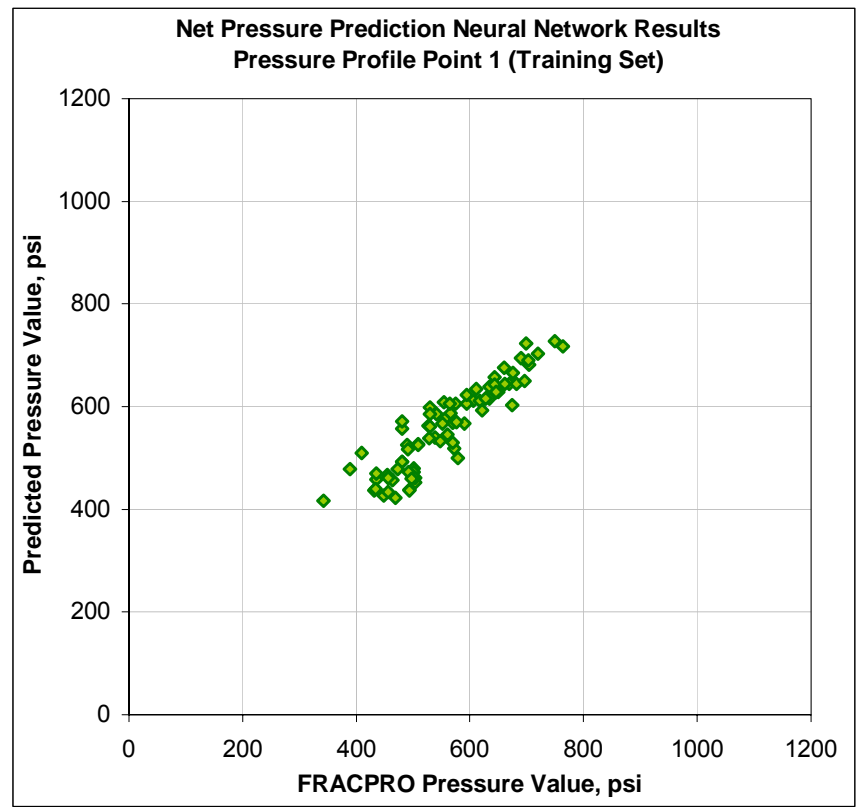

Figure 5-89: Net Pressure Neural Network Training Set Results for Pressure Point 1

Figure $5-90$ shows the calibration set results for pressure point $1 . R^{2}$ is $0.73, r^{2}$ is 0.75 , and the correlation coefficient is 0.86 .

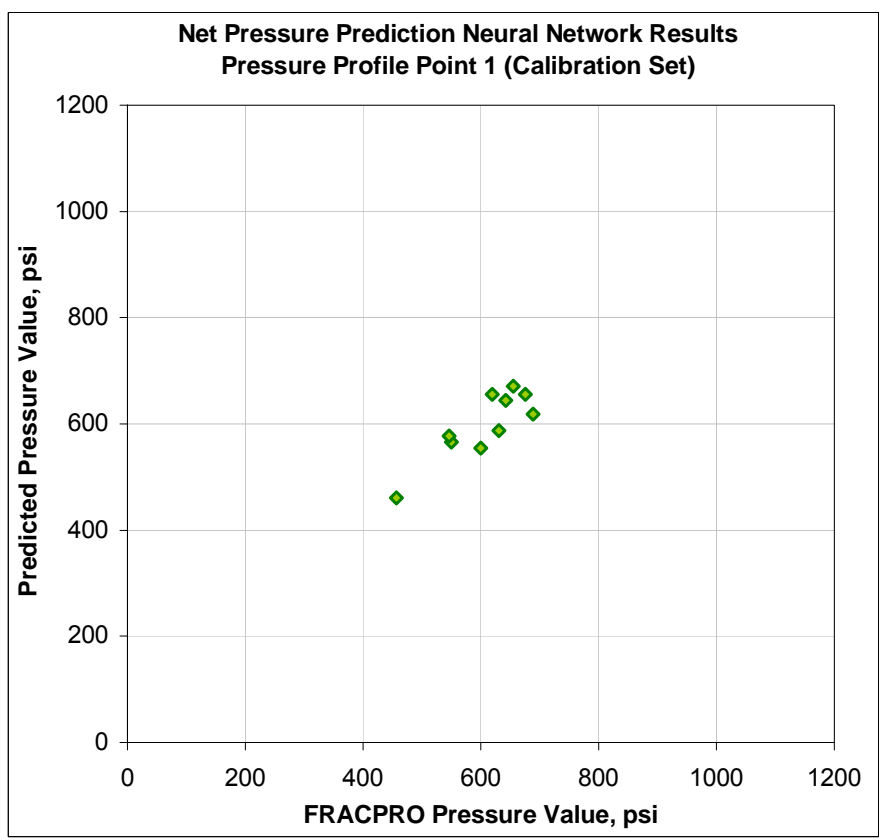

Figure 5-90: Net Pressure Neural Network Calibration Set Results for Pressure Point 1 
Figure 5-91 shows the verification set results for pressure point $1 . R^{2}$ is $0.81, r^{2}$ is 0.84 , and the correlation coefficient is 0.92 .

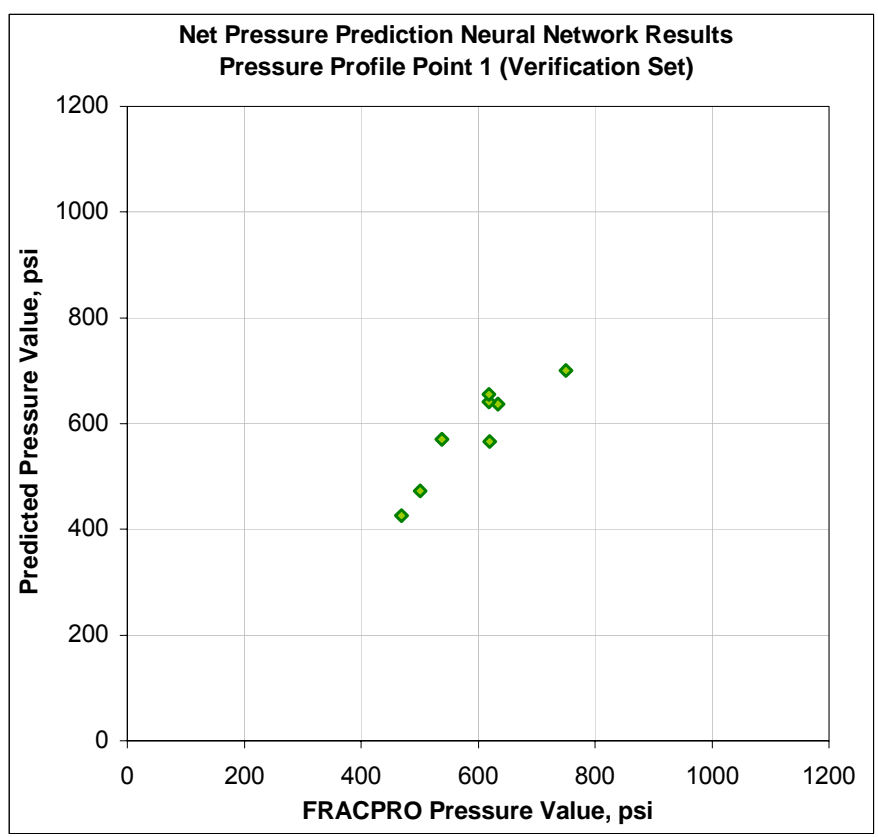

Figure 5-91: Net Pressure Neural Network Verification Set Results for Pressure Point 1

Figure 5-92 shows the training set results for pressure point $2 . R^{2}$ is $0.80, r^{2}$ is 0.80 , and the correlation coefficient is 0.89 .

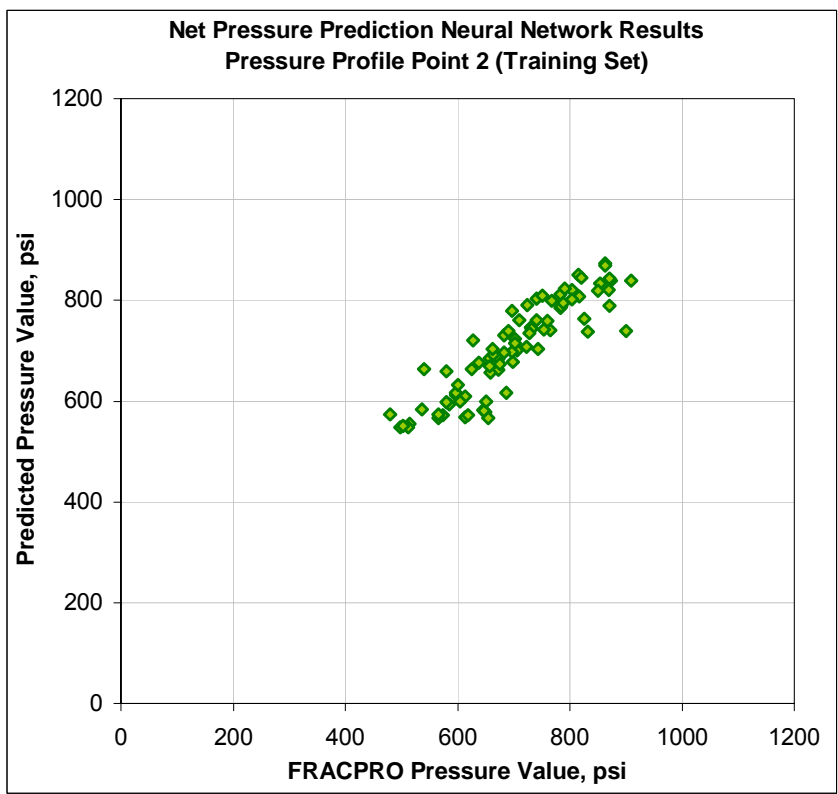

Figure 5-92: Net Pressure Neural Network Training Set Results for Pressure Point 2 
Figure 5-93 shows the calibration set results for pressure point $2 . R^{2}$ is $0.80, r^{2}$ is 0.84 , and the correlation coefficient is 0.92 .

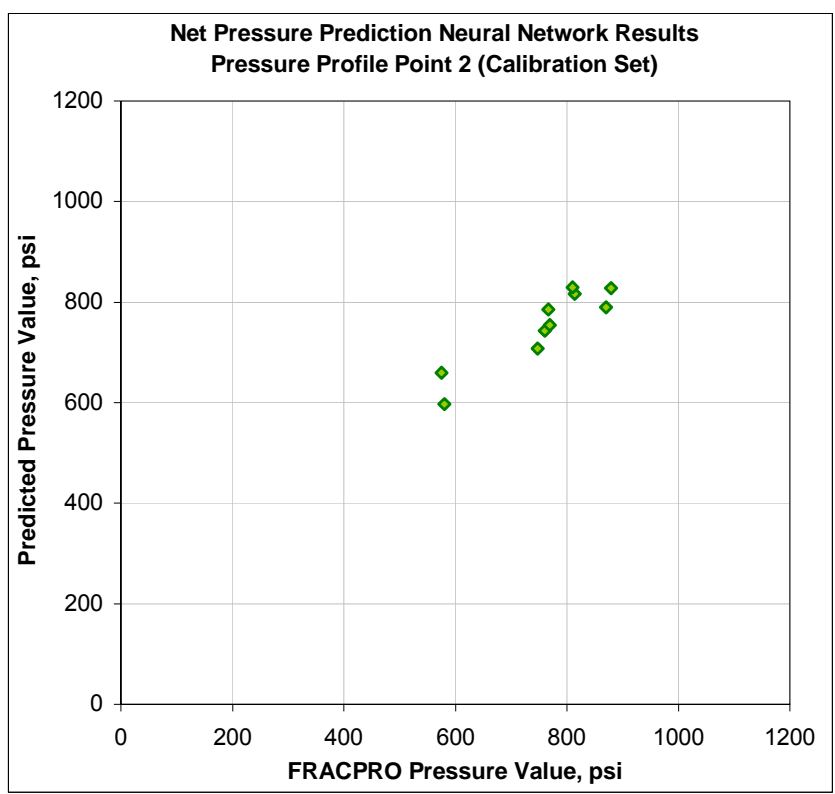

Figure 5-93: Net Pressure Neural Network Calibration Set Results for Pressure Point 2

Figure 5-94 shows the verification set results for pressure point $2 . R^{2}$ is $0.78, r^{2}$ is 0.81 , and the correlation coefficient is 0.90 .

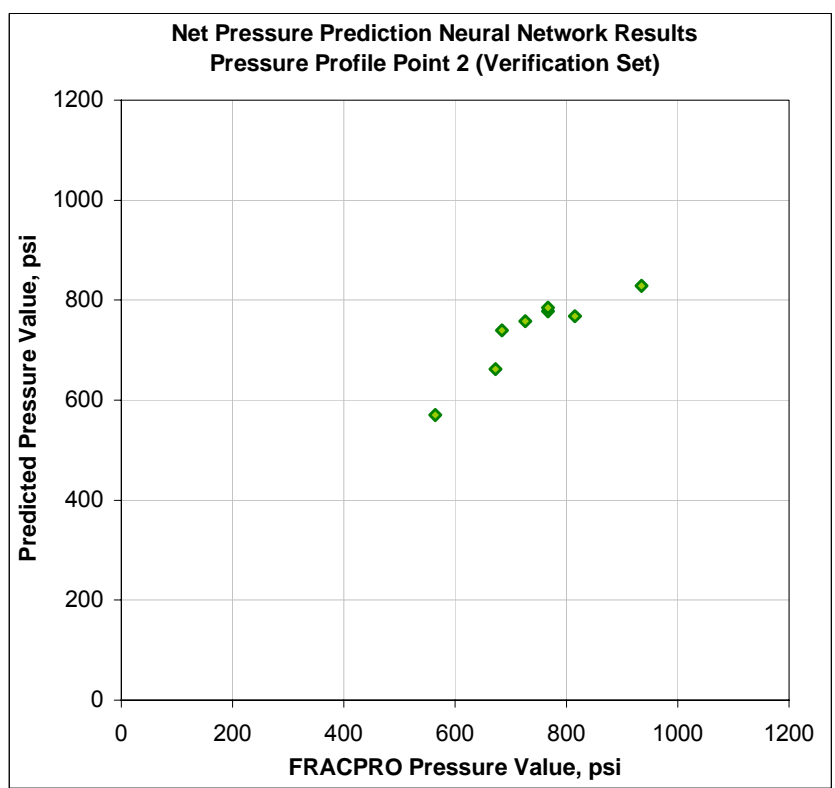

Figure 5-94: Net Pressure Neural Network Verification Set Results for Pressure Point 2 
Figure 5-95 shows the training set results for pressure point $3 . R^{2}$ is $0.81, r^{2}$ is 0.81 , and the correlation coefficient is 0.90 .

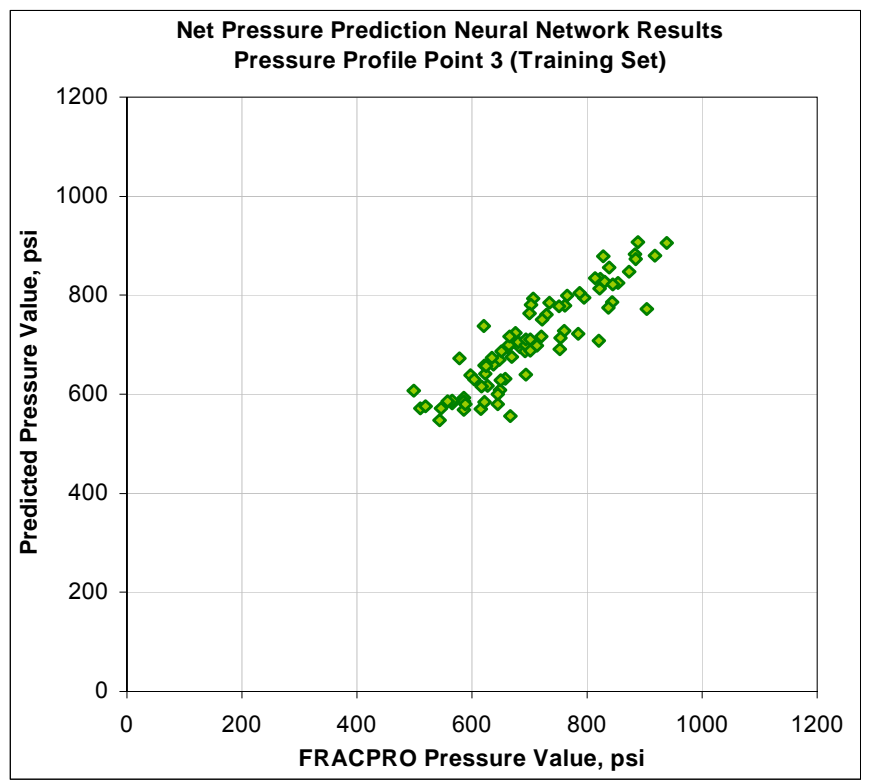

Figure 5-95: Net Pressure Neural Network Training Set Results for Pressure Point 3

Figure 5-96 shows the calibration set results for pressure point $3 . R^{2}$ is $0.82, r^{2}$ is 0.85 , and the correlation coefficient is 0.92 .

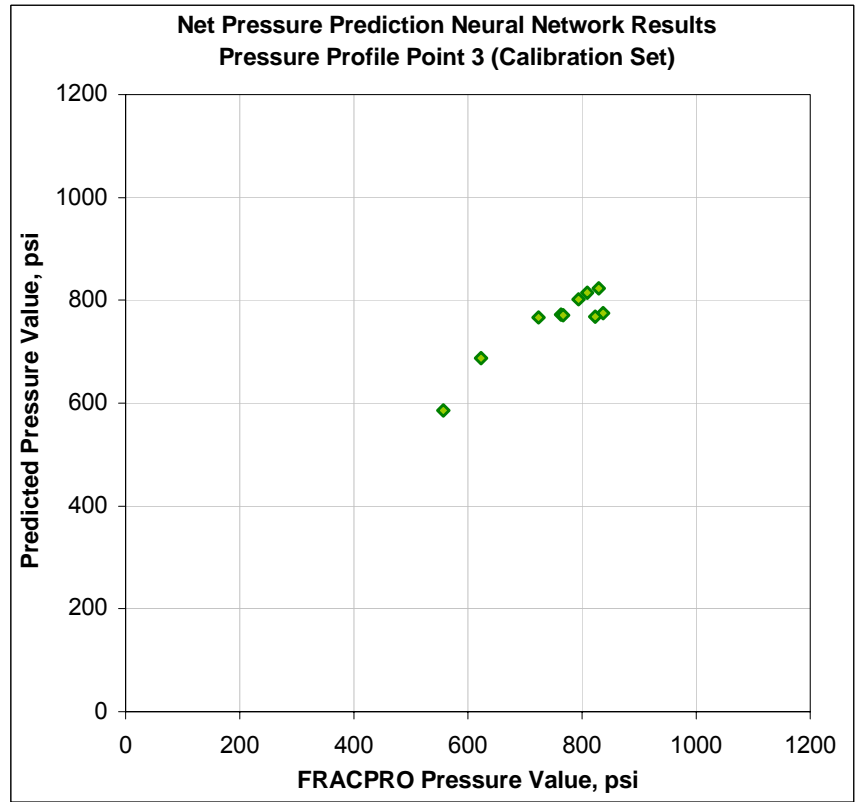

Figure 5-96: Net Pressure Neural Network Calibration Set Results for Pressure Point 3 
Figure 5-97 shows the verification set results for pressure point $3 . R^{2}$ is $0.79, r^{2}$ is 0.81 , and the correlation coefficient is 0.90 .

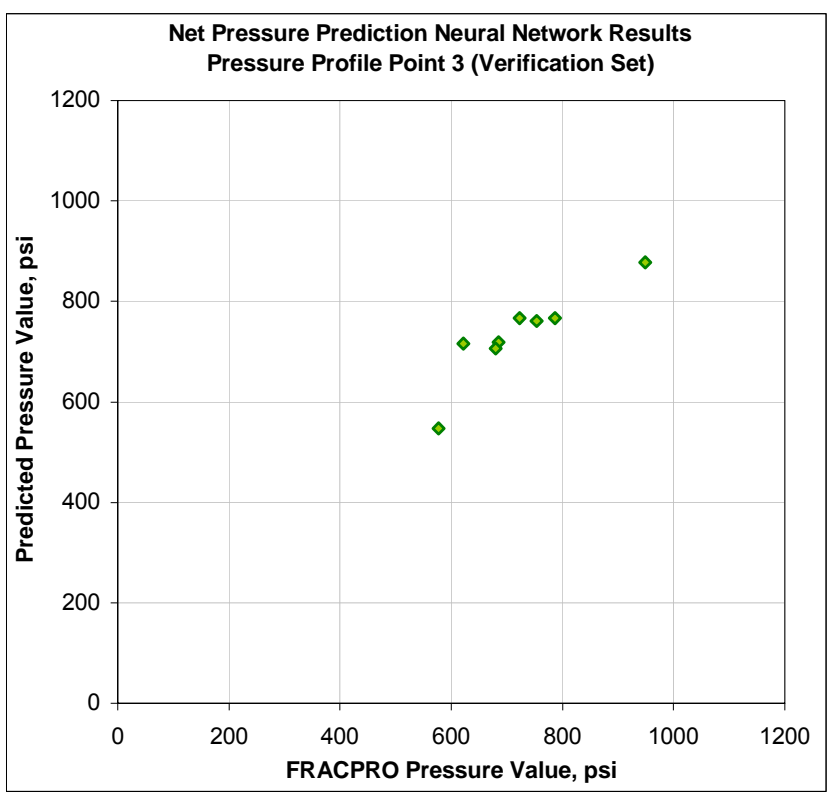

Figure 5-97: Net Pressure Neural Network Verification Set Results for Pressure Point 3

Figure 5-98 shows the training set results for pressure point $4 . R^{2}$ is $0.82, r^{2}$ is 0.82 , and the correlation coefficient is 0.91 .

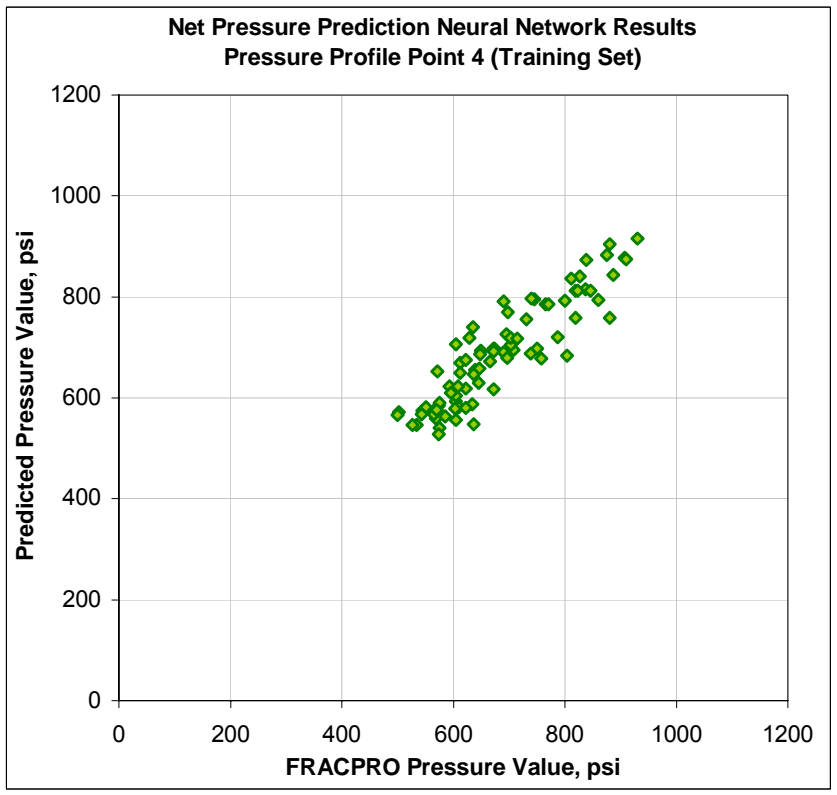

Figure 5-98: Net Pressure Neural Network Training Set Results for Pressure Point 4 
Figure 5-99 shows the calibration set results for pressure point $4 . R^{2}$ is $0.85, r^{2}$ is 0.87 , and the correlation coefficient is 0.92 .

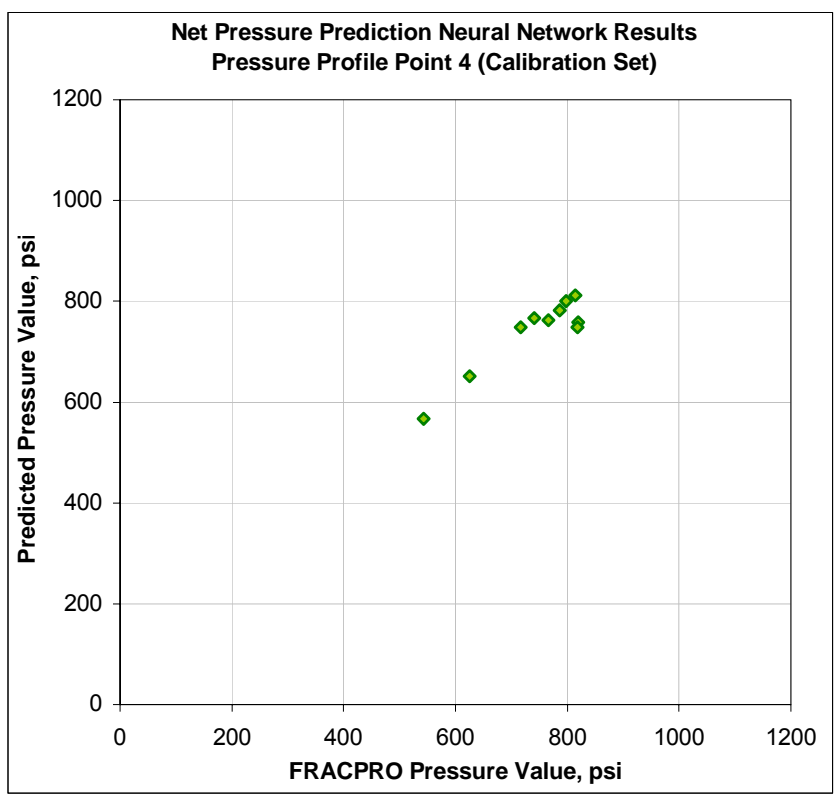

Figure 5-99: Net Pressure Neural Network Calibration Set Results for Pressure Point 4

Figure $5-100$ shows the verification set results for pressure point $4 . R^{2}$ is $0.86, r^{2}$ is 0.92 , and the correlation coefficient is 0.96 .

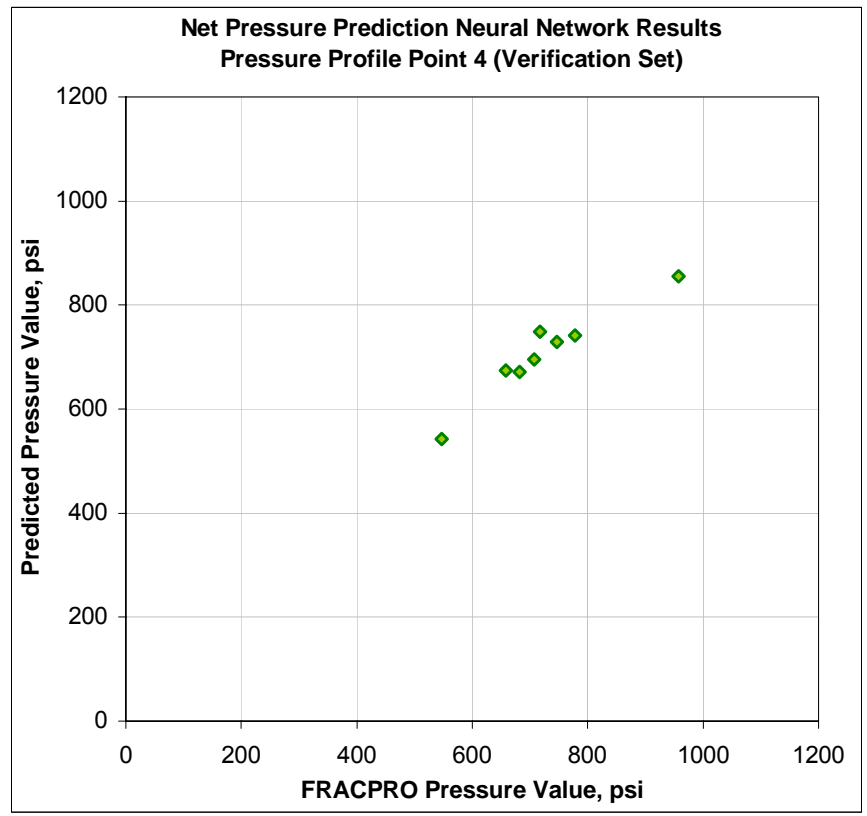

Figure 5-100: Net Pressure Neural Network Verification Set Results for Pressure Point 4 
Figure $5-101$ shows the training set results for pressure point $5 . R^{2}$ is $0.83, r^{2}$ is 0.83 , and the correlation coefficient is 0.91 .

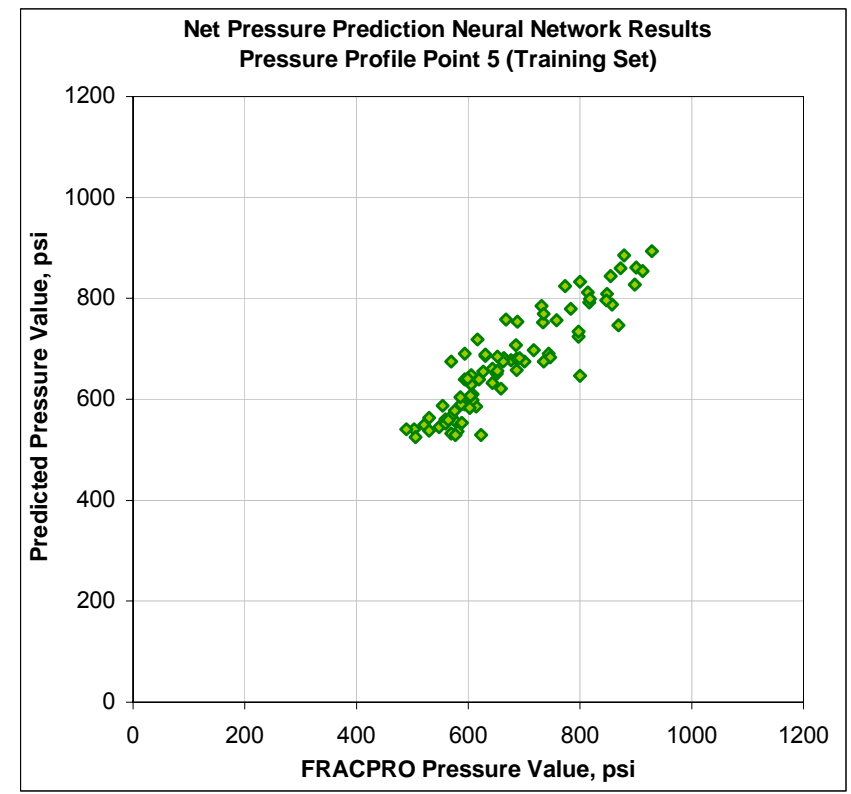

Figure 5-101: Net Pressure Neural Network Training Set Results for Pressure Point 5

Figure $5-102$ shows the calibration set results for pressure point $5 . R^{2}$ is $0.84, r^{2}$ is 0.86 , and the correlation coefficient is 0.93 .

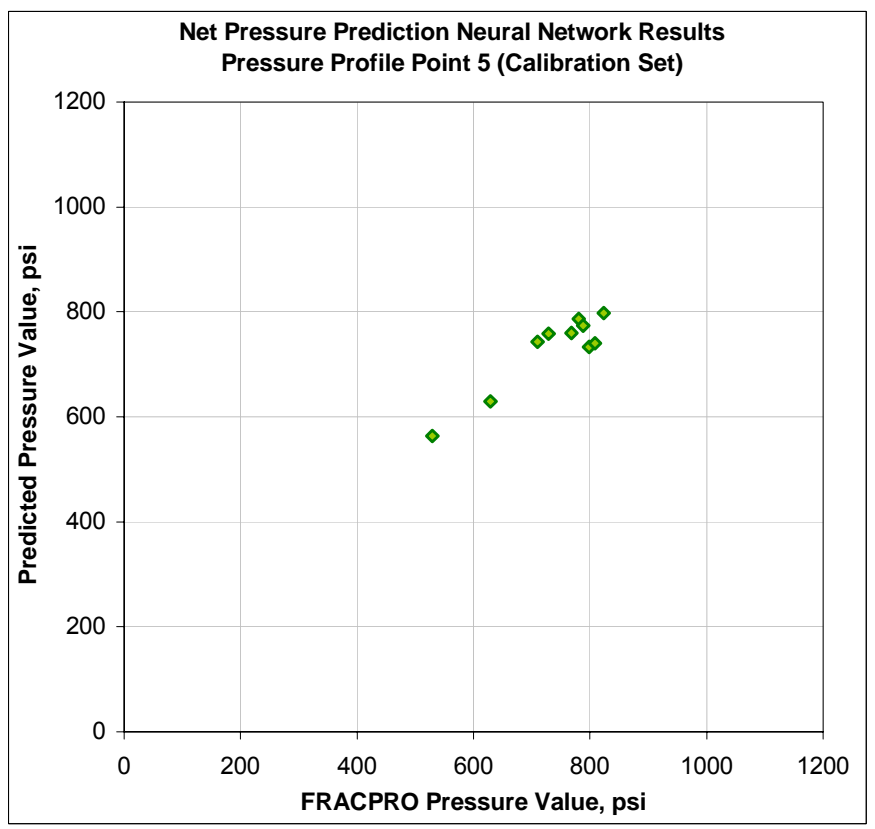

Figure 5-102: Net Pressure Neural Network Calibration Set Results for Pressure Point 5 
Figure 5-103 shows the verification set results for pressure point $5 . R^{2}$ is $0.82, r^{2}$ is 0.87 , and the correlation coefficient is 0.93 .

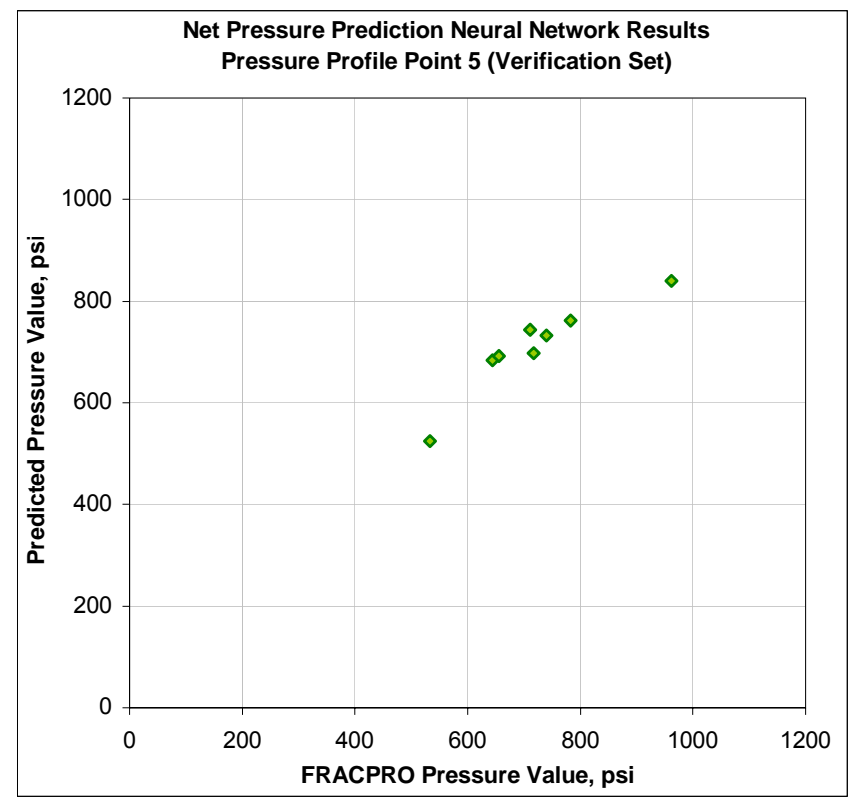

Figure 5-103: Net Pressure Neural Network Verification Set Results for Pressure Point 5

Figure 5-104 shows the training set results for pressure point $6 . R^{2}$ is $0.83, r^{2}$ is 0.83 , and the correlation coefficient is 0.91 .

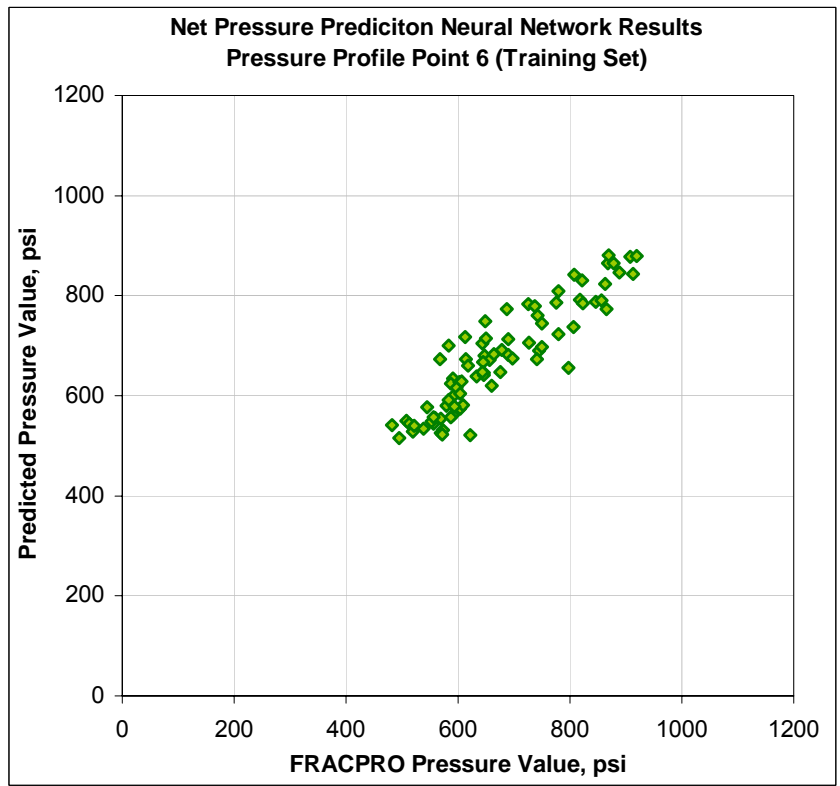

Figure 5-104: Net Pressure Neural Network Training Set Results for Pressure Point 6 
Figure $5-105$ shows the calibration set results for pressure point $6 . R^{2}$ is $0.85, r^{2}$ is 0.86 , and the correlation coefficient is 0.93 .

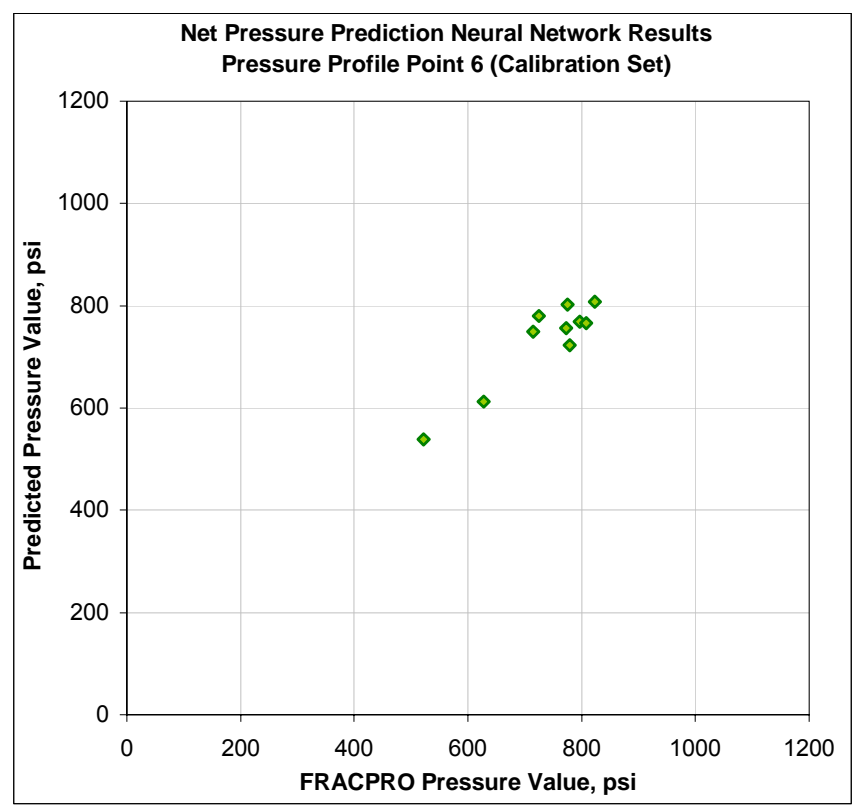

Figure 5-105: Net Pressure Neural Network Calibration Set Results for Pressure Point 6

Figure 5-106 shows the verification set results for pressure point $6 . R^{2}$ is $0.86, r^{2}$ is 0.89 , and the correlation coefficient is 0.94 .

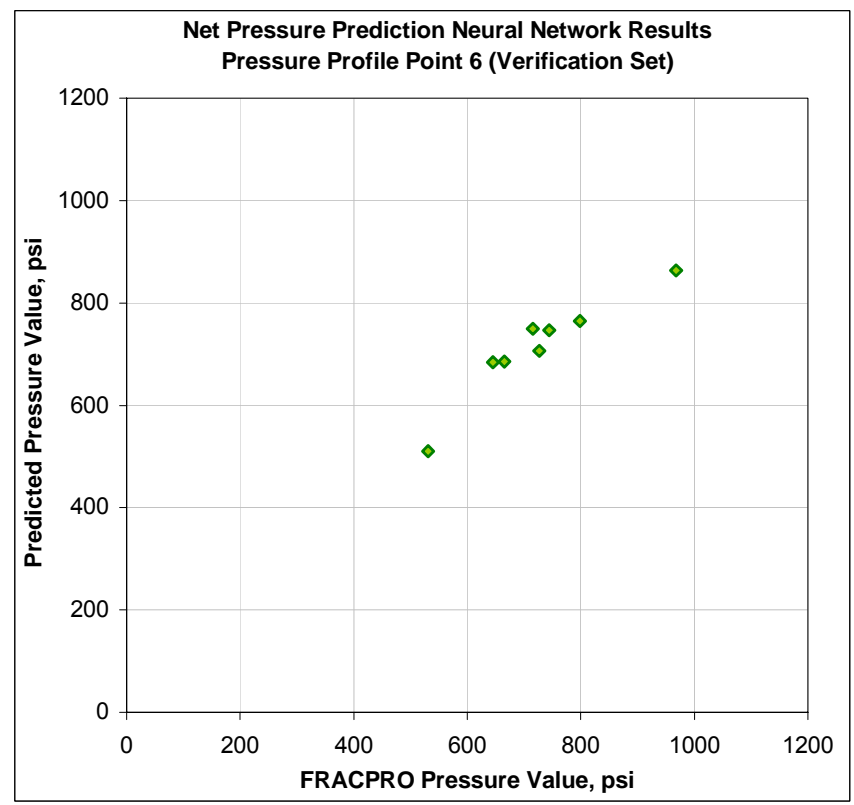

Figure 5-106: Net Pressure Neural Network Verification Set Results for Pressure Point 6 
Figure $5-107$ shows the training set results for pressure point $7 . R^{2}$ is $0.82, r^{2}$ is 0.82 , and the correlation coefficient is 0.91 .

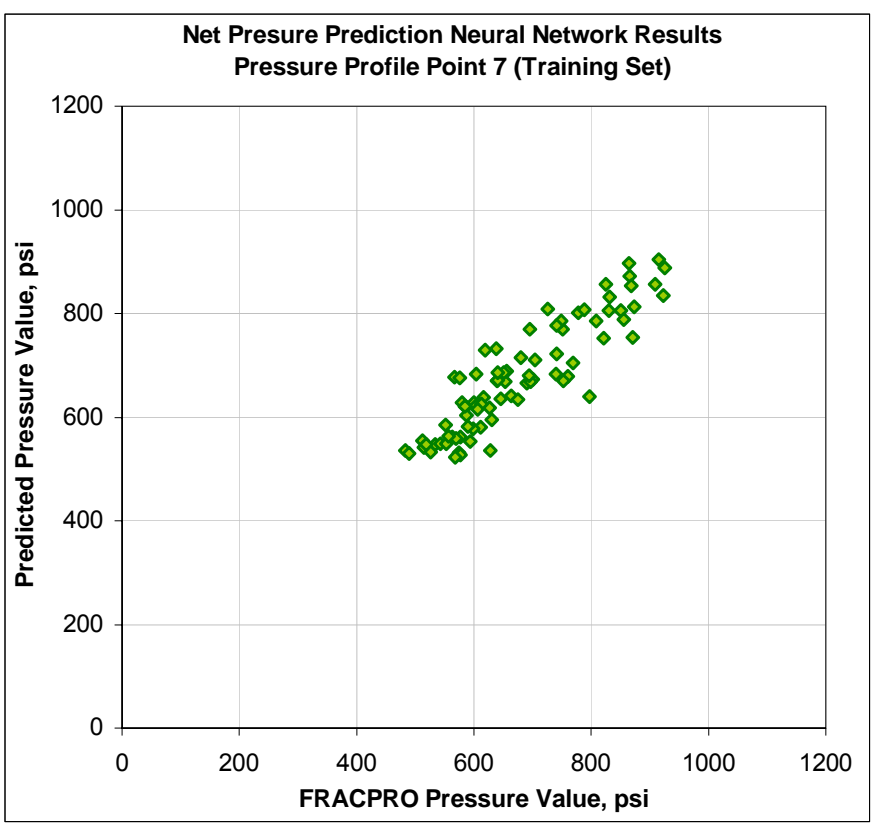

Figure 5-107: Net Pressure Neural Network Training Set Results for Pressure Point 7

Figure $5-108$ shows the calibration set results for pressure point $7 . R^{2}$ is $0.83, r^{2}$ is 0.83 , and the correlation coefficient is 0.91 .

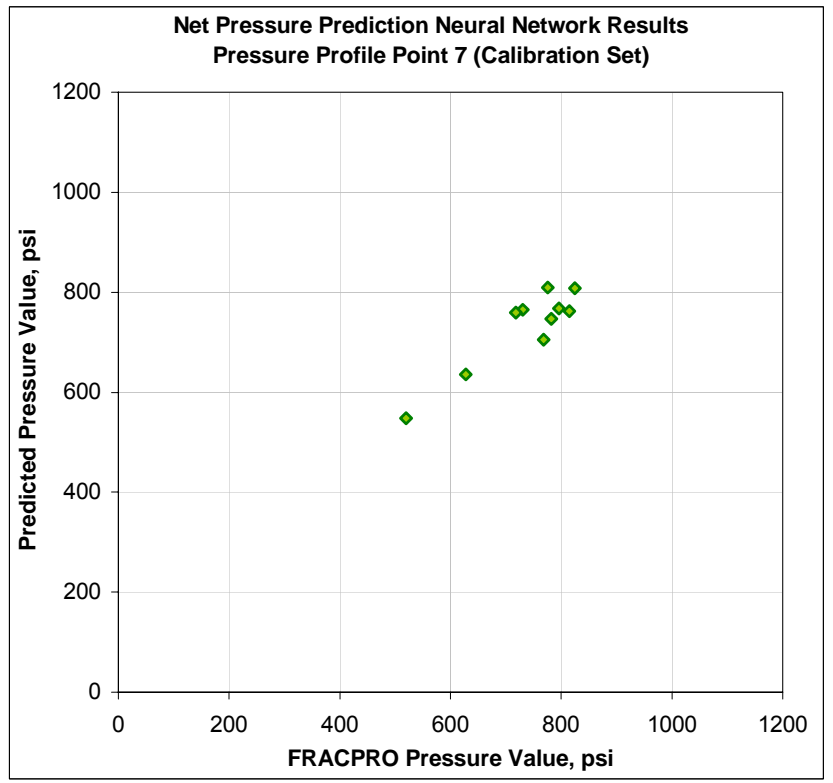

Figure 5-108: Net Pressure Neural Network Calibration Set Results for Pressure Point 7 
Figure 5-109 shows the verification set results for pressure point $7 . R^{2}$ is $0.85, r^{2}$ is 0.89 , and the correlation coefficient is 0.94 .

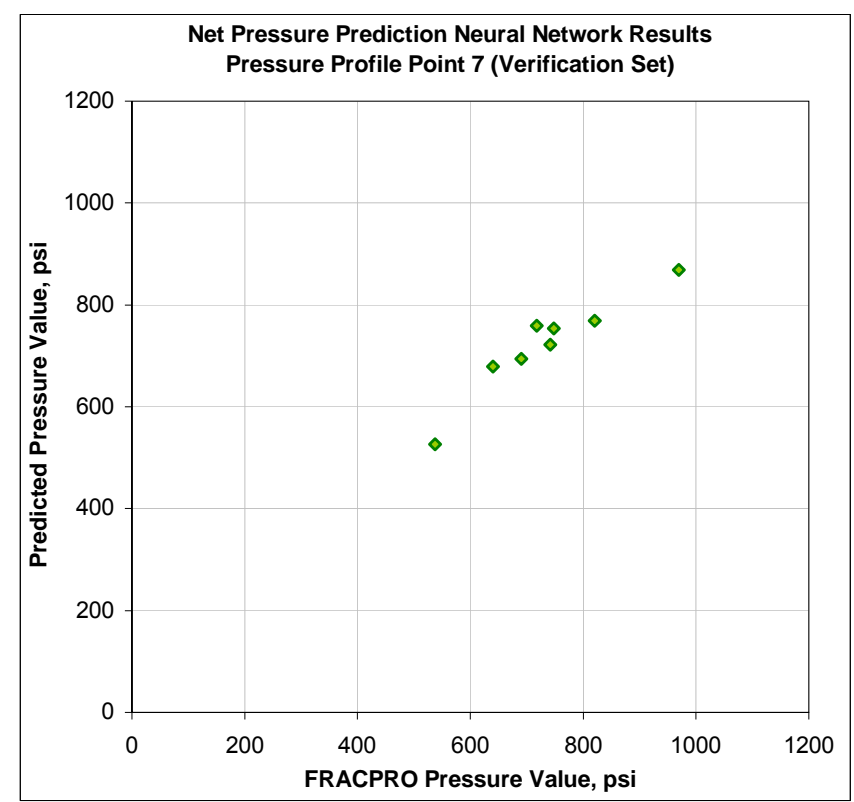

Figure 5-109: Net Pressure Neural Network Verification Set Results for Pressure Point 7

Figure $5-110$ shows the training set results for pressure point $8 . R^{2}$ is $0.83, r^{2}$ is 0.84 , and the correlation coefficient is 0.91 .

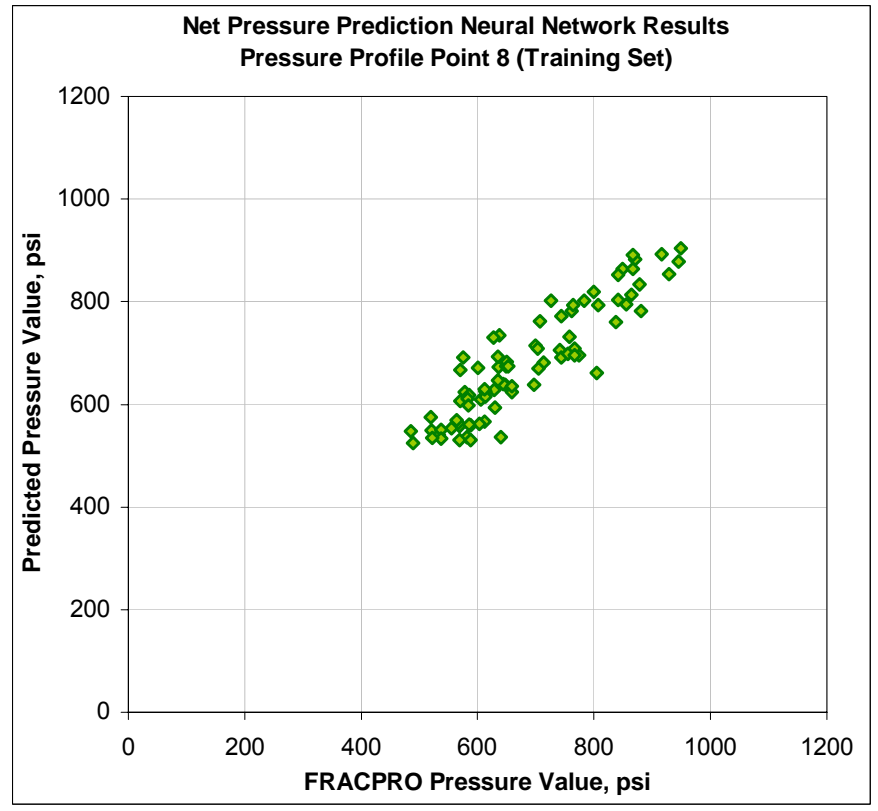

Figure 5-110: Net Pressure Neural Network Training Set Results for Pressure Point 8 
Figure $5-111$ shows the calibration set results for pressure point $8 . R^{2}$ is $0.79, r^{2}$ is 0.80 , and the correlation coefficient is 0.89 .

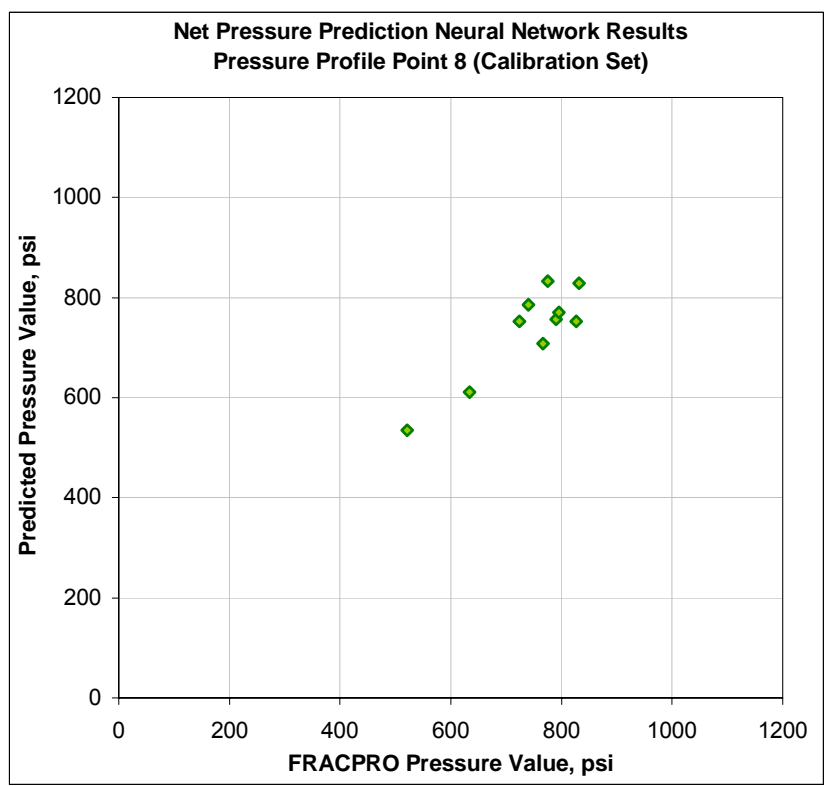

Figure 5-111: Net Pressure Neural Network Calibration Set Results for Pressure Point 8

Figure $5-112$ shows the verification set results for pressure point $8 . R^{2}$ is $0.84, r^{2}$ is 0.89 , and the correlation coefficient is 0.94 .

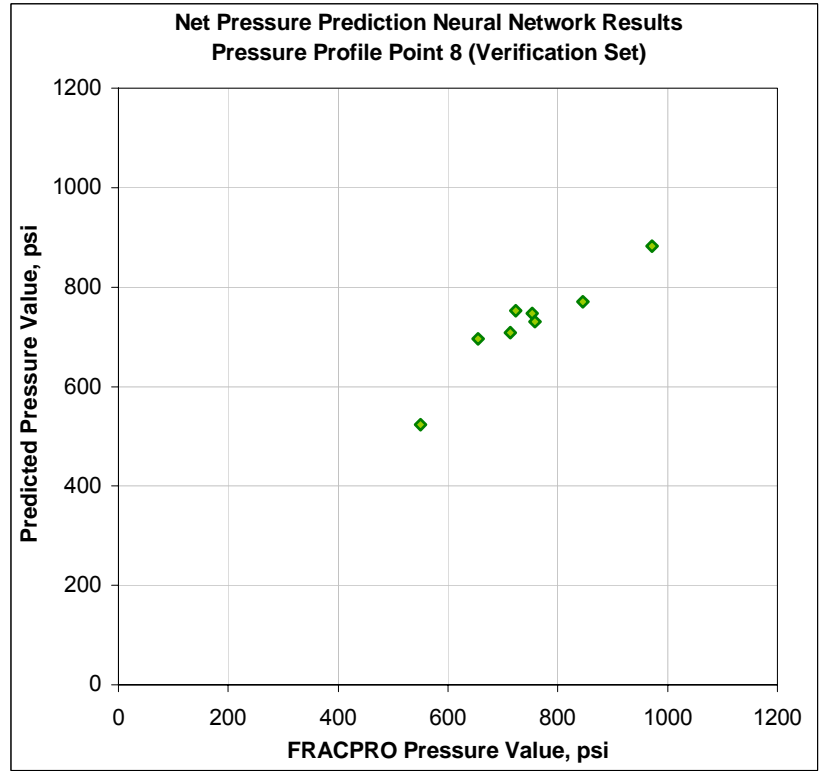

Figure 5-112: Net Pressure Neural Network Verification Set Results for Pressure Point 8 
Figure $5-113$ shows the training set results for pressure point $9 . R^{2}$ is $0.81, r^{2}$ is 0.81 , and the correlation coefficient is 0.90 .

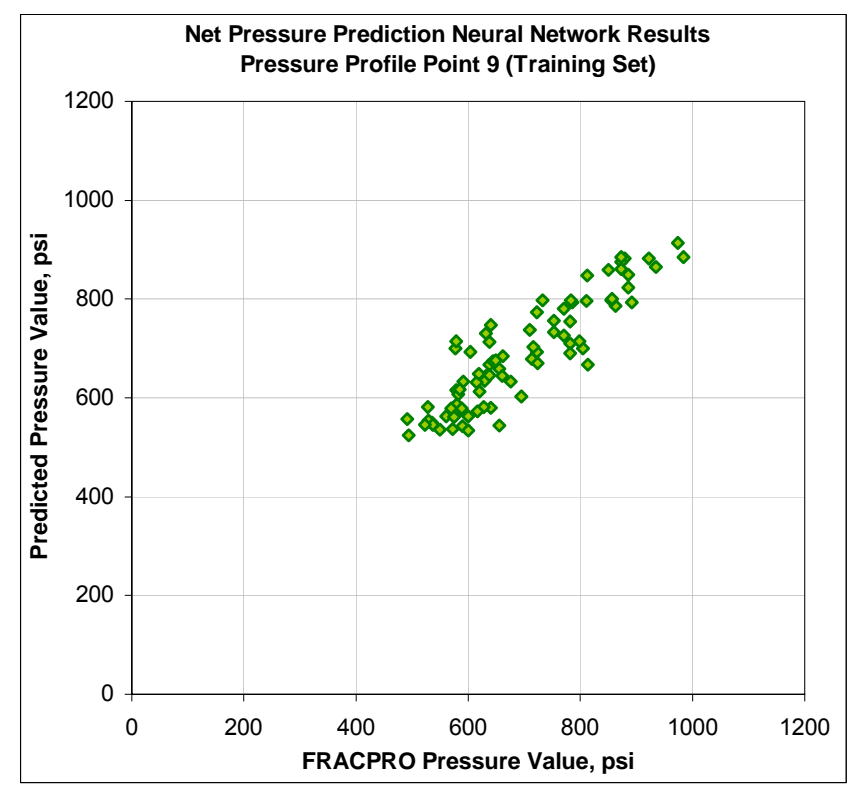

Figure 5-113: Net Pressure Neural Network Training Set Results for Pressure Point 9

Figure $5-114$ shows the calibration set results for pressure point $9 . R^{2}$ is $0.81, r^{2}$ is 0.81 , and the correlation coefficient is 0.90 .

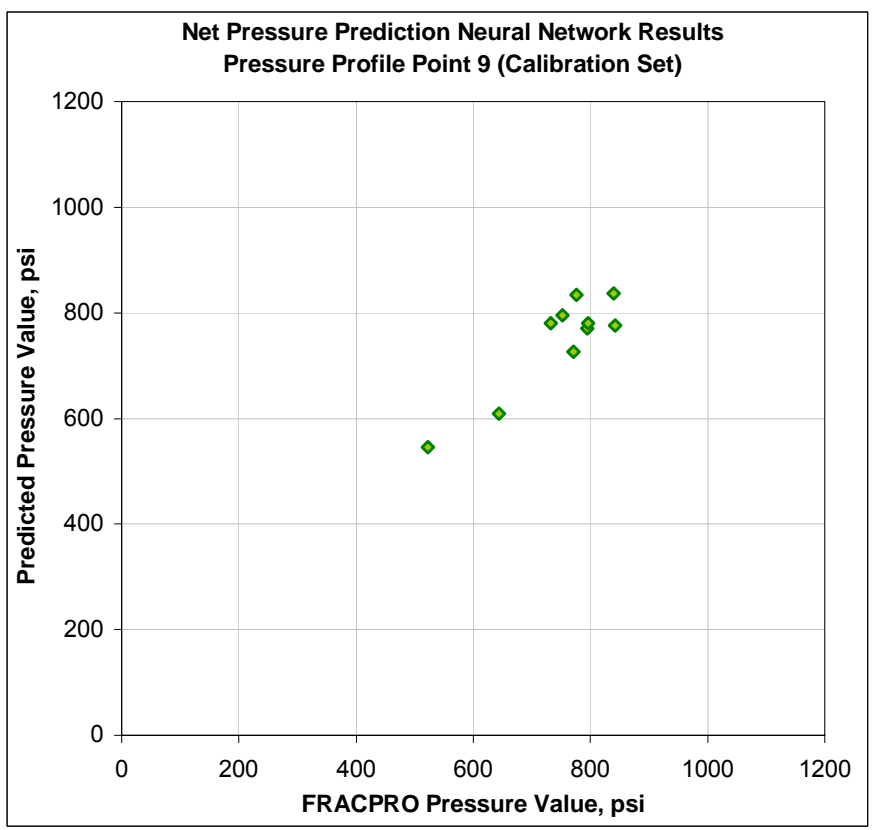

Figure 5-114: Net Pressure Neural Network Calibration Set Results for Pressure Point 9 
Figure $5-115$ shows the verification set results for pressure point $9 . R^{2}$ is $0.82, r^{2}$ is 0.85 , and the correlation coefficient is 0.92 .

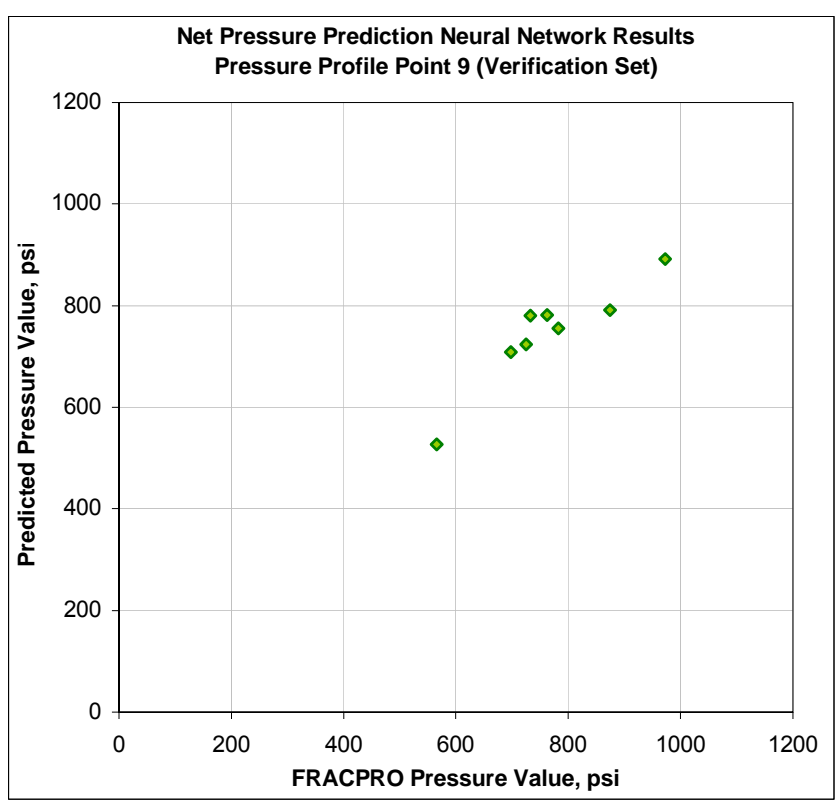

Figure 5-115: Net Pressure Neural Network Verification Set Results for Pressure Point 9

Figure $5-116$ shows the training set results for pressure point $10 . R^{2}$ is $0.79, r^{2}$ is 0.79 , and the correlation coefficient is 0.89 .

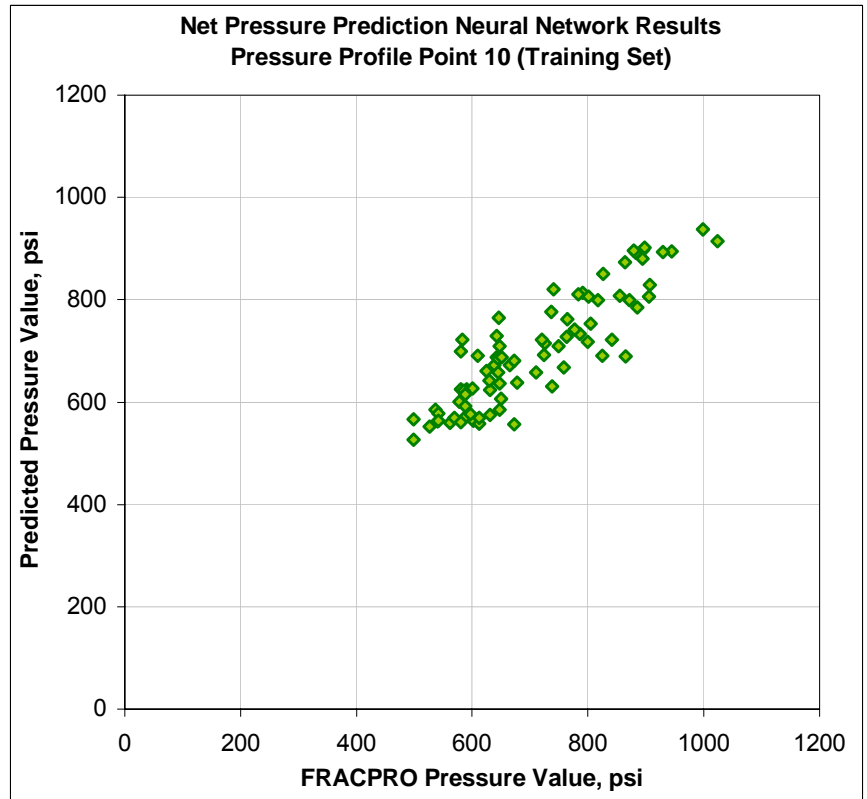

Figure 5-116: Net Pressure Neural Network Training Set Results for Pressure Point 10 
Figure $5-117$ shows the calibration set results for pressure point $10 . R^{2}$ is $0.79, r^{2}$ is 0.80 , and the correlation coefficient is 0.90 .

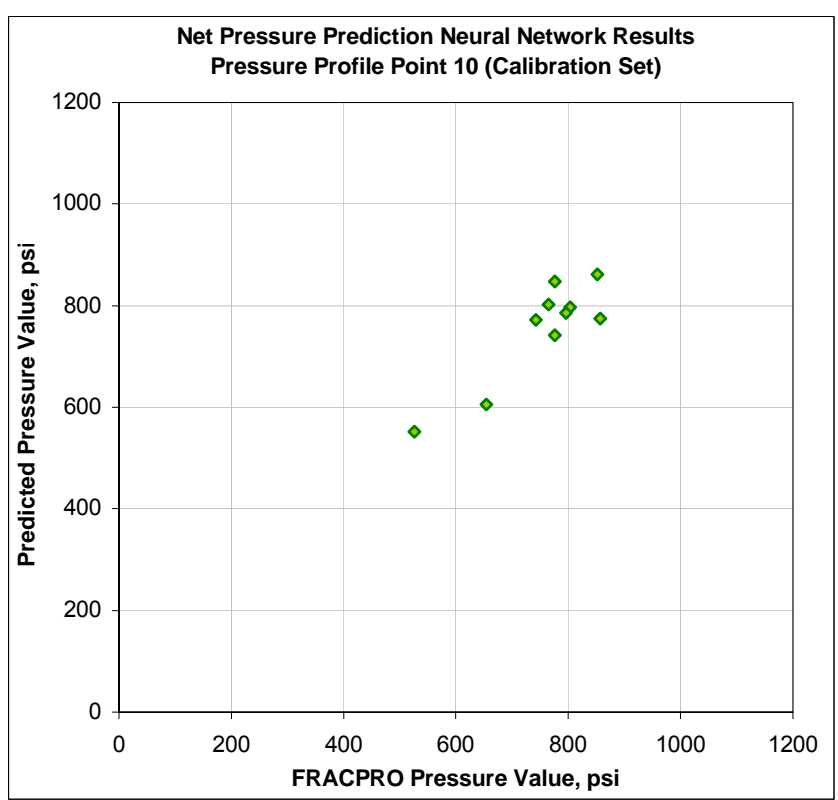

Figure 5-117: Net Pressure Neural Network Calibration Set Results for Pressure Point 10

Figure $5-118$ shows the verification set results for pressure point $10 . R^{2}$ is $0.76, r^{2}$ is 0.88 , and the correlation coefficient is 0.94 .

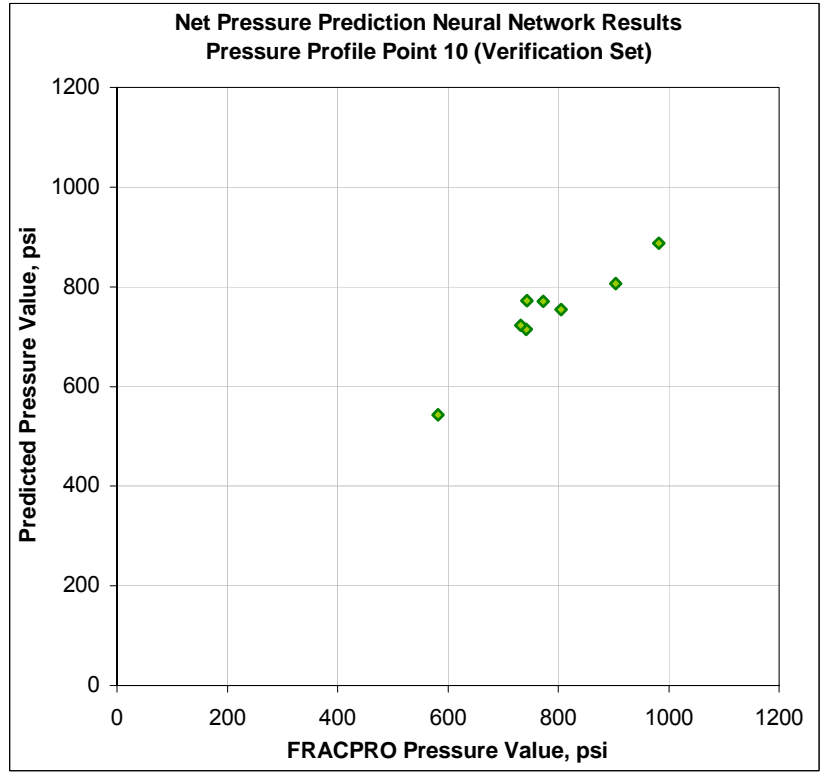

Figure 5-118: Net Pressure Neural Network Verification Set Results for Pressure Point 10 
The details of the results for each of the 10 points output pressure in the training, calibration, and verification sets can be seen in Table 5-12.

\begin{tabular}{|c|c|c|c|c|c|c|c|c|c|c|}
\hline & $\begin{array}{c}\text { Pressure } \\
\text { Profile } \\
\text { Point } 1\end{array}$ & $\begin{array}{c}\text { Pressure } \\
\text { Profile } \\
\text { Point } 2\end{array}$ & $\begin{array}{c}\text { Pressure } \\
\text { Profile } \\
\text { Point } 3\end{array}$ & $\begin{array}{l}\text { Pressure } \\
\text { Profile } \\
\text { Point } 4\end{array}$ & $\begin{array}{c}\text { Pressure } \\
\text { Profile } \\
\text { Point } 5\end{array}$ & $\begin{array}{c}\text { Pressure } \\
\text { Profile } \\
\text { Point } 6\end{array}$ & $\begin{array}{c}\text { Pressure } \\
\text { Profile } \\
\text { Point } 7\end{array}$ & $\begin{array}{c}\text { Pressure } \\
\text { Profile } \\
\text { Point } 8\end{array}$ & $\begin{array}{c}\text { Pressure } \\
\text { Profile } \\
\text { Point } 9\end{array}$ & $\begin{array}{c}\text { Pressure } \\
\text { Profile } \\
\text { Point } 10\end{array}$ \\
\hline \multicolumn{11}{|l|}{ Training Set (82 cases) } \\
\hline $\mathrm{R}^{2}$ & 0.8317 & 0.7993 & 0.8120 & 0.8230 & 0.8314 & 0.8332 & 0.8188 & 0.8341 & 0.8051 & 0.7865 \\
\hline$r^{2}$ & 0.8320 & 0.8008 & 0.8139 & 0.8242 & 0.8324 & 0.8333 & 0.8189 & 0.8356 & 0.8097 & 0.7924 \\
\hline Correlation Coefficient, $r$ & 0.9121 & 0.8949 & 0.9022 & 0.9079 & 0.9123 & 0.9128 & 0.9050 & 0.9141 & 0.8998 & 0.8902 \\
\hline \multicolumn{11}{|l|}{ Callbration Set (10 cases) } \\
\hline$R^{2}$ & 0.7331 & 0.8024 & 0.8220 & 0.8502 & 0.8346 & 0.8546 & 0.8285 & 0.7864 & 0.8068 & 0.7885 \\
\hline$r^{2}$ & 0.7469 & 0.8410 & 0.8533 & 0.8659 & 0.8578 & 0.8564 & 0.8349 & 0.8004 & 0.8131 & 0.8028 \\
\hline Correlation Coefficient, $r$ & 0.8642 & 0.9171 & 0.9238 & 0.9305 & 0.9262 & 0.9254 & 0.9138 & 0.8946 & 0.9017 & 0.8960 \\
\hline \multicolumn{11}{|l|}{ Verification Set (8 cases) } \\
\hline $\mathrm{R}^{2}$ & 0.8050 & 0.7830 & 0.7924 & 0.8552 & 0.8178 & 0.8576 & 0.8535 & 0.8394 & 0.8197 & 0.7577 \\
\hline$r^{2}$ & 0.8420 & 0.8074 & 0.8103 & 0.9222 & 0.8657 & 0.8885 & 0.8926 & 0.8901 & 0.8522 & 0.8775 \\
\hline Correlation Coefficient, $r$ & 0.9176 & 0.8986 & 0.9002 & 0.9603 & 0.9304 & 0.9426 & 0.9448 & 0.9434 & 0.9232 & 0.9368 \\
\hline
\end{tabular}

Table 5-12: Net Pressure Prediction Neural Network Results

Table 5-12 gives the $\mathrm{R}^{2}$ and correlation coefficients for each of the pressure points. It can be seen that the predication performance of the neural network is weaker at the end points (first two and last two points). Some of these points even have $R^{2}$ values under 0.8 in the calibration and verification sets. However, correlation coefficients show higher values, in most cases above 0.9. The data indicates that the middle pressure points have a better correlation in regard to the neural network prediction capability. These pressure points have higher correlation coefficients and $\mathrm{R}^{2}$ values around 0.8 .

\subsubsection{Summary}

The net pressure neural network was trained with only 100 data cases. Even with the low number of cases, the network has proven good accuracy in predicting the pressure points and the resulting pressure profiles.

Different types of neural networks were tried. Since the performance of the network is weaker for the end points, neural networks were developed that predict one point at a time in an attempt to improve performance. However, even with only one output, the performance did not improve. Moreover, a better match was observed when reconstructing the pressure signal using the 10-point neural network compared to the match observed from the 1-point networks. Similar observations were made after attempts using two neural networks of five outputs each.

After additional examination, it was concluded that using all 10 pressure points simultaneously enables the neural network to achieve a better correlation between points and 
more accurate shape of the signal. This characteristic was not achieved when using separate one-point prediction neural networks.

In Figure 5-89 through Figure 5-118, each pressure point is plotted versus the actual value separately. Unfortunately, this does not provide a clear picture of the 10-point pressure signal results. Eight cases used in the verification set (not in the training process) are presented to examine the neural network pressure prediction capability. In Figure 5-119 through Figure $5-126$, the FRACPRO pressure profile obtained using the equivalent four-layer profile and neural network predicted pressure profile is plotted.

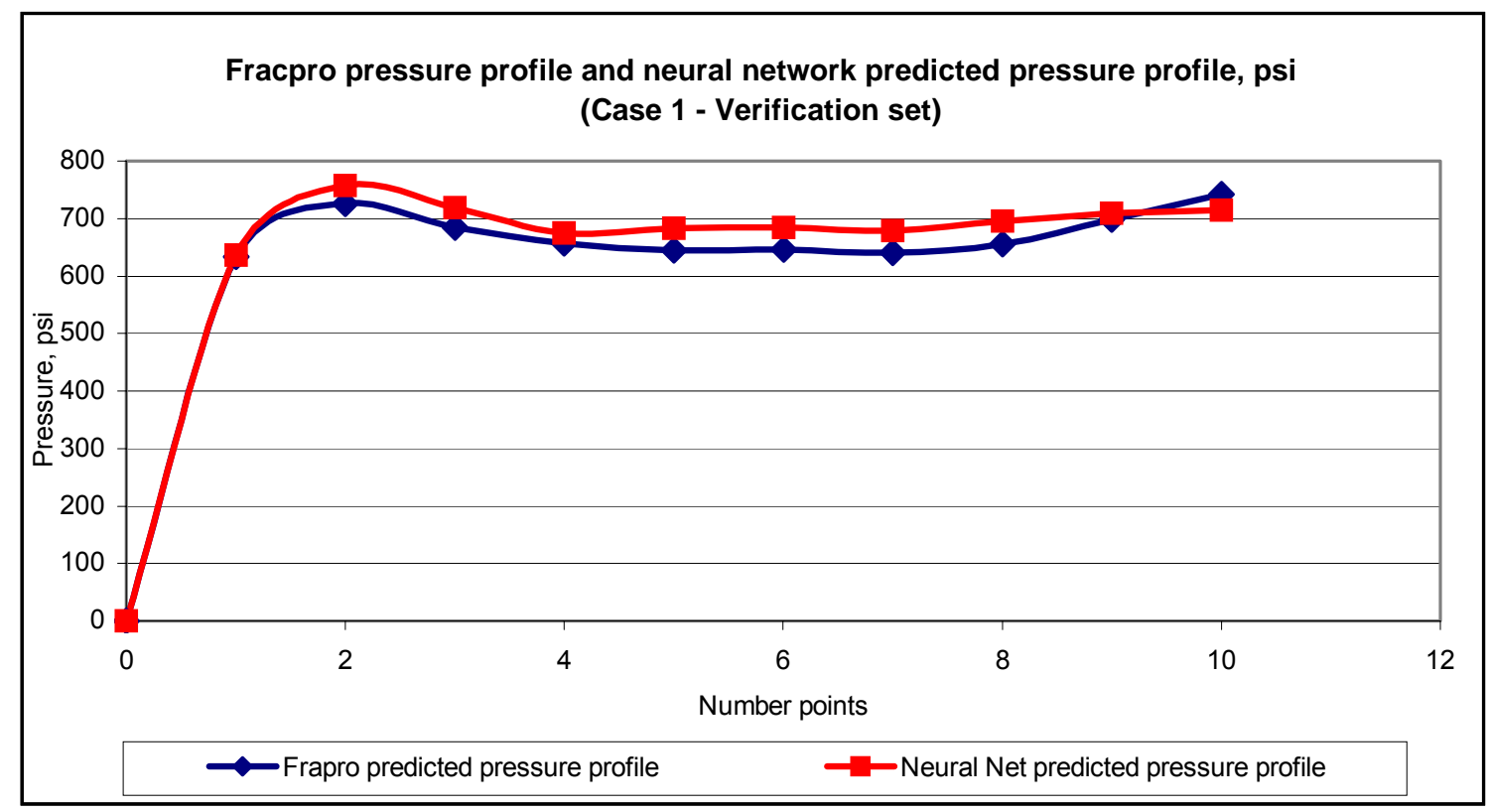

Figure 5-119: Case 1 FRACPRO and Neural Network Pressure Profiles 


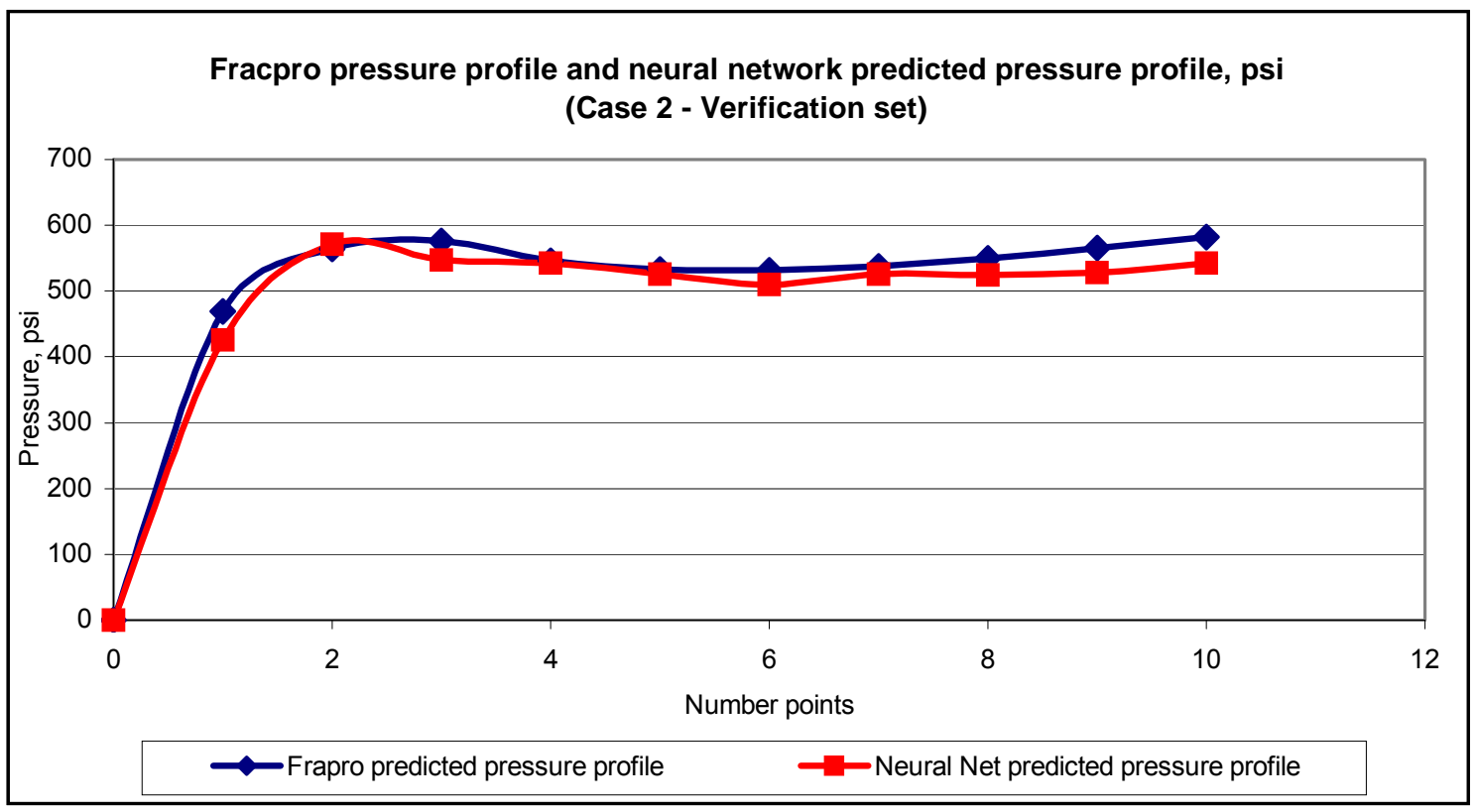

Figure 5-120: Case 2 FRACPRO and Neural Network Pressure Profiles

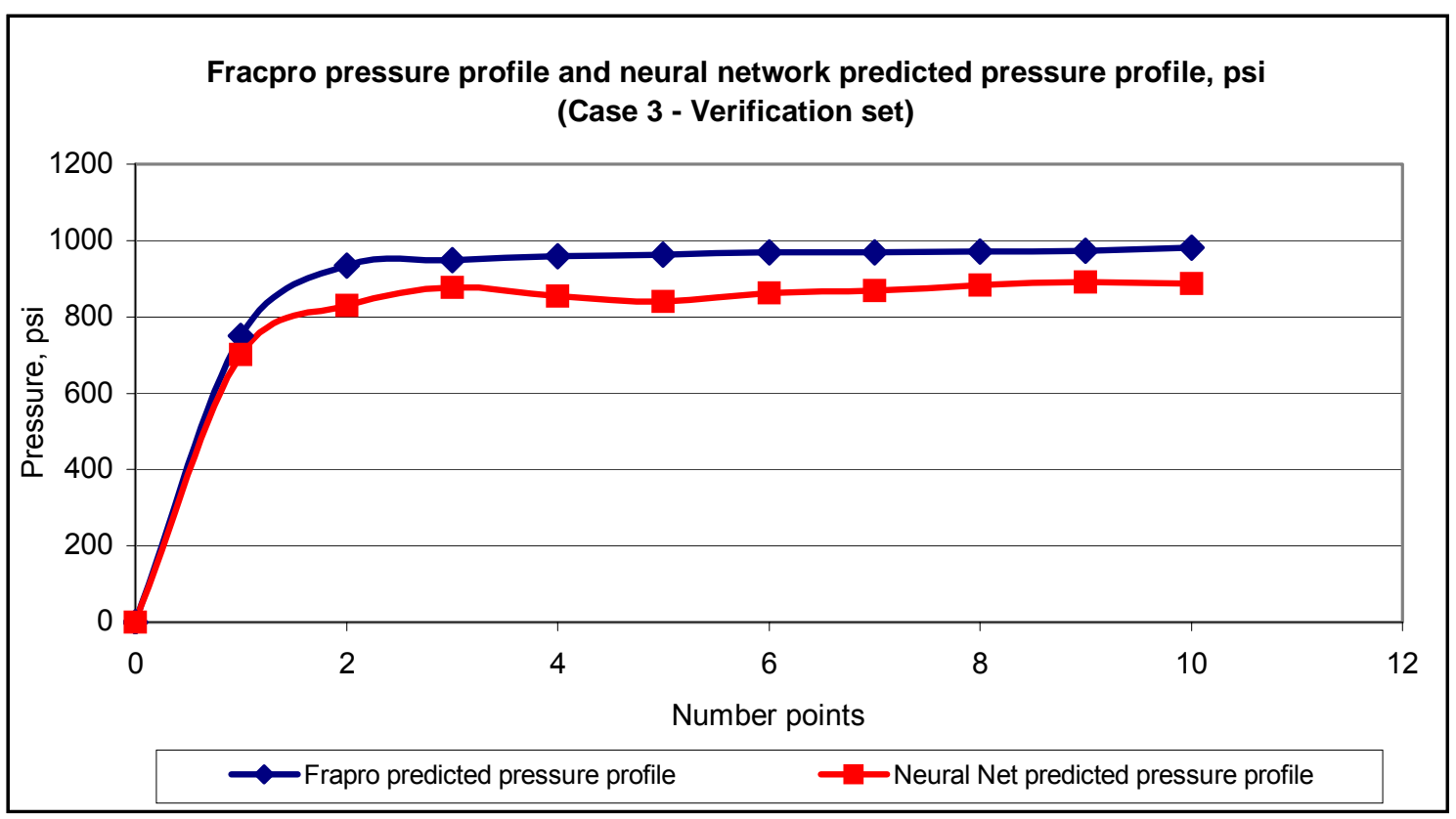

Figure 5-121: Case 3 FRACPRO and Neural Network Pressure Profiles 


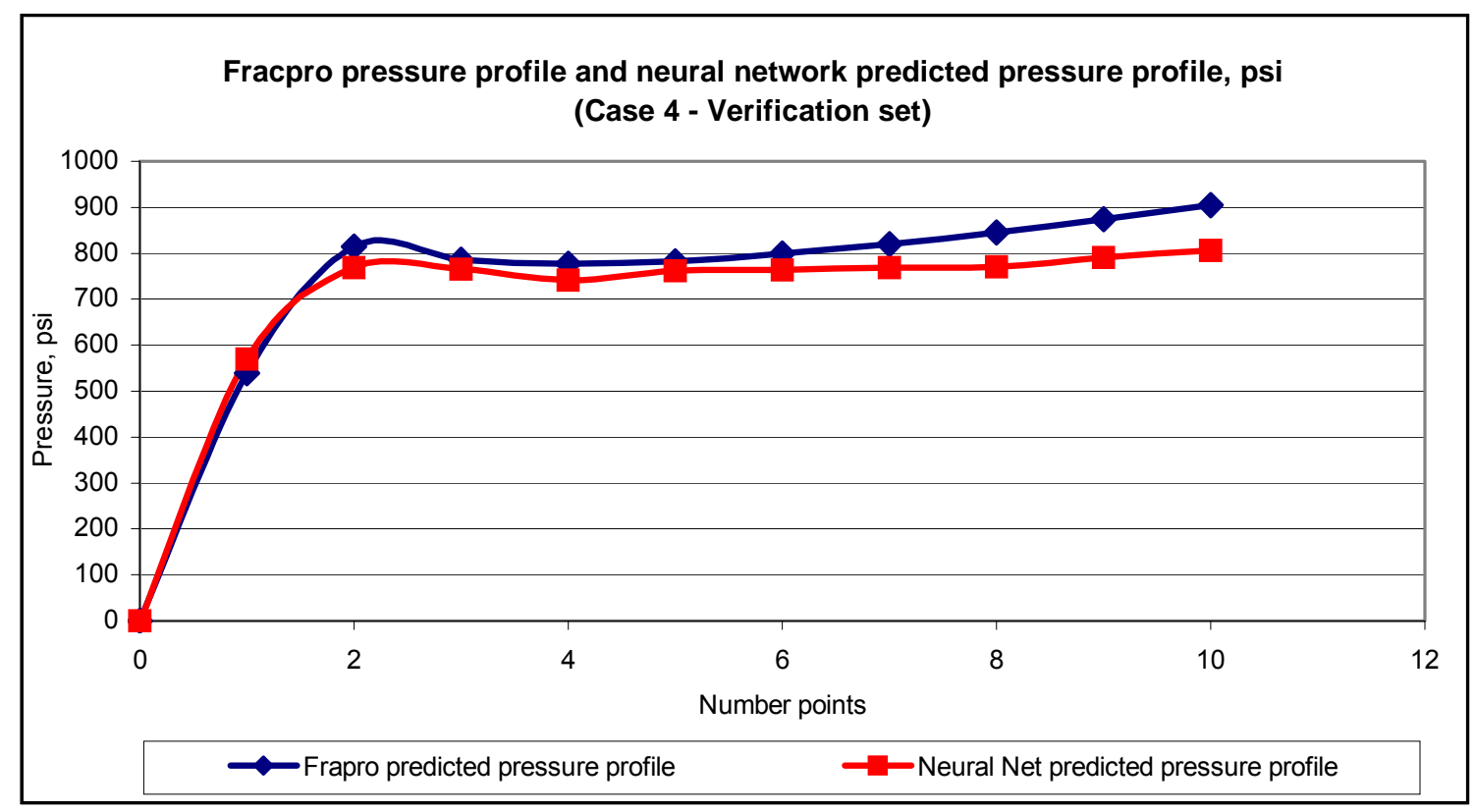

Figure 5-122: Case 4 FRACPRO and Neural Network Pressure Profiles

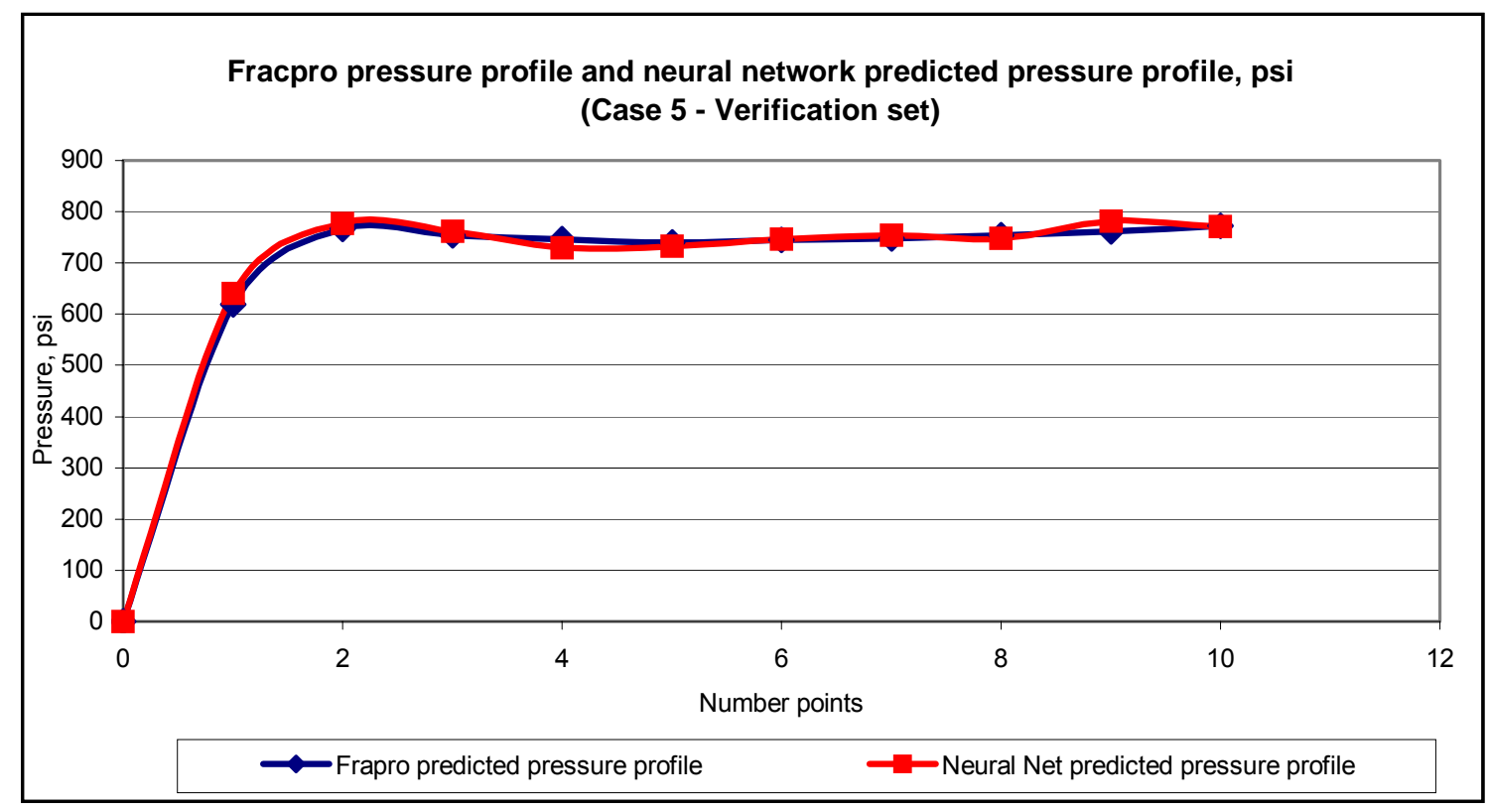

Figure 5-123: Case 5 FRACPRO and Neural Network Pressure Profiles 


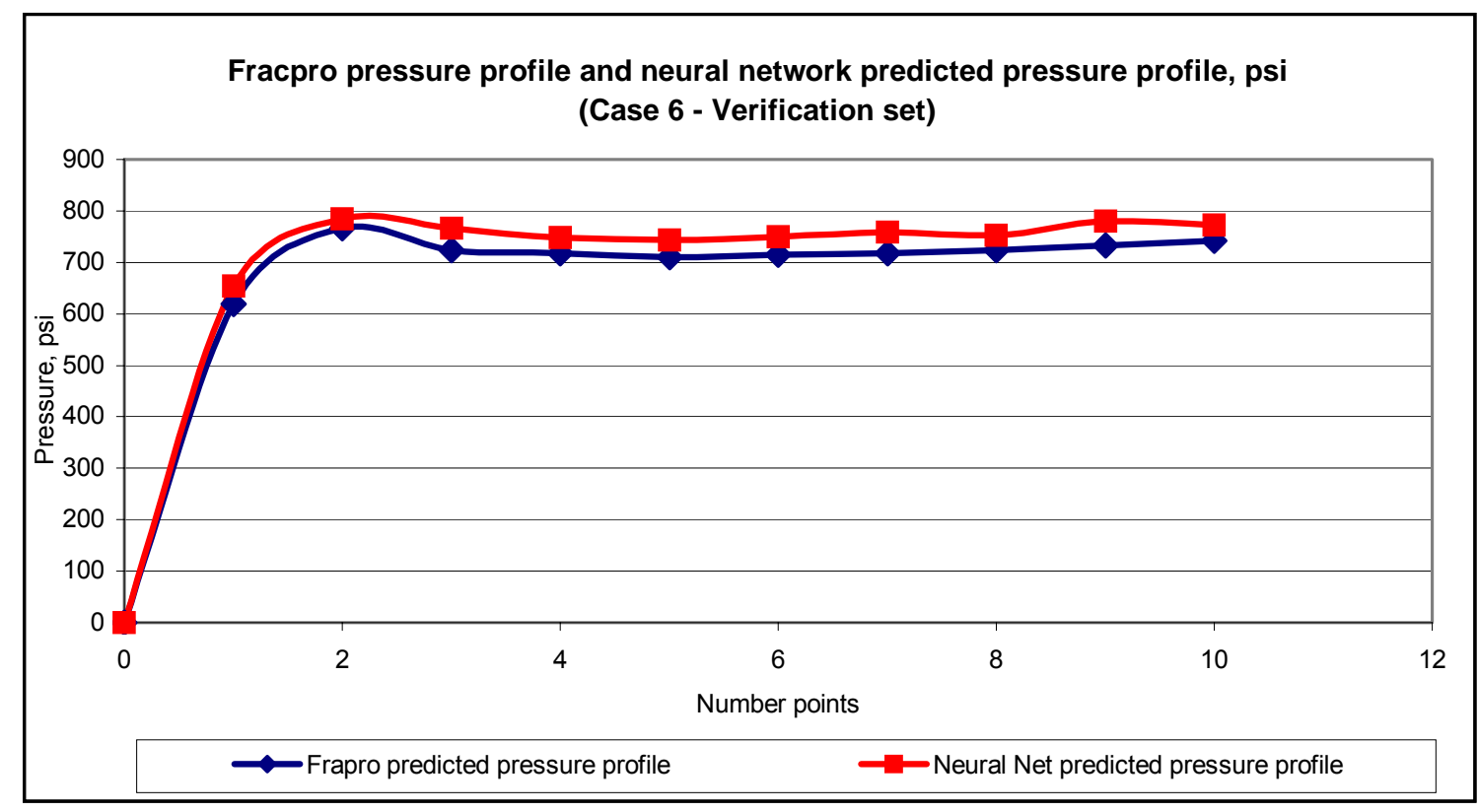

Figure 5-124: Case 6 FRACPRO and Neural Network Pressure Profiles

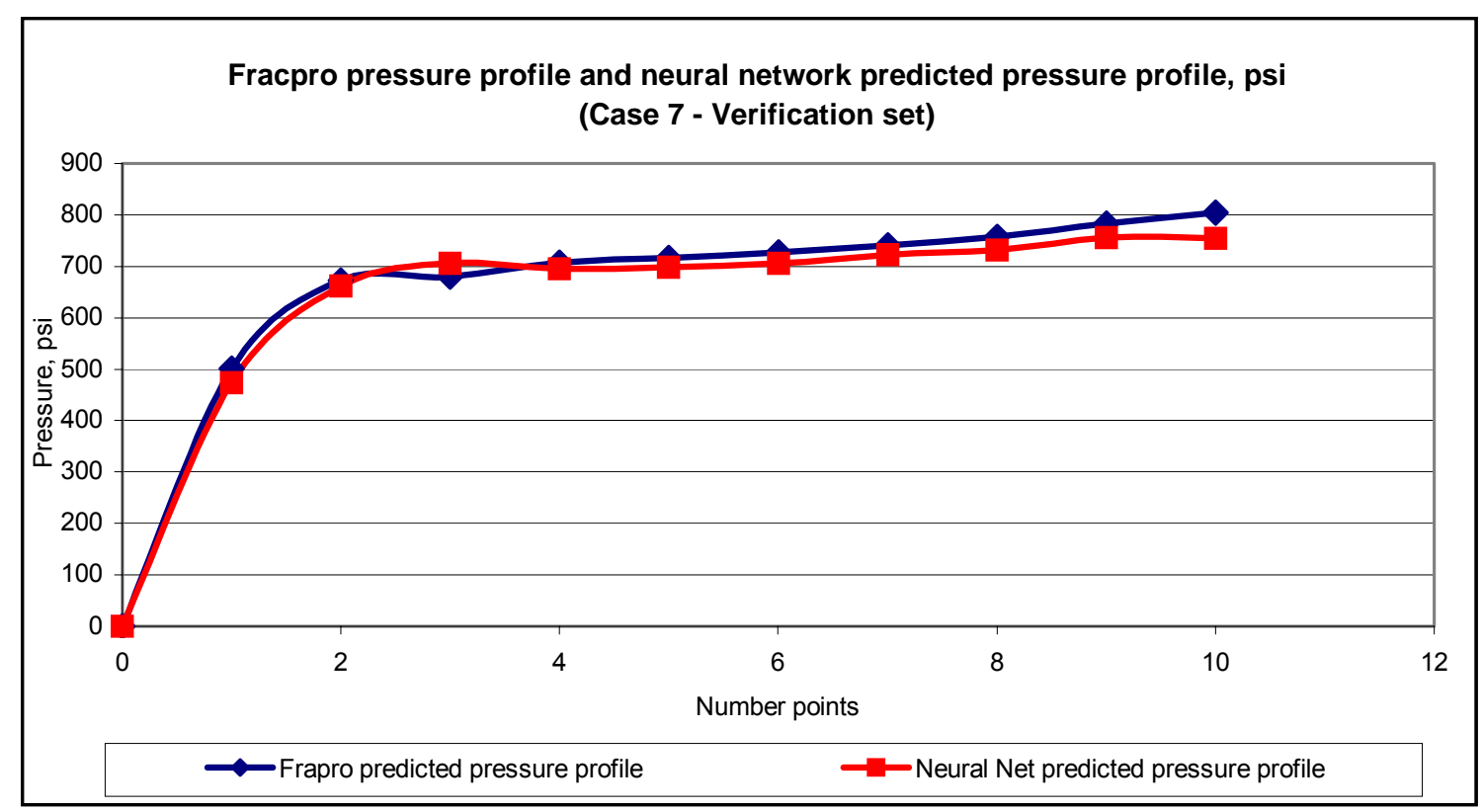

Figure 5-125: Case 7 FRACPRO and Neural Network Pressure Profiles 


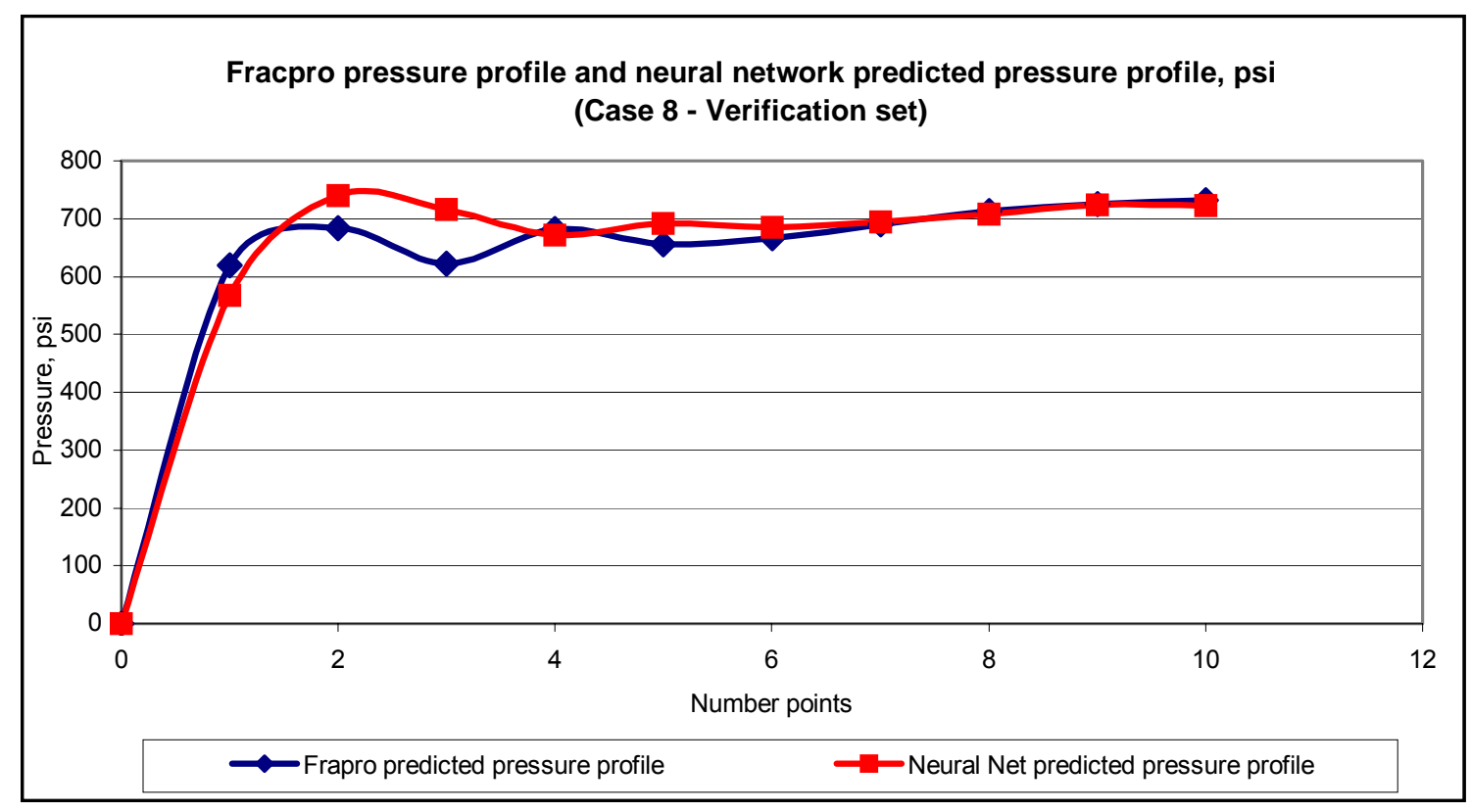

Figure 5-126: Case 8 FRACPRO and Neural Network Pressure Profiles

Figure 5-119 through Figure 5-126 graphically show the two pressure profiles, one from FRACPRO and the other generated by the neural network. The first point in each of the eight cases is called pressure point one. Similarly, for the remaining points to pressure point ten. In each pressure point set, there is at least one point where the neural network prediction significantly different than the actual value. For example, the neural network prediction of point seven is very close to the actual value in seven out of eight cases. However, in Case 3 (Figure $5-121$ ), point seven falls below the actual by about $70 \mathrm{psi}$. This is more than enough to cause the $\mathrm{R}^{2}$ for point seven to drop to 0.85 . Point 10 falls short in three cases (2, 3 and 4$)$, which drops the $\mathrm{R}^{2}$ to 0.76 . In other words, a few points in a set may tend to imply that the neural network is not accurate. However, the accuracy should not be judged by single point prediction since the output of the system is the entire signal. The closer the neural network pressure profile is to the shape and values of the FRACPRO pressure profile, the better the accuracy. Cases 1 through 8 show that, with exception of case 3 , the pressure profiles generated by the neural network are very close to the FRACPRO generated profiles in values and shape. Even for Case 3, the shape of the neural network profile is very close to the FRACPRO profile.

The selected net pressure neural network provided good results when tested against FRACPRO generated data. Moreover, although the $\mathrm{R}^{2}$ values are not exceptional, the correlation coefficients are above 0.9 , with some up to 0.96 . This proves the capability of reproducing the shape of the signal. Similar results were observed when working with logs, where neural networks were very capable of identifying layer signatures while falling short in predicting their 
picks. As a result, the $\mathrm{R}^{2}$ values were low, even though the neural networks were considered good performers.

\subsection{Neuro-Genetic Model Verification}

The optimum fracture treatment design is found through the use of a two-step method that integrates neural networks with genetic algorithms. The methodology presented in section 4.13 is implemented in a computer program that automatically runs different scenarios and provides optimum solutions. Since this study addresses tight sands, the tool was designed such that the optimization search is performed on fracture length. The fracture conductivity is calculated from the dimensionless coefficient.

\subsubsection{Tool Design vs FRACPRO vs Actual for Treatment Schedule}

To prove the accuracy of the proposed methodology, a comparison between the treatment schedule from a fracturing job performed by Halliburton and the treatment design generated by the computer program was initiated.

The original job is a ramp treatment schedule, performed at a depth of $11,440 \mathrm{ft}$ in a tight gas reservoir. The job was designed to create a propped fracture length of $450 \mathrm{ft}$. The other characteristics of the treatment, fluid volumes, proppant concentration, and pumping rate are presented in Table 5-13. Since the fracture propped length of the original job was 450 feet, the same value was set as the target for the optimization criteria. The tool was run several times, and three examples of ramp treatment schedules were selected. The characteristics of these examples are included in Table 5-13. The FRACPRO simulator was run using the three selected treatments, and the results for each treatment were recorded. These results are presented in comparison with the output from the optimization tool.

\begin{tabular}{|c|c|c|c|c|c|c|c|c|c|c|}
\hline & \multirow[b]{3}{*}{ Case \# } & \multirow{2}{*}{\multicolumn{5}{|c|}{ Treatment Characteristics }} & \multirow{2}{*}{\multicolumn{2}{|c|}{$\begin{array}{c}\text { NN \& GA } \\
\text { Predictions }\end{array}$}} & \multirow{2}{*}{\multicolumn{2}{|c|}{ FRACPRO Results }} \\
\hline & & & & & & & & & & \\
\hline & & $\begin{array}{c}\text { Total } \\
\text { Fluid } \\
\text { Volume } \\
\text { (gal) }\end{array}$ & \begin{tabular}{|c} 
Pad \\
Fluid \\
Volume \\
(gal)
\end{tabular} & $\begin{array}{l}\text { Starting } \\
\text { Propp } \\
\text { Conc } \\
\text { (ppg) }\end{array}$ & $\begin{array}{l}\text { Final } \\
\text { Propp } \\
\text { Conc } \\
\text { (ppg) }\end{array}$ & $\begin{array}{l}\text { Pump } \\
\text { Rate } \\
\text { (bpm) }\end{array}$ & $\begin{array}{c}\text { Propp } \\
\text { Length } \\
\text { (ft) }\end{array}$ & \begin{tabular}{|c|} 
Frac \\
Conduct \\
ivity \\
(mD-ft)
\end{tabular} & $\begin{array}{c}\text { Propp } \\
\text { Length } \\
\text { (ft) }\end{array}$ & $\begin{array}{c}\text { Frac } \\
\text { Conducti } \\
\text { vity } \\
\text { (mD-ft) }\end{array}$ \\
\hline Original Job & & 59929 & 21504 & 1 & 7.5 & 20 & & & 451.6 & 315 \\
\hline NN \& GA & Case 1 & 72041 & 28974 & 1 & 9 & 23 & 450.3 & 203.87 & 453.5 & 359 \\
\hline Optimum & Case 2 & 65843 & 20940 & 1 & 7.5 & 20 & 450.8 & 192.28 & 458.1 & 309 \\
\hline Ireatments & Case 3 & 61674 & 39448 & 1 & 6 & 29 & 449.1 & 154.1 & 455.5 & 232 \\
\hline
\end{tabular}

Table 5-13: Original Ramp Treatment with Optimum Suggested Treatments 
The optimization routine suggested treatment designs with fracture propped length virtually equal to the desired value. The fracture propped length created by FRACPRO, when the suggested optimum treatment is run, is very close to the desired value. The largest difference between the original target and the suggested design propped length when run in FRACPRO is approximately 8 feet. This corresponds to a percentage error of $2 \%$.

Note that each suggested optimum treatment has different characteristics:

- The treatments have various total fluid and pad volumes.

- The treatments have different final proppant concentrations.

- The treatments suggest different injection rates.

The second example tests the accuracy of the system for an eight-stage treatment design. The original job was pumped in the Teapot Formation in Kaye Field, Oklahoma. The treatment induced a fracture with a $403 \mathrm{ft}$ length. Since it believed that the treatment was designed for a $400 \mathrm{ft}$ fracture length, this value was set as the optimization criteria for the tool. The optimization module was run for each of the three eight-stage treatment types.

The optimum treatments suggested for each type of eight-stage job are presented in Table 5-14 along with the original Halliburton treatment. The table also shows the comparison between the fracture length suggested by the optimization routine, the fracture length generated by FRACPRO, and the fracture length of the original treatment. The three types of suggested eight stage treatments provided very close fracture length values compared FRACPRO. However the proppant concentration values are all higher than the original treatment.

\begin{tabular}{|c|c|c|c|c|c|c|c|c|c|c|c|c|}
\hline & \multirow{2}{*}{\multicolumn{3}{|c|}{ Original Treatment }} & \multicolumn{9}{|c|}{ NN\&GA Optimum Suggested Treatment } \\
\hline & & & & \multicolumn{3}{|c|}{ Type I } & \multicolumn{3}{|c|}{ Type II } & \multicolumn{3}{|c|}{ Type III } \\
\hline & $\begin{array}{c}\text { Fluid } \\
\text { Volume }\end{array}$ & $\begin{array}{c}\text { Pump } \\
\text { Rate }\end{array}$ & $\begin{array}{l}\text { Propp } \\
\text { Conc }\end{array}$ & $\begin{array}{l}\text { Fluid } \\
\text { Volume }\end{array}$ & $\begin{array}{c}\text { Pump } \\
\text { Rate }\end{array}$ & $\begin{array}{l}\text { Propp } \\
\text { Conc }\end{array}$ & $\begin{array}{c}\text { Fluid } \\
\text { Volume }\end{array}$ & $\begin{array}{c}\text { Pump } \\
\text { Rate }\end{array}$ & $\begin{array}{l}\text { Propp } \\
\text { Conc }\end{array}$ & $\begin{array}{c}\text { Fluid } \\
\text { Volume }\end{array}$ & $\begin{array}{c}\text { Pump } \\
\text { Rate }\end{array}$ & $\begin{array}{l}\text { Propp } \\
\text { Conc }\end{array}$ \\
\hline & gal & bmp & $\mathrm{ppg}$ & gal & bmp & ppg & gal & bmp & ppg & gal & bmp & ppg \\
\hline Stage 1 & 20,762 & 20 & 0 & 27,054 & 20 & 0 & 22,945 & 24 & 0 & 22,548 & 22 & 0 \\
\hline Stage 2 & 2,585 & 20 & 1 & 4,340 & 20 & 1.3 & 4,593 & 24 & 1 & 4,573 & 22 & 1.1 \\
\hline Stage 3 & 4,571 & 20 & 2 & 4,340 & 20 & 2.6 & 5,741 & 24 & 2 & 5,716 & 22 & 2.2 \\
\hline Stage 4 & 7,224 & 20 & 3 & 6,511 & 20 & 3.9 & 6,890 & 24 & 3 & 6,859 & 22 & 3.3 \\
\hline Stage 5 & 9,152 & 20 & 4 & 6,511 & 20 & 5.2 & 8,038 & 24 & 4 & 9,146 & 22 & 4.4 \\
\hline Stage 6 & 10,950 & 20 & 5 & 8,681 & 20 & 6.5 & 9,186 & 24 & 5 & 10,289 & 22 & 5.5 \\
\hline Stage 7 & 5,088 & 20 & 6 & 8,681 & 20 & 7.8 & 11,483 & 24 & 6 & 11,289 & 22 & 6.6 \\
\hline Stage 8 & 8,886 & 20 & 0 & 10,851 & 20 & 9.1 & 6,890 & 24 & 7 & 4,573 & 22 & 7.7 \\
\hline Flush & & & & 2,000 & 20 & 0 & 2,000 & 24 & 0 & 2,000 & 22 & 0 \\
\hline Total & 69,218 & & & 78,969 & & & 77,766 & & & 76,993 & & \\
\hline \multicolumn{13}{|c|}{ Fracture Propped Length } \\
\hline NN\&GA Prediction & & - & & \multirow{2}{*}{\multicolumn{3}{|c|}{$\begin{array}{c}400.5 \\
400.19 \\
\end{array}$}} & \multirow{2}{*}{\multicolumn{3}{|c|}{$\begin{array}{r}399.5 \\
394.04 \\
\end{array}$}} & \multirow{2}{*}{\multicolumn{3}{|c|}{$\begin{array}{c}400.2 \\
404.25\end{array}$}} \\
\hline FRACPRO Results & & 03.71 & & & & & & & & & & \\
\hline \multicolumn{13}{|c|}{ Proppant Concentration } \\
\hline NN\&GA Prediction & \multirow{2}{*}{\multicolumn{3}{|c|}{$\begin{array}{c}- \\
0.74\end{array}$}} & \multirow{2}{*}{\multicolumn{3}{|c|}{$\begin{array}{l}1.21 \\
1.07\end{array}$}} & \multirow{2}{*}{\multicolumn{3}{|c|}{$\begin{array}{l}0.91 \\
0.81\end{array}$}} & \multicolumn{3}{|c|}{1.4} \\
\hline FRACPRO Results & & & & & & & & & & & 1.2 & \\
\hline
\end{tabular}

Table 5-14: Original Halliburton Treatment with Eight Stage Optimum Suggested Treatments 
Note that the provided Halliburton treatment actually consists of seven stages, and the fracturing fluid used was slightly different than the one used in training the neural network. Since the original treatment consisted of seven stages, a six-stage design was also generated for comparison. Table 5-15 shows the suggested six-stage optimum treatment along with the original Halliburton treatment.

\begin{tabular}{|c|c|c|c|c|c|c|}
\hline & \multicolumn{3}{|c|}{ Original Treatment } & \multicolumn{3}{|c|}{$\begin{array}{l}\text { NN\&GA Optimum } \\
\text { Suggested Treatment }\end{array}$} \\
\hline & $\begin{array}{l}\text { Fluid } \\
\text { Volume }\end{array}$ & $\begin{array}{l}\text { Pump } \\
\text { Rate }\end{array}$ & $\begin{array}{l}\text { Propp } \\
\text { Conc }\end{array}$ & $\begin{array}{l}\text { Fluid } \\
\text { Volume }\end{array}$ & $\begin{array}{l}\text { Pump } \\
\text { Rate }\end{array}$ & $\begin{array}{l}\text { Propp } \\
\text { Conc }\end{array}$ \\
\hline & gal & bmp & $\mathrm{ppg}$ & gal & $\mathrm{bmp}$ & $\mathrm{ppg}$ \\
\hline Stage 1 & 20,762 & 20 & 0 & 26,269 & 28 & 0 \\
\hline Stage 2 & 2,585 & 20 & 1 & 6,565 & 28 & 1 \\
\hline Stage 3 & 4,571 & 20 & 2 & 6,565 & 28 & 2.8 \\
\hline Stage 4 & 7,224 & 20 & 3 & 9,848 & 28 & 4.5 \\
\hline Stage 5 & 9,152 & 20 & 4 & 13,131 & 28 & 6.3 \\
\hline Stage 6 & 10,950 & 20 & 5 & 16,413 & 28 & 8 \\
\hline Stage 7 & 5,088 & 20 & 6 & 2,000 & 28 & 0 \\
\hline $\begin{array}{r}\text { Stage } 8 \\
\text { Flush }\end{array}$ & 8,886 & 20 & 0 & & & \\
\hline Total & 69,218 & & & 80,791 & & \\
\hline \multicolumn{7}{|c|}{ Fracture Propped Length } \\
\hline NN\&GA Prediction & \multicolumn{3}{|c|}{-} & \multicolumn{3}{|c|}{400.8} \\
\hline FRACPRO Results & \multicolumn{3}{|c|}{403.71} & \multicolumn{3}{|c|}{396.7} \\
\hline \multicolumn{7}{|c|}{ Proppant Concentration } \\
\hline NN\&GA Prediction & \multirow{2}{*}{\multicolumn{3}{|c|}{$\begin{array}{c}- \\
0.74\end{array}$}} & \multicolumn{3}{|c|}{1.02} \\
\hline FRACPRO Results & & & & \multicolumn{3}{|c|}{0.96} \\
\hline
\end{tabular}

Table 5-15:Original Halliburton Treatment with Six Stage Optimum Suggested Treatment

All treatments suggested by the optimization tool created a fracture propped length very close to the desired when run on FRACPRO. However, the system tends to provide slightly larger treatments, with higher total volumes and either more proppant or higher final proppant concentration.

Overall, the intent of a tool to design fracturing treatments was achieved. Using this tool, engineers can select the treatment that best fits their needs from a large variety of available treatment designs.

\subsubsection{Design versus FRACPRO for Net Pressure Prediction}

The accuracy of the pressure prediction module was tested using the three ramp treatment designs suggested in Table 5-13. The net pressure profile was recorded when each of the ramp treatments were run with FRACPRO. This profile was compared with the net pressure 
profile created by the two neural networks (net pressure neural network and calibration neural network).

Figure 5-127 through Figure 5-129 shows the comparison of the FRACPRO pressure profile and the system predicted profile.

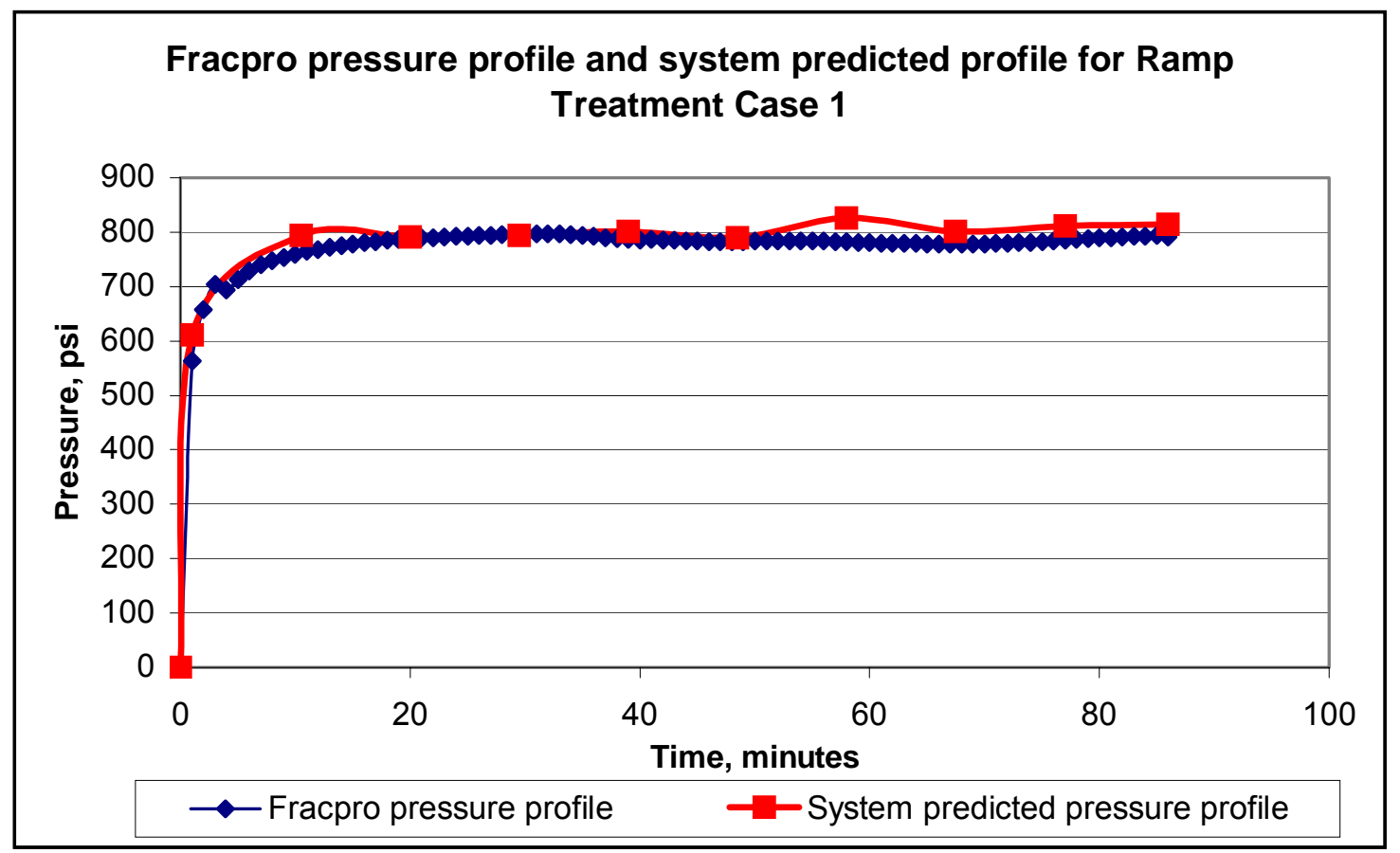

Figure 5-127: FRACPRO and System Generated Pressure Profile for Ramp Case 1

Figure 5-127 shows the best match among the three cases. It corresponds to the largest treatment suggested by the optimization tool. 
Fracpro pressure profile and system predicted profile for Ramp Treatment Case 2

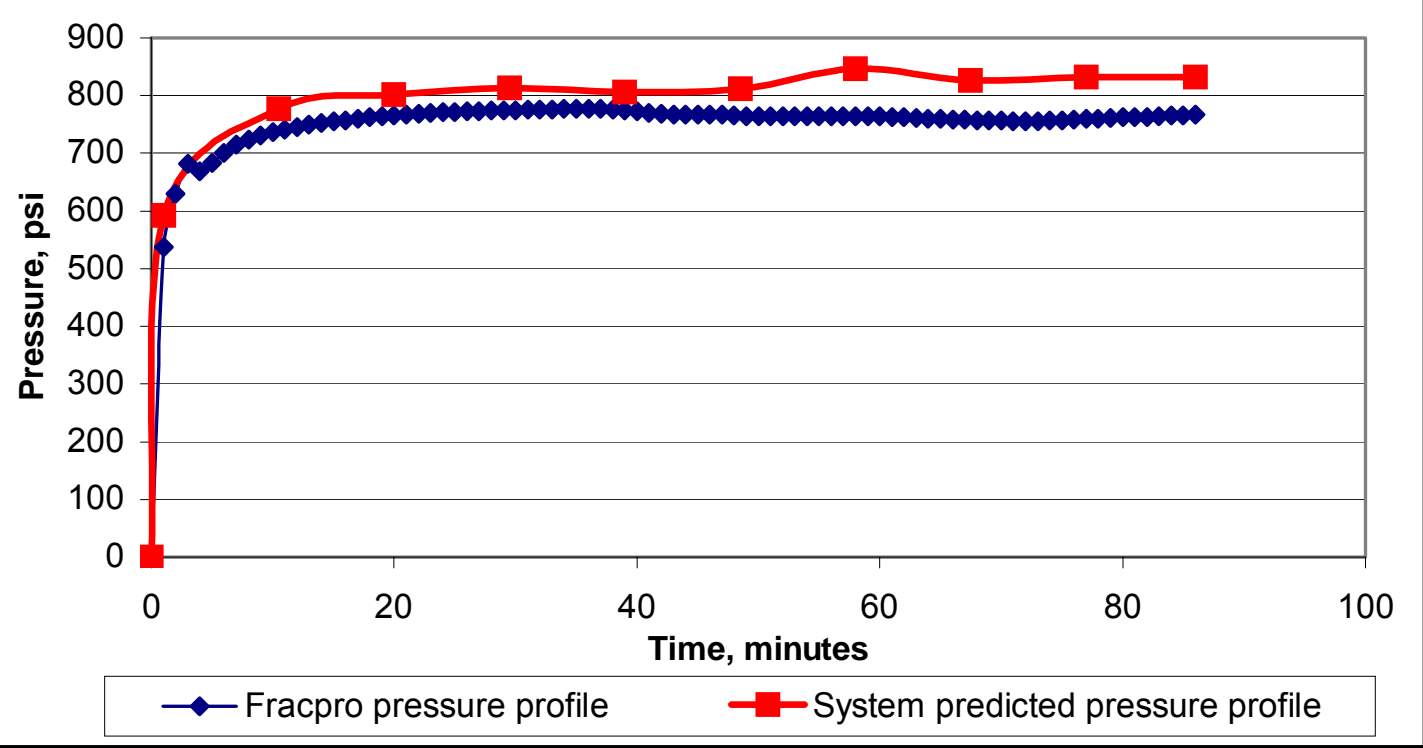

Figure 5-128: FRACPRO and System Generated Pressure Profile for Ramp Case 2

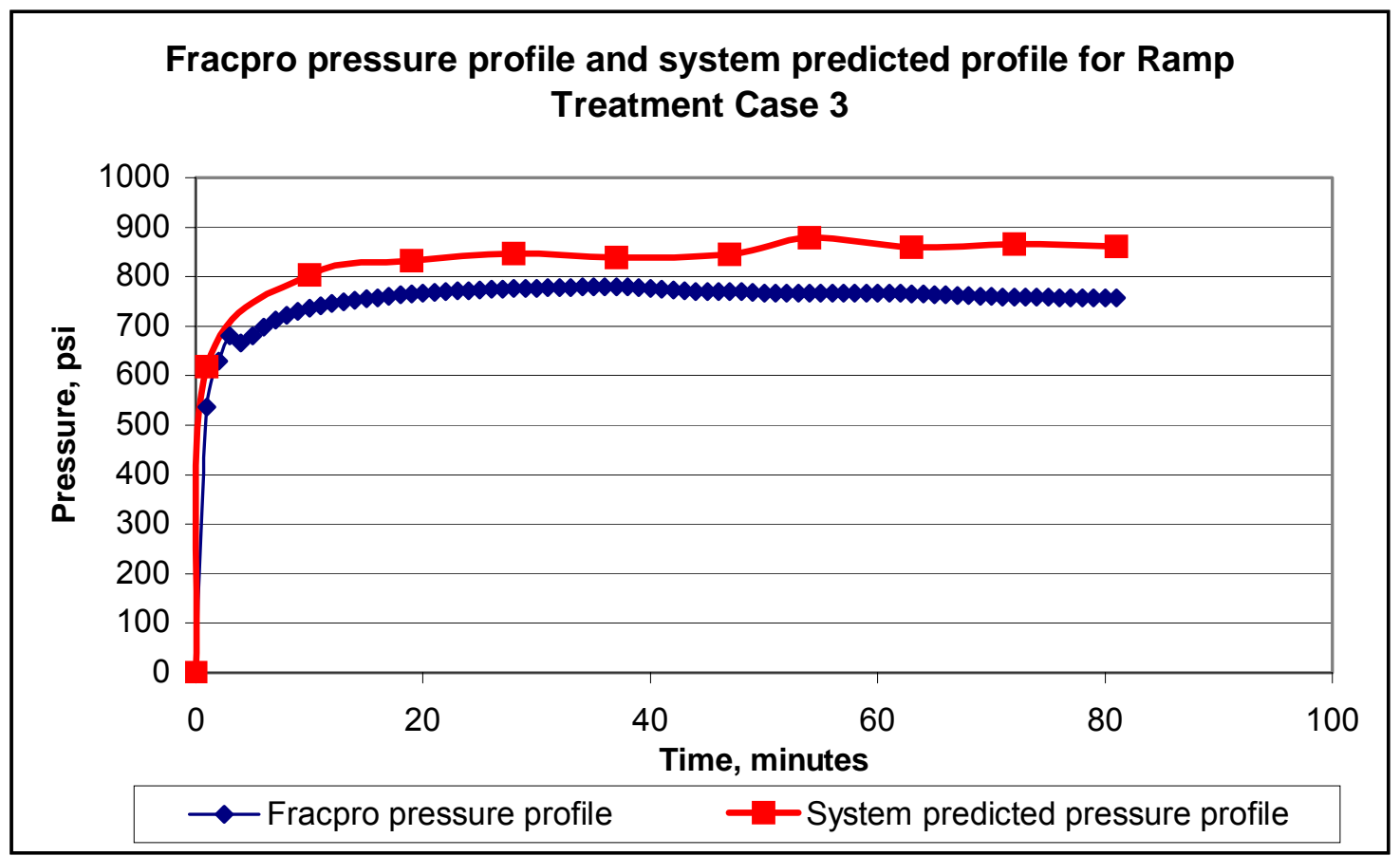

Figure 5-129: FRACPRO and System Generated Pressure Profile for Ramp Case 3 
In Figure 5-128, the system predicted pressure profile is above the FRACPRO pressure profile. This case corresponds to a job very similar to the original one, except that a larger fluid volume was injected.

In the last case, Figure 5-129, the system overestimates the pressure profile. In this case, the fluid volume was closest to the original job, but the treatment had a much lower final proppant concentration. 


\section{CHAPTER 6: FUTURE RESEARCH}

1. The methodology presented in this study proposed an equivalent four-layer stress profile to deal with the large amount of data necessary for complex reservoir characterization. This equivalent four-layer profile proved very effective in preserving the geometry of the fracture when used in the simulation. However, a significant gap was observed between the net pressure profile using the original lithology and the pressure profile using the resulting equivalent stress profile. In this study, the gap was corrected using a calibration neural network trained using only formation characteristics. Further research should be conducted into the cause of this gap. There is potential to develop a new equivalent layer profile model that may predict the correct net pressure profile without the use of a calibration neural network.

2. In section 4.12.2.2 The Eight Stage Treatment, Table 4-17 and Figure 4-14 proved that neural network performance improves with the number of cases. Since, only 200 cases were used to train each of the eight-stage treatment types, it is proposed that new types of neural networks use at least 500 generated cases. If needed, more cases should be generated for each type of eight-stage treatment and the neural network retrained. Additionally, the dimensionless conductivity ratio should be included in the data collected.

3. The neural network for predicting the net pressure profile was trained using only 100 cases. The intention was to research the possibility of predicting the net pressure profile using neural networks. The performance of the neural networks is very acceptable considering the low number of cases used in training. However, to improve its performance, more cases should be generated. Furthermore, it appears that the accuracy of the neural network lessens at the extremities of the stress profile (first and last few points). It is proposed to use more points, particularly to define the first $10-15$ minutes of the pressure signal.

4. Finally, future work should focus on reproducing this study using actual field data. Major companies possess databases with significant amounts of data required to reproduce this study. Fields where more than 80 wells are frac'ed every year, like Lost Hills, California, would provide a great source of data for both treatment schedules and pressure profiles. Not only could the application of this research facilitate the design of optimum fracturing treatments, it also has the potential of identifying other best practices that could lead to major savings for the company. 


\section{CHAPTER 7: SOFTWARE IMPLEMENATION}

The methodology presented in this study was incorporated into a computer application. This application is intended to be a companion tool for any commercial hydraulic fracture simulator to assist engineers in optimizing fracturing treatment schedules. Figure 7-1 shows the start-up form of the application. The user can select an already existing well or load data for a new well.

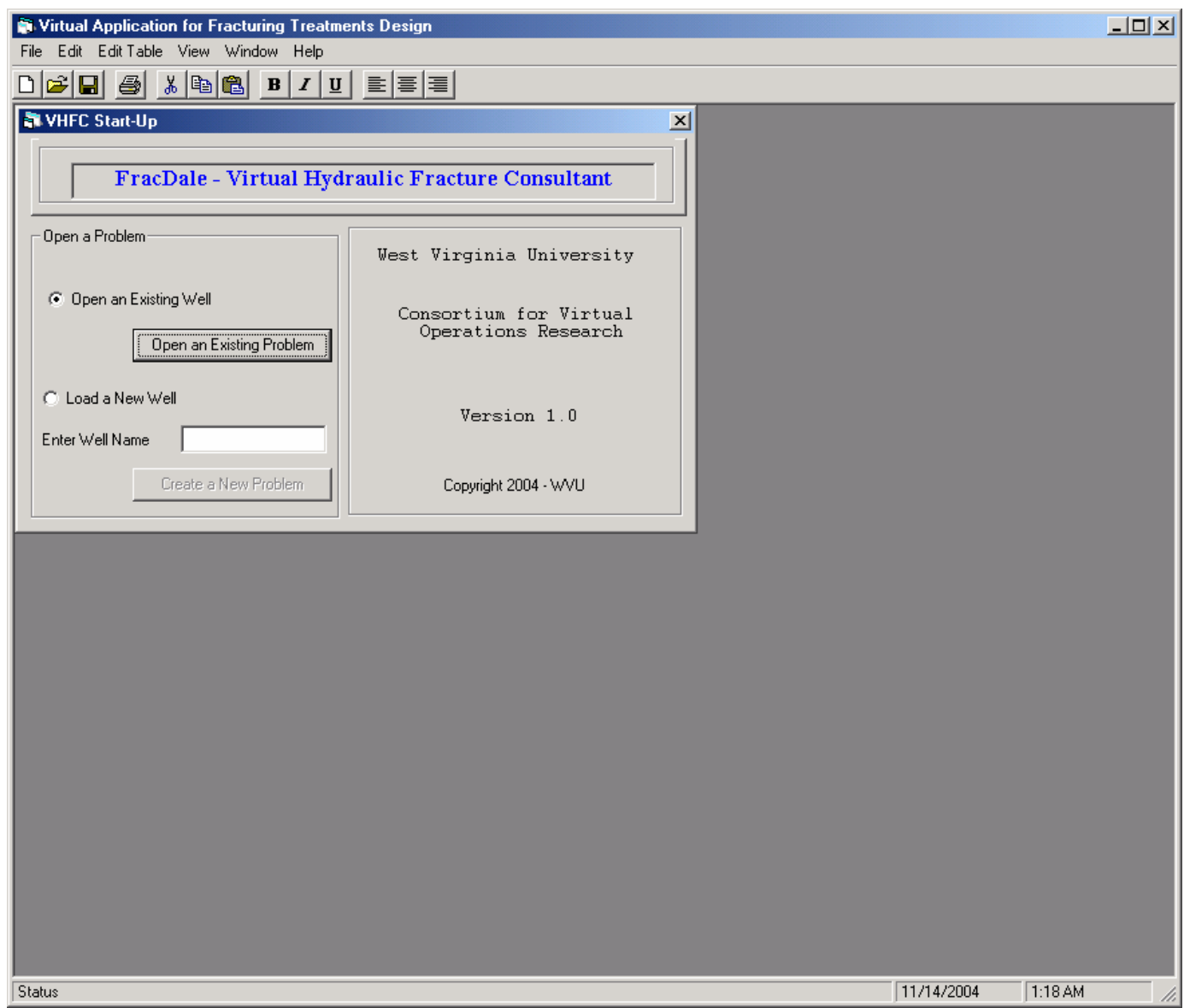

Figure 7-1: Start-Up Form 


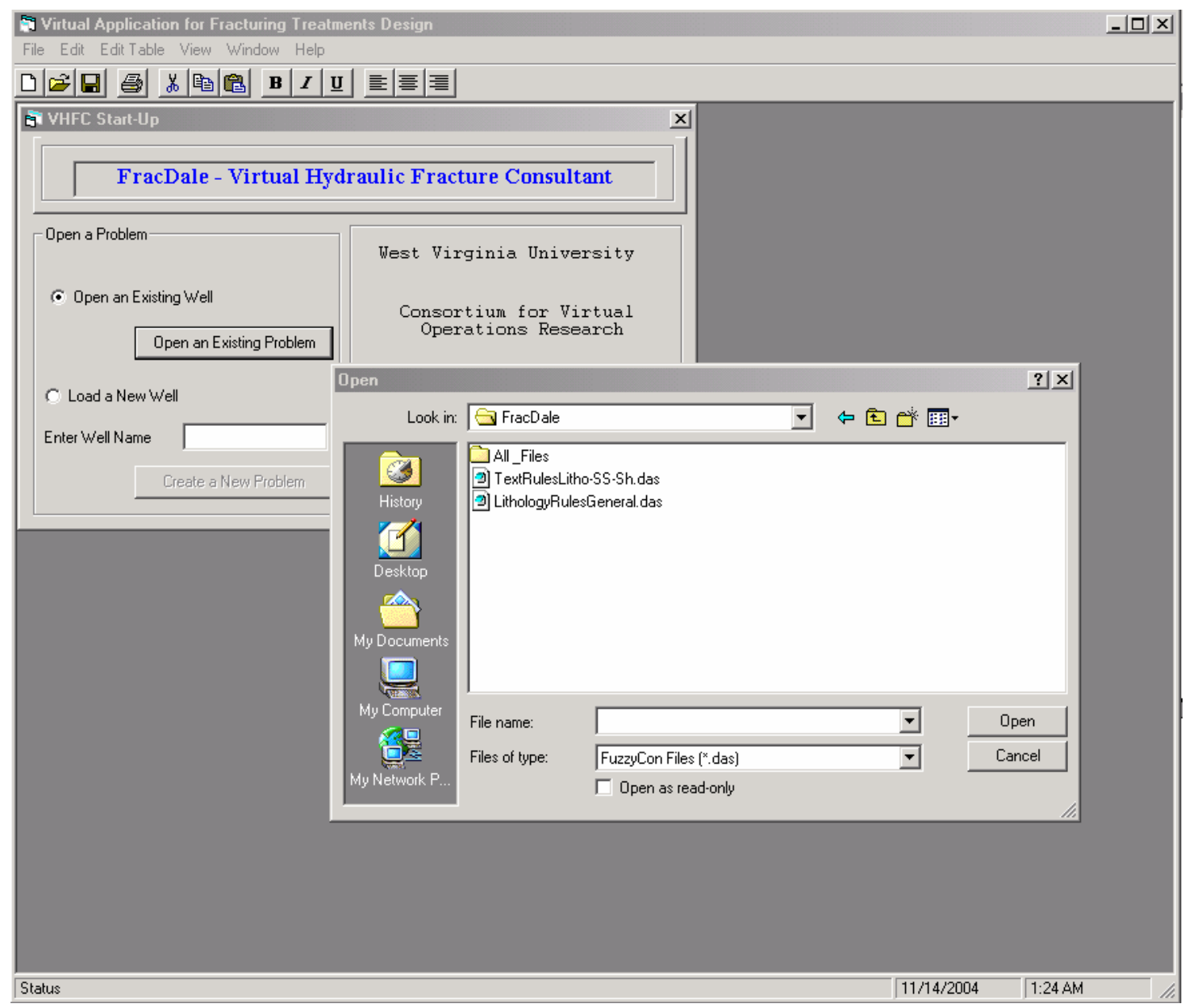

Figure 7-2: Loading a Well File

Figure 7-2 shows the process of opening a well file. This screen is the same whether the user is loading a pre-existing file or creating a new well and importing log data from an external file. The application allows the user to import data from text files, Microsoft Excel files, or databases. 


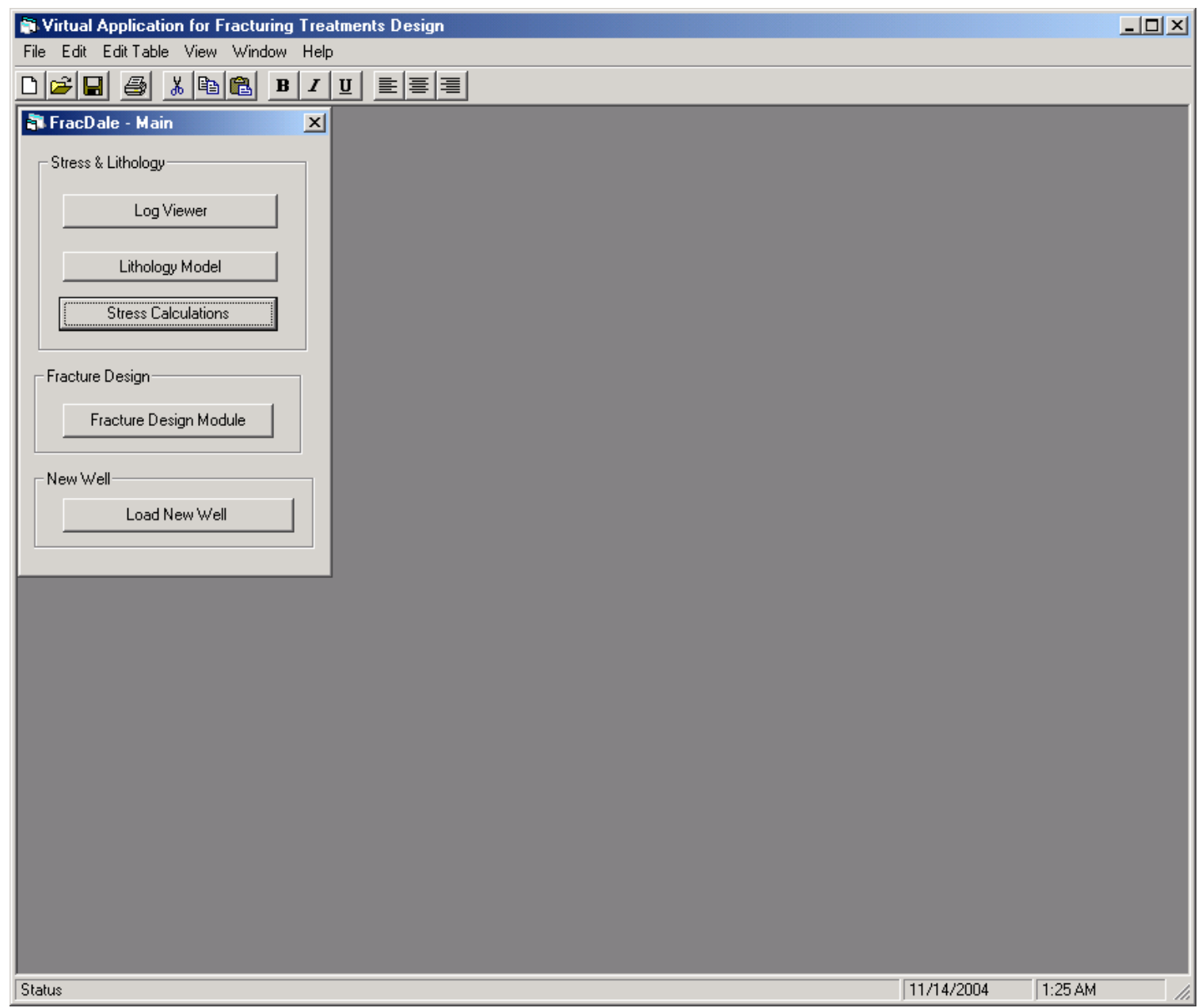

Figure 7-3: Main Console Form

Figure 7-3 shows the Main Console of the program. From this form, users have access to all modules of the program at any time. This form is active any time the program is running. The main modules are the Stress \& Lithology Modules, which includes the Log Viewer, Lithology Model, Stress Calculations, and Fracture Design Module. Typically the normal flow process starts with Log Viewer model. 


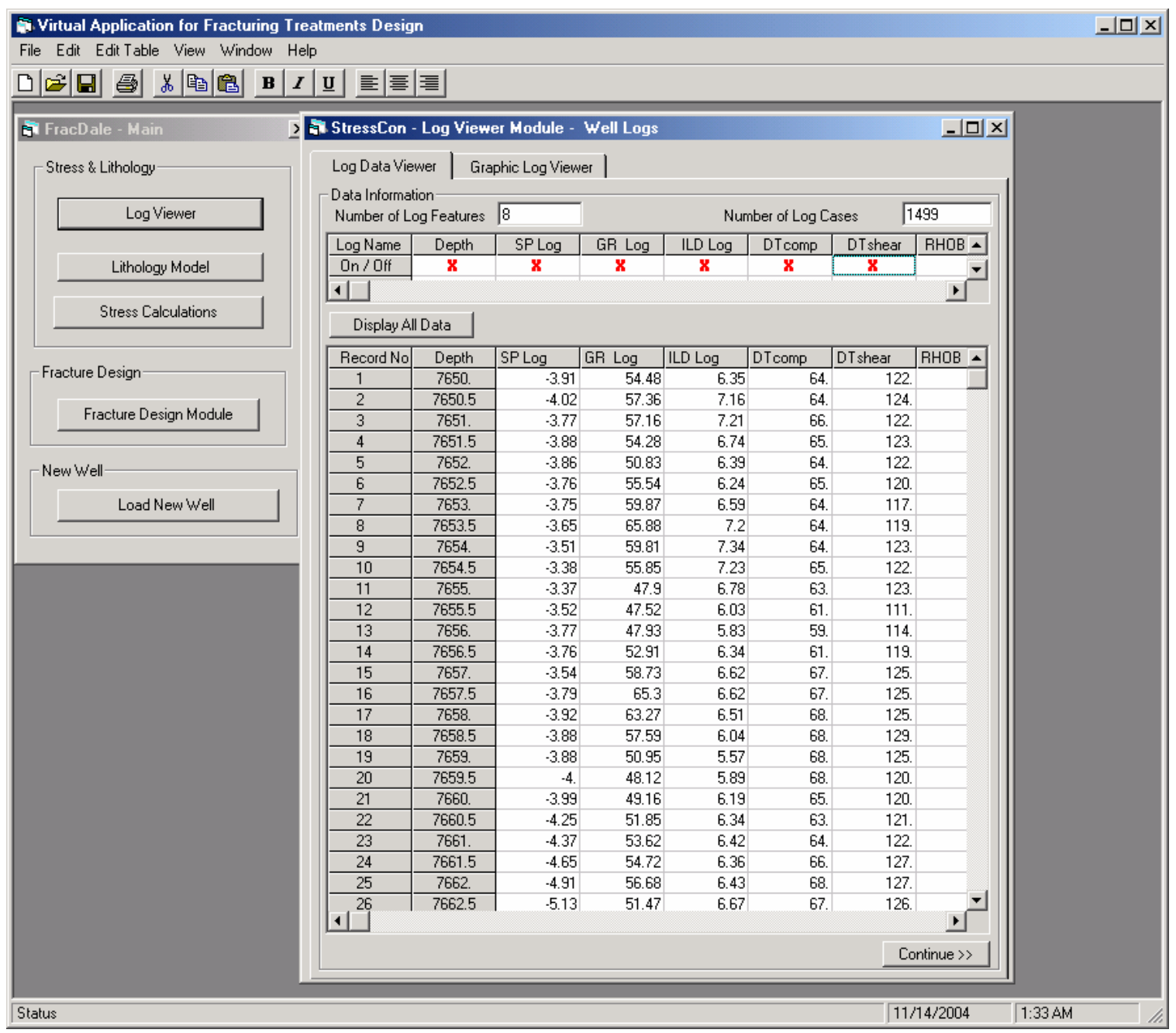

Figure 7-4: Log Viewer Model Form - Log Data Viewer Tab

Figure 7-4 shows the Log Viewer form, which has two tabs. In the first tab, log data is presented in table format. The log values are available for visual analysis. From the top table, the user can select the logs he or she wants to plot. Once the desired logs are selected they will be plotted on the second tab. One can return to this tab at any time to select a different combination of logs or to plot one log at a time. 


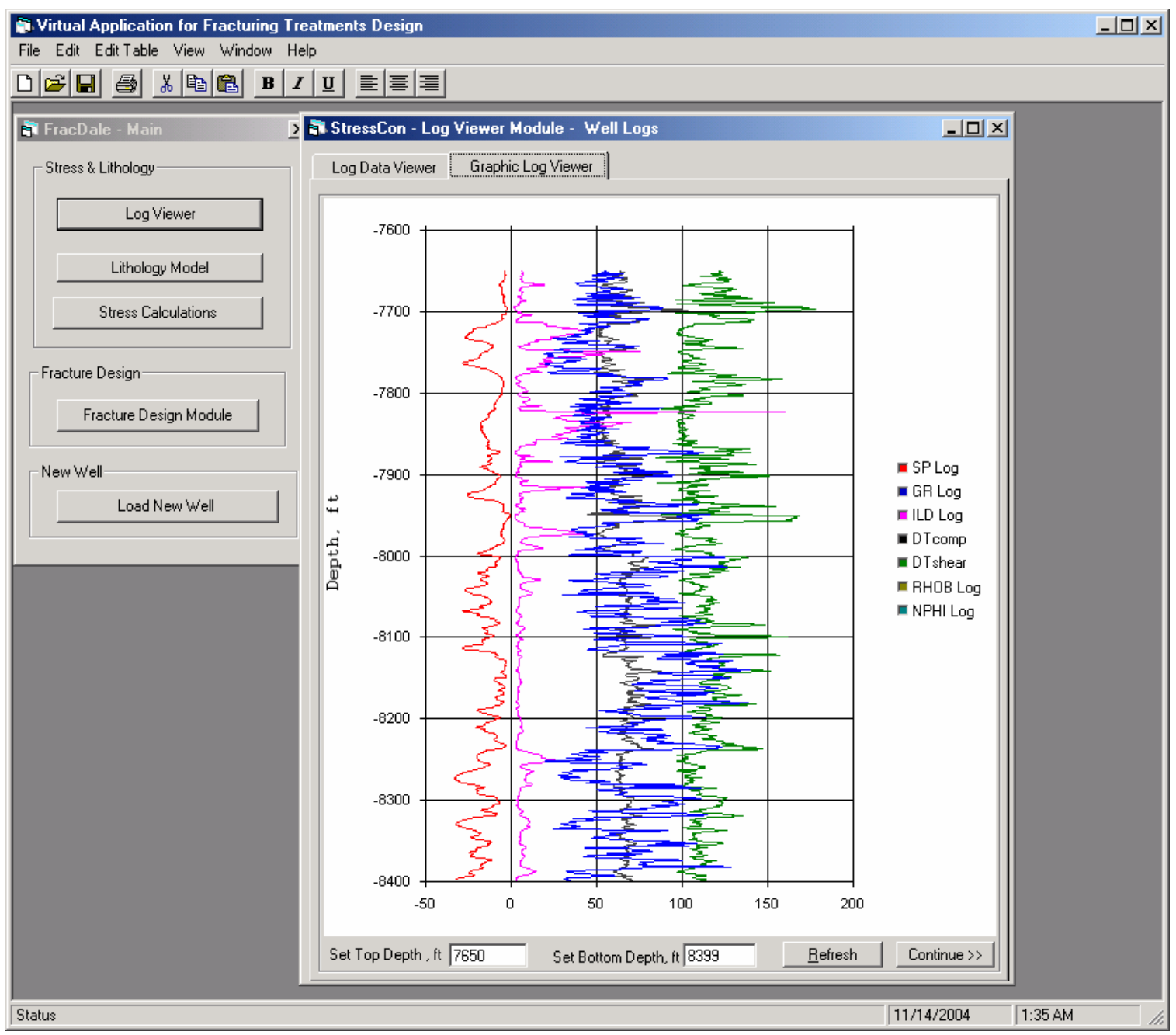

Figure 7-5: Log Viewer Model Form - Graph Log Viewer Tab

Figure 7-5 shows the plot of the selected logs from the first tab. The legend indicates which logs were selected for analysis. The scale of the graph can be changed to accommodate the desired intervals. 


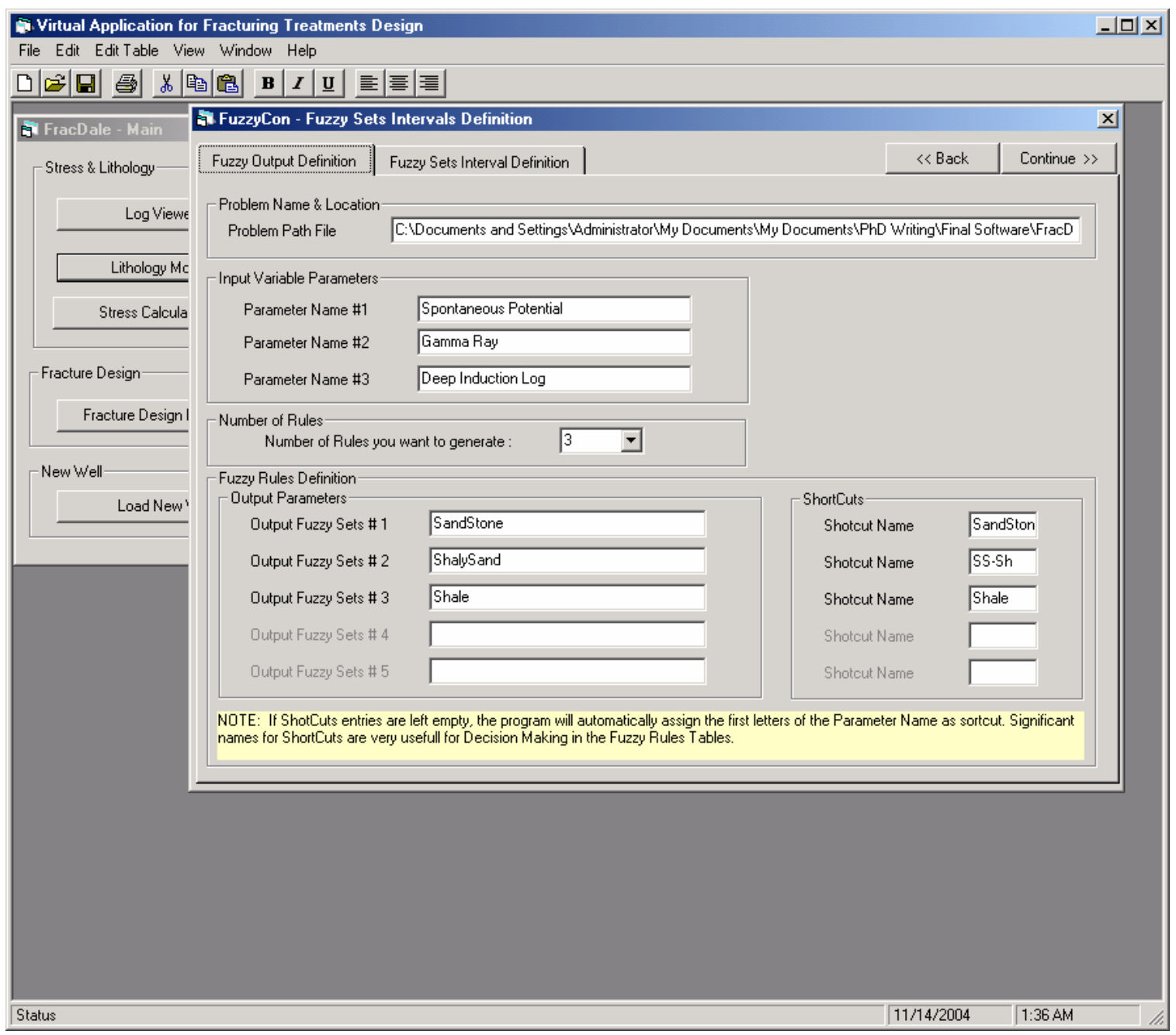

Figure 7-6: Lithology Model Design Form - Input/Output Definition Tab

Figure 7-6 shows the Design form of the Lithology Model. The analysis is done with defined fuzzy lithology rules loaded from an external file. However the user can design his/her own rules as well. The form includes two tabs, Fuzzy Input/Output Definition and Fuzzy Sets Interval Definition. Figure 7-6 shows the first tab where the user enters the names of the input parameters and the outcomes of the system. 


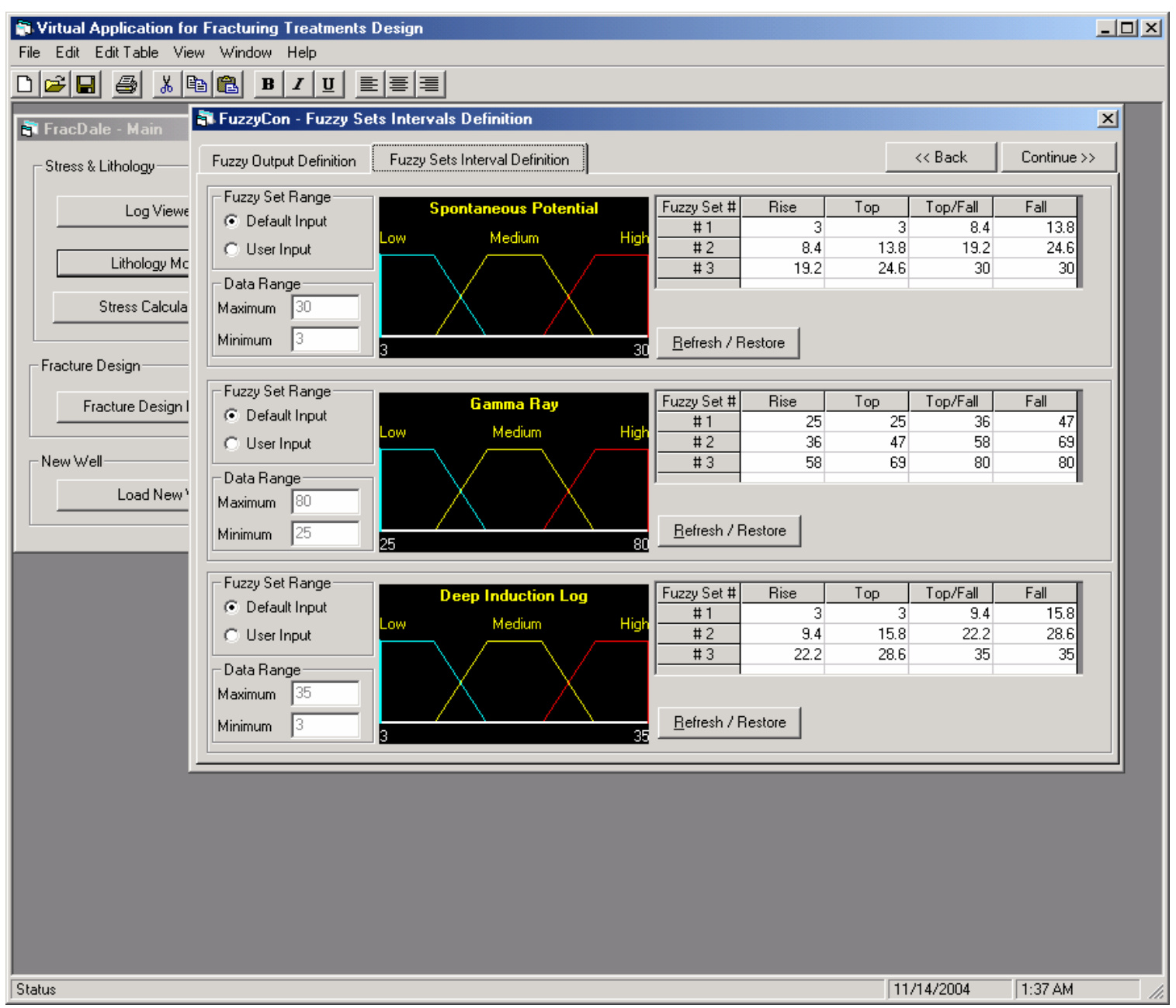

Figure 7-7: Lithology Model Design Form - Fuzzy Sets Interval Definition Tab

Figure 7-7 shows the second tab of Lithology Model Design form entitled Fuzzy Sets Interval Definition. As the name implies, the intervals for the fuzzy sets are entered in this section. The default is the trapezoidal fuzzy set. However, each one can be modified to reflect specific cases. The user can access this form at any time during fuzzy design. 


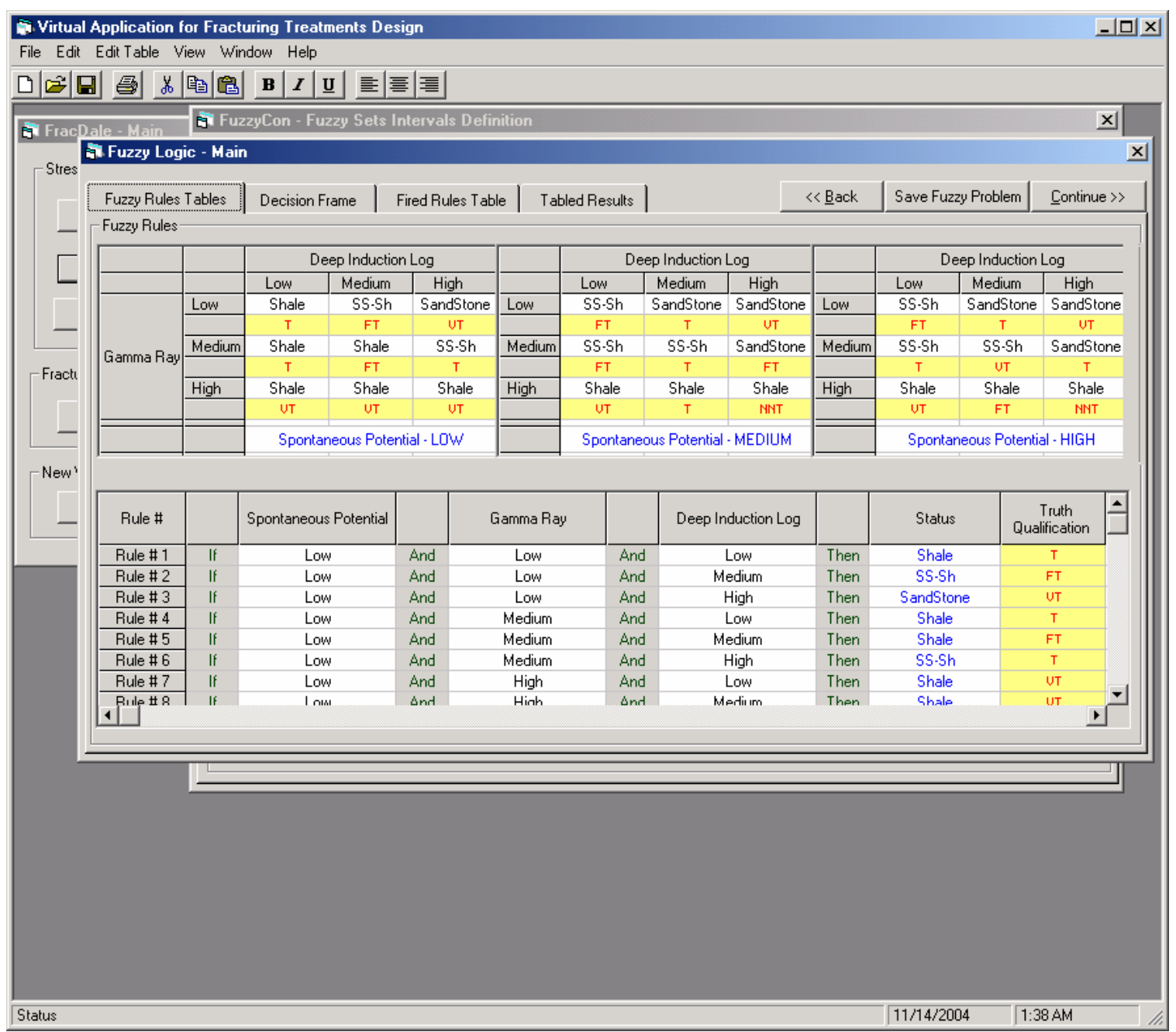

Figure 7-8: Lithology Model Main Form - Fuzzy Rules Table Tab

Figure 7-8 shows the main form of the Lithology Model. This form consists of four tabs: Fuzzy Rules Table, Decision Frame, Fired Rules Table, and Tabled Results. Figure 7-8 presents the first tab, Fuzzy Rules Table. The rules are set in the three-grid matrix using data previously entered in the Lithology Model Design form, Input/Output Definition Tab (Figure 7-6). If a well file is loaded the rules are already present in the grids; otherwise, the grids are empty and the user is required to build the rules. The next table shows the rules in line format. 


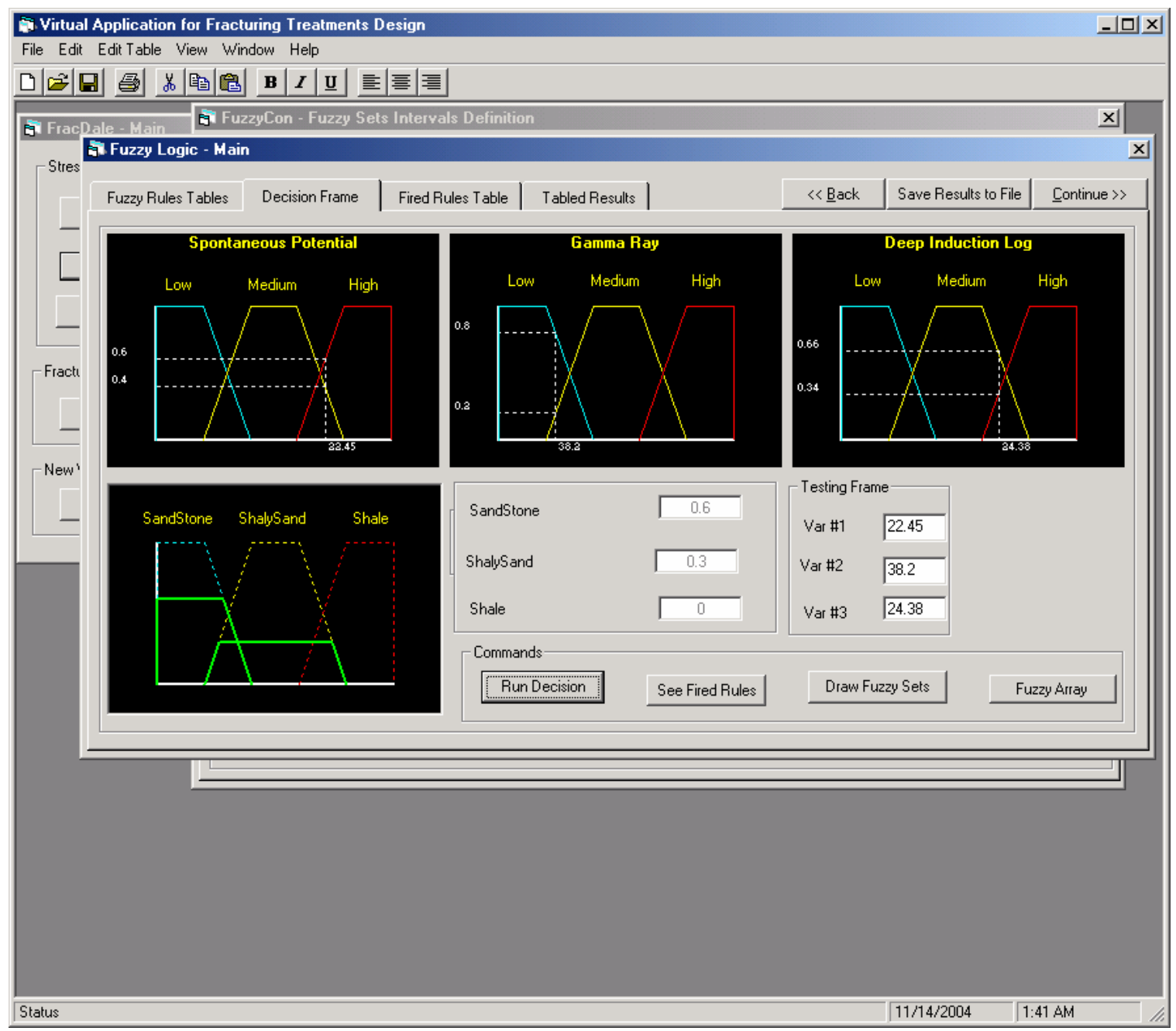

Figure 7-9: Lithology Model Main Form - Decision Frame Tab

Figure 7-9 shows the Decision Frame tab of the main form in the Lithology Model. This tab illustrates the decision process. Fuzzy logic decision is a parallel process where multiple rules are fired in the same time. Figure 7-9 shows an example of a decision where each input value belongs to more than one set. The outcome of the system is given in degrees of membership, graphically presented in the plot on the bottom of the screenshot. The user can make decisions firing cases one by one or the entire set of cases at once. To fire all cases, the Fuzzy Array command button is used. 


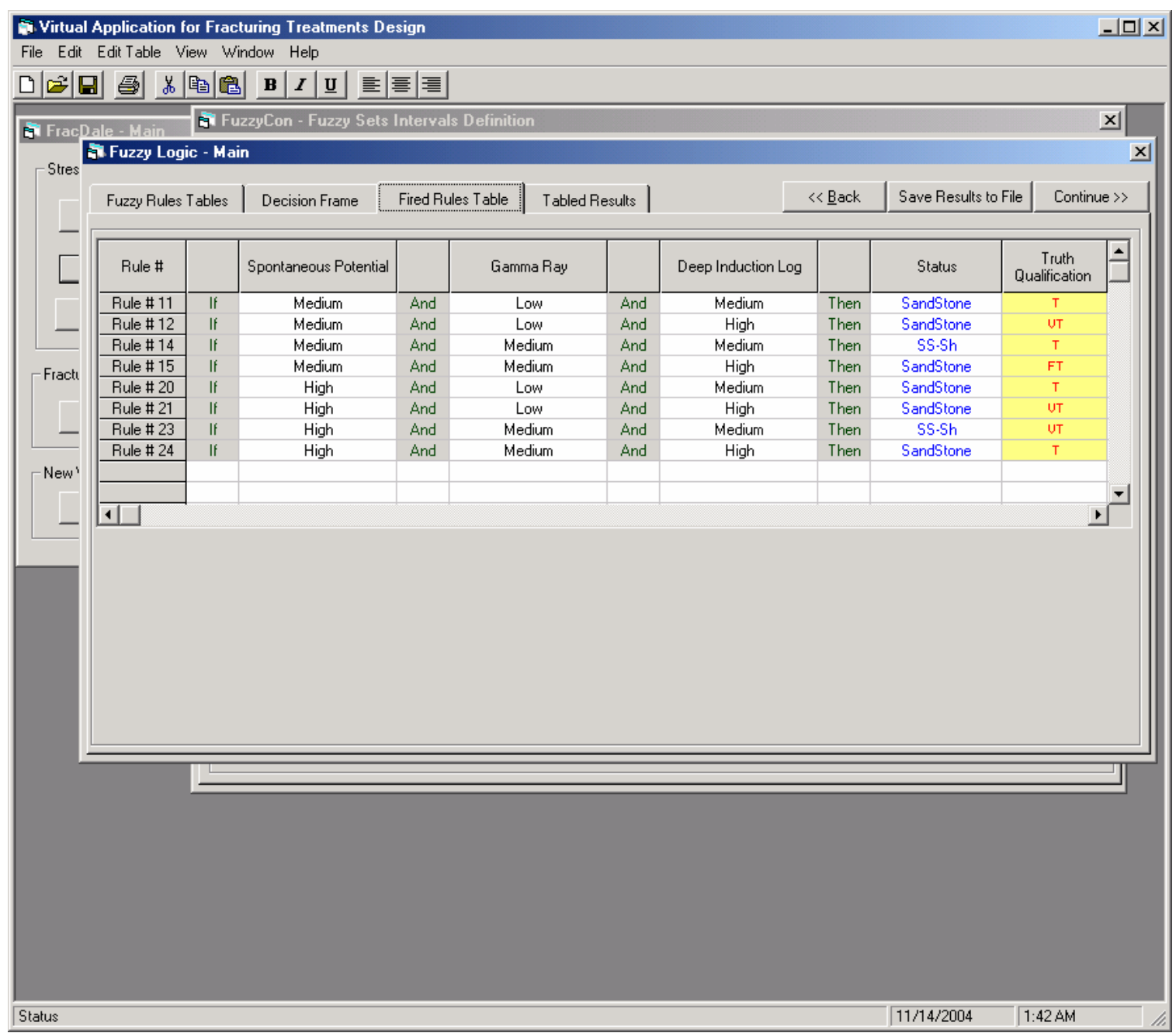

Figure 7-10: Lithology Model Main Form - Fired Rules Table Tab

Figure 7-10 shows the Fired Rules Table tab of the main form in the Lithology Model. This tab shows all the rules that were fired for the example presented in the previous tab. This information is typically used for a single case decision where in-depth analysis is required. 


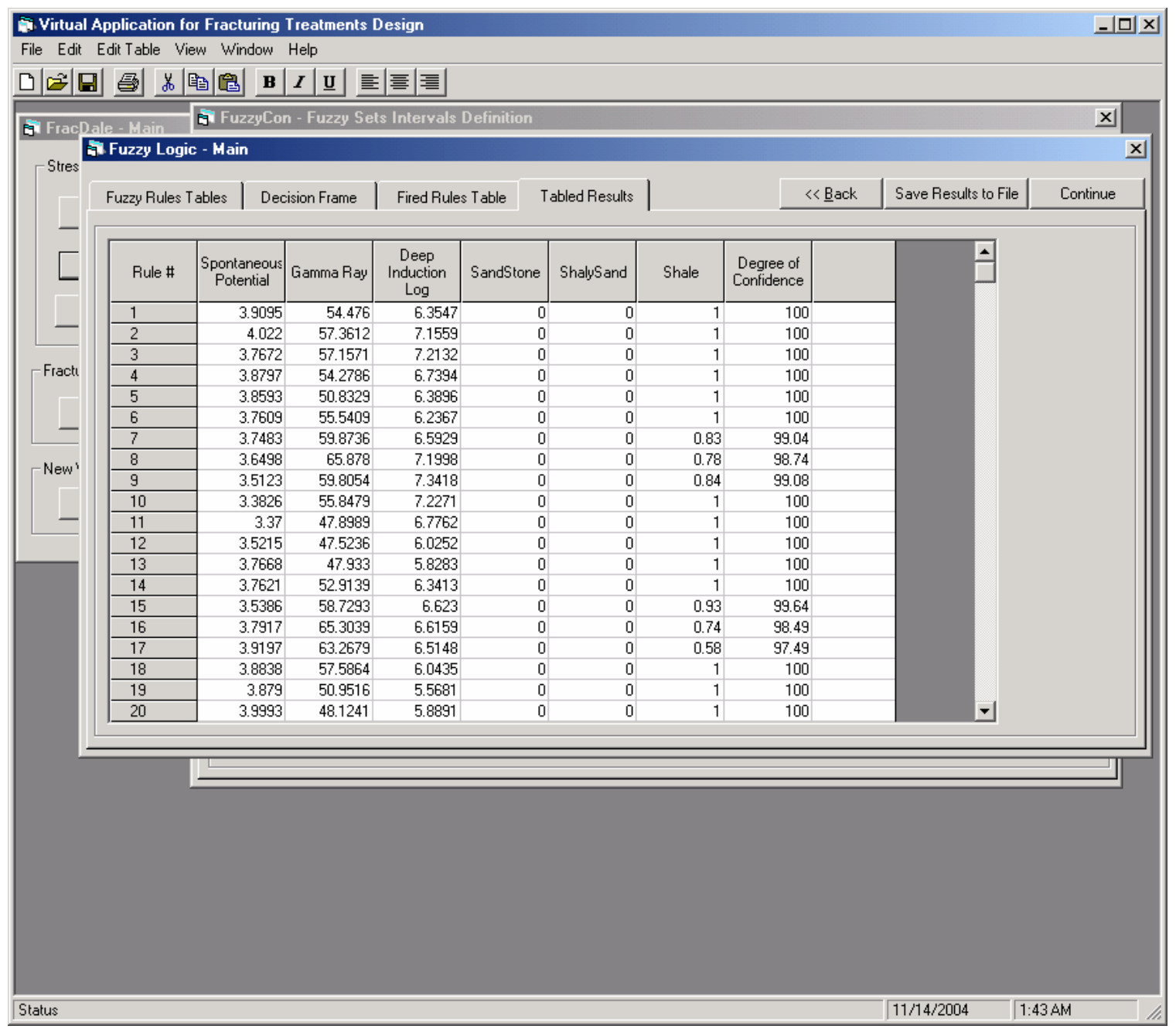

Figure 7-11: Lithology Model Main Form - Tabled Results Tab

Figure 7-11 shows the Tabled Results tab of the main form in the Lithology Model. The table includes all cases from the data set involved in the decision process. The outcome of the decision is presented in columns four, five, and six, while the degree of confidence for each decision is given in column seven. This information can be used as is or as the input for a different process. In this application, the outcome of the decision process is used with a classic stress equation to calculate the stress profile. 


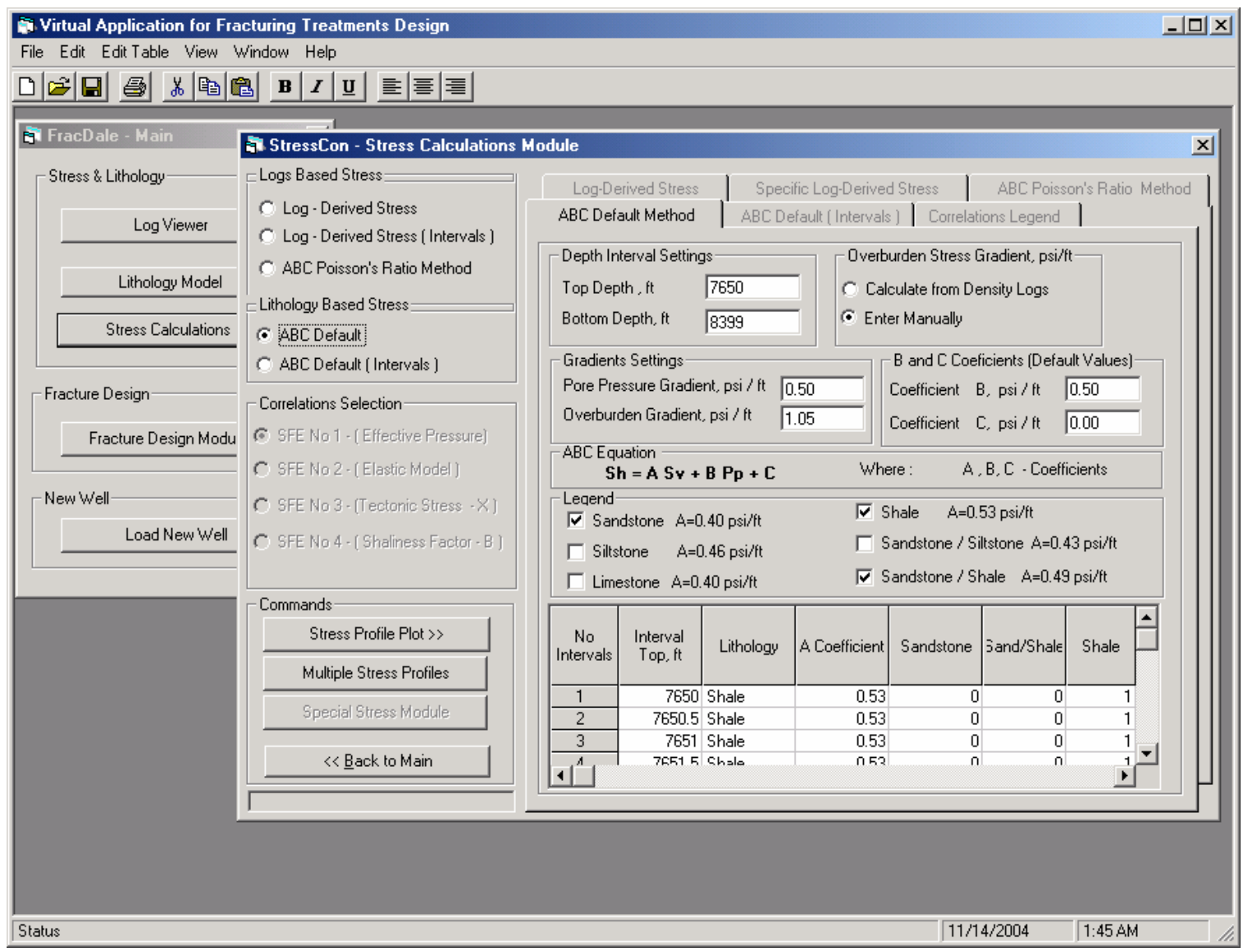

Figure 7-12: Stress Calculation Module Form - ABC Default Method Tab

Figure 7-12 shows the Stress Calculation Module Main form. This module includes all available techniques and equations for stress calculations. On the left side of the form, a radio button is provided for each model. By selecting a model, a tab is activated on the right part of the screen that has the details regarding the model along with the requested and default information. Figure 7-12 presents the ABC Default Model selection, since this model was used in the study. The inner ABC Default tab shows information regarding data and gradients used in calculations. The table from the bottom of the screen displays the lithology identification interpretation as an outcome of the fuzzy lithology system. A routine assigns the corresponding lithology coefficient and the calculated stress. 


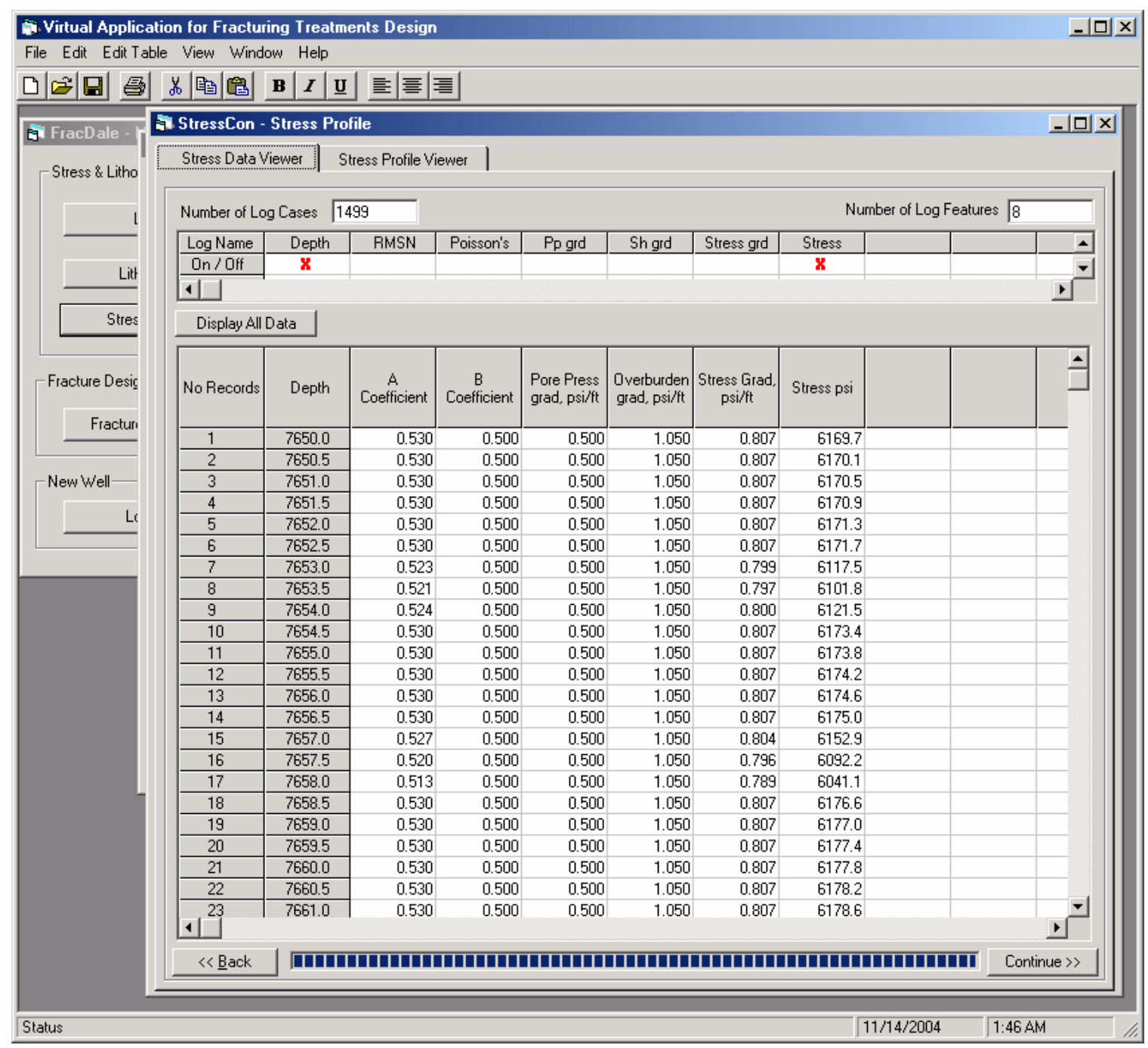

Figure 7-13: Stress Profile Form - Stress Data Viewer Tab

Figure 7-13 shows the Stress Profile form. This form presents two tabs: Stress Data Viewer and Stress Profile Viewer. The results of the stress calculation done in the Stress Calculation Module form are imported into the Stress Data Viewer tab of this form. Depths, Poisson's coefficients, overburden gradients, stress values, and others are displayed in table format. Similar to the Log Viewer form, the user can select which parameter to plot. The stress profile presents the most interest for graphic visualization since this processing will be a direct input into the fracture treatment optimization. 


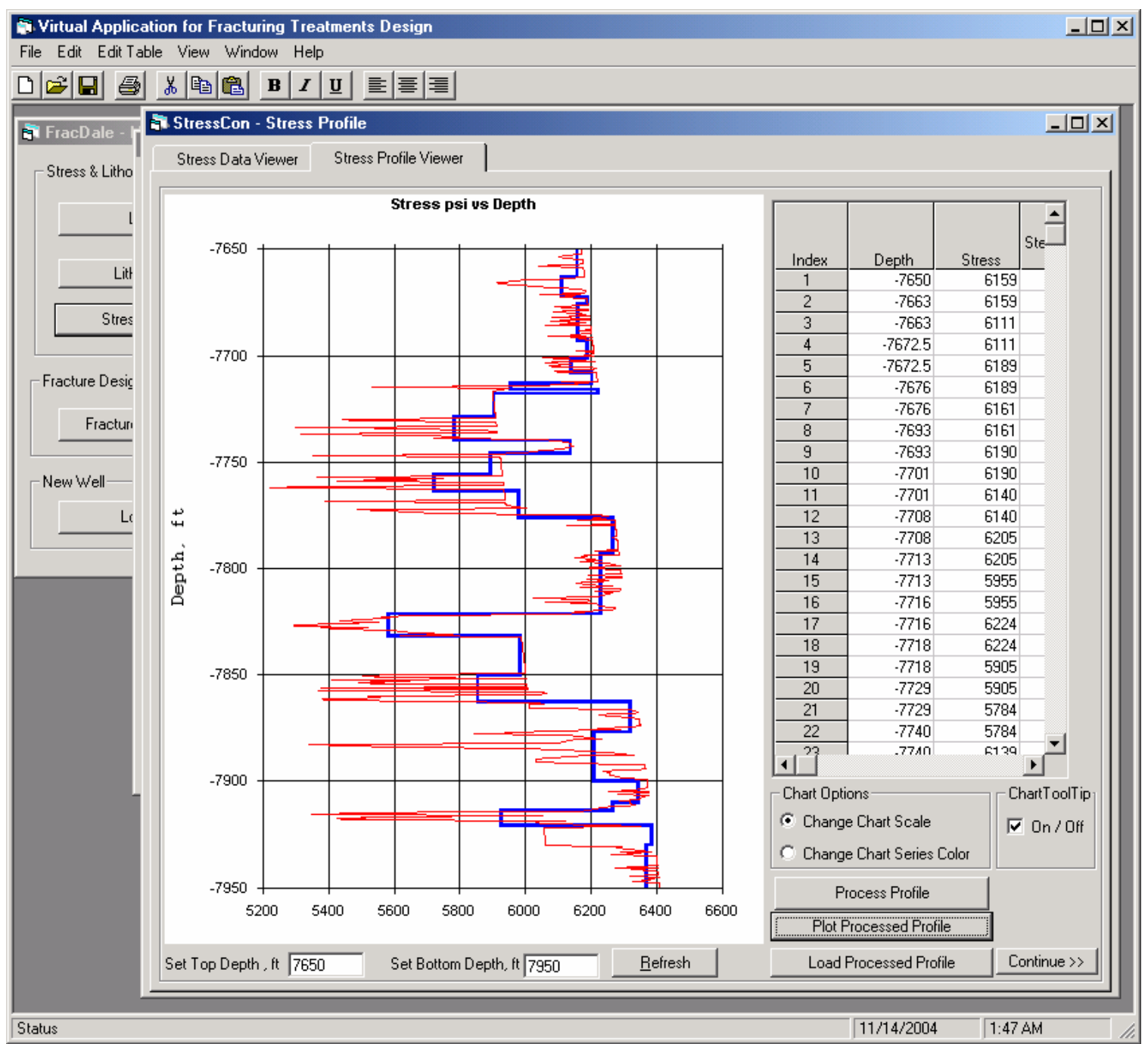

Figure 7-14: Stress Profile Form - Stress Data Viewer Tab

Figure 7-14 shows the Stress Profile Viewer under the Stress Profile form. This tab displays the graphical representation of the stress profile, or any other parameter selected in the previous tab. The stress profile processing is completed during this step. At the right side of the form, a table allows the user to visually define the formation with similar stress characteristics. If an existing well is loaded, this file already exists and can be loaded; otherwise, the user can enter the depths for each potential layer. The application will calculate an average stress for the selected interval and plot the processed profile on the chart. The processed profile is then loaded in the fracture design module where it will be used to derive the four-layer equivalent stress profile. 


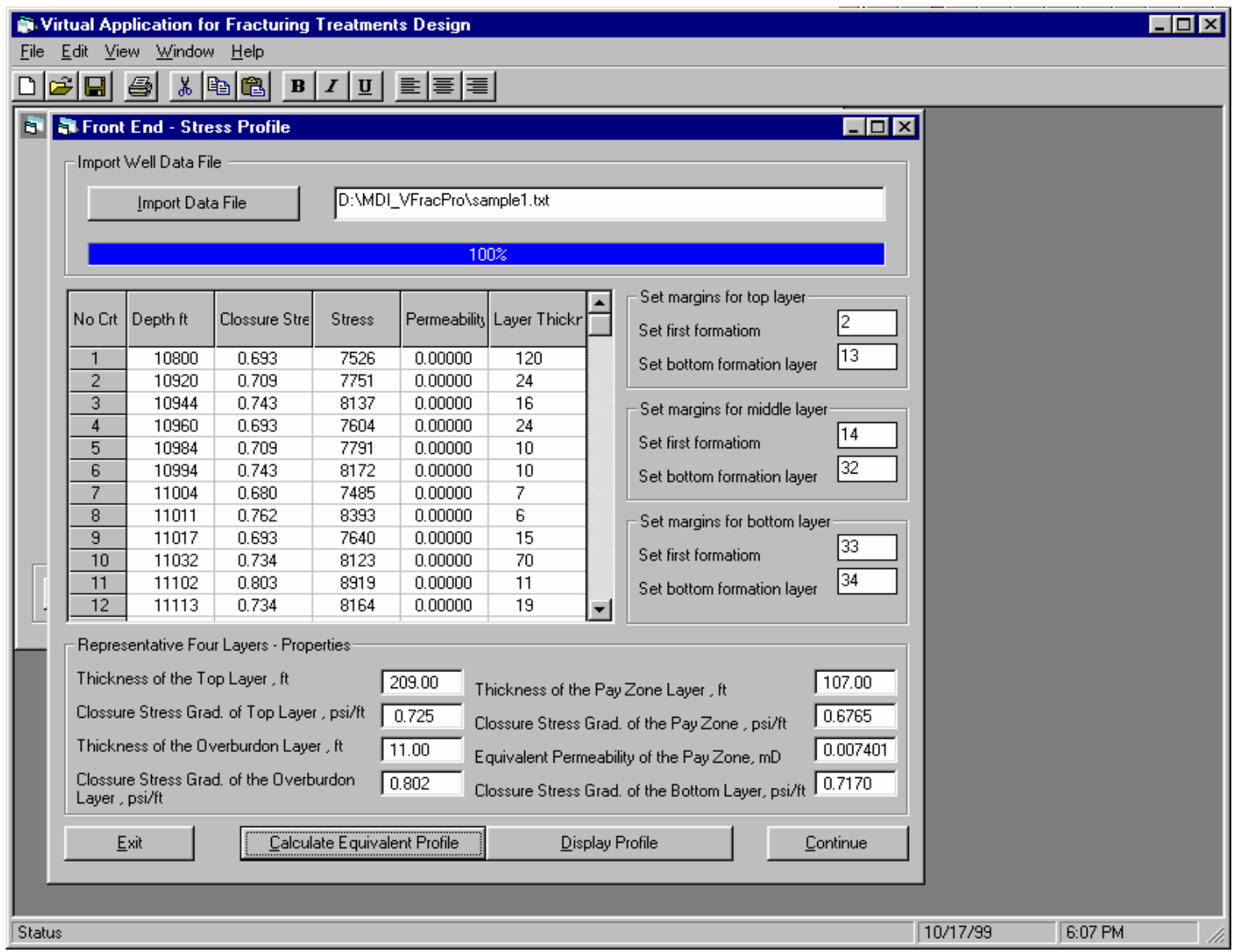

Figure 7-15: Equivalent Stress Profile Form

Figure 7-15 shows the Equivalent Stress Profile form. This form allows for the user of multiple data source for stress profiling. If a stress profile was already processed with this application, it can be loaded and used for optimization. If a processed stress profile is available from an external source, such as Excel or text files, it can be loaded into the application at this point. The processed stress profile is displayed in the table at the left side of the form. The application identifies the production zones based on permeability contrasts and automatically calculates the equivalent four-layer stress profile. 
File Edit View Window $\underline{\text { Help }}$

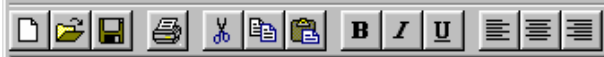

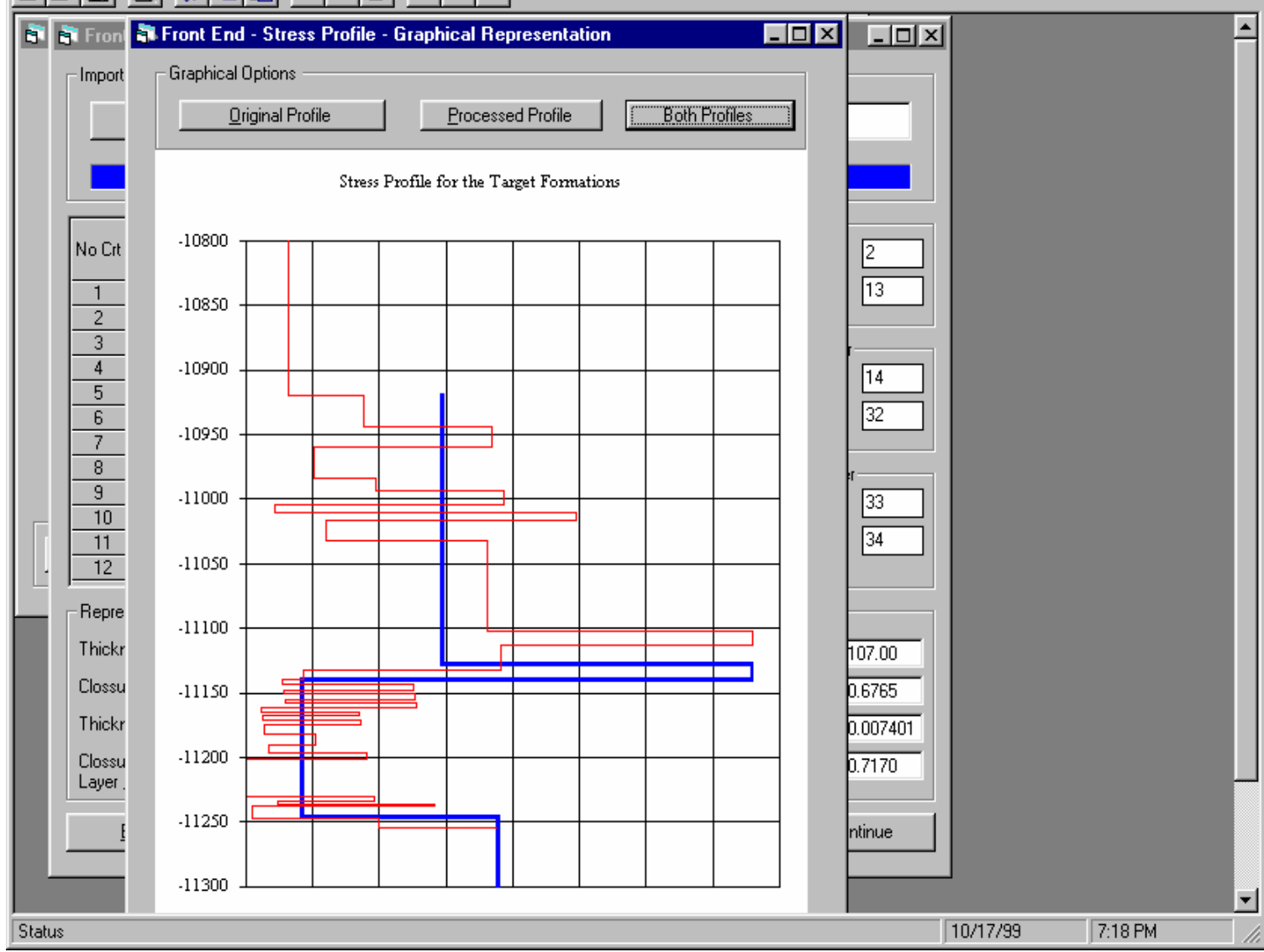

Figure 7-16: Equivalent Stress Profile Form

Figure 7-16 shows the graphical representation of the equivalent four-layer stress profile. The form allows for plotting the original profile and the equivalent profile separately. In addition, both profiles can be plotted in the same time. Next, the equivalent four-layer profile is sent to the main fracture design optimization treatment form, where it is used as direct input in the optimization process. 


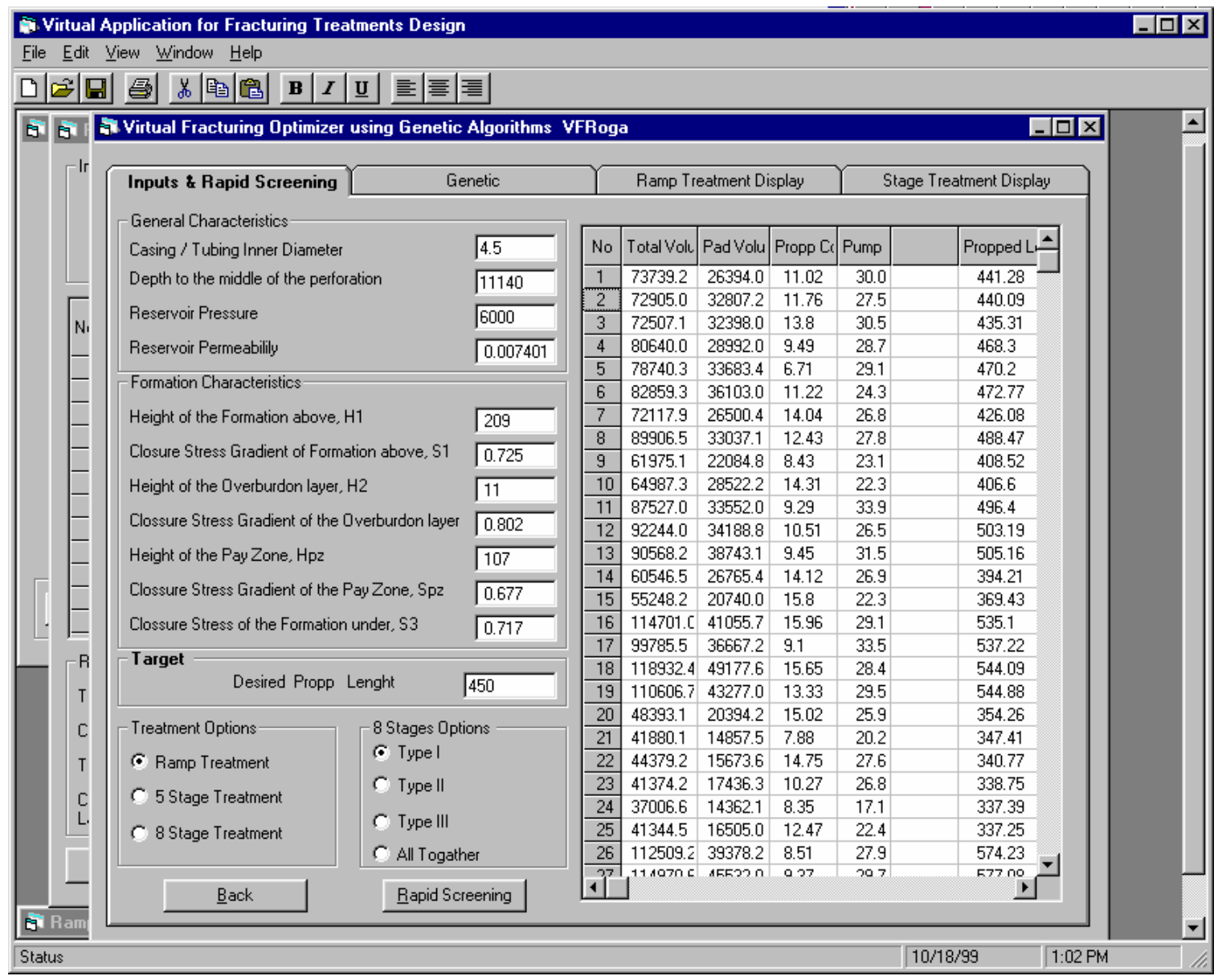

Figure 7-17: Main Optimization Form - Rapid Screening Tab

Figure 7-17 shows the Main Optimization form. This form consists of four tabs. The current figure presents the Rapid Screening tab. The left side of the tab shows the reservoir inputs loaded from the previous form. The types of treatments available for optimization are given at the bottom left of the form. The selection can be done using the radio buttons. A rapid screening represents the first generation of the genetic optimization. An initial population is generated and the output is ranked from the best to the weakest after each case is run through the fitness function. The ranked list is displayed in table format at the right side of the tab. The user can visually analyze each job and the predicted outcome. Double-clicking on each job index brings up the next form. 


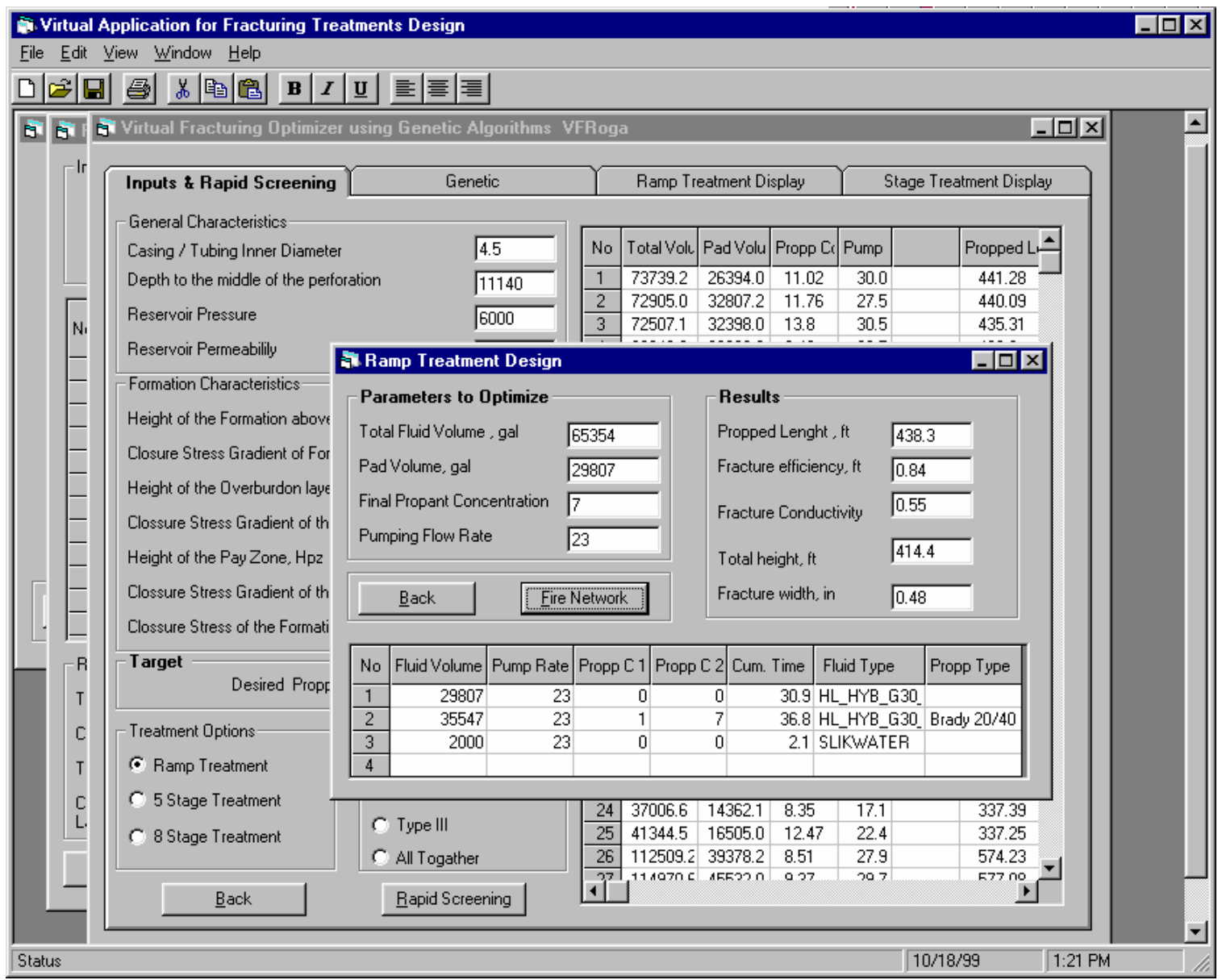

Figure 7-18: Ramp Treatment Form

Figure 7-18 shows the Ramp Treatment form. This form displays the description of the ramp treatment suggested by the application in detail. Each of the treatments displayed in the Main Optimization form, Rapid Screening Tab, can be further analyzed in this form. The treatment design can manually be modified, and the neural network can be fired again for the altered treatment design. The rapid screening provides a fast and easy way to manually design optimum treatments. 


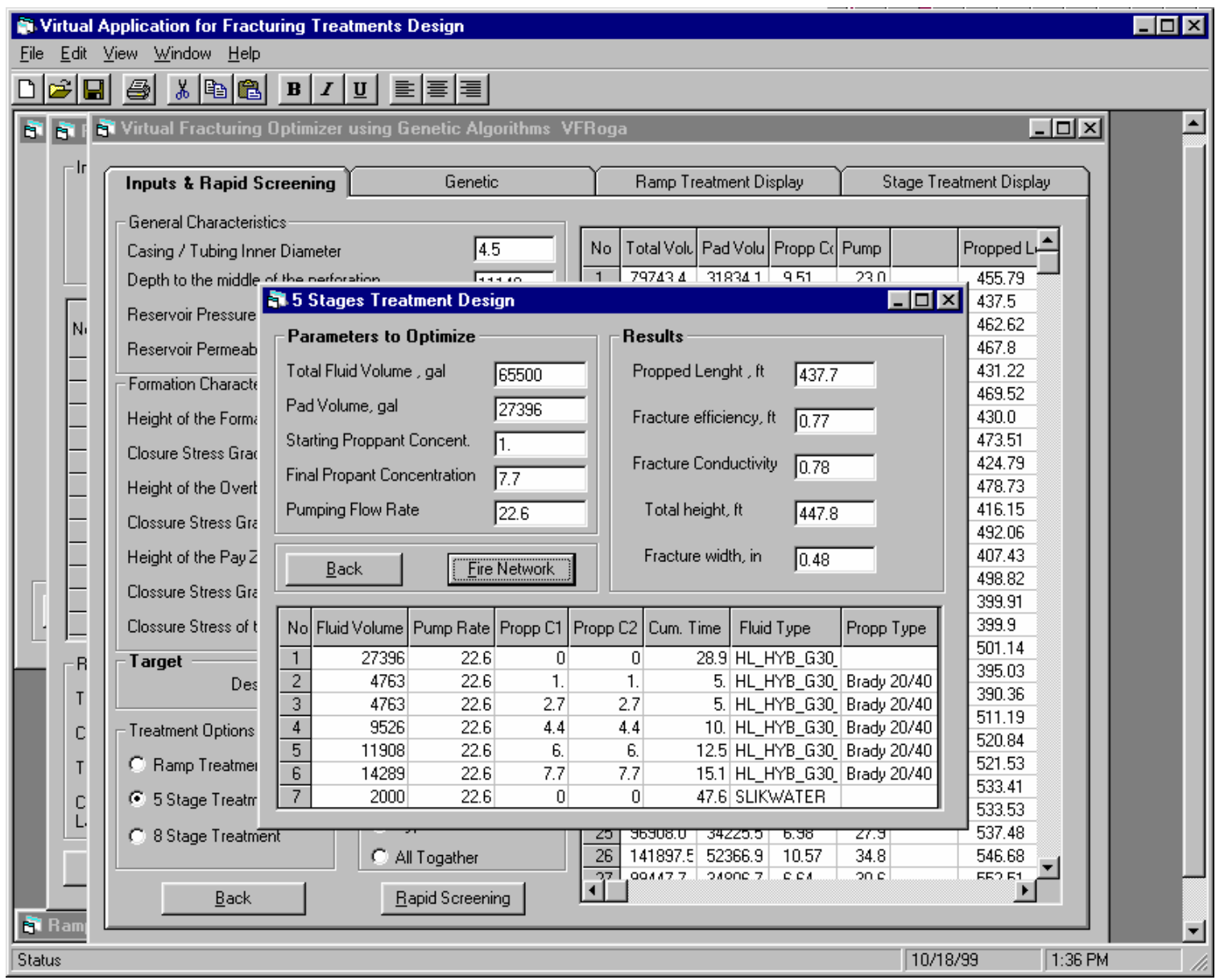

Figure 7-19: Five Stage Treatment Design Form

Figure 7-19 shows the Five Stage Treatment Design form. This form comes up when the Five Stage Treatment radio button is selected. Moreover, this form is identical to the ramp treatment form with the exception that it designs five stage treatments. Each of the treatments displayed in the Main Optimization form, Rapid Screening Tab, can be further analyzed in this form. The treatment design can manually be modified, and the neural network can be fired again for the altered treatment design. 


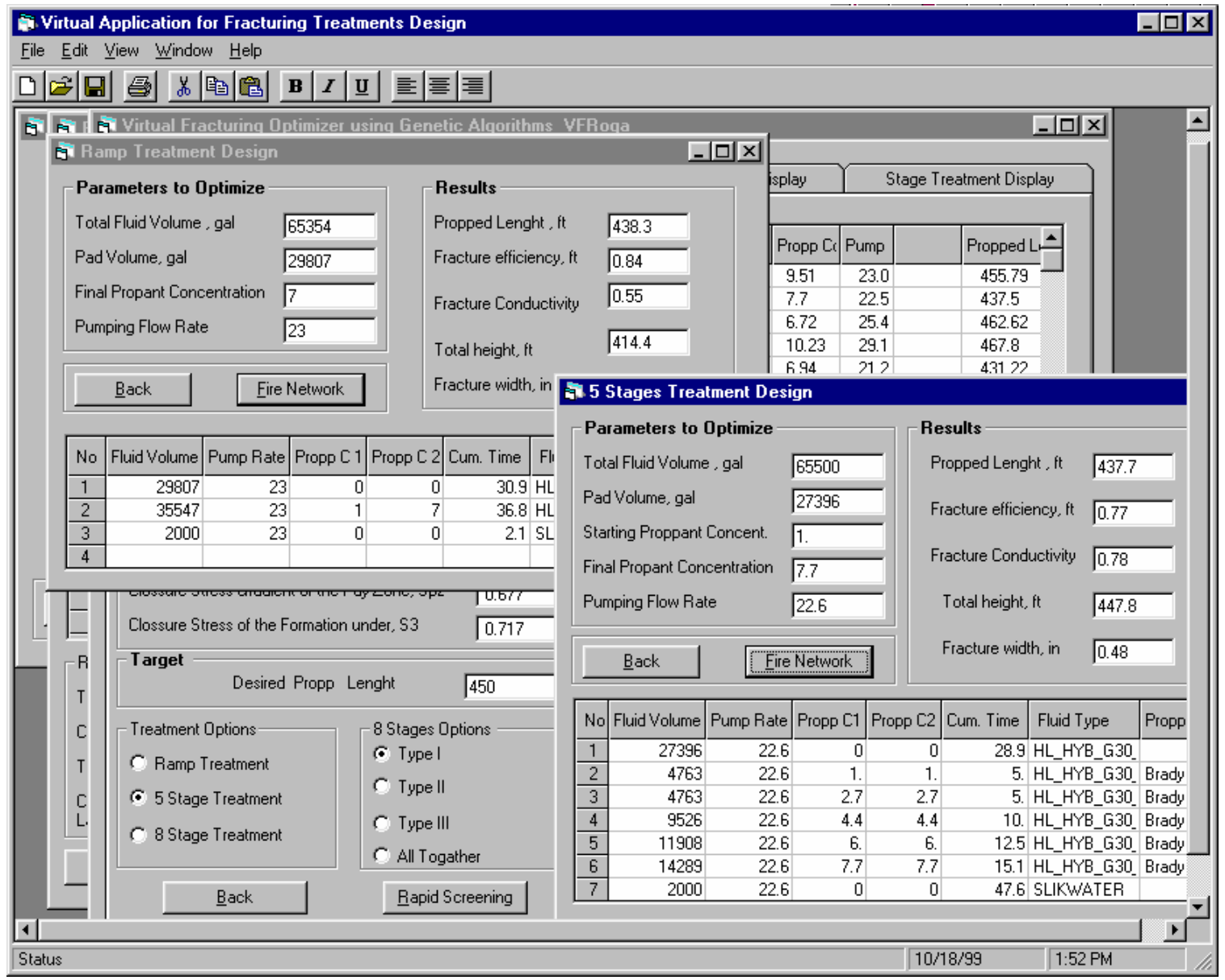

Figure 7-20: Multiple Screens Comparison - Ramp Treatment \& Five Stage Treatment

Figure 7-20 shows two screens where the same fracture target lengths are compared. One screen, at the top left shows the optimum ramp treatment while the second screen at the bottom right shows the five-stage treatment. This capability of the application makes comparing different treatments very easy. 


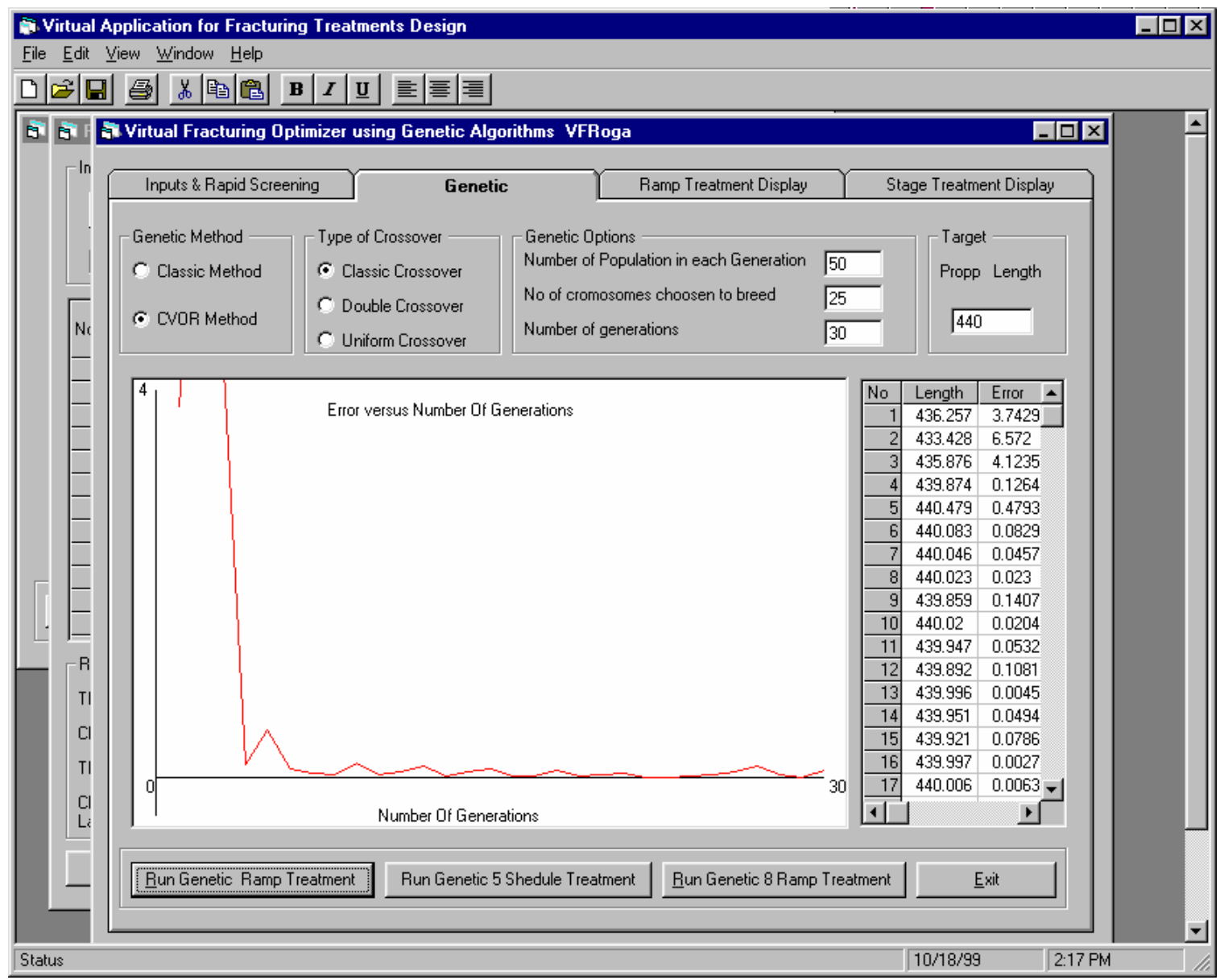

Figure 7-21: Main Optimization Form - Genetic Tab

Figure 7-21 shows the Main Optimization form, Genetic tab. This form was designed specifically for optimization using genetic algorithms. The typical genetic parameters are given at the top of the form. The user can modify these parameters as desired. The convergence speed is measured graphically using a plot. The plot shows the error between best solution and target versus the number of generations. The solutions of the final population are ranked ascending in the table at the right side of the tab. 


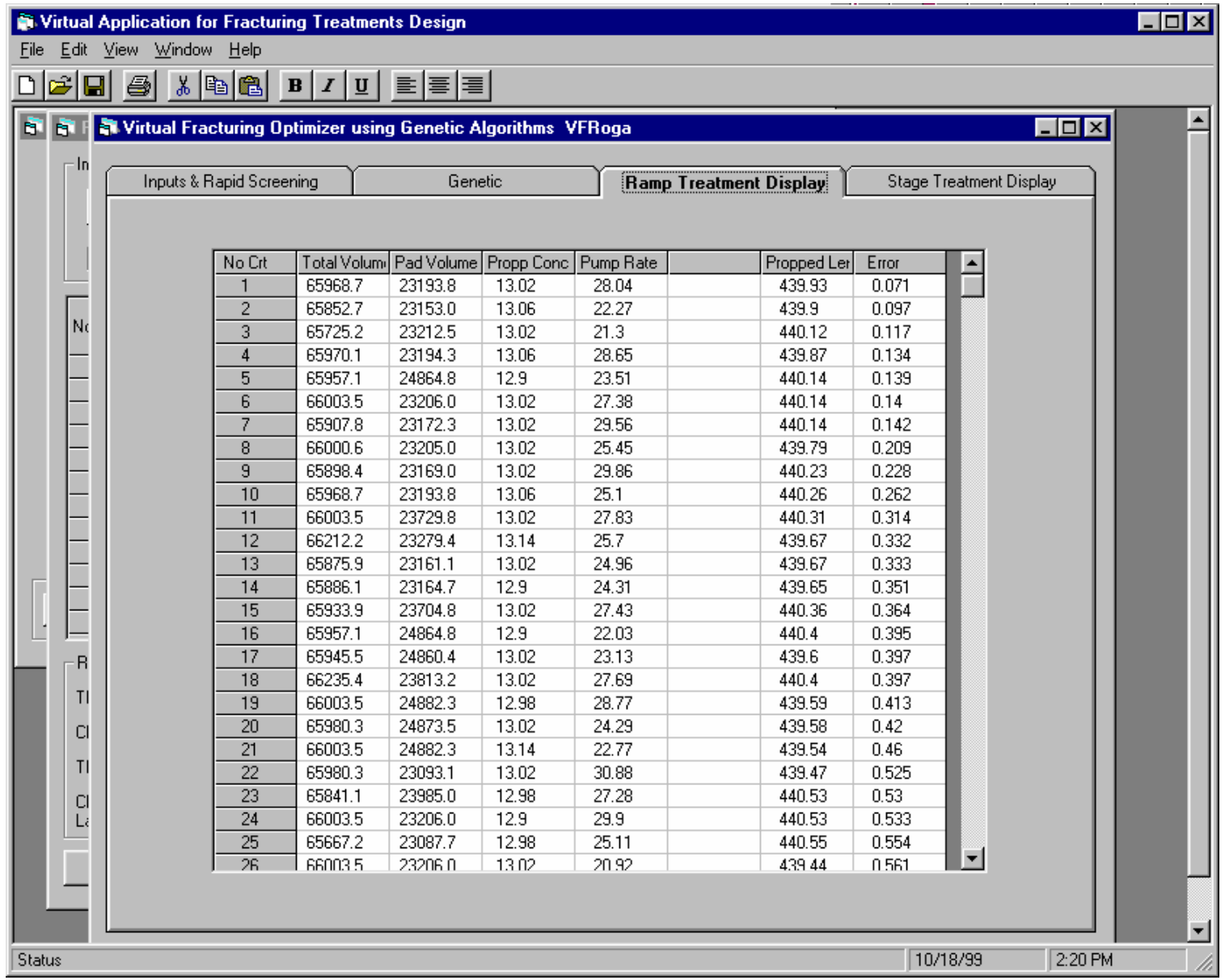

Figure 7-22: Main Optimization Form - Ramp Treatment Display Tab

Figure 7-22 shows the Main Optimization form, Ramp Treatment Display tab. This screen shows the best solutions suggested by the optimization routine. These solutions are displayed in table format. Double-clicking on the solution index number brings up the ramp treatment form where the user can visualize the detailed treatment schedule. A similar tab is available for stage treatments schedules. 


\section{CHAPTER 8: CONCLUSIONS}

1. Optimum treatment design can be achieved using a combination of artificial intelligent tools without the use of a traditional hydraulic fracture simulator. The study proved that three basic logs, together with a series of artificial intelligence tools, are sufficient to provide robust initial optimum solution for a treatment design.

2. Stress profiles can be sufficiently determined using a fuzzy expert system for lithology identification together with a basic stress equation. The fuzzy expert system makes use of three basic logs (spontaneous potential, gamma ray, and resisitivity) or any other available logs. The power of the fuzzy logic lithology identification system is particularly compelling when sonic logs are not available.

3. A new methodology to reduce the complex lithology profile to an equivalent four-layer stress profile was introduced. This reduced the large amount of data necessary for complex reservoir characterization to eight main parameters. The equivalent four-layer profile proved very efficient in preserving the fracture geometry during simulation.

4. A neuro-genetic optimization procedure was developed to design optimum fracture treatments. The procedure integrates power and speed of neural networks with the fast convergence of genetic algorithms to provide solution to a complex four-parameter optimization problem.

5. A one-dimensional modified version of vector quantization was used to compress the large number of data recordings from the pressure profile to ten pressure points. This compression allowed the use of neural networks for pressure profile prediction. Without this reduction, the large amount of data would have made impossible to train a predictive model.

6. Two neural networks were successfully developed to predict net pressure profile developed during a fracture treatment. One neural network compensates for the difference between the pressure profile obtained from the original lithology and the pressure profile generated using equivalent four-layer profile. The other neural network is used for net pressure profile prediction. Combined, they provide the expected net pressure profile equivalent to that resulting from the original lithology.

7. The methodology was tested for an example treatment that was designed by Halliburton experts. The treatment design suggested by applying this methodology was found to be in good agreement with the Halliburton-designed treatment. 
8. A new computer application was developed to address the continuous effort towards fracture optimization. This application integrated the methodology presented in this study into a userfriendly computer application intended to be a companion tool to any fracture simulation software.

9. The modular implementation of the developed software permits altering the neural networks without modifying the source code. This allows the program to be used in any field or with data from simulators other than FRACPRO.

10. The methodology used in this study can be used with other simulators or reproduced using real field data. Since the development of this methodology was focused on tight gas sands, it is directly applicable to similar formations. However, the methodology is flexible enough to be applied to any kind of field where fracturing is utilized to increase performance. 


\section{BIBLIOGRAPHY}

1. Department of Energy, Contract EPA-816-D-02-006: Evaluation of Impacts to Underground Sources of Drinking water by Underground Hydraulic Fracturing of Coalbed Methane Reservoirs. (2002) Appendix A.

2. Hykin S.: Neural Networks: A Comprehensive Foundation. New York: Macmillan College Publishing Inc, 1994.

3. Mohaghegh, S.D.: "Virtual Intelligence Applications in Petroleum Engineering: Part 1 Artificial Neural Networks," JPT (September 2000) 64-73.

4. Nelson, M. and Illigworth, W.I.: A Practical Guide to Neural Nets. Addison-Wesley Publishing Company Inc, 1992.

5. Mohaghegh, S.D.: "Virtual Intelligence Applications in Petroleum Engineering: Part 2 Evolutionary Computing," JPT (October 2000) 40-46.

6. Michalewicz, Z.: Genetic Algorithms + Data Structures = Evolutionary Programs. Verlag, Berlin, Springer, 1992.

7. Mohaghegh, S.D.: "Virtual Intelligence Applications in Petroleum Engineering: Part 3 - Fuzzy Logic," JPT (November 2000) 82-87.

8. Mohaghegh, S., Platon, V., and Ameri, S.: "Candidate Selection for Stimulation of Gas Storage Wells Using Available Data with Neueral Networks and Genetic Algorithms," paper SPE 51080 presented at the 1998 SPE Eastern Regional Meeting, Pittsburgh, Pennsylvania, 9-11 November.

9. FRACPAC Completion Services: Stimulation and Sand-Control Techniques for HighPermeability Oil and Gas Wells. Halliburton Energy Services, Houston, Texas, 1994.

10. CER Corporation and S.A. Holditch \& Associates: Staged Field Experiment No. 2: Application of Advanced Geological, Petrophysical and Engineering Technologies to Evaluate and Improve Gas Recovery from Low Permeability Sandstone Reservoirs - Volume I. Gas Research Institute, Topical Report No. GRI-89/0140, Contract No. 5088-211-1682 (1989).

11. CER Corporation and S.A. Holditch \& Associates: Staged Field Experiment No. 2: Application of Advanced Geological, Petrophysical and Engineering Technologies to Evaluate and Improve Gas Recovery from Low Permeability Sandstone Reservoirs - Volume II. Gas Research Institute, Topical Report No. GRI-90/0093, Contract No. 5088-211-1682 (1990).

12. CER Corporation and S.A. Holditch \& Associates: Staged Field Experiment No. 3: Application of Advanced Technologies in Tight Gas Sandstone - Travis Peak and Cotton Valley 
Formations, Waskom Field, Harrison County, Texas. Gas Research Institute, Topical Report No. GRI-91/0048, Contract No. 5090-211-1940 (1991).

13. CER Corporation and S.A. Holditch \& Associates: Staged Field Experiment No. 3: Application of Advanced Technologies in Tight Gas Sandstone - Frontier Formation, Chimney Butters Field, Sublette County, Wyoming. Gas Research Institute, Topical Report No. GRI-92/0394, Contract No. 5091-211-2130 (1992).

14. Nolte, K.G.: "Principles for Fracture Design Based on Pressure Analysis," paper SPE 10911, SPEPE (February 1988).

15. Nordgren, R.P.: "Propagation of a Vertical Hydraulic Fracture," SPEJ (August 1972) 306.

16. Prats, M., Hazebroek, P., and Strickler, W.R.: "Effect of Vertical Fractures on Reservoir Behavior-Compressible-Fluid Case," SPEJ (June 1962) 87.

17. Evaluation of the $A B C$ Stress Derivations Methodology. Bragan \& Associates, Final Report GRI-95/0353, Contract No. 5094-2203086, Las Vegas, Nevada (June 1995).

18. Khristianovic, S.A. and Zheltov, Y.P.: "Formation of Vertical Fractures by Means of Highly Viscous Liquids," presented at the World Petroleum Congress, Rome (1955).

19. Economides, J.M. and Nolte, K.G.: Reservoir Stimulation, second edition, Schlumberger Educational Services, Houston, Texas (1989).

20. Meyer, B.R.: "Design Formulae for 2-D and 3-D Vertical Hydraulic Fractures: Model Comparison and Parametric Studies," paper SPE 15240 presented at the 1986 Unconventional Gas Technology Symposium of the Society of Petroleum Engineers, Louisville, Kentucky, 18-21 May.

21. Geertsma, J. and de Klerk, F.: "A Rapid Method of Predicting Width and Extend of Hydraulically Induced Fractures," JPT (December 1969) 1571.

22. Perkins, T.K. and Kern, L.R.: "Widths of Hydraulic Fractures," JPT (1961) 937.

23. Cleary, M.P.: "Analysis of Mechanisms and Procedures for Producing Favourable Shapes of Hydraulic Fractures," paper SPE 9260 presented at the 1980 Annual Fall Conference and Exhibition of the Society Petroleum Engineers of AIME, Dallas, Texas, 21-24 September.

24. Cleary, M.P.: "The Engineering of Hydraulic Fractures-State of the Art and Technology of the Future," JPT (January 1988) 13-21.

25. Cleary, M.P.: "Hydraulic Fracturing in Medium-High Permeability Reservoir: Methodology and Economic Advantages of Properly-Executed Jobs," paper SPE 28918 presented the EUROPEC 94, London, United Kingdom, 25-27 October. 
26. FRACPRO: Hydraulic Fracture Treatment Design and Analysis User Manual, version 8.1, Resources Engineering Systems, Inc. (RES).

27. Warpinski, N.R. et al: "Comparison Study of Hydraulic Fracturing Models - Test Case: GRI Stage Field Experiment No. 3," paper SPE 25890, SPEPF (February 1994).

28. Cherkassky, Vladimir and Mulier, Filip: Learning From Data, Concepts, Theory and Methods. John Wiley \& Sons, Inc, New York (1998).

29. Linde, Y., Buzo, A., and Gray, R.M.: "An Algorithm for Vector Quantizer Design,"presented at the IEEE Trans. on Comm., Vol. COM-28 (January 1980) 84-95.

30. Gray, R.M.: “Vector Quantization," IEEE ASSP Magazine, Vol. 1 (April 1984) 4-29.

31. Mohaghegh, S., Goddard, C., Popa, A., Ameri, S., and Bhuiyan, M.: "Reservoir Characterization Through Synthetic Logs," paper SPE 65675 presented at the 2000 SPE Eastern Regional Conference and Exhibition, Morgantown, West Virginia, October 17-19.

32. Mohaghegh, S., Koperna, G., Popa, A. S., and Hill, D. G.: "Reducing the Cost of Field-Scale Log Analysis Using Virtual Intelligence Techniques," paper SPE 57454 presented at the 1999 SPE Eastern Regional Conference and Exhibition, Charleston, West Virginia, 21-22 October 21-22.

33. Mohaghegh, S.D. et al: "Determining In-Situ Stress Profiles From Logs," paper SPE 90070 presented at the 2004 SPE Annual Technical Conference and Exhibition, Houston, Texas, 26-29 September.

34. Popa, A., Mohaghegh, S.D., Gaskari, R., and Ameri, S.: "Identification of Contaminated Data in Hydraulic Fracturing Databases: Application to the Codell Formation in the DJ Basin," paper SPE 83446 presented at the 2003 SPE Western Regional Conference and Exhibition, Long Beach, California, 20-24 May.

35. Mohaghegh, S., Popa, A., and Ameri, S.: "Intelligent Systems Can Design Optimum Fracturing Jobs," paper SPE 57433 presented at the 1999 SPE Eastern Regional Meeting, Charleston, West Virginia, 20-22 October.

36. Mohaghegh, S. Popa, A., Gaskari, R., and Ameri, S: "Identifying Successful Practices in Hydraulic Fracturing Using Intelligence Data Mining Tools; Application to the Codell Formation in the DJ Basin," paper SPE 77597 presented at the 2002 SPE Annual Conference and Exhibition, San Antonio, Texas, 29 September-2 October.

37. Mohaghegh, S. Popa, A., Gaskari, R., and Ameri, S: "Identifying Best Practices in Hydraulic Fracturing Using Virtual Intelligence Techniques," paper SPE 72385 presented at the 2001 SPE Eastern Regional Conference and Exhibition, North Canton, Ohio, 17-19 October. 
38. Mohaghegh, S., Mohamad, K., Popa, A. S., and Ameri, S.: "Performance Drivers in Restimulation of Gas Storage Wells," paper SPE 57453 presented at the 1999 SPE Eastern Regional Conference and Exhibition, Charleston, West Virginia, October 21-22.

39. Jikich, S, Popa. A: "Hyperbolic Decline Parameters Identification Using Optimization Procedures", paper SPE 65634 presented at the 2000 SPE Eastern Regional Conference and Exhibition, Morgantown, West Virginia, 17-19 October.

40. Cinco-Ley H., Samaniego F., "Transient Pressure Analysis for Fractured Wells", Journal of Petroleum Technology (September 1981) 1747-1766.Trans. AIME.

41. Centilmen, A., Ertekin, T., and Grader A.S.: "Applications of Neural Networks in Multiwell Field Development," paper SPE 56433 presented at the 1999 SPE Annual Technical Conference and Exhibition, Houston, Texas, 3-6 October.

42. Al- Wadahi, M., Grader, A.S., and Ertekin, T.: "An Investigation of Three-Phase CounterCurrent Flow Using X-Ray Computerized Tomography and Neuro-Simulation Modeling," paper SPE 63146 presented at the 2000 SPE Annual Technical Conference and Exhibition, Dallas, Texas, 1-4 October.

43. Chang, P.C. and Gray, R.M.: "Gradient Algorithms for Designing Predictive Vector Quantizers," IEEE Transactions ASSP, Vol. ASSP-34 (August 1986) 679-690. 
APPENDIX 


\begin{tabular}{|c|c|c|c|c|c|c|c|c|c|c|c|c|c|c|c|c|c|c|c|}
\hline 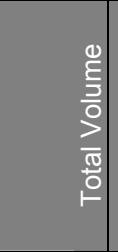 & $\begin{array}{l}\stackrel{0}{E} \\
\frac{5}{5} \\
0 \\
\frac{0}{\sigma} \\
0\end{array}$ & 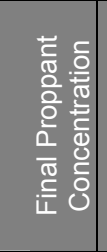 & 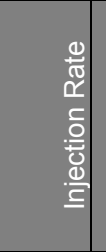 & 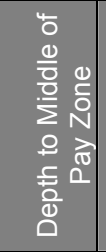 & 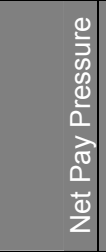 & 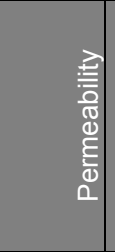 & 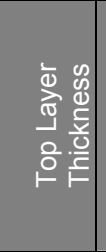 & 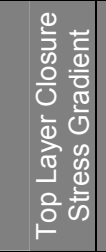 & 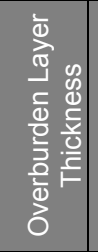 & 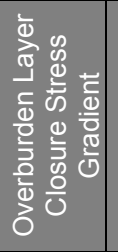 & 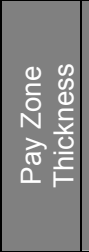 & 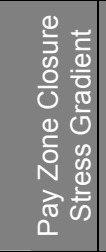 & 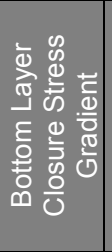 & 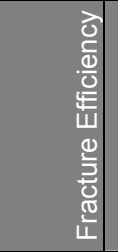 & 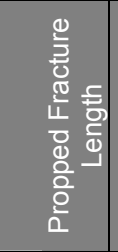 & 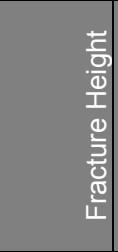 & 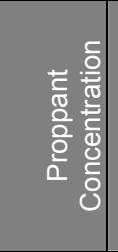 & 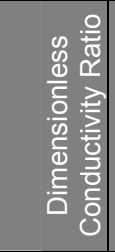 & 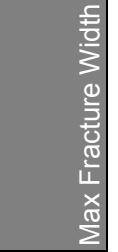 \\
\hline input & input & input & input & input & input & input & input & input & input & input & input & input & input & output & output & output & output & output & output \\
\hline 188,201 & 76,582 & 9.8 & 30 & 8,236 & 3,769 & 0.0461 & 228 & 0.761 & 12 & 0.792 & 122 & 0.706 & 0.790 & 0.680 & 432.80 & 635.63 & 1.33 & 20.03 & 0.64 \\
\hline 188,201 & 76,582 & 9.8 & 30 & 8,236 & 3,769 & 0.0086 & 228 & 0.790 & 12 & 0.823 & 122 & 0.695 & 0.764 & 0.800 & 610.92 & 582.81 & 1.03 & 51.62 & 0.69 \\
\hline 120,214 & 47,534 & 13.3 & 28 & 5,438 & 1,982 & 0.0464 & 174 & 0.738 & 20 & 0.752 & 93 & 0.683 & 0.778 & 0.750 & 349.98 & 548.67 & 1.59 & 63.21 & 0.65 \\
\hline 120,214 & 47,534 & 13.3 & 28 & 5,438 & 1,982 & 0.0066 & 174 & 0.752 & 20 & 0.789 & 93 & 0.683 & 0.738 & 0.890 & 370.45 & 543.68 & 1.51 & 359.88 & 0.66 \\
\hline 16,821 & 927 & 9.9 & 23 & 7,500 & 2,918 & 0.0367 & 176 & 0.731 & 13 & 0.774 & 117 & 0.693 & 0.769 & .460 & 152.62 & 251.68 & 0.84 & 48.15 & 0.31 \\
\hline 16,821 & 6,927 & 9.9 & 23 & 7,500 & 2,918 & 0.0033 & 176 & 0.731 & 13 & 0.774 & 117 & 0.693 & 0.769 & 0.810 & 172.44 & 267.87 & 0.70 & 227.91 & 0.35 \\
\hline 39,351 & 15,540 & 14.5 & 22 & 7,202 & 3,068 & 0.0231 & 180 & 0.742 & 29 & 0.798 & 84 & 0.661 & 0.747 & 0.710 & 260.11 & 324.15 & 1.26 & 82.05 & 0.53 \\
\hline 131,874 & 55,101 & 7.5 & 22 & 8,142 & 2,276 & 0.0430 & 187 & 0.764 & 27 & 0.799 & 134 & 0.653 & 0.769 & 0.540 & 615.29 & 409.62 & 0.78 & 27.18 & 0.63 \\
\hline 131,874 & 55,101 & 7.5 & 25 & 8,142 & 2,276 & 0.0089 & 187 & 0.788 & 27 & 0.823 & 134 & 0.710 & 0.769 & 0.710 & 492.92 & 532.68 & 0.75 & 46.78 & 0.57 \\
\hline 178,443 & 74,888 & 6.6 & 25 & 5,640 & 1,146 & 0.0172 & 212 & 0.780 & 20 & 0.801 & 85 & 0.697 & 0.733 & 0.880 & 419.53 & 669.99 & 0.85 & 57.73 & 0.60 \\
\hline 149,220 & 60,097 & 14.0 & 21 & 5,854 & 2,208 & 0.0023 & 189 & 0.755 & 31 & 0.799 & 98 & 0.669 & 0.758 & 0.920 & 442.73 & 532.72 & 1.63 & 84.90 & 0.75 \\
\hline 149,220 & 60,097 & 14.0 & 21 & 5,854 & 2,208 & 0.0023 & 189 & 0.764 & 31 & 0.786 & 98 & 0.650 & 0.720 & 0.640 & 463.69 & 550.29 & 1.51 & 25.86 & 0.67 \\
\hline 23,341 & 409 & 9.8 & 16 & 6,250 & 2,553 & 0.0177 & 199 & 0.758 & 11 & 0.815 & 90 & 0.689 & 0.785 & 0.690 & 198.24 & 281.93 & 0.82 & 106.93 & 0.40 \\
\hline 150,488 & 54,975 & 9.1 & 22 & 6,870 & 2,863 & 0.0165 & 181 & 0.741 & 34 & 0.803 & 62 & 0.673 & 0.731 & 0.880 & 471.05 & 615.24 & 0.99 & 52.85 & 0.58 \\
\hline 150,317 & 53,030 & 13.5 & 26 & 7,790 & 2,509 & 0.0039 & 172 & 0.781 & 29 & 0.846 & 57 & 0.670 & 0.769 & 0.920 & 501.03 & 602.23 & 1.35 & 204.66 & 0.62 \\
\hline 98,898 & 42,715 & 6.0 & 18 & 6,953 & 2,553 & 0.0071 & 170 & 0.762 & 30 & 0.782 & 105 & 0.700 & 0.736 & 0.810 & 384.67 & 527.63 & 0.60 & 70.82 & 0.47 \\
\hline 61,055 & 26,072 & 7.6 & 17 & 5,921 & 2,223 & 0.0073 & 199 & 0.750 & 19 & 0.841 & 111 & 0.691 & 0.748 & 0.800 & 305.57 & 444.76 & 0.69 & 146.42 & 0.44 \\
\hline 86,515 & 33,288 & 15.6 & 18 & 7,787 & 3,082 & 0.0402 & 170 & 0.777 & 22 & 0.783 & 106 & 0.701 & 0.767 & 0.540 & 371.34 & 447.50 & 1.49 & 36.40 & 0.58 \\
\hline 152,750 & 58,126 & 13.7 & 28 & 5,388 & 1,557 & 0.0336 & 179 & 0.746 & 31 & 0.762 & 90 & 0.663 & 0.777 & 0.770 & 386.56 & 493.59 & 1.78 & 94.33 & 0.72 \\
\hline 147,295 & 56,116 & 10.8 & 27 & 8,278 & 3,900 & 0.0182 & 208 & 0.774 & 11 & 0.799 & 121 & 0.690 & 0.742 & 0.720 & 503.93 & 559.76 & 1.15 & 36.49 & 0.64 \\
\hline 147,295 & 56,116 & 10.8 & 27 & 8,278 & 3,900 & 0.0066 & 208 & 0.743 & 11 & 0.780 & 121 & 0.700 & 0.737 & 0.850 & 443.59 & 622.57 & 1.17 & 109.71 & 0.60 \\
\hline 37,569 & 15,725 & 6.7 & 21 & 7,542 & 2,172 & 0.0300 & 234 & 0.786 & 14 & 0.816 & 106 & 0.686 & 0.772 & 0.480 & 272.13 & 320.59 & 0.57 & 24.80 & 0.39 \\
\hline 31,461 & 12,495 & 6.4 & 19 & 8,387 & 2,960 & 0.0165 & 180 & 0.744 & 29 & 0.808 & 117 & 0.698 & 0.746 & 0.560 & 241.70 & 341.63 & 0.53 & 37.36 & 0.34 \\
\hline 31,461 & 12,495 & 6.4 & 19 & 8,387 & 2,960 & 0.0052 & 180 & 0.744 & 29 & 0.766 & 117 & 0.660 & 0.720 & 0.720 & 267.20 & 305.33 & 0.53 & 100.95 & 0.40 \\
\hline 188,307 & 74,838 & 14.2 & 21 & 5,998 & 1,772 & 0.0337 & 231 & 0.765 & 14 & 0.829 & 71 & 0.675 & 0.746 & 0.930 & 500.54 & 640.58 & 1.53 & 217.54 & 0.70 \\
\hline 188,307 & 74,838 & 14.2 & 21 & 5,998 & 1,772 & 0.0500 & 231 & 0.765 & 14 & 0.790 & 100 & 0.698 & 0.740 & 0.690 & 443.39 & 652.04 & 1.70 & 42.71 & 0.68 \\
\hline 161,689 & 65,428 & 11.5 & 26 & 6,360 & 2,618 & 0.0146 & 190 & 0.737 & 22 & 0.778 & 123 & 0.672 & 0.743 & 0.780 & 428.70 & 566.49 & 1.46 & 116.06 & 0.71 \\
\hline 161,689 & 65,428 & 11.5 & 26 & 6,360 & 2,618 & 0.0023 & 190 & 0.780 & 22 & 0.830 & 123 & 0.702 & 0.765 & 0.900 & 487.69 & 603.91 & 1.20 & 245.62 & 0.65 \\
\hline 136,794 & 51,211 & 11.1 & 25 & 6,686 & 2,015 & 0.0364 & 205 & 0.733 & 10 & 0.804 & 81 & 0.677 & 0.759 & 0.760 & 374.47 & 569.05 & 1.43 & 49.42 & 0.68 \\
\hline 136,794 & 51,211 & 11.1 & 25 & 6,686 & 2,015 & 0.0042 & 205 & 0.765 & 10 & 0.824 & 81 & 0.707 & 0.767 & 0.900 & 370.21| & 526.94 & 1.56 & 367.52 & 0.77 \\
\hline
\end{tabular}

Table A - 1: Data Generated for Ramp Treatment 


\begin{tabular}{|c|c|c|c|c|c|c|c|c|c|c|c|c|c|c|c|c|c|c|c|}
\hline 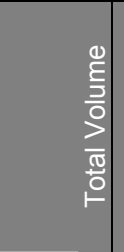 & 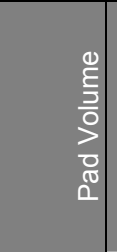 & 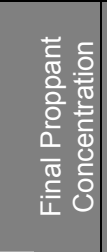 & 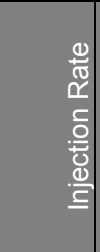 & 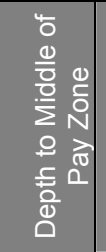 & 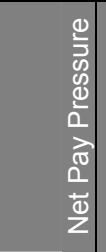 & $\begin{array}{l}\frac{\lambda}{2} \\
\frac{0}{0} \\
\frac{0}{\pi} \\
\frac{0}{0} \\
\frac{5}{0} \\
0\end{array}$ & 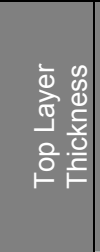 & 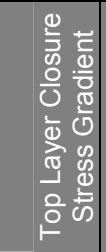 & 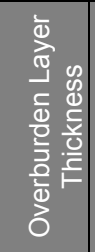 & 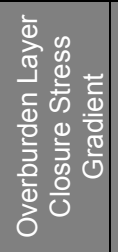 & 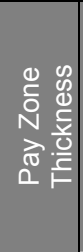 & 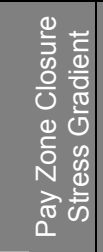 & 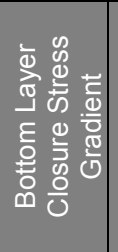 & 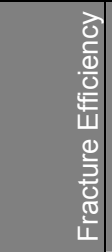 & 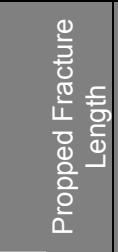 & 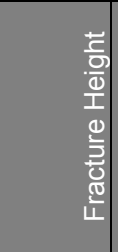 & 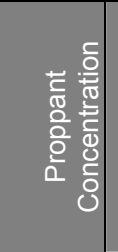 & 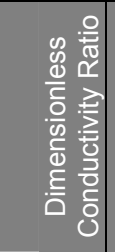 & 훈 \\
\hline input & input & input & input & input & input & input & input & input & input & input & input & input & input & output & output & output & output & output & output \\
\hline 24,567 & 9,152 & 10.4 & 18 & 6,266 & 2,228 & 0.0104 & 205 & 0.750 & 25 & 0.788 & 101 & 0.707 & 0.778 & 0.710 & 197.43 & 310.71 & 0.86 & 185.52 & 0.39 \\
\hline 126,108 & 48,743 & 12.2 & 20 & 6,758 & 2,803 & 0.0041 & 174 & 0.767 & 23 & 0.819 & 132 & 0.704 & 0.734 & 0.860 & 400.81 & 565.73 & 1.30 & 292.48 & 0.61 \\
\hline 119,135 & 51,634 & 15.5 & 27 & 6,176 & 2,181 & 0.0681 & 229 & 0.786 & 23 & 0.837 & 91 & 0.703 & 0.783 & 0.870 & 403.46 & 516.18 & 1.52 & 289.80 & 0.65 \\
\hline 119,135 & 51,634 & 15.5 & 27 & 6,176 & 2,181 & 0.0681 & 229 & 0.749 & 23 & 0.799 & 91 & 0.670 & 0.745 & 0.640 & 380.26 & 512.93 & 1.62 & 35.07 & 0.62 \\
\hline 149,673 & 56,005 & 15.9 & 30 & 7,715 & 2,925 & 0.0114 & 211 & 0.760 & 23 & 0.783 & 122 & 0.662 & 0.767 & 0.730 & 540.75 & 01.45 & 1.66 & 95.62 & 0.74 \\
\hline 149,673 & 56,005 & 15.9 & 30 & 7,715 & 2,925 & 0.0114 & 211 & 0.800 & 23 & 0.833 & 92 & 0.710 & 0.840 & 0.830 & 404.57 & 556.16 & 2.00 & 134.35 & 0.74 \\
\hline 95,141 & 36,899 & 15.6 & 29 & 6,718 & 2,071 & 0.0170 & 176 & 0.776 & 30 & 0.844 & 127 & 0.663 & 0.730 & 0.680 & 369.93 & 435.01 & 1.70 & 128.90 & 0.68 \\
\hline 95,141 & 36,899 & 15.6 & 29 & 6,718 & 2,071 & 0.0033 & 176 & 0.763 & 30 & 0.804 & 127 & 0.697 & 0.737 & 0.860 & 343.51 & 487.94 & 1.63 & 252.43 & 0.65 \\
\hline 101,619 & 35,902 & 11.7 & 20 & 5,047 & 2,390 & 0.0100 & 208 & 0.783 & 21 & 0.791 & 60 & 0.675 & 0.746 & 0.740 & 379.34 & 530.26 & 1.20 & 23.68 & 0.59 \\
\hline 34,401 & 13,459 & 15.7 & 17 & 7,126 & 3,420 & 0.1215 & 197 & 0.767 & 24 & 0.776 & 126 & 0.658 & 0.752 & 0.270 & 200.64 & 314.97 & 1.56 & 31.65 & 0.42 \\
\hline 73,631 & 29,186 & 15.1 & 28 & 8,196 & 3,128 & 0.0295 & 228 & 0.725 & 35 & 0.847 & 112 & 0.681 & 0.749 & 0.590 & 350.89 & 400.68 & 1.45 & 45.81 & 0.59 \\
\hline 73,631 & 29,186 & 15.1 & 28 & 8,196 & 3,128 & 0.0069 & 228 & 0.742 & 35 & 0.790 & 112 & 0.690 & 0.720 & 0.810 & 308.72 & 442.68 & 1.49 & 192.47 & 0.59 \\
\hline 136,740 & 47,946 & 8.5 & 29 & 7,317 & 3,635 & 0.0012 & 195 & 0.789 & 12 & 0.800 & 65 & 0.702 & 0.765 & 0.960 & 494.34 & 604.95 & 0.85 & 438.10 & 0.59 \\
\hline 185,498 & 76,789 & 8.3 & 18 & 7,871 & 3,265 & 0.0042 & 193 & 0.764 & 31 & 0.816 & 119 & 0.685 & 0.737 & 0.860 & 561.29 & 619.19 & 0.90 & 103.89 & 0.62 \\
\hline 20,186 & 8,467 & 6.3 & 23 & 7,364 & 3,576 & 0.0491 & 222 & 0.743 & 31 & 0.800 & 57 & 0.666 & 0.779 & 0.620 & 219.77 & 258.21 & 0.47 & 14.70 & 0.33 \\
\hline 143,944 & 62,412 & 15.3 & 29 & 6,036 & 2,006 & 0.0386 & 193 & 0.748 & 12 & 0.811 & 113 & 0.701 & 0.785 & 0.800 & 345.85 & 491.31 & 2.20 & 215.16 & 0.86 \\
\hline 137,148 & 59,667 & 11.7 & 26 & 8,111 & 3,188 & 0.0073 & 195 & 0.744 & 24 & 0.751 & 68 & 0.673 & 0.736 & 0.880 & 448.96 & 546.84 & 1.16 & 95.87 & 0.62 \\
\hline 190,430 & 79,607 & 13.3 & 30 & 7,277 & 3,531 & 0.0235 & 185 & 0.754 & 19 & 0.783 & 104 & 0.678 & 0.783 & 0.800 & 432.88 & 628.84 & 1.71 & 57.93 & 0.70 \\
\hline 190,430 & 79,607 & 13.3 & 30 & 7,277 & 3,531 & 0.0054 & 185 & 0.790 & 19 & 0.820 & 104 & 0.710 & 0.783 & 0.870 & 560.66 & 602.57 & 1.38 & 137.05 & 0.70 \\
\hline 95,259 & 36,174 & 12.9 & 20 & 6,557 & 1,784 & 0.0073 & 202 & 0.779 & 34 & 0.829 & 54 & 0.686 & 0.786 & 0.880 & 382.48 & 446.01 & 1.41 & 222.65 & 0.67 \\
\hline 31,651 & 12,212 & 8.5 & 28 & 5,744 & 1,982 & 0.0818 & 226 & 0.777 & 35 & 0.802 & 104 & 0.673 & 0.738 & 0.390 & 217.36 & 323.65 & 0.79 & 32.23 & 0.36 \\
\hline 16,202 & 6,348 & 6.9 & 16 & 7,364 & 2,463 & 0.0154 & 233 & 0.762 & 27 & 0.788 & 89 & 0.667 & 0.779 & 0.530 & 208.45 & 229.02 & 0.51 & 53.95 & 0.34 \\
\hline 62,537 & 22,554 & 14.0 & 17 & 6,227 & 2,550 & 0.0818 & 188 & 0.798 & 24 & 0.830 & 88 & 0.710 & 0.780 & 0.520 & 294.46 & 412.32 & 1.42 & 35.28 & 0.54 \\
\hline 72,823 & 31,706 & 12.3 & 24 & 7,852 & 3,287 & 0.0157 & 203 & 0.757 & 34 & 0.761 & 53 & 0.662 & 0.741 & 0.840 & 370.19 & 415.61 & 1.04 & 50.65 & 0.54 \\
\hline 81,375 & 30,527 & 8.4 & 22 & 8,259 & 2,981 & 0.0089 & 213 & 0.760 & 29 & 0.804 & 84 & 0.710 & 0.738 & 0.840 & 350.25 & 503.40 & 0.82 & 67.76 & 0.48 \\
\hline 30,061 & 12,457 & 12.8 & 15 & 6,730 & 2,123 & 0.0112 & 180 & 0.735 & 23 & 0.810 & 56 & 0.683 & 0.745 & 0.800 & 227.63 & 322.35 & 0.97 & 146.75 & 0.43 \\
\hline 175,697 & 69,827 & 7.1 & 27 & 5,123 & 2,080 & 0.0575 & 180 & 0.777 & 22 & 0.826 & 60 & 0.700 & 0.797 & 0.830 & 406.44 & 692.34 & 0.93 & 26.23 & 0.64 \\
\hline 30,150 & 11,060 & 13.0 & 19 & 7,101 & 3,530 & 0.0818 & 210 & 0.781 & 22 & 0.834 & 63 & 0.692 & 0.767 & 0.600 & 213.45 & 323.61 & 1.12 & 23.17 & 0.43 \\
\hline 86,222 & 32,587 & 8.1 & 23 & 7,941 & 2,086 & 0.0058 & 205 & 0.764 & 16 & 0.756 & 97 & 0.682 & 0.769 & 0.790 & 414.82 & 466.12 & 0.77 & 97.02 & 0.56 \\
\hline 105,574 & 37,646 & 10.3 & 25 & 6,704 & 2,697 & 0.0124 & 229 & 0.755 & 18 & 0.807 & 61 & 0.672 & 0.777 & 0.890 & 351.94 & 539.44 & 1.20 & 122.42 & 0.62 \\
\hline
\end{tabular}

Table A-1: Data Generated for Ramp Treatment, continued 


\begin{tabular}{|c|c|c|c|c|c|c|c|c|c|c|c|c|c|c|c|c|c|c|c|}
\hline 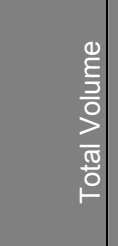 & \begin{tabular}{l|}
$\frac{0}{2}$ \\
$\frac{1}{3}$ \\
0 \\
0 \\
0 \\
0 \\
0
\end{tabular} & 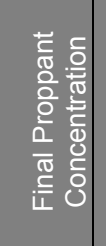 & 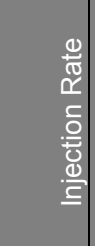 & 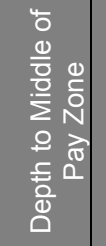 & 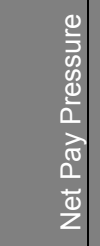 & 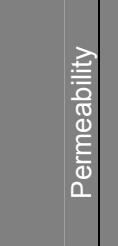 & 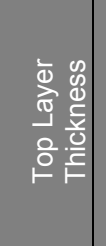 & 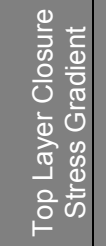 & 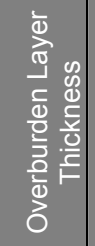 & 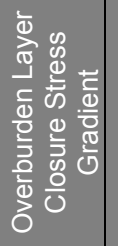 & 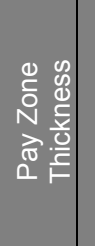 & 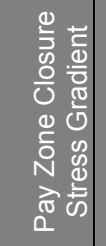 & 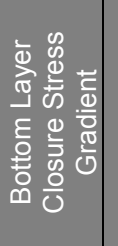 & 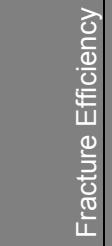 & 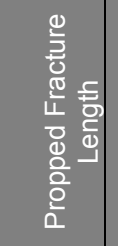 & 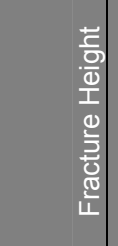 & 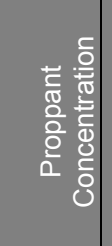 & 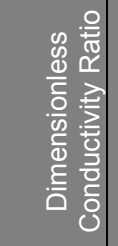 & $\frac{0}{5}$ \\
\hline input & input & input & input & input & input & input & input & input & input & input & input & input & input & output & output & output & output & output & output \\
\hline 105,574 & 37,646 & 10.3 & 25 & 6,704 & 2,697 & 0.0076 & 229 & 0.755 & 18 & 0.807 & 61 & 0.692 & 0.780 & 0.860 & 338.17 & 503.42 & 1.34 & 217.97 & \\
\hline 57,575 & 25,302 & 13.7 & 19 & 7,173 & 3,209 & 0.0076 & 227 & 0.768 & 12 & 0.809 & 107 & 0.699 & 0.765 & .790 & 291.34 & 390.10 & 1.20 & 167.05 & .53 \\
\hline 60,595 & 26,596 & 14.4 & 25 & 8,011 & 3,088 & 0.0488 & 177 & 0.767 & 29 & 0.811 & 128 & 0.703 & 0.733 & 0.480 & 262.03 & 393.77 & 1.43 & 35.80 & \\
\hline 13,578 & ,997 & 13.2 & 19 & 6,547 & 2,297 & 0.0075 & 190 & 0.775 & 14 & 0.838 & 112 & 0.707 & 0.786 & 0.640 & 158.12 & 239.93 & 0.92 & 294.69 & \\
\hline 56,398 & 23,307 & 13.0 & 21 & 6,597 & 2,645 & 0.0315 & 232 & 0.758 & 24 & 0.822 & 134 & 0.685 & 0.762 & 0.540 & 277.74 & 86.87 & 1.25 & 70.20 & \\
\hline 61,335 & 24,189 & 11.6 & 18 & 6,569 & 2,701 & 0.0012 & 208 & 0.740 & 23 & 0.819 & 131 & 0.665 & 0.735 & 0.900 & 329.57 & 422.36 & 0.99 & 116.70 & .53 \\
\hline 106,559 & 46,581 & 9.7 & 26 & 5,178 & 1,814 & 0.0159 & 172 & 0.782 & 33 & 0.795 & 78 & 0.670 & 0.771 & 0.830 & 402.60 & 492.32 & 0.97 & 1.19 & 5 \\
\hline 100,951 & 43,256 & 9.1 & 27 & 5,590 & 2,238 & 0.0062 & 237 & 0.769 & 21 & 0.819 & 73 & 0.700 & 0.765 & 0.910 & 353.97 & 517.38 & 0.95 & 218.77 & .56 \\
\hline 94,702 & 35,525 & 12.5 & 28 & 7,882 & 2,594 & 0.0197 & 233 & 0.776 & 18 & 0.782 & 55 & 0.695 & 0.747 & 0.840 & 365.31 & 97.90 & 1.28 & 52.53 & 6 \\
\hline 94,702 & 35,525 & 12.5 & 28 & 7,882 & 2,594 & 0.0900 & 233 & 0.776 & 18 & 0.782 & 95 & 0.706 & 0.790 & 0.490 & 363.24 & 446.63 & 1.43 & 16.28 & 0.55 \\
\hline 26,126 & 364 & 8.7 & 21 & 6,660 & 2,500 & 0.0213 & 174 & 0.723 & 30 & 0.759 & 85 & 0.657 & 0.750 & .650 & 233.03 & 308.64 & 0.69 & 67.74 & 0.3 \\
\hline 175,793 & 67,485 & 11.7 & 30 & 7,037 & 3,336 & 0.0737 & 211 & 0.739 & 27 & 0.753 & 83 & 0.689 & 0.724 & 0.740 & 445.63 & 656.13 & 1.39 & $16.67 \mid$ & 0.62 \\
\hline 184,517 & 70,474 & 10.9 & 30 & 5,707 & 2,393 & 0.0053 & 201 & 0.767 & 19 & 0.834 & 105 & 0.694 & 0.749 & .900 & 478.23 & 58.69 & 1.28 & 270.32 & 6 \\
\hline 98,653 & 42,370 & 13.1 & 26 & 6,459 & 1,484 & 0.0018 & 194 & 0.760 & 28 & 0.781 & 96 & 0.675 & 0.743 & 0.920 & 382.93 & 480.07 & 1.26 & 244.92 & 0.61 \\
\hline 36,753 & 15,639 & 6.4 & 20 & 7,022 & 3,508 & 0.0277 & 202 & 0.773 & 31 & 0.813 & 56 & 0.707 & 0.745 & 0.810 & 220.78 & 356.71 & 0.61 & 28.72 & $0.4 C$ \\
\hline 117,093 & 49,780 & 7.2 & 27 & 6,457 & 2,530 & 0.0874 & 220 & 0.789 & 32 & 0.770 & 99 & 0.675 & 0.771 & 0.600 & 359.59 & 518.53 & 0.90 & 15.19 & 0.56 \\
\hline 117,093 & 49,780 & 7.2 & 27 & 6,457 & 2,530 & 0.0020 & 220 & 0.789 & 32 & 0.770 & 99 & 0.675 & 0.771 & 0.930 & 366.44 & 543.11 & 0.85 & 181.26 & 0.58 \\
\hline 149,133 & 60,213 & 15.6 & 30 & 7,098 & 3,579 & 0.0465 & 230 & 0.751 & 15 & 0.809 & 89 & 0.687 & 0.746 & 0.750 & 429.54 & 578.49 & 1.68 & 31.77 & 0.66 \\
\hline 30,961 & 13,082 & 7.3 & 16 & 5,938 & 2,731 & 0.0040 & 205 & 0.768 & 31 & 0.788 & 133 & 0.701 & 0.766 & .800 & 224.51 & 30.74 & 0.61 & 296.44 & 4 \\
\hline 141,814 & 51,837 & 12.6 & 30 & 7,251 & 3,550 & 0.0330 & 225 & 0.736 & 14 & 0.763 & 110 & 0.673 & 0.724 & 0.740 & 433.98 & 88.38 & 1.40 & 38.14 & 0.64 \\
\hline 191,842 & 70,965 & 12.6 & 30 & 7,793 & 3,547 & 0.0459 & 182 & 0.755 & 20 & 0.787 & 119 & 0.710 & 0.773 & 0.720 & 419.20 & 652.66 & 1.75 & 29.89 & 0.74 \\
\hline 191,842 &, 965 & 12.6 & 30 & 7,793 & 3,547 & 0.0010 & 182 & 0.755 & 20 & 0.787 & 119 & 0.680 & 0.720 & 0.950 & 511.23 & 28.24 & 1.49 & 234.38 & 0.74 \\
\hline 82,366 & 30,446 & 9.7 & 25 & 6,023 & 2,847 & 0.0153 & 239 & 0.800 & 28 & 0.840 & 96 & 0.718 & 0.800 & 0.800 & 359.11 & 479.06 & 0.97 & 86.78 & 0.55 \\
\hline 88,841 & 33,114 & 8.7 & 25 & 5,311 & 1,871 & 0.0382 & 197 & 0.782 & 19 & 0.775 & 61 & 0.677 & 0.750 & 0.810 & 355.52 & 503.58 & 0.91 & 43.02 & 0.53 \\
\hline 39,182 & 17,138 & 12.8 & 18 & 7,733 & 3,747 & 0.0046 & 195 & 0.754 & 22 & 0.776 & 109 & 0.691 & 0.727 & 0.850 & 244.86 & 366.30 & 0.98 & 227.28 & 0.4 \\
\hline 108,011 & 38,862 & 6.8 & 27 & 6,504 & 2,314 & 0.0135 & 207 & 0.730 & 19 & 0.752 & 94 & 0.685 & 0.723 & 0.860 & 376.51 & 580.56 & 0.76 & 67.39 & .52 \\
\hline 140,287 & 57,933 & 10.6 & 28 & 6,027 & 2,501 & 0.0065 & 227 & 0.762 & 32 & 0.783 & 95 & 0.678 & 0.742 & 0.900 & 440.22 & 570.88 & 1.13 & 189.72 & 0.62 \\
\hline 84,646 & 34,494 & 12.3 & 25 & 8,004 & 3,832 & 0.0322 & 204 & 0.774 & 18 & 0.838 & 128 & 0.705 & 0.788 & 0.650 & 376.98 & 440.18 & 1.18 & 27.70 & 0.56 \\
\hline 84,646 & 34,494 & 12.3 & 25 & 8,004 & 3,832 & 0.0032 & 204 & 0.763 & 18 & 0.787 & 128 & 0.681 & 0.732 & 0.890 & 365.86 & 437.54 & 1.22 & 71.10 & \\
\hline 84,646 & 34,494 & 12.3 & 25 & 8,004 & 3,832 & 0.0010 & 204 & 0.764 & 18 & 0.787 & 128 & 0.674 & 0.748 & 0.930 & 409.66 & 451.12 & $1.06 \mid$ & 175.77 & \\
\hline
\end{tabular}

Table A-1: Data Generated for Ramp Treatment, continued 


\begin{tabular}{|c|c|c|c|c|c|c|c|c|c|c|c|c|c|c|c|c|c|c|c|}
\hline \begin{tabular}{|l|}
$\stackrel{0}{E}$ \\
$\frac{1}{3}$ \\
$\frac{0}{0}$ \\
$\frac{\pi}{0}$ \\
$\stackrel{0}{\circ}$
\end{tabular} & 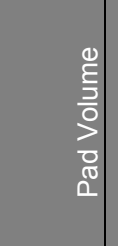 & 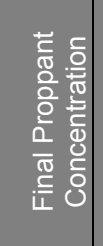 & 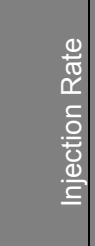 & 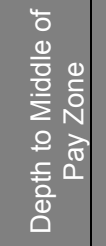 & 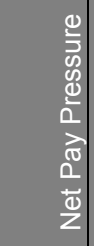 & $\begin{array}{l}\frac{\lambda}{3} \\
\frac{7}{\overline{0}} \\
\frac{0}{10} \\
\frac{0}{0} \\
0 \\
0\end{array}$ & 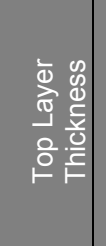 & 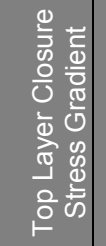 & 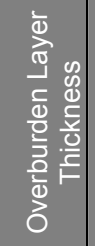 & 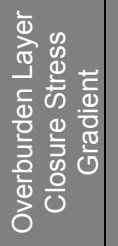 & 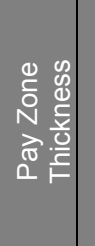 & 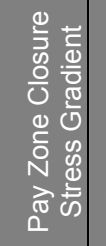 & 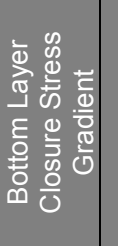 & 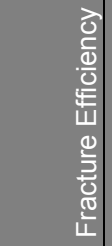 & 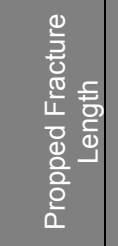 & 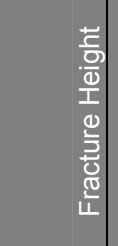 & 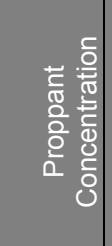 & 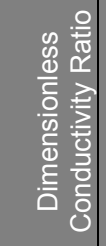 & 은 \\
\hline input & input & input & input & input & input & input & input & input & input & input & input & input & input & output & output & output & output & output & output \\
\hline 120,306 & 43,572 & 10.2 & 29 & 7,732 & 2,548 & 0.0127 & 205 & 0.744 & 18 & 0.750 & 68 & 0.702 & 0.726 & 0.890 & 387.53 & 591.86 & 1.12 & 67.43 & \\
\hline 88,488 & 31,631 & 6.8 & 27 & 6,867 & 2,720 & 0.0272 & 200 & 0.764 & 11 & 0.785 & 52 & 0.684 & 0.745 & .870 & 385.67 & 16.86 & 0.69 & 25.55 & \\
\hline 116,859 & 43,581 & 6.8 & 27 & 7,763 & 1,990 & 0.0097 & 228 & 0.722 & 31 & 0.791 & 64 & 0.653 & 0.748 & 0.870 & 453.44 & 86.00 & 0.80 & 59.34 & .6 \\
\hline 87,206 & 34,011 & 14.6 & 25 & 7,443 & 3,191 & 0.0032 & 179 & 0.763 & 23 & 0.784 & 128 & 0.674 & 0.774 & 0.870 & 395.77 & 440.87 & 1.36 & 336.48 & .64 \\
\hline 143,203 & 58,662 & 15.1 & 30 & 5,105 & 2,112 & 0.0099 & 194 & 0.736 & 33 & 0.781 & 124 & 0.699 & 0.724 & .870 & 355.39 & 01.69 & 1.81 & 15.81 & 7 \\
\hline 157,542 & 58,527 & 12.7 & 30 & 6,699 & 3,041 & 0.0439 & 233 & 0.747 & 20 & 0.793 & 70 & 0.704 & 0.737 & 0.850 & 388.57 & 616.64 & 1.64 & 38.44 & .71 \\
\hline 77,706 & 33,359 & 11.7 & 28 & 7,209 & 3,550 & 0.0066 & 230 & 0.785 & 24 & 0.839 & 133 & 0.720 & 0.787 & 0.830 & 335.76 & |1.62 & 1.12 & 148.74 & 5 \\
\hline 80,226 & 28,440 & 8.2 & 25 & 5,568 & 2,500 & 0.0213 & 174 & 0.763 & 27 & 0.838 & 98 & 0.658 & 0.789 & 0.780 & 375.92 & 434.75 & 0.89 & 72.81 & .58 \\
\hline 138,120 & 57,846 & 6.9 & 28 & 6,810 & 3,224 & 0.0224 & 186 & 0.733 & 31 & 0.744 & 99 & 0.674 & 0.740 & 0.820 & 393.54 & 88.77 & 0.84 & 38.25 & 5 \\
\hline 116,101 & 50,486 & 8.7 & 30 & 7,627 & 2,920 & 0.0311 & 227 & 0.773 & 16 & 0.799 & 101 & 0.704 & 0.758 & 0.730 & 409.57 & 531.81 & 0.88 & 20.60 & 0.54 \\
\hline 136,922 & 48,387 & 14.8 & 35 & 6,681 & 1,950 & 0.0494 & 184 & 0.726 & 15 & 0.817 & 70 & 0.692 & 0.748 & .890 & 425.37 & 59.85 & 1.68 & 173.14 & .71 \\
\hline 38,283 & 16,051 & 13.3 & 16 & 5,493 & 1,405 & 0.0161 & 230 & 0.792 & 11 & 0.831 & 83 & 0.720 & 0.788 & 0.780 & 227.89 & 352.16 & 1.15 & 162.09 & 0.48 \\
\hline 12,688 & ,545 & 15.0 & 19 & 7,494 & 2,510 & 0.0016 & 188 & 0.752 & 33 & 0.793 & 56 & 0.691 & 0.769 & .900 & 173.07 & 34.28 & 0.80 & 295.49 & \\
\hline 31,986 & 12,228 & 9.5 & 20 & 6,858 & 1,511 & 0.0372 & 172 & 0.768 & 21 & 0.794 & 94 & 0.685 & 0.728 & 0.590 & 224.31 & 328.82 & 0.85 & 42.44 & 0.40 \\
\hline 21,852 & ,054 & 10.8 & 18 & 7,193 & 3,586 & 0.0519 & 186 & 0.742 & 27 & 0.762 & 59 & 0.651 & 0.736 & 0.670 & 215.80 & 281.45 & 0.80 & 29.19 & 38 \\
\hline 18,944 & ,705 & 11.2 & 20 & 6,775 & 2,930 & 0.0094 & 222 & 0.753 & 32 & 0.781 & 131| & 0.704 & 0.764 & 0.680 & 178.10 & 284.91 & 0.87 & 190.45 & 0.36 \\
\hline 31,808 & 13,850 & 7.6 & 17 & 6,265 & 2,838 & 0.0646 & 193 & 0.799 & 26 & 0.821 & 114 & 0.684 & 0.738 & 0.460 & 221.83 & 11.16 & 0.69 & 24.65 & .36 \\
\hline 182,194 & 68,142 & 9.1 & 35 & 5,519 & 2,836 & 0.0045 & 226 & 0.773 & 17 & 0.807 & 82 & 0.700 & 0.766 & 0.950 & 418.09 & 9.72 & 1.30 & 377.49 & 0.75 \\
\hline 184,981 & 67,117 & 14.7 & 35 & 7,912 & 3,528 & 0.0095 & 193 & 0.758 & 17 & 0.786 & 125 & 0.694 & 0.749 & 0.830 & 509.34 & 25.73 & 1.66 & 105.77 & .73 \\
\hline 142,162 & 50,021 & 8.3 & 17 & 5,648 & 2,835 & 0.0183 & 171 & .745 & 12 & 0.787 & 54 & 0.681 & 0.737 & 0.930 & 384.33 & 577.56 & 1.17 & 94.78 & 0.69 \\
\hline 127,344 & 50,742 & 6.5 & 26 & 6,142 & 2,638 & 0.0074 & 205 & 0.761 & 22 & 0.793 & 51 & 0.696 & 0.788 & 0.940 & 369.13 & 647.00 & 0.74 & 123.35 & 0.60 \\
\hline 73,020 & 29,796 & 9.6 & 25 & 5,235 & 1,991 & 0.0310 & 175 & 0.793 & 19 & 0.837 & 83 & 0.694 & 0.759 & 0.780 & 315.61 & 45.55 & 0.98 & 59.78 & 0.53 \\
\hline 116,066 & 44,766 & 8.0 & 28 & 8,171 & 3,989 & 0.0072 & 171 & 0.747 & 33 & 0.757 & 80 & 0.673 & 0.721 & 0.900 & 450.82 & 33.68 & 0.81 & 66.89 & 0.56 \\
\hline 128,685 & 55,350 & 12.8 & 32 & 6,379 & 2,666 & 0.0047 & 238 & 0.768 & 33 & 0.823 & 110 & 0.698 & 0.781 & 0.890 & 392.38 & 512.13 & 1.47 & 330.31 & 0.69 \\
\hline 101,034 & 35,409 & 7.5 & 26 & 7,549 & 3,743 & 0.0197 & 203 & 0.782 & 24 & 0.813 & 121 & 0.684 & 0.741 & 0.740 & 425.06 & 480.99 & 0.83 & 33.22 & $.5 \varepsilon$ \\
\hline 13,189 & 044 & 7.0 & 15 & 6,852 & 2,548 & 0.0054 & 233 & 0.737 & 21 & 0.785 & 95 & 0.685 & 0.725 & 0.750 & 165.98 & 255.58 & 0.45 & 161.79 & 0.27 \\
\hline 138,962 & 56,770 & 12.2 & 30 & 7,788 & 3,600 & 0.0230 & 171 & 0.781 & 35 & 0.804 & 58 & 0.696 & 0.730 & 0.880 & 376.50 & 552.93 & 1.52 & 45.92 & 0.6 \\
\hline 11,412 & 939 & 7.4 & 15 & 6,272 & 2,804 & 0.0251 & 228 & 0.749 & 31 & 0.770 & 61 & 0.677 & 0.741 & 0.540 & 145.75 & 211.24 & 0.54 & 26.98| & 0.26 \\
\hline 73,657 & 27,986 & 10.9 & 24 & 5,493 & 2,492 & 0.0135 & 214 & 0.754 & 30 & 0.787 & 92 & 0.698 & 0.740 & 0.850 & 301.42 & 476.60 & 1.12 & 157.08 & \\
\hline 90,516 & 36,317 & 6.4 & 29 & 6,263 & 3,234 & 0.0045 & 225 & 0.756 & 27 & 0.782 & 102 & 0.688 & 0.741 & 0.920 & 357.80 & 507.58 & 0.68 & 178.70 & \\
\hline
\end{tabular}

Table A-1: Data Generated for Ramp Treatment, continued 


\begin{tabular}{|c|c|c|c|c|c|c|c|c|c|c|c|c|c|c|c|c|c|c|c|}
\hline 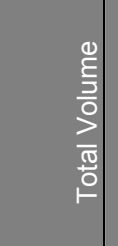 & \begin{tabular}{l|}
$\frac{0}{2}$ \\
$\frac{3}{0}$ \\
0 \\
$\frac{0}{0}$ \\
$\frac{0}{0}$
\end{tabular} & 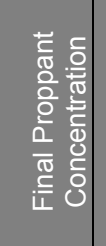 & 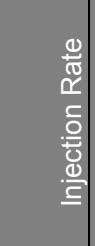 & 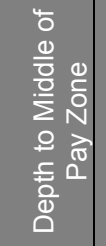 & \begin{tabular}{l|l}
$\frac{1}{5}$ & \\
0 & \\
0 & \\
0 & \\
0 & \\
ते & \\
0 & \\
$\frac{0}{2}$ &
\end{tabular} & 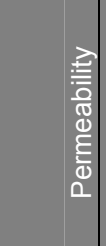 & 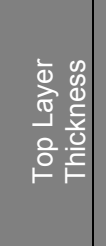 & 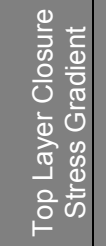 & 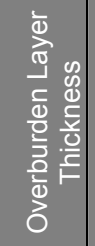 & 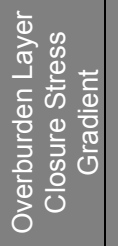 & 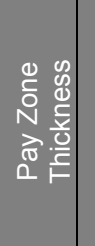 & 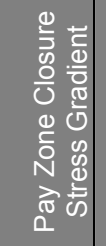 & 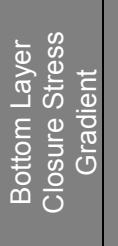 & 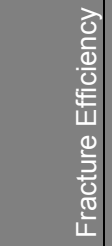 & 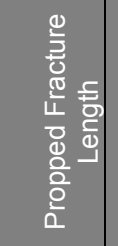 & 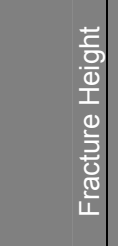 & 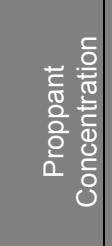 & 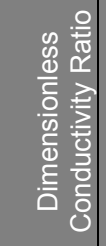 & 흔 \\
\hline input & input & input & input & input & input & input & input & input & input & input & input & input & input & output & output & output & output & output & output \\
\hline 167,771 & 69,779 & 7.5 & 36 & 7,695 & 3,613 & 0.0098 & 207 & 0.782 & 25 & 0.820 & 113 & 0.698 & 0.772 & 0.840 & 553.21 & 587.78 & 0.78 & 41.14 & 0.62 \\
\hline 140,220 & 53,034 & 9.9 & 35 & 7,950 & 3,482 & 0.0110 & 233 & 0.771 & 17 & 0.774 & 94 & 0.674 & 0.731 & .860 & 467.94 & 37.05 & 1.13 & 62.37 & \\
\hline 59,263 & 25,194 & 15.0 & 21 & 5,986 & 1,593 & 0.0086 & 179 & 0.722 & 14 & 0.740 & 68 & 0.652 & 0.730 & 0.890 & 286.22 & 407.65 & 1.34 & 81.51 & 5 \\
\hline 181,169 & 72,607 & 7.7 & 40 & 7,237 & 3,076 & 0.0271 & 174 & 0.768 & 16 & 0.792 & 115 & 0.704 & 0.785 & 0.790 & 433.81 & 632.14 & 1.05 & 29.30 & .65 \\
\hline 181,169 & 72,607 & 7.7 & 40 & 7,237 & 3,076 & 0.0072 & 174 & 0.768 & 16 & 0.792 & 115 & 0.690 & 0.745 & .880 & 510.84 & 20.16 & 0.91 & 75.68 & 6 \\
\hline 145,050 & 53,324 & 6.8 & 35 & 7,998 & 3,420 & 0.0020 & 171 & 0.766 & 15 & 0.801 & 114 & 0.720 & 0.772 & 0.920 & 384.67 & 40.60 & 1.06 & 242.68 & .75 \\
\hline 72,451 & 29,798 & 7.5 & 28 & 8,309 & 3,814 & 0.0077 & 234 & 0.782 & 31 & 0.804 & 58 & 0.709 & 0.776 & 0.900 & 368.89 & 63.86 & 0.65 & 53.56 & 4 \\
\hline 82,045 & 28,755 & 8.6 & 28 & 5,776 & 2,546 & 0.0055 & 178 & 0.767 & 29 & 0.803 & 99 & 0.693 & 0.763 & 0.890 & 356.27 & 489.52 & 0.89 & 243.08 & 0.55 \\
\hline 79,855 & 33,033 & 9.1 & 26 & 8,284 & 2,915 & 0.0425 & 236 & 0.777 & 24 & 0.793 & 125 & 0.657 & 0.761 & 0.530 & 436.37 & 53.51 & 0.91 & 16.43 & .5 \\
\hline 96,072 & 37,124 & 7.5 & 30 & 8,093 & 3,825 & 0.0236 & 220 & 0.753 & 34 & 0.793 & 100 & 0.697 & 0.744 & 0.920 & 384.14 & 519.64 & 0.71 & 198.62 & 0.51 \\
\hline 67,889 & 29,547 & 7.9 & 25 & 8,264 & 3,620 & 0.0398 & 224 & 0.767 & 17 & 0.794 & 90 & 0.698 & 0.758 & .850 & 313.57 & 428.07 & 0.77 & 58.19 & $.4 \mathrm{~S}$ \\
\hline 46,261 & 19,267 & 6.3 & 24 & 7,417 & 3,056 & 0.0245 & 211 & 0.729 & 23 & 0.821 & 99 & 0.683 & 0.734 & 0.790 & 251.97 & 383.11 & 0.63 & 65.76 & 0.41 \\
\hline 47,798 & 19,218 & 6.9 & 26 & 5,457 & $2,321 \mid$ & 0.0181 & 184 & 0.741 & 21 & 0.750 & 89 & 0.687 & 0.718 & .900 & 228.30 & 89.35 & 0.78 & 97.00 & 0.5 \\
\hline 63,096 & 27,325 & 8.4 & 26 & 5,066 & 2,078 & 0.0471 & 193 & 0.722 & 18 & 0.767 & 59 & 0.684 & 0.725 & 0.900 & 247.42 & 428.87 & 0.92 & 44.56 & 0.55 \\
\hline 60,607 & 22,873 & 6.7 & 17 & 7,817 & 2,615 & 0.0055 & 201 & 0.734 & 27 & 0.827 & 87 & 0.686 & 0.723 & 0.910 & 314.68 & 449.88 & 0.63 & 104.47 & 0.46 \\
\hline 56,885 & 23,983 & 8.0 & 17 & 6,239 & $2,011 \mid$ & 0.0290 & 173 & 0.739 & 26 & 0.794 & 81 & 0.684 & 0.738 & 0.870 & 286.51 & 418.81 & 0.75 & 40.47| & 0.48 \\
\hline 45,557 & 19,542 & 7.0 & 20 & 5,898 & 1,917 & 0.0310 & 205 & 0.753 & 22 & 0.803 & 75 & 0.675 & 0.735 & 0.810 & 259.42 & 381.39 & 0.64 & 38.04 & .43 \\
\hline 46,644 & 17,192 & 7.2 & 26 & 6,233 & 2,529 & 0.0497 & 171 & 0.752 & 30 & 0.802 & 76 & 0.667 & 0.743 & 0.740 & 286.99 & 394.69 & 0.65 & 24.36 & 0.43 \\
\hline 64,505 & 26,504 & 7.8 & 22 & 5,568 & 2,741 & 0.0314 & 183 & 0.744 & 31 & 0.789 & 99 & 0.695 & 0.743 & 0.860 & 281.36 & 447.82 & 0.81 & 49.56 & 0.51 \\
\hline 48,005 & 20,351 & 6.3 & 22 & 5,333 & 1,820 & 0.0303 & 174 & 0.728 & 18 & 0.755 & 89 & 0.683 & 0.720 & 0.820 & 249.26 & 413.88 & 0.60 & 43.67 & 0.40 \\
\hline 56,279 & 24,458 & 7.0 & 24 & 6,429 & 1,984 & 0.0327 & 213 & 0.739 & 14 & 0.805 & 80 & 0.680 & 0.732 & 0.800 & 286.94 & 425.32 & 0.64 & 30.84 & 0.43 \\
\hline 57,098 & 21,031 & 6.9 & 24 & 7,829 & 2,736 & 0.0222 & 240 & 0.734 & 30 & 0.812 & 83 & 0.672 & 0.743 & 0.810 & 331.72 & 423.26 & 0.62 & 26.59 & 0.46 \\
\hline 64,367 & 27,231 & 7.5 & 19 & 6,734 & 2,572 & 0.0184 & 183 & 0.746 & 16 & 0.806 & 79 & 0.682 & 0.742 & 0.840 & 312.36 & 433.99 & 0.71 & 48.49 & 0.49 \\
\hline 59,496 & 25,774 & 6.6 & 18 & 6,801 & 2,670 & 0.0394 & 213 & 0.731 & 30 & 0.768 & 106 & 0.668 & 0.746 & 0.550 & 295.31 & 408.45 & 0.65 & 28.26 & 0.45 \\
\hline 43,341 & 18,802 & 7.6 & 22 & 5,035 & 2,448 & 0.0164 & 236 & 0.759 & 27 & 0.776 & 97 & 0.670 & 0.730 & 0.740 & 236.66 & 366.56 & 0.74 & 113.38 & 0.44 \\
\hline 61,683 & 26,548 & 6.9 & 20 & 6,415 & 2,547 & 0.0204 & 226 & 0.744 & 23 & 0.807 & 66 & 0.685 & 0.734 & 0.900 & 282.03 & 430.02 & 0.70 & 48.80 & 0.49 \\
\hline 53,458 & 19,938 & 7.2 & 15 & 5,006 & 2,309 & 0.0259 & 189 & 0.758 & 23 & 0.767 & 86 & 0.693 & 0.722 & 0.810 & 255.28 & 426.33 & 0.77 & 77.57 & 0.49 \\
\hline 139,524 & 57,390 & 7.5 & 30 & 8,455 & 2,287 & 0.0063 & 177 & 0.758 & 16 & 0.814 & 91 & 0.698 & 0.746 & 0.930 & 480.08 & 588.97 & 0.75 & 66.75 & 0.56 \\
\hline 40,681 & 16,186 & 13.5 & 18 & 10,435 & 4,921 & 0.0228 & 182 & 0.748 & 28 & 0.806 & 114 & 0.710 & 0.764 & 0.750 & 295.46 & 361.22 & 0.97 & 32.01 & 0.4 \\
\hline 57,199 & 22,632 & 15.0 & 28 & 11,411 & 5,176 & 0.0110 & 188 & 0.751 & 28 & 0.769 & 63 & 0.687 & 0.745 & 0.910 & 342.18 & 357.39 & 0.84 & 20.47 & 0.4 \\
\hline
\end{tabular}

Table A-1: Data Generated for Ramp Treatment, continued 


\begin{tabular}{|c|c|c|c|c|c|c|c|c|c|c|c|c|c|c|c|c|c|c|c|}
\hline \begin{tabular}{l|}
$\frac{0}{\Xi}$ \\
$\frac{3}{0}$ \\
$\frac{0}{0}$ \\
$\frac{\pi}{0}$ \\
$\frac{0}{\circ}$
\end{tabular} & \begin{tabular}{l|}
0 \\
है \\
3 \\
0 \\
2 \\
0 \\
0 \\
0
\end{tabular} & 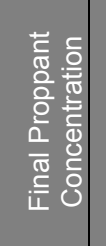 & 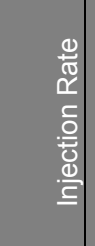 & 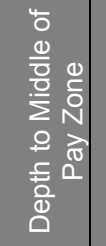 & \begin{tabular}{l|l}
$\frac{0}{3}$ \\
0 \\
0 \\
0 \\
0 \\
$\frac{1}{2}$ \\
$\frac{\pi}{\pi}$ \\
$\frac{1}{0}$ \\
$\frac{0}{2}$ \\
$z$
\end{tabular} & 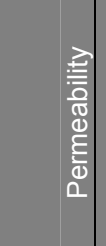 & 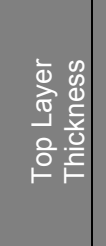 & 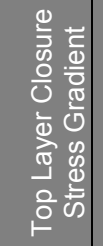 & 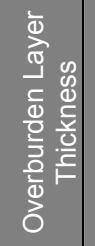 & 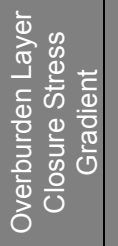 & 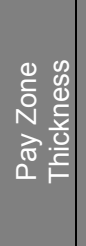 & 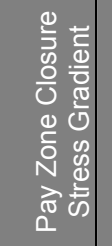 & 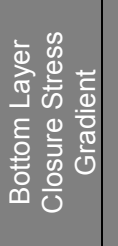 & 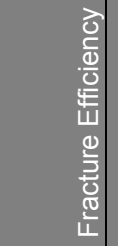 & 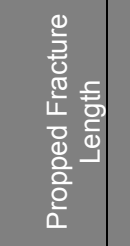 & 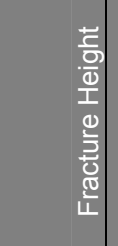 & 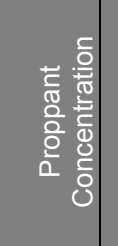 & 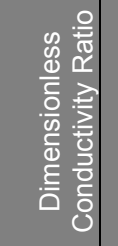 & \\
\hline input & input & input & input & input & input & input & input & input & input & input & input & input & input & output & output & output & output & output & output \\
\hline 171,255 & 62,924 & 15.7 & 38 & 8,649 & 3,942 & 0.0088 & 197 & 0.746 & 17 & 0.760 & 116 & 0.684 & 0.734 & 0.920 & 500.01 & 604.88 & 1.69 & 130.55 & 7. \\
\hline 171,255 & 62,924 & 8.7 & 38 & 8,649 & 3,942 & 0.0345 & 197 & 0.788 & 17 & 0.835 & 116 & 0.723 & 0.753 & 850 & 489.05 & 626.15 & 1.04 & 18.46 & \\
\hline 38,750 & 16,670 & 7.7 & 19 & 9,951 & 3,340 & 0.0085 & 209 & 0.771 & 30 & 0.805 & 95 & 0.686 & 0.776 & 810 & 353.36 & 304.31 & 0.55 & 40.96 & 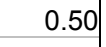 \\
\hline 38,750 & 16,670 & 7.7 & 19 & 9,951 & 3,340 & 0.0645 & 209 & 0.771 & 30 & 0.805 & 95 & 0.714 & 0.776 & 610 & 285.14 & 334.44 & 0.61 & 8.20 & \\
\hline 196,759 & 69,742 & 11.8 & 24 & 0,666 & 4,663 & 0092 & 235 & 0.761 & 12 & 0.809 & 123 & 0.690 & 0.736 & .900 & 619.98 & 17.87 & 1.25 & 46.91 & 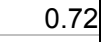 \\
\hline 83,282 & 29,407 & 6.5 & 29 & 11,147 & 3,916 & 0.0021 & 175 & 0.743 & 26 & 0.784 & 110 & 0.679 & 0.744 & 0.920 & 517.03 & 481.02 & 0.50 & 89.00 & \\
\hline 83,282 & 29,407 & 6.5 & 29 & 11,147 & 3,916 & 0.0740 & 175 & 0.743 & 26 & 0.784 & 110 & 0.692 & 0.744 & 640 & 442.47 & 481.47 & 0.58 & 3.96 & 45 \\
\hline 35,863 & 12,782 & 12.8 & 18 & 11,220 & 5,280 & 0.0086 & 187 & 0.806 & 21 & 0.841 & 51 & 0.730 & 0.772 & 0.930 & 241.67 & 384.68 & 0.99 & 67.71 & 43 \\
\hline 43,136 & 16,869 & 8.7 & 16 & 10,876 & 4,693 & 0.0022 & 189 & 0.786 & 30 & 0.806 & 129 & 0.712 & 0.787 & .880 & 385.02 & .58 & 0.62 & 19.93 & \\
\hline 43,136 & 16,869 & 8.7 & 16 & 10,876 & 4,693 & 0.0090 & 189 & 0.786 & 30 & 0.806 & 129 & 0.730 & 0.787 & 0.800 & 329.22 & 365.17 & 0.63 & 34.79 & .44 \\
\hline 40,865 & 15,714 & 13.9 & 22 & 9,868 & 4,920 & 0.0122 & 206 & 0.764 & 23 & 0.768 & 101 & 0.660 & 0.716 & .850 & 306.53 & 41.70 & 1.05 & 3.42 & \\
\hline 104,089 & 39,938 & 9.6 & 29 & 10,516 & 3,548 & 0.0862 & 202 & 0.753 & 13 & 0.785 & 130 & 0.690 & 0.739 & 0.590 & 443.16 & 483.58 & 0.95 & 6.58 & .55 \\
\hline 147,768 & ,406 & 15.5 & 35 & 10,510 & 3,717 & 0.0064 & 196 & 0.731 & 13 & 0.754 & 98 & 0.671 & 0.734 & .930 & 464.97| & 93.81 & 1.70 & 127.22 & 77 \\
\hline 79,792 & 33,417 & 13.6 & 26 & 10,329 & 5,019 & 0.0255 & 224 & 0.799 & 28 & 0.849 & 104 & 0.731 & 0.782 & 0.800 & 391.86 & 417.81 & 1.19 & 23.09 & .58 \\
\hline 55,586 & 22,762 & 8.2 & 21 & 10,642 & 4,037 & 0.0092 & 233 & 0.764 & 14 & 0.789 & 63 & 0.688 & 0.796 & 0.830 & 296.77 & 418.31 & 0.73 & 57.38 & 49 \\
\hline 55,586 & 22,762 & 8.2 & 21 & 10,642 & 4,037 & 0.0754 & 233 & 0.764 & 14 & 0.763 & 63 & 0.688 & 0.731 & 0.830 & 308.99 & 404.93 & 0.73 & 50.93 & 0.47 \\
\hline 165,073 & 60,059 & 6.7 & 35 & 10,144 & 4,217 & 0.0410 & 215 & 0.745 & 27 & 0.788 & 52 & 0.700 & 0.749 & 0.760 & 443.45 & 558.12 & 1.00 & 4.85 & 65 \\
\hline 165,073 & 60,059 & 6.7 & 35 & 10,144 & 4,217 & 0.0041 & 215 & 0.745 & 27 & 0.788 & 52 & 0.691 & 0.738 & 0.910 & 573.97 & 0.54 & 0.67 & 70.06 & 0.55 \\
\hline 95,475 & 35,074 & 10.1 & 27 & 8,739 & 3,775 & 0.0067 & 171 & 0.755 & 20 & 0.772 & 53 & 0.670 & 0.737 & 0.890 & 453.87 & 7.43 & 0.90 & 101.97 & .5 \\
\hline 79,143 & 32,975 & 16.0 & 26 & 11,303 & 5,423 & 0.0076 & 210 & 0.736 & 19 & 0.793 & 68 & 0.687 & 0.755 & 0.850 & 435.14 & 435.50 & 1.16 & 3.33 & .56 \\
\hline 128,638 & 53,480 & 13.3 & 24 & 10,796 & 4,406 & 0.0055 & 240 & 0.784 & 27 & 0.826 & 76 & 0.716 & 0.781 & 0.860 & 541.50 & 524.57 & 1.08 & 66.85 & $.5 \mathrm{~s}$ \\
\hline , 740 & 33,417 & 6.8 & 28 & 9,519 & 4,593 & 0.0074 & 212 & 0.754 & 35 & 0.806 & 100 & 0.675 & 0.761 & 0.880 & 494.63 & 451.18 & 0.55 & 40.35 & 0.52 \\
\hline 117,993 & 47,268 & 11.4 & 26 & 9,644 & 4,102 & 0.0028 & 239 & 0.805 & 23 & 0.844 & 88 & 0.730 & 0.781 & 0.910 & 453.09 & 514.10 & 1.11| & 194.76 & 0.62 \\
\hline 70,961 & 27,443 & 7.4 & 26 & 8,884 & 3,881 & 0.0315 & 230 & 0.752 & 25 & 0.829 & 61 & 0.673 & 0.748 & 0.780 & 394.48 & 444.82 & 0.64 & 17.12 & 0.48 \\
\hline 88,264 & 35,276 & 6.6 & 30 & 10,423 & 4,215 & 0.0093 & 229 & 0.792 & 19 & 0.823 & 95 & 0.701 & 0.770 & 0.780 & 447.93 & 413.86 & 0.66 & 33.24 & 0.59 \\
\hline 30,555 & 12,236 & 7.8 & 17 & 10,617 & 5,307 & 0.0034 & 219 & 0.738 & 21 & 0.758 & 131 & 0.674 & 0.727 & 0.780 & 275.96 & 312.89 & 0.56 & 124.85 & 0.40 \\
\hline 58,529 & 22,207 & 13.8 & 24 & 11,184 & 4,873 & 0.0158 & 199 & 0.756 & 21 & 0.781 & 131 & 0.695 & 0.761 & 0.590 & 351.88 & 358.98 & 1.22 & 46.75 & .53 \\
\hline 166,733 & 72,293 & 15.1 & 38 & 11,471 & 5,640 & 0.0061 & 233 & 0.745 & 31 & 0.772 & 87 & 0.685 & 0.755 & 0.890 & 459.18 & 463.93 & 2.03 & 124.03 & 0.90 \\
\hline 133,751 & 57,853 & 9.5 & 30 & 9,198 & 4,020 & 0.0094 & 229 & 0.784 & 21 & 0.840 & 100 & 0.720 & 0.766 & 0.830 & 467.12 & 2.87 & 0.96 & 4.56 & \\
\hline 133,751 & 57,853 & 9.5 & 30 & 9,198 & 4,020 & 0.0182 & 229 & 0.766 & 21 & 0.780 & 100 & 0.685 & 0.744 & 0.760 & 500.08 & 520.36 & 0.92 & 28.96 & \\
\hline
\end{tabular}

Table A-1: Data Generated for Ramp Treatment, continued 


\begin{tabular}{|c|c|c|c|c|c|c|c|c|c|c|c|c|c|c|c|c|c|c|c|}
\hline \begin{tabular}{|l|}
$\stackrel{0}{E}$ \\
$\frac{1}{3}$ \\
$\frac{0}{0}$ \\
$\frac{\pi}{0}$ \\
$\stackrel{0}{\circ}$
\end{tabular} & \begin{tabular}{l|}
$\frac{0}{2}$ \\
$\frac{3}{0}$ \\
0 \\
$\frac{0}{0}$ \\
$\frac{0}{0}$
\end{tabular} & 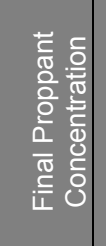 & 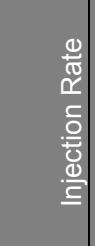 & 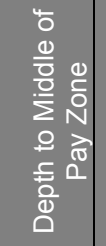 & 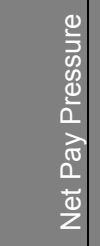 & 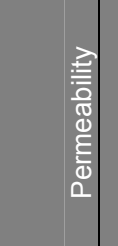 & 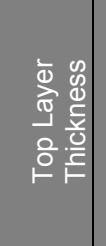 & 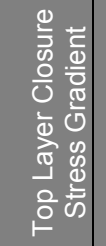 & 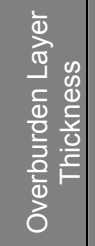 & 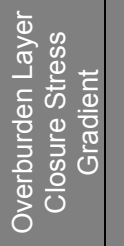 & 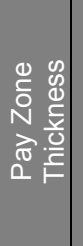 & 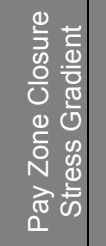 & 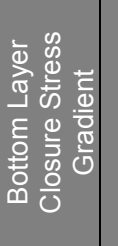 & 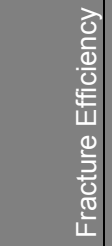 & 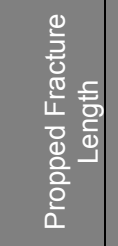 & 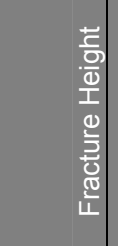 & 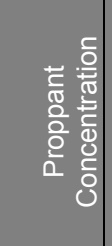 & 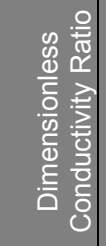 & 훈 \\
\hline input & input & input & input & input & input & input & input & input & input & input & input & input & input & output & output & output & output & output & output \\
\hline 72,811 & 26,280 & 12.7 & 25 & 11,666 & 5,503 & 0.0421 & 200 & 0.751 & 29 & 0.771 & 128 & 0.661 & 0.758 & 0.390 & 460.02 & 294.34 & 1.37 & 18.01 & 0.56 \\
\hline 104,389 & 39,533 & 7.0 & 30 & 9,322 & 4,082 & 0.0948 & 196 & 0.763 & 32 & 0.784 & 55 & 0.682 & 0.744 & .920 & 470.93 & 25.17 & 0.64 & 77.86 & \\
\hline 189,868 & 72,636 & 12.8 & 22 & 10,588 & 4,633 & 0.0083 & 173 & 0.745 & 20 & 0.769 & 88 & 0.676 & 0.732 & 0.860 & 613.96 & 598.85 & 1.28 & 58.85 & 6 \\
\hline 72,807 & 29,437 & 6.6 & 23 & 9,922 & 4,086 & 0.0058 & 172 & 0.781 & 24 & 0.806 & 66 & 0.696 & 0.742 & 0.890 & 307.95 & 462.41 & 0.71 & 85.30 & .50 \\
\hline 81,903 & 33,985 & 15.5 & 25 & 10,139 & 4,460 & 0.0083 & 180 & 0.744 & 30 & 0.781 & 101 & 0.688 & 0.720 & 810 & 358.50 & 4.75 & $1.41 \mid$ & 12.61 & 5 \\
\hline 178,493 & 72,197 & 7.5 & 15 & 8,729 & 3,510 & 0.0396 & 232 & 0.753 & 31 & 0.775 & 95 & 0.699 & 0.743 & 0.760 & 496.96 & 746.63 & 0.86 & 14.46 & 0.57 \\
\hline 144,946 & 57,063 & 16.0 & 36 & 9,727 & 3,537 & 0.0057 & 210 & 0.741 & 22 & 0.791 & 76 & 0.687 & 0.737 & .890 & 467.37 & 63.07 & 1.60 & 63.91 & 67 \\
\hline 11,476 & 936 & 7.3 & 28 & 9,835 & 4,026 & 0.0062 & 175 & 0.795 & 20 & 0.844 & 132 & 0.690 & 0.801 & 0.490 & 201.28 & 149.52 & 0.55 & 95.24 & .27 \\
\hline 11,476 & ,936 & 7.3 & 28 & 9,835 & 4,026 & 0.0128 & 175 & 0.741 & 20 & 0.761 & 132 & 0.694 & 0.719 & 0.450 & 143.92 & 35.26 & 0.49 & 58.21 & .2 \\
\hline 86,957 & 36,307 & 6.4 & 28 & 9,244 & 3,924 & 0.0067 & 231 & 0.724 & 28 & 0.746 & 50 & 0.656 & 0.715 & 0.880 & 410.83 & 494.88 & 0.57 & 34.27 & 0.46 \\
\hline 199,821 & 75,420 & 9.4 & 40 & , ,958 & 4,927 & 0.0083 & 206 & 0.724 & 26 & 0.742 & 121 & 0.652 & 0.728 & 0.800 & 769.72 & 48.86 & 0.91 & 39.04 & 0.72 \\
\hline 199,821 & 75,420 & 9.4 & 40 & 10,958 & 4,927 & 0.0500 & 206 & 0.724 & 26 & 0.742 & 121 & 0.690 & 0.728 & 0.650 & 542.78 & 659.28 & 1.08 & 6.56 & 0.61 \\
\hline 154,934 & 66,681 & 14.4 & 38 & , ,491 & 4,065 & 0.0049 & 239 & 0.764 & 30 & 0.813 & 86 & 0.708 & 0.745 & .890 & 471.01 & 74.43 & 1.44 & 121.56 & .64 \\
\hline 26,065 & 10,634 & 8.8 & 18 & 11,242 & 4,702 & 0.0085 & 234 & 0.745 & 34 & 0.772 & 68 & 0.673 & 0.731 & 0.740 & 284.77 & 295.04 & 0.55 & 40.74 & 0.38 \\
\hline 124,702 & 45,628 & 6.6 & 30 & 11,026 & 4,066 & 0.0684 & 189 & 0.736 & 15 & 0.751 & 129 & 0.662 & 0.710 & 0.420 & 487.51 & 481.19 & 0.79 & 7.08 & .53 \\
\hline 108,160 & 43,969 & 7.4 & 28 & 9,940 & 4,538 & 0.0054 & 236 & 0.756 & 12 & 0.777 & 58 & 0.683 & 0.763 & 0.920 & 370.48 & 451.91 & 0.98 & 120.59 & 0.73 \\
\hline 175,326 & 70,282 & 7.3 & 40 & $\mid, 316$ & 5,510 & 0.0281 & 207 & 0.768 & 19 & 0.800 & 65 & 0.698 & 0.750 & 0.820 & 610.51 & 608.92 & $0.71 \mid$ & 7.02 & .57 \\
\hline 194,065 & 83,698 & 8.4 & 40 & 11,394 & 5,284 & 0.0914 & 233 & 0.763 & 27 & 0.800 & 109 & 0.672 & 0.752 & 0.470 & 719.95 & 486.74 & 0.90 & 3.37 & 0.67 \\
\hline 119,126 & 42,630 & 6.3 & 32 & 11,185 & 4,477 & 0.0125 & 221 & 0.745 & 33 & 0.768 & 102 & 0.687 & 0.727 & .900 & 506.70 & 570.94 & 0.60 & 86.64 & 0.53 \\
\hline 53,846 & 20,676 & 10.5 & 27 & 9,129 & 3,257 & 0.0053 & 237 & 0.795 & 29 & 0.811 & 114 & 0.717 & 0.752 & 0.800 & 302.35 & 75.08 & 1.01 & 168.19 & 0.51 \\
\hline 22,982 & 433 & 12.8 & 19 & ,424 & 4,210 & 0.0699 & 238 & 0.743 & 22 & 0.775 & 77 & 0.657 & 0.735 & 0.420 & 234.47 & 251.93 & 0.99 & 22.75 & 0.35 \\
\hline 42,396 & 17,571 & 14.3 & 20 & 10,892 & 4,898 & 0.0089 & 175 & 0.726 & 30 & 0.732 & 103 & 0.658 & 0.714 & 0.720 & 311.95 & 320.78 & 1.09 & 88.57 & 0.50 \\
\hline 32,311 & 12,034 & 9.2 & 21 & 11,156 & 4,463 & 0.0158 & 174 & 0.770 & 23 & 0.820 & 65 & 0.690 & 0.760 & 0.680 & 318.71 & 303.49 & 0.64 & 24.79 & 0.43 \\
\hline 21,127 & 8,011 & 10.5 & 16 & 0,722 & 5,311 & 0.0062 & 224 & 0.801 & 18 & 0.832 & 114 & 0.730 & 0.770 & 0.680 & 230.70 & 281.27 & 0.69 & 83.36 & 0.36 \\
\hline 21,127 & 8,011 & 10.5 & 16 & 10,722 & 5,311 & 0.0500 & 224 & 0.751 & 18 & 0.774 & 114 & 0.684 & 0.743 & 0.320 & 218.83 & 261.42 & 0.79 & 20.82 & 0.31 \\
\hline 39,143 & 14,201 & 7.8 & 22 & 11,359 & 4,779 & 0.0091 & 233 & 0.777 & 12 & 0.798 & 133 & 0.697 & 0.755 & 0.610 & 327.13 & 298.45 & 0.69 & 41.88 & 0.44 \\
\hline 61,471 & 23,387 & 11.5 & 25 & 9,383 & 3,225 & 0.0081 & 227 & 0.742 & 10 & 0.792 & 84 & 0.678 & 0.735 & 0.810 & 356.43 & 431.90 & 0.61 & 61.62 & 0.46 \\
\hline 61,471 & 23,387 & 11.5 & 25 & 9,383 & 3,225 & 0.0800 & 227 & 0.742 & 10 & 0.767 & 84 & 0.691 & 0.735 & 0.530 & 309.29 & 531.41 & 0.69 & 9.71 & 0.42 \\
\hline 68,061 & 27,092 & 14.3 & 26 & ,743 & 5,465 & 0.0044 & 179 & 0.733 & 31 & 0.754 & 56 & 0.660 & 0.717 & 0.910 & 385.66 & 426.16 & 1.10 & 110.93 & 0. \\
\hline 68,061 & 27,092 & 14.3 & 26 & 11,743 & 5,465 & 0.0076 & 179 & 0.733 & 31 & 0.754 & 96 & 0.678 & 0.717 & 0.800 & $348.77 \mid$ & 405.35 & $1.27 \mid$ & 80.63 & \\
\hline
\end{tabular}

Table A-1: Data Generated for Ramp Treatment, continued 


\begin{tabular}{|c|c|c|c|c|c|c|c|c|c|c|c|c|c|c|c|c|c|c|c|}
\hline \begin{tabular}{|l|l|}
$\frac{0}{g}$ \\
$\frac{3}{0}$ \\
$\frac{2}{0}$ \\
$\frac{\pi}{0}$
\end{tabular} & \begin{tabular}{l|l}
0 \\
$E$ \\
3 \\
0 \\
2 \\
0 \\
0 \\
0
\end{tabular} & 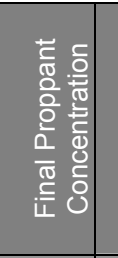 & 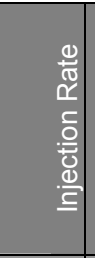 & 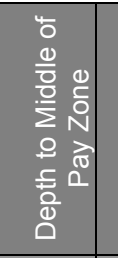 & \begin{tabular}{l|l}
$\frac{0}{3}$ \\
0 \\
0 \\
0 \\
$\frac{2}{2}$ \\
$\frac{1}{0}$ \\
$\frac{1}{0}$ \\
$\frac{1}{2}$
\end{tabular} & 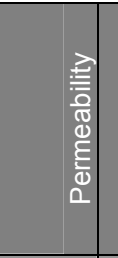 & 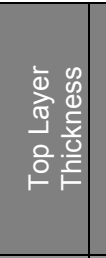 & 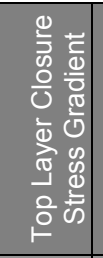 & 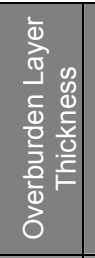 & 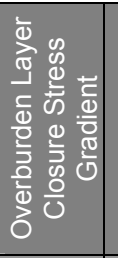 & 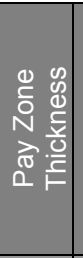 & 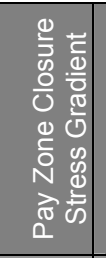 & 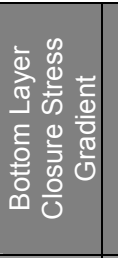 & 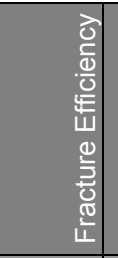 & 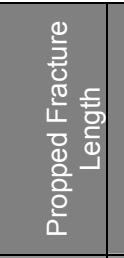 & 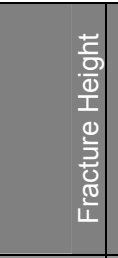 & 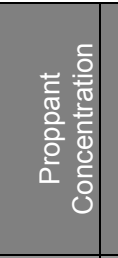 & 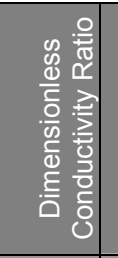 & \\
\hline input & input & input & input & input & input & input & input & input & input & input & input & input & input & output & output & output & output & output & output \\
\hline 15,398 & 5,782 & 7.2 & 16 & 11,166 & 5,482 & 0.0365 & 236 & 0.737 & 31 & 0.752 & 118 & 0.686 & 0.727 & 0.650 & 8.08 & 265.99 & 0.44 & 56.95 & \\
\hline 141,458 & 168 & 6.1 & 30 & 1,620 & 4,090 & 0.0071 & 188 & 786 & 15 & 0.818 & 65 & 0.718 & 0.780 & 880 & 575.64 & 4.39 & 0.58 & 20.56 & \\
\hline 12,409 & 4,583 & 7.2 & 15 & 11,013 & 4,893 & 0.0089 & 192 & 0.776 & 13 & 0.791 & 93 & 0.700 & 0.730 & 0.600 & 179.21 & 223.12 & 0.41 & 39.84 & \\
\hline 78,756 & 33,740 & 9.5 & 27 & 11,039 & 5,338 & 0.0024 & 234 & 0.802 & 29 & 0.858 & 124 & 0.725 & 0.787 & 0.830 & 453.46 & 381.85 & 0.81 & 102.39 & \\
\hline 37,936 & 16,192 & 13.7 & 20 & 10,198 & 4,837 & 0.0463 & 189 & 0.751 & 31 & 0.774 & 102 & 0.680 & 0.738 & 0.770 & 292.64 & 315.12 & 1.00 & 123.93 & \\
\hline 54,743 & 23,822 & 9.0 & 22 & 0,392 & 3,841 & 0.0147 & 186 & 0.791 & 20 & 0.845 & 71 & 0.730 & 0.789 & 800 & 359.37 & 7.23 & 0.64 & 22.72 & \\
\hline 87,330 & 32,523 & 14.6 & 30 & 10,611 & 4,774 & 0.0325 & 205 & 0.740 & 23 & 0.761 & 84 & 0.668 & 0.722 & 0.710 & 404.20 & 427.03 & 1.41 & 28.26 & 62 \\
\hline 78,331 & 32,794 & 6.5 & 28 & 9,246 & 3,891 & 0.0032 & 188 & 0.769 & 29 & 0.820 & 73 & 0.706 & 0.764 & 0.910 & 329.20 & 77.56 & 0.67 & 151.58 & .55 \\
\hline 21,702 & 321 & 13.2 & 16 & 8,852 & 3,299 & 0.0184 & 204 & 0.787 & 30 & 0.779 & 60 & 0.694 & 0.739 & 650 & 245.78 & 242.49 & 0.85 & 56.73 & 40 \\
\hline 38,905 & 3,772 & 8.0 & 22 & 10,922 & 4,620 & 0.0076 & 213 & 0.736 & 30 & 0.754 & 122 & 0.666 & 0.717 & .700 & 314.94 & 327.81 & 0.67 & 34.47 & \\
\hline 121,606 & ,939 & 15.0 & 32 & 10,469 & 4,707 & 0.0826 & 180 & 0.737 & 21 & 0.754 & 113 & 0.661 & 0.757 & 450 & 497.04 & 412.69 & 1.64 & 3.45 & 65 \\
\hline 121,606 & ,939 & 15.0 & 32 & 10,469 & 4,707 & 0.0826 & 180 & 0.737 & 21 & 0.754 & 113 & 0.690 & 0.720 & .540 & 400.61 & 29.16 & 1.59 & $\mid 3.71$ & \\
\hline 104,305 & 298 & 8.2 & 30 & 10,454 & 468 & 0.0135 & 230 & 0.786 & 13 & 0.854 & 123 & 0.733 & 0.774 & 730 & 446.46 & 520.65 & 0.75 & 5.28 & \\
\hline 43,325 & 15,718 & 10.7 & 20 & 9,140 & 3,789 & 0.0055 & 215 & 0.754 & 14 & 0.761 & 73 & 0.709 & 0.738 & 0.870 & 268.34 & 430.99 & 0.83 & 157.60 & 42 \\
\hline 58,181 &, 554 & 13.1 & 24 & ,571 & 5,303 & 0681 & 203 & 0.809 & 28 & 0.838 & 58 & 0.727 & 0.787 & 590 & 1.74 & & 1.12 & 7.50 & 50 \\
\hline 98,179 & 42,854 & 9.0 & 30 & 10,141 & 3,994 & 0.0063 & 212 & 0.734 & 27 & 0.766 & 67 & 0.697 & 0.715 & 0.910 & 323.35 & 520.32 & 0.94 & 5.99 & .50 \\
\hline 158,277 & 761 & 8.0 & 35 & 0,035 & 3,853 & 081 & 196 & 0.753 & 34 & 785 & 83 & 0.701 & 0.735 & 870 & 499.44 & .22 & 0.83 & 47.16 & 55 \\
\hline 89,123 & 2,471 & 14.3 & 29 & 10,914 & 370 & 0.0471 & 216 & 0.800 & 25 & 0.819 & 83 & 0.731 & 0.764 & 0.660 & 336.07 & 464.28 & 1.60 & 19.78 & 63 \\
\hline 59,171 &, 185 & 13.6 & 28 & 8,577 & 4,235 & 051 & 192 & 0.721 & 27 & 0.746 & 112 & 0.689 & 0.771 & 880 & 265.30 & .78 & 1.29 & 312.77 & 56 \\
\hline 19,182 & 279 & 9.8 & 15 & 9,187 & 3,607 & 0.0076 & 189 & 0.756 & 35 & 0.775 & 121 & 0.692 & 0.748 & 0.660 & 210.12 & 266.85 & 0.63 & 105.31 & .34 \\
\hline 149,260 & & 11.9 & 40 & 8,669 & 3,837 & 0.0025 & 204 & 0.783 & 27 & 0.804 & 94 & 0.720 & 0.770 & 930 & 478.76 & 6.50 & 1.26 & 322.73 & \\
\hline 126,972 & ,, 894 & 16.0 & 35 & 11,575 & 5,188 & 0.0098 & 231 & 0.798 & 27 & 0.847 & 113 & 0.734 & 0.790 & 0.760 & 578.42 & 3.85 & 1.57 & 50.34 & .72 \\
\hline 177,441 &,+ 106 & 11.4 & 40 & 8,898 & 3,587 & 181 & 208 & 0.764 & 13 & 0.782 & 71 & 0.708 & 0.739 & 0.870 & 455.97 & & 1.31 & 44.69 & .64 \\
\hline 109,515 & 45,574 & 6.4 & 28 & 9,862 & 3,963 & 0.0976 & 199 & 0.732 & 34 & 0.740 & 65 & 0.693 & 0.718 & 0.690 & 393.19 & 562.24 & 0.66 & 4.72 & .44 \\
\hline 109,515 & 45,574 & 6.4 & 28 & 9,862 & 3,963 & 0.0032 & 199 & 0.732 & 34 & 0.740 & 105 & 0.680 & 0.718 & .880 & 442.22 & & 0.61 & 105.60 & .50 \\
\hline 171,735 & 65,155 & 8.7 & 40 & 8,784 & 3,482 & 0.0049 & 220 & 0.781 & 25 & 0.809 & 123 & 0.717 & 0.763 & 0.860 & 551.18 & & 0.94 & 5.39 & 65 \\
\hline 42,094 & 15,663 & 15.1 & 26 & 11,140 & 5,135 & 0.0056 & 197 & 0.750 & 33 & 0.773 & 130 & 0.688 & 0.724 & 0.720 & 295.90 & 0.13 & 1.21 & 143.22 & .49 \\
\hline 168,027 & 64,774 & 7.0 & 23 & 10,482 & 4,136 & 0.0066 & 187 & 0.785 & 23 & 0.824 & 76 & 0.723 & 0.791 & 0.890 & 459.44 & 496.46 & 1.11 & 72.95 & \\
\hline 13,172 & 4,760 & 8.2 & 16 & 8,554 & 3,878 & 0.0900 & 211 & 0.771 & 14 & 0.799 & 56 & 0.680 & 0.741 & 0.420 & 181.16 & 222.33 & 0.59 & 14.21 & $.2 \varepsilon$ \\
\hline 182,182 & 75,172 & 13.6 & 40 & 8,724 & 4,259 & 0.0511 & 198 & 0.756 & 15 & 0.782 & 119 & 0.673 & 0.729 & 0.680 & 522.40 & 572.28 & 1.51 & 21.01 & 0.7 \\
\hline
\end{tabular}

Table A-1: Data Generated for Ramp Treatment, continued 


\begin{tabular}{|c|c|c|c|c|c|c|c|c|c|c|c|c|c|c|c|c|c|c|c|}
\hline 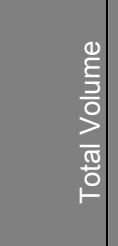 & 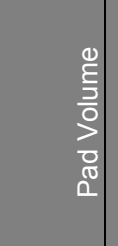 & 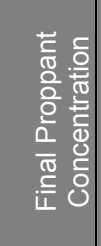 & 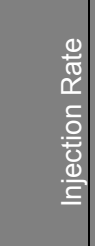 & 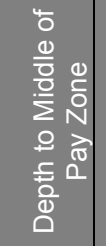 & 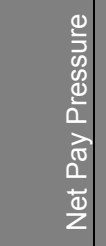 & 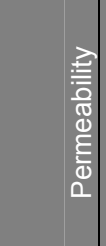 & 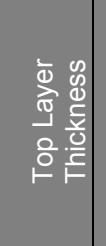 & 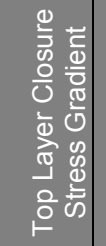 & 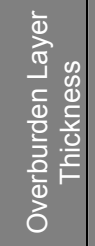 & 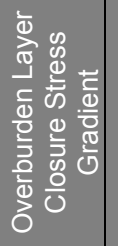 & 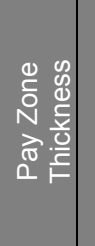 & 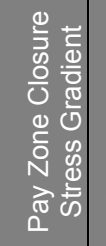 & 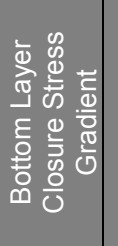 & 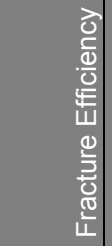 & 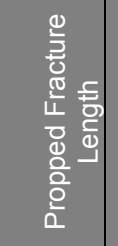 & 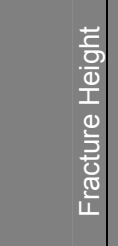 & 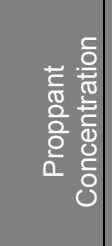 & 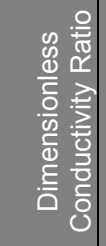 & $\frac{0}{5}$ \\
\hline input & input & input & input & input & input & input & input & input & input & input & input & input & input & output & output & output & output & output & output \\
\hline 182,182 & 75,172 & 13.6 & 40 & 8,724 & 4,259 & 0.0098 & 198 & 0.756 & 15 & 0.782 & 119 & 0.700 & 0.729 & 0.860 & 462.43 & 619.58 & 1.58 & 104.49 & $.6 \mathrm{~s}$ \\
\hline 136,821 & 55,713 & 9.7 & 36 & 9,696 & 4,335 & 0.0084 & 238 & 0.808 & 33 & 0.849 & 90 & 0.735 & 0.796 & .850 & 506.76 & 46.57 & 0.94 & 49.79 & .50 \\
\hline 167,912 & 67,518 & 15.2 & 39 & 8,467 & 4,134 & 0.0131 & 185 & 0.766 & 25 & 0.803 & 125 & 0.707 & 0.750 & 0.820 & 463.46 & 593.09 & 1.69 & 92.22 & .7 \\
\hline 23,600 & ,821 & 11.0 & 16 & 9,251 & 3,155 & 0.0185 & 181 & 0.806 & 17 & 0.768 & 51 & 0.690 & 0.733 & 0.750 & 213.69 & 18.42 & 0.71 & 50.56 & .35 \\
\hline 144,599 & 56,904 & 8.3 & 37 & 8,968 & 4,066 & 0.0037 & 201 & 0.799 & 25 & 0.852 & 80 & 0.740 & 0.774 & .930 & 435.69 & 4.59 & .92 & 44.29 & 5 \\
\hline 155,578 & 65,481 & 16.0 & 40 & 11,563 & 5,410 & 0.0037 & 202 & 0.757 & 12 & 0.788 & 81 & 0.694 & 0.749 & 0.910 & 549.99 & 557.19 & 1.41 & 110.13 & .67 \\
\hline 121,199 & 48,643 & 9.0 & 29 & 1,440 & 5,047 & 0.0043 & 193 & 0.773 & 20 & 0.801 & 56 & 0.709 & 0.799 & .940 & 384.97 & |0.34 & .93 & 99.72 & 5 \\
\hline 197,651 & 85,781 & 8.0 & 40 & 9,060 & 4,428 & 0.0032 & 197 & 0.749 & 25 & 0.766 & 130 & 0.667 & 0.744 & 0.890 & 660.56 & 568.85 & 0.81 & 109.84 & .68 \\
\hline 114,232 & 40,234 & 9.2 & 30 & ,517 & 4,665 & 0.0910 & 183 & 0.783 & 27 & 0.824 & 92 & 0.716 & 0.800 & .490 & 472.13 & 34.75 & .10 & 7.91 & 6 \\
\hline 148,091 & 64,048 & 7.6 & 38 & 11,310 & 5,057 & 0.0051 & 201 & 0.789 & 34 & 0.812 & 131 & 0.736 & 0.794 & 0.830 & 496.92 & 510.71 & 0.87 & 51.52 & 0.64 \\
\hline 49,590 & 18,477 & 9.2 & 22 & 8,906 & 3,543 & 0.0062 & 187 & 0.759 & 13 & 0.802 & 89 & 0.711 & 0.775 & 0.820 & 292.46 & 85.40 & 0.85 & 41.84 & .50 \\
\hline 57,098 & 21,623 & 10.1 & 20 & 11,085 & 4,318 & 0.0444 & 234 & 0.802 & 27 & 0.829 & 79 & 0.733 & 0.772 & 0.580 & 319.82 & 393.46 & 0.94 & 12.43 & 0.47 \\
\hline 152,846 & 53,742 & 12.2 & 37 & 9,974 & 3,507 & 0.0145 & 202 & 0.749 & 25 & 0.786 & 102 & 0.712 & 0.736 & 0.800 & 444.70 & 32.04 & .36 & 55.32 & .61 \\
\hline 91,784 & 39,534 & 7.2 & 30 & 11,287 & 4,381 & 0.0092 & 171 & 0.783 & 28 & 0.813 & 104 & 0.696 & 0.750 & 0.740 & 455.71 & 385.61 & 0.91 & 34.91 & 0.59 \\
\hline 161,485 & 66,077 & 12.0 & 40 & 9,228 & 3,514 & 0.0047 & 193 & 0.774 & 33 & 0.800 & 65 & 0.680 & 0.775 & 0.910 & 503.10 & 74.96 & 1.52 & 185.50 & 0.84 \\
\hline 149,671 & 65,267 & 10.3 & 39 & 10,229 & 4,051 & 0.0084 & 216 & 0.754 & 25 & 0.783 & 70 & 0.674 & 0.740 & 0.870 & 556.23 & 540.72 & 0.94 & 46.37| & 0.61 \\
\hline 125,414 & 49,873 & 11.1 & 35 & 8,941 & 3,834 & 0.0073 & 186 & 0.724 & 33 & 0.771 & 95 & 0.670 & 0.747 & 0.860 & 463.38 & 486.93 & 1.20 & 114.72 & .68 \\
\hline 21,588 & 502 & 12.1 & 15 & 11,154 & 5,299 & 0.0067 & 227 & 0.737 & 20 & 0.754 & 117 & 0.672 & 0.718 & 0.660 & $235.91 \mid$ & 275.93 & 0.77 & 69.87 & 0.37 \\
\hline 49,614 & $\mid, 218$ & 14.5 & 24 & 8,747 & 3,761 & 0.0106 & 209 & 0.754 & 26 & 0.795 & 58 & 0.720 & 0.743 & 1.870 & 249.31 & 2.41 & 1.20 & 92.45 & .46 \\
\hline 113,083 & 47,454 & 12.0 & 32 & 9,859 & 4,230 & 0.0049 & 221 & 0.784 & 28 & 0.831 & 86 & 0.713 & 0.764 & 0.880 & 450.85 & 499.54 & 1.11 & 77.62 & 0.60 \\
\hline 123,326 & 45,977 & 13.1 & 33 & 10,365 & 4,575 & 0.0034 & 199 & 0.799 & 11 & 0.832 & 76 & 0.723 & 0.729 & 0.900 & 531.91 & 528.72 & 1.13 & 93.01 & 0.63 \\
\hline 51,518 & 20,935 & 8.2 & 21 & 10,838 & 5,307 & 0.0187 & 198 & 0.761 & 22 & 0.801 & 110 & 0.701 & 0.745 & 0.640 & 332.36 & 370.32 & 0.70 & 16.81 & 0.44 \\
\hline 51,518 & 20,935 & 8.2 & 21 & 10,838 & 5,307 & 0.0074 & 198 & 0.761 & 22 & 0.801 & 110 & 0.680 & 0.745 & 0.730 & 387.89 & 324.40 & 0.68 & 34.33 & 0.50 \\
\hline 45,586 & 18,476 & 13.4 & 23 & 9,384 & 3,948 & 0.0022 & 221 & 0.767 & 22 & 0.817 & 106 & 0.668 & 0.770 & 0.840 & 369.79 & 12.33 & 0.98 & 252.64 & 0.56 \\
\hline 110,794 & 40,917 & 9.5 & 34 & 10,763 & 4,385 & 0.0047 & 219 & 0.802 & 23 & 0.847 & 107 & 0.730 & 0.795 & 0.820 & 527.10 & 493.66 & 0.84 & 47.26 & 0.58 \\
\hline 119,631 & 48,027 & 6.1 & 35 & 8,767 & 4,221 & 0.0357 & 172 & 0.733 & 14 & 0.742 & 124 & 0.650 & 0.718 & 0.680 & 495.09 & 503.42 & 0.63 & 14.37 & 0.56 \\
\hline 076 & 911 & 7.8 & 15 & 8,838 & 3,557 & 0.0052 & 179 & 0.750 & 24 & 0.800 & 76 & 0.695 & 0.719 & 0.770 & 166.30 & 258.87 & 0.51 & 121.50 & 0.28 \\
\hline 103,480 & 44,815 & 8.8 & 34 & 8,473 & 3,713 & 0.0120 & 174 & 0.748 & 11 & 0.768 & 108 & 0.706 & 0.733 & 0.830 & 356.93 & 530.06 & 0.92 & $65.37 \mid$ & 0.53 \\
\hline ,543 & 18,230 & 12.2 & 25 & 9,289 & 4,053 & 0.0045 & 209 & 0.758 & 15 & 0.793 & 118 & 0.686 & 0.751 & 0.800 & 309.24 & 361.74 & 1.02 & 210.72 & 0.5 \\
\hline 138,288 & 60,813 & 10.2 & 27 & 10,557 & 4,091 & 0.0091 & 191| & 0.733 & 27 & 0.741 & 105 & 0.677 & 0.726 & 0.820 & 489.87| & 538.70 & 0.98 & 48.27 & \\
\hline
\end{tabular}

Table A-1: Data Generated for Ramp Treatment, continued 


\begin{tabular}{|c|c|c|c|c|c|c|c|c|c|c|c|c|c|c|c|c|c|c|c|}
\hline 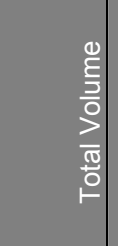 & \begin{tabular}{l|}
$\frac{0}{2}$ \\
$\frac{1}{3}$ \\
0 \\
0 \\
0 \\
0 \\
0
\end{tabular} & 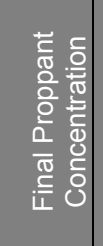 & 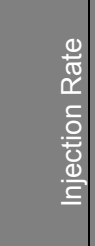 & 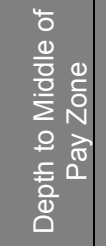 & 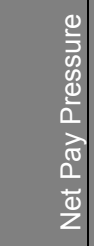 & 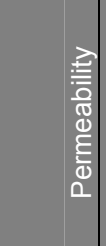 & 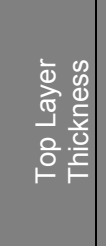 & 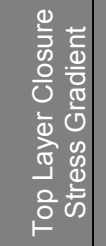 & 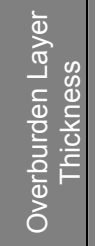 & 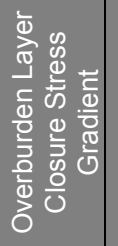 & 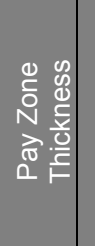 & 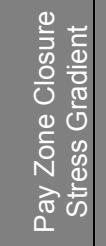 & 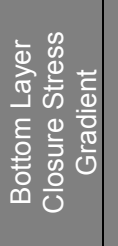 & 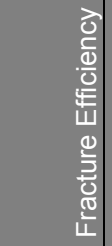 & 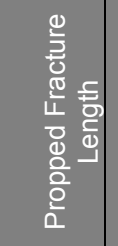 & 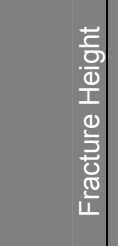 & 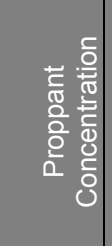 & 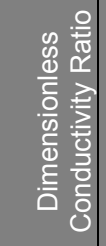 & $\frac{0}{5}$ \\
\hline input & input & input & input & input & input & input & input & input & input & input & input & input & input & output & output & output & output & output & output \\
\hline 59,716 & 21,522 & 11.4 & 27 & 8,695 & 3,928 & 0.0105 & 190 & 0.805 & 30 & 0.844 & 116 & 0.717 & 0.778 & 0.740 & 345.40 & 377.07 & 1.08 & 95.40 & .5 \\
\hline 180,044 & 75,239 & 9.6 & 40 & 10,492 & 4,555 & 0.0073 & 228 & 0.768 & 20 & 0.801 & 102 & 0.707 & 0.740 & 0.850 & 572.58 & 570.16 & 1.02 & 49.09 & \\
\hline 27,366 & 11,778 & 14.5 & 20 & 10,549 & 3,934 & 0.0066 & 177 & 0.765 & 22 & 0.770 & 72 & 0.688 & 0.725 & 0.800 & 207.98 & 298.66 & 1.10 & 52.30 & \\
\hline 80,595 & 28,896 & 6.4 & 33 & 9,289 & 3,680 & 0.0088 & 236 & 0.769 & 11 & 0.772 & 81 & 0.696 & 0.753 & 0.850 & 389.43 & 477.24 & 0.62 & 50.06 & \\
\hline 136,558 & 59,271 & 8.2 & 35 & 8,621 & 3,568 & 0.0057 & 223 & 0.755 & 12 & 0.782 & 95 & 0.681 & 0.723 & 0.890 & 441.89 & 543.27 & 0.90 & 105.54 & \\
\hline 42,743 & 15,914 & 7.6 & 22 & 8,800 & 4,005 & 0.0166 & 219 & 0.734 & 18 & 0.743 & 56 & 0.666 & 0.720 & 0.840 & 281.25 & 393.73 & 0.64 & 46.26 & .44 \\
\hline 86,122 & 4,006 & 9.8 & 31 & 9,200 & 3,779 & 0.0281 & 221 & 0.746 & 33 & 0.772 & 70 & 0.670 & 0.733 & .770 & 405.78 & 61.66 & 0.90 & 24.79 & \\
\hline 88,324 & 35,336 & 13.9 & 32 & 10,756 & 4,580 & 0.0065 & 174 & 0.783 & 16 & 0.804 & 86 & 0.705 & 0.804 & 0.840 & 380.51 & 363.02 & 1.64 & 132.49 & 0.78 \\
\hline 88,324 & 35,336 & 13.9 & 32 & 0,756 & 4,580 & 0.0025 & 174 & 0.783 & 16 & 0.804 & 86 & 0.735 & 0.804 & .920 & 328.89 & 17.80 & 1.33 & 277.18 & 5 \\
\hline 114,673 & 43,526 & 8.8 & 32 & 10,877 & 4,836 & 0.0369 & 175 & 0.770 & 26 & 0.800 & 124 & 0.704 & 0.759 & 0.550 & 502.62 & 496.36 & 0.84 & 10.89 & 0.54 \\
\hline 104,896 & 41,437 & 7.5 & 30 & 8,578 & 3,362 & 0.0056 & 210 & 0.805 & 28 & 0.848 & 72 & 0.728 & 0.797 & .890 & 466.59 & 14.99 & 0.68 & 76.86 & 5 \\
\hline 141,028 & 61,396 & 13.6 & 36 & 9,593 & 4,005 & 0.0129 & 212 & 0.742 & 24 & 0.785 & 51 & 0.668 & 0.736 & 0.910 & 411.83 & 574.19 & 1.43 & 70.15 & .65 \\
\hline 12,935 & 525 & 6.2 & 15 & 0,789 & 4,714 & 0.0051 & 184 & 0.769 & 22 & 0.809 & 99 & 0.681 & 0.746 & .600 & 238.73 & 56.47 & 0.43 & 59.66 & 30 \\
\hline 32,439 & 12,036 & 14.5 & 19 & 10,813 & 4,659 & 0.0163 & 192 & 0.739 & 21 & 0.773 & 89 & 0.668 & 0.740 & 0.620 & 292.06 & 284.25 & 1.08 & 56.17 & 0.47 \\
\hline 26,817 & 10,103 & 6.9 & 16 & 9,477 & 4,484 & 0.0071 & 219 & 0.777 & 30 & 0.808 & 120 & 0.698 & 0.746 & 0.690 & 259.15 & .96 & 0.53 & 75.54 & .37 \\
\hline 31,172 & 11,044 & 13.1 & 18 & 9,348 & 3,581 & 0.0043 & 233 & 0.769 & 34 & 0.795 & 107 & 0.719 & 0.765 & 0.770 & 252.12 & 349.55 & 0.93 & 238.99 & 0.43 \\
\hline 13,726 & 933 & 10.1 & 15 & 0,771 & 4,682 & 0.0854 & 218 & 0.731 & 23 & 0.759 & 104 & 0.669 & 0.718 & 0.120 & 165.24 & 226.30 & 0.77 & 17.90 & .20 \\
\hline 195,617 & 1,194 & 12.0 & 40 & 10,185 & 4,051 & 0.0092 & 212 & 0.755 & 21 & 0.831 & 79 & 0.688 & 0.733 & 0.870 & 582.84 & 641.00 & 1.27 & 58.97| & 0.67 \\
\hline 154,234 & $2,517 \mid$ & 9.8 & 38 & 11,257 & 4,698 & 0.0046 & 202 & 0.728 & 35 & 0.764 & 125 & 0.710 & 0.734 & .840 & 529.73 & 38.60 & 1.04 & 84.20 & 0.6 \\
\hline , 691 & 9,149 & 14.9 & 28 & 9,206 & 3,870 & 0.0228 & 230 & .758 & 23 & 0.780 & 115 & 0.702 & 0.778 & 0.670 & 347.88 & 409.33 & 1.52 & 62.82 & 0.60 \\
\hline 167,458 & 65,418 & 15.9 & 26 & 11,522 & 4,984 & 0.0103 & 238 & 0.802 & 34 & 0.840 & 76 & 0.735 & 0.804 & 0.840 & 553.26 & 522.60 & 1.69 & 47.17 & 0.76 \\
\hline 151,750 &, 161 & 9.1 & 38 & 9,563 & 4,300 & 0.0367 & 199 & 0.790 & 12 & 0.821 & 103 & 0.682 & 0.754 & 0.680 & 557.43 & 488.09 & 0.99 & 13.88 & 0.68 \\
\hline 75,989 & 9,956 & 10.3 & 26 & 9,296 & 4,027 & 0.0162 & 202 & 0.795 & 15 & 0.786 & 133 & 0.668 & 0.724 & 0.670 & 375.43 & 411.64 & 1.00 & 53.60 & 0.55 \\
\hline 44,681 & 16,366 & 8.8 & 25 & 8,762 & 3,932 & 0.0084 & 223 & 0.736 & 30 & 0.744 & 76 & 0.655 & 0.741 & 0.830 & 326.38 & 365.95 & 0.70 & 94.99 & 0.48 \\
\hline 21,352 & 8,575 & 9.7 & 19 & 10,207 & 4,260 & 0.0023 & 179 & 0.755 & 22 & 0.775 & 91 & 0.684 & 0.738 & 0.810 & 242.25 & 279.80 & 0.60 & 185.64 & 0.37 \\
\hline 1,046 & 25,689 & 8.8 & 25 & 11,138 & 4,069 & 0.0088 & 175 & 0.749 & 31 & 0.788 & 102 & 0.668 & 0.771 & 0.680 & 526.16 & 351.90 & 0.72 & 37.01 & 0.53 \\
\hline 162,938 & 70,300 & 14.2 & 39 & 10,584 & 5,243 & 0.0674 & 188 & 0.772 & 34 & 0.798 & 122 & 0.709 & 0.758 & 0.560 & 508.94 & 545.52 & 1.45 & 10.18 & 0.6 \\
\hline 97,667 & 39,900 & 11.1 & 31 & 9,531 & 4,062 & 0.0038 & 216 & 0.757 & 30 & 0.766 & 95 & 0.696 & 0.734 & 0.890 & 391.28 & 491.35 & 1.08 & 80.61| & 0.57 \\
\hline .904 & 497 & 8.3 & 17 & 968 & 3,599 & 0.0028 & 197 & 0.753 & 25 & 0.783 & 105 & 0.670 & 0.744 & 0.790 & 258.24 & 270.71 & 0.54 & 180.67 & 0.3 \\
\hline 141,878 & 51,762 & 9.4 & 38 & 8,780 & 3,798 & 0.0153 & 234 & 0.742 & 26 & 0.742 & 68 & 0.688 & 0.748 & 0.890 & 406.00 & 621.53 & 1.12 & 59.67 & \\
\hline
\end{tabular}

Table A-1: Data Generated for Ramp Treatment, continued 


\begin{tabular}{|c|c|c|c|c|c|c|c|c|c|c|c|c|c|c|c|c|c|c|c|}
\hline 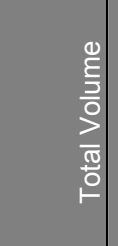 & \begin{tabular}{l|}
$\frac{0}{2}$ \\
$\frac{3}{0}$ \\
0 \\
$\frac{0}{0}$ \\
$\frac{0}{0}$
\end{tabular} & 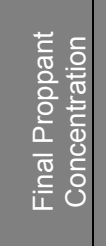 & 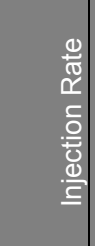 & 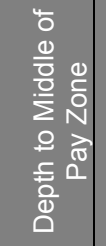 & \begin{tabular}{l|l}
$\frac{0}{3}$ \\
0 \\
0 \\
0 \\
$\frac{0}{0}$ \\
$\frac{1}{c}$ \\
$\frac{1}{0}$ \\
$\frac{0}{2}$
\end{tabular} & 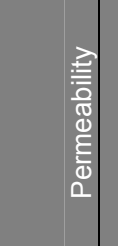 & 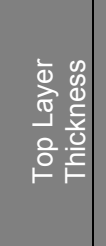 & 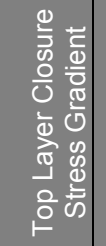 & 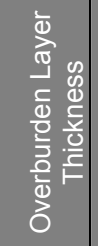 & 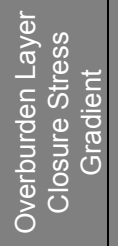 & 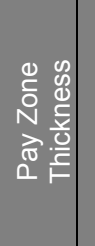 & 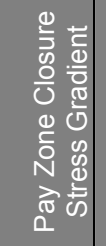 & 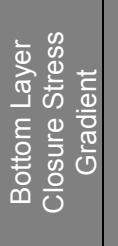 & 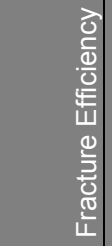 & 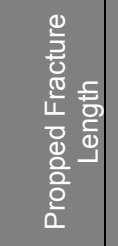 & 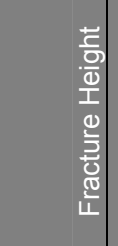 & 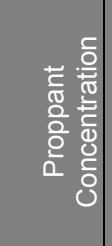 & 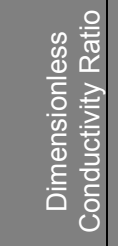 & $\frac{0}{5}$ \\
\hline input & input & input & input & input & input & input & input & input & input & input & input & input & input & output & output & output & output & output & output \\
\hline 45,404 & 19,150 & 7.3 & 24 & 10,080 & 4,090 & 0.0476 & 224 & 0.798 & 25 & 0.819 & 98 & 0.723 & 0.763 & 0.490 & 287.78 & 344.00 & 0.67 & 11.90 & \\
\hline 45,404 & 19,150 & 7.3 & 24 & 10,080 & 4,090 & 0.0059 & 224 & 0.741 & 25 & 0.761 & 98 & 0.681 & 0.751 & .790 & 320.46 & 361.18 & 0.57 & 71.79 & \\
\hline 187,857 & 68,691 & 15.1 & 40 & 10,188 & 4,748 & 0.0863 & 217 & 0.752 & 31 & 0.775 & 131 & 0.696 & 0.764 & 0.530 & 481.87 & 534.31 & 2.13 & 16.32 & .7 \\
\hline 58,493 & 21,005 & 9.0 & 25 & 8,417 & 3,526 & 0.0422 & 235 & 0.737 & 35 & 0.742 & 63 & 0.702 & 0.718 & 0.770 & 288.14 & 460.15 & 0.85 & 25.35 & .44 \\
\hline 58,493 & 21,005 & 9.0 & 25 & 8,417 & 3,526 & 0.0098 & 235 & 0.737 & 35 & 0.742 & 63 & 0.671 & 0.718 & 870 & 324.51 & 43.39 & 0.79 & 96.11 & 4 \\
\hline 152,619 & 61,151 & 12.5 & 39 & 9,632 & 3,713 & 0.0202 & 214 & 0.781 & 13 & 0.809 & 115 & 0.712 & 0.794 & 0.730 & 441.52 & 465.83 & 1.75 & 54.81 & .83 \\
\hline 108,600 & 4,235 & 8.6 & 32 & 1,179 & 4,555 & 0.0078 & 178 & 0.765 & 24 & 0.770 & 70 & 0.693 & 0.731 & 0.890 & 354.08 & 524.12 & 1.00 & 59.02 & 6 \\
\hline 75,628 & 29,170 & 11.2 & 28 & 10,397 & 4,254 & 0.0044 & 228 & 0.804 & 24 & 0.809 & 119 & 0.713 & 0.763 & 0.810 & 399.07 & 377.85 & 1.11 & 126.37 & .60 \\
\hline 81,277 & ,367 & 9.2 & 30 & 9,219 & 3,390 & 0.0102 & 205 & 0.740 & 14 & 0.766 & 129 & 0.698 & 0.779 & .770 & 323.21 & .39 & 1.00 & 93.02 & \\
\hline 195,439 & 78,878 & 7.9 & 40 & 10,231 & 4,398 & 0.0140 & 219 & 0.763 & 14 & 0.785 & 93 & 0.665 & 0.784 & 0.820 & 509.29 & 427.35 & 1.45 & 53.32 & 1.03 \\
\hline 154,683 & 64,752 & 8.4 & 37 & 9,611 & 4,576 & 0.0096 & 182 & 0.781 & 11 & 0.797 & 54 & 0.711 & 0.793 & .930 & 408.81| & 51.80 & 0.96 & 59.63 & 6 \\
\hline 167,242 & 65,536 & 6.4 & 38 & 10,946 & 4,045 & 0.0364 & 226 & 0.758 & 20 & 0.758 & 90 & 0.670 & 0.718 & 0.750 & 437.25 & 582.47 & 0.91 & 12.46 & 0.69 \\
\hline 36,086 & 4,126 & 13.7 & 21 & 0,906 & 4,600 & 0.0055 & 170 & 0.758 & 25 & 0.768 & 72 & 0.697 & 0.772 & 0.810 & 313.18 & 31.97 & 0.89 & 100.25 & .45 \\
\hline 172,797 & 73,620 & 7.2 & 40 & 10,077 & 3,796 & 0.0404 & 198 & 0.743 & 11 & 0.775 & 112 & 0.668 & 0.748 & 0.620 & 581.80 & 520.37 & 0.82 & 10.47 & 0.64 \\
\hline 27,147 & 10,077 & 9.9 & 19 & 9,266 & 3,290 & 0.0166 & 213 & 0.753 & 30 & 0.769 & 109 & 0.653 & 0.740 & 0.520 & 273.13 & 55.15 & 0.79 & 61.94 & .42 \\
\hline 27,147 & 10,077 & 9.9 & 19 & 9,266 & 3,290 & 0.0040 & 213 & 0.753 & 30 & 0.769 & 109 & 0.680 & 0.765 & 0.750 & 272.39 & 3.30 & 0.69 & 187.51 & 0.42 \\
\hline 93,268 & ,348 & 12.5 & 30 & 9,718 & 4,102 & 0.0085 & 209 & 0.739 & 19 & 0.747 & 126 & 0.676 & 0.722 & .780 & 397.77 & 468.74 & 1.22 & 103.82 & .60 \\
\hline 60,775 & ,338 & 15.8 & 28 & 10,128 & 4,856 & 0.0132 & 204 & 0.764 & 34 & 0.781 & 68 & 0.670 & 0.737 & 0.820 & 351.84 & .25 & 1.38 & 73.84 & 0.60 \\
\hline 180,481 & 74,375 & 12.2 & 40 & 1,871 . & 4,486 & 0.0119 & 207 & 0.755 & 25 & 0.785 & 78 & 0.671 & 0.746 & .790 & 728.03 & 44.89 & 1.03 & 20.45 & .67 \\
\hline 144,812 & 56,971 & 7.3 & 37 & 5,148 & 5,822 & 0.0080 & 219 & .740 & 24 & 0.777 & 131 & 0.685 & 0.740 & 0.660 & 744.06 & 488.36 & 0.61 & 9.19 & 0.57 \\
\hline 1,515 & 51,207 & 8.6 & 36 & 3,380 & 5,136 & 0.0055 & 194 & 0.788 & 31 & 0.828 & 52 & 0.726 & 0.803 & 0.900 & 360.25 & 381.48 & 1.69 & 61.99 & 1.03 \\
\hline 146,625 & ,507 & 14.5 & 38 & 12,333 & 5,134 & 0.0196 & 174 & 0.743 & 14 & 0.763 & 56 & 0.693 & 0.731 & 0.850 & 489.53 & 60.76 & 1.33 & 9.08 & 0.58 \\
\hline 132,076 & .350 & 11.7 & 36 & 12,982 & 4,854 & 0.0085 & 233 & 0.775 & 20 & 0.816 & 128 & 0.714 & 0.780 & 0.690 & 604.82 & 474.32 & 1.04 & 26.09 & 0.62 \\
\hline 164,986 & 69,016 & 14.3 & 39 & 12,634 & 5,401 & 0.0271 & 196 & 0.738 & 22 & 0.800 & 78 & 0.667 & 0.736 & 0.720 & 687.06 & 520.12 & 1.18 & 10.02 & 0.65 \\
\hline 20,323 & 8,547 & 9.4 & 16 & 14,704 & 5,699 & 0.0074 & 200 & 0.790 & 16 & 0.817 & 133 & 0.702 & 0.732 & 0.470 & 236.20 & 234.10 & 0.63 & 28.80 & 0.29 \\
\hline 20,323 & 8,547 & 9.4 & 16 & 14,704 & 5,699 & 0.0111 & 200 & 0.790 & 16 & 0.817 & 133 & 0.695 & 0.766 & 0.300 & 274.14 & 159.80 & 0.80 & 23.60 & 0.27 \\
\hline 97,606 & 1,526 & 7.9 & 30 & 14,692 & 6,228 & 0.0088 & 200 & 0.809 & 15 & 0.842 & 75 & 0.736 & 0.781 & 0.830 & 373.27 & 468.66 & 0.86 & 16.14 & 0.60 \\
\hline 72,073 & 29,033 & 8.8 & 25 & 12,538 & 4,554 & 0.0059 & 220 & 0.764 & 12 & 0.786 & 113 & 0.687 & 0.735 & 0.710 & 463.75 & 359.13 & 0.76 & 38.16 & 0.52 \\
\hline 72,073 & 29,033 & 8.8 & 25 & 12,538 & 4,554 & 0.0115 & 220 & 0.754 & 12 & 0.786 & 113 & 0.699 & 0.741 & 0.640 & 420.74 & 417.92 & 0.72 & 21.91 & 0.4 \\
\hline 46,649 & 19,737 & 11.1 & 17 & 13,987 & 5,978 & 0.0080 & 177 & 0.784 & 11 & 0.817 & 77 & 0.715 & 0.769 & 0.720 & 370.29 & 325.16 & 0.79 & 21.90 & 0.4 \\
\hline
\end{tabular}

Table A-1: Data Generated for Ramp Treatment, continued 


\begin{tabular}{|c|c|c|c|c|c|c|c|c|c|c|c|c|c|c|c|c|c|c|c|}
\hline 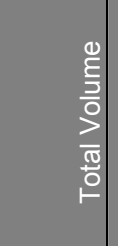 & 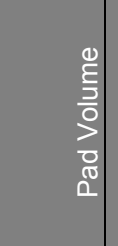 & 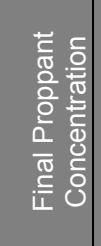 & 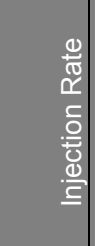 & 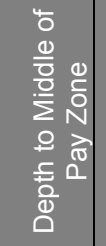 & 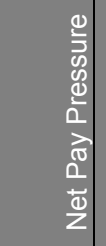 & $\begin{array}{l}\frac{\lambda}{3} \\
\frac{7}{\overline{0}} \\
\frac{0}{10} \\
\frac{0}{0} \\
0 \\
0\end{array}$ & 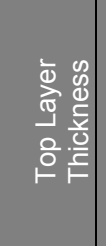 & 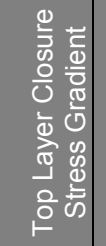 & 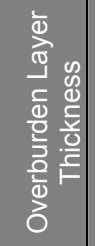 & 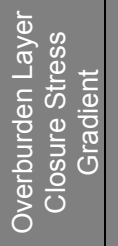 & 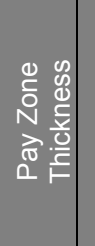 & 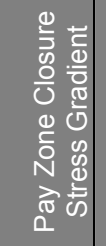 & 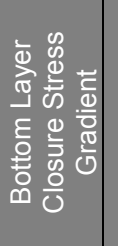 & 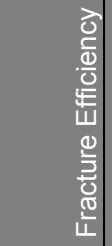 & 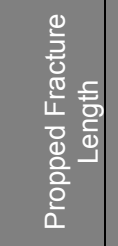 & 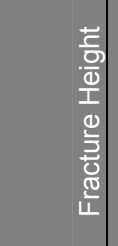 & 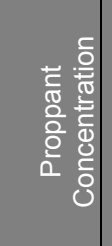 & 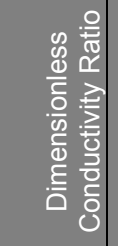 & 은 \\
\hline input & input & input & input & input & input & input & input & input & input & input & input & input & input & output & output & output & output & output & output \\
\hline 46,649 & 19,737 & 11.1 & 17 & 13,987 & 5,978 & 0.0250 & 177 & 0.756 & 11 & 0.817 & 77 & 0.715 & 0.742 & 0.630 & 302.95 & 374.13 & 0.84 & 10.08 & .40 \\
\hline 106,076 & 43,585 & 14.6 & 31 & 13,320 & 5,786 & 0.0228 & 217 & 0.734 & 26 & 0.747 & 103 & 0.665 & 0.722 & 610 & 522.16 & 412.70 & 1.29 & 16.17 & 63 \\
\hline 106,076 & 43,585 & 14.6 & 31 & 13,320 & 5,786 & 0.0090 & 217 & 0.734 & 26 & 0.747 & 103 & 0.686 & 0.722 & 0.780 & 457.63 & 491.12 & 1.24 & 37.04 & 5 \\
\hline 95,578 & 40,890 & 6.8 & 33 & 14,079 & 5,612 & 0.0069 & 196 & 0.738 & 29 & 0.791 & 119 & 0.706 & 0.786 & 0.680 & 601.32 & 61.99 & 0.60 & 13.25 & .49 \\
\hline 186,182 & 78,456 & 6.3 & 40 & 11,937 & 4,608 & 0.0466 & 214 & 0.801 & 28 & 0.850 & 99 & 0.740 & 0.810 & .580 & 588.53 & 3.48 & 0.80 & 4.71 & 6 \\
\hline 10,653 & 4,151 & 10.5 & 15 & 11,810 & 5,235 & 0.0094 & 198 & 0.746 & 19 & 0.751 & 59 & 0.694 & 0.719 & 0.730 & 167.59 & 34.84 & 0.57 & 46.59 & .27 \\
\hline 106,219 & 7,811 & 15.3 & 31 & 12,003 & 5,358 & 0.0143 & 195 & 0.760 & 17 & 0.767 & 111 & 0.680 & 0.747 & 0.680 & 528.30 & 12.31 & 1.46 & 35.98 & .7 \\
\hline 38,040 & 16,634 & 7.9 & 26 & 14,022 & 5,307 & 0.0072 & 210 & 0.733 & 14 & 0.746 & 109 & 0.677 & 0.726 & 0.630 & 335.93 & 297.25 & 0.58 & 23.96 & .41 \\
\hline 119,040 & 3,592 & 6.0 & 30 & 4,891 & 6,245 & 0.0227 & 181 & 0.760 & 22 & 0.786 & 115 & 0.714 & 0.746 & 0.580 & 564.81 & .98 & .53 & 3.76 & .47 \\
\hline 175,946 & 71,448 & 8.8 & 39 & 12,675 & 4,530 & 0.0336 & 211 & 0.756 & 19 & 0.792 & 112 & 0.710 & 0.748 & 0.610 & 572.40 & 622.95 & 0.86 & 5.67 & 0.54 \\
\hline 56,847 & 22,587 & 7.5 & 25 & 12,059 & 5,433 & 0.0063 & 211 & 0.722 & 16 & 0.760 & 117 & 0.675 & 0.720 & .760 & 364.32 & 386.49 & 0.63 & 2.55 & 0.47 \\
\hline 124,196 & 48,385 & 15.9 & 34 & 13,654 & 6,319 & 0.0563 & 171 & 0.745 & 18 & 0.782 & 98 & 0.691 & 0.756 & 0.500 & 538.96 & 442.90 & 1.52 & 7.32 & 0.61 \\
\hline 167,725 & 4,002 & 8.2 & 38 & 4,249 & 6,985 & 0.0095 & 190 & 0.721 & 13 & 0.781 & 96 & 0.705 & 0.756 & .820 & 610.12 & 37.10 & $0.81 \mid$ & |1.13 & .61 \\
\hline 41,749 & 15,111 & 12.5 & 23 & 14,149 & 5,330 & 0.0091 & 178 & 0.783 & 33 & 0.796 & 100 & 0.701 & 0.768 & 0.550 & 420.87 & 254.17 & 0.97 & 26.08 & 0.49 \\
\hline 66,477 & 23,764 & 10.6 & 25 & 14,511 & 6,980 & 0.0078 & 236 & 0.752 & 25 & 0.781 & 96 & 0.713 & 0.744 & 0.770 & 384.79 & 457.52 & 0.83 & 24.17 & 0.47 \\
\hline 169,012 & 72,006 & 8.9 & 37 & 13,871 & 5,820 & 0.0251 & 204 & 0.766 & 15 & 0.793 & 67 & 0.684 & 0.765 & 0.680 & 865.06 & 513.50 & 0.65 & 3.49 & 0.60 \\
\hline 18,186 & 695 & 7.9 & 16 & 12,891 & 4,925 & 0.0035 & 187 & 0.772 & 26 & 0.789 & 127 & 0.697 & 0.723 & 0.660 & 228.24 & 257.13 & 0.52 & 76.98 & 0.30 \\
\hline 179,209 & 77,654 & 8.3 & 40 & 14,367 & 6,217 & 0.0031 & 222 & 0.781 & 25 & 0.801 & 123 & 0.722 & 0.757 & 0.840 & 640.59 & 547.67 & 0.61 & 27.68 & 0.61 \\
\hline 150,935 & 56,036 & 9.4 & 37 & 12,961 & 5,444 & 0.0864 & 171 & 0.759 & 29 & 0.766 & 53 & 0.674 & 0.774 & .720 & 471.08 & 72.66 & 1.10 & 4.30 & 0.66 \\
\hline 95,010 & 38,180 & 13.5 & 31 & 14,062 & 5,245 & 0.0926 & 186 & 0.746 & 32 & 0.767 & 64 & 0.687 & 0.718 & 0.860 & 340.28 & 20.12 & 1.34 & 40.62 & 0.57 \\
\hline 3,687 & 12,508 & 8.8 & 21 & 12,515 & 5,431 & 0.0069 & 210 & 0.780 & 22 & 0.833 & 68 & 0.726 & 0.790 & 0.770 & 322.77 & 52.09 & 0.55 & 31.43 & 0.39 \\
\hline 117,187 & 49,854 & 9.8 & 32 & 14,134 & 5,371 & 0.0078 & 173 & 0.737 & 11 & 0.764 & 122 & 0.697 & 0.759 & 0.780 & 344.32 & 371.16 & 1.70 & 60.18 & 0.89 \\
\hline 5,255 & 34,793 & 9.6 & 29 & 14,964 & 7,234 & 0.0089 & 236 & 0.805 & 22 & 0.805 & 125 & 0.720 & 0.785 & 0.590 & 614.14 & 277.98 & 0.93 & 13.02 & 0.57 \\
\hline 154,171 & 57,263 & 6.7 & 35 & 12,341 & 5,288 & 0.0022 & 195 & 0.764 & 32 & 0.783 & 85 & 0.694 & 0.751 & 0.900 & 696.20 & 574.45 & 0.57 & 52.16 & 0.58 \\
\hline 124,121 & 45,837 & 6.1 & 32 & 13,785 & 5,699 & 0.0063 & 239 & 0.743 & 12 & 0.770 & 108 & 0.702 & 0.730 & 0.810 & 521.23 & 590.93 & 0.55 & 17.51 & 0.48 \\
\hline 127,385 & 53,649 & 12.2 & 32 & 13,234 & 5,814 & 0.0201 & 239 & 0.751 & 32 & 0.777 & 112 & 0.680 & 0.781 & 0.580 & 620.76 & 358.07 & 1.28 & 15.37 & 0.67 \\
\hline 5,363 & 27,957 & 15.1 & 27 & 12,474 & 6,135 & 0.0079 & 203 & 0.744 & 18 & 0.769 & 67 & 0.668 & 0.728 & 0.850 & 433.75 & 409.38 & 1.22 & 54.66 & 0.61 \\
\hline 146,797 & 58,465 & 13.7| & 27 & 14,363 & 5,764 & 0.0075 & 216 & 0.786 & 34 & 0.812 & 128 & 0.727 & 0.764 & 0.710 & 580.08 & 494.97 & 1.30 & 25.00 & 0.65 \\
\hline 75,058 & 32,801 & 8.5 & 25 & 13,621 & 5,219 & 0.0051 & 229 & 0.754 & 14 & 0.785 & 129 & 0.692 & 0.735 & 0.710 & 443.44 & 371.26 & 0.73 & 33.04| & 0.5 \\
\hline 97,455 & 42,038 & 6.5 & 30 & 13,340 & 5,208 & 0.0891 & 195 & 0.792 & 21 & 0.836 & 98 & 0.736 & 0.810 & 0.740 & 424.03 & 358.77 & 0.83 & 20.77 & 0.0 \\
\hline
\end{tabular}

Table A-1: Data Generated for Ramp Treatment, continued 


\begin{tabular}{|c|c|c|c|c|c|c|c|c|c|c|c|c|c|c|c|c|c|c|c|}
\hline 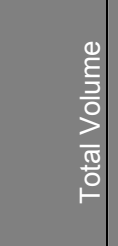 & \begin{tabular}{l|}
$\frac{0}{2}$ \\
$\frac{3}{0}$ \\
0 \\
$\frac{0}{0}$ \\
$\frac{0}{0}$
\end{tabular} & 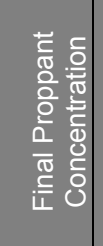 & 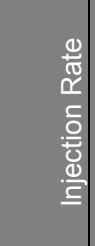 & 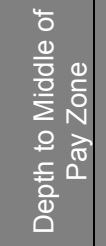 & 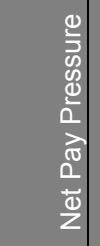 & 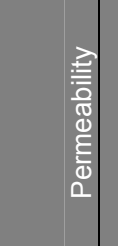 & 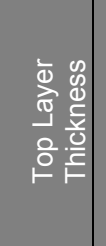 & 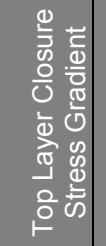 & 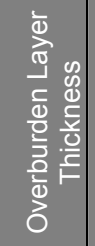 & 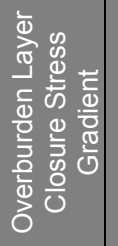 & 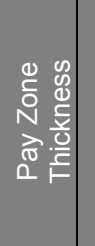 & 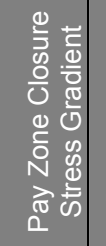 & 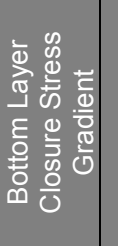 & 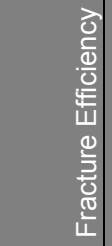 & 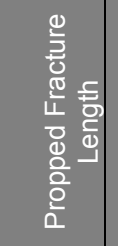 & 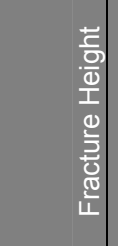 & 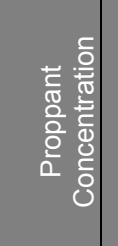 & 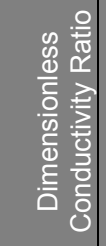 & $\frac{0}{5}$ \\
\hline input & input & input & input & input & input & input & input & input & input & input & input & input & input & output & output & output & output & output & output \\
\hline 83,829 & 32,387 & 6.5 & 28 & 13,397 & 5,494 & 0.0075 & 177 & 0.729 & 12 & 0.766 & 112 & 0.662 & 0.723 & 0.710 & 555.02 & 392.71 & 0.54 & $17.67 \mid$ & 5 \\
\hline 54,021 & 20,718 & 14.6 & 20 & 11,806 & 5,134 & 0.0094 & 239 & 0.763 & 22 & 0.790 & 79 & 0.672 & 0.736 & .750 & 384.95 & 327.55 & 1.18 & 55.43 & 57 \\
\hline 20,795 & ,376 & 9.9 & 25 & 12,080 & 4,406 & 0.0071 & 174 & 0.740 & 23 & 0.754 & 133 & 0.673 & 0.716 & 0.540 & 233.62 & 271.27 & 0.69 & 70.50 & .32 \\
\hline 15,346 & 685 & 14.7 & 15 & 14,898 & 6,519 & 0.0051 & 202 & 0.755 & 26 & 0.794 & 78 & 0.713 & 0.769 & 0.660 & 241.02 & 231.35 & 0.68 & 36.92 & .32 \\
\hline 68,906 & 8,872 & 9.6 & 26 & 3,736 & 5,581 & 0.0076 & 184 & 0.752 & 30 & 0.780 & 131 & 0.683 & 0.726 & 640 & 438.63 & 35.02 & 0.85 & 27.77 & 5 \\
\hline 135,000 & 49,343 & 8.1 & 35 & 14,090 & 5,893 & 0.0400 & 173 & 0.790 & 12 & 0.846 & 134 & 0.720 & 0.792 & 0.360 & 640.69 & 374.10 & 0.98 & 4.61 & .58 \\
\hline 123,715 & 44,384 & 7.1 & 34 & 3,061 & 5,829 & 0.0169 & 205 & 0.728 & 16 & 0.746 & 87 & 0.672 & 0.718 & 0.760 & 579.59 & 49.99 & 0.61 & 9.32 & 5 \\
\hline 154,008 & 56,066 & 10.3 & 37 & 14,714 & 5,798 & 0.0039 & 235 & 0.758 & 11 & 0.788 & 60 & 0.701 & 0.735 & 0.930 & 423.66 & 680.01 & 1.14 & 53.23 & .61 \\
\hline 24,224 & 668 & 14.0 & 17 & 4,140 & 6,261 & 0.0045 & 175 & 0.753 & 31 & 0.789 & 120 & 0.666 & 0.727 & 0.620 & 319.40 & 203.38 & 1.01 & 78.25 & .43 \\
\hline 124,891 & 53,166 & 15.0 & 36 & 14,724 & 5,837 & 0.0089 & 223 & 0.750 & 27 & 0.774 & 72 & 0.688 & 0.761 & 0.820 & 464.25 & 388.70 & 1.80 & 37.62 & 0.84 \\
\hline 96,011 & 36,044 & 13.3 & 32 & 3,715 & 5,625 & 0.0122 & 217 & 0.744 & 29 & 0.799 & 118 & 0.683 & 0.737 & .640 & 522.85 & 16.88 & 1.14 & 23.36 & .66 \\
\hline 67,907 & 24,349 & 14.1 & 29 & 14,211 & 5,341 & 0.0037 & 180 & 0.745 & 31 & 0.746 & 56 & 0.690 & 0.728 & 0.910 & 323.49 & 487.29 & 1.19 & 93.46 & 0.56 \\
\hline 16,862 & 769 & 10.2 & 15 & 2,168 & 5,152 & 0.0075 & 226 & 0.746 & 18 & 0.780 & 97 & 0.658 & 0.718 & .580 & 250.57 & 207.55 & 0.63 & 50.33 & .36 \\
\hline 51,761 & 19,026 & 10.3 & 25 & 14,085 & 6,828 & 0.0087 & 214 & 0.745 & 26 & 0.769 & 131 & 0.683 & 0.750 & 0.620 & 430.36 & 303.21 & 0.84 & 25.42 & 0.51 \\
\hline 28,685 & 11,764 & 13.6 & 17 & 12,474 & 5,583 & 0.0089 & 216 & 0.768 & 17 & 0.808 & 55 & 0.688 & 0.773 & 0.740 & 336.72 & 283.33 & 0.74 & 34.36 & 0.43 \\
\hline 188,966 & 81,357 & 11.6 & 40 & 13,195 & 6,174 & 0.0138 & 177 & 0.761 & 20 & 0.802 & 119 & 0.698 & 0.757 & 0.750 & 674.88 & 557.28 & 1.06 & 17.02 & 0.65 \\
\hline 105,531 & 39,776 & 14.9 & 35 & 1,867 & 5,593 & 0.0645 & 188 & 0.758 & 16 & 0.791 & 115 & 0.686 & 0.730 & 0.810 & 455.57 & 436.34 & 1.50 & 82.60 & .68 \\
\hline 105,104 & 41,496 & 9.9 & 34 & 13,801 & 6,120 & 0.0212 & 199 & 0.780 & 13 & 0.824 & 63 & 0.721 & 0.794 & 0.800 & 350.81 & 348.32 & 1.69 & 21.53 & 0.93 \\
\hline 143,279 & 51,081 & 8.5 & 36 & 14,664 & 5,952 & 0.0345 & 238 & 0.750 & 23 & 0.767 & 99 & 0.678 & 0.747 & 510 & 741.39 & 439.50 & 0.81 & 3.89 & 0.63 \\
\hline 169,371 & 4,343 & 14.0 & 38 & 2,761 & 5,365 & 0.0068 & 203 & .753 & 21 & 0.784 & 95 & 0.691 & 0.722 & 0.870 & 441.51 & 10.18 & 1.69 & 73.71 & 0.74 \\
\hline 9,882 & 28,178 & 12.5 & 28 & 11,995 & 5,420 & 0.0060 & 184 & 0.760 & 12 & 0.794 & 129 & 0.696 & 0.803 & 0.730 & 413.23 & 314.56 & 1.27 & 80.07 & 0.62 \\
\hline 172,000 & 66,797 & 9.4 & 39 & 12,809 & 5,825 & 0.0156 & 199 & 0.760 & 12 & 0.760 & 100 & 0.670 & 0.746 & 0.700 & 811.60 & 473.81 & 0.85 & 10.27 & 0.71 \\
\hline 172,000 & 66,797 & 9.4 & 39 & 12,809 & 5,825 & 0.0088 & 199 & 0.760 & 12 & 0.760 & 100 & 0.690 & 0.750 & 0.800 & 663.11 & 586.23 & 0.85 & 19.26 & 0.62 \\
\hline 12,370 & 5,330 & 7.0 & 15 & 13,423 & 6,340 & 0.0097 & 181 & 0.777 & 34 & 0.811 & 127 & 0.695 & 0.739 & 0.420 & 202.66 & 176.31 & 0.46 & 23.68 & 0.23 \\
\hline 91,850 & 9,961 & 15.5 & 31 & 12,310 & 6,195 & 0.0354 & 199 & 0.747 & 35 & 0.770 & 120 & 0.677 & 0.724 & 0.560 & 440.99 & 396.74 & 1.39 & 25.23 & 0.58 \\
\hline 144,609 & 1,386 & 6.2 & 35 & 13,739 & 5,172 & 0.0064 & 203 & 0.760 & 22 & 0.794 & 92 & 0.691 & 0.789 & 0.830 & 430.92 & 310.28 & 1.38 & 51.84 & 1.08 \\
\hline 62,540 & 27,514 & 11.8 & 27 & 4,193 & 6,305 & 0.0197 & 221 & 0.779 & 22 & 0.847 & 67 & 0.736 & 0.777 & 0.720 & 378.80 & 420.09 & 0.82 & 8.77 & 0.43 \\
\hline 23,593 & 9,198 & 8.6 & 18 & 13,172 & 6,509 & 0.0084 & 219 & 0.751 & 29 & 0.791 & 50 & 0.701 & 0.769 & 0.810 & 255.22 & 271.05 & 0.59 & 31.32 & 0.40 \\
\hline 69,810 & 29,877 & 11.6 & 26 & 12,105 & 5,798 & 0.0048 & 196 & 0.789 & 26 & 0.789 & 84 & 0.687 & 0.754 & 0.830 & 424.38 & 330.18 & 1.06 & 67.70 & 0.6 \\
\hline 133,252 & 53,330 & 9.8 & 34 & 13,369 & 5,822 & 0.0075 & 210 & 0.784 & 30 & 0.858 & 126 & 0.730 & 0.809 & 0.720 & $541.71 \mid$ & 410.19 & 1.16 & 30.65 & \\
\hline
\end{tabular}

Table A-1: Data Generated for Ramp Treatment, continued 


\begin{tabular}{|c|c|c|c|c|c|c|c|c|c|c|c|c|c|c|c|c|c|c|c|}
\hline 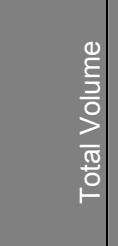 & 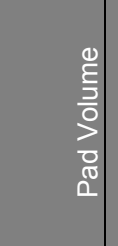 & 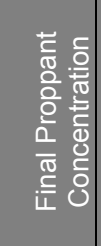 & 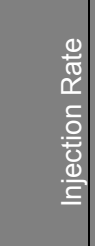 & 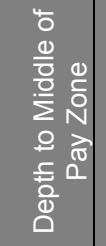 & 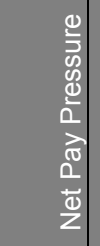 & 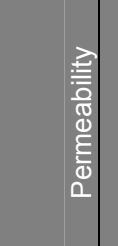 & 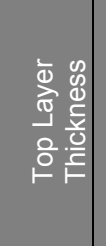 & 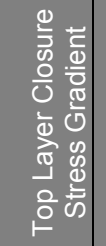 & 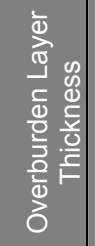 & 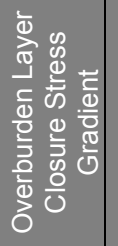 & 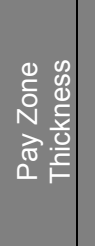 & 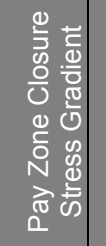 & 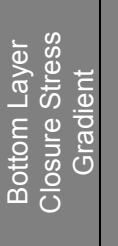 & 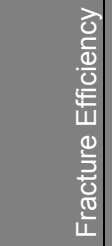 & 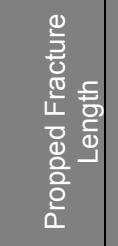 & 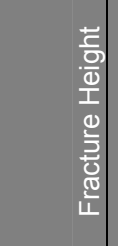 & 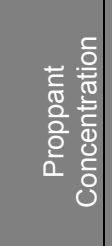 & 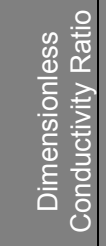 & $\frac{0}{5}$ \\
\hline input & input & input & input & input & input & input & input & input & input & input & input & input & input & output & output & output & output & output & output \\
\hline 46,776 & 19,366 & 6.7 & 22 & 14,980 & 6,672 & 0.0158 & 227 & 0.745 & 17 & 0.765 & 65 & 0.664 & 0.742 & 0.620 & 497.82 & 273.84 & 0.47 & 5.35 & .50 \\
\hline 101,827 & 43,356 & 9.7 & 32 & 12,576 & 5,418 & 0.0491 & 198 & 0.770 & 24 & 0.809 & 80 & 0.711 & 0.760 & 600 & 480.87 & 479.12 & 0.81 & 4.96 & 49 \\
\hline 95,046 & 40,672 & 7.4 & 30 & 14,126 & 5,969 & 0.0091 & 196 & 0.749 & 13 & 0.785 & 122 & 0.708 & 0.806 & 0.770 & 358.92 & 69.37 & 0.82 & 21.90 & .47 \\
\hline 95,046 & 40,672 & 7.4 & 30 & 14,126 & 5,969 & 0.0061 & 196 & 0.749 & 13 & 0.785 & 122 & 0.690 & 0.755 & 0.710 & 575.80 & 388.75 & 0.62 & 16.85 & .52 \\
\hline 54,681 & 22,762 & 12.2 & 24 & 12,602 & 4,330 & 0.0102 & 207 & 0.748 & 15 & 0.776 & 134 & 0.687 & 0.719 & .600 & 334.95 & 43.65 & 1.07 & 46.72 & 4 \\
\hline 85,354 & 35,687 & 6.1 & 28 & 12,429 & 5,501 & 0.0014 & 180 & 0.774 & 29 & 0.777 & 79 & 0.684 & 0.795 & 0.890 & 589.69 & 315.61 & 0.57 & 90.70 & .61 \\
\hline 73,956 & 29,267 & 7.1 & 28 & 1,948 & 5,294 & 0.0079 & 215 & 0.754 & 28 & 0.783 & 93 & 0.700 & 0.723 & 0.840 & 319.51| & 60.71 & 0.75 & 41.30 & 4 \\
\hline 73,956 & 29,267 & 7.1 & 28 & 11,948 & 5,294 & 0.0040 & 215 & 0.754 & 28 & 0.775 & 93 & 0.680 & 0.731 & 0.850 & 438.06 & 411.04 & 0.61 & 50.75 & 0.5 \\
\hline 38,773 & 5,177 & 10.0 & 23 & 3,860 & 6,507 & 0.0084 & 177 & 0.722 & 21 & 0.803 & 99 & 0.686 & 0.740 & 0.660 & 362.36 & 1.25 & 0.74 & 24.77 & \\
\hline 152,774 & 54,855 & 8.1 & 36 & 14,791 & 5,855 & 0.0144 & 216 & 0.767 & 30 & 0.792 & 85 & 0.700 & 0.764 & 0.680 & 826.76 & 530.45 & 0.61 & 4.96 & $0.5 \varepsilon$ \\
\hline 173,174 & 71,709 & 12.4 & 38 & 13,825 & 6,585 & 0.0276 & 186 & 0.809 & 25 & 0.856 & 106 & 0.736 & 0.810 & 0.730 & 851.19 & 485.15 & 0.96 & 13.43 & 0.6 \\
\hline 140,710 & 56,606 & 16.0 & 36 & 12,398 & 5,776 & 0.0203 & 176 & 0.781 & 27 & 0.817 & 110 & 0.717 & 0.779 & 0.660 & 580.02 & 491.12 & 1.42 & 18.27| & .66 \\
\hline 88,998 & 33,678 & 11.9 & 29 & 2,035 & 5,086 & 0.0732 & 233 & 0.761 & 21 & 0.800 & 116 & 0.670 & 0.763 & 300 & 479.52 & 04.92 & 1.43 & 9.44 & 5 \\
\hline 88,998 & 33,678 & 11.9 & 29 & 12,035 & 5,086 & 0.0067 & 233 & 0.756 & 21 & 0.795 & 116 & 0.692 & 0.763 & 0.750 & 480.75 & 408.83 & 1.06 & 54.41 & 0.60 \\
\hline 82,572 & 35,138 & 14.3 & 29 & 14,062 & 6,755 & 0.0099 & 188 & 0.744 & 21 & 0.760 & 130 & 0.677 & 0.772 & 0.620 & 523.57 & 293.81 & 1.33 & 28.49 & $0.6 \mathrm{C}$ \\
\hline 137,626 & 48,971 & 7.8 & 27 & 12,883 & 5,313 & 0.0022 & 183 & 0.756 & 33 & 0.793 & 122 & 0.688 & 0.798 & 0.870 & 514.22 & 329.92 & 1.39 & 168.76 & 0.96 \\
\hline 92,356 & 35,507 & 11.3 & 31 & 12,958 & 5,983 & 0.0168 & 188 & 0.782 & 33 & 0.821 & 98 & 0.696 & 0.756 & 0.640 & 535.21 & 73.77| & 1.02 & 14.20 & .61 \\
\hline 56,696 & 20,847 & 8.7 & 26 & 12,264 & 5,260 & 0.0043 & 194 & 0.753 & 22 & 0.775 & 68 & 0.688 & 0.742 & 0.860 & 399.92 & 428.78 & 0.61 & 50.45 & 0.46 \\
\hline 114,109 & 45,626 & 11.8 & 33 & 13,002 & 4,582 & 0.0094 & 214 & 0.795 & 22 & 0.836 & 52 & 0.730 & 0.775 & .890 & 359.55 & 72.95 & 1.25 & 37.55 & 0.62 \\
\hline 95,604 & 38,227 & 7.5 & 30 & 13,380 & 5,722 & 0.0081 & 230 & 0.733 & 10 & 0.762 & 118 & 0.668 & 0.733 & 0.710 & 547.87 & 398.85 & 0.68 & 20.29 & 0.61 \\
\hline 95,604 & 38,227 & 7.5 & 30 & 13,380 & 5,722 & 0.0150 & 230 & 0.733 & 10 & 0.733 & 118 & 0.684 & 0.722 & 0.680 & 454.86 & 482.36 & 0.67 & 12.50 & 0.50 \\
\hline 11,812 & 831| & 9.1 & 15 & 12,653 & 5,719 & 0.0056 & 213 & 0.762 & 10 & 0.806 & 72 & 0.700 & 0.784 & 0.660 & 229.00 & 196.60 & 0.46 & 39.72 & 0.29 \\
\hline 28,280 & 10,746 & 14.6 & 20 & 14,088 & 5,452 & 0.0251 & 213 & 0.774 & 27 & 0.797 & 130 & 0.712 & 0.756 & 0.280 & 253.44 & 260.99 & 1.16 & 21.27 & 0.35 \\
\hline 40,027 & 16,514 & 8.4 & 20 & 13,944 & 6,953 & 0.0081 & 228 & 0.755 & 28 & 0.786 & 82 & 0.675 & 0.734 & 0.710 & 396.11 & 279.13 & 0.59 & 18.02 & 0.46 \\
\hline 38,341 & 16,266 & 7.4 & 18 & 14,562 & 6,232 & 0.0072 & 187 & 0.787 & 28 & 0.819 & 110 & 0.688 & 0.733 & 0.660 & 326.77 & 251.08 & $0.67 \mid$ & 26.33 & 0.44 \\
\hline 38,341 & 16,266 & 7.4 & 18 & 14,562 & 6,232 & 0.0100 & 187 & 0.747 & 28 & 0.773 & 110 & 0.670 & 0.728 & 0.510 & 414.33 & 223.41 & 0.59 & 13.94 & 0.40 \\
\hline 81,918 & 34,518 & 9.9 & 28 & 13,712 & 6,436 & 0.0237 & 222 & 0.755 & 26 & 0.779 & 94 & 0.685 & 0.774 & 0.570 & 543.35 & 336.20 & 0.84 & 8.32 & 0.53 \\
\hline 77,125 & 31,620 & 13.4 & 28 & 13,578 & 5,080 & 0.0089 & 213 & 0.747 & 31 & 0.755 & 98 & 0.693 & 0.715 & 0.780 & 327.43 & 430.68 & 1.34 & 48.75 & 0.56 \\
\hline 39,245 & 17,077 & 12.4 & 20 & 14,297 & 5,176 & 0.0286 & 190 & 0.760 & 22 & 0.781 & 88 & 0.678 & 0.737 & 0.360 & 373.81 & 247.52 & 0.92 & 10.49 & 0.4 \\
\hline 130,155 & 55,871 & 14.4 & 35 & 12,015 & 6,075 & 0.0333 & 228 & 0.731 & 10 & 0.776 & $51 \mid$ & 0.683 & 0.723 & 0.850 & 459.85 & 558.79 & 1.28 & $13.01 \mid$ & \\
\hline
\end{tabular}

Table A-1: Data Generated for Ramp Treatment, continued 


\begin{tabular}{|c|c|c|c|c|c|c|c|c|c|c|c|c|c|c|c|c|c|c|c|}
\hline \begin{tabular}{|l|}
$\stackrel{0}{E}$ \\
$\frac{1}{3}$ \\
$\frac{0}{0}$ \\
$\frac{\pi}{0}$ \\
$\stackrel{0}{\circ}$
\end{tabular} & \begin{tabular}{l|}
$\frac{0}{2}$ \\
$\frac{1}{3}$ \\
0 \\
0 \\
0 \\
0 \\
0
\end{tabular} & 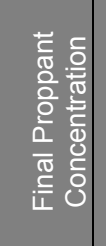 & 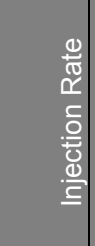 & 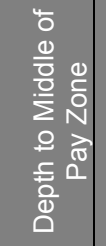 & \begin{tabular}{l|l}
$\frac{1}{5}$ & \\
0 & \\
0 & \\
0 & \\
0 & \\
ते & \\
0 & \\
$\frac{0}{2}$ &
\end{tabular} & 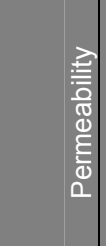 & 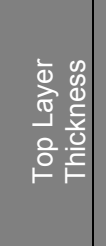 & 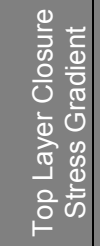 & 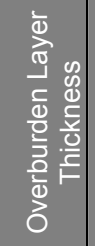 & 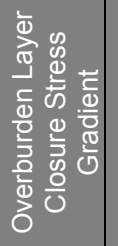 & 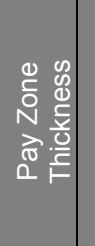 & 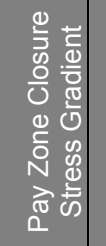 & 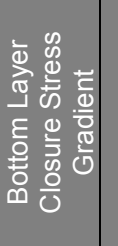 & 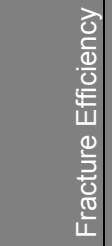 & 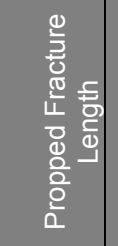 & 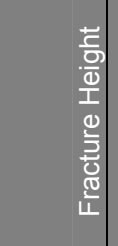 & 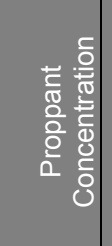 & 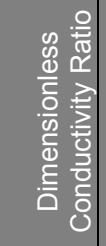 & $\frac{5}{5}$ \\
\hline input & input & input & input & input & input & input & input & input & input & input & input & input & input & output & output & output & output & output & output \\
\hline 140,412 & 49,844 & 8.2 & 36 & 13,509 & 6,844 & 0.0046 & 208 & 0.723 & 22 & 0.743 & 107 & 0.663 & 0.715 & 0.850 & 668.64 & 549.07 & 0.69 & 29.58 & 0.60 \\
\hline 58,027 & ,199 & 6.5 & 26 & 12,734 & 4,909 & 0.0510 & 203 & 0.775 & 11 & 0.786 & 114 & 0.689 & 0.745 & 0.330 & 383.79 & 327.74 & 0.62 & 5.60 & .42 \\
\hline 178,715 & 74,774 & 14.8 & 40 & 13,789 & 6,047 & 0.0168 & 232 & 0.768 & 30 & 0.782 & 95 & 0.701 & 0.770 & 0.700 & 713.89 & 501.54 & 1.31 & 12.22 & 6 \\
\hline 186,378 & 4,945 & 11.5 & 40 & 14,386 & 6,020 & 0.0058 & 202 & 0.760 & 18 & 0.760 & 83 & 0.697 & 0.752 & 0.810 & 770.47 & 559.87 & 0.95 & 18.84 & 66 \\
\hline 32,895 & .547 & 6.4 & 27 & 12,353 & 5,093 & 0.0087 & 203 & 0.758 & 27 & 0.776 & 73 & 0.708 & 0.765 & 0.720 & 318.00 & 18.99 & 0.41 & 20.81| & 3 \\
\hline 187,279 & 69,991 & 12.7 & 40 & 14,841 & 7,441 & 0.0091 & 237 & 0.808 & 11 & 0.838 & 77 & 0.740 & 0.812 & 0.820 & 637.85 & 469.54 & 1.56 & 19.63 & .87 \\
\hline 70,177 & 25,647 & 8.8 & 29 & 4,821 & 6,919 & 0.0049 & 212 & 0.806 & 25 & 0.841 & 76 & 0.733 & 0.804 & 0.790 & 552.13 & 382.38 & 0.61 & 16.25 & 5 \\
\hline 40,790 & 14,778 & 7.0 & 23 & 12,034 & 5,274 & 0.0079 & 212 & 0.775 & 19 & 0.800 & 86 & 0.707 & 0.758 & 0.750 & 244.17 & 361.68 & 0.51 & 27.15 & .42 \\
\hline 191,023 & ;,871 & 9.9 & 40 & 2,938 & 5,757 & 0.0234 & 217 & 0.785 & 28 & 0.818 & 70 & 0.706 & 0.768 & 0.760 & 751.06 & .42 & .85 & 6.12 & 6 \\
\hline 40,946 & 16,973 & 6.7 & 19 & 13,124 & 5,466 & 0.0107 & 198 & 0.735 & 33 & 0.775 & 107 & 0.680 & 0.741 & 0.600 & 369.90 & 311.88 & 0.49 & 16.48 & 0.43 \\
\hline 165,566 & 57,994 & 9.3 & 39 & 13,633 & 5,208 & 0.0057 & 232 & 0.762 & 12 & 0.779 & 120 & 0.676 & 0.740 & .750 & 772.75 & 35.14 & 0.98 & 38.36 & 0.72 \\
\hline 47,590 & 20,854 & 9.1 & 24 & 13,158 & 4,851 & 0.0044 & 182 & 0.753 & 32 & 0.790 & 86 & 0.688 & 0.747 & 0.770 & 391.05| & 338.59 & 0.61 & 40.15 & 0.47 \\
\hline 119,983 & 40,722 & 14.8 & 32 & 4,348 & 6,601 & 0.0021 & 230 & 0.768 & 20 & 0.798 & 105 & 0.699 & 0.771 & 0.850 & 680.05 & 426.03 & 1.23 & 82.27| & .71 \\
\hline 119,983 & 40,722 & 14.8 & 32 & 14,348 & 6,601 & 0.0099 & 230 & 0.768 & 20 & 0.798 & 105 & 0.712 & 0.771 & 0.720 & 606.15 & 480.18 & 1.22 & 20.61 & 0.63 \\
\hline 144,904 & 54,421 & 10.2 & 36 & 14,770 & 5,523 & 0.0408 & 187 & 0.724 & 32 & 0.775 & 61 & 0.692 & 0.727 & 0.650 & 546.12 & 01.29 & 0.92 & 4.07 & 0.5 \\
\hline 55,065 & 22,758 & 10.6 & 26 & 13,735 & 6,035 & 0.0129 & 227 & 0.756 & 34 & 0.788 & 73 & 0.688 & 0.781 & 0.710 & 383.72 & 277.06 & 1.03 & 23.73 & 0.60 \\
\hline 55,065 & 22,758 & 10.6 & 26 & 3,735 & 6,035 & 0.0080 & 227 & 0.756 & 34 & 0.788 & 73 & 0.670 & 0.741 & 0.740 & 468.36 & 310.48 & 0.76 & 22.20 & .53 \\
\hline 13,844 & 347 & 13.6 & 23 & 12,400 & 5,462 & 0.0046 & 220 & 0.750 & 30 & 0.750 & 52 & 0.668 & 0.750 & 0.780 & 249.38 & 219.99 & 0.64 & 75.41 & 0.37 \\
\hline 144,657 & 52,414 & 14.8 & 37 & 12,084 & 5,112 & 0.0059 & 216 & 0.746 & 15 & 0.781 & 132 & 0.692 & 0.732 & .800 & 523.62 & 4.49 & 1.43 & 76.36 & .67 \\
\hline 136,804 & 56,240 & 10.1 & 26 & 12,156 & 5,598 & 0.0372 & 183 & .768 & 26 & 0.789 & 124 & 0.672 & 0.732 & 0.530 & 603.52 & 405.79 & 1.09 & 9.14 & 0.65 \\
\hline 198,572 & 1,462 & 16.0 & 40 & 5,079 & 6,797 & 0.0066 & 173 & 0.771 & 31 & 0.801 & 97 & 0.705 & 0.785 & 0.780 & 735.07 & 404.59 & 1.89 & 31.13 & 0.88 \\
\hline,+ 485 & , ,322 & 13.4 & 30 & 4,313 & 7,102 & 0.0089 & 205 & 0.798 & 33 & 0.847 & 134 & 0.732 & 0.809 & 0.670 & 406.54 & 325.56 & 1.38 & 31.65 & 0.69 \\
\hline 101,421 & 37,762 & 11.0 & 30 & 12,035 & 5,018 & 0.0026 & 175 & 0.766 & 34 & 0.777 & 65 & 0.717 & 0.755 & 0.920 & 450.84 & 558.83 & 0.89 & 112.58 & 0.51 \\
\hline 17,663 & ,888 & 8.1 & 15 & 13,882 & 6,265 & 0.0079 & 207 & 0.768 & 28 & 0.787 & 95 & 0.689 & 0.768 & 0.560 & 256.58 & 184.25 & 0.61 & 28.84 & 0.39 \\
\hline 46,436 & 17,064 & 7.6 & 20 & 12,393 & 5,277 & 0.0066 & 178 & 0.741 & 16 & 0.772 & 126 & 0.672 & 0.775 & 0.630 & 426.56 & 268.72 & 0.67 & 39.55 & 0.44 \\
\hline, 004 & 5,384 & 10.9 & 26 & 13,873 & 5,036 & 0.0173 & 214 & 0.797 & 27 & 0.844 & 103 & 0.739 & 0.805 & 0.500 & 441.26 & 346.49 & 0.85 & 11.27 & 0.48 \\
\hline 191,880 & 67,358 & 9.2 & 40 & 14,637 & 6,270 & 0.0075 & 208 & 0.759 & 20 & 0.782 & 92 & 0.677 & 0.736 & 0.790 & 849.59 & 516.97 & 0.86 & 12.64 & 0.70 \\
\hline 98,219 & 39,436 & 11.6 & 32 & 13,653 & 6,100 & 0.0232 & 204 & 0.773 & 18 & 0.799 & 52 & 0.688 & 0.772 & 0.740 & 621.41 & 432.03 & 0.81 & 6.66 & 0.57 \\
\hline 24,951 & 8,837 & 12.7 & 17 & 13,471 & 6,127 & 0.0082 & 219 & 0.741 & 34 & 0.780 & 94 & 0.678 & 0.726 & 0.630 & 291.44 & 270.13 & 0.81 & 41.44| & 0.3 \\
\hline 34,720 & 13,518 & 7.1 & 23 & 13,132 & 5,382 & 0.0259 & 187 & 0.762 & 18 & 0.796 & 132 & 0.705 & 0.758 & 0.350 & 294.42 & 299.84 & 0.59 & 11.21 & 0.3 \\
\hline
\end{tabular}

Table A-1: Data Generated for Ramp Treatment, continued 


\begin{tabular}{|c|c|c|c|c|c|c|c|c|c|c|c|c|c|c|c|c|c|c|c|}
\hline 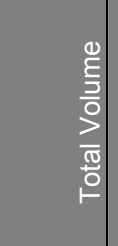 & 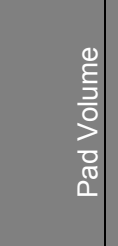 & 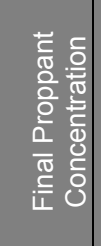 & 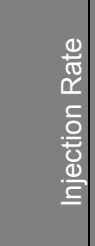 & 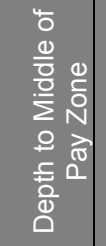 & 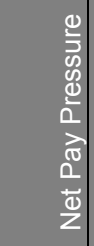 & 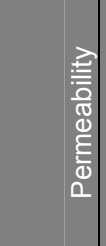 & 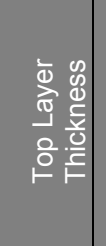 & 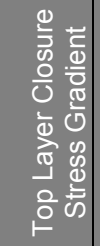 & 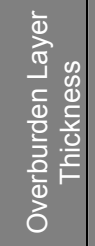 & 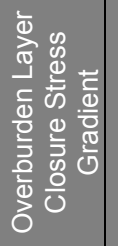 & 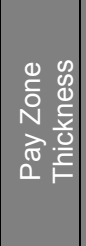 & 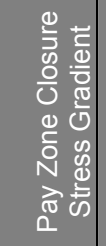 & 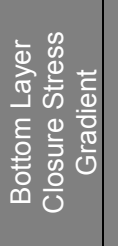 & 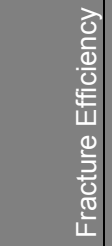 & 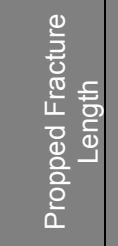 & 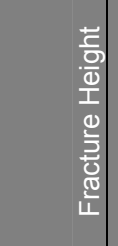 & 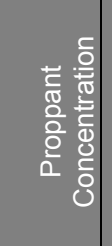 & 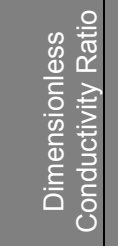 & $\frac{0}{5}$ \\
\hline input & input & input & input & input & input & input & input & input & input & input & input & input & input & output & output & output & output & output & output \\
\hline 83,189 & 31,047 & 8.1 & 29 & 14,640 & 5,982 & 0.0074 & 181 & 0.801 & 22 & 0.830 & 109 & 0.728 & 0.764 & 0.730 & 421.75 & 408.89 & 0.83 & 19.30 & 5 \\
\hline 183,380 & 9,841 & 11.5 & 40 & 13,200 & 5,763 & 0.0512 & 186 & 0.756 & 11 & 0.789 & 111 & 0.721 & 0.766 & 640 & 399.00 & 481.46 & 1.98 & 11.71 & 88 \\
\hline 168,000 & 62,160 & 7.5 & 40 & 13,200 & 5,763 & 0.0100 & 186 & 0.756 & 11 & 0.766 & 111 & 0.707 & 0.746 & 0.780 & 620.36 & 615.26 & 0.72 & 14.11 & 5 \\
\hline 77,968 & 34,255 & 6.3 & 27 & 13,439 & 6,309 & 0.0472 & 214 & 0.791 & 21 & 0.820 & 89 & 0.714 & 0.773 & 0.470 & 497.15 & 361.44 & 0.54 & 2.77 & .47 \\
\hline 98,955 & 35,313 & 11.0 & 29 & 12,253 & 5,715 & 0.0821 & 205 & 0.781 & 25 & 0.812 & 82 & 0.721 & 0.761 & .550 & 434.65 & 78.87| & 1.08 & 5.28 & 5 \\
\hline 89,309 & 34,200 & 14.3 & 28 & 13,966 & 6,894 & 0.0664 & 219 & 0.749 & 14 & 0.755 & 87 & 0.711 & 0.741 & 0.570 & 392.15 & 487.17 & 1.26 & 5.49 & 0.50 \\
\hline 40,500 & ,363 & 12.6 & 21 & 11,898 & 5,223 & 0.0548 & 197 & 0.746 & 17 & 0.784 & 79 & 0.679 & 0.729 & .480 & 302.50 & 20.82 & 0.98 & 11.50 & 4 \\
\hline 119,239 & 52,425 & 16.0 & 33 & 14,467 & 6,292 & 0.0056 & 218 & 0.733 & 23 & 0.756 & 75 & 0.674 & 0.727 & 0.840 & 570.30 & 492.73 & 1.14 & 33.25 & 0.59 \\
\hline 90,308 & 39,520 & 8.7 & 17 & 2,793 & 5,657 & 0.0275 & 177 & 0.785 & 19 & 0.812 & 86 & 0.692 & 0.789 & .550 & 607.86 & 22.66 & 0.75 & 6.62 & 5 \\
\hline 157,550 & 56,310 & 10.8 & 35 & 14,078 & 6,559 & 0.0968 & 229 & 0.768 & 29 & 0.789 & 84 & 0.687 & 0.738 & 0.580 & 485.56 & 559.79 & 1.30 & 3.60 & 0.73 \\
\hline 116,384 & 45,603 & 8.0 & 37 & 12,442 & 5,258 & 0.0159 & 177 & 0.803 & 31 & 0.829 & 95 & 0.720 & 0.810 & .650 & 666.96 & 393.32 & 0.74 & 0.15 & $.5 \varsigma$ \\
\hline 72,664 & 28,201 & 6.3 & 26 & 15,098 & 7,363 & 0.0506 & 207 & 0.755 & 28 & 0.771 & 62 & 0.683 & 0.757 & 0.540 & $567.07 \mid$ & 368.82 & 0.47 & 1.62 & 0.51 \\
\hline 135,642 &, 121 & 8.6 & 36 & 2,062 & 4,820 & 0.0364 & 231 & 0.746 & 25 & 0.777 & 112 & 0.689 & 0.739 & 590 & 546.06 & 8.90 & 0.83 & 8.12 & .55 \\
\hline 76,422 & 27,738 & 14.0 & 28 & 13,758 & 5,922 & 0.0365 & 206 & 0.738 & 19 & 0.785 & 69 & 0.671 & 0.733 & 0.620 & 463.91 & 400.08 & 1.13 & 9.48 & 0.55 \\
\hline 96,239 & 35,443 & 7.6 & 22 & 3,908 & 6,278 & 0.0893 & 193 & 0.727 & 12 & 0.744 & 71 & 0.686 & 0.722 & 0.570 & 464.20 & 525.71 & 0.65 & 2.10 & .43 \\
\hline 131,473 & 55,585 & 12.5 & 36 & 14,904 & 6,509 & 0.0669 & 189 & 0.731 & 29 & 0.768 & 119 & 0.655 & 0.744 & 0.260 & 667.26 & 290.42 & 1.54 & 4.70 & 0.59 \\
\hline 118,514 & 47,027 & 9.0 & 36 & 3,350 & 6,504 & 0.0269 & 175 & 0.779 & 28 & 0.822 & 78 & 0.699 & 0.803 & 0.680 & 523.61 & 326.24 & 1.25 & 11.53 & 0.80 \\
\hline 51,562 & 0,580 & 6.9 & 24 & 12,235 & $5,981 \mid$ & 0.0165 & 206 & 0.728 & 24 & 0.828 & 60 & 0.652 & 0.717 & 0.820 & 393.62 & 2.10 & 0.49 & 11.72 & 0.46 \\
\hline 64,918 & 28,243 & 13.9 & 30 & 4,995 & 6,055 & 0.0504 & 216 & 0.739 & 33 & 0.772 & 94 & 0.660 & 0.743 & .310 & 473.01 & 89.03 & 1.13 & 5.53 & 0.51 \\
\hline 58,824 & 20,776 & 12.2 & 24 & 2,005 & 5,626 & 0.0302 & 180 & .770 & 22 & 0.770 & 59 & 0.695 & 0.745 & 0.730 & 365.01 & 395.99 & 1.01 & 15.14 & 0.52 \\
\hline 145,432 & 62,032 & 9.6 & 38 & 5,044 & 6,451 & 0.0565 & 197 & 0.739 & 10 & 0.787 & 87 & 0.690 & 0.730 & 0.570 & 587.40 & 556.15 & 0.81 & 2.28 & 0.51 \\
\hline 198,939 & 2,945 & 14.2 & 40 & 12,570 & 5,842 & 0.0104 & 184 & 0.751 & 12 & 0.782 & 61 & 0.699 & 0.743 & 0.870 & 590.22 & 613.01 & 1.40 & 30.68 & 0.67 \\
\hline $\mid, 474$ &, 102 & 11.9 & 29 & 11,866 & 5,142 & 0.0675 & 194 & 0.774 & 32 & 0.818 & 103 & 0.729 & 0.788 & 0.430 & 365.54 & 412.07 & 1.09 & 8.35 & 0.46 \\
\hline 157,301 & 62,969 & 9.7 & 37 & 14,020 & 6,586 & 0.0085 & 208 & 0.784 & 14 & 0.826 & 91 & 0.716 & 0.802 & 0.810 & 497.40 & 364.60 & 1.66 & 25.39 & 0.98 \\
\hline 41,604 & 17,439 & 8.7 & 19 & 13,034 & 5,589 & 0.0353 & 171 & 0.762 & 28 & 0.779 & 110 & 0.685 & 0.739 & 0.330 & 350.90 & 278.60 & 0.71 & 9.55 & $0.3 s$ \\
\hline ,644 & 21,867 & 11.3 & 25 & 12,169 & 5,640 & 0.0066 & 205 & 0.752 & 12 & 0.772 & 110 & 0.664 & 0.713 & 0.750 & 367.20 & 321.28 & 1.00 & 61.86 & 0.53 \\
\hline 117,630 & 4,171 & 8.8 & 30 & 13,654 & 5,910 & 0.0088 & 223 & 0.748 & 22 & 0.781 & 89 & 0.682 & 0.755 & 0.741 & 666.70 & 449.20 & 0.72 & 15.76 & 0.58 \\
\hline 101,769 & 40,673 & 12.4 & 30 & 12,282 & 5,508 & 0.0159 & 189 & 0.735 & 27 & 0.776 & 115 & 0.674 & 0.739 & 0.702 & 529.70 & 454.90 & 1.02 & 21.44 & 0.57 \\
\hline 7,629 & 31,388 & 12.8 & 28 & 12,388 & 5,289 & 0.0011 & 216 & 0.762 & 13 & 0.786 & 121 & 0.704 & 0.734 & 0.905 & 373.60 & 500.50 & 1.20 & 39.90 & 0.5 \\
\hline 197,756 & 81,813 & 9.9 & 40 & 14,828 & 6,188 & 0.0043 & 205 & 0.798 & 13 & 0.835 & 74 & 0.734 & 0.810 & 0.900 & 452.17 & 373.04 & 2.23 & 76.67 & \\
\hline
\end{tabular}

Table A-1: Data Generated for Ramp Treatment, continued 


\begin{tabular}{|c|c|c|c|c|c|c|c|c|c|c|c|c|c|c|c|c|c|c|c|}
\hline \begin{tabular}{l|}
$\frac{0}{\Xi}$ \\
$\frac{3}{0}$ \\
$\frac{0}{0}$ \\
$\frac{\pi}{0}$ \\
$\frac{0}{\circ}$
\end{tabular} & \begin{tabular}{l|}
0 \\
है \\
3 \\
0 \\
2 \\
0 \\
0 \\
0
\end{tabular} & 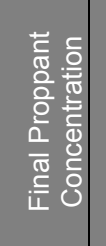 & 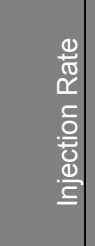 & 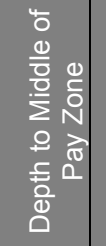 & 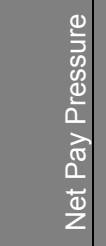 & 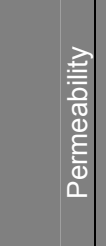 & 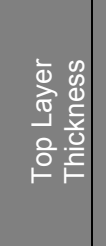 & 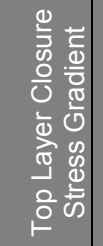 & 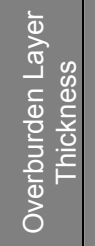 & 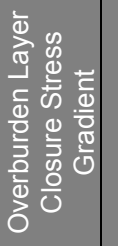 & 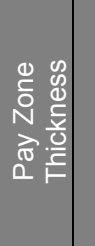 & 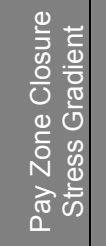 & 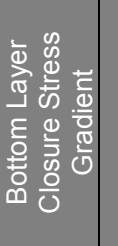 & 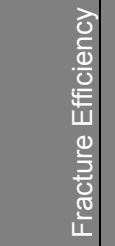 & 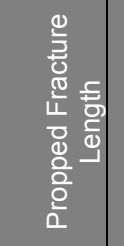 & 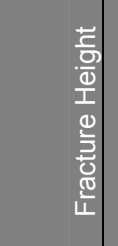 & 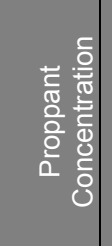 & 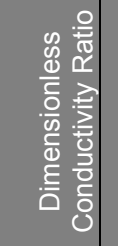 & \\
\hline input & input & input & input & input & input & input & input & input & input & input & input & input & input & output & output & output & output & output & output \\
\hline 107,238 & 42,922 & 8.5 & 21 & 5,068 & 2,069 & 0.0109 & 204 & 0.735 & 10 & 0.804 & 100 & 0.654 & 0.738 & 0.780 & 387.10 & 531.87 & 0.90 & 150.37 & 5 \\
\hline 107,238 & 42,922 & 8.5 & 21 & 5,068 & 2,069 & 0.0500 & 204 & 0.750 & 10 & 0.844 & 100 & 0.690 & 0.738 & 610 & 345.26 & 548.80 & 0.97 & 43.33 & \\
\hline 178,443 & 74,888 & 6.6 & 30 & 5,640 & 1,146 & 0.0078 & 212 & 0.764 & 20 & 0.786 & 85 & 0.650 & 0.720 & .920 & 495.35 & 605.55 & 0.79 & 106.92 & 6 \\
\hline 103,751 & 45,037 & 13.8 & 29 & 7,390 & 2,046 & 0.0411 & 234 & 0.751 & 32 & 0.777 & 51 & 0.710 & 0.730 & .790 & 380.22 & 482.08 & 1.39 & 30.44 & \\
\hline 98,898 & 42,715 & 6.0 & 18 & 6,953 & 2,553 & 0071 & 170 & 0.787 & 30 & 0.842 & 105 & 0.684 & 0.764 & 770 & 476.34 & 8.28 & 0.53 & 55.61 & \\
\hline 177,570 & 70,775 & 12.1 & 30 & 6,859 & 2,547 & 0.0012 & 179 & 0.756 & 16 & 0.794 & 130 & 0.662 & 0.780 & 0.920 & 452.90 & 464.69 & 1.94 & 141.42 & T \\
\hline 27,805 & 10,824 & 11.1 & 26 & 5,138 & 2,102 & 0.0111 & 201 & 0.789 & 12 & 0.810 & 119 & 0.696 & 0.752 & 710 & 200.97 & 6.82 & 0.95 & 57.58 & 42 \\
\hline 123,789 & 43,874 & 16.0 & 29 & 8,373 & 3,622 & 0.0268 & 219 & 0.741 & 33 & 0.750 & 83 & 0.706 & 0.730 & 0.790 & 373.16 & 579.16 & 1.78 & 50.63 & 64 \\
\hline 123,789 & 43,874 & 16.0 & 29 & 8,373 & 3,622 & 0.0023 & 219 & 0.790 & 33 & 0.833 & 83 & 0.710 & 0.760 & .920 & 417.34 & .33 & 1.69 & 40.03 & 70 \\
\hline 34,022 & 14,266 & 6.3 & 22 & 6,709 & 2,647 & 0.0365 & 173 & 0.749 & 20 & 0.836 & 64 & 0.671 & 0.787 & 0.680 & 250.09 & 311.40 & 0.58 & 26.86 & .41 \\
\hline 34,022 & 14,266 & 6.3 & 22 & 6,709 & 2,647 & 0.0365 & 173 & 0.749 & 20 & 0.808 & 64 & 0.683 & 0.732 & 0.720 & 229.05 & 344.98 & 0.57 & 25.87 & 37 \\
\hline 73,142 & 29,174 & 7.4 & 20 & 6,270 & 2,687 & 0.0241 & 195 & 0.759 & 11 & 0.802 & 115 & 0.695 & 0.758 & 0.680 & 331.79 & 471.47 & 0.73 & 45.18 & .46 \\
\hline 73,142 &, 174 & 7.4 & 20 & 6,270 & 2,687 & 0.0241 & 195 & 0.762 & 11 & 0.782 & 115 & 0.660 & 0.732 & 680 & 48.98 & 35.64 & 0.75 & 47.32 & \\
\hline 31,723 & 13,395 & 7.3 & 17 & 5,544 & 2,019 & 0.0454 & 192 & 0.772 & 24 & 0.844 & 112 & 0.674 & 0.738 & 0.480 & 218.56 & 322.37 & 0.64 & 41.42 & .35 \\
\hline 135,988 & 50,101 & 7.2 & 29 & 6,321 & 2,173 & 0.0069 & 195 & 0.749 & 28 & 0.799 & 92 & 0.670 & 0.745 & 0.870 & 466.64 & 581.09 & 0.82 & 25.97 & 5 \\
\hline 135,988 & 50,101 & 7.2 & 29 & 6,321 & 2,173 & 0.0069 & 195 & 0.767 & 28 & 0.819 & 92 & 0.704 & 0.734 & 0.880 & 413.81 & 607.47 & 0.89 & 133.96 & 0.57 \\
\hline 164,619 & 59,908 & 9.0 & 30 & 7,795 & 3,621 & 0.0340 & 220 & 0.761 & 25 & 0.796 & 69 & 0.704 & 0.746 & 0.820 & 481.01 & 651.64 & 1.01 & 18.98| & 58 \\
\hline 34,401 & 13,459 & 15.7 & 17 & 7,126 & 3,420 & 0.0010 & 197 & 0.767 & 24 & 0.776 & 126 & 0.658 & 0.752 & 0.890 & 256.85 & 63 & 1.13 & 140.43 & 0.49 \\
\hline 28,261 & 10,653 & 6.6 & 18 & 6,888 & 2,724 & 0.0354 & 216 & 0.776 & 27 & 0.793 & 134 & 0.699 & 0.781 & .410 & 222.50 & 321.56 & 0.57 & 32.75 & .32 \\
\hline 185,510 & 75,695 & 13.1 & 30 & 7,487 & 1,978 & 0.0059 & 227 & 0.748 & 26 & 0.754 & 85 & 0.707 & 0.730 & 900 & 397.24 & 1.14 & 1.54 & 198.11 & 0.73 \\
\hline 120,923 & 45,143 & 7.8 & 28 & 7,040 & 2,385 & 0.0140 & 214 & 0.770 & 12 & 0.834 & 126 & 0.709 & 0.790 & 0.740 & 384.06 & 501.60 & 1.08 & 73.55 & .66 \\
\hline 22,042 & 171 & 15.2 & 15 & 7,311 & 2,288 & 0.0072 & 177 & 0.740 & 20 & 0.764 & 52 & 0.703 & 0.739 & 0.840 & 191.59 & 321.69 & 1.02 & 225.31 & 0.39 \\
\hline 65,387 & 27,331 & 11.5 & 20 & 8,324 & 3,814 & 0.0105 & 229 & 0.736 & 18 & 0.757 & 114 & 0.650 & 0.776 & 0.710 & 363.17 & 334.87 & 1.15 & 90.72 & 0.60 \\
\hline 175,697 & 69,827 & 7.1 & 27 & 5,123 & 2,080 & 0.0057 & 180 & 0.760 & 22 & 0.780 & 60 & 0.671 & 0.740 & 0.890 & 489.18 & 646.39 & 0.83 & 184.31 & 0.61 \\
\hline 24,376 & 10,491 & 8.4 & 20 & 7,295 & 2,927 & 0.0022 & 228 & 0.757 & 11 & 0.836 & 116 & 0.687 & 0.736 & 0.820 & 216.91 & 285.24 & 0.63 & 342.59 & 0.38 \\
\hline 77,837 & 31,127 & 7.6 & 22 & 5,937 & 1,270 & 0.0100 & 213 & 0.732 & 12 & 0.821 & 80 & 0.668 & 0.771 & 0.870 & 288.05 & 453.30 & 0.94 & 169.27 & .59 \\
\hline 98,653 & 42,370 & 13.1 & 26 & 6,459 & 1,484 & 0.0018 & 194 & 0.770 & 28 & 0.764 & 96 & 0.675 & 0.722 & 0.930 & 328.75 & 476.07 & 1.48 & 269.96 & .65 \\
\hline 179,024 & 63,574 & 15.9 & 40 & 6,369 & 2,598 & 0.0066 & 184 & 0.779 & 19 & 0.817 & 103 & 0.701 & 0.767 & 0.900 & 471.61 & 610.67 & 1.93 & 279.08 & .79 \\
\hline, 287 & 57,933 & 10.6 & 28 & 6,027 & 2,501 & 0.0647 & 227 & 0.726 & 32 & 0.741 & 95 & 0.684 & 0.719 & 0.760 & 378.25 & 606.05 & 1.24 & 26.82 & \\
\hline 140,287 & 57,933 & 10.6 & 28 & 6,027 & $2,501 \mid$ & 0.0065 & 227 & 0.800 & 32 & 0.840 & 95 & 0.720 & 0.820 & 0.900 & 373.09 & 555.49 & 1.37| & 249.80 & \\
\hline
\end{tabular}

Table A-1: Data Generated for Ramp Treatment, continued 


\begin{tabular}{|c|c|c|c|c|c|c|c|c|c|c|c|c|c|c|c|c|c|c|c|}
\hline \begin{tabular}{l|}
$\frac{0}{\Xi}$ \\
$\frac{3}{0}$ \\
$\frac{0}{0}$ \\
$\frac{\pi}{0}$ \\
$\frac{0}{\circ}$
\end{tabular} & \begin{tabular}{l|}
0 \\
है \\
3 \\
0 \\
2 \\
0 \\
0 \\
0
\end{tabular} & 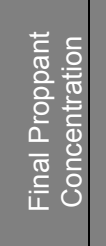 & 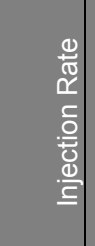 & 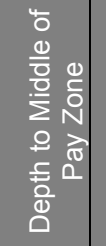 & \begin{tabular}{l|l}
$\frac{0}{3}$ \\
0 \\
0 \\
0 \\
0 \\
$\frac{1}{2}$ \\
$\frac{\pi}{\pi}$ \\
$\frac{1}{0}$ \\
$\frac{0}{2}$ \\
$z$
\end{tabular} & 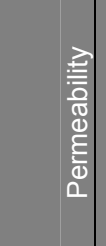 & 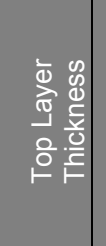 & 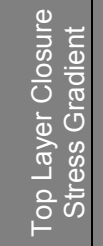 & 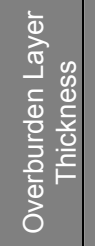 & 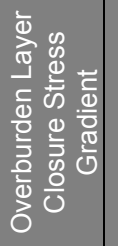 & 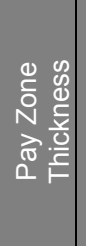 & 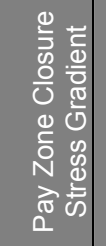 & 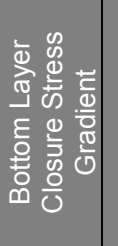 & 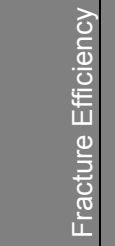 & 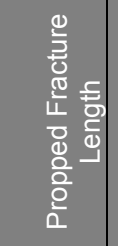 & 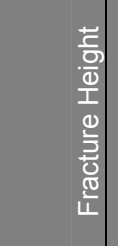 & 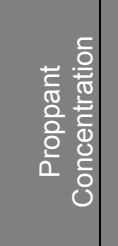 & 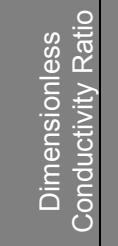 & \\
\hline input & input & input & input & input & input & input & input & input & input & input & input & input & input & output & output & output & output & output & output \\
\hline 14,030 & 6,004 & 7.9 & 28 & 6,644 & 2,094 & 0.0232 & 198 & 0.766 & 21 & 0.779 & 103 & 0.672 & 0.751 & 0.510 & 159.68 & 219.97 & 0.62 & 62.98 & 26 \\
\hline 199,989 & 87,746 & 10.1 & 35 & 5,991 & 2,392 & 0.0085 & 184 & 0.775 & 32 & 0.792 & 105 & 0.700 & 0.780 & 900 & 437.76 & 642.79 & 1.32 & 160.30 & \\
\hline 68,869 & 29,072 & 8.8 & 21 & 6,563 & 2,947 & 0.0389 & 174 & 0.764 & 34 & 0.772 & 103 & 0.704 & 0.727 & 610 & 286.84 & 51.50 & 0.91 & 15.54 & 46 \\
\hline 29,985 & 11,078 & 11.5 & 19 & 5,705 & 2,374 & 0.0049 & 189 & 0.756 & 29 & 0.780 & 72 & 0.665 & 0.767 & .890 & 244.12 & 333.68 & 0.86 & 395.75 & \\
\hline 87,206 & 34,011 & 14.6 & 25 & 7,443 & 3,191 & 0437 & 179 & 0.753 & 23 & 0.833 & 128 & 0.690 & 0.775 & 460 & 372.54 & 0.79 & 1.55 & 20.92 & \\
\hline 153,909 & 58,282 & 15.2 & 40 & 8,205 & 2,559 & 0.0084 & 210 & 0.753 & 18 & 0.802 & 101 & 0.666 & 0.760 & .840 & 457.76 & 510.94 & 1.89 & 149.60 & 82 \\
\hline 80,226 & 28,440 & 8.2 & 25 & 5,568 & 2,500 & 0.0020 & 174 & 0.763 & 27 & 0.838 & 98 & 0.720 & 0.789 & .940 & 298.84 & 97.77 & 0.97 & 267.26 & 6 \\
\hline 74,578 & 27,827 & 10.6 & 27 & 7,204 & 3,087 & 0.0891 & 209 & 0.751 & 32 & 0.769 & 122 & 0.676 & 0.757 & 0.500 & 326.82 & 428.41 & 1.16 & 17.91 & \\
\hline 74,522 & , 104 & 14.1 & 30 & 7,886 & 3,935 & 0.0053 & 216 & 0.743 & 29 & 0.754 & 77 & 0.690 & 0.745 & 910 & 327.31 & 7.28 & 1.26 & 202.60 & \\
\hline 166,000 & 71,348 & 10.4 & 35 & 6,319 & 2,679 & 0.0123 & 170 & 0.785 & 34 & 0.820 & 85 & 0.708 & 0.749 & 0.890 & 434.64 & 596.37 & 1.24 & 90.37 & .65 \\
\hline 77,599 & 33,481 & 9.1 & 24 & 6,985 & 2,750 & 0.0062 & 237 & 0.737 & 16 & 0.751 & 87 & 0.670 & 0.748 & .880 & 322.41 & 60.58 & 0.90 & 162.14 & \\
\hline 182,194 & 68,142 & 9.1 & 35 & 5,519 & 2,836 & 0.0045 & 226 & 0.743 & 17 & 0.766 & 82 & 0.675 & 0.718 & 0.950 & 460.08 & 675.69 & 1.12 & 305.42 & .64 \\
\hline 153,438 & 63,730 & 7.6 & 35 & 7,738 & 3,663 & 0.0257 & 237 & 0.781 & 27 & 0.830 & 70 & 0.706 & 0.788 & .850 & 417.79 & 9.10 & 1.10 & 29.19 & 75 \\
\hline 61,724 & 23,475 & 15.6 & 19 & 5,866 & 2,255 & 0.0295 & 213 & 0.747 & 28 & 0.792 & 57 & 0.657 & 0.744 & 0.810 & 309.06 & 415.81 & 1.41 & 91.02 & .56 \\
\hline 167,771 & 69,779 & 7.5 & 36 & 7,695 & 3,613 & 0.0800 & 207 & 0.782 & 25 & 0.820 & 113 & 0.698 & 0.772 & 0.610 & 537.13 & 585.02 & 0.81 & 6.41 & .58 \\
\hline 29,324 & 10,279 & 7.6 & 18 & 7,273 & 2,663 & 0.0067 & 214 & 0.756 & 34 & 0.795 & 101 & 0.696 & 0.749 & 0.420 & 223.08 & 330.67 & 0.67 & 19.12 & 0.34 \\
\hline 43,280 & 16,305 & 6.7 & 27 & 5,175 & 1,971 & 0.0079 & 237 & 0.748 & 28 & 0.802 & 82 & 0.678 & 0.732 & 0.820 & 242.40 & 387.01 & 0.68 & 210.52 & .44 \\
\hline 57,150 & 21,812 & 6.8 & 28 & 5,693 & 2,813 & 0.0342 & 212 & 0.747 & 34 & 0.814 & 76 & 0.683 & 0.727 & 0.910 & 272.20 & 422.22 & 0.74 & 41.57 & 0.51 \\
\hline 46,020 & 16,425 & 6.3 & 19 & 6,872 & 1,985 & 0.0033 & 180 & 0.747 & 31 & 0.784 & 71 & 0.674 & 0.738 & 0.940 & 297.21 & 4.10 & 0.56 & 216.12 & .46 \\
\hline 54,853 & 21,224 & 7.1 & 27 & 6,672 & 2,820 & 0.0035 & 187 & 0.739 & 34 & 0.769 & 105 & 0.668 & 0.736 & 0.940 & 281.93 & 438.08 & 0.67 & 250.21 & 0.48 \\
\hline 58,855 & 21,050 & 6.7 & 18 & 5,726 & 2,000 & 0.0084 & 222 & 0.751 & 34 & 0.768 & 63 & 0.671 & 0.735 & 0.930 & 302.76 & 443.56 & 0.67 & |43.45 & .50 \\
\hline 64,287 & 27,282 & 7.4 & 21 & 7,799 & 3,194 & 0.0027 & 212 & 0.747 & 10 & 0.803 & 99 & 0.688 & 0.734 & 0.870 & 306.99 & 426.46 & 0.71 & 230.88 & 0.50 \\
\hline 57,791 & 24,137 & 6.4 & 24 & 7,716 & 3,000 & 0.0251 & 216 & 0.733 & 31 & 0.763 & 79 & 0.667 & 0.745 & 0.820 & 319.82 & 401.06 & 0.60 & 22.38 & 0.48 \\
\hline 131,540 & 57,607 & 10.1 & 23 & 9,919 & 4,566 & 0.0099 & 213 & 0.734 & 26 & 0.788 & 130 & 0.687 & 0.725 & 0.900 & 447.92 & 562.16 & 0.97 & 51.62 & 0.57 \\
\hline 141,795 & 50,306 & 7.5 & 33 & 11,587 & 5,719 & 0.0043 & 190 & 0.778 & 21 & 0.823 & $131 \mid$ & 0.718 & 0.767 & 0.910 & 609.47 & 579.83 & 0.67 & 39.61 & 0.59 \\
\hline 184,445 & 73,527 & 10.7 & 40 & 10,952 & 5,163 & 0.0396 & 196 & 0.774 & 28 & 0.797 & 51 & 0.706 & 0.740 & .870 & 440.98 & 637.59 & 1.37 & 3.67 & 0.65 \\
\hline 121,311 & 48,249 & 12.2 & 30 & 9,857 & 4,552 & 0.0082 & 202 & 0.783 & 30 & 0.833 & 130 & 0.725 & 0.784 & 0.770 & 481.60 & 519.74 & 1.13 & 70.76 & 0.59 \\
\hline ,348 & 38,652 & 7.2 & 30 & 10,291 & 4,682 & 0.0275 & 191 & 0.782 & 23 & 0.818 & 76 & 0.718 & 0.748 & 0.800 & 352.22 & 516.31 & 0.83 & 18.12 & .54 \\
\hline 172,898 & 74,331 & 12.8 & 22 & 10,642 & 4,480 & 0.0085 & 186 & 0.782 & 12 & 0.815 & 82 & 0.707 & 0.759 & 0.860 & 560.90 & 559.88 & 1.26 & 48.92 & .67 \\
\hline 90,772 & 36,800 & 12.2 & 30 & 8,438 & 4,018 & 0.0073 & 203 & 0.746 & 17 & 0.765 & 94 & 0.679 & 0.733 & 0.870 & 381.94| & 474.36 & 1.15 & 147.30 & \\
\hline
\end{tabular}

Table A-1: Data Generated for Ramp Treatment, continued 


\begin{tabular}{|c|c|c|c|c|c|c|c|c|c|c|c|c|c|c|c|c|c|c|c|}
\hline \begin{tabular}{|l|}
$\stackrel{0}{E}$ \\
$\frac{1}{3}$ \\
$\frac{0}{0}$ \\
$\frac{\pi}{0}$ \\
$\stackrel{0}{\circ}$
\end{tabular} & \begin{tabular}{l|}
$\frac{0}{2}$ \\
$\frac{3}{0}$ \\
0 \\
$\frac{0}{0}$ \\
$\frac{0}{0}$
\end{tabular} & 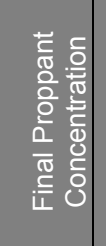 & 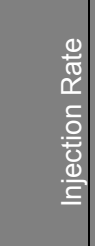 & 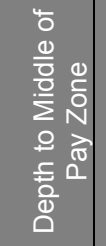 & 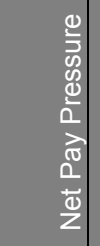 & 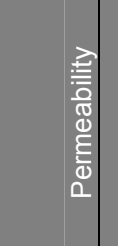 & 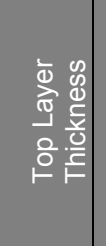 & 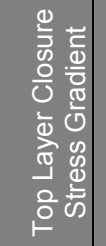 & 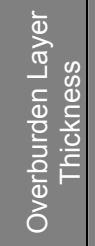 & 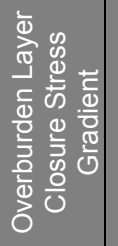 & 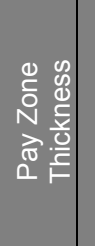 & 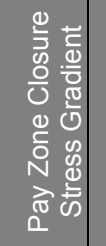 & 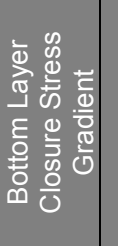 & 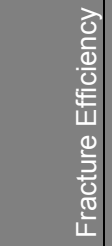 & 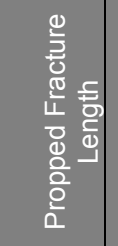 & 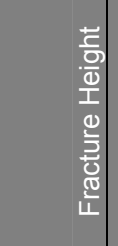 & 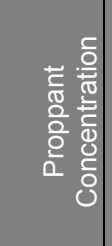 & 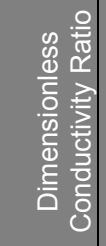 & $\frac{5}{5}$ \\
\hline input & input & input & input & input & input & input & input & input & input & input & input & input & input & output & output & output & output & output & output \\
\hline 195,831 & 73,060 & 6.3 & 19 & 9,994 & 4,830 & 0.0038 & 212 & 0.781 & 23 & 0.846 & 93 & 0.724 & 0.795 & 0.920 & 469.13 & 515.75 & 1.14 & 146.23 & 0.96 \\
\hline 197,729 & 80,180 & 12.7 & 40 & 9,575 & 4,296 & 0.0062 & 230 & 0.759 & 29 & 0.788 & 100 & 0.702 & 0.742 & 0.880 & 569.24 & 627.00 & 1.32 & 99.11 & 68 \\
\hline 124,702 & 45,628 & 10.1 & 30 & 11,026 & 4,066 & 0.0096 & 189 & 0.768 & 15 & 0.791 & 89 & 0.695 & 0.740 & 0.800 & 516.96 & 515.58 & 0.98 & 38.64 & 6 \\
\hline 41,446 & 18,027 & 13.0 & 20 & 11,356 & 4,605 & 0.0155 & 215 & 0.768 & 23 & 0.791 & 107 & 0.691 & 0.754 & 0.550 & 315.61 & 294.33 & 0.96 & 35.59 & .45 \\
\hline 78,756 & 33,740 & 9.5 & 27 & 1,039 & 5,338 & 0.0010 & 234 & 0.741 & 29 & 0.762 & 124 & 0.683 & 0.729 & 0.750 & 391.10 & 23.95 & 0.58 & 42.29 & כ \\
\hline 98,179 & 42,854 & 7.7 & 30 & 10,141 & 3,994 & 0.0554 & 212 & 0.734 & 27 & 0.766 & 67 & 0.675 & 0.735 & 0.710 & 367.62 & 427.53 & 0.93 & 12.20 & .61 \\
\hline 19,159 & 171 & 7.7 & 15 & 1,779 & 5,558 & 0.0538 & 196 & 0.762 & 12 & 0.799 & 133 & 0.719 & 0.755 & 190 & 182.44 & 32.73 & ).61| & 13.01 & 2 \\
\hline 164,007 & 72,005 & 14.9 & 39 & 9,112 & 4,030 & 0.0314 & 186 & 0.761 & 22 & 0.789 & 106 & 0.672 & 0.733 & 0.700 & 556.38 & 530.76 & 1.42 & 25.53 & .67 \\
\hline 90,027 & 36,172 & 11.7 & 30 & 9,772 & 4,467 & 0.0031 & 200 & 0.724 & 31 & 0.735 & 81 & 0.668 & 0.728 & .920 & 399.68 & .31 & 1.00 & 213.23 & 5 \\
\hline 90,027 & 36,172 & 11.7 & 30 & 9,772 & 4,467 & 0.1000 & 200 & 0.724 & 31 & 0.735 & 81 & 0.682 & 0.728 & 0.630 & 350.60 & 494.10 & 1.16 & 10.21 & .51 \\
\hline 117,249 & 43,798 & 6.8 & 34 & 8,584 & 3,637 & 0.0022 & 213 & 0.730 & 22 & 0.745 & 120 & 0.695 & 0.727 & 0.930 & 399.52 & 97.35 & 0.74 & 268.19 & 5 \\
\hline 129,951 & 48,674 & 7.2 & 35 & 9,539 & 3,981 & 0.0043 & 174 & 0.764 & 16 & 0.785 & 56 & 0.684 & 0.743 & 0.930 & 510.99 & 560.23 & 0.71 & 84.64| & 0.56 \\
\hline 80,075 & 29,321 & 7.8 & 27 & 1,715 & 4,429 & 0.0017 & 214 & 0.779 & 27 & 0.852 & 56 & 0.672 & 0.757 & .930 & 398.40 & 469.21 & 0.73 & 182.05 & \\
\hline 174,549 & 74,764 & 6.5 & 40 & 9,112 & 2,858 & 0.0929 & 229 & 0.784 & 33 & 0.822 & 55 & 0.715 & 0.763 & 0.800 & 460.30 & 665.15 & 0.75 & 4.78 & 0.55 \\
\hline 182,575 & 79,810 & 15.8 & 40 & 1,499 & 5,424 & 0.0093 & 215 & 0.739 & 21 & 0.750 & 128 & 0.685 & 0.739 & 0.800 & 533.72 & 47.63 & 1.67 & 44.88 & 0.73 \\
\hline 114,673 & 43,526 & 8.8 & 32 & 10,877 & 4,836 & 0.0040 & 175 & 0.770 & 26 & 0.800 & 124 & 0.680 & 0.759 & 0.800 & 621.04 & 418.53 & 0.81 & 67.90 & 0.65 \\
\hline 6,691 & 29,149 & 6.2 & 28 & 9,206 & 3,870 & 0.0028 & 230 & 0.769 & 23 & 0.780 & 115 & 0.672 & 0.741 & 0.860 & 426.01 & 403.79 & $0.61 \mid$ & 144.55 & 0.57 \\
\hline 103,715 & 36,373 & 7.3 & 20 & 10,147 & 3,458 & 0.0075 & 229 & 0.780 & 19 & 0.858 & 81 & 0.695 & 0.777 & 0.810 & 573.13 & 493.57 & 0.60 & 36.05 & 0.55 \\
\hline 21,352 & 575 & 9.7 & 19 & 10,207 & 4,260 & 0.0665 & 179 & 0.726 & 22 & 0.740 & 91 & 0.673 & 0.722 & 350 & 200.18 & 272.38 & 0.75 & 16.99 & 2 \\
\hline 16,230 & 230 & 7.2 & 16 & 286 & 4,339 & 0.0075 & 174 & 0.791 & 27 & 0.839 & 131 & 0.726 & 0.750 & 0.620 & 186.98 & 268.76 & 0.49 & 85.74 & 0.28 \\
\hline 167,242 & 65,536 & 6.4 & 38 & 0,946 & 4,045 & 0.0029 & 226 & 0.748 & 20 & 0.777 & 90 & 0.663 & 0.745 & 0.890 & 657.22 & 523.32 & 0.67 & 75.72 & 0.70 \\
\hline 0,775 & 24,338 & 15.8 & 28 & 10,128 & 4,856 & 0.0088 & 204 & 0.790 & 34 & 0.841 & 68 & 0.728 & 0.810 & 0.840 & 347.92 & 378.09 & 1.33 & 95.76 & 0.57 \\
\hline 121,578 & 51,226 & 13.8 & 34 & 12,006 & 5,988 & 0.0063 & 230 & 0.761 & 27 & 0.806 & 68 & 0.711 & 0.760 & 0.890 & 499.03 & 548.43 & 1.10 & 50.24 & 0.55 \\
\hline 144,812 & 56,971 & 7.3 & 37 & 15,148 & 5,822 & 0.0654 & 219 & 0.759 & 24 & 0.777 & 131 & 0.685 & 0.725 & 0.310 & 579.29 & 406.14 & 0.94 & 2.80 & 0.52 \\
\hline 87,209 & 32,744 & 16.0 & 30 & 12,676 & 5,495 & 0.0042 & 229 & 0.750 & 25 & 0.765 & 78 & 0.678 & 0.738 & 0.860 & 459.15 & 437.23 & 1.31| & 97.12 & 0.64 \\
\hline 77,117 & 28,579 & 7.7 & 26 & 14,340 & 6,560 & 0.0062 & 215 & 0.791 & 18 & 0.826 & 84 & 0.727 & 0.778 & 0.780 & 495.62 & 418.42 & 0.61 & 15.90 & 0.52 \\
\hline 63,845 & 24,662 & 8.3 & 24 & 12,421 & 5,319 & 0.0075 & 188 & 0.741 & 23 & 0.762 & 130 & 0.703 & 0.733 & 0.720 & 358.38 & 443.90 & 0.69 & 37.20 & 0.43 \\
\hline 92,403 & 36,355 & 9.6 & 31 & 14,244 & 6,971 & 0.0106 & 189 & 0.767 & 32 & 0.786 & 134 & 0.729 & 0.757 & 0.700 & 439.84| & 496.57 & $0.81 \mid$ & 15.11 & 0.48 \\
\hline 67,870 & 27,288 & 10.8 & 26 & |924 & 5,046 & 0.0130 & 213 & 0.756 & 20 & 0.771 & 131 & 0.680 & 0.780 & 0.560 & 467.30 & 302.44 & 1.01 & 30.46 & 0.5 \\
\hline 17,917 & 7,144 & 12.6 & 16 & 14,591 & 5,612 & 0.0045 & $197 \mid$ & 0.758 & 13 & 0.770 & 122 & 0.701 & 0.732 & 0.590 & 218.77 & 250.94 & 0.75 & 57.95 & \\
\hline
\end{tabular}

Table A-1: Data Generated for Ramp Treatment, continued 


\begin{tabular}{|c|c|c|c|c|c|c|c|c|c|c|c|c|c|c|c|c|c|c|c|}
\hline 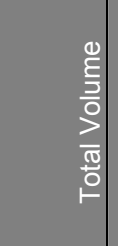 & 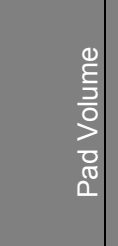 & 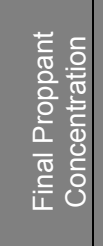 & 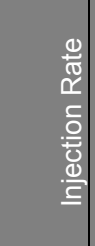 & 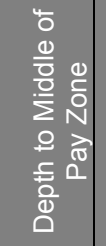 & \begin{tabular}{l|l}
$\frac{1}{5}$ & \\
0 & \\
0 & \\
0 & \\
0 & \\
ते & \\
0 & \\
$\frac{0}{2}$ &
\end{tabular} & 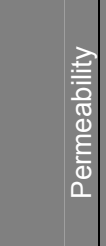 & 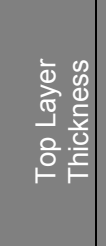 & 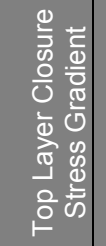 & 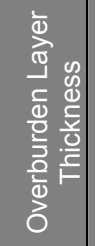 & 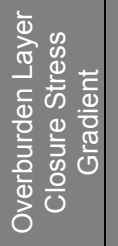 & 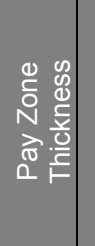 & 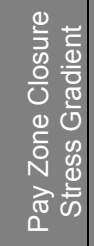 & 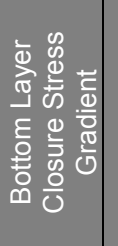 & 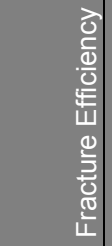 & 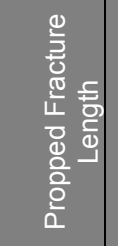 & 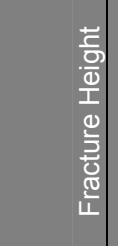 & 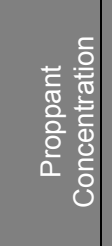 & 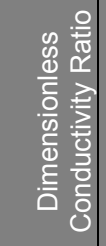 & $\frac{5}{5}$ \\
\hline input & input & input & input & input & input & input & input & input & input & input & input & input & input & output & output & output & output & output & output \\
\hline 198,609 & 70,236 & 8.3 & 40 & 14,206 & 5,218 & 0.0281 & 173 & 0.790 & 17 & 0.832 & 93 & 0.724 & 0.791 & 0.570 & 880.35 & 563.46 & 0.73 & 3.25 & .61 \\
\hline 99,885 & 38,093 & 8.4 & 32 & 14,938 & 6,521 & 0.0173 & 182 & 0.767 & 35 & 0.795 & 117 & 0.687 & 0.751 & .540 & 622.92 & 372.91 & 0.75 & 6.66 & \\
\hline 188,074 & 81,062 & 10.5 & 23 & 15,170 & 6,375 & 0.0108 & 206 & 0.739 & 29 & 0.778 & 78 & 0.705 & 0.749 & 0.850 & 446.14 & 482.94 & 1.69 & 25.87 & 8 \\
\hline 188,074 & 81,062 & 10.5 & 23 & 15,170 & 6,375 & 0.0108 & 206 & 0.739 & 29 & 0.778 & 78 & 0.670 & 0.730 & 0.770 & 864.14 & 556.66 & 0.76 & 6.85 & 60 \\
\hline 105,104 & 41,496 & 9.9 & 34 & 3,801 & 6,120 & 0.0212 & 199 & 0.780 & 13 & 0.824 & 63 & 0.711 & 0.774 & .740 & 597.00 & 63.42 & .72 & 5.76 & \\
\hline 140,522 & 60,992 & 8.2 & 34 & 13,864 & 6,625 & 0.0059 & 227 & 0.733 & 27 & 0.757 & 73 & 0.670 & 0.718 & 0.870 & 617.28 & 535.88 & 0.67 & 18.48 & .55 \\
\hline 14,895 & 502 & 10.9 & 16 & 13,109 & 6,232 & 0.0046 & 194 & 0.745 & 10 & 0.780 & 56 & 0.676 & 0.742 & 0.790 & 241.97 & 29.25 & 0.52 & 48.54 & 3 \\
\hline 101,381 & 42,331 & 12.5 & 30 & 12,317 & 4,706 & 0.0069 & 238 & 0.770 & 18 & 0.811 & 70 & 0.709 & 0.765 & 0.840 & 496.35 & 492.55 & 0.95 & 37.72 & 0.54 \\
\hline 37,526 & 13,886 & 8.5 & 28 &,+ 131 & 6,254 & 0.0369 & 176 & 0.784 & 16 & 0.811 & 97 & 0.708 & 0.763 & .350 & 356.17 & 269.51 & .70 & 6.15 & 3 \\
\hline 77,539 & 29,568 & 15.1 & 27 & 13,493 & 6,095 & 0.0184 & 232 & 0.792 & 16 & 0.795 & 97 & 0.712 & 0.767 & 0.720 & 473.89 & 355.01 & 1.30 & 35.30 & .62 \\
\hline 16,463 & 241 & 7.1 & 17 & 3,804 & 6,221 & 0.0149 & 220 & 0.754 & 34 & 0.788 & 76 & 0.684 & 0.725 & 0.620 & 191.97| & 50.07 & 0.50 & 16.66 & 3 \\
\hline 26,459 & 9,866 & 9.4 & 19 & 12,471 & 4,772 & 0.0039 & 216 & 0.746 & 23 & 0.772 & 117 & 0.655 & 0.718 & 0.670 & 315.66 & 235.42 & 0.68 & 82.13 & 0.42 \\
\hline 164,467 & 70,312 & 7.9 & 38 & 3,423 & 5,370 & 0.0078 & 187 & 0.750 & 24 & 0.785 & 82 & 0.687 & 0.719 & 0.880 & 430.75 & 99.02 & 0.99 & 29.32 & 0.62 \\
\hline 162,036 & 67,463 & 11.3 & 28 & 11,915 & 4,743 & 0.0159 & 222 & 0.746 & 32 & 0.782 & 100 & 0.680 & 0.726 & 0.750 & 574.96 & 554.58 & 1.07 & 19.27 & 0.63 \\
\hline 33,060 & 12,905 & 8.0 & 18 & 3,145 & 7,203 & 0.0059 & 177 & 0.762 & 34 & 0.766 & 130 & 0.687 & 0.754 & 0.600 & 365.16 & 240.42 & 0.62 & 35.31 & .4 \\
\hline 144,168 & 59,473 & 12.8 & 36 & 14,106 & 5,706 & 0.0147 & 217 & 0.757 & 33 & 0.782 & 92 & 0.686 & 0.768 & 0.660 & 714.25 & 424.42 & 1.12 & 12.16 & 0.66 \\
\hline 117,352 & 47,589 & 8.0 & 32 & 3,436 & 6,096 & 0.0947 & 196 & 0.757 & 21 & 0.757 & 57 & 0.684 & 0.729 & 0.710 & 388.10 & 534.55 & 0.92 & 3.03 & .5 \\
\hline 160,104 & 66,044 & 13.0 & 34 & 12,279 & 4,780 & 0.0083 & 238 & 0.736 & 31 & 0.755 & 105 & 0.668 & 0.719 & 0.780 & 614.55 & 536.80 & 1.16 & 35.80 & 0.65 \\
\hline 84,687 & 30,246 & 6.1 & 20 & 2,188 & 5,933 & 0.0769 & 188 & 0.807 & 24 & 0.842 & 83 & 0.709 & 0.763 & .860 & 355.21 & 460.17 & 0.72 & 38.30 & .5 \\
\hline 111,874 & 44,767 & 7.6 & 33 & 1,999 & 5,076 & 0.0143 & 180 & 0.753 & 15 & 0.763 & 87 & 0.661 & 0.770 & 0.710 & 618.55 & 54.07 & 0.80 & 16.47 & 0.67 \\
\hline 17,186 & 148 & 9.9 & 15 & 12,882 & 6,553 & 0.0026 & 187 & 0.746 & 28 & 0.847 & 111 & 0.665 & 0.720 & 0.750 & 266.13 & 216.67 & 0.60 & 108.94 & 0.38 \\
\hline 95,395 & 40,767 & 7.4 & 34 & 12,283 & 6,128 & 0.0082 & 179 & 0.730 & 10 & 0.766 & 132 & 0.695 & 0.727 & 0.800 & 386.99 & 516.02 & 0.70 & 29.91| & 0.47 \\
\hline 183,786 & 71,066 & 12.8 & 40 & 11,980 & 4,343 & 0.0092 & 233 & 0.737 & 27 & 0.769 & 110 & 0.690 & 0.725 & 0.790 & 610.51 & 615.24 & 1.13 & 34.50 & 0.64 \\
\hline 83,740 & 32,774 & 10.5 & 28 & 12,832 & 5,043 & 0.0075 & 213 & 0.800 & 11 & 0.835 & 99 & 0.736 & 0.806 & 0.722 & 497.10 & 411.50 & 0.85 & 26.76 & 0.55 \\
\hline 187,802 & 73,241 & 7.9 & 40 & 14,368 & 6,209 & 0.0051 & 220 & 0.744 & 10 & 0.786 & 109 & 0.683 & 0.739 & 0.803 & 826.00 & 575.60 & 0.65 & 15.04 & 0.62 \\
\hline 143,130 & 59,149 & 7.0 & 30 & 7,190 & 3,008 & 0.0026 & 223 & 0.756 & 18 & 0.762 & 57 & 0.688 & 0.737 & 0.960 & 449.48 & 605.50 & 0.76 & 192.71 & 0.55 \\
\hline 190,339 & 69,496 & 10.5 & 40 & ,715 & 3,286 & 0.0173 & 208 & 0.742 & 18 & 0.757 & 95 & 0.655 & 0.729 & 0.840 & 544.49 & 636.84 & 1.19 & 42.09 & 0.69 \\
\hline 90,516 & 36,317 & 6.4 & 29 & 6,263 & 3,234 & 0.0466 & 225 & 0.800 & 27 & 0.837 & 102 & 0.720 & 0.783 & 0.730 & 361.16 & 494.77| & 0.69 & 17.84| & 0.50 \\
\hline 115,178 & 50,068 & 10.7 & 17 & 6,455 & 2,434 & 0.0485 & 220 & 0.775 & 21 & 0.806 & 126 & 0.705 & 0.736 & 0.650 & 345.30 & 517.67 & 1.26 & 32.69 & 0.5 \\
\hline 23,486 & 8,936 & 6.9 & 17 & 8,153 & 4,056 & 0.0392 & 204 & 0.747 & 33 & 0.763 & 108 & 0.700 & 0.755 & 0.510 & 207.13 & 306.03 & 0.54 & 19.95 & \\
\hline
\end{tabular}

Table A-1: Data Generated for Ramp Treatment, continued 


\begin{tabular}{|c|c|c|c|c|c|c|c|c|c|c|c|c|c|c|c|c|c|c|c|}
\hline 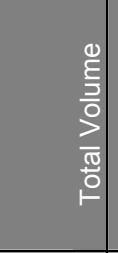 & \begin{tabular}{l|}
0 \\
$\frac{0}{3}$ \\
0 \\
2 \\
0 \\
$\frac{0}{0}$ \\
0
\end{tabular} & 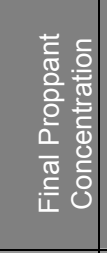 & \begin{tabular}{l|}
$\frac{0}{\pi 0}$ \\
$\frac{1}{\alpha}$ \\
$\frac{c}{0}$ \\
$\frac{0}{0}$ \\
$\frac{0}{c}$ \\
$\frac{1}{c}$
\end{tabular} & 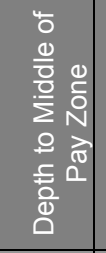 & 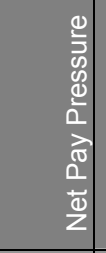 & 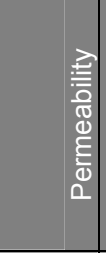 & 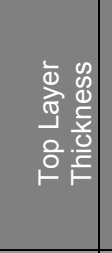 & 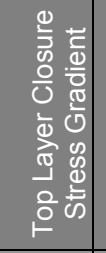 & 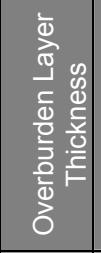 & 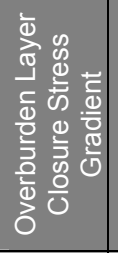 & 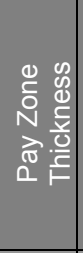 & 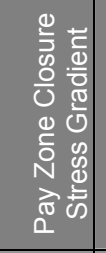 & 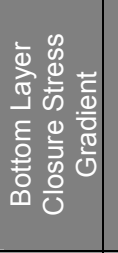 & 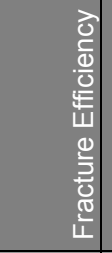 & 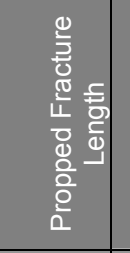 & 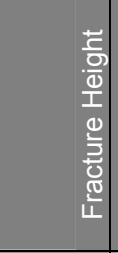 & 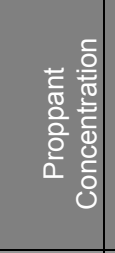 & 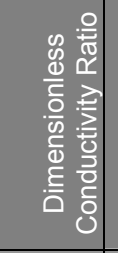 & $\div$ \\
\hline input & input & input & input & input & input & input & input & input & input & input & input & input & input & output & output & output & output & output & output \\
\hline 72,178 & 31,305 & 8.5 & 26 & 8,385 & 4,000 & 0.0076 & 197 & 0.781 & 25 & 0.817 & 70 & 0.717 & 0.753 & 0.900 & 290.79 & 462.69 & 0.88 & 83.47 & 051 \\
\hline 28,271 & 10,074 & 11.9 & 18 & 8,379 & 4,043 & 0.0196 & 200 & 0.738 & 11 & 0.760 & 55 & 0.678 & 0.742 & 0.800 & 235.33 & 345.12 & 0.85 & 51.25 & .42 \\
\hline 145,050 & 53,324 & 6.8 & 35 & 7,998 & 3,420 & 0.0381 & 171 & 0.766 & 15 & 0.801 & 114 & 0.689 & 0.772 & 0.680 & 482.69 & 528.88 & 0.86 & 15.49 & \\
\hline 121,654 & 49,707 & 14.5 & 18 & 6,051 & 2,967 & 0.0385 & 178 & 0.778 & 28 & 0.813 & 61 & 0.721 & 0.763 & 0.850 & 368.94 & 547.70 & 1.58 & 50.91 & .63 \\
\hline 193,889 & 68,500 & 6.4 & 40 & 6,712 & 2,660 & 0.0122 & 184 & 0.743 & 23 & 0.773 & 124 & 0.706 & 0.735 & 0.860 & 448.44 & 713.16 & 0.89 & 64.65 & .64 \\
\hline 151,222 & 56,039 & 11.1 & 35 & 7,077 & 2,243 & 0.0096 & 237 & 0.765 & 29 & 0.786 & 91 & 0.699 & 0.764 & 0.890 & 417.14 & 596.33 & 1.37 & 117.28 & 0.70 \\
\hline 101,406 & 40,871 & 15.3 & 15 & 7,580 & 2,095 & 0.0162 & 216 & 0.770 & 11 & 0.818 & 75 & 0.687 & 0.743 & 0.840 & 372.97 & 486.59 & 1.55 & 77.59 & \\
\hline 80,812 & 33,819 & 15.6 & 28 & 6,376 & 3,000 & 0.0314 & 227 & 0.742 & 28 & 0.758 & 115 & 0.707 & 0.760 & 0.690 & 286.35 & 467.30 & 1.62 & 50.81 & 0.60 \\
\hline 158,087 & 58,326 & 9.1 & 27 & 5,998 & 2,344 & 0.0073 & 202 & 0.724 & 16 & 0.845 & 89 & 0.692 & 0.757 & 0.890 & 406.56 & 575.87 & 1.28 & 222.66 & .74 \\
\hline 95,629 & 33,626 & 8.8 & 30 & 6,354 & 2,185 & 0.0135 & 206 & 0.761 & 28 & 0.785 & 107 & 0.684 & 0.756 & 0.800 & 385.65 & 508.90 & 0.94 & 88.60 & 0.57 \\
\hline 74,410 & 28,380 & 10.3 & 27 & 6,926 & 2,797 & 0.0185 & 185 & 0.769 & 30 & 0.800 & 60 & 0.716 & 0.755 & 0.910 & 319.29 & 477.69 & 1.02 & 120.59 & .52 \\
\hline 87,192 & 33,397 & 6.6 & 26 & 7,045 & 2,548 & 0.0463 & 230 & 0.780 & 34 & 0.825 & 98 & 0.696 & 0.751 & 0.650 & 366.92 & 478.97 & 0.72 & 16.15 & 0.50 \\
\hline 53,715 & 19,751 & 7.8 & 15 & 7,473 & 3,562 & 0.0417 & 219 & 0.730 & 25 & 0.777 & 84 & 0.676 & 0.733 & 0.600 & 294.36 & 418.01 & 0.74 & 24.61 & .45 \\
\hline 46,781 & 17,624 & 7.5 & 27 & 7,065 & 2,528 & 0.0313 & 238 & 0.739 & 13 & 0.800 & 79 & 0.671 & 0.738 & 0.620 & 268.06 & 391.29 & 0.72 & 36.33 & 0.43 \\
\hline 63,974 & 25,440 & 7.0 & 25 & 6,546 & 2,523 & 0.0082 & 218 & 0.737 & 17 & 0.773 & 64 & 0.670 & 0.755 & 0.940 & 269.04 & 456.78 & 0.77 & 147.43 & 0.55 \\
\hline 60,476 & 21,267 & 6.4 & 15 & 5,894 & 1,886 & 0.0302 & 223 & 0.757 & 32 & 0.787 & 96 & 0.670 & 0.743 & 0.770 & 326.93 & 433.68 & 0.63 & 38.16 & 0.50 \\
\hline 52,204 & 19,080 & 7.7 & 25 & 6,095 & 2,807 & 0.0463 & 184 & 0.736 & 15 & 0.786 & 90 & 0.680 & 0.739 & 0.810 & 230.67 & 422.39 & 0.74 & 30.12 & 0.47 \\
\hline 134,577 & 49,431 & 11.5 & 34 & 11,244 & 5,331 & 0.0181 & 173 & 0.755 & 18 & 0.778 & 128 & 0.684 & 0.736 & 0.680 & 554.10 & 506.55 & 1.11 & 23.84 & .64 \\
\hline 85,455 & 30,269 & 9.2 & 28 & 10,422 & 4,626 & 0.0099 & 220 & 0.775 & 20 & 0.800 & 116 & 0.710 & 0.788 & 0.730 & 451.31 & 434.39 & 0.86 & 45.67 & 0.56 \\
\hline 41,190 & 14,417 & 13.8 & 23 & 9,610 & 3,943 & 0.0103 & 219 & 0.741 & 12 & 0.773 & 79 & 0.689 & 0.748 & 0.790 & 272.87 & 375.54 & 1.12 & 116.12 & .49 \\
\hline 120,297 & 52,477 & 8.5 & 33 & 10,886 & 4,741 & 0.0031 & 232 & 0.749 & 19 & 0.781 & 94 & 0.671 & 0.760 & 0.880 & 510.31 & 420.34 & 0.91 & 115.05 & 0.69 \\
\hline 22,188 & 9,662 & 7.6 & 16 & 8,645 & 3,553 & 0.0072 & 232 & 0.738 & 20 & 0.750 & 58 & 0.671 & 0.720 & 0.830 & 221.19 & 307.68 & 0.43 & 80.42 & .33 \\
\hline 74,119 & 30,297 & 12.7 & 28 & 11,409 & 5,000 & 0.0066 & 233 & 0.770 & 16 & 0.798 & 107 & 0.695 & 0.744 & 0.780 & 393.80 & 384.97 & 1.15 & 72.44 & 0.58 \\
\hline 179,486 & 64,235 & 15.5 & 40 & 9,204 & 3,827 & 0.0393 & 187 & 0.761 & 20 & 0.784 & 132 & 0.663 & 0.727 & 0.620 & 557.78 & 530.68 & 1.83 & 32.15 & .80 \\
\hline 161,567 & 59,776 & 10.1 & 39 & 10,908 & 4,737 & 0.0048 & 189 & 0.793 & 35 & 0.828 & 99 & 0.718 & 0.767 & 0.870 & 590.96 & 561.26 & 1.01 & 62.63 & 0.65 \\
\hline 94,245 & 39,055 & 7.5 & 31 & 11,655 & 3,922 & 0.0100 & 178 & 0.756 & 27 & 0.763 & 52 & 0.702 & 0.766 & 0.890 & 349.74 & 556.24 & 0.73 & 32.35 & 0.49 \\
\hline 51,250 & 17,978 & 11.0 & 24 & 8,469 & 4,045 & 0.0167 & 192 & 0.721 & 30 & 0.725 & 61 & 0.660 & 0.718 & 0.840 & 328.67 & 422.57 & 0.85 & 65.15 & 0.45 \\
\hline 49,614 & 21,218 & 14.5 & 24 & 8,747 & 3,761 & 0.0500 & 209 & 0.769 & 26 & 0.821 & 138 & 0.715 & 0.766 & 0.440 & 253.83 & 366.05 & 1.36 & 39.45 & 0.46 \\
\hline 65,926 & 24,260 & 14.3 & 25 & 10,119 & 3,288 & 0.0156 & 184 & 0.732 & 21 & 0.755 & 111 & 0.694 & 0.782 & 0.710 & 289.52 & 431.48 & 1.47 & 89.96 & 0.55 \\
\hline 13,172 & 4,760 & 8.2 & 16 & 8,554 & 3,878 & 0.0093 & 211 & 0.771 & 14 & 0.799 & 56 & 0.703 & 0.741 & 0.790 & 181.06 & 265.52 & 0.49 & 82.84 & 0.20 \\
\hline
\end{tabular}

Table A-1: Data Generated for Ramp Treatment, continued 


\begin{tabular}{|c|c|c|c|c|c|c|c|c|c|c|c|c|c|c|c|c|c|c|c|}
\hline \begin{tabular}{|l|}
$\frac{0}{2}$ \\
$\frac{2}{0}$ \\
$\frac{2}{2}$ \\
$\frac{\pi}{0}$ \\
\end{tabular} & \begin{tabular}{l|l}
0 \\
$\xi$ \\
3 \\
0 \\
0 \\
0 \\
0 \\
0
\end{tabular} & 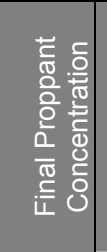 & 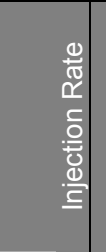 & 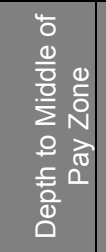 & 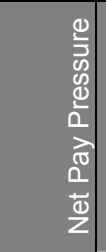 & 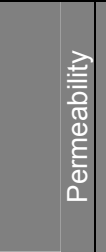 & 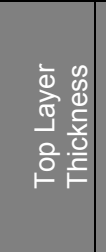 & 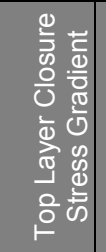 & 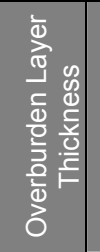 & 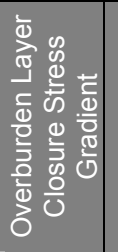 & 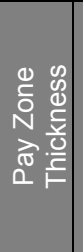 & 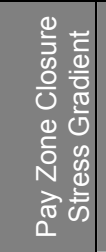 & 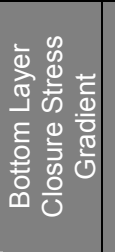 & 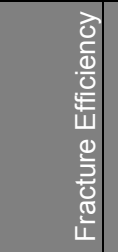 & 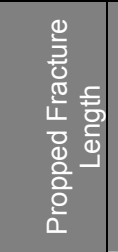 & 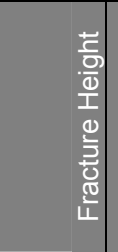 & 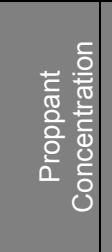 & 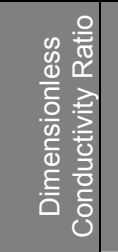 & \\
\hline input & input & input & input & input & input & input & input & input & input & input & input & input & input & output & output & output & output & output & output \\
\hline 26,443 & 10,717 & 7.1 & 18 & 8,619 & 3,514 & 0.0057 & 203 & 0.737 & 26 & 0.764 & 111 & 0.709 & 0.795 & 0.740 & 247.10 & 313.09 & 0.51 & 120.27 & 037 \\
\hline 38,905 & 13,772 & 8.0 & 22 & 10,922 & 4,620 & 0.0022 & 213 & 0.756 & 30 & 0.780 & 122 & 0.693 & 0.748 & 0.820 & 323.04 & 358.58 & 0.60 & 164.49 & 0.4 \\
\hline 108,731 & 43,810 & 16.0 & 29 & 10,665 & 4,610 & 0.0096 & 198 & 0.772 & 13 & 0.807 & 89 & 0.700 & 0.768 & 0.790 & 491.33 & 466.79 & 1.36 & 62.22 & \\
\hline 13,484 & 5,576 & 8.2 & 16 & 11,652 & 4,566 & 0.0014 & 209 & 0.752 & 29 & 0.794 & 105 & 0.683 & 0.732 & 0.780 & 223.27 & 215.94 & 0.46 & 189.27 & 32 \\
\hline 188,001 & 69,195 & 6.4 & 40 & 9,448 & 4,284 & 0.0055 & 189 & 0.795 & 28 & 0.828 & 51 & 0.730 & 0.762 & 0.930 & 448.89 & 696.80 & 0.87 & 82.48 & \\
\hline 66,648 & 27,583 & 7.7 & 26 & 11,407 & 5,218 & 0.0033 & 224 & 0.769 & 25 & 0.794 & 91 & 0.696 & 0.739 & 0.880 & 318.81 & 433.18 & 0.74 & 101.44 & .52 \\
\hline 72,811 & 26,280 & 12.7 & 25 & 11,666 & 5,503 & 0.0200 & 200 & 0.741 & 29 & 0.750 & 128 & 0.661 & 0.717 & 0.590 & 430.10 & 364.13 & 1.18 & 30.71 & \\
\hline 63,904 & 23,454 & 9.0 & 33 & 13,581 & 4,800 & 0.0092 & 214 & 0.744 & 27 & 0.784 & 65 & 0.691 & 0.750 & 0.780 & 441.30 & 434.80 & 0.63 & 14.54 & 0.45 \\
\hline 131,847 & 54,885 & 15.2 & 33 & 12,633 & 5,383 & 0.0134 & 218 & 0.759 & 31 & 0.786 & 121 & 0.677 & 0.742 & 0.636 & 617.00 & 406.10 & 1.41 & 27.09 & 70 \\
\hline 163,288 & 62,450 & 6.9 & 36 & 12,389 & 6,155 & 0.0279 & 180 & 0.764 & 17 & 0.764 & 105 & 0.716 & 0.762 & 0.712 & 521.80 & 604.20 & 0.77 & 7.90 & 0.58 \\
\hline 125,329 & 45,593 & 14.3 & 33 & 14,288 & 5,619 & 0.0077 & 172 & 0.728 & 24 & 0.749 & 90 & 0.680 & 0.726 & 0.786 & 562.10 & 516.90 & 1.19 & 30.57 & 60 \\
\hline 72,764 & 28,687 & 8.8 & 27 & 13,231 & 5,878 & 0.0245 & 236 & 0.759 & 14 & 0.798 & 51 & 0.730 & 0.767 & 0.814 & 296.40 & 363.10 & 1.21 & 19.52 & 0.72 \\
\hline 85,069 & 34,904 & 10.9 & 26 & 13,737 & 6,087 & 0.0075 & 238 & 0.746 & 25 & 0.800 & 65 & 0.670 & 0.726 & 0.830 & 465.00 & 415.30 & 0.91 & 20.27 & 65 \\
\hline 42,478 & 15,271 & 8.5 & 24 & 12,243 & 4,611 & 0.0173 & 187 & 0.741 & 10 & 0.767 & 113 & 0.687 & 0.732 & 0.562 & 312.90 & 354.20 & 0.70 & 22.71 & .41 \\
\hline 60,306 & 26,086 & 14.5 & 29 & 14,526 & 5,297 & 0.0096 & 219 & 0.759 & 23 & 0.794 & 86 & 0.671 & 0.733 & 0.653 & 455.80 & 284.80 & 1.16 & 24.66 & .57 \\
\hline 92,967 & 36,445 & 7.0 & 26 & 12,578 & 5,876 & 0.0085 & 233 & 0.735 & 30 & 0.761 & 124 & 0.674 & 0.710 & 0.740 & 467.00 & 447.20 & 0.66 & 24.19 & 0.51 \\
\hline 97,897 & 42,612 & 12.0 & 31 & 14,708 & 5,366 & 0.0122 & 233 & 0.758 & 30 & 0.785 & 89 & 0.692 & 0.749 & 0.660 & 551.40 & 400.90 & 0.94 & 12.18 & 0.58 \\
\hline 59,244 & 25,005 & 15.0 & 25 & 12,385 & 5,664 & 0.0098 & 183 & 0.808 & 14 & 0.845 & 73 & 0.734 & 0.792 & 0.770 & 376.99 & 354.24 & 1.16 & 37.77 & .55 \\
\hline 16,862 & 6,769 & 10.2 & 15 & 12,168 & 5,152 & 0.0075 & 226 & 0.746 & 18 & 0.780 & 97 & 0.685 & 0.728 & 0.610 & 222.18 & 249.57 & 0.59 & 49.79 & 0.32 \\
\hline 47,262 & 17,139 & 11.5 & 21 & 14,195 & 6,662 & 0.0195 & 191 & 0.764 & 25 & 0.785 & 66 & 0.691 & 0.741 & 0.710 & 325.15 & 357.92 & 0.94 & 14.21 & 0.49 \\
\hline 158,009 & 58,547 & 6.1 & 36 & 13,559 & 5,917 & 0.0437 & 210 & 0.747 & 14 & 0.762 & 108 & 0.702 & 0.741 & 0.590 & 597.05 & 612.62 & 0.59 & 3.06 & 0.51 \\
\hline 58,789 & 22,477 & 12.8 & 28 & 12,071 & 5,567 & 0.0094 & 175 & 0.761 & 12 & 0.789 & 77 & 0.692 & 0.756 & 0.790 & 399.85 & 390.38 & 0.94 & 38.47 & 0.52 \\
\hline 132,726 & 53,099 & 10.4 & 35 & 13,772 & 5,370 & 0.0231 & 191 & 0.742 & 15 & 0.783 & 111 & 0.693 & 0.757 & 0.610 & 559.54 & 533.81 & 0.90 & 8.71 & 0.54 \\
\hline 72,636 & 28,066 & 9.8 & 28 & 13,146 & 6,259 & 0.0034 & 236 & 0.793 & 12 & 0.827 & 64 & 0.725 & 0.802 & 0.880 & 453.95 & 417.29 & 0.76 & 52.39 & 0.56 \\
\hline 78,995 & 30,523 & 6.4 & 23 & 15,116 & 6,122 & 0.0289 & 221 & 0.759 & 34 & 0.775 & 131 & 0.687 & 0.743 & 0.330 & 533.14 & 302.30 & 0.68 & 4.72 & 0.44 \\
\hline 22,895 & 9,260 & 7.7 & 17 & 12,821 & 4,469 & 0.0058 & 199 & 0.781 & 21 & 0.811 & 121 & 0.711 & 0.757 & 0.560 & 259.65 & 251.29 & 0.54 & 40.51 & 0.36 \\
\hline 67,775 & 26,579 & 12.1 & 27 & 14,417 & 5,777 & 0.0376 & 229 & 0.742 & 26 & 0.766 & 67 & 0.701 & 0.734 & 0.650 & 366.20 & 436.69 & 0.98 & 6.84 & 0.45 \\
\hline 111,207 & 44,736 & 13.4 & 31 & 15,034 & 6,549 & 0.0198 & 230 & 0.768 & 12 & 0.812 & 78 & 0.710 & 0.779 & 0.710 & 446.59 & 382.34 & 1.61 & 14.99 & 0.77 \\
\hline 125,772 & 55,148 & 9.6 & 35 & 12,195 & 5,620 & 0.0179 & 220 & 0.806 & 27 & 0.843 & 85 & 0.724 & 0.791 & 0.720 & 592.28 & 465.92 & 0.81 & 9.96 & 0.60 \\
\hline 77,992 & 28,393 & 8.3 & 25 & 8,393 & 4,189 & 0.0082 & 192 & 0.727 & 24 & 0.845 & 125 & 0.698 & 0.781 & 0.770 & 437.00 & 401.91 & 0.80 & 59.02 & 0.59 \\
\hline
\end{tabular}

Table A-1: Data Generated for Ramp Treatment, continued 


\begin{tabular}{|c|c|c|c|c|c|c|c|c|c|c|c|c|c|c|c|c|c|c|c|c|c|c|}
\hline 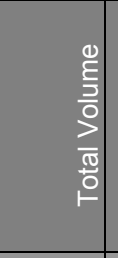 & 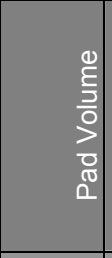 & 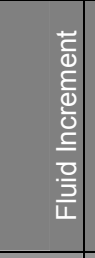 & 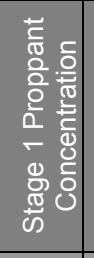 & 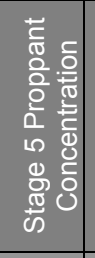 & 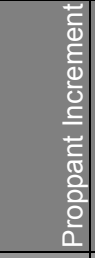 & 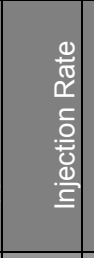 & 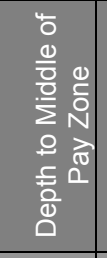 & \begin{tabular}{l|l}
0 & \\
$\overline{3}$ & \\
0 & \\
0 & \\
0 & \\
0 & \\
0 & \\
0 & \\
& \\
2 &
\end{tabular} & 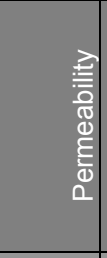 & 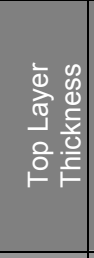 & 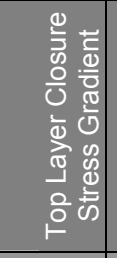 & 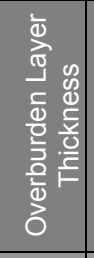 & 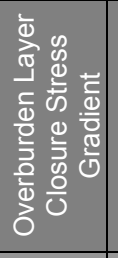 & 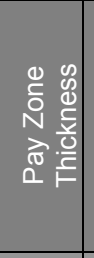 & 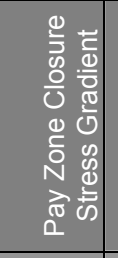 & 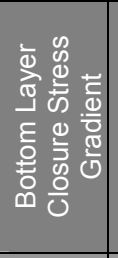 & 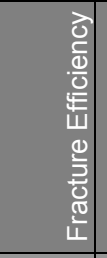 & 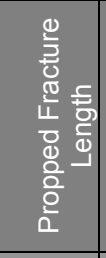 & 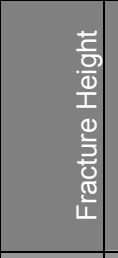 & 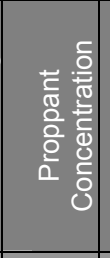 & 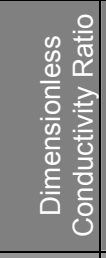 & \\
\hline input & input & input & input & input & input & input & input & input & input & input & input & input & input & input & input & input & output & output & output & output & output & output \\
\hline 88,692 & 34,851 & 3,365 & 2.0 & 9.2 & 1.8 & 27 & 8,064 & 3,386 & 0.0077 & 219 & 0.770 & 16 & 0.791 & 129 & 0.673 & 0.745 & 0.79 & 395.54 & \begin{tabular}{|l|}
454.47 \\
\end{tabular} & \begin{tabular}{|l|}
1.24 \\
\end{tabular} & 177.47 & \\
\hline 191,270 & 76,844 & 7,152 & 1.0 & 7.0 & 1.5 & 40 & 6,420 & 2,666 & 0.0087 & 217 & 0.782 & 13 & 0.823 & 98 & 0.668 & 0.753 & 0.89 & 510.46 & 628.12 & 1.08 & 155.59 & .74 \\
\hline 167,331 & 9,140 & 6,137 & 1.5 & 7.5 & 1.5 & 36 & 6,149 & 2,736 & 0.0448 & 224 & 0.738 & 25 & 0.771 & 105 & 0.65 & 0.721 & 0.92 & 430.25 & 600.22 & 1.24 & $\mid 13.36$ & \\
\hline 101,823 & 42,426 & 3,712 & 2.0 & 9.6 & 1.9 & 30 & 6,846 & 3,004 & 0.0160 & 223 & 0.753 & 10 & 0.785 & 71 & 0.677 & 0.744 & 0.88 & 321.18 & 514.49 & 1.55 & 175.57 & 0.68 \\
\hline 48,253 & 18,700 & 1,847 & 2.0 & 9.6 & 1.9 & 22 & 6,314 & 2,815 & 0.0602 & 170 & 0.760 & 16 & 0.799 & 91 & 0.681 & 0.740 & 0.64 & 255.92 & $|391.63|$ & 1.27 & 62.60 & .52 \\
\hline 27,236 & 11,440 & 987 & 1.5 & 7.5 & 1.5 & 17 & 6,661 & 2,403 & 0.0197 & 215 & 0.810 & 28 & 0.856 & 74 & 0.727 & 0.796 & 0.70 & 219.95 & 307.97 & 0.78 & 60.77 & 0.39 \\
\hline 16,528 & 7,069 & 591 & 1.5 & 6.3 & 1.2 & 15 & 7,376 & 3,534 & 0.0072 & 235 & 0.766 & 12 & 0.774 & 50 & 0.67 & 0.732 & 0.86 & 192.80 & |261.17| & 0.53 & 100.78 & .33 \\
\hline 27,760 & 10,520 & 1,077 & 1.5 & 9.5 & 2.0 & 20 & 5,422 & 2,100 & 0.0588 & 187 & 0.735 & 25 & 0.774 & 54 & 0.662 & 0.753 & 0.73 & 202.41 & 316.52 & 1.12 & 59.59 & 0.44 \\
\hline 173,821 & 72,413 & 6,338 & 2.0 & 8.4 & 1.6 & 38 & 5,407 & 2,460 & 0.0202 & 201 & 0.788 & 31 & 0.820 & 134 & 0.691 & 0.777 & 0.81 & 422.28 & 591.88 & 1.55 & 07.65 & .76 \\
\hline 104,788 & 43,737 & 3,816 & 2.0 & 9.6 & 1.9 & 32 & 8,004 & 4,036 & 0.0015 & 220 & 0.790 & 12 & 0.845 & 104 & 0.663 & 0.765 & 0.93 & 474.68 & 456.65 & 1.18 & 424.82 & 0.71 \\
\hline 180,025 & 75,718 & 6,519 & 2.0 & 8.8 & 1.7 & 39 & 7,175 & 2,998 & 0.0713 & 223 & 0.745 & 23 & 0.808 & 73 & 0.663 & 0.723 & 0.78 & 454.46 & 627.18 & 1.46 & 17.10 & .69 \\
\hline 59,386 & 25,650 & 2,108 & 1.0 & 9.8 & 2.2 & 25 & 8,168 & 3,832 & 0.0136 & 233 & 0.753 & 34 & 0.758 & 79 & 0.676 & 0.741 & 0.82 & 309.23 & \begin{tabular}{|l|}
410.67 \\
\end{tabular} & 1.09 & 69.21 & 0.53 \\
\hline 94,060 & 41,354 & 3,294 & 2.0 & 8.0 & 1.5 & 29 & 8,276 & 4,095 & 0.0428 & 217 & 0.803 & 24 & 0.852 & 123 & 0.698 & 0.799 & 0.57 & 423.87 & 404.53 & 1.12 & 17.89 & 0.61 \\
\hline 108,578 & 46,831 & 3,859 & 1.5 & 7.5 & 1.5 & 24 & 7,672 & 3,797 & 0.0082 & 235 & 0.761 & 31 & 0.796 & 126 & 0.689 & 0.734 & 0.84 & 382.60 & 549.40 & 0.99 & 87.68 & 0.56 \\
\hline 122,925 & 44,117 & 4,925 & 1.5 & 6.7 & 1.3 & 23 & 7,000 & 2,942 & 0.0442 & 234 & 0.750 & 30 & 0.782 & 71 & 0.673 & 0.743 & 0.83 & 362.34 & \begin{tabular}{|l|}
534.51 \\
\end{tabular} & 1.23 & 40.39 & 0.71 \\
\hline 139,728 & 53,214 & 5,407 & 1.5 & 8.7 & 1.8 & 34 & 6,663 & 3,171 & 0.0075 & 226 & 0.768 & 14 & 0.768 & 87 & 0.651 & 0.764 & 0.90 & 432.84 & 569.74 & 1.34 & 187.83 & 0.74 \\
\hline 175,550 & 62,789 & 7,048 & 1.0 & 9.4 & 2.1 & 39 & 5,214 & 2,031 & 0.0877 & 230 & 0.768 & 34 & 0.814 & 66 & 0.697 & 0.753 & 0.83 & 376.89 & 660.49 & 1.81 & 34.27 & 0.79 \\
\hline 90,824 & 38,781 & 3,253 & 1.0 & 7.0 & 1.5 & 30 & 7,310 & 3,340 & 0.0098 & 170 & 0.792 & 31 & 0.830 & 66 & 0.7 & 0.762 & 0.89 & 376.75 & \begin{tabular}{|l|}
498.04 \\
\end{tabular} & 0.84 & 63.36 & .54 \\
\hline 165,516 & 71,887 & 5,852 & 1.0 & 6.6 & 1.4 & 35 & 8,226 & 4,013 & 0.0221 & 239 & 0.763 & 35 & 0.793 & 88 & 0.68 & 0.728 & 0.84 & 457.29 & 649.41 & 0.90 & 22.45 & 0.60 \\
\hline 154,202 & 65,967 & 5,515 & 1.0 & 6.6 & 1.4 & 36 & 7,605 & 3,608 & 0.0746 & 172 & 0.802 & 34 & 0.841 & 126 & 0.676 & 0.797 & 0.81 & 620.73 & 485.89 & 0.84 & 52.56 & 0.71 \\
\hline 40,846 & 15,071 & 1,611 & 1.0 & 9.8 & 2.2 & 23 & 7,413 & 3,109 & 0.0213 & 215 & 0.765 & 28 & 0.813 & 51 & 0.656 & 0.738 & 0.82 & 290.88 & 362.92 & 0.66 & 36.20 & 0.45 \\
\hline 43,430 & 17,781 & 1,603 & 1.5 & 7.1 & 1.4 & 20 & 7,409 & 3,498 & 0.0073 & 197 & 0.772 & 24 & 0.805 & 100 & 0.683 & 0.757 & 0.81 & 292.73 & \begin{tabular}{|l|}
361.37 \\
\end{tabular} & 0.77 & 108.92 & 0.47 \\
\hline 92,675 & 33,081 & 3,725 & 2.0 & 8.8 & 1.7 & 27 & 6,885 & 2,776 & 0.0576 & 184 & 0.760 & 35 & 0.807 & 71 & 0.667 & 0.766 & 0.73 & 350.41 & 431.68 & 1.56 & 36.45 & 0.72 \\
\hline 94,157 & 33,027 & 3,821 & 2.0 & 10.8 & 2.2 & 30 & 7,714 & 3,022 & 0.0049 & 210 & 0.749 & 11 & 0.796 & 134 & 0.659 & 0.728 & 0.84 & 384.65 & \begin{tabular}{|l|}
482.47 \\
\end{tabular} & 1.57 & 278.28 & 0.70 \\
\hline 72,096 & 29,227 & 2,679 & 1.0 & 9.8 & 2.2 & 28 & 5,995 & 3,002 & 0.0015 & 236 & 0.768 & 33 & 0.768 & 112 & 0.694 & 0.750 & 0.94 & 301.18 & 470.75 & 1.25 & 266.97 & 0.58 \\
\hline 72,096 & 29,227 & 2,679 & 1.0 & 9.8 & 2.2 & 28 & 5,995 & 3,002 & 0.0100 & 236 & 0.768 & 33 & 0.768 & 112 & 0.66 & 0.750 & 0.85 & 320.04 & 431.56 & 1.29 & 208.09 & 0.62 \\
\hline 175,294 & 74,707 & 6,287 & 2.0 & 10.0 & 2.0 & 39 & 7,392 & 3,264 & 0.0062 & 205 & 0.775 & 11 & 0.776 & 127 & 0.686 & 0.778 & 0.87 & 448.87 & 598.01 & 1.67 & 184.08 & 0.77 \\
\hline 72,688 & 29,095 & 2,725 & 2.0 & 6.8 & 1.2 & 26 & 6,433 & 3,058 & 0.0176 & 217 & 0.800 & 15 & 0.836 & 58 & 0.716 & 0.790 & 0.89 & 286.51 & 427.15 & 1.14 & 87.01 & 0.63 \\
\hline 64,194 & 23,156 & 2,565 & 1.5 & 9.5 & 2.0 & 26 & 7,319 & 3,523 & 0.0072 & 174 & 0.754 & 18 & 0.770 & 77 & 0.668 & 0.721 & 0.89 & 318.03 & 443.69 & 1.20 & 177.33 & 0.57 \\
\hline 86,684 & 30,752 & 3,496 & 1.5 & $6.7 \mid$ & 1.3 & 28 & 8,313 & 4,038 & 0.0285 & 183 & 0.793 & 18 & 0.840 & 83 & 0.724 & 0.796 & 0.77 & 340.78 & | 439.93| & 1.13 & 31.46 & 0.65 \\
\hline
\end{tabular}

Table A - 2: Data Generated for Six Stage Treatment 


\begin{tabular}{|c|c|c|c|c|c|c|c|c|c|c|c|c|c|c|c|c|c|c|c|c|c|c|}
\hline 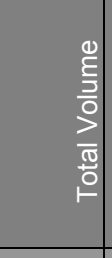 & 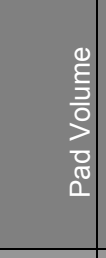 & 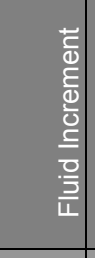 & 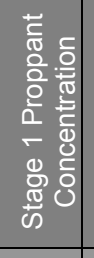 & 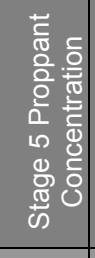 & 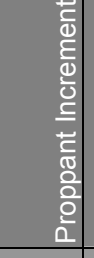 & 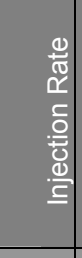 & 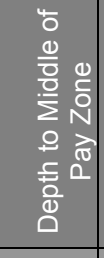 & 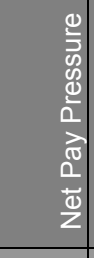 & 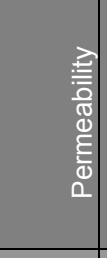 & 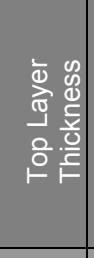 & 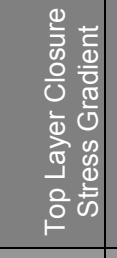 & 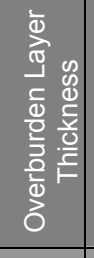 & 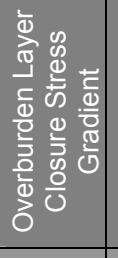 & 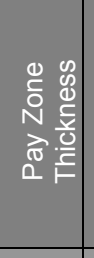 & 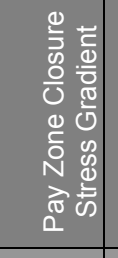 & 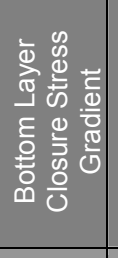 & 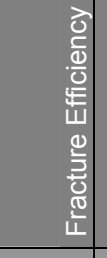 & 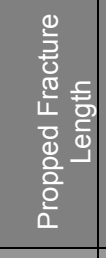 & 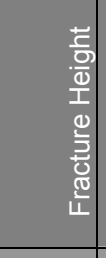 & 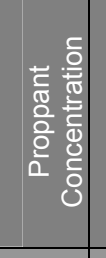 & 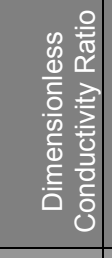 & 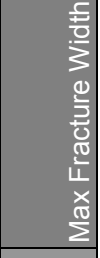 \\
\hline input & input & input & input & input & input & input & input & input & input & input & input & input & input & input & input & input & output & output & output & output & output & I nutti \\
\hline 127,892 & 50,786 & 4,819 & 2.0 & 6.0 & 1.0 & 34 & 6,270 & 3,018 & 0.0076 & 182 & 0.746 & 29 & 0.789 & 127 & 0.675 & 0.726 & 0.88 & 400.84 & 591.91 & 0.93 & 140.96 & 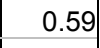 \\
\hline 25,295 & 10,756 & 909 & 1.0 & 8.2 & 1.8 & 17 & 6,617 & 3,114 & 0055 & 228 & 0.760 & 27 & 0.760 & 51 & 0.669 & 0.763 & 0.90 & 209.17 & 305.31 & 0.73 & 23.70 & 40 \\
\hline 51,639 & 18,462 & 2,074 & 2.0 & 8.4 & 1.6 & 24 & 6,393 & 2,977 & 0.0360 & 192 & 0.783 & 33 & 0.814 & 72 & 0.708 & 0.753 & 0.77 & 270.62 & 414.81 & 1.13 & 51.60 & 51 \\
\hline 57,418 & 22,110 & 2,207 & 1.5 & 6.7 & 1.3 & 15 & 6,835 & 3,296 & 0.0091 & 229 & 0.741 & 18 & 0.795 & 88 & 0.663 & 0.724 & 0.86 & 303.81 & 432.14 & 0.81 & 17.81 & .50 \\
\hline 192,068 & 67,787 & 7,768 & 2.0 & 8.4 & 1.6 & 40 & 7,410 & 3,512 & 0.0044 & 239 & 0.800 & 25 & 0.836 & 113 & 0.699 & 0.810 & 0.90 & 463.58 & 97.86 & 2.06 & 26.27 & $0 /$ \\
\hline 113,767 & 46,837 & ,183 & 1.0 & 9.0 & 2.0 & 30 & 6,242 & 3,065 & 0.0421 & 216 & 0.757 & 26 & 0.796 & 55 & 0.658 & 0.786 & 0.87 & 373.39 & 558.88 & 1.23 & 38.21 & .62 \\
\hline 167,327 & 1,179 & 634 & 1.5 & 9.5 & 2.0 & 37 & 8,265 & 3,887 & 0135 & 221 & 0.767 & 29 & 0.789 & 115 & 0.683 & 0.746 & 0.94 & 91.81 & 619.30 & 1.60 & 69.39 & 81 \\
\hline 16,628 & 7,118 & 594 & 1.5 & 6.7 & 1.3 & 16 & 6,830 & 2,854 & 0.0075 & 230 & 0.736 & 29 & 0.770 & 59 & 0.668 & 0.718 & 0.84 & 182.98 & 274.71 & 0.57 & 136.62 & ).31 \\
\hline 186,129 & 68,403 & 358 & 2.0 & 7.6 & 1.4 & 38 & 7,928 & 3,874 & 0.0326 & 182 & 0.765 & 25 & 0.787 & 103 & 0.664 & 0.747 & 0.76 & 41.62 & 572.33 & 1.33 & 25.01 & .77 \\
\hline 196,711 & 77,037 & 7,480 & 1.0 & 8.2 & 1.8 & 40 & 6,686 & 2,907 & 0.0094 & 204 & 0.750 & 11 & 0.806 & 116 & 0.68 & 0.732 & 0.88 & 459.02 & 642.34 & 1.42 & 140.29 & 0.77 \\
\hline 46,697 & 19,200 & 719 & 2.0 & 8.0 & 1.5 & 21 & 5,217 & 2,791 & 0047 & 208 & 0.751 & 17 & 0.809 & 121 & .664 & 0.729 & 0.89 & 254.14 & 394.28 & 1.01 & 95.04 & 51 \\
\hline 108,509 & 42,380 & 4,133 & 1.5 & 7.5 & 1.5 & 32 & 7,876 & 3,745 & 0.0707 & 236 & 0.772 & 19 & 0.817 & 60 & 0.694 & 0.753 & 0.79 & 373.31 & 528.02 & 1.12 & 12.71 & 0.61 \\
\hline 147,603 & 4,413 & 824 & 2.0 & 8.8 & 1.7 & 36 & $5,931 \mid$ & 3,009 & 0439 & 211 & 0.800 & 12 & 0.839 & 75 & 0.709 & 0.771 & 0.84 & 393.44 & 587.39 & 1.61 & 47.69 & .75 \\
\hline 65,424 & 26,285 & 2,446 & 1.5 & 8.7 & 1.8 & 24 & 6,012 & 3,038 & 0.0168 & 191 & 0.820 & 16 & 0.859 & 100 & 0.729 & 0.822 & 0.79 & 311.68 & 431.90 & 1.11 & 97.50 & 0.56 \\
\hline 78,561 & 30,989 & 2,973 & 1.5 & 7.5 & 1.5 & 24 & 5,930 & 1,983 & 0.0075 & 190 & 0.743 & 16 & 0.780 & 117 & 0.663 & 0.730 & 0.83 & 334.55 & 486.65 & 0.98 & 210.89 & .54 \\
\hline 39,809 & 15,277 & 1,533 & 2.0 & 6.4 & 1.1 & 19 & 7,285 & 2,948 & 0.0109 & 214 & 0.780 & 17 & 0.819 & 108 & 0.662 & 0.760 & 0.71 & 297.54 & 317.69 & 0.79 & 87.83 & 0.50 \\
\hline 54,769 & 23,039 & 1,983 & 1.5 & 8.3 & 1.7 & 26 & 7,716 & 2,027 & 0.0063 & 236 & 0.752 & 20 & 0.752 & 122 & 0.67 & 0.727 & 0.81 & 285.13 & 394.06 & 1.03 & 166.04 & .53 \\
\hline 95,473 & 38,871 & 3,538 & 2.0 & 7.2 & 1.3 & 31 & 7,066 & 1,751 & 0.0229 & 214 & 0.766 & 19 & 0.793 & 78 & 0.684 & 0.737 & 0.82 & 360.49 & 517.69 & 1.01 & 42.36 & 0.56 \\
\hline 95,473 & 38,871 & 538 & 1.5 & 7.5 & 1.5 & 31 & 7,066 & 1,751 & 0060 & 214 & 0.766 & 19 & 0.793 & 78 & 0.658 & 0.737 & 0.89 & 392.61 & 494.29 & 0.97 & 147.97 & .61 \\
\hline 71,673 & 28,925 & 2,672 & 1.5 & 7.5 & 1.5 & 28 & 6,620 & 3,741 & 0529 & 206 & 0.794 & 12 & 0.838 & 96 & 0.729 & 0.775 & 0.74 & 00.61 & 73.01 & 1.00 & 23.52 & 0.51 \\
\hline 120,936 & 44,271 & 4,792 & 1.5 & 9.1 & 1.9 & 34 & 5,436 & 2,760 & 0.0014 & 225 & 0.800 & 25 & 0.830 & 59 & 0.724 & 0.803 & 0.97 & 366.31 & 572.53 & 1.45 & 287.00 & 0.70 \\
\hline 110,535 & 45,964 & 4,036 & 2.0 & 6.8 & 1.2 & 30 & 6,318 & 3,236 & 0.0077 & 178 & 0.800 & 29 & 0.800 & 116 & 0.711 & 0.780 & 0.87 & 84.21 & 7.65 & 1.00 & 134.14 & .61 \\
\hline 75,819 & 28,867 & 2,934 & 1.0 & 6.2 & 1.3 & 26 & 7,474 & 2,959 & 0.0343 & 191 & 0.769 & 30 & 0.800 & 96 & 0.669 & 0.733 & 0.68 & 372.48 & 447.72 & 0.76 & 20.64 & 0.51 \\
\hline 68,086 & 27,685 & 2,525 & 1.5 & 8.3 & 1.7 & 24 & 5,232 & 2,301 & 0.0081 & 233 & 0.782 & 14 & 0.827 & 51 & 0.661 & 0.774 & 0.93 & 283.35 & 13.69 & 1.26 & 28.64 & 0.66 \\
\hline 68,086 & 27,685 & 2,525 & 1.5 & 8.3 & 1.7 & 24 & 5,232 & 2,301 & 0.0110 & 233 & 0.750 & 14 & 0.800 & 51 & 0.661 & 0.731 & 0.92 & 285.27 & 458.33 & 1.13 & 215.48 & 0.57 \\
\hline 182,226 & 72,229 & 6,875 & 1.5 & 8.3 & 1.7 & 37 & 7,251 & 2,993 & 0.0082 & 220 & 0.768 & 33 & 0.789 & 65 & 0.692 & 0.742 & 0.93 & 437.93 & 35.63 & 1.45 & 24.91 & .73 \\
\hline 70,960 & 25,832 & 2,821 & 2.0 & 8.8 & 1.7 & 30 & 5,116 & 2,554 & 0.0114 & 199 & 0.800 & 26 & 0.800 & 111 & 0.655 & 0.772 & 0.84 & 333.38 & 428.27 & 1.26 & 222.06 & 0.65 \\
\hline 32,203 & 12,292 & 1,244 & 2.0 & 6.8 & 1.2 & 19 & 5,424 & 2,106 & 0.0044 & 177 & 0.774 & 11 & 0.813 & 69 & 0.708 & 0.754 & 0.90 & 223.03 & 371.05 & 0.77 & 00.52 & 0.41 \\
\hline 75,563 & 29,370 & 2,887 & 2.0 & 8.0 & 1.5 & 26 & 6,837 & 2,871 & 0.0032 & 205 & 0.814 & 28 & 0.855 & 93 & 0.711 & 0.809 & 0.89 & 350.47 & 3.90 & 1.19 & 362.63 & 0.66 \\
\hline $124,341 \mid$ & 43,555 & 5,049 & 1.0 & 8.2 & 1.8 & 30| & 8,271 & $4,047 \mid$ & 0.0117 & 212 & 0.740 & $23 \mid$ & 0.777 & 116 & 0.679 & 0.733 & 0.83 & 406.04 & 561.65 & $1.24 \mid$ & 78.22 & 0.6 \\
\hline
\end{tabular}

Table A-2: Data Generated for Six Stage Treatment, continued 


\begin{tabular}{|c|c|c|c|c|c|c|c|c|c|c|c|c|c|c|c|c|c|c|c|c|c|c|}
\hline 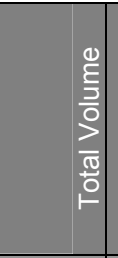 & 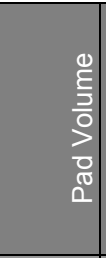 & 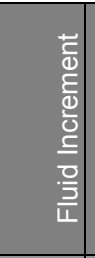 & 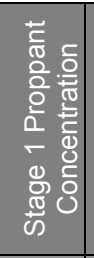 & 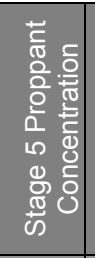 & 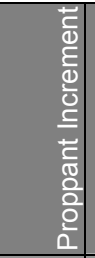 & 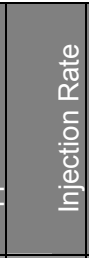 & 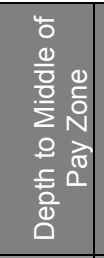 & 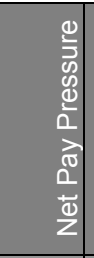 & 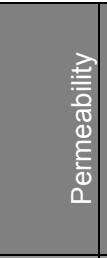 & 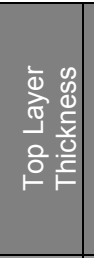 & 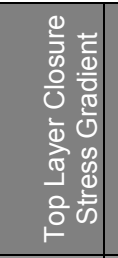 & 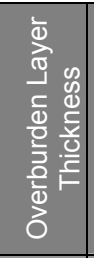 & 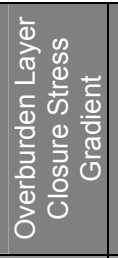 & 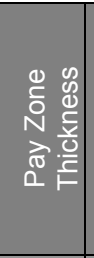 & 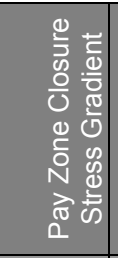 & 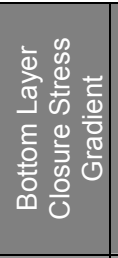 & 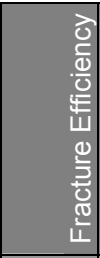 & 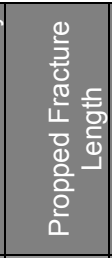 & \begin{tabular}{l|l}
$\frac{7}{0}$ \\
$\frac{0}{0}$ \\
$\frac{1}{1}$ \\
0 \\
$\frac{0}{3}$ \\
$\frac{0}{0}$ \\
$\frac{\pi}{4}$ \\
$\frac{1}{1}$
\end{tabular} & 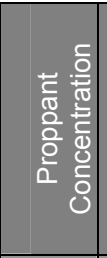 & 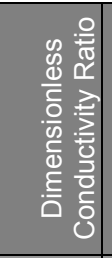 & . \\
\hline input & input & input & input & input & input & input & input & input & input & input & input & input & input & input & input & input & output & output & utput & output & output & output \\
\hline 133,685 & 8,297 & 5,337 & 2.0 & 9.6 & 1.9 & 34 & 7,329 & 3,497 & 0.0077 & 185 & 0.765 & 20 & 0.804 & 103 & 0.666 & 0.751 & 0.87 & 38.74 & 22.28 & 1.60 & 9.34 & \\
\hline 188,759 & 0,603 & 385 & 1.0 & 7.0 & 1.5 & 39 & 7,260 & 450 & 0.0078 & 208 & .780 & 30 & 0.794 & 63 & 0.691 & 0.758 & 0.93 & 471.27 & 9.95 & 1.18 & 101.86 & \\
\hline 128,414 &, 907 & 844 & 1.5 & 7.1 & 1.4 & 31 & 6,908 & 396 & 0.0904 & 198 & .755 & 30 & 0.778 & 93 & 0.666 & 0.736 & .68 & 435.80 & 559.33 & 1.01 & 1.49 & \\
\hline 132,994 & 7,705 & 706 & 2.0 & 10.8 & 2.2 & 32 & 6,570 & 2,521 & 0.0549 & 240 & 0.786 & 29 & 0.827 & 116 & 0.681 & 0.751 & 0.64 & 399.90 & 3.54 & 1.79 & 8.08 & \\
\hline 30,147 & 2,067 & 130 & 1.5 & 7.5 & 1.5 & 18 & 7,186 & 3,517 & 0.0024 & 204 & 0.734 & 17 & 0.734 & 64 & 0.65 & 0.734 & 0.93 & 237.38 & 352.58 & 0.72 & 439.22 & \\
\hline 43,431 &, 060 & 523 & 1.5 & 8.7 & 1.8 & 25 & 7,993 & 677 & 0.0448 & 236 & 0.759 & 19 & 0.799 & 121 & 0.676 & 0.738 & 0.54 & 250.88 & 42.41 & 1.08 & 29.68 & 1 \\
\hline 167,067 & 9,721 & 084 & 1.0 & 9.0 & 2.0 & 37 & 7,401 & 103 & 0.0194 & 186 & 0.786 & 11 & 0.831 & 88 & 0.655 & 0.767 & 0.80 & 554.70 & \begin{tabular}{|l|}
537.14 \\
\end{tabular} & 1.25 & 40.30 & 0.7 \\
\hline 146,938 & 7,198 & 609 & 1.0 & 9.0 & 2.0 & 33 & 5,299 & 2,517 & 0.0080 & 170 & 0.743 & 16 & 0.782 & 98 & 0.652 & 0.723 & 0.91 & \begin{tabular}{|l|}
401.75 \\
\end{tabular} & 37.15 & 1.45 & 282.61 & 0.72 \\
\hline 102,382 & 38,468 & 995 & 2.0 & 6.0 & 1.0 & 27 & 7,110 & 2,917 & 0.0346 & 223 & 0.755 & 32 & 0.800 & 99 & 0.689 & 0.762 & 0.67 & \begin{tabular}{|l|}
352.02 \\
\end{tabular} & 484.22 & 1.07 & 22.14 & .61 \\
\hline 109,815 & 47,791 & 876 & 1.0 & 8.6 & 1.9 & 30 & 7,727 & 041 & 0.0031 & 232 & 0.754 & 27 & 0.769 & 123 & 0.671 & 0.737 & 0.88 & 400.83 & 6.27 & 1.08 & 241.98 & .62 \\
\hline 11,435 &, 795 & 415 & 1.0 & 5.8 & 1.2 & 15 & 7,652 & 632 & 0.0049 & 207 & .733 & 18 & 0.762 & 113 & 651 & 0.714 & .67 & 5.30 & 07.51 & 0.46 & 3.42 & 0.27 \\
\hline 60,058 & 22,146 & 2,370 & 1.5 & 6.7 & 1.3 & 27 & 6,972 & 2,893 & 0.0058 & 236 & 0.792 & 17 & 0.792 & 63 & .691 & 0.768 & 0.90 & 317.30 & 437.69 & 0.83 & 152.50 & 0.54 \\
\hline 121,250 & 3,410 & 553 & 1.0 & 8.6 & 1.9 & 33 & 7,605 & 541 & 0.0182 & 193 & .753 & 18 & 0.778 & 78 & 0.675 & 0.737 & 0.86 & 389.58 & 3.31 & 1.28 & 56.07 & 0.67 \\
\hline 87,407 & 33,169 & 390 & 2.0 & 9.2 & 1.8 & 28 & 6,924 & 2,712 & 0.0244 & 238 & 0.761 & 21 & 0.786 & 73 & 0.65 & 0.743 & 0.80 & 369.17 & 459.30 & 1.32 & 64.87 & 0.66 \\
\hline 198,340 & 4,682 & 729 & 2.0 & 10.0 & 2.0 & 40 & ,371 & 418 & 0.0061 & & 744 & 32 & 0.768 & 105 & 0.654 & .728 & .93 & 422.51 & 70.37 & 1.94 & 5.58 & .83 \\
\hline 91,474 & 37,990 & 3,343 & 1.5 & 7.5 & 1.5 & 29 & 7,685 & 2,929 & 0.0017 & 229 & 0.812 & 24 & 0.850 & 74 & 0.719 & 0.830 & 0.94 & \begin{tabular}{|l|}
354.37 \\
\end{tabular} & 401.72 & 1.26 & 545.36 & 0.76 \\
\hline ,474 & ,990 & 343 & 1.5 & 7.5 & 1.5 & 29 & 7,685 & 929 & 0.0017 & 229 & .770 & 24 & 0.770 & 74 & 0.673 & 0.760 & 0.94 & \begin{tabular}{|l|}
372.24 \\
\end{tabular} & 8.09 & 0.97 & 428.30 & .60 \\
\hline 171,302 & 70,674 & 6,289 & 1.5 & 6.7 & 1.3 & 40 & 5,393 & 2,588 & 0.0212 & 197 & 0.753 & 15 & 0.797 & 121 & 0.69 & 0.736 & 0.85 & 383.46 & 631.82 & 1.26 & 88.39 & 0.70 \\
\hline 185,771 & 69,343 & 277 & 1.0 & 6.6 & 1.4 & 40 & 6,221 & 2,257 & 0.0015 & 189 & .745 & 25 & 0.850 & 121 & 0.675 & .770 & 0.93 & \begin{tabular}{|l|}
$\mid 571.75$ \\
\end{tabular} & 37.49 & 0.91 & 468.21 & 0.75 \\
\hline 172,291 & 64,630 & 6,729 & 2.0 & 7.2 & 1.3 & 40 & 8,336 & 3,700 & 0.0490 & 198 & 0.772 & 28 & 0.798 & 68 & 0.67 & 0.771 & 0.91 & 493.36 & 482.82 & 1.50 & 170.74 & 0.91 \\
\hline 12,951 & 5,150 & 488 & 1.0 & 6.2 & 1.3 & 15 & 5,485 & 2,331 & 0.0088 & 235 & .776 & 19 & 0.793 & 58 & 0.682 & 0.746 & 0.82 & 157.69 & 237.49 & 0.50 & 173.90 & 0.30 \\
\hline 38,916 & 16,388 & ,408 & 1.0 & 7.4 & 1.6 & 20 & 7,236 & 3,000 & 0.0206 & 221 & 0.790 & 20 & 0.827 & 135 & 0.694 & 0.741 & 0.60 & 244.62 & 345.63 & 0.84 & 55.71 & 0.43 \\
\hline 124,069 & 50,252 & 614 & 2.0 & 7.6 & 1.4 & 20 & 7,653 & 3,211 & 0.0067 & 208 & .757 & 20 & 0.783 & 72 & 0.675 & 0.752 & 0.91 & 832.63 & 489.83 & 1.38 & 1.95 & 0.77 \\
\hline 75,636 & 30,988 & 790 & 2.0 & 8.0 & 1.5 & 27 & 5,654 & 2,439 & 0.0159 & 204 & .800 & 13 & 0.816 & 115 & 0.71 & 0.784 & .79 & 313.62 & 464.00 & 1.12 & 17.63 & 0.56 \\
\hline 72,183 & 29,467 & 2,670 & 1.5 & 7.1 & 1.4 & 26 & 7,425 & 2,664 & 0.0052 & 235 & 0.762 & 29 & 0.781 & 96 & 0.65 & 0.742 & 0.84 & 390.71 & 416.40 & 0.79 & 127.81 & 0.57 \\
\hline 78,096 & 27,672 & 3,152 & 1.0 & 7.4 & 1.6 & 28 & 6,526 & 3,066 & 0.0914 & 222 & 0.743 & 28 & 0.771 & 74 & 0.66 & 0.725 & 0.72 & 340.53 & 487.29 & 0.97 & 16.40 & 0.53 \\
\hline 116,223 & 44,374 & 4,491 & 1.5 & 9.5 & 2.0 & 32 & 7,874 & 3,645 & 0.0059 & 180 & 0.762 & 14 & 0.783 & 91 & 0.659 & 0.762 & 0.88 & 421.28 & 444.14 & 1.59 & 197.57 & 0.81 \\
\hline 63,572 & 23,744 & 2,489 & 2.0 & 8.4 & 1.6 & 24 & 5,055 & 2,507 & 0.0065 & 221 & 0.777 & 14 & 0.804 & 87 & 0.674 & 0.757 & 0.90 & 302.78 & 446.72 & 1.12 & 364.33 & 0.57 \\
\hline 22,408 & 7,862 & 909 & 2.0 & 6.4 & 1.1 & 17 & 6,124 & 2,993 & 0.0210 & 228 & 0.747 & 25 & 0.782 & 115 & 0.671 & 0.740 & 0.65 & |199.08 & 308.26 & 0.68 & 85.97 & 0.3 \\
\hline 172,553 & 67,458 & 6,568 & 2.0 & 10.4 & 2.1 & 38 & 6,342 & 2,846 & 0.0128 & 195 & 0.769 & 19 & 0.811 & 91 & 0.689 & 0.744 & 0.89 & |413.62 & 617.31 & 1.90 & 162.21 & 0.7 \\
\hline
\end{tabular}

Table A-2: Data Generated for Six Stage Treatment, continued 


\begin{tabular}{|c|c|c|c|c|c|c|c|c|c|c|c|c|c|c|c|c|c|c|c|c|c|c|}
\hline 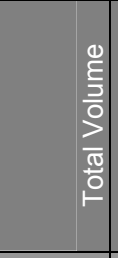 & 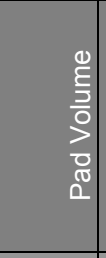 & 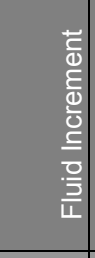 & 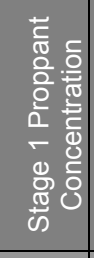 & 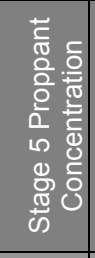 & 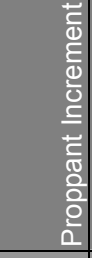 & 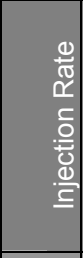 & 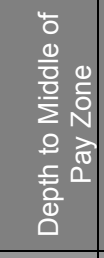 & 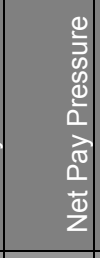 & 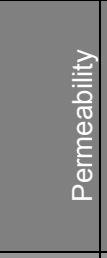 & 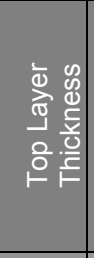 & 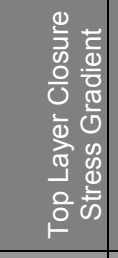 & 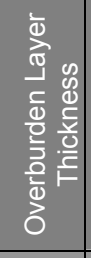 & 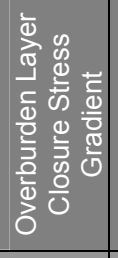 & 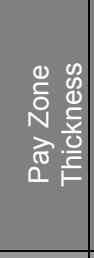 & 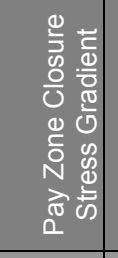 & 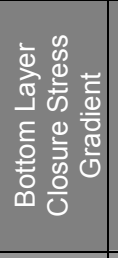 & 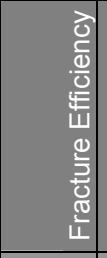 & 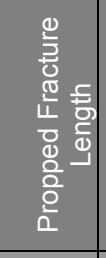 & \begin{tabular}{l|l}
$\frac{7}{0}$ \\
$\frac{0}{0}$ \\
$\frac{1}{1}$ \\
0 \\
$\frac{0}{3}$ \\
$\frac{3}{0}$ \\
$\frac{0}{4}$ \\
$\frac{\pi}{4}$
\end{tabular} & 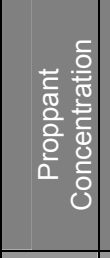 & 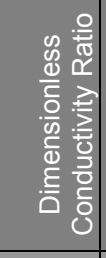 & \\
\hline input & input & input & input & input & input & input & input & input & input & input & input & input & input & input & input & input & output & output & output & output & output & output \\
\hline 156,511 & 63,727 & 5,799 & 1.0 & 7.4 & 1.6 & 35 & 7,974 & 4,003 & 0.0130 & 188 & 0.764 & 12 & 0.784 & 68 & 0.677 & 0.756 & 0.90 & 437.74 & 17.89 & 1.30 & 64.05 & .7 \\
\hline 57,828 & 24,294 & 2,096 & 1.5 & 6.7 & 1.3 & 24 & 5,025 & 2,031 & 0.0297 & 187 & 0.751 & 18 & 0.785 & 122 & 0.677 & 0.760 & 0.70 & 270.00 & 32.06 & 0.87 & 72.00 & .47 \\
\hline 104,355 & 44,047 & 3,769 & 2.0 & 8.8 & 1.7 & 30 & 6,739 & 2,964 & 0.0061 & 187 & 0.761 & 28 & 0.769 & 104 & 0.653 & 0.756 & 0.87 & 388.36 & 479.77 & 1.29 & 226.57 & 6 \\
\hline 16,438 & 6,875 & 598 & 1.5 & 9.9 & 2.1 & 16 & 8,212 & 3,369 & 0.0333 & 234 & 0.750 & 33 & 0.755 & 81 & 0.68 & 0.739 & 0.52 & 171.58 & 240.46 & 0.96 & 46.40 & \\
\hline 61,164 & 22,966 & 2,387 & 1.5 & 6.3 & 1.2 & 30 & 7,621 & 3,811 & 0.0219 & 205 & 0.774 & 33 & 0.811 & 98 & 0.681 & 0.760 & 0.84 & 359.49 & 419.38 & 0.72 & 75.48 & \\
\hline 29,610 & 1,197 & 1,151 & 1.0 & 7.8 & 1.7 & 19 & 5,786 & 2,486 & 0.0083 & 186 & 0.751 & 17 & 0.784 & 132 & 0.676 & 0.719 & 0.76 & 205.36 & 341.28 & 0.88 & 268.86 & .42 \\
\hline 153,157 & 0,133 & 814 & 1.5 & 7.5 & 1.5 & 34 & 7,706 & 3,252 & 0.0456 & 199 & 0.732 & 35 & 0.775 & 118 & 0.667 & 0.758 & 0.69 & 401.84 & 11.60 & 1.51 & 29.40 & 7 \\
\hline 141,748 & 54,604 & 5,447 & 2.0 & 10.0 & 2.0 & 34 & 6,927 & 2,938 & 0.0205 & 237 & 0.780 & 22 & 0.813 & 98 & 0.705 & 0.748 & 0.83 & 391.20 & 598.64 & 1.66 & 74.20 & .70 \\
\hline 141,748 & 4,604 & 447 & 2.0 & 10.0 & 2.0 & 34 & 6,927 & 2,938 & 0.0089 & 237 & .750 & 22 & 0.813 & 98 & 0.67 & 0.748 & 0.88 & 386.41 & 533.87 & 1.88 & 221.67 & 8 \\
\hline 193,646 & 75,250 & 7,400 & 1.5 & 9.1 & 1.9 & 40 & 8,146 & 4,085 & 0.0638 & 230 & 0.810 & 27 & 0.848 & 89 & 0.705 & 0.789 & 0.81 & 549.47 & 604.94 & 1.42 & 30.06 & 0.77 \\
\hline 49,113 & 8,130 & ,936 & 1.5 & 5.9 & 1.1 & 23 & 5,822 & 2,382 & 0.0088 & 196 & 0.760 & 11 & 0.760 & 90 & 0.661 & 0.755 & 0.84 & 281.13 & 99.51 & 1.10 & 252.47 & 54 \\
\hline 107,314 & 42,306 & 4,063 & 1.5 & 7.1 & 1.4 & 32 & 5,610 & 2,094 & 0.0685 & 184 & 0.744 & 15 & 0.807 & 133 & 0.678 & 0.755 & 0.62 & 342.96 & 535.76 & 1.12 & 31.88 & 0.56 \\
\hline 90,013 & 38,842 & , 198 & 1.5 & 7.9 & 1.6 & 18 & 6,364 & 120 & 0113 & 205 & .727 & 13 & 0.751 & 70 & 0.65 & 0.715 & 0.97 & 317.47 & 1.44 & 1.13 & 142.68 & $.5 \mathrm{~s}$ \\
\hline 16,987 & 6,292 & 668 & 2.0 & 8.0 & 1.5 & 17 & 7,095 & 2,653 & 0.0038 & 216 & 0.749 & 32 & 0.758 & 72 & 0.674 & 0.747 & 0.84 & 201.45 & 275.94 & 0.70 & 308.93 & 0.36 \\
\hline 30,029 & 1,541 & 155 & 1.5 & 8.3 & 1.7 & 18 & 6,721 & ,218 & 0.0079 & 220 & 0.762 & 13 & 0.755 & 129 & 0.726 & 0.762 & 0.76 & 224.79 & 3.12 & 0.90 & 10.69 & .44 \\
\hline 108,394 & 6,597 & 3,862 & 1.0 & 8.2 & 1.8 & 21 & 7,004 & \begin{tabular}{|l|} 
\\
\end{tabular}, 054 & 0.0052 & 183 & 0.794 & 24 & 0.838 & 105 & 0.726 & 0.801 & 0.89 & 339.96 & 469.10 & 1.35 & 228.95 & 0.71 \\
\hline 108,394 & 6,597 & 3,862 & 1.0 & 8.2 & 1.8 & 21 & 7,004 & , 054 & 0.0700 & 183 & 0.794 & 24 & 0.838 & 105 & 0.726 & 0.801 & 0.87 & 382.01 & |446.93 & 1.26 & 202.28 & 0.72 \\
\hline 80,953 & 0,503 & 3,153 & 1.5 & 6.3 & 1.2 & 26 & 7,441 & 2,820 & 0.0404 & 176 & 0.766 & 25 & 0.816 & 111 & 0.661 & 0.745 & 0.59 & 404.91 & 439.69 & 0.81 & 19.48 & 0.53 \\
\hline 73,745 & 26,926 & 2,926 & 1.0 & 7.8 & 1.7 & 25 & 7,222 & ,332 & 0.0094 & 225 & 0.763 & 27 & 0.796 & 125 & 0.69 & 0.746 & 0.80 & 338.78 & 475.94 & 0.97 & 103.30 & 0.54 \\
\hline 44,310 & 7,914 & 650 & 2.0 & 8 & 1.5 & 21 & 8,009 & 4,045 & 0.0226 & 231 & .776 & 31 & 0.770 & 70 & 0.675 & 0.755 & 0.77 & 288.62 & 53.30 & 0.94 & 40.56 & 0.51 \\
\hline 194,045 & 5,332 & 7,420 & 2.0 & 8.8 & 1.7 & 20 & 7,434 & \begin{tabular}{|l|}
3,678 \\
\end{tabular} & 0.0100 & 227 & 0.786 & 28 & 0.793 & 85 & 0.676 & 0.756 & 0.90 & 452.50 & 563.00 & 1.85 & 131.44 & 0.91 \\
\hline 123,330 & 6,910 & 4,776 & 2.0 & 7.6 & 1.4 & 28 & 5,730 & 2,203 & 0.0070 & 189 & .788 & 30 & 0.854 & 132 & 0.702 & 0.790 & 0.84 & 388.24 & 538.82 & 1.33 & 265.88 & 0.68 \\
\hline 136,317 & 56,542 & 4,986 & 1.5 & 7.5 & 1.5 & 30 & 8,272 & 3,687 & 0.0184 & 187 & 0.781 & 11 & 0.782 & 120 & 0.667 & 0.750 & 0.71 & 538.05 & 495.89 & 1.00 & 27.27 & 0.67 \\
\hline 38,769 & 16,387 & | 1,399 & 1.5 & 8.7 & 1.8 & 19 & 6,263 & 2,886 & 0.0050 & 204 & .784 & 23 & 0.848 & 52 & 0.686 & 0.739 & 0.93 & 223.47 & |373.44 & 1.03 & 61.05 & 0.46 \\
\hline 91,395 & 39,453 & 3,246 & 1.5 & 9.1 & 1.9 & 27 & 5,786 & 2,059 & 0.0067 & 208 & 0.767 & 15 & 0.789 & 94 & 0.685 & 0.757 & 0.88 & 321.96 & 502.15 & 1.28 & 297.09 & 0.62 \\
\hline 112,541 & 45,228 & 4,207 & 1.0 & 9.8 & 2.2 & 29 & 6,348 & 2,739 & 0.0379 & 179 & 0.757 & 30 & 0.787 & 89 & 0.675 & 0.747 & 0.77 & 372.39 & 523.09 & 1.43 & 50.40 & 0.65 \\
\hline 172,148 & 61,618 & 6,908 & 1.0 & 7.8 & 1.7 & 36 & 8,047 & 3,448 & 0.0112 & 207 & 0.774 & 26 & 0.801 & 131 & 0.67 & 0.766 & 0.78 & 577.43 & 559.86 & 1.14 & 56.83 & 0.75 \\
\hline 119,806 & 47,120 & 4,543 & 1.5 & 8.3 & 1.7 & 30 & 5,330 & $\mid 2,636$ & 0.0098 & 234 & .736 & 27 & 0.770 & 77 & 0.65 & 0.716 & 0.92 & 357.95 & |553.64 & 1.34 & 237.34 & 0.68 \\
\hline 168,472 & 70,442 & 6,127 & 2.0 & 6.4 & 1.1 & 38 & 8,373 & 4,261 & 0.0603 & 239 & .783 & 25 & 0.825 & 64 & .705 & 0.771 & 0.82 & 420.00 & |553.14 & 1.32 & 13.17| & 0.76 \\
\hline $136,695 \mid$ & 51,597 & $\mid 5,319$ & 1.5 & 9.1 & 1.9 & 32 & 7,666 & $\mid 3,188$ & 0.0381 & 223 & 0.764 & 28 & 0.805 & 73 & 0.658 & 0.734 & 0.78 & 493.86 & | 577.30| & 1.00 & 17.90 & \\
\hline
\end{tabular}

Table A-2: Data Generated for Six Stage Treatment, continued 


\begin{tabular}{|c|c|c|c|c|c|c|c|c|c|c|c|c|c|c|c|c|c|c|c|c|c|c|}
\hline 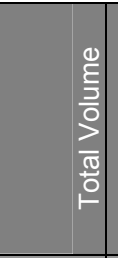 & 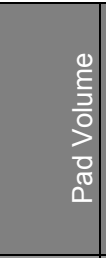 & 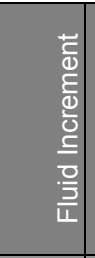 & 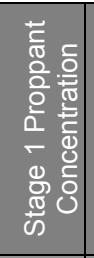 & 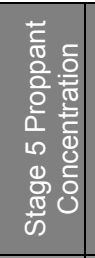 & 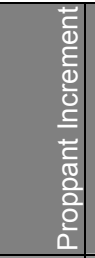 & 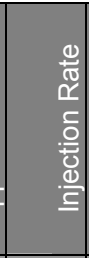 & 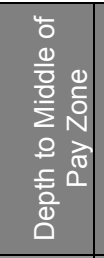 & 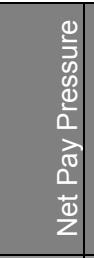 & 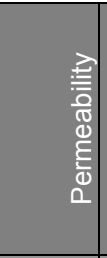 & 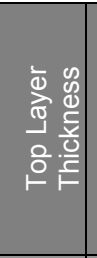 & 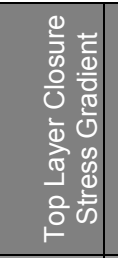 & 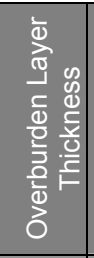 & 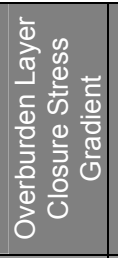 & 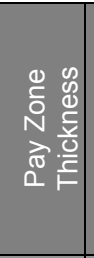 & 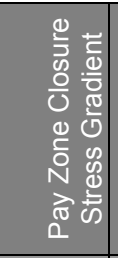 & 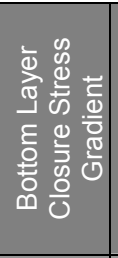 & 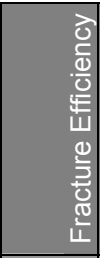 & 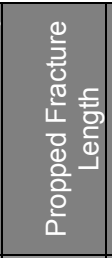 & \begin{tabular}{l|l}
$\frac{7}{0}$ & \\
$\frac{0}{0}$ & \\
$\frac{1}{1}$ & \\
$\frac{0}{3}$ & \\
$\frac{3}{0}$ & \\
$\frac{\pi}{4}$ & \\
$\frac{1}{4}$ &
\end{tabular} & 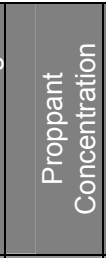 & 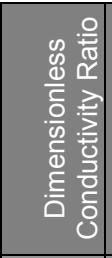 & . \\
\hline input & input & input & input & input & input & input & input & input & input & input & input & input & input & input & input & input & output & output & utput & output & output & output \\
\hline 83,269 & 33,529 & 3,109 & 1.5 & 7.5 & 1.5 & 28 & 7,717 & 3,370 & 0.0085 & 199 & 0.750 & 19 & 0.796 & 124 & 0.685 & 0.729 & 0.71 & 46.52 & 38.14 & 0.98 & 5.85 & \\
\hline 83,269 &, 529 & 109 & 1.5 & 7.5 & 1.5 & 28 & 7,717 & 370 & 0.0200 & 199 & .750 & 19 & 0.796 & 124 & 0.665 & 0.729 & 0.79 & 374.86 & 57.00 & 0.97 & 77.49 & \\
\hline 183,199 & 78,135 & 567 & 2.0 & 9.2 & 1.8 & 40 & 6,024 & 2,933 & 0.0221 & 218 & 0.776 & 32 & 0.809 & 132 & 0.686 & 0.756 & 0.81 & \begin{tabular}{|l|}
464.26 \\
\end{tabular} & 620.39 & 1.51 & 74.86 & .73 \\
\hline 113,140 & 9,061 & 005 & 1.0 & 7.0 & 1.5 & 32 & 7,056 & 2,941 & 0.0797 & 187 & 0.745 & 25 & 0.820 & 78 & 0.657 & 0.733 & 0.70 & 413.19 & 25.73 & 0.89 & 11.10 & \\
\hline 56,758 & 4,942 & 988 & 2.0 & 9.6 & 1.9 & 18 & 6,601 & 2,918 & 0.0655 & 178 & 0.810 & 24 & 0.833 & 77 & 0.697 & 0.791 & 0.61 & 307.41 & \begin{tabular}{|l|}
376.09 \\
\end{tabular} & 1.18 & 25.41 & 0 \\
\hline 119,927 & 611 & 707 & 1.0 & 7.4 & 1.6 & 29 & 7,190 & 117 & 0.0474 & 211 & .750 & 14 & 0.783 & 103 & 0.674 & 0.729 & 0.70 & 411.53 & \begin{tabular}{|l|}
576.27 \\
\end{tabular} & 1.01 & 20.56 & \\
\hline 40,213 & 14,121 & 1,631 & 1.5 & 9.5 & 2.0 & 24 & 6,366 & 3,174 & 0.0043 & 222 & 0.752 & 28 & 0.796 & 57 & 0.664 & 0.751 & 0.93 & 271.94 & 377.39 & 1.05 & 427.90 & .51 \\
\hline 22,122 & 8,546 & 849 & 1.0 & 7.8 & 1.7 & 17 & 8,008 & 3,348 & 0.0634 & 188 & 0.797 & 18 & 0.852 & 79 & 0.71 & 0.770 & 0.43 & 207.09 & \begin{tabular}{|l|}
274.27 \\
\end{tabular} & 0.80 & \begin{tabular}{|l|}
19.40 \\
\end{tabular} & 0.34 \\
\hline 13,316 & 793 & 533 & 1.0 & 5.8 & 1.2 & 15 & 6,664 & 509 & 0.0034 & 197 & .758 & 29 & 0.806 & 96 & 0.674 & 0.739 & .78 & 175.30 & 234.59 & .46 & 283.51 & 0.30 \\
\hline 195,925 & 83,815 & 7,007 & 2.0 & 9.6 & 1.9 & 40 & 7,812 & 2,866 & 0.0304 & 170 & 0.770 & 31 & 0.772 & 79 & 0.685 & 0.766 & 0.81 & 461.88 & 2.39 & 1.65 & 4.98 & .74 \\
\hline 195,925 & 3,815 & 007 & 2.0 & 9.6 & 1.9 & 40 & 7,812 & 2,866 & 0.0090 & 170 & .770 & 31 & 0.772 & 79 & 0.66 & 0.750 & .88 & 528.60 & 92.83 & 1.54 & 94.22 & 0.79 \\
\hline 1,267 & 0,969 & 894 & 1.0 & 9.0 & 2.0 & 22 & 8,130 & 3,586 & 0081 & 185 & .769 & 26 & 0.791 & 110 & 0.672 & 0.736 & 0.78 & 307.38 & 0.49 & 1.04 & 113.29 & 0.54 \\
\hline 115,970 & 46,968 & 4,313 & 2.0 & 9.6 & 1.9 & 31 & 8,345 & 3,694 & 0.0085 & 220 & 0.815 & 27 & 0.855 & 82 & 0.718 & 0.802 & 0.85 & |423.34 & 83.69 & 1.44 & \begin{tabular}{|r|}
97.26 \\
\end{tabular} & 0.72 \\
\hline 25,602 & 9,867 & 983 & 1.5 & 6.3 & 1.2 & 17 & 6,485 & 2,725 & 0.0077 & 228 & 0.781 & 20 & 0.827 & 123 & 0.702 & 0.769 & 0.72 & 214.07 & 313.38 & 0.67 & 160.54 & 0.38 \\
\hline 125,788 & 3,138 & 541 & 1.5 & 7.1 & 1.4 & 32 & 8,032 & 000 & 0.0674 & & 758 & 32 & 0.780 & 62 & 672 & 746 & .85 & 08.99 & 30 & .14 & 8.46 & .68 \\
\hline 167,240 & 63,085 & 510 & 1.0 & 7.4 & 1.6 & 37 & 7,992 & 3,826 & 0.0091 & 193 & 0.808 & 13 & 0.851 & 90 & 0.705 & 0.816 & 0.87 & 470.92 & 464.01 & 1.51 & 98.86 & 0.94 \\
\hline 165,864 & 65,147 & 6,295 & 2.0 & 10.0 & 2.0 & 36 & 6,238 & 678 & 0.0924 & 203 & .757 & 27 & 0.757 & 97 & 0.681 & 0.733 & 0.70 & 426.12 & 6.80 & .71 & 23.81 & .71 \\
\hline 23,392 & 8,354 & 940 & 1.0 & 8.6 & 1.9 & 18 & 7,439 & 3,209 & 0.0858 & 180 & 0.780 & 22 & 0.829 & 93 & 0.701 & 0.775 & 0.78 & 229.05 & 298.26 & 0.79 & 181.74 & 0.41 \\
\hline 6,043 & 3,564 & ,030 & 2.0 & 6.4 & 1.1 & 23 & 6,027 & 2,390 & 0.0141 & 227 & .752 & 24 & 0.758 & 86 & .671 & & 0.82 & 280.71 & 1.86 & 0.83 & 4.43 & 0.50 \\
\hline 193,972 & 75,701 & 392 & 2.0 & 10.0 & 2.0 & 36 & 7,747 & 3,384 & 0.0010 & 223 & 0.769 & 30 & 0.780 & 115 & 0.666 & 0.737 & 0.84 & 546.36 & 633.61 & 1.52 & \begin{tabular}{|l|}
87.16 \\
\end{tabular} & 0.77 \\
\hline 65,720 & 24,467 & 578 & 2.0 & 10.0 & 2.0 & 28 & 7,247 & 3,247 & 0.0924 & 222 & .752 & 25 & 0.831 & 68 & .652 & 0.755 & 0.88 & 334.35 & 9.66 & 1.37 & \begin{tabular}{|l|}
172.05 \\
\end{tabular} & 0.64 \\
\hline 151,075 & 66,255 & 301 & 1.5 & 8.3 & 1.7 & 35 & 6,878 & 2,124 & 0.0923 & 203 & .800 & 26 & 0.800 & 96 & 0.728 & 0.800 & .63 & 434.00 & 548.57 & 1.30 & \begin{tabular}{|r|}
11.58 \\
\end{tabular} & 0.66 \\
\hline 80,822 & 3,791 & 939 & 1.5 & 8.7 & 1.8 & 28 & 6,584 & 3,085 & 0.0072 & & .781 & 33 & 0.822 & 95 & 0.708 & 0.774 & 0.88 & 330.06 & 280.09 & 1.13 & 177.56 & 0.57 \\
\hline 76,245 & 30,990 & 828 & 2.0 & 9.2 & 1.8 & 26 & 5,931 & 2,488 & 0.0403 & 202 & .816 & 23 & 0.857 & 116 & 0.715 & 0.808 & 0.83 & 335.30 & 449.95 & 1.24 & 242.67 & 0.60 \\
\hline 156,790 & 58,927 & 116 & 1.5 & 7.5 & 1.5 & 36 & 5,969 & 2,619 & 0.0404 & 203 & 0.775 & 19 & 0.797 & 66 & 0.68 & 0.767 & 0.85 & 407.95 & 630.52 & 1.27 & 41.54 & 0.68 \\
\hline 128,118 & 54,073 & 628 & 2.0 & 8.0 & 1.5 & 32 & 5,819 & 2,207 & 0.0084 & 203 & 0.753 & 14 & 0.773 & 131 & 0.659 & 0.730 & 0.84 & 397.59 & 561.63 & 1.21 & \begin{tabular}{|l|}
193.72 \\
\end{tabular} & 0.64 \\
\hline 173,776 & 4,112 & 6,229 & 2.0 & 9.2 & 1.8 & 37 & 5,277 & 2,053 & 0.0258 & 206 & 0.740 & 14 & 0.816 & 66 & 0.681 & 0.766 & 0.89 & 384.72 & 626.63 & 1.71 & 101.34 & 0.7 \\
\hline 170,423 & 5,942 & 6,530 & 1.5 & 8.7 & 1.8 & 38 & 5,952 & 3,040 & 0.0462 & 239 & 0.800 & 33 & 0.858 & 109 & 0.716 & 0.806 & 0.90 & 432.00 & 579.46 & 1.59 & \begin{tabular}{|l|} 
\\
280.77 \\
\end{tabular} & 0.8 \\
\hline 40,758 & 14,899 & 616 & 1.0 & 6.2 & 1.3 & 22 & 6,312 & 3,040 & 0.0095 & 188 & 0.723 & 22 & 0.742 & 134 & 0.65 & .714 & 0.79 & 256.34 & 389.98 & 0.70 & 145.18 & 0.4 \\
\hline 73,420 & 31,104 & 2,645 & 2.0 & 8.8 & 1.7 & 30 & 5,045 & 2,445 & 0.0192 & 194| & 0.763 & 19 & 0.799 & 77 & 0.685 & 0.745 & 0.91 & 288.43 & 469.30 & 1.24 & $\mid$ & 0.5 \\
\hline
\end{tabular}

Table A-2: Data Generated for Six Stage Treatment, continued 


\begin{tabular}{|c|c|c|c|c|c|c|c|c|c|c|c|c|c|c|c|c|c|c|c|c|c|c|}
\hline 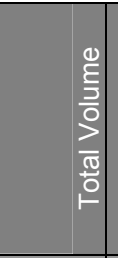 & 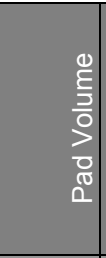 & 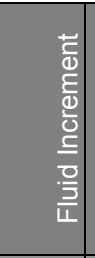 & 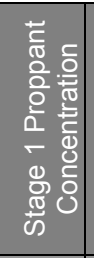 & 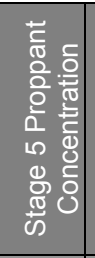 & 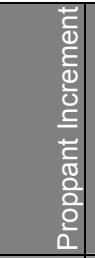 & 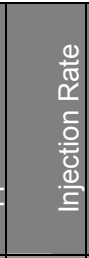 & 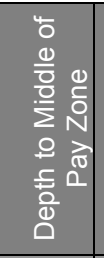 & 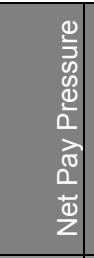 & 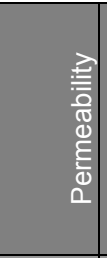 & 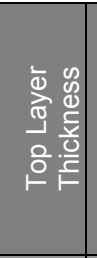 & 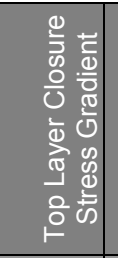 & 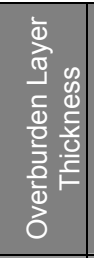 & 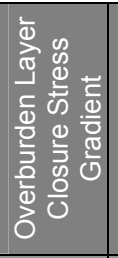 & 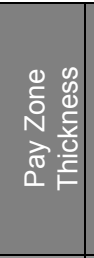 & 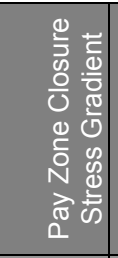 & 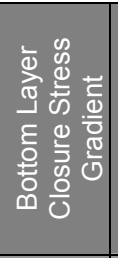 & 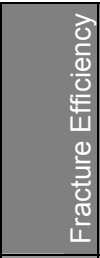 & 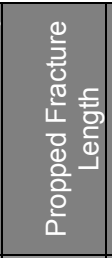 & \begin{tabular}{l|l}
$\frac{7}{0}$ \\
$\frac{0}{0}$ \\
$\frac{1}{1}$ \\
0 \\
$\frac{0}{3}$ \\
$\frac{0}{0}$ \\
$\frac{\pi}{4}$ \\
$\frac{1}{1}$
\end{tabular} & 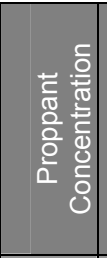 & 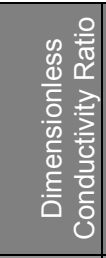 & . \\
\hline input & input & input & input & input & input & input & input & input & input & input & input & input & input & input & input & input & output & output & iutput & output & output & output \\
\hline 73,420 & 1,104 & 2,645 & 2.0 & 8.8 & 1.7 & 30 & 5,045 & 2,445 & 0.0400 & 194 & 0.763 & 19 & 0.799 & 77 & 0.65 & 0.745 & 0.82 & 07.10 & 46.08 & \begin{tabular}{|l|}
1.23 \\
\end{tabular} & 65.76 & \\
\hline 99,827 & 3,104 & 3,545 & 2.0 & 8.4 & 1.6 & 29 & 016 & 4,061 & 0.0034 & 224 & 0.748 & 12 & 0.749 & 100 & 0.666 & 0.732 & 0.92 & 71.16 & 92.04 & 1.18 & 246.72 & \\
\hline 24,563 & 9,068 & 968 & 1.0 & 6.6 & 1.4 & 18 & 5,838 & 2,804 & 0.0109 & 205 & .778 & 33 & 0.805 & 121 & 0.664 & 0.751 & 0.72 & 219.33 & 387.03 & 0.69 & 152.90 & \\
\hline 29,011 & 10,831 & 1,136 & 2.0 & 6.4 & 1.1 & 17 & 7,463 & 3,211 & 0.0436 & 208 & 0.750 & 15 & 0.781 & 106 & 0.651 & 0.736 & 0.48 & 243.38 & \begin{tabular}{|l|}
304.04 \\
\end{tabular} & 0.70 & 27.44 & \\
\hline 45,485 & 8,843 & 665 & 1.5 & 6.7 & 1.3 & 22 & 8,168 & 3,370 & 0.0062 & 234 & 0.804 & 16 & 0.850 & 75 & 0.686 & 0.809 & 0.82 & 330.99 & 311.47 & 0.77 & 96.46 & .U \\
\hline 118,445 & 0,784 & 229 & 1.5 & 9.5 & 2.0 & 31 & 6,147 & 134 & 0.0775 & 237 & 0.765 & 30 & 0.794 & 74 & .672 & 0.744 & 0.73 & 373.14 & 39.54 & 1.39 & 24.17 & \\
\hline 83,545 &, 755 & 3,237 & 1.5 & 9.5 & 2.0 & 27 & 5,398 & 2,439 & 0.0383 & 208 & 0.753 & 19 & 0.781 & 115 & 0.651 & 0.725 & .73 & 323.49 & $475.64 \mid$ & 1.39 & 77.31 & 0 \\
\hline 19,623 & 309 & 707 & 1.0 & 6.6 & 1.4 & 16 & 6,334 & 2,654 & 0.0915 & 179 & 0.795 & 28 & 0.832 & 103 & 0.674 & 0.774 & 0.27 & 175.87 & 241.62 & 0.67 & 24.32 & 0.28 \\
\hline 54,204 & ,647 & 2,035 & 1.5 & 7.5 & 1.5 & 26 & 8,132 & 385 & 0.0075 & 234 & 0.774 & 22 & 0.808 & 128 & 0.705 & 0.786 & 0.77 & 306.46 & 397.35 & 0.89 & 02.08 & .4 \\
\hline 17,043 & 043 & 625 & 1.0 & 7.0 & 1.5 & 15 & 6,874 & 2,809 & 0.0062 & 220 & 0.749 & 13 & 0.770 & 111 & 0.677 & 0.733 & 0.73 & 66.04 & 50.38 & 0.64 & 205.28 & .33 \\
\hline 29,981 & ,408 & 161 & 2.0 & 10.0 & 2.0 & 19 & ,419 & 757 & 0.0758 & 182 & .746 & 11 & 0.788 & 105 & 671 & 0.727 & .58 & 189.92 & 32.43 & .31 & 1.92 & 0.4 \\
\hline 28,479 & 1,364 & 070 & 2.0 & 7.2 & 1.3 & 17 & 7,626 & 3,730 & 0.0099 & 222 & 0.793 & 11 & 0.828 & 98 & 0.707 & 0.760 & 0.75 & 230.79 & 09.33 & 0.79 & 95.09 & 0.42 \\
\hline 26,935 & 10,354 & 1,036 & 1.5 & 7.5 & 1.5 & 18 & 7,916 & 2,210 & 0.0276 & 173 & 0.764 & 31 & 0.765 & 80 & 0.657 & 0.731 & 0.62 & 241.67 & 82.74 & 0.80 & 38.33 & 0.42 \\
\hline 12,118 & 4,815 & 456 & 1.0 & 9.0 & 2.0 & 15 & 6,541 & 2,605 & 0.0049 & 187 & 0.725 & 21 & 0.736 & 84 & 0.657 & 0.717 & 0.80 & 148.18 & 223.92 & 0.75 & 392.73 & 0.32 \\
\hline 40,314 & ,344 & 561 & 1.5 & 7.5 & 1.5 & 30 & 6,412 & 965 & 0137 & 186 & .756 & 30 & 0.807 & 85 & 0.674 & 0.752 & .80 & 73.09 & 373.46 & .82 & 2.21 & .46 \\
\hline 67,309 & 23,720 & 724 & 1.5 & 8.3 & 1.7 & 25 & 8,021 & 3,318 & 0.0442 & 218 & 0.809 & 30 & 0.817 & 62 & 0.688 & 0.810 & 0.70 & 354.87 & 393.41 & 1.14 & 23.64 & 0.63 \\
\hline 74,247 & 7,644 & 913 & 2.0 & 6.8 & 1.2 & 27 & 5,971 & 514 & 197 & 176 & .752 & 13 & 0.780 & 133 & .676 & 0.756 & 0.74 & 23.46 & .70 & 0.96 & 4.89 & .53 \\
\hline 77,596 & 29,415 & 3,011 & 1.0 & 8.2 & 1.8 & 28 & 6,561 & 2,489 & 0.0087 & 179 & 0.763 & 12 & 0.794 & 60 & 0.665 & 0.724 & 0.91 & 331.56 & 489.42 & 1.04 & 153.64 & 0.56 \\
\hline 74,783 & ,752 & 689 & 1.5 & 8.7 & 1.8 & 26 & 7,939 & 3,368 & 0.0901 & 230 & 789 & 29 & 0.820 & 88 & .677 & 40 & 0.53 & 361.95 & 396.10 & 1.14 & 12.06 & 0.56 \\
\hline 74,783 & 31,752 & 2,689 & 1.5 & 8.7 & 1.8 & 26 & 7,939 & 3,368 & 0.0100 & 230 & 0.770 & 29 & 0.800 & 88 & 0.7 & 0.760 & 0.83 & 332.82 & \begin{tabular}{|l|}
461.08 \\
\end{tabular} & 1.07 & 86.67 & 0.55 \\
\hline 37,409 & 4,262 & 447 & 2.0 & 10.0 & 2.0 & 20 & 6,580 & 3,103 & 0.0081 & 223 & .742 & 25 & 0.754 & 71 & 0.664 & 0.720 & 0.87 & 241.84 & 364.50 & 1.17 & 262.48 & 0.50 \\
\hline 182,362 & 74,303 & 6,754 & 2.0 & 8.8 & 1.7 & 38 & 6,110 & 2,801 & 0.0076 & 178 & 0.753 & 22 & 0.782 & 86 & 0.673 & 0.754 & 0.92 & 414.71 & 660.74 & 1.57 & 241.17 & 0.71 \\
\hline 12,037 & 4,549 & 468 & 1.0 & 5.8 & 1.2 & 15 & 6,004 & 2,351 & 0.0074 & 224 & 0.778 & 35 & 0.815 & 50 & 0.699 & 0.744 & 0.84 & 154.83 & 233.64 & 0.46 & 156.52 & 0.28 \\
\hline 176,221 & 65,616 & 6,913 & 2.0 & 6.0 & 1.0 & 38 & 6,181 & 2,819 & 0.0269 & 174 & 0.769 & 12 & 0.788 & 132 & 0.65 & 0.735 & 0.77 & 510.13 & 617.37 & 1.01 & 39.30 & 0.68 \\
\hline 103,796 & 40,077 & 3,982 & 1.0 & 8.2 & 1.8 & 30 & 5,976 & 2,589 & 0.0080 & 203 & 0.784 & 31 & 0.785 & 99 & 0.683 & 0.770 & 0.87 & 382.27 & 526.50 & 1.11 & 3.34 & 0.64 \\
\hline 130,770 & 50,270 & 5,031 & 2.0 & 7.6 & 1.4 & 33 & 6,698 & 3,006 & 0.0283 & 173 & 0.761 & 12 & 0.805 & 73 & 0.69 & 0.740 & 0.85 & 370.49 & 555.72 & 1.37 & 3.04 & 0.69 \\
\hline 92,719 & 40,469 & 3,266 & 1.0 & 8.6 & 1.9 & 30 & 5,343 & 2,446 & 0.0050 & 222 & 0.799 & 16 & 0.829 & 120 & 0.693 & 0.800 & 0.89 & 340.31 & 472.48 & 1.19 & 376.67 & 0.6 \\
\hline 54,421 & 19,628 & 2,175 & 1.0 & 6.6 & 1.4 & 22 & 6,739 & 2,925 & 0.0089 & 170 & 0.748 & 34 & 0.788 & 109 & 0.654 & 0.720 & 0.80 & 323.63 & 417.65 & 0.74 & 114.95 & $0.4 \mathrm{~S}$ \\
\hline 33,924 & 13,547 & 1,274 & 1.0 & 5.4 & 1.1 & 17 & 7,482 & 3,284 & & 174 & 0.750 & 23 & 0.774 & 97 & 0.657 & 0.738 & 0.56 & & & 0.46 & 16.71 & 0.3 \\
\hline 143,695 & 55,173 & 5,533 & 2.0 & 6.8 & 1.2 & 35 & 7,794 & 1,854 & 0.0099 & 176 & 0.792 & 19 & 0.824 & 64 & 0.671 & 0.768 & 0.90 & 508.65 & 510.95 & 1.08 & 62.62 & 0.7 \\
\hline
\end{tabular}

Table A-2: Data Generated for Six Stage Treatment, continued 


\begin{tabular}{|c|c|c|c|c|c|c|c|c|c|c|c|c|c|c|c|c|c|c|c|c|c|c|}
\hline \begin{tabular}{l|}
$\frac{0}{5}$ \\
$\frac{3}{0}$ \\
$\frac{1}{0}$ \\
$\frac{\pi}{0}$ \\
$\frac{0}{0}$
\end{tabular} & 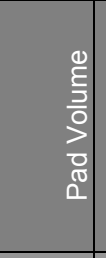 & 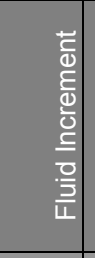 & 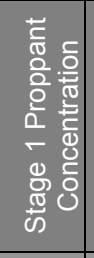 & 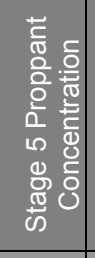 & 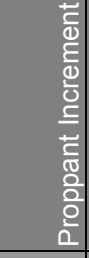 & 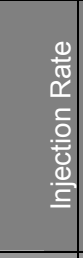 & 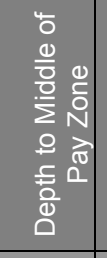 & \begin{tabular}{l|l}
$\frac{0}{3}$ & \\
0 & \\
0 & \\
$\frac{0}{2}$ & \\
$\frac{\pi}{0}$ & \\
$\frac{1}{0}$ & \\
$\frac{\Phi}{2}$ &
\end{tabular} & 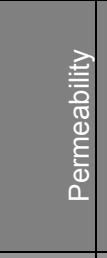 & 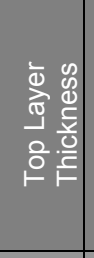 & 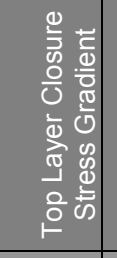 & 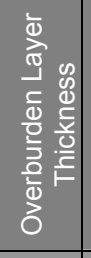 & 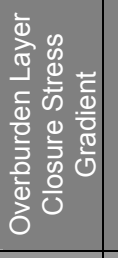 & 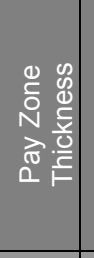 & 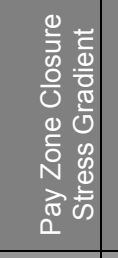 & 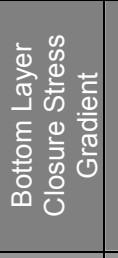 & 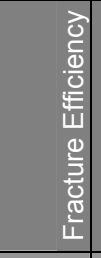 & 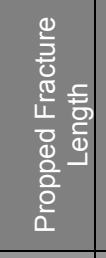 & 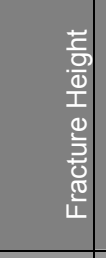 & 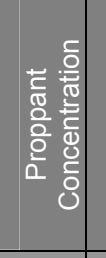 & 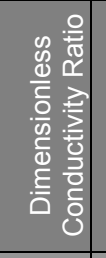 & \\
\hline input & input & input & input & input & input & input & input & input & input & input & put & input & input & input & input & input & output & output & utput & output & output & \\
\hline 190,722 & 75,303 & 7,214 & 2.0 & 10.0 & 2.0 & 40 & 7,904 & 130 & 0.0172 & 221 & 0.819 & 31 & 0.849 & 77 & 0.652 & 0.805 & 0.84 & 499.38 & 9.19 & 1.87 & 62.05 & 89 \\
\hline 102,916 & 37,532 & 087 & 1.5 & 7.5 & 1.5 & 30 & 6,382 & 678 & 0074 & 208 & 0.767 & 16 & 0.808 & 83 & 0.686 & 0.774 & 0.90 & 341.35 & 532.83 & 1.20 & 17.63 & - \\
\hline 102,916 & 37,532 & 087 & 1.5 & 7.5 & 1.5 & 30 & 6,382 & 678 & 0074 & 208 & 0.767 & 16 & 0.808 & 83 & 0.658 & 0.750 & 0.89 & 391.92 & 495.43 & 1.13 & 89.30 & 67 \\
\hline 60,037 & 25,382 & 2,166 & 1.0 & 8.2 & 1.8 & 23 & 6,742 & 2,947 & 0.0087 & 231 & 0.770 & 27 & 0.771 & 126 & 0.668 & 0.755 & 0.79 & 306.90 & 391.64 & 1.01 & 148.81 & \\
\hline 17,300 & 6,490 & 676 & 1.0 & 5.8 & 1.2 & 15 & 6,943 & 2,500 & 0123 & 171 & 0.750 & 25 & 0.764 & 91 & 0.69 & 0.723 & .67 & 175.74 & 252.31 & 0.54 & 91.76 & 32 \\
\hline 94,185 & 36,819 & 585 & 1.5 & 9.5 & 2.0 & 30 & 6,047 & 2,523 & 0.0048 & 195 & 0.770 & 23 & 0.799 & 116 & 0.682 & 0.767 & 0.88 & 346.59 & 489.83 & 1.40 & 19.02 & 67 \\
\hline 184,760 & 2,680 & 005 & 1.5 & 8.3 & 1.7 & 21 & 7,451 & $157 \mid$ & 0054 & 187 & 0.738 & 18 & 0.761 & 106 & 0.65 & 0.716 & 0.90 & 481.12 & 610.75 & 1.39 & 31.20 & 77 \\
\hline 80,585 & 31,636 & 3,059 & 2.0 & 8.4 & 1.6 & 28 & 7,108 & 2,793 & 0.0645 & 215 & 0.765 & 32 & 0.782 & 108 & 0.671 & 0.746 & 0.58 & 342.85 & 441.89 & 1.23 & 24.15 & .57 \\
\hline 19,227 & 7,247 & 749 & 2.0 & 6.8 & 1.2 & 17 & 6,802 & 2,496 & 0926 & 193 & 0.755 & 31 & 0.755 & 85 & 0.683 & 0.722 & 0.40 & 167.75 & 285.93 & 0.80 & 4.26 & 30 \\
\hline 158,466 & 61,272 & 6,075 & 1.5 & 6.3 & 1.2 & 36 & 6,822 & $2,621 \mid$ & 0.0186 & 183 & 0.763 & 34 & 0.784 & 111 & 0.667 & 0.740 & 0.79 & 489.12 & 599.36 & 0.95 & 44.02 & ).65 \\
\hline 175,812 & 6,924 & 181 & 1.5 & 7.9 & 1.6 & 39 & 5,899 & 2,268 & 0091 & 224 & 0.784 & 26 & 0.817 & 61 & 0.675 & 0.758 & 0.93 & 422.37 & 582.24 & 1.41 & 74.33 & 76 \\
\hline 49,123 & 21,449 & 1,730 & 1.0 & 6.6 & 1.4 & 23 & 6,620 & 3,011 & 0.0517 & 175 & 0.759 & 15 & 0.791 & 106 & 0.675 & 0.764 & 0.60 & 279.11 & 383.63 & 0.74 & 23.15 & ).43 \\
\hline 125,620 & 6,814 & 925 & 2.0 & 7.6 & 1.4 & 35 & 7,408 & 3,585 & 0546 & 228 & 0.784 & 20 & 0.798 & 63 & .665 & 0.760 & 0.80 & 419.46 & 500.30 & 1.31 & 20.24 & .74 \\
\hline 103,795 & 45,303 & 3,656 & 1.5 & 9.1 & 1.9 & 31 & 7,482 & 3,826 & 0.0133 & 227 & 0.772 & 23 & 0.799 & 115 & 0.676 & 0.756 & 0.81 & 400.58 & 493.74 & 1.17 & 66.24 & 0.62 \\
\hline 175,513 & 61,468 & 128 & 1.0 & 7.8 & 1.7 & 40 & 7,392 & 2,985 & 0.0558 & 203 & 0.811 & 32 & 0.851 & 115 & 0.714 & 0.793 & 0.64 & 513.16 & 600.34 & 1.07 & 3.52 & 65 \\
\hline 30,719 & 13,344 & 1,086 & 1.0 & 7.8 & 1.7 & 24 & 6,246 & 2,416 & 0.0706 & 211 & 0.767 & 20 & 0.797 & 113 & 0.685 & 0.730 & 0.75 & 207.38 & 318.09 & 0.88 & 161.94 & 0.42 \\
\hline 65,662 & 28,008 & 2,353 & 2.0 & 9.2 & 1.8 & 28 & 5,505 & 2,513 & 0.0082 & 188 & 0.797 & 22 & 0.822 & 84 & 0.723 & 0.788 & 0.89 & 279.10 & 447.32 & 1.25 & 271.64 & .57 \\
\hline 65,662 & 28,008 & 353 & 2.0 & 9.2 & 1.8 & 28 & 5,505 & 2,513 & 0.0082 & 188 & 0.797 & 22 & 0.822 & 84 & 0.69 & 0.788 & 0.88 & 304.79 & 422.80 & 1.21 & 256.28 & .59 \\
\hline 140,431 & 1,570 & 929 & 1.5 & 5.9 & 1.1 & 35 & 5,842 & 813 & 0715 & 193 & 0.779 & 31 & 0.779 & 127 & 682 & 0.779 & 0.66 & 406.83 & 9.12 & 0.94 & 18.10 & 61 \\
\hline 195,170 & 4,115 & 566 & 1.0 & 6.6 & 1.4 & 29 & 6,855 & 2,941 & 0.0481 & 193 & 758 & 32 & 0.791 & 89 & 0.671 & 0.747 & 0.86 & 468.39 & 601.68 & 1.23 & 59.86 & .79 \\
\hline 61,918 & 22,340 & 474 & 1.5 & 8.3 & 1.7 & 27 & 7,555 & 2,998 & 0.0750 & 211 & 0.764 & 12 & 0.781 & 51 & 0.656 & 0.727 & 0.91 & 326.66 & 448.35 & 0.98 & 125.72 & 0.55 \\
\hline 104,873 & 44,650 & 3,764 & 2.0 & 7.6 & 1.4 & 30 & 5,537 & 2,209 & 0.0423 & 207 & 0.817 & 30 & 0.817 & 135 & 0.684 & 0.800 & 0.62 & 390.89 & 469.38 & 1.15 & 42.93 & .62 \\
\hline 98,393 & 34,991 & 3,963 & 1.0 & 6.2 & 1.3 & 29 & 8,744 & 4,155 & 0.0066 & 201 & 0.752 & 18 & 0.780 & 56 & 0.683 & 0.758 & 0.93 & 363.25 & 600.10 & 0.79 & 176.43 & 0.50 \\
\hline 17,692 & 7,655 & 627 & 1.5 & 9.9 & 2.1 & 15 & 9,481 & 3,739 & 0.0095 & 202 & 0.790 & 15 & 0.852 & 58 & 0.73 & 0.782 & 0.75 & 196.85 & 265.41 & 0.75 & 86.75 & 34 \\
\hline 76,983 & 32,181 & 800 & 2.0 & 10.0 & 2.0 & 26 & 11,754 & 5,377 & 0.0081 & 205 & 0.755 & 14 & 0.804 & 99 & .676 & 0.723 & 0.78 & 396.46 & 425.44 & $1.17 \mid$ & 56.21 & .58 \\
\hline 106,730 & 39,066 & 4,229 & 1.0 & 7.0 & 1.5 & 30 & 9,789 & 3,776 & 0.0315 & 216 & 0.799 & 13 & 0.799 & 51 & 695 & 0.776 & 0.80 & 453.45 & 491.91 & 0.92 & 4.32 & 64 \\
\hline 174,450 & 68,218 & 6,640 & 2.0 & 8.4 & 1.6 & 39 & 9,818 & 3,832 & 0.0097 & 224 & 0.739 & 26 & 0.786 & 134 & 0.669 & 0.718 & 0.79 & 535.73 & 647.56 & 1.17 & 64.99 & ).66 \\
\hline 115,180 & 46,996 & 4,262 & 1.5 & 7.5 & 1.5 & 32 & 10,743 & 4,674 & 0.0248 & 224 & 0.740 & 30 & 0.740 & 98 & .654 & 0.729 & 0.70 & 487.21 & 470.12 & 0.99 & 20.88 & 0.64 \\
\hline 40,712 & 15,377 & 1,583 & 2.0 & 10.0 & 2.0 & 22 & 9,501 & 3,532 & 0.0099 & 174 & 741 & 31 & 0.779 & 107 & 678 & 0.746 & 0.70 & 287.81 & 1.72 & 1.11 & 120.75 & 4 \\
\hline $79,981 \mid$ & 33,003 & 2,936 & 1.0 & 7.0 & 1.5 & 28| & 11,274 & $4,961 \mid$ & 0.0487 & 226 & 0.748 & 18 & 0.799 & 56 & 0.688 & 0.740 & 0.75 & 352.92 & 456.68 & 0.88 & 9.78 & \\
\hline
\end{tabular}

Table A-2: Data Generated for Six Stage Treatment, continued 


\begin{tabular}{|c|c|c|c|c|c|c|c|c|c|c|c|c|c|c|c|c|c|c|c|c|c|c|}
\hline 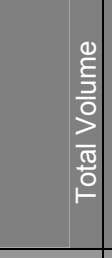 & $\begin{array}{l}\stackrel{0}{E} \\
\frac{3}{3} \\
0 \\
\frac{0}{\sigma} \\
0 \\
0\end{array}$ & 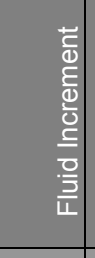 & 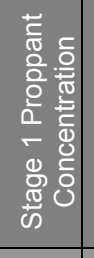 & 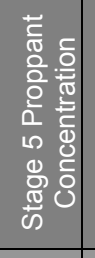 & 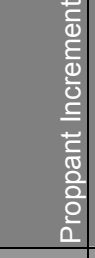 & 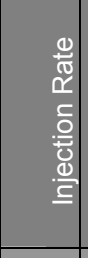 & 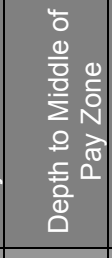 & 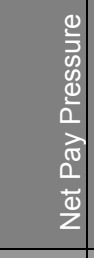 & 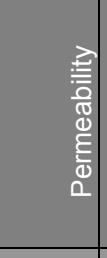 & 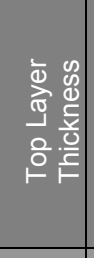 & 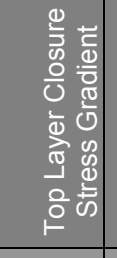 & 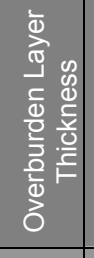 & 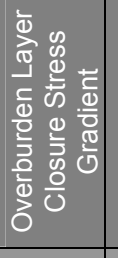 & 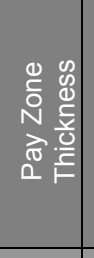 & 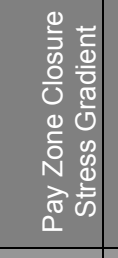 & 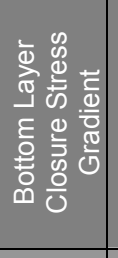 & 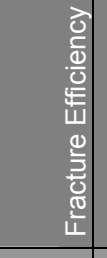 & 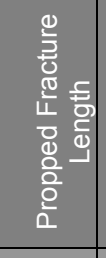 & 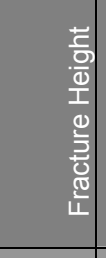 & 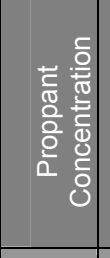 & 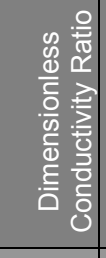 & \\
\hline input & input & input & input & input & input & input & input & input & input & input & input & input & input & input & input & input & output & output & output & output & output & output \\
\hline 169,657 & 69,956 & 6,231 & 1.5 & 9.5 & 2.0 & 38 & 10,776 & 4,427 & 0.0088 & 193 & 0.761 & 32 & 0.800 & 84 & 0.703 & 0.755 & 0.86 & 471.56 & 551.20 & 1.61 & 77.41 & .76 \\
\hline 169,657 & 69,956 & 6,231 & 1.5 & 9.5 & 2.0 & 38 & 10,776 & 4,427 & 0150 & 193 & 0.761 & 32 & 0.786 & 84 & 0.68 & 0.750 & 0.80 & 564.15 & 533.71 & 1.39 & 36.59 & .74 \\
\hline 155,303 & 63,736 & 5,723 & 1.0 & 7.0 & 1.5 & 35 & $\mid 10,892$ & 3,859 & 0.0698 & 202 & 0.810 & 27 & 0.833 & 75 & 0.723 & 0.799 & 0.57 & 579.04 & 525.53 & 0.91 & 4.76 & 62 \\
\hline 107,413 & 37,779 & 4,352 & 1.0 & 6.6 & 1.4 & 29 & 11,788 & 5,208 & 0.0174 & 195 & 0.800 & 28 & 0.839 & 97 & 0.739 & 0.785 & 0.70 & 514.06 & 553.67 & 0.70 & 12.51 & 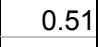 \\
\hline 190,522 & 67,270 & ,703 & 1.5 & 7.5 & 1.5 & 40 & 8,847 & 4,372 & 0.0046 & 230 & 0.763 & 34 & 0.793 & 85 & 0.67 & 0.735 & 0.92 & 564.56 & 632.38 & 1.16 & 47.57 & .73 \\
\hline 51,669 & 22,597 & 1,817 & 1.0 & 6.2 & 1.3 & 26 & $\mid 10,019$ & 4,941 & 0064 & 224 & 0.770 & 28 & 0.813 & 124 & 0.678 & 0.772 & 0.74 & 386.34 & 307.66 & 0.66 & 61.47 & .52 \\
\hline 125,339 & 51,044 & 4,643 & 1.5 & 8.7 & 1.8 & 30 & \begin{tabular}{|c|}
10,997 \\
\end{tabular} & ,842 & 0757 & 221 & 0.761 & 34 & 0.806 & 126 & 0.672 & 0.785 & 0.33 & 543.63 & 158.15 & 1.57 & $\mid 1.19$ & 63 \\
\hline 136,108 & 51,350 & 5,297 & 2.0 & 10.0 & 2.0 & 34 & 10,806 & 5,350 & 0.0085 & 212 & 0.784 & 24 & 0.784 & 128 & 0.71 & 0.751 & 0.81 & 453.06 & 579.51 & 1.43 & 70.30 & 1.68 \\
\hline 127,366 & 45,048 & 5,145 & 1.0 & 9.0 & 2.0 & 32 & 9,012 & ,862 & 0155 & 197 & 0.800 & 28 & 0.851 & 80 & 0.734 & 0.800 & 0.83 & 403.09 & 503.06 & 1.55 & 75.41 & .76 \\
\hline 101,532 & 40,464 & 3,817 & 2.0 & 10.8 & 2.2 & 29 & \begin{tabular}{|c|}
$\mid 10,784$ \\
\end{tabular} & 4,332 & 0.0230 & 206 & 0.817 & 10 & 0.844 & 78 & 0.721 & 0.800 & 0.69 & 483.57 & 457.86 & 1.32 & 23.78 & 0.66 \\
\hline 14,980 & 5,432 & 597 & 1.5 & 7.5 & 1.5 & 15 & 9,591 & ,430 & 0098 & 175 & 0.762 & 24 & 0.806 & 67 & 0.665 & 0.739 & 0.69 & 242.94 & 221.59 & 0.60 & 77.73 & 36 \\
\hline 12,718 & 4,621 & 506 & 1.5 & 7.1 & 1.4 & 16 & 9,723 & 3,755 & 0.0266 & 174 & 0.808 & 25 & 0.831 & 78 & 0.708 & 0.803 & 0.36 & 213.55 & 187.86 & 0.65 & 32.81 & ).34 \\
\hline 25,765 & 10,219 & 972 & 1.0 & 6.2 & 1.3 & 20 & 8,591 & 363 & 0.0078 & 197 & 0.752 & 28 & 0.781 & 57 & 0.686 & 0.725 & 0.87 & 218.02 & 324.50 & 0.53 & 92.87 & 35 \\
\hline 25,765 & 10,219 & 972 & 1.0 & 6.2 & 1.3 & 20 & 8,591 & 4,363 & 0.0078 & 197 & 0.752 & 28 & 0.770 & 57 & 0.667 & 0.746 & 0.86 & 259.14 & 307.86 & 0.53 & 88.96 & 0.41 \\
\hline 67,586 & 24,022 & 2,723 & 1.0 & 8.2 & 1.8 & 25 & 9,831 & 888 & 0.0113 & 232 & 0.805 & 18 & 0.853 & 79 & 0.737 & 0.794 & 0.81 & 360.56 & 451.87 & 0.93 & 54.52 & .54 \\
\hline 98,358 & 37,011 & 3,834 & 1.0 & 7.0 & 1.5 & 28 & $9,851 \mid$ & 3,936 & 0.0207 & 188 & 0.738 & 31 & 0.761 & 105 & 0.656 & 0.718 & 0.69 & 475.44 & 491.76 & 0.80 & 25.35 & 0.56 \\
\hline 62,729 & 25,364 & 2,335 & 2.0 & 10.8 & 2.2 & 26 & $|10,087|$ & 556 & 0479 & 219 & 0.760 & 21 & 0.796 & 106 & 0.699 & 0.748 & 0.56 & 304.68 & 404.42 & 1.45 & 27.96 & .54 \\
\hline 12,992 & 5,007 & 499 & 1.0 & 7.4 & 1.6 & 16 & 9,127 & 4,492 & 0.0049 & 191 & 0.810 & 19 & 0.810 & 51 & 0.714 & 0.780 & 0.84 & 188.21 & 218.95 & 0.54 & 131.15 & 0.34 \\
\hline 76,874 & 28,395 & 030 & 1.5 & 7.9 & 1.6 & 24 & \begin{tabular}{|l|}
10,777 \\
\end{tabular} & ,423 & 0098 & 192 & 0.758 & 25 & 0.775 & 132 & 0.663 & 0.731 & 0.66 & 465.96 & 350.15 & 1.04 & 57.37 & .60 \\
\hline 123,582 & 48,891 & 668 & 1.5 & 9.5 & 2.0 & 33 & \begin{tabular}{|l|}
10,609 \\
\end{tabular} & 4,845 & 0.0927 & 211 & 0.800 & 30 & 0.851 & 86 & 0.713 & 0.786 & 0.51 & 527.42 & 492.73 & 1.19 & 6.27 & 0.61 \\
\hline 181,340 & 71,809 & 6,846 & 2.0 & 10.0 & 2.0 & 40 & 10,292 & 4,738 & 0.0293 & 180 & 0.730 & 24 & 0.751 & 113 & 0.663 & 0.717 & 0.73 & 505.65 & 593.64 & 1.63 & 31.05 & 0.74 \\
\hline 181,624 & 65,899 & 7,233 & 1.5 & 8.3 & 1.7 & 38 & 11,728 & 3,881 & 0.0053 & 201 & 0.796 & 31 & 0.841 & 105 & 0.727 & 0.786 & 0.82 & 606.38 & 8.88 & 1.18 & 58.76 & 0.71 \\
\hline 74,044 & 29,683 & 2,773 & 1.5 & 7.9 & 1.6 & 28 & 11,079 & 4,805 & 0.0062 & 237 & 0.762 & 34 & 0.752 & 103 & 0.68 & 0.750 & 0.79 & 433.05 & 403.49 & 0.89 & 63.61 & 0.58 \\
\hline 69,767 & 27,882 & 2,618 & 1.5 & 8.3 & 1.7 & 26 & 8,434 & 3,508 & 0.0056 & 195 & 0.760 & 18 & 0.786 & 93 & 0.69 & 0.738 & 0.86 & 335.39 & 467.31 & 0.98 & 175.36 & 0.53 \\
\hline 151,126 & 59,569 & 5,722 & 1.0 & 8.2 & 1.8 & 35 & 11,681 & 3,967 & 0.0092 & 228 & 0.737 & 27 & 0.772 & 76 & 0.675 & 0.741 & 0.85 & 458.13 & 476.44 & 1.55 & 66.90 & 0.83 \\
\hline 40,321 & 15,786 & 1,533 & 1.0 & 6.6 & 1.4 & 21 & 9,361 & 3,733 & 0.0169 & 209 & 0.738 & 20 & 0.757 & 100 & 0.655 & 0.711 & 0.71 & 277.20 & 344.91 & 0.73 & 46.84 & 0.46 \\
\hline 67,758 & 27,749 & 2,501 & 2.0 & 8.4 & 1.6 & 26 & 10,269 & 4,599 & 0.0316 & 201 & 0.796 & 11 & 0.835 & 108 & 0.729 & 0.773 & 0.86 & 350.98 & 455.72 & 0.95 & 162.64 & 0.52 \\
\hline 67,758 & 27,749 & 2,501 & 2.0 & 8.4 & 1.6 & 26 & 10,269 & 4,599 & 0.0100 & 201 & 0.796 & 11 & 0.835 & 108 & 0.7 & 0.773 & 0.72 & 402.11 & 372.42 & 1.02 & 56.25 & 0.59 \\
\hline 197,190 & 72,144 & 7,815 & 1.5 & 7.5 & 1.5 & 18 & 8,508 & 3,858 & 0.0210 & 198 & 759 & 29 & 0.786 & 91 & 0.66 & 0.741 & 0.81 & 573.73 & 588.09 & 1.24 & 43.44| & 0.78 \\
\hline 102,109 & 37,453 & 4,041 & 1.5 & 8.3 & $1.7 \mid$ & 23 & 10,605 & 3,598 & 0.0122 & 222 & 0.788 & 12 & 0.825 & 63 & 0.688 & 0.762 & 0.81| & 487.26 & 484.74 & 1.00 & 37.78 & 0.0 \\
\hline
\end{tabular}

Table A-2: Data Generated for Six Stage Treatment, continued 


\begin{tabular}{|c|c|c|c|c|c|c|c|c|c|c|c|c|c|c|c|c|c|c|c|c|c|c|}
\hline 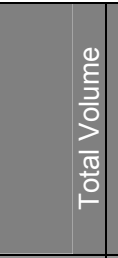 & 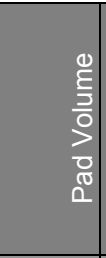 & 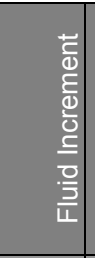 & 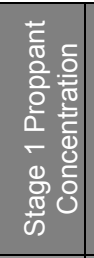 & 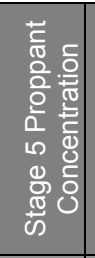 & 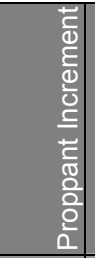 & 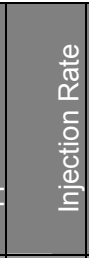 & 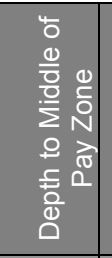 & 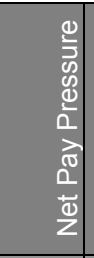 & 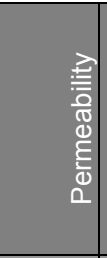 & 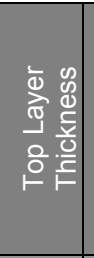 & 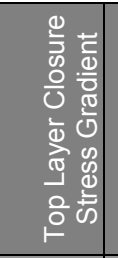 & 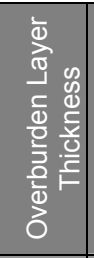 & 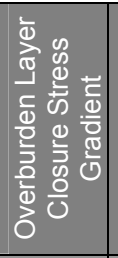 & 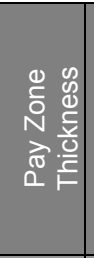 & 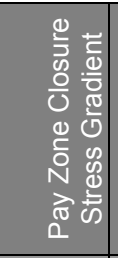 & 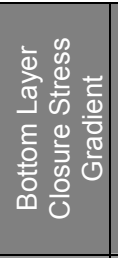 & 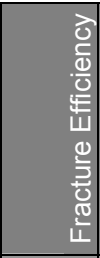 & 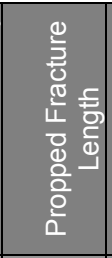 & \begin{tabular}{l|l}
$\frac{7}{0}$ & \\
$\frac{0}{0}$ & \\
$\frac{1}{1}$ & \\
$\frac{0}{3}$ & \\
$\frac{3}{0}$ & \\
$\frac{\pi}{4}$ & \\
$\frac{1}{4}$ &
\end{tabular} & 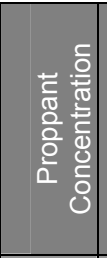 & 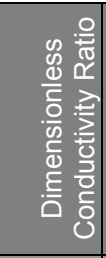 & . \\
\hline input & input & input & input & input & input & input & input & input & input & input & input & input & input & input & input & input & output & output & utput & output & sutput & output \\
\hline 44,601 & 7,227 & 1,711 & 1.0 & 7.0 & 1.5 & 20 & 8,840 & 544 & 0.0065 & 237 & 0.763 & 19 & 0.792 & 73 & 0.676 & 0.742 & 0.83 & 14.60 & 84.57 & 0.69 & 2.81 & \\
\hline 161,192 &, 842 & 647 & 1.0 & 6.2 & 1.3 & 36 & 8,988 & 639 & 0.0151 & 236 & .751 & 31 & 0.789 & 82 & 0.677 & 0.768 & 0.85 & 08.48 & 42.43 & 1.35 & 6.10 & \\
\hline 69,820 & 8,822 & 562 & 1.0 & 7.4 & 1.6 & 24 & ,349 & 4,456 & 0.0661 & 202 & 0.748 & 29 & 0.781 & 94 & 0.666 & 0.752 & .53 & 399.52 & 403.15 & 0.81 & 1.04 & \\
\hline 100,159 & 9,284 & 805 & 1.5 & 6.7 & 1.3 & 30 & 9,844 & 3,882 & 0.0059 & 205 & 0.805 & 32 & 0.857 & 69 & 0.722 & 0.808 & 0.87 & 422.62 & 119.90 & 1.04 & 7.73 & \\
\hline 120,479 & 9,182 & ,456 & 2.0 & 10.8 & 2.2 & 30 & 1,378 & 4,652 & 0.0126 & 206 & 0.763 & 31 & 0.797 & 76 & 0.687 & 0.738 & 0.82 & 428.76 & 565.40 & 1.40 & 46.30 & \\
\hline 157,973 & 3,982 & 874 & 1.0 & 6.2 & 1.3 & 36 & 0,459 & 506 & 0.0084 & 184 & .735 & 17 & 0.775 & 94 & 0.66 & 0.731 & 0.84 & 538.51 & 4.71 & 0.92 & 49.92 & .70 \\
\hline 74,648 & 8,930 & 857 & 1.5 & 8.3 & 1.7 & 28 & 11,759 & 4,850 & 0.0327 & 198 & 0.765 & 15 & 0.790 & 124 & 0.695 & 0.753 & 0.49 & 394.09 & \begin{tabular}{|l|}
398.03 \\
\end{tabular} & 1.06 & 16.29 & .5 \\
\hline 101,071 &, 146 & 995 & 2.0 & 10.0 & 2.0 & 30 & 9,313 & 3,774 & 0.0088 & 179 & 0.742 & 31 & 0.768 & 78 & 0.677 & 0.724 & 0.86 & 388.43 & 9.13 & 1.41 & 127.11 & .64 \\
\hline 169,536 & 0,380 & 822 & 2.0 & 10.0 & 2.0 & 35 & 10,146 & 5,029 & 0.0284 & 205 & 0.810 & 34 & 0.834 & 117 & 0.73 & 0.817 & .67 & 517.45 & 476.68 & 1.97 & 4.90 & .88 \\
\hline 105,355 & 0,101 & 078 & 1.0 & 6.2 & 1.3 & 28 & 9,771 & 384 & 0.0228 & 200 & 0.757 & 32 & 0.795 & 123 & 0.694 & 0.750 & .64 & 471.15 & 45.27 & 0.69 & 19.19 & .49 \\
\hline 60,301 & 5,056 & 140 & 1.0 & 6.2 & 1.3 & 24 & 1,783 & 4,707 & 0.0094 & 214 & .765 & 26 & 0.788 & 112 & 0.677 & 0.763 & .59 & 469.53 & 71.75 & 0.72 & 6.37 & .54 \\
\hline 145,654 & 9,585 & 379 & 2.0 & 10.0 & 2.0 & 24 & 11,398 & 4,801 & 0.0506 & 192 & 0.739 & 22 & 0.785 & 57 & 0.68 & 0.721 & 0.80 & 389.50 & 23.89 & 1.57 & 15.22 & 0.67 \\
\hline 95,451 &, 820 & 789 & 1.5 & 7.5 & 1.5 & 29 & 8,782 & 4,600 & 0.0072 & 235 & 0.786 & 22 & 0.816 & 88 & 0.704 & 0.766 & 0.87 & 406.36 & 03.12 & 0.99 & 04.87 & 0.61 \\
\hline 163,563 & 60,340 & 6,451 & 2.0 & 8.8 & 1.7 & 38 & 10,913 & 4,033 & 0.0064 & 237 & 0.735 & 19 & 0.750 & 122 & 0.666 & 0.717 & 0.81 & 512.67 & 595.03 & 1.34 & 95.41 & 0.72 \\
\hline 127,207 & 9,982 & 827 & 1.5 & 9.1 & 1.9 & 31 & 11,780 & 5,308 & 0.0065 & 240 & .754 & 28 & 0.772 & 111 & 0.66 & 747 & .78 & 549.32 & 8.44 & .24 & 1.23 & .73 \\
\hline 190,845 & 69,126 & 7,607 & 2.0 & 10.4 & 2.1 & 40 & 8,479 & 4,001 & 0.0172 & 193 & 0.799 & 25 & 0.837 & 82 & 0.725 & 0.767 & 0.87 & 458.28 & \begin{tabular}{|l|}
687.09 \\
\end{tabular} & 1.78 & 73.59 & 0.77 \\
\hline 190,845 &, 126 & 607 & 2.0 & 10.4 & 2.1 & 40 & 3,479 & 4,001 & 0.0072 & & .799 & 25 & 0.837 & 82 & 0.7 & 0.800 & .90 & |496.97| & 4.82 & 2.28 & 222.82 & .04 \\
\hline 101,068 & 4,020 & 3,566 & 1.0 & 7.0 & 1.5 & 30 & 10,159 & 4,325 & 0.0015 & 180 & 0.763 & 23 & 0.796 & 114 & 0.668 & 0.788 & 0.88 & 509.85 & 332.91 & 1.01 & 300.69 & 0.72 \\
\hline 82,026 &, 730 & 206 & 1.0 & 8.2 & 1.8 & 27 &, 301 & 4,059 & 0.0215 & 193 & .739 & 24 & 0.777 & 106 & 0.67 & 0.724 & 0.64 & 414.64 & 3.57 & 0.90 & 8.69 & .51 \\
\hline 97,300 & 40,876 & 3,527 & 2.0 & 10.4 & 2.1 & 28 & 10,435 & 4,364 & 0.0097 & 205 & 0.759 & 27 & 0.797 & 133 & 0.673 & 0.738 & 0.68 & 458.49 & \begin{tabular}{|l|}
445.04 \\
\end{tabular} & 1.28 & 70.39 & 0.63 \\
\hline 7,700 & 6,915 & 674 & 1.0 & 6.6 & 1.4 & 15 & 9,324 & 3,745 & 0.0035 & 192 & .762 & 12 & 0.792 & 83 & 0.678 & 0.737 & 0.76 & 220.87 & 41.12 & 0.51 & 166.68 & 0.34 \\
\hline 115,235 & 42,884 & 4,522 & 1.5 & 7.5 & 1.5 & 32 & 8,408 & 3,639 & 0.0188 & 233 & 0.753 & 30 & 0.807 & 103 & 0.686 & 0.762 & 0.77 & 382.82 & 478.12 & 1.32 & 76.44 & 0.71 \\
\hline 104,252 & 43,375 & 3,805 & 2.0 & 10.0 & 2.0 & 30 & 9,215 & 3,755 & 0.0058 & 217 & 0.800 & 24 & 0.800 & 130 & 0.699 & 0.780 & 0.77 & 464.02 & 463.38 & 1.26 & 129.72 & 0.67 \\
\hline 49,057 & 19,858 & 1,825 & 2.0 & 10.0 & 2.0 & 20 & 9,878 & 3,790 & 0.0085 & 228 & 0.749 & 11 & 0.772 & 67 & 0.683 & 0.731 & 0.82 & 301.19 & 409.91 & 1.06 & 105.15 & 0.49 \\
\hline 67,246 & 28,116 & 446 & 1.5 & 9.1 & 1.9 & 29 & 9,277 & 4,274 & 0.0770 & 229 & 0.766 & 29 & 0.819 & 114 & 0.687 & 0.766 & 0.46 & 317.39 & 400.00 & 1.23 & 17.50 & $0.4 \varsigma$ \\
\hline 148,238 & 56,237 & 750 & 1.0 & 9.4 & 2.1 & 25 & 8,940 & 3,722 & 0.0433 & 233 & 0.768 & 35 & 0.833 & 99 & 0.717 & 0.779 & 0.65 & 496.29 & 596.68 & 1.23 & 19.70 & 0.61 \\
\hline 97,423 & 41,777 & 3,478 & 1.5 & 8.7 & 1.8 & 26 & 11,151 & 3,661 & 0.0136 & 177 & 0.749 & 19 & 0.781 & 113 & 0.704 & 0.736 & 0.68 & 388.45 & 547.98 & 1.00 & 35.20 & 0.4 \\
\hline 127,934 & 51,769 & 4,760 & 2.0 & 10.0 & 2.0 & 32 & 10,859 & 5,411 & 0.0083 & 196 & 0.770 & 22 & 0.791 & 112 & 0.691 & 0.769 & 0.79 & 504.56 & 455.01 & 1.47 & 73.83 & 0.7 \\
\hline 40,881 & 15,968 & 1,557 & 1.5 & 7.5 & 1.5 & 21 & 11,511 & 4,898 & 0.0061 & 184 & 0.780 & 35 & 0.814 & 113 & 0.696 & 0.785 & 0.62 & 392.42 & & 0.81 & 56.75 & 0.4 \\
\hline 40,881 & 15,968 & 1,557 & 1.5 & 7.5 & 1.5 & 21 & $11,511 \mid$ & 4,898 & 0.0061 & 184| & 0.780 & 35 & $0.814 \mid$ & 113 & 0.73 & 0.785 & 0.68 & 320.67 & 375.65 & 0.68 & 54.39 & 0.4 \\
\hline
\end{tabular}

Table A-2: Data Generated for Six Stage Treatment, continued 


\begin{tabular}{|c|c|c|c|c|c|c|c|c|c|c|c|c|c|c|c|c|c|c|c|c|c|c|}
\hline \begin{tabular}{l|}
$\frac{\Phi}{\mathrm{E}}$ \\
$\frac{3}{0}$ \\
$\frac{\pi}{0}$ \\
$\frac{\pi}{0}$
\end{tabular} & $\begin{array}{l}\stackrel{0}{0} \\
\frac{5}{3} \\
\frac{3}{0} \\
\frac{1}{0} \\
\frac{0}{0} \\
0\end{array}$ & 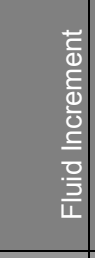 & 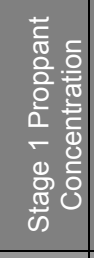 & 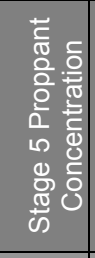 & 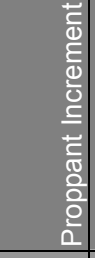 & 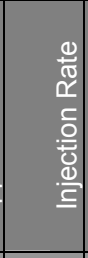 & 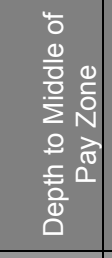 & 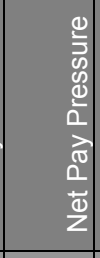 & 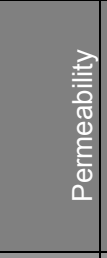 & 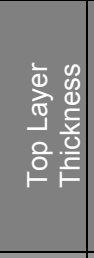 & 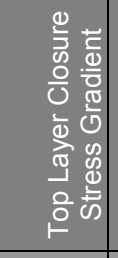 & 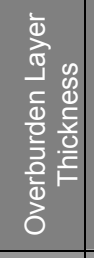 & 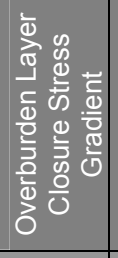 & 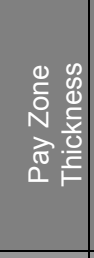 & 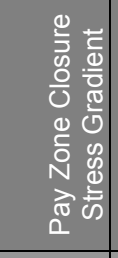 & 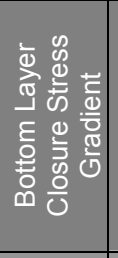 & 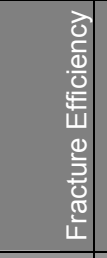 & 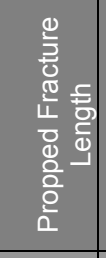 & \begin{tabular}{l|}
$\frac{}{0}$ \\
$\frac{0}{0}$ \\
$\frac{1}{1}$ \\
0 \\
$\frac{0}{3}$ \\
$\frac{3}{0}$ \\
$\frac{\pi}{\frac{\pi}{4}}$
\end{tabular} & 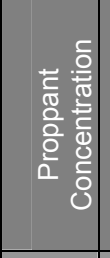 & 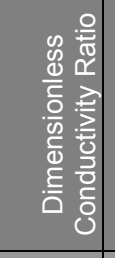 & \\
\hline input & input & input & input & input & input & input & input & input & input & input & input & input & input & input & input & input & output & output & output & output & output & output \\
\hline 82,151 & 32,381 & 3,111 & 1.0 & 7.0 & 1.5 & 30 & 0,597 & 4,276 & 0.0098 & 200 & 0.782 & 16 & 0.808 & 68 & 0.729 & 0.767 & 0.84 & 47.49 & 489.37 & 0.88 & 47.65 & \\
\hline 62,501 & 6,776 & 233 & 2.0 & 10.4 & 2.1 & 23 & 10,389 & 451 & 0.0156 & 197 & 0.756 & 22 & 0.778 & 98 & 0.664 & 0.735 & 0.61 & 374.89 & 353.21 & 1.24 & 55.53 & \\
\hline 130,004 & 6,403 & 600 & 1.5 & 9.5 & 2.0 & 34 & 8,916 & 245 & 0.0042 & 218 & 0.747 & 19 & 0.759 & 91 & 0.658 & 0.713 & 0.89 & 430.98 & 564.74 & 1.25 & 07.25 & \\
\hline 69,172 & 5,215 & 747 & 1.5 & 7.5 & 1.5 & 26 & 11,230 & 383 & 0.0055 & 210 & 0.810 & 17 & 0.847 & 60 & 0.728 & 0.820 & 0.86 & 340.25 & 332.10 & 1.29 & 113.74 & \\
\hline 142,866 & 57,845 & ,314 & 1.0 & 6.2 & 1.3 & 36 & 9,421 & 4,061 & 0.0078 & 199 & 0.784 & 34 & 0.812 & 52 & 0.701 & 0.803 & 0.92 & 432.87 & 652.48 & 0.82 & 64.40 & \\
\hline 166,886 & 2,388 & 531 & 1.0 & 7.0 & 1.5 & 38 & 10,008 & ,718 & 0.0061 & 206 & 0.772 & 35 & 0.803 & 113 & 0.71 & 0.755 & 0.86 & 517.86 & 38.32 & 0.96 & 72.39 & \\
\hline 187,736 & 2,941 & ,175 & 1.5 & 9.5 & 2.0 & 40 & 8,763 & 4,095 & 0.0049 & 214 & 0.763 & 30 & 0.763 & 118 & 0.672 & 0.756 & 0.88 & 528.68 & 614.54 & 1.46 & 191.92 & .18 \\
\hline 103,262 & 9,362 & 994 & 1.5 & 8.7 & 1.8 & 32 & 9,011 & |3,657 & 0.0037 & 194 & 0.772 & 12 & 0.784 & 117 & 0.687 & 0.770 & 0.85 & 412.54 & 449.25 & 1.32 & 265.91 & .72 \\
\hline 89,625 & 8,813 & 176 & 1.5 & 6.3 & 1.2 & 26 & 0,075 & ,767 & 0.0109 & 210 & 0.758 & 13 & 0.794 & 78 & 0.681 & 0.743 & 0.81 & 420.89 & 469.20 & 0.74 & 37.29 & \\
\hline 64,566 & 6,708 & ,366 & 1.0 & 6.6 & 1.4 & 24 & 10,138 & ,385 & 0.0319 & 221 & 0.753 & 29 & 0.794 & 63 & 0.668 & 0.719 & 0.75 & 333.06 & 50.83 & 0.72 & 16.41 & .46 \\
\hline 173,277 & 1,468 & ,988 & 2.0 & 10.8 & 2.2 & 37 & 8,946 & ,791 & 0.0064 & 219 & 0.775 & 32 & 0.798 & 101 & 0.686 & 0.780 & 0.87 & 483.16 & 521.82 & 2.12 & 232.47 & 94 \\
\hline 113,777 & 1,437 & 521 & 2.0 & 10.0 & 2.0 & 27 & 11,382 & 130 & 0.0235 & 217 & 0.744 & 27 & 0.785 & 63 & 0.667 & 0.753 & 0.78 & 428.45 & 399.12 & 1.88 & 41.62 & .85 \\
\hline 162,048 & 7,940 & 507 & 1.0 & 5.8 & 1.2 & 38 & 10,921 & 3,804 & 0.0188 & 235 & 0.759 & 18 & 0.796 & 94 & 0.697 & 0.741 & 0.83 & 536.58 & 638.06 & 0.77 & 35.65 & \\
\hline 99,232 & 39,588 & 3,728 & 1.5 & 7.5 & 1.5 & 17 & 11,132 & 5,234 & 0.0068 & 220 & 0.811 & 11 & 0.849 & 108 & 0.698 & 0.816 & 0.74 & 617.97 & 299.51 & 1.07 & 46.40 & 0.72 \\
\hline 147,231 & 8,795 & 527 & 1.5 & 6.3 & 1.2 & 25 & 8,458 & 4,042 & 0.0023 & 190 & 753 & 18 & 0.789 & 101 & 0.659 & 0.742 & 0.92 & 508.20 & 15.99 & 0.96 & 309.18 & 0.72 \\
\hline 174,671 & 68,343 & 6,646 & 2.0 & 10.8 & 2.2 & 40 & 10,334 & 4,618 & 0.0068 & 209 & 0.759 & 27 & 0.782 & 97 & 0.663 & 0.722 & 0.87 & 532.58 & 621.15 & 1.54 & 102.03 & 0.77 \\
\hline 92,169 & 2,514 & 728 & 1.5 & 7.5 & 1.5 & 28 & 11,440 & ,308 & 0.0524 & 212 & .764 & 19 & 0.781 & 106 & 0.679 & 0.746 & 0.44 & 69.41 & 8.28 & 1.06 & 10.78 & .57 \\
\hline 186,947 & 71,222 & 7,233 & 1.5 & 6.7 & 1.3 & 39 & 9,861 & 4,183 & 0.0033 & 211 & 0.766 & 10 & 0.789 & 84 & 0.683 & 0.760 & 0.92 & 532.17 & 526.36 & 1.25 & 185.90 & 0.86 \\
\hline 186,947 & 1,222 & 233 & 1.5 & 6.7 & 1.3 & 39 & 9,861 & 183 & 0.0100 & 211 & 766 & 10 & 0.789 & 84 & 0.66 & & 0.84 & 625.07 & 502.20 & 1.12 & 51.55 & .84 \\
\hline 49,932 & 20,317 & $|1,851|$ & 2.0 & 10.0 & 2.0 & 23 & 10,228 & 4,639 & 0.0074 & 204 & 0.775 & 29 & 0.800 & 76 & 0.672 & 0.735 & 0.81 & 335.99 & 330.14 & 1.18 & 107.81 & 0.57 \\
\hline 178,971 & 3,458 & ,595 & 1.0 & 7.4 & 1.6 & 38 & 10,478 & 4,239 & 0.0506 & 210 & .797 & 21 & 0.798 & 107 & 0.666 & 0.778 & 0.47 & 802.59 & 364.62 & 1.14 & 8.81 & .75 \\
\hline 59,437 & 25,376 & 2,129 & 2.0 & 10.4 & 2.1 & 23 & 9,785 & 3,410 & 0.0372 & 206 & .747 & 18 & 0.774 & 110 & 0.679 & 0.762 & 0.50 & 316.56 & 366.90 & 1.35 & 37.34 & .52 \\
\hline 32,289 & 3,545 & 171 & 2.0 & 6.0 & 1.0 & 19 & 10,340 & 3,558 & 0.0076 & 203 & .769 & 24 & 0.796 & 90 & 0.662 & 0.741 & 0.67 & 308.86 & 250.36 & 1.08 & 110.64 & .51 \\
\hline 189,393 & 1,301 & ,381 & 2.0 & 9.6 & 1.9 & 40 & 8,887 & 3,869 & 0.0807 & 204 & 0.766 & 30 & 0.810 & 79 & 0.677 & 0.764 & 0.70 & 492.31 & 522.37 & 1.97 & 18.76 & 0.89 \\
\hline 32,901 & 12,710 & 1,262 & 1.5 & 9.5 & 2.0 & 18 & 11,729 & 5,639 & 0.0165 & 193 & .767 & 19 & 0.830 & 85 & 0.707 & 0.801 & 0.59 & 307.52 & 265.92 & 1.01 & 34.40 & 0.48 \\
\hline 98,928 & 38,581 & 3,772 & 1.5 & 7.5 & 1.5 & 27 & 9,692 & 3,967 & 0.0136 & 179 & 0.731 & 33 & 0.736 & 59 & 0.659 & 0.729 & 0.86 & 393.52 & 549.84 & 0.93 & 51.09 & \\
\hline 133,229 & 47,216 & 5,376 & 2.0 & 8.0 & 1.5 & 34 & 10,019 & 5,006 & 0.0064 & 237 & 0.768 & 15 & 0.814 & 125 & 0.708 & 0.755 & 0.85 & 438.88 & 571.02 & 1.26 & 112.81 & 0.67 \\
\hline 157,918 & 61,484 & 6,027 & 1.0 & 7.0 & 1.5 & 32 & 11,344 & 4,732 & 0.0539 & 237 & 0.830 & 31 & 0.836 & 119 & 0.73 & 0.820 & 0.37 & 678.73 & 370.78 & 1.16 & 6.79 & 0.65 \\
\hline 18,161 & $7,851 \mid$ & 644 & 1.0 & 5.8 & 1.2 & 29 & 8,696 & 3,495 & 0.0028 & 225 & 0.764 & 15 & 0.817 & 132 & 0.688 & 0.731 & 0.75 & 194.01 & 254.65 & 0.47 & 230.39 & .3 \\
\hline 29,118 & $\mid 12,673$ & 1,028 & 1.5 & 7.5 & 1.5 & 19 & 9,653 & $\mid 4,463$ & 0.0078 & 208 & 0.744 & 13 & 0.748 & 79 & 0.665 & 0.727 & 0.79 & 257.40 & $|319.46|$ & 0.66 & 83.17 & \\
\hline
\end{tabular}

Table A-2: Data Generated for Six Stage Treatment, continued 


\begin{tabular}{|c|c|c|c|c|c|c|c|c|c|c|c|c|c|c|c|c|c|c|c|c|c|c|}
\hline 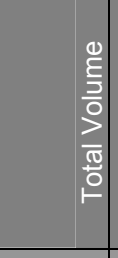 & 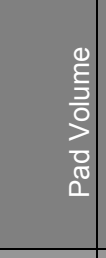 & 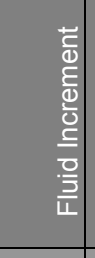 & 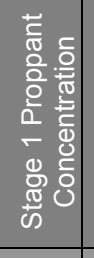 & 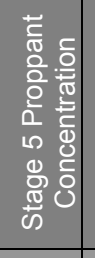 & 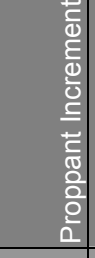 & 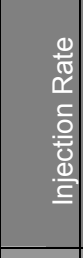 & 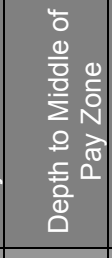 & 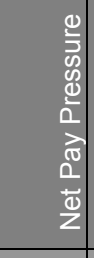 & 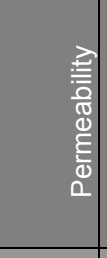 & 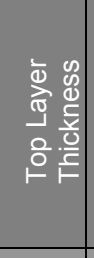 & 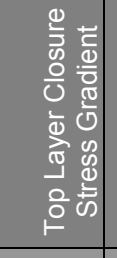 & 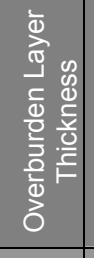 & 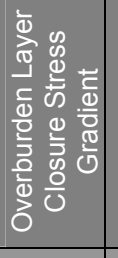 & 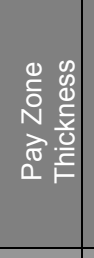 & 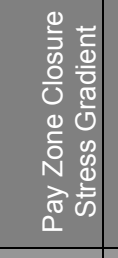 & 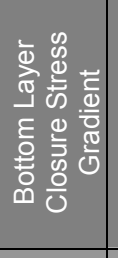 & 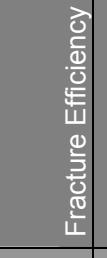 & 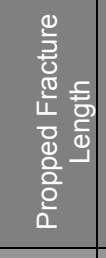 & 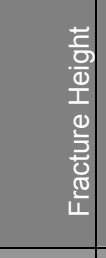 & 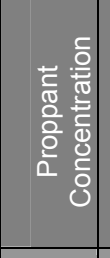 & 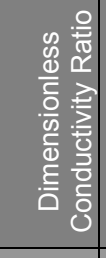 & \\
\hline input & input & input & input & input & input & input & input & input & input & input & input & input & input & input & input & input & output & output & output & output & output & output \\
\hline 35,574 & 12,931 & 1,415 & 1.5 & 8.3 & 1.7 & 19 & 10,236 & 4,999 & 0.0056 & 225 & 0.781 & 35 & 0.813 & 122 & 0.712 & 0.770 & 0.73 & 297.28 & 332.63 & 0.84 & 113.35 & 45 \\
\hline 63,518 & 27,689 & 2,239 & 1.0 & 9.0 & 2.0 & 25 & 9,784 & 4,688 & 0047 & 173 & 0.810 & 24 & 0.838 & 127 & 0.737 & 0.825 & 0.78 & 352.82 & 368.74 & 1.05 & 137.42 & .57 \\
\hline 118,230 & 49,996 & 265 & 1.5 & 7.5 & 1.5 & 29 & 9,217 & 3,627 & 0.0317 & 224 & 0.743 & 22 & 0.782 & 111 & 0.676 & 0.723 & 0.67 & 429.02 & 554.60 & 0.96 & 22.97 & 54 \\
\hline 41,979 & 17,553 & 1,527 & 2.0 & 6.0 & 1.0 & 20 & 9,714 & 4,025 & 0.0056 & 220 & 0.794 & 32 & 0.827 & 110 & 0.662 & 0.785 & 0.67 & 425.86 & 200.70 & 0.82 & 88.52 & 52 \\
\hline 170,978 & 74,466 & ,032 & 1.0 & 6.2 & 1.3 & 36 & 9,657 & ,745 & 0.0078 & 182 & 0.743 & 29 & 0.776 & 102 & 0.673 & 0.744 & 0.87 & 495.87 & 09.01 & 1.03 & 70.66 & .75 \\
\hline 149,265 & 53,385 & ,993 & 1.0 & 6.6 & 1.4 & 34 & $\mid 11,086$ & 4,775 & 0.0955 & 216 & 0.755 & 22 & 0.785 & 89 & 0.683 & 0.746 & 0.54 & 561.81 & 560.58 & 0.87 & 4.33 & .58 \\
\hline 112,220 & 4,101 & 257 & 1.5 & 8.3 & 1.7 & 30 & $\mid 11,796$ & ,038 & 0068 & 174 & 0.800 & 14 & 0.800 & 110 & 0.71 & 0.764 & 0.77 & 534.61 & 403.85 & 1.15 & 45.11 & 66 \\
\hline 99,277 & 38,469 & 3,800 & 2.0 & 8.0 & 1.5 & 28 & 11,326 & 4,059 & 0.0092 & 232 & 0.820 & 22 & 0.851 & 52 & 0.727 & 0.820 & 0.85 & 430.00 & 397.41 & 1.30 & 52.86 & .76 \\
\hline 135,818 & 53,772 & ,128 & 1.0 & 7.4 & 1.6 & 32 & $\mid 11,646$ & 5,487 & 0.0223 & 233 & 0.765 & 22 & 0.814 & 58 & 0.68 & 0.751 & 0.81 & 535.78 & 487.63 & 1.00 & 4.95 & 68 \\
\hline 134,321 & 55,470 & 4,928 & 2.0 & 10.0 & 2.0 & 25 & \begin{tabular}{|l|}
10,487 \\
\end{tabular} & 4,327 & 0.0026 & 208 & 0.730 & 32 & 0.760 & 132 & 0.66 & 0.719 & 0.86 & 471.73 & 543.00 & 1.37 & 269.69 & ).69 \\
\hline 68,704 & 29,932 & 2,423 & 2.0 & 8.0 & 1.5 & 26 & 9,340 & 031 & 0152 & 225 & 0.791 & 11 & 0.826 & 98 & .716 & 0.787 & 0.73 & 339.17 & 409.81 & 1.02 & 52.26 & 55 \\
\hline 187,205 & 73,227 & 7,124 & 2.0 & 10.0 & 2.0 & 38 & 9,797 & 4,341 & 0.0021 & 232 & 0.754 & 14 & 0.802 & 87 & 0.665 & 0.735 & 0.93 & 541.82 & 593.42 & 1.58 & 369.80 & 0.81 \\
\hline 194,481 & 76,127 & 397 & 1.5 & 7.5 & 1.5 & 40 & \begin{tabular}{|l|}
10,089 \\
\end{tabular} & ,236 & 0031 & 217 & 0.783 & 25 & 0.819 & 122 & 0.706 & 0.781 & 0.88 & 568.66 & 542.45 & 1.28 & 69.32 & 81 \\
\hline 36,855 & 15,740 & 1,320 & 1.5 & 9.5 & 2.0 & 19 & 11,202 & 4,181 & 0.0048 & 202 & 0.761 & 21 & 0.801 & 92 & 0.675 & 0.726 & 0.76 & 295.93 & 299.70 & 0.98 & 117.67 & 0.48 \\
\hline 124,663 & 49,764 & 4,681 & 1.5 & 8.7 & 1.8 & 30 & 11,508 & 4,620 & 0.0283 & 192 & 0.793 & 32 & 0.819 & 80 & 0.72 & 0.800 & 0.68 & 473.98 & 416.50 & 1.44 & 19.26 & .74 \\
\hline 56,700 & 20,438 & 2,266 & 1.5 & 7.5 & 1.5 & 25 & $\mid 11,591$ & 4,470 & 0.0052 & 233 & 0.810 & 31 & 0.831 & 132 & 0.729 & 0.822 & 0.67 & 424.25 & 308.57 & 0.92 & 67.39 & 0.55 \\
\hline 53,849 & 21,654 & 2,012 & 2.0 & 8.0 & 1.5 & 23 & 9,922 & ,454 & 0194 & 193 & 0.771 & 26 & 0.823 & 101 & 0.679 & 0.771 & 0.61 & 381.89 & 329.86 & 0.94 & 36.42 & .53 \\
\hline 56,547 & 22,151 & ,150 & 2.0 & 7.2 & 1.3 & 24 & 11,225 & 3,997 & 0.0228 & 196 & 0.757 & 23 & 0.757 & 122 & 0.66 & 0.723 & 0.78 & 405.09 & 292.60 & 0.96 & 157.95 & 0.57 \\
\hline 137,659 & 57,701 & ,997 & 2.0 & 10.0 & 2.0 & 35 & $\mid 11,149$ & ,399 & 0074 & 237 & 0.782 & 15 & 0.829 & 75 & .696 & 0.762 & 0.86 & 475.10 & 533.67 & 1.40 & 70.25 & 0.72 \\
\hline 41,130 & 14,578 & 1,660 & 1.5 & 7.5 & 1.5 & 21 & 9,851 & 511 & 0.0272 & 239 & 0.745 & 32 & 0.756 & 82 & 0.655 & 0.723 & 0.64 & 327.11 & 328.02 & 0.83 & 29.43 & 0.48 \\
\hline 155,273 & 65,754 & 5,595 & 2.0 & 10.0 & 2.0 & 37 & 8,857 & ,329 & 0.0020 & 178 & 0.785 & 18 & 0.797 & 63 & 0.699 & 0.770 & 0.96 & 457.05 & 666.16 & 1.31 & 401.12 & 0.65 \\
\hline 144,521 & 53,404 & 695 & 2.0 & 10.0 & 2.0 & 33 & ,734 & 4,832 & 0.0860 & 237 & 0.766 & 21 & 0.798 & 112 & 0.67 & 0.805 & 0.62 & 427.64 & 472.63 & 2.01 & 23.43 & 0.83 \\
\hline 39,765 & 15,562 & 1,513 & 2.0 & 8.8 & 1.7 & 20 & 10,636 & 4,443 & 0.0281 & 237 & 0.786 & 24 & 0.815 & 84 & 0.708 & 0.747 & 0.87 & 274.36 & 388.11 & 0.90 & 208.03 & 0.47 \\
\hline 60,643 & 21,623 & 2,439 & 1.0 & 9.0 & 2.0 & 24 & 11,116 & 4,630 & 0.0190 & 229 & 0.789 & 16 & 0.828 & 73 & 0.684 & 0.774 & 0.67 & 437.13 & 345.97 & 0.98 & 26.15 & 0.59 \\
\hline 182,800 & 80,319 & 6,405 & 1.0 & 6.2 & 1.3 & 40 & 9,365 & 4,323 & 0.0051 & 209 & 0.801 & 19 & 0.830 & 56 & 0.703 & 0.787 & 0.93 & 552.08 & 545.69 & 0.92 & 79.20 & 0.71 \\
\hline 73,652 & 26,278 & 2,961 & 1.0 & 7.0 & 1.5 & 27 & 11,434 & 4,592 & 0.0346 & 221 & 0.735 & 28 & 0.764 & 74 & 0.666 & 737 & 0.67 & 373.10 & 398.76 & 0.95 & 6.66 & .58 \\
\hline 70,437 & 26,705 & 2,733 & 1.5 & 7.5 & 1.5 & 26 & 9,034 & 4,348 & 0.0099 & 223 & 0.795 & 30 & 0.841 & 104 & 0.729 & 0.800 & 0.78 & 354.53 & 427.31 & 0.96 & 79.43 & 0.55 \\
\hline 70,437 & 26,705 & 2,733 & 1.5 & 7.5 & 1.5 & 26 & 9,034 & 4,348 & 0.0190 & 223 & 0.795 & 30 & 0.841 & 104 & 0.69 & 0.800 & 0.66 & 433.56 & 365.17 & 0.92 & 37.28 & .59 \\
\hline 69,649 & 27,369 & 2,642 & 2.0 & 10.0 & 2.0 & 23 & 11,014 & 5,249 & 077 & 192 & 0.751 & 32 & 0.806 & 101 & 0.68 & 0.751 & 0.40 & 390.91 & 350.90 & 1.36 & 12.54 & 0.57 \\
\hline 129,726 & 49,249 & 5,030 & 2.0 & 10.0 & 2.0 & 33 & 9,048 & 3,774 & 0.0239 & 195 & 0.820 & 28 & 0.831| & 106 & 0.713 & 0.811 & 0.70 & 434.73 & 434.76 & 1.90 & 60.53 & 0.8 \\
\hline
\end{tabular}

Table A-2: Data Generated for Six Stage Treatment, continued 


\begin{tabular}{|c|c|c|c|c|c|c|c|c|c|c|c|c|c|c|c|c|c|c|c|c|c|c|}
\hline 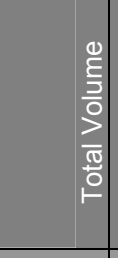 & 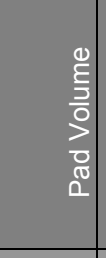 & 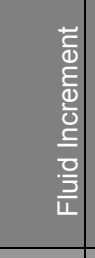 & 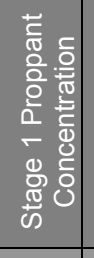 & 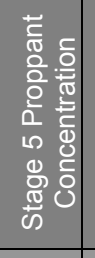 & 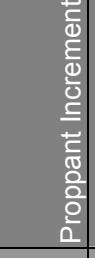 & 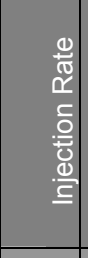 & 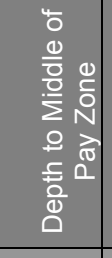 & 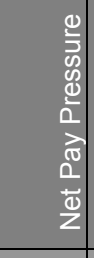 & 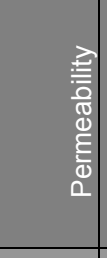 & 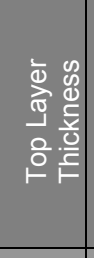 & 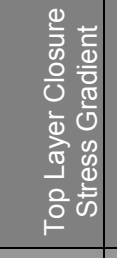 & 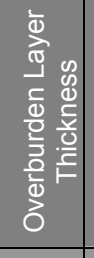 & 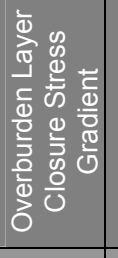 & 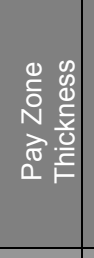 & 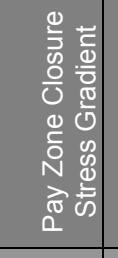 & 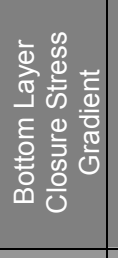 & 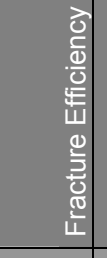 & 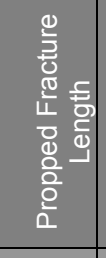 & 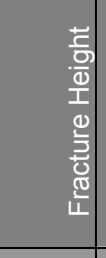 & 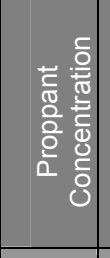 & 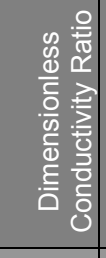 & \\
\hline input & input & input & input & input & input & input & input & input & input & input & input & input & input & input & input & input & output & output & output & output & output & output \\
\hline 49,689 & 21,686 & 1,750 & 2.0 & 10.8 & 2.2 & 20 & 10,409 & 4,660 & 0.0066 & 235 & 0.748 & 31 & 0.759 & 86 & 0.664 & 0.749 & 0.78 & 357.11 & 327.16 & 1.14 & 113.71 & \\
\hline 69,596 & 24,400 & 2,825 & 2.0 & 8.8 & 1.7 & 25 & 11,194 & 3,849 & 0.0893 & 174 & 0.782 & 27 & 0.831 & 97 & 0.686 & 0.776 & 0.27 & 381.58 & 283.79 & 1.65 & 13.43 & .55 \\
\hline 82,829 & 36,360 & 2,904 & 2.0 & 9.6 & 1.9 & 29 & 10,388 & 3,827 & 0.0044 & 197 & 0.767 & 15 & 0.786 & 123 & 0.689 & 0.731 & 0.82 & 364.35 & 456.37 & 1.20 & 61.29 & .59 \\
\hline 79,449 & 27,904 & 3,222 & 1.5 & 7.5 & 1.5 & 26 & 9,629 & 3,915 & 0.0084 & 178 & 0.800 & 25 & 0.838 & 107 & 0.702 & 0.810 & 0.72 & 482.42 & 373.71 & 0.95 & 69.02 & 63 \\
\hline 66,136 & 27,938 & 2,387 & 2.0 & 10.4 & 2.1 & 24 & 11,633 & 4,438 & 0.0034 & 235 & 0.830 & 31 & 0.830 & 112 & 0.73 & 0.830 & 0.73 & 477.45 & 359.05 & 1.37 & 17.62 & 65 \\
\hline 66,136 & 27,938 & 2,387 & 2.0 & 10.4 & 2.1 & 24 & 11,633 & 4,438 & 0.0200 & 235 & 0.790 & 31 & 0.820 & 112 & 0.73 & 0.780 & 0.57 & 350.35 & 400.73 & 1.25 & 29.98 & .52 \\
\hline 61,342 & 60,513 & ,302 & 2.0 & 9.2 & 1.8 & 35 & 10,626 & 5,134 & 0042 & 171 & 0.777 & 22 & 0.793 & 60 & 0.675 & 0.758 & 0.92 & 609.92 & 520.33 & 1.31 & 14.51 & .76 \\
\hline 45,959 & 19,629 & 1,646 & 1.0 & 6.6 & 1.4 & 22 & 10,251 & 4,691 & 0.0180 & 191 & 0.736 & 31 & 0.784 & 120 & 0.664 & 0.719 & 0.60 & 320.48 & 350.67 & 0.67 & 29.21 & .43 \\
\hline 96,124 & 33,947 & 3,886 & 1.5 & 9.5 & 2.0 & 27 & 9,036 & 171 & 0408 & 176 & 0.800 & 17 & 0.851 & 126 & 0.664 & 0.719 & 0.56 & 407.70 & 478.20 & 1.32 & 28.80 & 59 \\
\hline 183,701 & 78,149 & 6,597 & 2.0 & 10.0 & 2.0 & 39 & 10,191 & 4,421 & 0.0069 & 213 & 0.774 & 20 & 0.825 & 133 & 0.69 & 0.766 & 0.80 & 579.86 & 556.95 & 1.45 & 86.61 & 0.77 \\
\hline 18,913 & 7,021 & 743 & 1.5 & 5.9 & 1.1 & 24 & 8,690 & 3,405 & 0.0054 & 212 & 0.751 & 17 & 0.819 & 131 & 0.67 & 0.754 & 0.65 & 231.53 & 256.10 & 0.55 & 52.66 & 35 \\
\hline 122,633 & 44,973 & 4,854 & 1.5 & 6.3 & 1.2 & 30 & 11,659 & 4,859 & 0.0136 & 220 & 0.768 & 29 & 0.801 & 105 & 0.693 & 0.808 & 0.69 & 610.87 & 513.73 & 0.70 & 15.64 & .57 \\
\hline 101,716 & 6,185 &, 096 & 2.0 & 8.0 & 1.5 & 30 & 9,720 & ,216 & 0119 & 180 & 0.810 & 32 & 0.850 & 118 & 0.729 & 0.791 & 0.72 & 470.27 & 496.00 & 1.03 & 48.77 & 60 \\
\hline 29,840 & 10,792 & 1,191 & 1.0 & 7.0 & 1.5 & 16 & 10,282 & 3,743 & 0.0072 & 237 & 0.770 & 18 & 0.782 & 61 & 0.679 & 0.756 & 0.78 & 309.97 & 299.82 & 0.62 & 65.76 & 0.44 \\
\hline 29,840 & 10,792 & 1,191 & 1.0 & 7.0 & 1.5 & 16 & 10,282 & 3,743 & 0.0072 & 237 & 0.747 & 18 & 0.782 & 61 & 0.705 & 0.756 & 0.83 & 200.95 & 290.78 & 0.88 & 136.59 & .49 \\
\hline 168,510 & 66,691 & 6,364 & 1.5 & 7.5 & 1.5 & 37 & 9,019 & 4,018 & 0.0395 & 228 & 0.759 & 22 & 0.786 & 78 & 0.681 & 0.723 & 0.81 & 457.60 & 683.64 & 1.09 & 18.19 & 0.63 \\
\hline 124,834 & 50,796 & ,627 & 1.5 & 9.5 & 2.0 & 33 & 8,606 & 4,354 & 0108 & 213 & 0.721 & 25 & 0.762 & 71 & 0.668 & 0.718 & 0.90 & 392.59 & 521.84 & 1.49 & 131.82 & .71 \\
\hline 115,603 & 42,864 & ,546 & 1.0 & 7.0 & 1.5 & 29 & 9,897 & 4,138 & 0.0380 & 235 & 0.759 & 27 & 0.782 & 65 & 0.656 & 0.729 & 0.68 & 551.36 & 434.30 & 0.91 & 8.57 & 0.64 \\
\hline 113,915 & 48,319 & 4,100 & 1.5 & 7.5 & 1.5 & 31 & 1,779 & 5,741 & 0083 & 204 & 0.784 & 18 & 0.818 & 96 & 0.704 & 0.789 & 0.78 & 520.38 & 406.83 & 1.15 & 41.02 & .70 \\
\hline 157,572 & 57,689 & 6,243 & 2.0 & 10.4 & 2.1 & 36 & 10,669 & 4,958 & 0.0079 & 209 & 0.750 & 17 & 0.765 & 116 & 0.679 & 0.740 & 0.82 & 503.19 & 549.20 & 1.67 & 101.41 & 0.78 \\
\hline 186,839 & 78,230 & 6,788 & 1.5 & 7.5 & 1.5 & 19 & 10,709 & 4,578 & 0.0039 & 192 & 0.768 & 15 & 0.780 & 55 & 0.66 & 0.754 & 0.88 & 768.79 & 507.36 & 0.93 & 66.01 & 0.75 \\
\hline 149,847 & 60,898 & 5,559 & 2.0 & 10.4 & 2.1 & 34 & 11,238 & 4,241 & 0.0426 & 239 & 0.810 & 12 & 0.840 & 80 & 0.724 & 0.800 & 0.65 & 473.78 & 497.45 & 1.74 & 16.19 & 0.80 \\
\hline 190,559 & 83,105 & 6,716 & 1.0 & 6.2 & 1.3 & 40 & 8,913 & 3,646 & 0.0032 & 216 & 0.725 & 31 & 0.756 & 132 & 0.654 & 0.717 & 0.89 & 526.39 & 596.78 & 0.93 & 176.79 & 0.69 \\
\hline 156,628 & 64,221 & ,775 & 1.0 & 6.6 & 1.4 & 35 & 8,939 & 3,714 & 0.0175 & 227 & 0.746 & 17 & 0.781 & 65 & 0.669 & 0.720 & 0.88 & 439.68 & 640.59 & 0.94 & 37.16 & 0.63 \\
\hline 192,522 & 73,413 & 7,444 & 1.0 & 9.0 & 2.0 & 40 & 10,714 & 3,597 & 0.0279 & 199 & 0.779 & 13 & 0.781 & 105 & 0.684 & 0.792 & 0.63 & 624.58 & 505.87 & 1.44 & 21.80 & 0.81 \\
\hline 86,275 & 34,354 & 3,245 & 1.0 & 6.2 & 1.3 & 28 & 10,557 & 4,165 & 0.0079 & 193 & 0.820 & 24 & 0.820 & 66 & 0.73 & 830 & 0.84 & 408.03 & 471.31 & 0.73 & 2.11 & .55 \\
\hline 152,237 & 61,486 & 5,672 & 2.0 & 9.6 & 1.9 & 36 & 10,489 & 4,467 & 0.0174 & 193 & 0.801 & 21 & 0.841 & 120 & 0.695 & 0.810 & 0.65 & 661.10 & 408.14 & 1.44 & 36.76 & 0.79 \\
\hline 174,788 & 73,927 & 6,304 & 1.0 & 7.0 & 1.5 & 36 & 10,211 & 4,042 & 0.0077 & 189 & 0.761 & 16 & 0.791 & 97 & 0.694 & 0.751 & 0.84 & 525.74 & 568.40 & 1.02 & 57.80 & .68 \\
\hline 137,153 & 48,526 & & 1.5 & 7.5 & 1.5 & 33 & 11,216 & 4,075 & 720 & 206 & 0.744 & 34 & 0.775 & 112 & 0.681 & 0.747 & 0.45 & 515.16 & 501.98 & 1.14 & 8.31 & 0.58 \\
\hline 44,209 & 18,765 & 1,590 & 1.0 & 7.0 & 1.5 & 19 & 9,486 & 4,573 & 0.0081 & 222 & 0.784 & 25 & 0.784 & 55 & $0.674 \mid$ & 0.762 & 0.84 & 338.59 & 327.10 & 0.69 & 63.54 & 0.5 \\
\hline
\end{tabular}

Table A-2: Data Generated for Six Stage Treatment, continued 


\begin{tabular}{|c|c|c|c|c|c|c|c|c|c|c|c|c|c|c|c|c|c|c|c|c|c|c|}
\hline 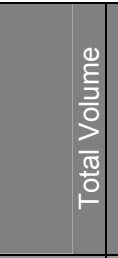 & 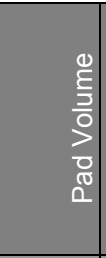 & 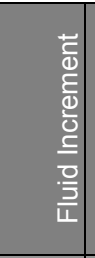 & 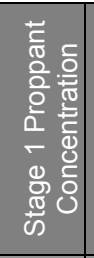 & 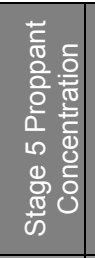 & 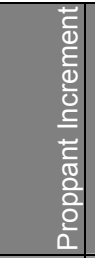 & 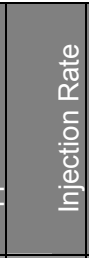 & 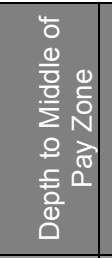 & 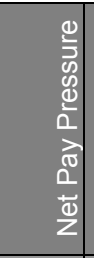 & 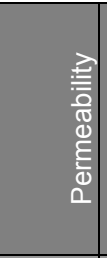 & 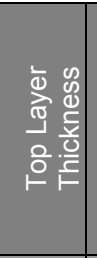 & 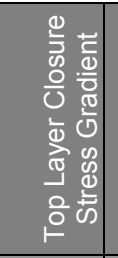 & 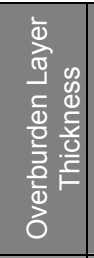 & 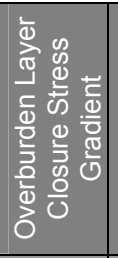 & 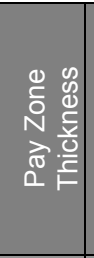 & 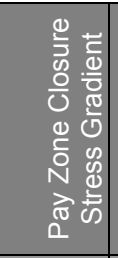 & 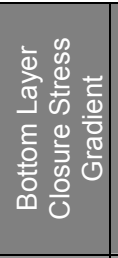 & 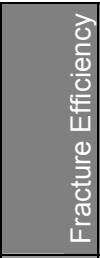 & 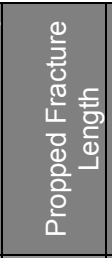 & \begin{tabular}{l|l}
$\frac{7}{0}$ & \\
$\frac{0}{0}$ & \\
$\frac{1}{1}$ & \\
$\frac{0}{3}$ & \\
$\frac{3}{0}$ & \\
$\frac{\pi}{4}$ & \\
$\frac{1}{4}$ &
\end{tabular} & 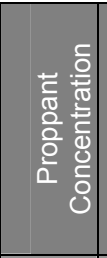 & 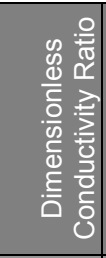 & . \\
\hline input & input & input & input & input & input & input & input & input & input & input & input & input & input & input & input & input & output & output & utput & output & output & output \\
\hline 198,452 & 79,847 & 7,413 & 2.0 & 10.0 & 2.0 & 37 & 8,898 & 3,835 & 0.0589 & 223 & 0.768 & 34 & 0.800 & 121 & 0.668 & 0.770 & 0.67 & 80.54 & 14.87 & 1.77 & 9.30 & \\
\hline 160,919 & 3,514 & 400 & 1.5 & 7.1 & 1.4 & 32 & 11,613 & 4,595 & 0.0088 & 231 & 0.777 & 23 & 0.821 & 100 & 0.709 & 0.780 & 0.78 & 48.41 & 94.75 & 1.20 & 43.48 & \\
\hline 84,838 & 5,099 & 109 & 1.5 & 7.5 & 1.5 & 26 & 9,132 & 4,220 & 0.0067 & 194 & 0.756 & 22 & 0.800 & 129 & 0.68 & 0.746 & 0.79 & 413.16 & 471.62 & 0.86 & 91.69 & \\
\hline 84,838 & 099 & 109 & 1.5 & 7.5 & 1.5 & 26 & 9,132 & 4,220 & 0.0110 & 194 & 0.756 & 22 & 0.790 & 129 & 0.65 & 0.736 & 0.72 & 442.14 & 379.71 & 0.99 & 64.06 & \\
\hline 112,191 & 2,213 & 374 & 1.0 & 6.2 & 1.3 & 31 & 9,185 & 4,720 & 0.0662 & 183 & 0.800 & 25 & 0.828 & 96 & 0.714 & 0.787 & 0.60 & 509.08 & 511.27 & 0.73 & 7.28 & \\
\hline 64,418 &, 685 & 608 & 2.0 & 10.0 & 2.0 & 24 & 1,549 & 626 & 0113 & 234 & .762 & 26 & 0.794 & 112 & .709 & 0.761 & .69 & 357.32 & \begin{tabular}{|l|}
429.03 \\
\end{tabular} & .20 & 54.83 & \\
\hline 107,540 & 2,430 & 069 & 1.5 & 7.9 & 1.6 & 30 & 9,494 & 4,727 & 0.0313 & 226 & 0.782 & 32 & 0.841 & 130 & 0.694 & 0.753 & .62 & 452.75 & 469.91 & 1.07 & 23.48 & .0 \\
\hline 22,483 &, 871 & 788 & 1.5 & 7.5 & 1.5 & 16 & 10,891 & 4,715 & 0.0051 & 190 & 0.755 & 23 & 0.810 & 102 & 0.664 & 0.726 & 0.68 & 266.97 & 0.70 & 0.73 & 96.11 & .40 \\
\hline 188,946 & 3,296 & 916 & 1.5 & 9.5 & 2.0 & 40 & 9,837 & 640 & 0.0373 & 239 & 0.764 & 25 & 0.799 & 65 & 0.673 & 0.737 & 0.81 & 453.29 & 652.76 & 1.55 & 25.09 & 0.77 \\
\hline 130,494 & 5,742 & 672 & 1.0 & 5.8 & 1.2 & 30 & 9,956 & 777 & 0.0072 & 220 & .801 & 14 & 0.822 & 76 & 0.7 & 0.786 & 0.83 & 579.69 & 36.94 & 0.66 & 35.55 & .62 \\
\hline 41,499 & 14,916 & 661 & 2.0 & 9.2 & 1.8 & 24 & 395 & 615 & 0.0568 & 214 & 749 & 12 & 0.778 & 97 & 0.686 & 0.735 & .56 & 263.83 & 5.33 & 1.18 & 6.94 & .48 \\
\hline 41,499 & 4,916 & 661 & 2.0 & 9.2 & 1.8 & 24 & 9,395 & 615 & 0.0100 & 214 & 0.749 & 12 & 0.778 & 97 & 0.66 & 0.735 & 0.76 & |312.67 & 20.93 & 1.10 & 109.03 & 0.55 \\
\hline 159,096 &, 017 & 442 & 1.5 & 7.5 & 1.5 & 32 & 9,867 & 4,682 & 0.0251 & 183 & 0.747 & 11 & 0.785 & 100 & 0.661 & 0.766 & 0.74 & 590.50 & |597.32 & 0.98 & 20.24 & 0.65 \\
\hline 63,543 & 25,763 & 361 & 2.0 & 9.2 & 1.8 & 24 & 9,103 & 3,664 & 0.0122 & 230 & 0.747 & 27 & 0.783 & 67 & 0.679 & 0.744 & 0.83 & 308.13 & 399.71 & 1.27 & 103.23 & 0.60 \\
\hline 65,468 & 4,381 & 568 & 1.5 & 8.7 & 1.8 & 27 & 587 & 001 & 0.0959 & 197 & 776 & 26 & 0.776 & 92 & .677 & 770 & .51 & 8.69 & 2.90 & .18 & 5.58 & .57 \\
\hline 32,873 & 13,169 & 1,232 & 1.5 & 8.7 & 1.8 & 18 & 9,638 & 4,081 & 0.0023 & 224 & 0.757 & 31 & 0.797 & 131 & 0.683 & 0.735 & 0.80 & 274.69 & 321.76 & 0.85 & 334.22 & 0.45 \\
\hline 162,815 & 132 & 168 & 1.5 & 7.5 & 1.5 & 34 & 8,857 & 3,577 & 0.0088 & 226 & .728 & 20 & 0.747 & 81 & 0.65 & 0.710 & .88 & 473.52 & 4.82 & .19 & 3.71 & .71 \\
\hline 113,047 & 41,883 & 448 & 1.5 & 8.3 & 1.7 & 28 & 11,634 & 3,949 & 0.0099 & 238 & 0.810 & 18 & 0.846 & 83 & 0.735 & 0.800 & 0.76 & 478.30 & 480.68 & 1.12 & 37.81 & 0.66 \\
\hline 177,334 & 5,432 & 931 & 1.5 & 7.5 & 1.5 & 38 & 10,359 & 4,589 & 0.0149 & 239 & .786 & 18 & 0.835 & 119 & 0.702 & .798 & 0.74 & 554.94 & 463.69 & 1.44 & 43.42 & 0.86 \\
\hline 61,381 & 26,314 & 192 & 2.0 & 10.8 & 2.2 & 18 & 9,555 & 4,240 & 0.0056 & 194 & 0.781 & 11 & 0.752 & 119 & 0.671 & 0.727 & 0.80 & 324.68 & \begin{tabular}{|l|}
393.86 \\
\end{tabular} & 1.31 & 192.41 & 0.58 \\
\hline 7,680 & 1,448 & 389 & 1.5 & 7.5 & 1.5 & 38 & 8,829 & 3,642 & 0.0260 & 200 & .744 & 21 & 0.766 & 75 & 0.665 & 0.722 & 0.82 & 448.71 & 573.92 & 1.12 & 32.44 & 0.65 \\
\hline 151,581 & 4,434 & 447 & 1.0 & 6.2 & 1.3 & 35 & 9,784 & 4,677 & 0.0477 & 197 & 0.774 & 16 & 0.774 & 126 & 0.686 & 0.786 & 0.80 & 585.07 & 523.67 & 0.78 & 42.05 & 0.65 \\
\hline 44,463 & 16,158 & 1,769 & 2.0 & 10.8 & 2.2 & 24 & 8,737 & 3,870 & 0.0108 & 218 & 0.804 & 35 & 0.809 & 51 & 0.721 & 0.782 & 0.86 & 289.46 & 8.58 & 1.23 & 117.87 & 0.53 \\
\hline 13,471 & 5,630 & 490 & 1.0 & 5.4 & 1.1 & 15 & 10,446 & 3,465 & 0.0056 & 236 & 0.765 & 16 & 0.814 & 104 & 0.676 & 0.765 & 0.54 & 230.47 & 166.42 & 0.48 & 75.15 & 0.31 \\
\hline 116,966 & 49,734 & ,202 & 1.5 & 9.1 & 1.9 & 29 & 10,768 & 4,759 & 0.0256 & 191 & 0.758 & 15 & 0.793 & 84 & 0.671 & 0.755 & 0.69 & 502.64 & 441.81 & 1.20 & 22.39 & 0.67 \\
\hline 42,603 & 16,148 & 653 & 1.5 & 7.5 & 1.5 & 21 & 11,265 & 5,224 & 0.0023 & 171 & 0.795 & 27 & 0.845 & 52 & 0.725 & 0.820 & 0.92 & 266.32 & 258.85 & 1.27 & 319.64 & 0.72 \\
\hline 52,419 & 18,705 & 2,107 & 1.0 & 5.4 & 1.1 & 23 & 9,058 & 4,228 & 0.0141 & 221 & 0.755 & 24 & 0.817 & 133 & 0.691 & 0.732 & 0.68 & 319.44 & 421.62 & 0.60 & 43.76 & 0.43 \\
\hline 52,419 & 18,705 & 2,107 & 1.0 & 5.4 & 1.1 & 23 & 9,058 & 4,228 & 0.0060 & 221 & 0.755 & 24 & 0.790 & 133 & 0.661 & 0.732 & 0.76 & 369.97 & 362.99 & 0.60 & 86.88 & 0.51 \\
\hline 48,800 & 19,737 & 1,816 & 2.0 & 8.0 & 1.5 & 23 & 11,259 & 4,308 & 0.0249 & 216 & 0.763 & 11 & 0.800 & 56 & 0.667 & 0.726 & 0.75 & & 9.99 & 0.99 & 25.27 & 0.5 \\
\hline 185,989 & 77,110 & 6,805 & 1.0 & 7.0 & 1.5 & 39 & 8,598 & 3,940 & 0.0079 & 216 & 0.779 & 24 & $0.804 \mid$ & 72 & 0.662 & 0.747 & 0.90 & 619.58 & 592.42 & 0.90 & 62.04| & $0.6 \varsigma$ \\
\hline
\end{tabular}

Table A-2: Data Generated for Six Stage Treatment, continued 


\begin{tabular}{|c|c|c|c|c|c|c|c|c|c|c|c|c|c|c|c|c|c|c|c|c|c|c|}
\hline \begin{tabular}{l|}
$\frac{\Phi}{\mathrm{E}}$ \\
$\frac{3}{0}$ \\
$\frac{\pi}{0}$ \\
$\frac{\pi}{0}$
\end{tabular} & $\begin{array}{l}\stackrel{0}{0} \\
\frac{5}{3} \\
\frac{3}{0} \\
\frac{1}{0} \\
\frac{0}{0} \\
0\end{array}$ & 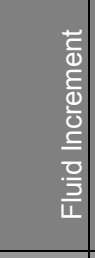 & 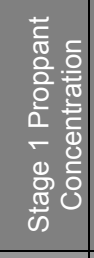 & 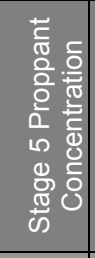 & 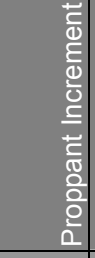 & 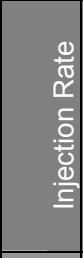 & 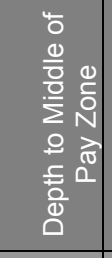 & 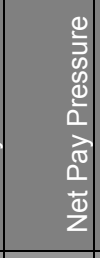 & 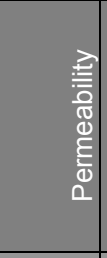 & 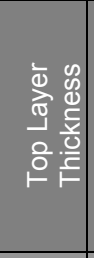 & 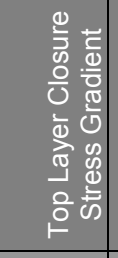 & 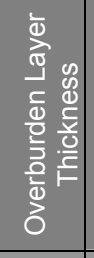 & 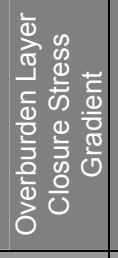 & 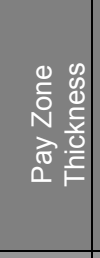 & 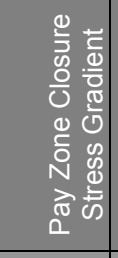 & 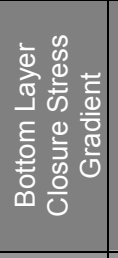 & 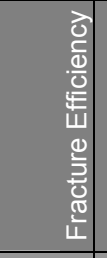 & 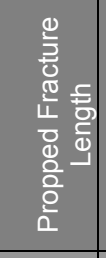 & \begin{tabular}{l|}
$\frac{}{0}$ \\
$\frac{0}{0}$ \\
$\frac{1}{1}$ \\
0 \\
$\frac{0}{3}$ \\
$\frac{3}{0}$ \\
$\frac{\pi}{\frac{\pi}{4}}$
\end{tabular} & 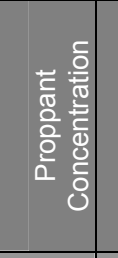 & 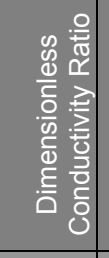 & \\
\hline input & input & input & input & input & input & input & input & input & input & input & input & input & input & input & input & input & output & output & output & output & output & output \\
\hline 94,834 & 39,373 & 3,466 & 1.5 & 7.9 & 1.6 & 32 & 0,172 & ,778 & 0.0382 & 237 & 0.779 & 27 & 0.810 & 78 & 0.711 & 0.761 & 0.69 & 96.11 & 6.46 & 0.97 & 5.23 & \\
\hline 90,144 & 6,736 & 3,338 & 1.5 & 7.1 & 1.4 & 24 & 9,968 & 561 & 0.0061 & 220 & 0.762 & 20 & 0.799 & 105 & 0.681 & 0.770 & 0.82 & 418.21 & 97.35 & 1.02 & 101.69 & \\
\hline 137,196 & 9,023 & 4,886 & 1.0 & 8.2 & 1.8 & 31 & 9,803 & 983 & 0.0168 & 208 & 0.773 & 29 & 0.821 & 84 & 0.716 & 0.752 & 0.80 & 435.68 & 577.61 & 1.09 & 36.03 & \\
\hline 112,916 & 1,396 & ,470 & 1.5 & 6.7 & 1.3 & 30 & 10,782 & 4,710 & 0.0082 & 238 & 0.731 & 22 & 0.757 & 67 & 0.657 & 0.715 & 0.88 & 451.66 & 513.51 & 0.93 & 59.60 & \\
\hline 181,047 & 5,961 & ,568 & 2.0 & 9.6 & 1.9 & 40 & 10,964 & \begin{tabular}{|l|}
5,079 \\
\end{tabular} & 0.0084 & 232 & 0.737 & 32 & 0.850 & 127 & 0.685 & 0.819 & 0.70 & 859.41 & 323.38 & 1.62 & 46.90 & \\
\hline 68,007 & 4,738 & ,704 & 1.0 & 7.0 & 1.5 & 27 & 8,973 & 265 & 0.0669 & 206 & 0.830 & 30 & 0.839 & 133 & 0.708 & 0.830 & .34 & 397.22 & 88.00 & 1.07 & 16.35 & \\
\hline 167,922 & 9,171 & ,172 & 2.0 & 10.0 & 2.0 & 34 & 9,842 & 941 & 0.0014 & 240 & 0.746 & 14 & 0.788 & 131 & 0.664 & 0.750 & 0.90 & 532.58 & 490.00 & 1.68 & 604.87 & \\
\hline 67,429 & 27,276 & 2,510 & 2.0 & 9.6 & 1.9 & 28 & 10,517 & 4,310 & 0.0168 & 213 & 0.759 & 21 & 0.795 & 73 & 0.677 & 0.765 & 0.76 & 337.86 & 347.91 & 1.46 & 62.19 & \\
\hline 70,962 & 9,166 & 2,612 & 1.0 & 7.0 & 1.5 & 26 & 10,128 & 067 & 0.0061 & 175 & 0.751 & 12 & 0.792 & 83 & 0.709 & 0.740 & 0.83 & 399.97 & 433.66 & 0.73 & 68.00 & \\
\hline 143,297 & 3,012 & 5,018 & 2.0 & 9.6 & 1.9 & 35 & 9,211 & 4,250 & 0.0056 & 221 & 0.807 & 11 & 0.833 & 117 & 0.72 & 0.797 & 0.84 & 465.47 & 08.28 & 1.46 & 143.33 & .75 \\
\hline 170,274 & 2,778 & ,719 & 2.0 & 10.0 & 2.0 & 40 & 10,832 & 4,763 & 0.0595 & 234 & 0.749 & 20 & 0.792 & 119 & 0.665 & 0.724 & 0.57 & 559.13 & 560.15 & 1.53 & 12.23 & .74 \\
\hline 30,456 & 1,423 & 1,190 & 2.0 & 10.4 & 2.1 & 18 & 9,891 & 4,128 & 0.0194 & 204 & 0.740 & 15 & 0.758 & 111 & 0.655 & 0.748 & 0.55 & 261.33 & 284.14 & 1.18 & 75.79 & .47 \\
\hline 96,458 & 9,567 &, 556 & 1.0 & 7.0 & 1.5 & 30 & 0,198 & 4,425 & 0.0391 & 229 & .761 & 20 & 0.788 & 80 & 0.698 & 0.750 & 0.71 & 381.78 & 497.05 & 0.91 & 15.17 & .55 \\
\hline 128,654 & 48,579 & 5,005 & 1.5 & 6.3 & 1.2 & 30 & 13,141 & 6,059 & 0.0642 & 215 & 0.788 & 12 & 0.799 & 116 & 0.688 & 0.763 & 0.34 & 622.61 & 326.17 & 1.13 & 3.48 & 0.60 \\
\hline 141,855 & 0,338 & 095 & 1.5 & 9.1 & 1.9 & 35 & 4,043 & 145 & 0.0071 & 196 & 750 & 13 & 0.821 & 124 & 0.674 & 730 & .72 & 60.07 & 88.63 & 1.12 & 17.87 & $6 s$ \\
\hline 72,754 & 27,943 & 2,801 & 1.5 & 6.7 & 1.3 & 28 & 15,188 & 6,617 & 0.0164 & 191 & 0.810 & 28 & 0.858 & 122 & 0.724 & 0.805 & 0.39 & 611.12 & 232.82 & 0.94 & 5.49 & 0.51 \\
\hline 72,754 & 27,943 & 2,801 & 1.5 & 6.7 & 1.3 & 28 & 5,188 & 617 & 164 & 191 & .810 & 28 & 0.858 & 122 & 0.735 & 0.805 & 0.41 & 83.69 & .63 & 0.85 & 5.04 & .53 \\
\hline 18,752 & 6,918 & 740 & 1.5 & 7.9 & 1.6 & 17 & 13,037 & ,708 & 0.0678 & 226 & 0.760 & 18 & 0.785 & 54 & 0.67 & 0.727 & 0.75 & 276.78 & 216.21 & 0.69 & 30.65 & .39 \\
\hline 121,447 & 47,620 & ,614 & 1.5 & 7.5 & 1.5 & 32 & 11,923 & ,929 & 0.0091 & 226 & .805 & 14 & 0.842 & 89 & 0.7 & 0.783 & 0.78 & 633.27 & 386.08 & 1.01 & 9.46 & .72 \\
\hline 84,208 & 33,863 & 3,147 & 1.5 & 6.7 & 1.3 & 27 & 14,628 & \begin{tabular}{|l|}
, 162 \\
\end{tabular} & 0.0096 & 204 & 0.724 & 19 & 0.769 & 120 & .663 & 0.718 & 0.66 & 561.11 & 331.09 & 0.81 & 11.12 & 0.56 \\
\hline 63,997 & 25,511 & 2,405 & 1.0 & 8.2 & 1.8 & 26 & 12,041 & 5,261 & 0.0077 & 205 & .755 & 23 & 0.800 & 89 & 0.675 & 0.726 & 0.78 & 403.84 & .79 & 1.04 & 38.00 & \\
\hline 133,526 & 53,220 & 5,019 & 1.0 & 7.0 & 1.5 & 34 & 13,570 & 6,619 & 0.0985 & 175 & .766 & 29 & 0.766 & 95 & 0.665 & 0.724 & 0.43 & 622.07 & 365.40 & 1.06 & 1.98 & .61 \\
\hline 22,145 & \begin{tabular}{|r|}
8,824 \\
\end{tabular} & 833 & 1.5 & 8.7 & 1.8 & 17 & $\mid 12,524$ & 5,882 & 0.0041 & 200 & .744 & 25 & 0.768 & 131 & 0.656 & 0.714 & 0.67 & 267.93 & 3.27 & 0.93 & 85.52 & .42 \\
\hline 41,886 & 18,373 & 1,470 & 1.0 & 8.2 & 1.8 & 20 & 12,838 & 6,274 & 0.0146 & 228 & 0.755 & 18 & 0.767 & 62 & 0.676 & 0.750 & 0.73 & 351.79 & 307.35 & 0.76 & 13.84 & 0.48 \\
\hline 107,394 & 38,682 & 4,294 & 2.0 & 8.0 & 1.5 & 30 & 14,970 & 6,767 & 0.0066 & 226 & 0.782 & 23 & 0.816 & 73 & 0.69 & 0.748 & 0.83 & 556.92 & 423.79 & 1.05 & 15.38 & 0.64 \\
\hline 90,211 & 38,770 & 3,215 & 1.0 & 5.8 & 1.2 & 29 & $\mid 13,873$ & 6,086 & 0.0485 & 186 & .765 & 21 & 0.801 & 115 & 0.7 & 0.748 & 0.39 & 489.74 & 395.05 & 0.67 & 2.64 & \\
\hline 131,337 & 52,810 & 4,908 & 2.0 & 6.0 & 1.0 & 31 & 14,907 & 7,425 & 0.0086 & 239 & 0.778 & 29 & 0.778 & 91 & 0.702 & 0.764 & 0.74 & 725.29 & 398.42 & 0.77 & 6.83 & 0.64 \\
\hline 70,972 & 28,733 & 2,640 & 2.0 & 9.6 & 1.9 & 26 & 12,009 & 5,581 & 0.0061 & 177 & 0.759 & 11 & 0.790 & 97 & 0.675 & 0.753 & 0.77 & 459.57 & 371.54 & 1.07 & 46.01 & \\
\hline 60,099 & 22,521 & & 2.0 & 8.8 & 1.7 & 24 & 15,026 & 6,959 & 0.0046 & 197 & 0.747 & 28 & 0.781 & 94 & 0.688 & 0.722 & 0.80 & & & 1.07 & 31.51 & .5 \\
\hline 191,058 & $\mid 80,385$ & 6,917 & 1.5 & 7.5 & 1.5 & 19 & $|13,635|$ & 6,630 & 0.0144 & 211 & 0.765 & 24 & 0.785 & 73 & 0.677 & 0.740 & 0.81 & 739.16 & $|549.25|$ & 0.91 & 6.32 & \\
\hline
\end{tabular}

Table A-2: Data Generated for Six Stage Treatment, continued 


\begin{tabular}{|c|c|c|c|c|c|c|c|c|c|c|c|c|c|c|c|c|c|c|c|c|c|c|}
\hline 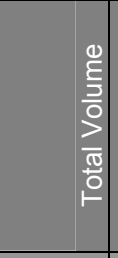 & 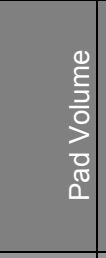 & 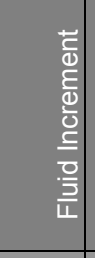 & 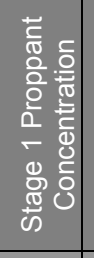 & 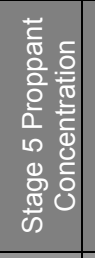 & 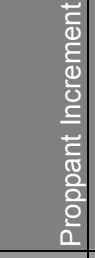 & 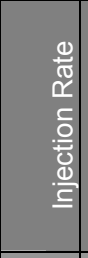 & 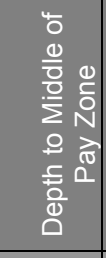 & 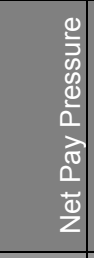 & 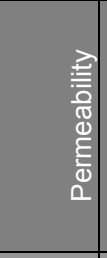 & 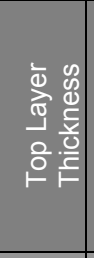 & 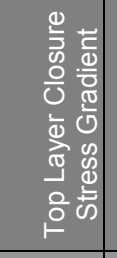 & 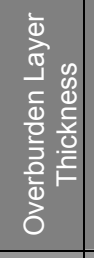 & 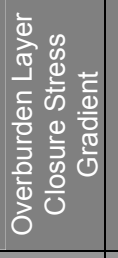 & 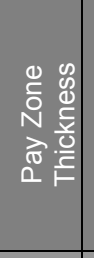 & 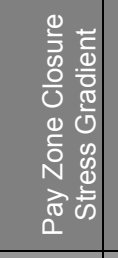 & 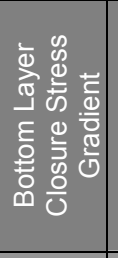 & 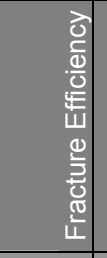 & 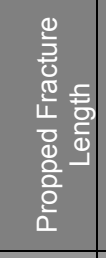 & \begin{tabular}{l|l}
$\frac{7}{0}$ \\
$\frac{0}{0}$ \\
$\frac{1}{1}$ \\
$\frac{10}{5}$ \\
$\frac{3}{0}$ \\
$\frac{\pi}{4}$ \\
$\frac{\pi}{4}$
\end{tabular} & 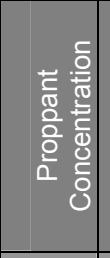 & 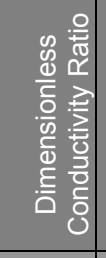 & \\
\hline input & input & input & input & input & input & input & input & input & input & input & input & input & input & input & input & input & output & output & output & output & output & output \\
\hline 136,466 & 49,386 & 5,443 & 2.0 & 9.6 & 1.9 & 32 & 12,875 & 6,228 & 0.0286 & 225 & 0.778 & 21 & 0.794 & 82 & 0.696 & 0.779 & 0.68 & 552.94 & 416.97 & 1.62 & 11.12 & .81 \\
\hline 55,024 & 22,940 & 005 & 1.0 & 7.8 & 1.7 & 24 & 15,000 & 501 & 0080 & 186 & 0.767 & 14 & 0.796 & 121 & 0.7 & 0.739 & 0.61 & 392.21 & 09.11 & 0.87 & 14.89 & .49 \\
\hline 113,497 & 47,620 & 4,117 & 2.0 & 9.2 & 1.8 & 29 & 14,181 & 6,977 & 0.0098 & 224 & 0.820 & 27 & 0.840 & 53 & 0.733 & 0.821 & 0.82 & 585.21 & 390.48 & 1.18 & 12.36 & .72 \\
\hline 143,666 & 60,164 & 5,219 & 2.0 & 10.4 & 2.1 & 35 & 14,834 & 6,631 & 0098 & 193 & 0.776 & 18 & 0.775 & 104 & 0.674 & 0.754 & 0.65 & 815.35 & 299.06 & 1.57 & 26.09 & \\
\hline 177,515 & 69,848 & 6,729 & 2.0 & 8.0 & 1.5 & 38 & 12,086 & ,931 & 0144 & 171 & 0.751 & 25 & 0.772 & 57 & 0.672 & 0.734 & 0.87 & 638.70 & 64.92 & 1.09 & 27.55 & 68 \\
\hline 184,572 & 80,419 & 6,510 & 1.0 & 6.6 & 1.4 & 40 & 11,956 & 5,598 & 0.0195 & 227 & 0.774 & 20 & 0.799 & 116 & 0.663 & 0.788 & 0.60 & 925.56 & 308.62 & 1.05 & 15.44 & .78 \\
\hline 112,669 & 48,223 & 4,028 & 2.0 & 6.4 & 1.1 & 30 & 13,178 & ,939 & 0.0521 & 189 & 0.755 & 28 & 0.778 & 122 & 0.686 & 0.745 & 0.40 & 537.64 & 404.59 & 0.89 & 6.81 & \\
\hline 108,037 & 43,704 & 4,021 & 2.0 & 6.4 & 1.1 & 29 & 13,265 & 6,614 & 0.0063 & 197 & 0.788 & 13 & 0.828 & 79 & 0.714 & 0.764 & 0.84 & 552.95 & 470.75 & 0.75 & 28.15 & .56 \\
\hline 15,316 & 5,989 & 583 & 1.0 & 5.8 & 1.2 & 15 & 13,521 & 164 & 0019 & 174 & 0.769 & 18 & 0.816 & 121 & .666 & 0.726 & 0.70 & 272.93 & 91.28 & 0.54 & 139.46 & .34 \\
\hline 35,290 & 13,252 & 1,377 & 2.0 & 8.4 & 1.6 & 20 & 12,998 & 5,985 & 0.0088 & 192 & 0.769 & 30 & 0.816 & 117 & 0.669 & 0.740 & 0.57 & 373.12 & 198.30 & 1.13 & 64.70 & .50 \\
\hline 23,678 & 9,740 & 871 & 2.0 & 7.2 & 1.3 & 17 & 12,363 & 164 & .0051 & 221 & 0.767 & 18 & 0.791 & 127 & 0.68 & 0.745 & 0.64 & 284.76 & 198.94 & 0.83 & 101.19 & 40 \\
\hline 199,962 & 71,246 & 8,045 & 1.5 & 7.5 & 1.5 & 40 & 14,489 & 6,887 & 0.0083 & 213 & 0.757 & 35 & 0.784 & 112 & 0.672 & 0.733 & 0.74 & 922.74 & 451.05 & 1.03 & 18.93 & .75 \\
\hline 155,859 & 54,907 & 6,309 & 2.0 & 8.8 & 1.7) & 38 & 13,521 & 6,294 & 0816 & 193 & 0.760 & 17 & 0.760 & 133 & 0.677 & 0.757 & 0.32 & 611.66 & 298.40 & 2.19 & 10.45 & .74 \\
\hline 177,555 & 76,901 & 6,291 & 2.0 & 9.2 & 1.8 & 37 & 13,771 & 5,991 & 0.0261 & 204 & 0.752 & 23 & 0.797 & 105 & 0.687 & 0.735 & 0.63 & 652.71 & 594.92 & 1.07 & 10.20 & 0.62 \\
\hline 23,051 & 8,843 & 888 & 1.5 & 7.9 & 1.6 & 16 & 15,055 & 7,015 & 0.0364 & 236 & 0.793 & 21 & 0.846 & 52 & 0.72 & 0.824 & 0.40 & 343.74 & 227.02 & 0.60 & 5.45 & 35 \\
\hline 39,798 & 14,322 & 1,592 & 1.5 & 7.1 & 1.4 & 21 & 13,348 & 6,058 & 0.0094 & 216 & 0.737 & 13 & 0.772 & 54 & 0.668 & 0.740 & 0.81 & 353.44 & 358.08 & 0.63 & 31.21 & 0.44 \\
\hline 160,639 & 62,733 & 6,119 & 1.0 & 9.0 & 2.0 & 36 & 12,091 & 5,220 & 0095 & 224 & 0.751 & 24 & 0.802 & 88 & 0.678 & 0.727 & 0.83 & 573.42 & 642.66 & 1.01 & 41.77| & .62 \\
\hline 46,619 & 19,662 & 1,685 & 1.5 & 9.5 & 2.0 & 22 & 13,736 & 6,660 & 0.0962 & 171 & 0.760 & 12 & 0.784 & 127 & 0.683 & 0.724 & 0.57 & 334.66 & 285.37 & 1.16 & 53.99 & 0.49 \\
\hline 30,505 & 13,412 & 1,068 & 1.5 & 7.5 & 1.5 & 22 & 12,289 & 5,641 & 0175 & 232 & 0.771 & 22 & 0.815 & 132 & 0.709 & 0.752 & 0.47 & 255.02 & 294.28 & 0.75 & 32.21 & .36 \\
\hline 66,638 & 24,106 & 2,658 & 1.5 & 8.7 & 1.8 & 26 & 14,648 & 6,337 & 0.0072 & 226 & 0.770 & 34 & 0.792 & 69 & 0.678 & 0.767 & 0.72 & 620.66 & 288.70 & 0.89 & 27.30 & 0.60 \\
\hline 66,638 & 24,106 & 2,658 & 1.5 & 8.7 & 1.8 & 26 & 14,648 & 6,337 & 0.0072 & 226 & 0.760 & 34 & 0.780 & 69 & 0.694 & 0.750 & 0.79 & 445.42 & 401.33 & 0.89 & 34.86 & 0.54 \\
\hline 113,183 & 43,732 & 4,341 & 1.5 & 9.9 & 2.1 & 28 & 12,011 & ,044 & 0.0254 & 187 & 0.736 & 35 & 0.761 & 118 & 0.673 & 0.717 & 0.60 & 479.38 & 530.46 & 1.17 & 27.30 & 0.58 \\
\hline 166,794 & 60,902 & 6,618 & 1.0 & 7.0 & 1.5 & 31 & 14,228 & 6,242 & 0.0431 & 203 & 0.760 & 26 & 0.782 & 62 & 0.66 & 0.735 & 0.64 & 884.41 & 476.61 & 0.75 & 3.39 & 0.70 \\
\hline 26,289 & 9,742 & 1,034 & 2.0 & 6.8 & 1.2 & 18 & 13,580 & 6,079 & 0.0085 & 237 & 0.775 & 15 & 0.792 & 99 & 0.715 & 0.746 & 0.64 & 263.30 & 283.72 & 0.69 & +1.04 & 0.37 \\
\hline 187,858 & 78,668 & 6,824 & 1.5 & 8.3 & 1.7 & 40 & 12,073 & 5,911 & 0.0079 & 198 & 0.755 & 29 & 0.787 & 113 & 0.67 & 0.770 & 0.79 & 707.30 & 408.10 & 1.39 & 58.59 & 0.86 \\
\hline 157,950 & 64,934 & 5,813 & 2.0 & 10.0 & 2.0 & 38 & 13,753 & 6,139 & 0.0184 & 177 & 0.760 & 22 & 0.760 & 80 & 0.69 & 0.740 & 0.76 & 552.40 & 569.02 & 1.31 & 9.17 & .69 \\
\hline 77,929 & 29,772 & 3,010 & 1.0 & 7.8 & 1.7 & 28 & 14,874 & 7,272 & 0.0174 & 214 & 0.757 & 27 & 0.788 & 71 & 0.686 & 0.739 & 0.71 & 526.31 & 349.58 & 0.86 & 11.24 & 0.56 \\
\hline 40,985 & 14,665 & 1,645 & 1.0 & 7.4 & 1.6 & 20 & 13,893 & 6,177 & 0.0095 & 234 & 0.754 & 16 & 0.772 & 55 & 0.69 & 0.751 & 0.79 & 302.33 & 306.55 & 0.80 & 10.33 & .51 \\
\hline 44,906 & 18,939 & 1,623 & 2.0 & 10.0 & 2.0 & 22 & 12,673 & 5,446 & 0.0678 & 239 & 768 & 31 & 0.768 & 84 & 0.67 & 0.745 & 0.32 & 360.18 & 53.91 & 1.26 & 13.72 & 0.48 \\
\hline 144,556 & 59,965 & 5,287 & 2.0 & 8.0 & 1.5 & $37 \mid$ & 13,634 & 6,524 & 0.0096 & 228 & 0.809 & 26 & 0.849 & 76 & 0.728 & 0.784 & 0.83| & 583.60 & 554.33 & 0.95 & 19.93 & 0.6 \\
\hline
\end{tabular}

Table A-2: Data Generated for Six Stage Treatment, continued 


\begin{tabular}{|c|c|c|c|c|c|c|c|c|c|c|c|c|c|c|c|c|c|c|c|c|c|c|}
\hline 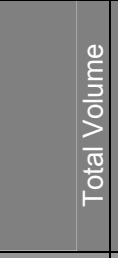 & 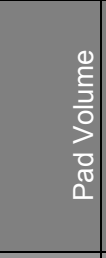 & 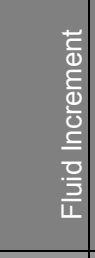 & 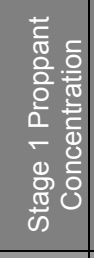 & 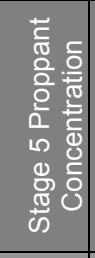 & 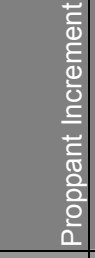 & 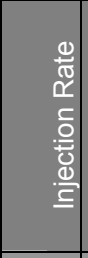 & 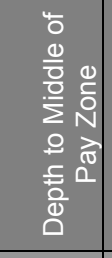 & 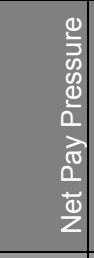 & 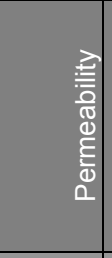 & 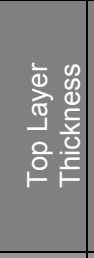 & 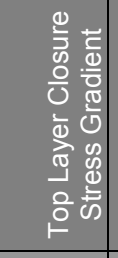 & 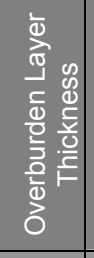 & 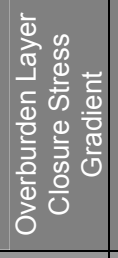 & 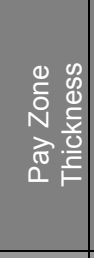 & 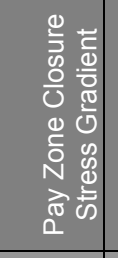 & 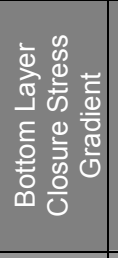 & 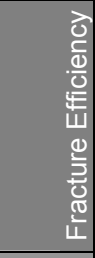 & 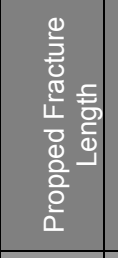 & \begin{tabular}{l|l}
$\frac{7}{0}$ \\
$\frac{0}{0}$ \\
$\frac{1}{1}$ \\
0 \\
$\frac{0}{3}$ \\
$\frac{3}{0}$ \\
$\frac{0}{4}$ \\
$\frac{\pi}{4}$
\end{tabular} & 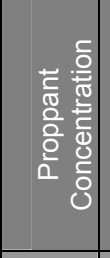 & 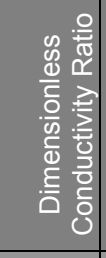 & \\
\hline input & input & input & input & input & input & input & input & input & input & input & input & input & input & input & input & input & output & output & utput & output & output & output \\
\hline 132,820 & 47,349 & 5,342 & 2.0 & 8.4 & 1.6 & 32 & 12,122 & 5,135 & 0.0490 & 176 & 0.739 & 23 & 0.744 & 102 & 0.659 & 0.716 & 0.54 & 589.79 & 496.36 & 1.11 & 12.29 & .6 \\
\hline 70,182 & 5,479 & 2,794 & 1.0 & 7.8 & 1.7 & 25 & 14,403 & 6,822 & 0.0166 & 215 & 0.739 & 19 & 0.798 & 113 & 0.682 & 0.767 & 0.50 & 576.35 & 274.65 & 0.94 & 6.59 & .57 \\
\hline 26,733 & 10,917 & 988 & 1.5 & 7.5 & 1.5 & 15 & 13,185 & 5,749 & 0.0073 & 202 & 0.761 & 25 & 0.798 & 52 & 0.7 & 0.741 & 0.82 & 261.77 & 342.94 & 0.58 & 41.39 & 3 \\
\hline 122,435 & 46,409 & 4,752 & 1.5 & 8.7 & 1.8 & 34 & 12,252 & 5,631 & 0.0374 & 176 & 0.788 & 20 & 0.830 & 110 & 0.713 & 0.764 & 0.68 & 541.46 & 515.36 & 1.04 & 22.46 & .62 \\
\hline 150,870 & 59,217 & ,728 & 1.0 & 8.2 & 1.8 & 33 & 14,806 & 6,518 & 0100 & 191 & 0.766 & 22 & 0.800 & 129 & 0.69 & 0.766 & 0.59 & 842.74 & 41.49 & 1.10 & 18.30 & 6 \\
\hline 22,626 & 9,177 & 841 & 1.0 & 7.0 & 1.5 & 16 & 14,793 & 6,900 & 0.0066 & 199 & 0.724 & 33 & 0.746 & 90 & 0.655 & 0.710 & 0.62 & 298.43 & 85.56 & 0.60 & 35.60 & .37 \\
\hline 60,820 & 5,859 & 2,185 & 1.0 & 7.8 & 1.7 & 24 & 2,679 & 6,050 & 0.0994 & 227 & .820 & 30 & 0.850 & 94 & 0.732 & 0.813 & 0.22 & 405.62 & 67.15 & 1.08 & 5.80 & 3 \\
\hline 90,239 & 33,590 & 3,541 & 1.5 & 7.5 & 1.5 & 28 & $\mid 12,496$ & 5,613 & 0.0887 & 173 & 0.770 & 20 & 0.770 & 108 & 0.67 & 0.792 & 0.74 & 443.81 & 437.84 & 0.97 & 50.44 & .61 \\
\hline 91,913 & 5,364 &, 534 & 1.0 & 7.4 & 1.6 & 30 & 4,996 & 319 & 0635 & 177 & 0.749 & 25 & 0.755 & 90 & 0.671 & 0.751 & 0.45 & 550.58 & 07.64 & 0.79 & 3.49 & .5 \\
\hline 55,843 & 22,222 & 2,101 & 2.0 & 6.8 & 1.2 & 24 & 13,045 & 5,720 & 0.0212 & 198 & 0.798 & 33 & 0.837 & 122 & 0.719 & 0.793 & 0.41 & 444.94 & 284.82 & 0.84 & 16.95 & 0.48 \\
\hline 146,251 & 4,035 & 5,763 & 1.0 & 6.2 & 1.3 & 34 & 3,109 & ,408 & 0.0084 & 203 & 0.750 & 14 & 0.780 & 85 & 0.714 & 0.743 & 0.85 & 501.81 & 49.90 & 0.90 & 35.33 & 66 \\
\hline 174,584 & 5,367 & 6,201 & 2.0 & 6.8 & 1.2 & 38 & 14,373 & 7,144 & 0.0050 & 192 & 0.769 & 20 & 0.796 & 74 & 0.674 & 0.737 & 0.90 & 603.53 & 513.38 & 1.01 & 34.55 & 0.74 \\
\hline 29,174 & 1,584 & ,099 & 2.0 & 6.8 & 1.2 & 18 & 4,198 & 6,865 & 0.0053 & 236 & .732 & 12 & 0.785 & 128 & 0.665 & 0.712 & 0.64 & 310.32 & 36.56 & 0.74 & 58.66 & .41 \\
\hline 30,589 & 11,287 & 1,206 & 1.0 & 7.0 & 1.5 & 21 & $\mid 14,514$ & 6,384 & 0.0076 & 224 & 0.800 & 23 & 0.820 & 98 & 0.728 & 0.779 & 0.59 & 319.02 & 221.66 & 0.64 & 26.63 & 0.43 \\
\hline 190,821 & 8,147 & ,667 & 1.0 & 7.4 & 1.6 & 23 & 2,201 & ,484 & 0.0065 & 222 & 0.777 & 20 & 0.805 & 56 & 0.692 & 0.750 & 0.92 & 587.09 & 720.28 & 0.92 & 48.17 & .64 \\
\hline 61,807 & 21,811 & 2,500 & 1.0 & 6.2 & 1.3 & 23 & 11,847 & 4,978 & 0.0605 & 171 & 0.798 & 11 & 0.811 & 71 & 0.695 & 0.765 & 0.80 & 486.65 & 342.86 & 0.65 & 47.55 & 0.54 \\
\hline 10,544 & 4,524 & 376 & 2.0 & 9.2 & 1.8 & 15 & 12,035 & 5,266 & 0.0046 & 187 & .751 & 32 & 0.773 & 90 & 0.672 & 0.743 & 0.66 & 193.69 & 177.11 & 0.74 & 151.30 & .33 \\
\hline 96,107 & 5,425 & 3,793 & 2.0 & 6.8 & 1.2 & 30 & 11,944 & 5,763 & 0.0542 & 209 & 0.735 & 30 & 0.746 & 89 & 0.655 & 0.714 & 0.60 & 501.35 & 474.71 & 0.81 & 9.23 & 0.53 \\
\hline 51,652 &, 241 & 2,088 & 2.0 & 9.2 & 1.8 & 27 & 3,290 & 6,510 & 0.0766 & 205 & 0.786 & 13 & 0.810 & 58 & 0.686 & 0.765 & 0.74 & 423.73 & 309.96 & 1.04 & 24.67 & .5 \\
\hline 86,527 & 3,029 & ,344 & 2.0 & 10.0 & 2.0 & 27 & 14,933 & 6,648 & 0.0319 & 196 & .782 & 29 & 0.828 & 129 & 0.71 & 0.800 & 0.31 & 511.98 & |253.24 & 1.81 & |4.11 & 0.59 \\
\hline 181,941 & 1,919 & 6,876 & 1.5 & 7.5 & 1.5 & 28 & 3,913 & 6,201 & 0.0110 & 170 & 0.787 & 29 & 0.813 & 122 & 0.684 & 0.786 & 0.86 & 975.07 & 323.17 & 1.16 & 138.64 & 0.81 \\
\hline 181,941 & 1,919 & 6,876 & 1.5 & 7.5 & 1.5 & 28 & 13,913 & 6,201 & 0.0500 & 170 & 0.787 & 29 & 0.813 & 122 & 0.72 & 0.786 & 0.46 & 688.97 & 536.37 & 0.99 & 4.80 & 0.62 \\
\hline 55,767 & 21,134 & 2,165 & 2.0 & 6.0 & 1.0 & 25 & $\mid 12,468$ & $\mid 5,327$ & 0.0034 & 221 & 0.772 & 23 & 0.800 & 123 & 0.706 & 0.750 & 0.78 & $|379.25|$ & $|366.65|$ & 0.71 & 91.88 & 0.50 \\
\hline 162,809 & 64,016 & 175 & 1.5 & 8.7 & 1.8 & 36 & $|14,017|$ & 6,251 & 0.0082 & 211 & .731 & 19 & 0.757 & 113 & 0.651 & 0.715 & 0.73 & $\mid 771.83$ & 450.06 & 1.08 & 27.85 & 0.72 \\
\hline 183,464 & 69,200 & | 141 & 1.5 & 7.5 & 1.5 & 40 & $\mid 14,977$ & 7,125 & 0.0093 & 221 & 0.762 & 16 & 0.796 & 94 & 0.691 & 0.749 & 0.78 & | 772.87| & 546.45 & 0.90 & 13.98 & 0.66 \\
\hline 87,086 & 0,515 & ,536 & 2.0 & 8.4 & 1.6 & 27 & $\mid 14,834$ & 7,144 & 0.0114 & 176 & .786 & 29 & 0.811 & 101 & 0.712 & 0.770 & 0.64 & 592.99 & 351.95 & 1.02 & 18.54 & 0.61 \\
\hline 150,266 & 59,538 & 5,671 & 1.5 & 9.9 & 2.1 & 38 & 14,489 & 6,942 & 0.0330 & 179 & .741 & 13 & 0.755 & 78 & 0.683 & 0.732 & 0.71 & 576.25 & 530.77 & 1.27 & 9.76 & 0.66 \\
\hline 61,746 & 22,893 & 2,428 & 1.5 & 8.3 & 1.7 & 25 & $\mid 13,195$ & $\mid 6,438$ & 0.0093 & 238 & .788 & 20 & 0.800 & 80 & 0.706 & 0.744 & 0.79 & 380.90 & |430.64 & 0.86 & 36.67 & 0.50 \\
\hline 147,246 & 62,951 & 5,268 & 2.0 & 6.4 & 1.1 & 34 & $\mid 12,411$ & 5,994 & 0.0112 & 209 & .754 & 30 & 0.775 & 99 & 0.682 & 0.755 & 0.79 & 586.37| & |457.85 & 0.95 & 35.40 & .6 \\
\hline 169,729 & 72,310 & 6,089 & 1.5 & 7.5 & 1.5 & 38 & $|13,275|$ & |6,592 & 0.0251 & 193 & 0.728 & 15 & $0.741 \mid$ & 89 & $0.667 \mid$ & 0.714 & 0.74 & $|708.17|$ & | 607.84 & 1.09 & 12.57 & \\
\hline
\end{tabular}

Table A-2: Data Generated for Six Stage Treatment, continued 


\begin{tabular}{|c|c|c|c|c|c|c|c|c|c|c|c|c|c|c|c|c|c|c|c|c|c|c|}
\hline 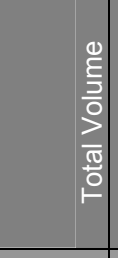 & 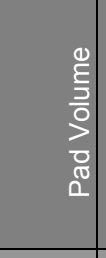 & 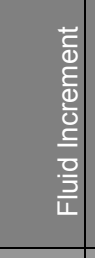 & 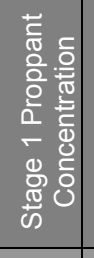 & 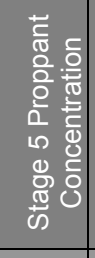 & 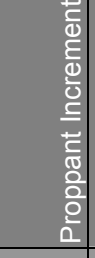 & 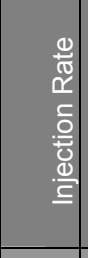 & 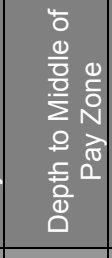 & 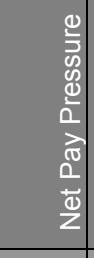 & 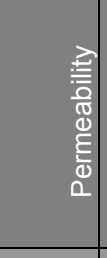 & 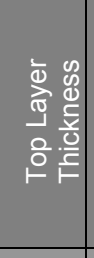 & 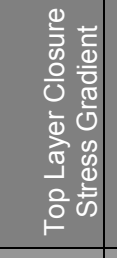 & 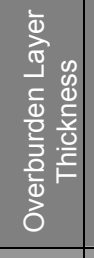 & 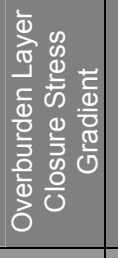 & 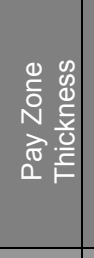 & 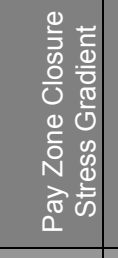 & 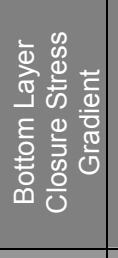 & 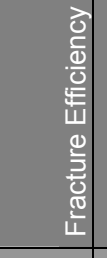 & 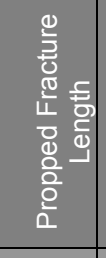 & 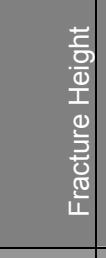 & 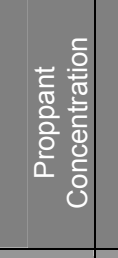 & 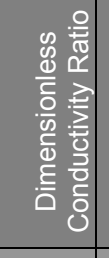 & \\
\hline input & input & input & input & input & input & input & input & input & input & input & input & input & input & input & input & input & output & output & utput & output & output & Outhi \\
\hline 137,308 & 48,675 & 5,540 & 1.5 & 7.5 & 1.5 & 35 & 13,765 & 6,568 & 0.0076 & 221 & 0.747 & 12 & 0.788 & 116 & 0.676 & 0.750 & 0.75 & 649.30 & 29.70 & 1.05 & 36.51 & .72 \\
\hline 68,446 & 26,951 & 2,593 & 1.5 & 9.5 & 2.0 & 26 & 12,300 & 5,873 & 0243 & 184 & 0.770 & 12 & 0.770 & 63 & 0.696 & 0.756 & 0.75 & 359.21 & 41.11 & 1.08 & 25.50 & .57 \\
\hline 27,338 & 11,395 & 996 & 1.5 & 5.9 & 1.1 & 18 & 14,740 & 6,871 & 0.0075 & 204 & 0.764 & 14 & 0.783 & 106 & 0.674 & 0.738 & 0.59 & 307.62 & 224.49 & 0.60 & 26.20 & 37 \\
\hline 74,720 & 29,191 & 2,846 & 2.0 & 7.2 & 1.3 & 27 & \begin{tabular}{|l|}
12,957 \\
\end{tabular} & 6,277 & 0.0220 & 191 & 0.779 & 25 & 0.850 & 110 & 0.697 & 0.765 & 0.53 & 523.54 & 330.15 & 0.88 & 14.94 & .55 \\
\hline 14,636 & 6,367 & 517 & 2.0 & 10.0 & 2.0 & 17 & 13,787 & 969 & 0.0111 & 193 & 0.747 & 23 & 0.774 & 114 & 0.688 & 0.729 & 0.44 & 193.21 & 09.08 & 0.88 & 54.98 & 30 \\
\hline 180,408 & 67,604 & 7,050 & 1.5 & 7.5 & 1.5 & 40 & \begin{tabular}{|l|}
14,364 \\
\end{tabular} & 6,613 & 0.0089 & 222 & 0.811 & 23 & 0.844 & 51 & 0.733 & 0.820 & 0.89 & 502.81 & 407.23 & 1.83 & 45.36 & .10 \\
\hline 124,281 & 4,082 & 012 & 1.0 & 5.8 & 1.2 & 33 & \begin{tabular}{|l|}
13,527 \\
\end{tabular} & 915 & 0072 & 194 & 0.743 & 23 & 0.755 & 101 & 0.689 & 0.728 & 0.78 & 629.83 & 518.00 & 0.62 & 22.62 & 57 \\
\hline 96,919 & 34,225 & 3,918 & 1.0 & 8.6 & 1.9 & 30 & \begin{tabular}{|l|}
14,997 \\
\end{tabular} & 7,185 & 0.0335 & 238 & 0.819 & 15 & 0.858 & 119 & 0.732 & 0.806 & 0.35 & 604.28 & 280.96 & 1.34 & 8.21 & .58 \\
\hline 39,101 & 13,914 & 574 & 1.0 & 6.6 & 1.4 & 21 & 12,114 & 851 & 0091 & 227 & 0.772 & 15 & 0.807 & 56 & 0.683 & 0.750 & 0.81 & 359.77 & 19.65 & 0.63 & 39.65 & .47 \\
\hline 32,800 & 12,292 & 1,282 & 1.5 & 7.5 & 1.5 & 19 & $\mid 12,702$ & 5,620 & 0.0391 & 234 & 0.740 & 19 & 0.773 & 94 & 0.659 & 0.724 & 0.37 & 327.88 & 265.78 & 0.78 & 16.44 & 0.44 \\
\hline 64,640 & 25,154 & 2,468 & 2.0 & 8.0 & 1.5 & 28 & $|12,367|$ & 5,962 & 0.0722 & 187 & 0.749 & 25 & 0.791 & 84 & 0.681 & 0.756 & 0.79 & 433.10 & 367.12 & 0.91 & 60.42 & 58 \\
\hline 34,184 & 14,960 & 1,201 & 1.5 & 5.9 & 1.1 & 20 & $\mid 13,147$ & 6,520 & 0.0090 & 209 & 0.731 & 17 & 0.732 & 69 & 0.685 & 0.717 & 0.80 & 280.51 & 356.72 & 0.52 & 28.56 & .36 \\
\hline 13,384 & 658 & 483 & 1.0 & 5.8 & 1.2 & 15 & $\mid 14,126$ & ,827 & 0730 & 235 & 0.735 & 33 & 0.751 & 110 & .657 & 713 & 0.51 & 227.43 & 138.43 & 0.53 & 38.40 & .28 \\
\hline 95,300 & 39,509 & 3,487 & 2.0 & 8.8 & 1.7 & 29 & $|14,507|$ & 7,230 & 0.0083 & 232 & 0.820 & 26 & 0.820 & 134 & 0.735 & 0.820 & 0.59 & 642.94 & 257.83 & 1.33 & 27.26 & 0.63 \\
\hline 95,300 & 39,509 & 3,487 & 2.0 & 8.8 & 1.7 & 29 & $|14,507|$ & 7,230 & 0.0083 & 232 & 0.780 & 26 & 0.780 & 134 & 0.73 & 0.780 & 0.70 & 429.69 & 448.79 & 1.14 & 34.39 & .59 \\
\hline 65,751 & 26,099 & 2,478 & 1.5 & 7.5 & 1.5 & 25 & $\mid 14,647$ & 6,537 & 0.0272 & 170 & 0.750 & 27 & 0.819 & 88 & 0.684 & 0.768 & 0.47 & 528.38 & 323.56 & 0.77 & 8.62 & 0.49 \\
\hline 65,751 & 26,099 & 2,478 & 1.5 & 7.5 & 1.5 & 25 & $\mid 14,647$ & 6,537 & 0272 & 170 & 0.750 & 27 & 0.778 & 88 & 0.699 & 0.731 & 0.59 & 400.97 & 435.29 & 0.75 & 8.98 & .44 \\
\hline 125,871 & 3,910 & 498 & 2.0 & 6.4 & 1.1 & 34 & $|14,165|$ & 6,495 & 0.0162 & 225 & 0.744 & 23 & 0.786 & 89 & 0.687 & 0.729 & 0.74 & 563.43 & 546.16 & 0.70 & 10.31 & 0.52 \\
\hline 99,223 & 43,403 & ,489 & 2.0 & 8.8 & 1.7 & 31 & 14,826 & 7,285 & 0.0063 & 195 & 0.776 & 18 & 0.811 & 89 & 0.72 & 0.770 & 0.78 & 548.36 & 429.71 & 0.93 & 27.09 & .59 \\
\hline 29,848 & 11,482 & ,148 & 2.0 & 7.6 & 1.4 & 19 & 14,468 & 7,109 & 0.0074 & 179 & 0.770 & 12 & 0.800 & 79 & 0.696 & 0.769 & 0.67 & 367.72 & 230.77 & 0.74 & 32.20 & 0.45 \\
\hline 29,851 & 10,687 & 198 & 1.0 & 7.8 & 1.7 & 18 & 13,763 & 6,120 & 0.0185 & 178 & 0.740 & 18 & 0.766 & 134 & 0.671 & 0.721 & 0.39 & 294.24 & 264.02 & 0.80 & 28.57 & .37 \\
\hline 192,767 & 72,861 & 7,494 & 2.0 & 10.8 & 2.2 & 38 & 12,934 & 5,590 & 0.0119 & 200 & 0.757 & 10 & 0.772 & 55 & 0.655 & 0.733 & 0.88 & 599.85 & 658.53 & 1.44 & 38.66 & 0.75 \\
\hline 167,438 & 66,247 & 6,324 & 2.0 & 10.0 & 2.0 & 36 & 13,039 & 6,251 & 0.0030 & 230 & 0.768 & 22 & 0.798 & 127 & 0.671 & 0.751 & 0.81 & 804.11 & 361.20 & 1.55 & 113.17 & 0.84 \\
\hline 76,019 & 29,705 & 2,895 & 2.0 & 9.2 & 1.8 & 25 & 12,828 & 6,276 & 0.0096 & 233 & 0.752 & 32 & 0.771 & 56 & 0.688 & 0.729 & 0.88 & 343.21 & 516.32 & 1.08 & 55.82 & 0.55 \\
\hline 104,014 & 45,159 & 3,678 & 1.0 & 9.0 & 2.0 & 32 & 13,199 & 6,043 & 0.0354 & 202 & 0.750 & 22 & 0.784 & 105 & 0.698 & 0.717 & 0.67 & 363.29 & 554.88 & 1.11 & 13.66 & 0.51 \\
\hline 45,048 & 19,270 & 1,611 & 1.0 & 9.0 & 2.0 & 23 & 13,389 & 6,487 & 0.0082 & 196 & 0.758 & 25 & 0.775 & 123 & 0.71 & 734 & 0.66 & 329.12 & 310.64 & 0.95 & 49.97 & .48 \\
\hline 181,866 & 77,049 & 6,551 & 1.0 & 6.2 & 1.3 & 39 & 14,939 & 6,819 & 0.0077 & 239 & 0.777 & 15 & 0.828 & 96 & 0.697 & 0.778 & 0.73 & 968.85 & 440.04 & 0.66 & 9.80 & 0.65 \\
\hline 61,114 & 23,758 & 2,335 & 2.0 & 9.6 & 1.9 & 28 & $\mid 14,187$ & 6,261 & 0.0090 & 188 & 0.742 & 22 & 0.783 & 75 & 0.68 & 0.744 & 0.77 & 389.96 & 360.29 & 1.13 & 47.20 & .58 \\
\hline 143,658 & 52,017 & 5,728 & 2.0 & 10.0 & 2.0 & 35 & 13,617 & 5,973 & 0.0086 & 178 & 740 & 13 & 0.799 & 69 & 0.698 & 0.731 & 0.87 & 445.72 & 573.56 & 1.59 & 65.19 & .70 \\
\hline 175,473 & 67,087 & 6,774 & 1.5 & 5.9 & 1.1| & 39 & $|14,897|$ & 6,802 & 0.0122 & 235 & 0.769 & 23 & 0.822 & 113 & 0.715 & 0.768 & $0.71 \mid$ & 729.75 & 624.51 & 0.64 & 8.10 & 0.5 \\
\hline
\end{tabular}

Table A-2: Data Generated for Six Stage Treatment, continued 


\begin{tabular}{|c|c|c|c|c|c|c|c|c|c|c|c|c|c|c|c|c|c|c|c|c|c|c|}
\hline 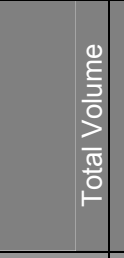 & $\begin{array}{l}\stackrel{0}{E} \\
\frac{3}{3} \\
0 \\
\frac{0}{\sigma} \\
0 \\
0\end{array}$ & 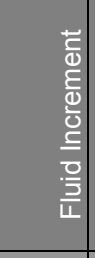 & 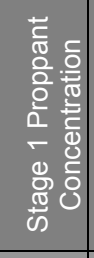 & 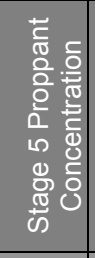 & 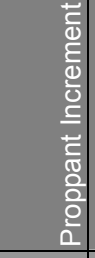 & 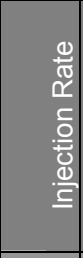 & 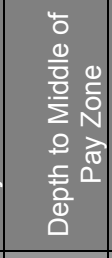 & 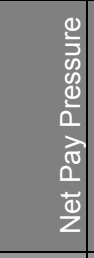 & 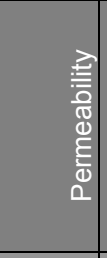 & 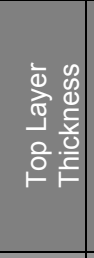 & 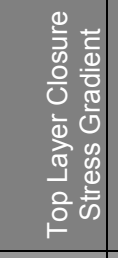 & 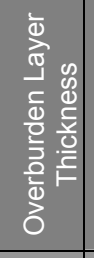 & 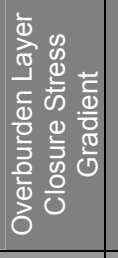 & 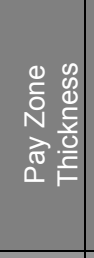 & 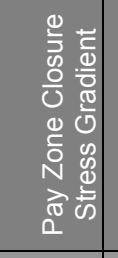 & 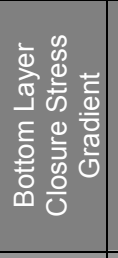 & 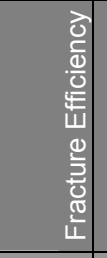 & 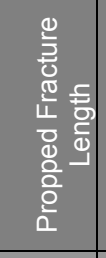 & \begin{tabular}{l|l}
$\frac{\pi}{0}$ & \\
$\frac{0}{0}$ & \\
$\frac{1}{1}$ & \\
0 & \\
$\frac{0}{0}$ & \\
$\frac{\pi}{0}$ & \\
$\frac{\pi}{4}$ &
\end{tabular} & 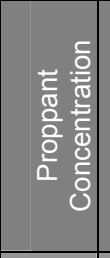 & 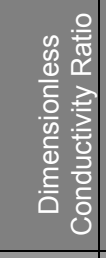 & 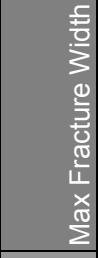 \\
\hline input & input & input & input & input & input & input & input & input & input & input & input & input & input & input & input & input & output & output & output & output & output & output \\
\hline 160,390 & 66,278 & 5,882 & 1.0 & 8.6 & 1.9 & 35 & 14,258 & 6,791 & 0477 & 225 & 0.820 & 18 & 0.851 & 95 & 0.725 & 0.821 & 0.39 & 58.05 & 19.87 & 1.25 & 4.69 & \\
\hline 97,150 & 8,084 & 692 & 1.5 & 7.5 & 1.5 & 30 & 13,998 & 371 & 0586 & 191 & 0.800 & 10 & 0.840 & 96 & 0.729 & 0.815 & 0.39 & 522.55 & 342.81 & 1.10 & 5.82 & \\
\hline 187,284 & 5,234 & 003 & 1.5 & 6.3 & 1.2 & 40 & 14,081 & 6,802 & 0.0071 & 212 & 0.732 & 16 & 0.773 & 64 & 0.687 & 0.730 & 0.91 & 578.55 & 696.23 & 0.79 & 23.84 & \\
\hline 29,364 & 0,733 & 164 & 1.0 & 5.8 & 1.2 & 18 & 12,674 & 5,764 & .0157 & 231 & 0.739 & 34 & 0.759 & 100 & 0.678 & 0.725 & 0.55 & 283.79 & 258.30 & 0.56 & 29.09 & \\
\hline 111,345 & 43,375 & 4,248 & 2.0 & 8.0 & 1.5 & 31 & 13,190 & 5,965 & 0.0425 & 173 & 0.765 & 16 & 0.765 & 80 & 0.689 & 0.751 & 0.60 & 566.96 & 475.67 & 0.92 & 7.31 & 58 \\
\hline 18,301 & 7,120 & 699 & 2.0 & 8.0 & 1.5 & 16 & 14,681 & ,774 & 0075 & 226 & 0.777 & 22 & 0.799 & 73 & 0.683 & 0.763 & 0.56 & 337.90 & 148.49 & 0.78 & 33.85 & \\
\hline 190,839 & 83,231 & 6,726 & 1.5 & 7.5 & 1.5 & 28 & 14,219 & 6,505 & 0.0052 & 181 & 0.757 & 24 & 0.795 & 106 & 0.692 & 0.746 & 0.81 & 771.69 & 563.11 & 0.82 & 25.62 & \\
\hline 54,663 & 2,357 & 019 & 2.0 & 7.6 & 1.4 & 27 & 12,121 & 5,946 & .0242 & 220 & 0.798 & 29 & 0.825 & 97 & 0.716 & 0.785 & 0.57 & 393.99 & 325.21 & 0.88 & 19.06 & .52 \\
\hline 106,727 & 9,900 & 177 & 1.5 & 9.1 & 1.9 & 30 & 14,611 & 6,560 & 0.0370 & 185 & 0.730 & 35 & 0.770 & 109 & 0.652 & 0.718 & 0.42 & 645.54 & 341.13 & 1.20 & 9.89 & .60 \\
\hline 53,538 & 19,025 & 2,157 & 2.0 & 8.4 & 1.6 & 25 & 13,349 & 5,920 & 0.0305 & 170 & 0.743 & 14 & 0.779 & 74 & 0.686 & 0.737 & 0.64 & 345.37 & 381.30 & 1.00 & 17.91 & .50 \\
\hline 75,160 & 27,545 & 2,976 & 1.5 & 7.1 & 1.4 & 28 & $14,923 \mid$ & 6,627 & 0100 & 182 & 0.729 & 33 & 0.766 & 115 & 0.679 & 0.719 & 0.67 & 480.90 & 451.86 & 0.69 & 19.19 & \\
\hline 132,604 & 55,798 & 4,800 & 1.5 & 9.5 & 2.0 & 34 & 14,245 & ,796 & 0096 & 175 & 0.820 & 24 & 0.845 & 62 & 0.728 & 0.817 & 0.79 & 753.77 & 376.82 & 1.11 & 16.53 & \\
\hline 47,365 & 17,462 & 1,869 & 2.0 & 8.8 & 1.7 & 24 & $|13,464|$ & 657 & 0.0071 & 237 & 0.765 & 21 & 0.792 & 83 & 0.685 & 0.764 & 0.74 & 425.08 & 296.92 & 0.93 & 49.81 & 0.55 \\
\hline 44,286 & 16,572 & 1,732 & 1.0 & 6.6 & 1.4 & 24 & 12,674 & 5,792 & 0.0095 & 213 & 0.780 & 23 & 0.799 & 66 & 0.71 & 0.768 & 0.78 & 357.48 & 360.59 & 0.72 & 34.69 & .47 \\
\hline 85,625 & 1,309 & ,395 & 1.5 & 7.5 & 1.5 & 24 & 11,850 & ,216 & 0.0510 & 196 & 0.734 & 23 & 0.772 & 97 & 0.676 & 0.717 & 0.77 & 407.97 & 467.60 & 0.97 & 6.10 & 0.55 \\
\hline 119,340 & 42,076 & 4,829 & 2.0 & 10.0 & 2.0 & 31 & \begin{tabular}{|l|}
14,454 \\
\end{tabular} & 7,083 & 0.0076 & 239 & 0.757 & 29 & 0.788 & 100 & 0.689 & 0.744 & 0.76 & 617.59 & 497.00 & 1.11 & 33.39 & 0.65 \\
\hline 80,784 & 1,961 & 051 & 2.0 & 10.8 & 2.2 & 25 & $\mid 12,735$ & 5,432 & 0.0421 & 186 & 0.801 & 16 & 0.835 & 82 & 0.74 & 0.780 & 0.68 & 386.76 & 465.97 & 1.36 & 5.12 & .55 \\
\hline 80,784 & 31,961 & ,051 & 2.0 & 10.8 & 2.2 & 25 & 12,735 & 5,432 & 0.0090 & 186 & 0.801 & 16 & 0.835 & 82 & 0.731 & 0.790 & 0.76 & 417.67 & 436.10 & 1.35 & 58.53 & 0.61 \\
\hline 86,893 & 32,446 & 403 & 2.0 & 9.6 & 1.9 & 27 & 13,296 & 5,488 & 0055 & 196 & 0.741 & 34 & 0.765 & 103 & 0.696 & 45 & 0.80 & 385.89 & 427.37 & 1.40 & 110.86 & 0.64 \\
\hline 33,342 & 12,414 & 1,308 & 1.5 & 7.5 & 1.5 & 18 & $\mid 15,004$ & 5,639 & 0.0063 & 192 & 0.772 & 16 & 0.811 & 53 & 0.722 & 0.765 & 0.80 & 290.44 & 338.17 & 0.67 & 32.96 & 0.40 \\
\hline 337 & 67,816 & 783 & 1.0 & 7.0 & 1.5 & 40 & 11,903 & 5,619 & 0.0063 & 234 & 0.752 & 20 & 0.791 & 56 & 0.69 & 0.736 & 0.88 & 562.45 & 636.30 & 0.92 & 57.62 & .64 \\
\hline 176,337 & 67,816 & 6,783 & 1.0 & 7.0 & 1.5 & 40 & 11,903 & 5,619 & 0.0288 & 234 & 0.741 & 20 & 0.770 & 56 & 0.67 & 0.736 & 0.76 & 569.63 & 543.91 & 1.06 & 17.25 & .73 \\
\hline 168,726 & 61,220 & 6,719 & 1.5 & 7.5 & 1.5 & 33 & 14,207 & 6,526 & 0.0066 & 181 & 0.780 & 32 & 0.780 & 94 & 0.678 & 0.750 & 0.79 & 818.76 & 427.15 & 1.02 & 24.28 & .74 \\
\hline 161,772 & 61,957 & 6,238 & 1.0 & 9.0 & 2.0 & 38 & 14,831 & 6,869 & 0.0955 & 233 & 0.744 & 30 & 0.783 & 78 & 0.681 & 0.725 & 0.58 & 589.30 & 641.39 & 1.00 & 2.20 & .58 \\
\hline 52,385 & 20,310 & 2,005 & 1.0 & 7.0 & 1.5 & 25 & 14,874 & 6,992 & 0.0109 & 216 & 0.776 & 25 & 0.817 & 59 & 0.678 & 0.772 & 0.78 & 353.47 & 353.34 & 0.67 & 17.33 & 0.47 \\
\hline 164,702 & 72,459 & 5,765 & 2.0 & 9.2 & 1.8 & 38 & 13,056 & 6,518 & 0.0084 & 201 & 0.737 & 21 & 0.761 & 76 & 0.677 & 0.784 & 0.90 & 467.44 & 670.42 & 1.21 & 47.93 & .63 \\
\hline 131,217 & 57,151 & 4,629 & 1.0 & 9.0 & 2.0 & 33 & 13,284 & 5,390 & 0.0066 & 182 & 0.782 & 21 & 0.822 & 67 & 0.725 & 0.799 & 0.88 & 367.66 & 349.32 & 2.20 & 123.17 & 1.07 \\
\hline 131,217 & 57,151 & 4,629 & 1.0 & 9.0 & 2.0 & 33 & 13,284 & 5,390 & 0.0066 & 182 & 0.782 & 21 & 0.822 & 67 & 0.707 & 0.762 & 0.85 & 558.59 & 544.06 & 0.93 & 33.49 & .59 \\
\hline 23,450 & 8,965 & 905 & 1.5 & 7.5 & 1.5 & 17 & 14,679 & 6,951 & 0.0074 & 207 & 0.748 & 27 & 0.770 & 112 & 0.684 & 0.728 & 0.58 & 284.43 & 221.54 & 0.71 & 38.35 & .39 \\
\hline 188,248 & 68,518 & 7,483 & 1.0 & 8.6 & 1.9 & 40 & 12,064 & 5,267 & 0.0493 & 232 & 0.766 & 27 & 0.786 & 115 & 0.684 & 0.735 & 0.57 & 657.36 & \begin{tabular}{|l|}
584.07 \\
\end{tabular} & 1.14 & 10.01 & .70 \\
\hline 55,664 & 20,813 & 2,178 & 1.0 & 7.8 & $1.7 \mid$ & 24 & $\mid 13,207$ & 5,456 & 0.0053 & 207 & 0.772 & 14 & 0.818 & 83 & 0.71 & 0.785 & 0.80 & 328.37 & 322.86 & 1.07 & 94.40 & 0.62 \\
\hline
\end{tabular}

Table A-2: Data Generated for Six Stage Treatment, continued 


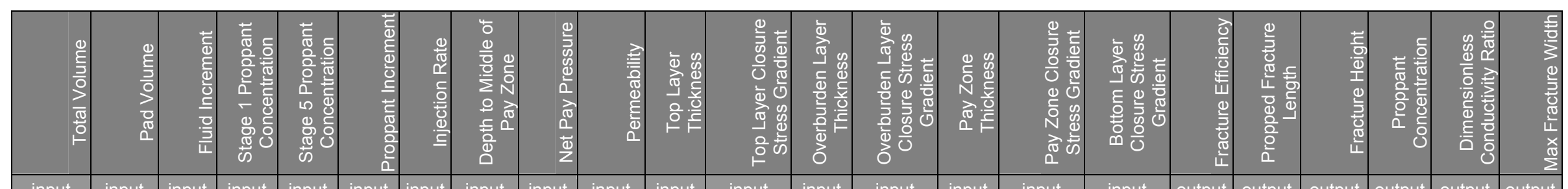

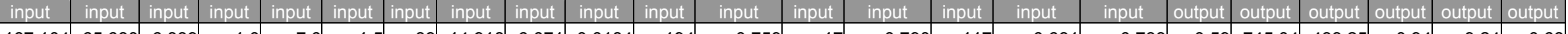

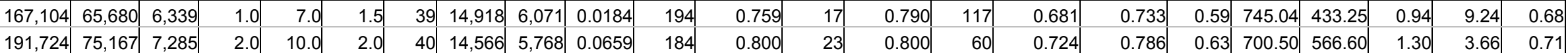

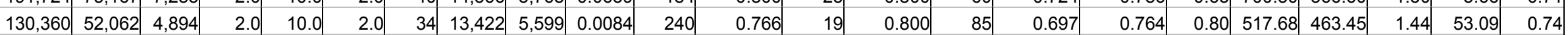

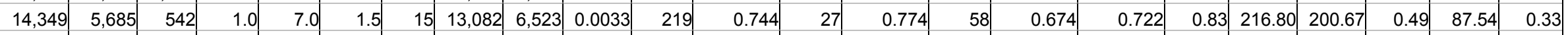

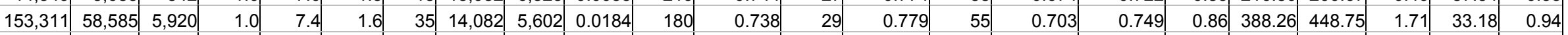

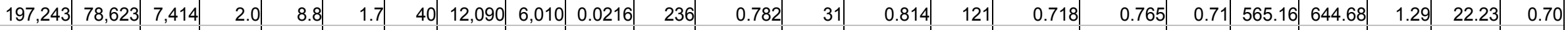

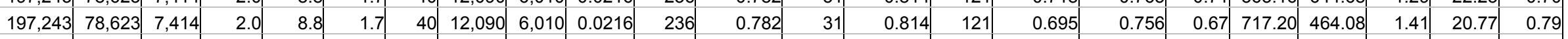

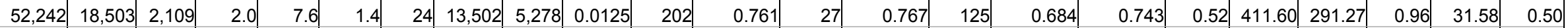

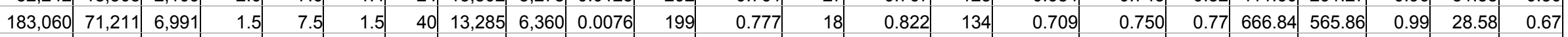

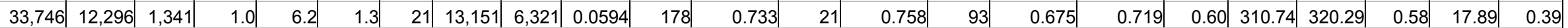

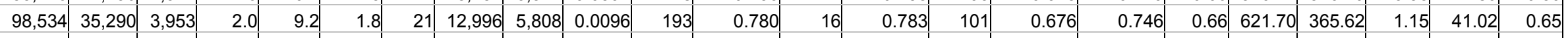

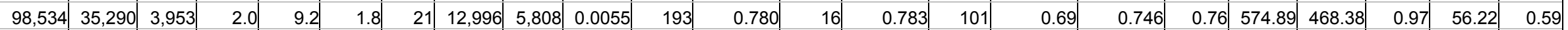

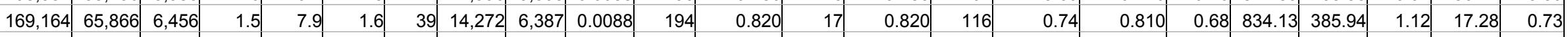

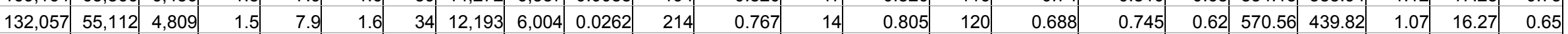

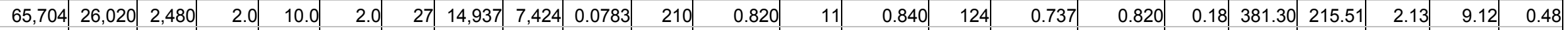

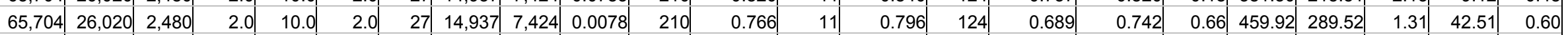

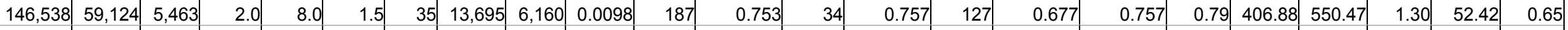

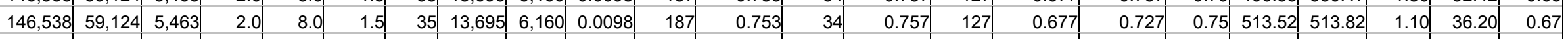

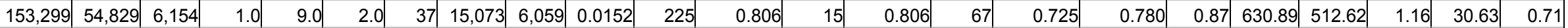

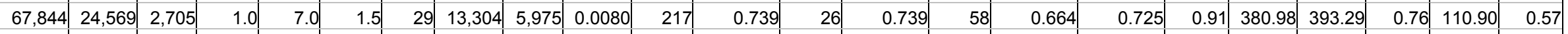

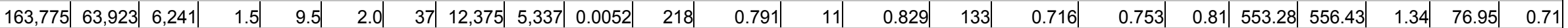

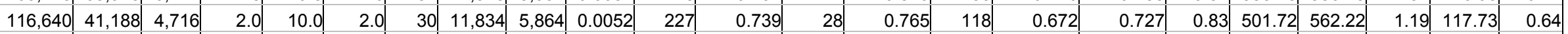

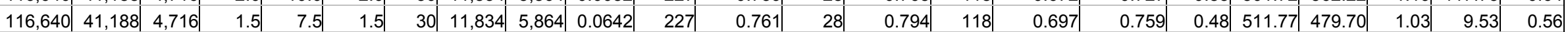

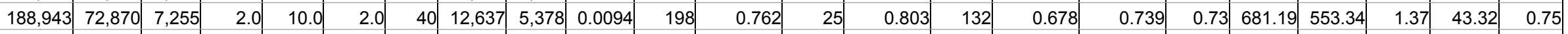

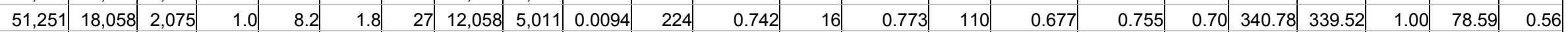

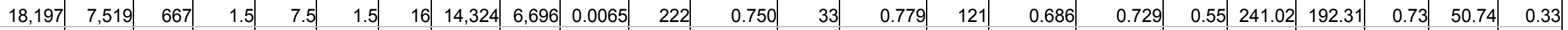

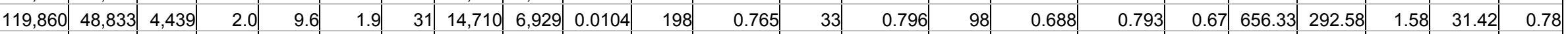

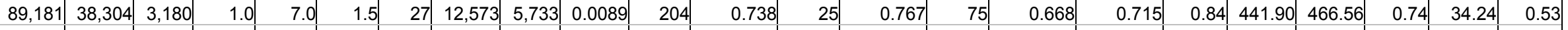

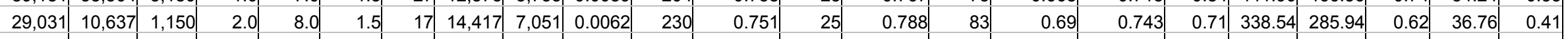

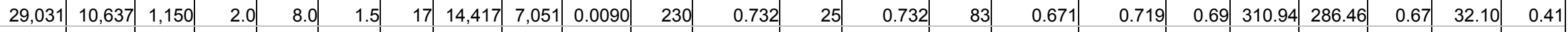

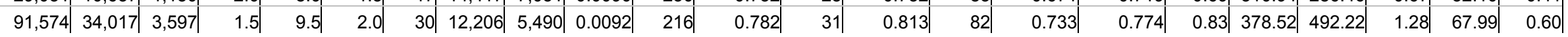

Table A-2: Data Generated for Six Stage Treatment, continued 


\begin{tabular}{|c|c|c|c|c|c|c|c|c|c|c|c|c|c|c|c|c|c|c|c|c|c|c|}
\hline 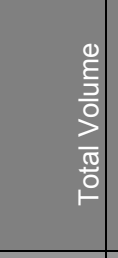 & $\begin{array}{l}\frac{0}{E} \\
\frac{1}{3} \\
0 \\
0 \\
\frac{0}{0} \\
0\end{array}$ & 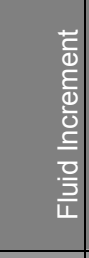 & 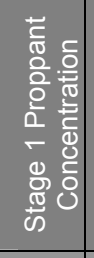 & 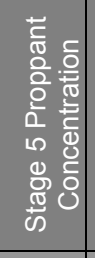 & 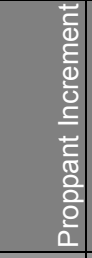 & \begin{tabular}{l|}
$\frac{0}{\pi}$ \\
$\square$ \\
$\frac{1}{c}$ \\
$\frac{0}{0}$ \\
$\frac{0}{0}$ \\
$\frac{0}{c}$
\end{tabular} & 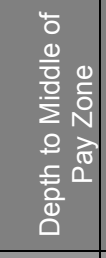 & \begin{tabular}{l|l}
$\frac{0}{3}$ & \\
0 & \\
0 & \\
$\frac{0}{2}$ & \\
$\frac{\pi}{0}$ & \\
$\frac{1}{0}$ & \\
$\frac{\Phi}{2}$ &
\end{tabular} & 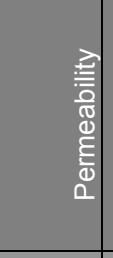 & 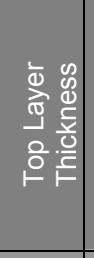 & 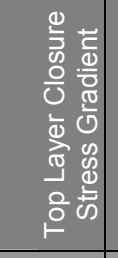 & 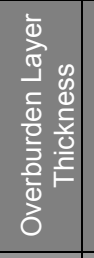 & 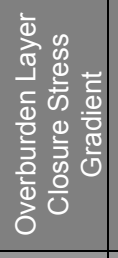 & 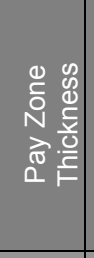 & 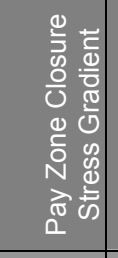 & 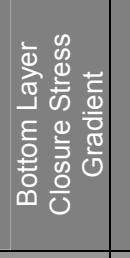 & 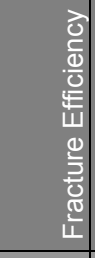 & 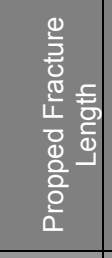 & 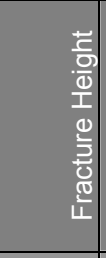 & 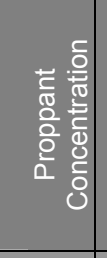 & 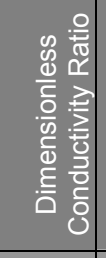 & $\begin{array}{l}\frac{5}{5} \\
\frac{5}{3} \\
0 \\
\frac{1}{3} \\
\frac{3}{0} \\
\frac{\pi}{20} \\
\frac{0}{4}\end{array}$ \\
\hline input & input & input & input & input & input & input & input & input & input & input & input & input & input & input & input & input & output & output & utput & output & output & output \\
\hline 91,574 & 34,017 & 3,597 & 1.5 & 9.5 & 2.0 & 30 & 12,206 & 5,490 & 0.0070 & 216 & 0.790 & 31 & 0.830 & 82 & 0.733 & 0.800 & 0.83 & 400.37| & 98.46 & 1.49 & 98.51 & .73 \\
\hline 91,574 & 34,017 & 3,597 & 1.5 & 9.5 & 2.0 & 30 & 12,206 & 5,490 & 0.0070 & 216 & 0.790 & 31 & 0.830 & 82 & 0.7 & 0.780 & 0.79 & 512.23 & 16.59 & 1.11 & 65.07 & .67 \\
\hline 112,561 & 7,054 & 4,094 & 1.5 & 7.5 & 1.5 & 30 & 12,431 & 4,853 & 0.0066 & 196 & .758 & 27 & 0.781 & 119 & 0.684 & 0.729 & 0.76 & 494.41 & 75.61 & 1.15 & 67.41 & .63 \\
\hline 108,469 & 39,590 & 4,305 & 1.5 & 9.5 & 2.0 & 31 & 13,156 & 5,529 & 0.0318 & 174 & 0.792 & 16 & 0.825 & 55 & 0.726 & 0.769 & 0.79 & 400.61 & 84.61 & 1.22 & 14.72 & .60 \\
\hline 82,028 & 34,929 & 2,944 & 1.0 & 7.0 & 1.5 & 29 & 15,159 & 7,360 & 0.0597 & 202 & 0.741 & 27 & 799 & 73 & 0.66 & 0.732 & .45 & 610.99 & 8.69 & 0.68 & 2.88 & .53 \\
\hline 82,028 & 34,929 & 2,944 & 1.5 & 7.5 & 1.5 & 29 & 15,159 & 7,360 & 0.0100 & 202 & 0.741 & 27 & 0.776 & 73 & 0.681 & 0.727 & 0.79 & 506.81 & 441.07 & 0.57 & 11.46 & .48 \\
\hline 92,673 & 40,241 & 3,277 & 2.0 & 10.0 & 2.0 & 28 & 14,177 & 7,066 & 0.0109 & 237 & 0.764 & 20 & 0.784 & 135 & .687 & 0.755 & 0.62 & $\mid 558.87$ & 303.63 & $1.37 \mid$ & 34.58 & .64 \\
\hline 92,673 & 40,241 & 3,277 & 2.0 & 8.0 & 1.5 & 28 & 14,177 & 7,066 & 0.0050 & 237 & 0.764 & 20 & 0.784 & 135 & 0.702 & 0.742 & 0.76 & 468.34 & 405.33 & 1.00 & 48.71 & 0.58 \\
\hline 105,837 & 4,424 & 3,838 & 1.0 & 10.8 & 2.2 & 30 & 14,224 & 6,990 & 0.0082 & 177 & 0.736 & 11 & 0.741 & 77 & .669 & 0.720 & 0.83 & $\mid 490.16$ & 6.51 & 1.26 & 4.90 & .63 \\
\hline 71,082 & 31,006 & 2,505 & 1.0 & 7.0 & 1.5 & 26 & 13,639 & 6,405 & 0.0710 & 211 & 0.767 & 34 & 0.810 & 131 & 0.692 & 0.760 & 0.20 & 424.21 & 263.13 & 1.08 & 7.23 & 0.40 \\
\hline 71,082 & 31,006 & 2,505 & 1.0 & 7.0 & 1.5 & 26 & 3,639 & 6,405 & 0.0071 & 211 & .767 & 34 & 0.810 & 131 & 0.716 & 0.745 & 0.71 & 388.98 & 425.81 & 0.73 & 32.78 & .46 \\
\hline 173,074 & 64,946 & 6,758 & 2.0 & 10.4 & 2.1 & 30 & 14,353 & 5,715 & 0.0032 & 213 & 0.758 & 30 & 0.777 & 80 & 0.679 & 0.760 & 0.86 & 726.58 & 449.18 & 1.52 & 86.85 & 0.84 \\
\hline 144,963 & 54,414 & 659 & 1.0 & 7.8 & 1.7 & 35 & 12,956 & 5,064 & 0.0034 & 224 & 0.800 & 35 & 0.825 & 71 & 0.725 & 0.780 & 0.88 & 640.78 & 572.84 & 0.80 & 52.23 & .60 \\
\hline 16,226 & 5,972 & 641 & 1.0 & 7.0 & 1.5 & 15 & 14,638 & 5,622 & 0.0086 & 172 & 0.745 & 32 & 0.765 & 68 & 0.681 & 0.758 & 0.49 & 303.01 & 154.40 & 0.54 & 27.18 & 0.34 \\
\hline 161,441 & 60,613 & ,302 & 2.0 & 10.8 & 2.3 & 37 & 13,480 & 5,054 & 0.0088 & 236 & 0.757 & 13 & 0.759 & 79 & 0.683 & 0.744 & 0.82 & 561.39 & 536.36 & 1.67 & 57.20 & 0.81 \\
\hline 14,329 & 5,956 & 523 & 1.5 & 7.5 & 1.5 & 16 & 12,758 & 5,465 & 0.0041 & 186 & 0.766 & 31 & 0.797 & 84 & 0.706 & 0.743 & 0.71 & 208.61 & 203.60 & 0.64 & 98.71 & 0.34 \\
\hline 119,014 & 9,771 & 328 & 2.0 & 12.0 & 2.5 & 30 & 13,715 & 5,318 & 0.0147 & 179 & 0.791 & 23 & 0.797 & 108 & 0.717 & 0.764 & 0.64 & 522.59 & 470.19 & 1.47 & 27.40 & 0.67 \\
\hline 125,610 & 52,655 & 4,560 & 2.0 & 10.0 & 2.0 & 32 & 14,204 & 6,575 & 0.0053 & 201 & 0.755 & 25 & 0.789 & 120 & 0.68 & 0.721 & 0.79 & 540.90 & 433.28 & 1.38 & 59.34| & 0.71 \\
\hline 74,077 & 28,646 & 2,839 & 1.5 & 7.5 & 1.5 & 26 & 13,138 & 5,510 & 0.0092 & 194 & 0.732 & 13 & 0.763 & 91 & 0.667 & 0.716 & 0.75 & 448.92 & 435.82 & 0.78 & 34.35 & 0.52 \\
\hline 39,936 & 16,115 & 489 & 1.0 & 7.0 & 1.5 & 24 & 12,661 & 5,592 & 0.0057 & 179 & .759 & 33 & 0.759 & 88 & 0.663 & 0.713 & 0.77 & 341.33 & 263.73 & 0.79 & 70.21 & 0.48 \\
\hline 81,866 & 34,383 & 2,968 & 2.0 & 10.0 & 2.0 & 28 & 12,844 & 4,683 & 0.0113 & 180 & 0.796 & 31 & 0.810 & 88 & 0.686 & 0.769 & 0.67 & 557.23 & 308.84 & 1.23 & 35.48 & 0.66 \\
\hline 81,866 & 34,383 & 2,968 & 2.0 & 10.0 & 2.0 & 28 & 12,844 & 4,683 & 0.0080 & 180 & 0.796 & 31 & 0.810 & 88 & 0.706 & 0.769 & 0.75 & |490.84 & 365.28 & 1.18 & 54.41 & 0.66 \\
\hline 163,612 & 68,097 & 5,970 & 2.0 & 10.8 & 2.2 & 37 & 13,963 & 5,337 & 0.0037 & 216 & 0.760 & 11 & 0.794 & 124 & 0.668 & 0.744 & 0.76 & 786.70 & 346.83 & 1.66 & 85.01 & 0.83 \\
\hline 53,531 & 19,551 & 2,124 & 1.5 & 9.5 & 2.0 & 25 & 13,010 & 5,574 & 0.0099 & 232 & 0.750 & 12 & 0.787 & 71 & 0.67 & 0.731 & 0.76 & 386.48 & 359.39 & 1.01 & 50.06 & 0.55 \\
\hline 24,016 & 10,322 & 856 & 1.5 & 7.5 & 1.5 & 19 & 13,264 & 5,029 & 0.0051 & 171 & 0.740 & 24 & 0.760 & 66 & 0.669 & 0.714 & 0.80 & 266.48 & 250.78 & 0.68 & 66.01 & 0.40 \\
\hline 48,466 & 18,684 & 1,861 & 1.0 & 7.4 & 1.6 & 23 & 12,054 & 5,936 & 0.0018 & 227 & 0.804 & 11 & 0.842 & 132 & 0.726 & 0.784 & 0.82 & 378.17 & 305.89 & 0.82 & 193.62 & 0.52 \\
\hline 65,049 & 25,865 & 2,449 & 1.5 & 7.5 & 1.5 & 24 & 14,965 & 7,131 & 0.0086 & 184 & 0.746 & 15 & 0.779 & 71 & 0.672 & 0.749 & 0.75 & 498.66 & 324.27 & 0.79 & 23.02 & 0.57 \\
\hline 0,933 & 37,865 & 3,317 & 1.5 & 7.5 & 1.5 & 27 & 13,927 & 5,726 & 0.0691 & 177 & 0.761 & 21 & 0.783 & 74 & 0.678 & 0.755 & 0.73 & | $643.74 \mid$ & 316.86 & 0.86 & 21.60 & 0.60 \\
\hline 126,660 & 54,398 & 4,516 & 1.0 & 7.0 & 1.5 & 35 & 15,013 & 5,615 & 0.0085 & 184 & 0.730 & 11 & 0.754 & 69 & 0.666 & 0.721 & 0.80 & $\mid 608.16$ & 493.30 & 0.72 & 16.65 & 0.58 \\
\hline 103 & 30,023 & 3,068 & 1.5 & 7.1 & 1.4 & 27 & 13,804 & 6,050 & 0.0075 & 228 & 0.754 & 24 & 0.788 & 72 & 0.679 & 0.751 & 0.79 & |491.50 & 386.43 & 0.82 & 33.99 & 0.59 \\
\hline 107,734 & 41,750 & 4,124 & 1.5 & 9.1 & 1.9 & 20 & 12,124 & 5,962 & 0.0180 & 229 & 0.801 & 12 & 0.850 & 124 & 0.719 & 0.796 & 0.61 & 539.80 & 398.87 & 1.22 & 27.66 & 0.66 \\
\hline
\end{tabular}

Table A-2: Data Generated for Six Stage Treatment, continued 


\begin{tabular}{|c|c|c|c|c|c|c|c|c|c|c|c|c|c|c|c|c|c|c|c|}
\hline \begin{tabular}{l|}
$\frac{0}{5}$ \\
$\frac{3}{0}$ \\
$\frac{0}{0}$ \\
$\frac{\pi}{0}$ \\
\end{tabular} & $\begin{array}{l}\text { है } \\
\frac{5}{3} \\
\frac{3}{0} \\
\frac{0}{0} \\
0 \\
0\end{array}$ & 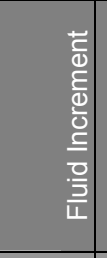 & 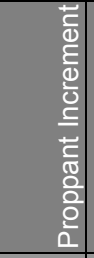 & 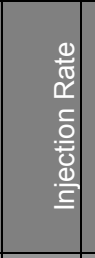 & 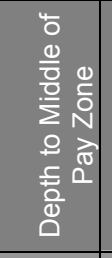 & 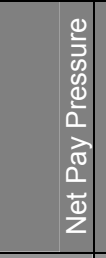 & 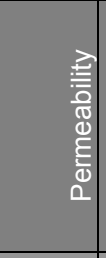 & 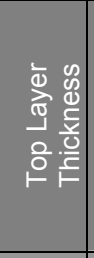 & 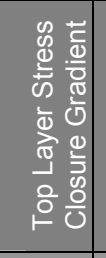 & 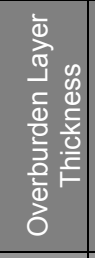 & 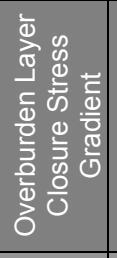 & 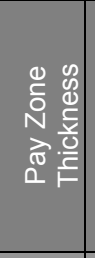 & 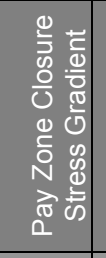 & 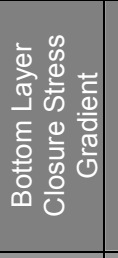 & 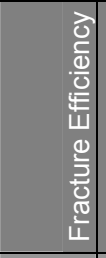 & 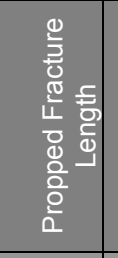 & 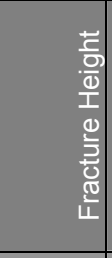 & 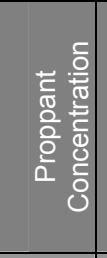 & \\
\hline input & input & input & input & input & input & input & input & input & input & input & input & input & input & input & output & output & output & output & output \\
\hline 96,569 & 38,287 & 2,534 & 1.3 & 30 & 5,572 & 2,482 & 0.0119 & 182 & 0.750 & 13 & 0.801 & 117 & 0.680 & 0.764 & \begin{tabular}{|l|}
0.88 \\
\end{tabular} & 311.40 & 474.82 & 1.52 & .7 \\
\hline 37,356 & 11,554 & 1,122 & 1.0 & 15 & 13,321 & 6,435 & 0.0205 & 178 & 0.758 & 36 & 0.762 & 132 & 0.679 & 0.766 & 0.53 & 362.95 & 227.19 & 0.86 & 0.51 \\
\hline 37,356 & 11,554 & 1,122 & 1.0 & 1 & 13,321 & 6,435 & 0.0205 & 178 & 0.778 & 36 & 0.832 & 132 & 0.720 & 0.796 & 0.59 & 283.27 & 349.79 & 0.72 & .4 \\
\hline 75,897 & 29,751 & 2,006 & 1.0 & 28 & 6,603 & 2,772 & 0.0157 & 225 & 0.739 & 25 & 0.750 & 88 & 0.701 & 0.729 & 0.88 & 285.49 & 538.68 & 1.01 & 0.52 \\
\hline 98,274 & 32,194 & 2,873 & 1.0 & 32 & 11,747 & 5,515 & 0.0050 & 176 & 0.768 & 17 & 0.782 & 120 & 0.712 & 0.730 & 0.88 & 380.81 & 502.88 & 1.01 & 0.62 \\
\hline 158,845 & 49,756 & 4,743 & 1.3 & 38 & 15,021 & 6,611 & 0.0214 & 193 & 0.727 & 13 & 0.734 & 104 & 0.685 & 0.724 & 0.76 & 486.02 & 595.64 & 1.61 & 0.75 \\
\hline 31,685 & 9,884 & 948 & 1.1 & 19 & 5,645 & 2,556 & 0.0028 & 224 & 0.718 & 42 & 0.742 & 65 & 0.696 & 0.715 & 0.96 & 202.30 & 378.90 & 1.05 & .54 \\
\hline 34,322 & 12,333 & 956 & 1.2 & 22 & 15,053 & 6,985 & 0.0106 & 232 & 0.776 & 31 & 0.833 & 98 & 0.682 & 0.742 & 0.71 & 354.86 & 177.67 & 1.14 & 0.60 \\
\hline 126,759 & 49,130 & 3,375 & 1.5 & 27 & 11,558 & 4,949 & 0.0276 & 209 & 0.730 & 29 & 0.777 & 124 & 0.686 & 0.723 & 0.68 & 440.35 & 578.00 & 1.37 & .60 \\
\hline 66,538 & 24,128 & 1,844 & 1.5 & 30 & 14,789 & 6,665 & 0.0144 & 231 & 0.758 & 38 & 0.758 & 58 & 0.710 & 0.742 & 0.86 & 311.55 & 447.21 & 1.18 & 0.56 \\
\hline 158,609 & 52,390 & 4,618 & 1.5 & 40 & 12,912 & 5,631 & 0.0230 & 223 & 0.789 & 16 & 0.868 & 78 & 0.719 & 0.816 & 0.83 & 380.32 & 343.51 & 1.80 & 1.54 \\
\hline 158,609 & 52,390 & 4,618 & 1.5 & 40 & 12,912 & 5,631 & 0.0230 & 223 & 0.770 & 16 & 0.868 & 78 & 0.710 & 0.760 & 0.81 & 517.13 & 587.90 & 1.63 & 0.75 \\
\hline 117,823 & 43,153 & 3,247 & 1.0 & 35 & 5,477 & 2,378 & 0.0724 & 182 & 0.747 & 56 & 0.793 & 93 & 0.684 & 0.728 & 0.88 & 349.08 & 560.76 & 1.09 & 0.66 \\
\hline 140,826 & 46,874 & 4,085 & 1.3 & 40 & 7,880 & 3,552 & 0.0091 & 224 & 0.763 & 27 & 0.763 & 93 & 0.700 & 0.736 & 0.92 & 380.19 & 614.27 & 1.59 & 0.75 \\
\hline 102,227 & 42,658 & 2,590 & 1.2 & 33 & 11,797 & 5,793 & 0.0048 & 240 & 0.721 & 11 & 0.768 & 61 & 0.661 & 0.717 & 0.94 & 383.73 & 469.88 & 1.19 & 0.67 \\
\hline 126,596 & 48,209 & 3,408 & 1.1 & 36 & 5,141 & 1,746 & 0.0076 & 196 & 0.769 & 34 & 0.792 & 79 & 0.709 & 0.745 & 0.94 & 332.09 & 575.64 & 1.48 & 0.71 \\
\hline 89,155 & 30,896 & 2,533 & 1.0 & 33 & 6,451 & 2,239 & 0.0452 & 191 & 0.705 & 45 & 0.733 & 87 & 0.643 & 0.716 & 0.81 & 305.73 & 477.80 & 1.42 & 0.70 \\
\hline 31,969 & 12,709 & 837 & 1.0 & 25 & 12,053 & 6,142 & 0.0029 & 207 & 0.747 & 18 & 0.769 & 129 & 0.705 & 0.729 & 0.87 & 221.00 & 323.18 & 0.72 & 0.44 \\
\hline 31,969 & 12,709 & 837 & 1.0 & 25 & 12,053 & 6,142 & 0.0029 & 207 & 0.747 & 18 & 0.769 & 129 & 0.725 & 0.739 & 0.88 & 204.26 & 358.37 & 0.71 & 0.41 \\
\hline 90,178 & 27,234 & 2,737 & 1.0 & 30 & 14,463 & 6,977 & 0.0114 & 192 & 0.788 & 54 & 0.843 & 99 & 0.730 & 0.763 & 0.82 & 402.34 & 518.04 & 1.08 & 0.65 \\
\hline 39,970 & 16,781 & 1,008 & 1.5 & 17 & 8,394 & 3,771 & 0.0148 & 220 & 0.741 & 19 & 0.759 & 70 & 0.680 & 0.720 & 0.85 & 234.77 & 357.72 & 1.28 & 0.55 \\
\hline 151,081 & 54,449 & 4,201 & 1.0 & 38 & 10,277 & 4,392 & 0.0093 & 200 & 0.796 & 28 & 0.852 & 81 & 0.724 & 0.769 & 0.90 & 465.52 & 629.15 & 0.94 & 0.66 \\
\hline 151,081 & 54,449 & 4,201 & 1.0 & 38 & 10,277 & 4,392 & 0.0093 & 200 & 0.796 & 28 & 0.796 & 81 & 0.700 & 0.769 & 0.87 & 579.19 & 532.32 & 0.89 & 0.71 \\
\hline 107,264 & 39,514 & 2,946 & 1.0 & 29 & 5,094 & 1,652 & 0.0031 & 217 & 0.750 & 24 & 0.776 & 63 & 0.680 & 0.743 & 0.96 & 316.06 & 549.26 & 1.29 & 0.68 \\
\hline 84,334 & 30,612 & 2,336 & 1.0 & 28 & 11,138 & 4,655 & 0.0065 & 177 & 0.728 & 30 & 0.739 & 98 & 0.682 & 0.720 & 0.87 & 375.98 & 484.25 & 0.84 & 0.57 \\
\hline 25,960 & 8,952 & 739 & 1.1 & 15 & 7,054 & 2,592 & 0.0647 & 233 & 0.729 & 28 & 0.760 & 80 & 0.675 & 0.730 & 0.66 & 200.21 & 312.29 & 0.87 & 0.49 \\
\hline 25,960 & 8,952 & 739 & 1.1 & 15 & 7,054 & 2,592 & 0.0647 & 233 & 0.729 & 28 & 0.750 & 80 & 0.690 & 0.730 & 0.68 & 183.35 & 325.57 & 0.92 & 0.48 \\
\hline 109,625 & 40,000 & 3,027 & 1.3 & 34 & 8,500 & 3,661 & 0.0404 & 188 & 0.733 & 35 & 0.773 & 132 & 0.685 & 0.734 & 0.67 & 247.72 & 515.21 & 1.66 & 0.70 \\
\hline 143,102 & 44,697 & 4,278 & 1.5 & 40 & 12,252 & 5,757 & 0.0068 & 175 & 0.788 & 13 & 0.825 & 54 & 0.711 & 0.778 & 0.92 & 499.57 & 494.89 & 1.85 & 0.87 \\
\hline 83,966 & 28,172 & 2,426 & 1.1 & 27 & 7,299 & 2,533 & 0.0047 & $177 \mid$ & 0.718 & 18 & 0.714 & 88 & 0.660 & 0.705 & 0.90 & 337.58 & 527.88 & 1.01 & 0.57 \\
\hline
\end{tabular}

Table A - 3: Data Generated for Eight Stage Treatment Type I 


\begin{tabular}{|c|c|c|c|c|c|c|c|c|c|c|c|c|c|c|c|c|c|c|c|}
\hline 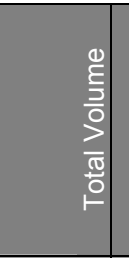 & 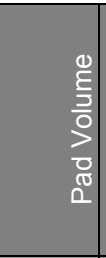 & 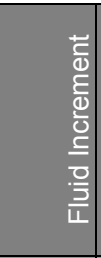 & 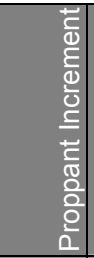 & 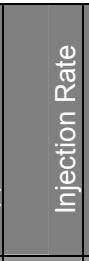 & 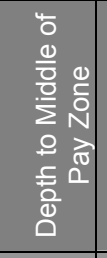 & 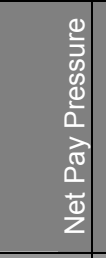 & 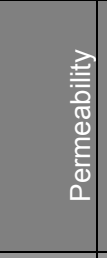 & 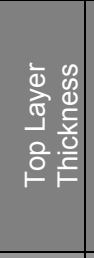 & 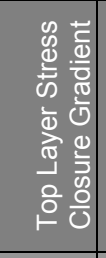 & 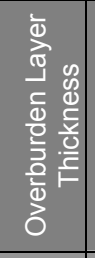 & 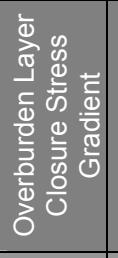 & 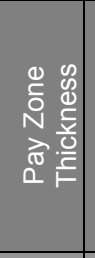 & 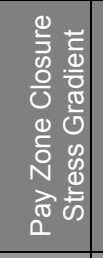 & 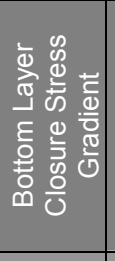 & 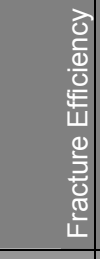 & 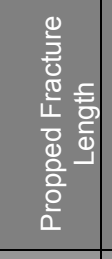 & 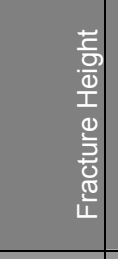 & 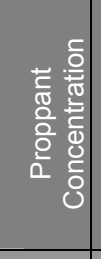 & 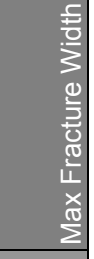 \\
\hline input & input & input & input & input & input & input & input & input & input & input & input & input & input & input & output & output & output & output & output \\
\hline 20,073 & 7,777 & 535 & 1.0 & 18 & 5,950 & 2,498 & 0.0277 & 235 & 0.727 & 18 & 0.781 & 99 & 0.683 & 0.725 & 0.64 & 163.65 & 284.63 & 0.71 & 0.33 \\
\hline 111,577 & 33,743 & 3,384 & 1.5 & 35 & 9,205 & 4,170 & 0.0065 & 179 & 0.754 & 18 & 0.827 & 65 & 0.703 & 0.746 & 0.92 & 360.53 & 520.94 & 1.83 & 0.77 \\
\hline 51,721 & 20,141 & 1,373 & 1.0 & 26 & 8,096 & 3,248 & 0.0231 & 230 & 0.724 & 20 & 0.778 & 73 & 0.655 & 0.718 & 0.78 & 300.09 & 419.16 & 0.73 & 0.46 \\
\hline 112,175 & 48,219 & 2,781 & 1.5 & 27 & 8,208 & 3,814 & 0.0255 & 187 & 0.748 & 32 & 0.808 & 74 & 0.690 & 0.740 & 0.82 & 384.14 & 539.91 & 1.36 & 0.59 \\
\hline 60,558 & 24,269 & 1,578 & 1.0 & 25 & 10,789 & 5,075 & 0.0729 & 195 & 0.764 & 22 & 0.813 & 79 & 0.709 & 0.750 & 0.57 & 338.79 & 439.52 & 0.71 & 0.41 \\
\hline 54,223 & 17,794 & 1,584 & 1.2 & 21 & 6,753 & 2,847 & 0.0309 & 203 & 0.781 & 35 & 0.786 & 98 & 0.725 & 0.766 & 0.70 & 276.23 & 445.24 & 1.04 & 0.48 \\
\hline 40,716 & 13,864 & 1,167 & 1.1 & 17 & 9,268 & 3,697 & 0.0579 & 231 & 0.720 & 17 & 0.779 & 65 & 0.663 & 0.717 & 0.61 & 281.18 & 386.85 & 0.79 & 0.41 \\
\hline 155,035 & 65,837 & 3,878 & 1.2 & 40 & 7,076 & 2,585 & 0.0045 & 171 & 0.761 & 28 & 0.816 & 109 & 0.719 & 0.769 & 0.91 & 346.17 & 530.37 & 1.71 & 0.86 \\
\hline 139,116 & 56,853 & 3,577 & 1.4 & 36 & 9,518 & 3,982 & 0.0178 & 204 & 0.723 & 36 & 0.757 & 73 & 0.670 & 0.714 & 0.86 & 388.51 & 551.14 & 1.58 & 0.71 \\
\hline 56,578 & 23,839 & 1,423 & 1.1 & 28 & 13,445 & 6,142 & 0.0161 & 171 & 0.716 & 22 & 0.769 & 134 & 0.652 & 0.709 & 0.61 & 409.31 & 289.15 & 0.89 & 0.51 \\
\hline 156,768 & 49,007 & 4,685 & 1.5 & 40 & 10,615 & 2,485 & 0.0414 & 207 & 0.729 & 14 & 0.782 & 93 & 0.675 & 0.726 & 0.69 & 429.86 & 543.42 & 0.98 & 0.54 \\
\hline 99,524 & 41,564 & 2,520 & 1.3 & 36 & 10,458 & 4,702 & 0.0065 & 194 & 0.729 & 28 & 0.810 & 78 & 0.666 & 0.730 & 0.88 & 407.96 & 473.20 & 1.14 & 0.61 \\
\hline 138,147 & 47,893 & 3,924 & 1.2 & 39 & 13,268 & 6,374 & 0.0073 & 172 & 0.765 & 34 & 0.822 & 133 & 0.719 & 0.746 & 0.79 & 520.23 & 635.81 & 0.96 & 0.57 \\
\hline 56,473 & 21,659 & 1,514 & 1.0 & 24 & 8,159 & 3,844 & 0.0972 & 204 & 0.734 & 39 & 0.733 & 114 & 0.678 & 0.721 & 0.50 & 278.94 & 428.12 & 0.86 & 0.42 \\
\hline 56,473 & 21,659 & 1,514 & 1.2 & 21 & 13,232 & 6,000 & 0.0089 & 226 & 0.704 & 23 & 0.761 & 107 & 0.649 & 0.704 & 0.70 & 408.27 & 390.49 & 0.77 & 0.48 \\
\hline 47,806 & 15,488 & 1,405 & 1.0 & 19 & 14,687 & 7,317 & 0.0333 & 195 & 0.741 & 20 & 0.822 & 72 & 0.696 & 0.744 & 0.58 & 356.18 & 364.90 & 0.67 & 0.42 \\
\hline 90,198 & 38,777 & 2,236 & 1.3 & 29 & 9,836 & 4,870 & 0.0027 & 175 & 0.726 & 10 & 0.726 & 90 & 0.643 & 0.722 & 0.91 & 416.36 & 465.56 & 1.01 & 0.62 \\
\hline 63,571 & 18,444 & 1,962 & 1.2 & 26 & 13,890 & 6,774 & 0.0055 & 210 & 0.707 & 38 & 0.751 & 83 & 0.658 & 0.704 & 0.89 & 408.75 & 441.35 & 0.88 & 0.53 \\
\hline 63,571 & 18,444 & 1,962 & 1.0 & 28 & 5,396 & 1,833 & 0.0757 & 238 & 0.788 & 33 & 0.838 & 82 & 0.694 & 0.746 & 0.68 & 295.56 & 471.33 & 0.95 & 0.51 \\
\hline 127,387 & 51,962 & 3,279 & 1.0 & 27 & 7,542 & 2,786 & 0.0332 & 216 & 0.739 & 26 & 0.806 & 65 & 0.700 & 0.738 & 0.85 & 342.81 & 547.15 & 1.18 & 0.68 \\
\hline 91,245 & 37,946 & 2,317 & 1.0 & 30 & 10,055 & 4,387 & 0.0297 & 176 & 0.733 & 19 & 0.788 & 91 & 0.683 & 0.821 & 0.73 & 377.28 & 526.29 & 0.79 & 0.49 \\
\hline 32,010 & 13,751 & 794 & 1.1 & 17 & 10,889 & 4,813 & 0.0105 & 184 & 0.712 & 16 & 0.756 & 102 & 0.676 & 0.710 & 0.71 & 242.83 & 372.25 & 0.65 & 0.34 \\
\hline 75,747 & 23,778 & 2,260 & 1.0 & 25 & 5,118 & 2,070 & 0.0678 & 185 & 0.753 & 26 & 0.772 & 124 & 0.687 & 0.732 & 0.62 & 306.55 & 506.71 & 0.98 & 0.51 \\
\hline 75,747 & 23,778 & 2,260 & 1.1 & 31 & 12,048 & 4,505 & 0.0085 & 224 & 0.708 & 27 & 0.781 & 134 & 0.656 & 0.707 & 0.70 & 423.16 & 474.43 & 0.80 & 0.51 \\
\hline 101,470 & 30,675 & 3,078 & 1.3 & 32 & 15,127 & 7,192 & 0.0123 & 237 & 0.749 & 39 & 0.806 & 98 & 0.710 & 0.744 & 0.74 & 468.71 & 559.93 & 1.03 & 0.54 \\
\hline 133,070 & 52,874 & 3,487 & 1.2 & 37 & 6,357 & 2,447 & 0.0085 & 187 & 0.745 & 32 & 0.745 & 106 & 0.696 & 0.745 & 0.88 & 365.48 & 600.33 & 1.29 & 0.66 \\
\hline 159,630 & 52,005 & 4,679 & 1.1 & 40 & 7,847 & 3,911 & 0.0050 & 199 & 0.715 & 22 & 0.753 & 101 & 0.651 & 0.712 & 0.93 & 405.42 & 567.72 & 1.51 & 0.85 \\
\hline 140,614 & 48,526 & 4,004 & 1.0 & 37 & 9,297 & 4,375 & 0.0352 & 232 & 0.730 & 28 & 0.789 & 91 & 0.672 & 0.722 & 0.78 & 439.26 & 585.03 & 1.05 & 0.63 \\
\hline 83,548 & 30,075 & 2,325 & 1.1 & 32 & 6,901 & 3,182 & 0.0071 & 211 & 0.731 & 37 & 0.775 & 135 & 0.680 & 0.718 & 0.87 & 343.51 & 544.18 & 0.92 & 0.51 \\
\hline 80,951 & 34,392 & 2,024 & 1.0 & 26 & 10,881 & 3,441 & 0.0174 & 189 & 0.743 & 20 & 0.842 & 128 & 0.711 & 0.749 & 0.64 & 320.95 & 485.30 & 0.88 & 0.47 \\
\hline
\end{tabular}

Table A-3: Data Generated for Eight Stage Treatment Type I, continued 


\begin{tabular}{|c|c|c|c|c|c|c|c|c|c|c|c|c|c|c|c|c|c|c|c|}
\hline \begin{tabular}{l|l}
$\frac{0}{3}$ \\
$\frac{3}{0}$ \\
$\frac{0}{0}$ \\
$\frac{\pi}{0}$ \\
$\vdash$
\end{tabular} & \begin{tabular}{l|}
$\frac{1}{E}$ \\
$\frac{3}{3}$ \\
0 \\
$\frac{0}{0}$ \\
0 \\
0
\end{tabular} & 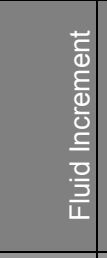 & 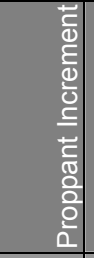 & 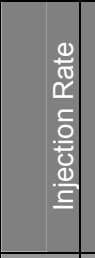 & 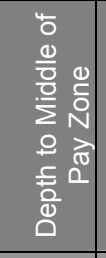 & 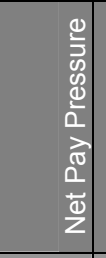 & 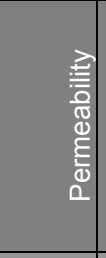 & 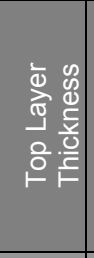 & 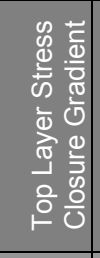 & 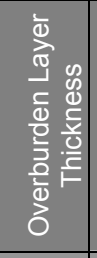 & 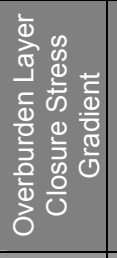 & 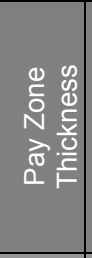 & 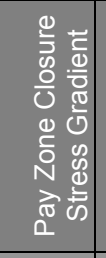 & 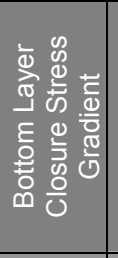 & 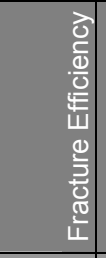 & 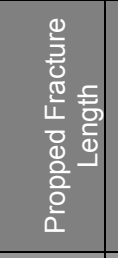 & 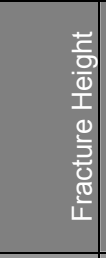 & 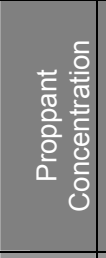 & \\
\hline input & input & input & input & input & input & input & input & input & input & input & input & input & input & input & output & output & output & output & utpl \\
\hline 82,354 & 31,867 & 2,195 & 1.2 & 28 & 8,726 & 4,309 & 0.0568 & 180 & 0.741 & 26 & 0.741 & 125 & 0.653 & 0.733 & 0.56 & 392.97 & 436.26 & 1.04 & \\
\hline 81,323 & 28,375 & 2,302 & 1.1 & 30 & 11,989 & 5,441 & 0.0283 & 171 & 0.733 & 19 & 0.731 & 55 & 0.663 & 0.729 & 0.80 & 381.38 & 491.50 & 0.91 & \\
\hline 114,520 & 42,181 & 3,145 & 1.0 & 22 & 5,957 & 2,216 & 0.0046 & 198 & 0.714 & 19 & 0.795 & 100 & 0.647 & 0.715 & 0.91 & 358.16 & 533.95 & 1.11 & \\
\hline 102,640 & 39,499 & 2,745 & 1.0 & 29 & 10,895 & 5,186 & 0.0957 & 199 & 0.756 & 40 & 0.842 & 105 & 0.671 & 0.729 & 0.49 & 471.74 & 514.48 & 0.76 & \\
\hline 81,199 & 30,767 & 2,193 & 1.0 & 26 & 12,319 & 5,895 & 0.0101 & 185 & 0.739 & 16 & 0.820 & 111 & 0.673 & 0.730 & 0.73 & 485.63 & 446.16 & 0.68 & \\
\hline 36,077 & 12,254 & 1,036 & 1.0 & 20 & 7,415 & 3,993 & 0.0697 & 214 & 0.748 & 38 & 0.859 & 123 & 0.702 & 0.750 & 0.47 & 247.47 & 363.00 & 0.78 & \\
\hline 107,728 & 37,945 & 3,034 & 1.0 & 28 & 11,842 & 5,275 & 0.0262 & 185 & 0.750 & 29 & 0.785 & 73 & 0.710 & 0.740 & 0.77 & 402.06 & 549.61 & 0.93 & \\
\hline 107,728 & 37,945 & 3,034 & 1.3 & 33 & 13,173 & 5,275 & 0.0118 & 222 & 0.713 & 31 & 0.769 & 129 & 0.655 & 0.714 & 0.67 & 532.86 & 493.35 & 1.01 & \\
\hline 38,430 & 15,952 & 977 & 1.0 & 27 & 9,348 & 4,194 & 0.0041 & 190 & 0.751 & 14 & 0.818 & 74 & 0.725 & 0.770 & 0.88 & 210.09 & 322.18 & 0.90 & \\
\hline 64,773 & 22,781 & 1,826 & 1.1 & 25 & 10,555 & 4,885 & 0.0075 & 214 & 0.723 & 46 & 0.748 & 60 & 0.676 & 0.715 & 0.90 & 295.60 & 436.79 & 1.05 & 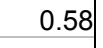 \\
\hline 42,511 & 16,679 & 1,123 & 1.0 & 28 & 15,014 & 7,024 & 0.0052 & 237 & 0.760 & 38 & 0.760 & 71 & 0.715 & 0.750 & 0.82 & 306.37 & 371.79 & 0.61 & \\
\hline 39,375 & 11,446 & 1,214 & 1.3 & 19 & 8,463 & 4,245 & 0.0311 & 215 & 0.727 & 30 & 0.772 & 100 & 0.690 & 0.724 & 0.59 & 252.93 & 397.66 & 0.75 &. \\
\hline 147,989 & 56,644 & 3,971 & 1.2 & 40 & 10,108 & 4,388 & 0.0089 & 192 & 0.766 & 36 & 0.843 & 85 & 0.719 & 0.760 & 0.87 & 472.93 & 595.57 & 1.14 & 6 \\
\hline 130,306 & 42,326 & 3,825 & 1.3 & 38 & 10,539 & 4,376 & 0.0014 & 179 & 0.730 & 20 & 0.791 & 66 & 0.703 & 0.741 & 0.98 & 323.55 & 492.46 & 2.10 & \\
\hline 130,306 & 42,326 & 3,825 & 1.0 & 38 & 10,539 & 4,376 & 0.0014 & 179 & 0.735 & 20 & 0.790 & 66 & 0.680 & 0.731 & 0.97 & 432.67 & 509.69 & 1.17 & 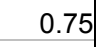 \\
\hline 82,716 & 35,349 & 2,059 & 1.1 & 26 & 13,743 & 6,503 & 0.0087 & 189 & 0.752 & 29 & 0.807 & 93 & 0.705 & 0.751 & 0.79 & 423.51 & 466.02 & 0.77 & \\
\hline 118,245 & 37,385 & 3,516 & 1.1 & 35 & 6,753 & 2,814 & 0.0198 & 225 & 0.738 & 20 & 0.815 & 65 & 0.642 & 0.708 & 0.89 & 369.43 & 517.94 & 1.37 & 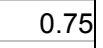 \\
\hline 143,466 & 59,884 & 3,634 & 1.3 & 40 & 12,532 & 5,453 & 0.0643 & 213 & 0.765 & 14 & 0.849 & 130 & 0.700 & 0.755 & 0.44 & 540.54 & 5.23 & 1.14 & \\
\hline 69,029 & 21,969 & 2,046 & 1.0 & 25 & 13,454 & 6,394 & 0.0088 & 204 & 0.729 & 38 & 0.741 & 104 & 0.674 & 0.733 & 0.74 & 425.10 & 389.67 & 0.83 & 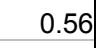 \\
\hline 76,174 & 26,516 & 2,159 & 1.2 & 30 & 14,381 & 6,719 & 0.0824 & 233 & 0.739 & 15 & 0.807 & 72 & 0.688 & 0.730 & 0.56 & 399.32 & 463.36 & 0.94 & \\
\hline 85,528 & 25,285 & 2,619 & 1.0 & 32 & 7,149 & 3,422 & 0.0284 & 205 & 0.765 & 33 & 0.765 & 80 & 0.710 & 0.776 & 0.58 & 306.08 & 514.60 & 1.34 & \\
\hline 88,268 & 26,818 & 2,672 & 1.2 & 27 & 7,137 & 2,911 & 0.0074 & 173 & 0.720 & 37 & 0.800 & 109 & 0.660 & 0.712 & 0.85 & 379.80 & 541.87 & 1.05 & 0.0 \\
\hline 117,128 & 34,790 & 3,580 & 1.3 & 33 & 9,307 & 3,891 & 0.0139 & 192 & 0.721 & 33 & 0.788 & 109 & 0.660 & 0.718 & 0.78 & 462.60 & 558.55 & 1.18 & \\
\hline 151,417 & 57,233 & 4,095 & 1.2 & 40 & 10,001 & 4,409 & 0.0069 & 226 & 0.744 & 14 & 0.744 & 80 & 0.677 & 0.825 & 0.90 & 448.55 & 653.35 & 1.13 & 0.6 \\
\hline 151,417 & 57,233 & 4,095 & 1.0 & 40 & 12,380 & 5,597 & 0.0976 & 240 & 0.718 & 15 & 0.789 & 79 & 0.650 & 0.717 & 0.62 & 518.00 & 510.36 & 1.04 & \\
\hline 49,438 & 18,568 & 1,342 & 1.1 & 22 & 13,557 & 6,743 & 0.0093 & 222 & 0.752 & 36 & 0.828 & 114 & 0.717 & 0.764 & 0.66 & 379.36 & 422.04 & 0.62 & 0.4 \\
\hline
\end{tabular}

Table A-3: Data Generated for Eight Stage Treatment Type I, continued 


\begin{tabular}{|c|c|c|c|c|c|c|c|c|c|c|c|c|c|c|c|c|c|c|c|}
\hline \begin{tabular}{l|}
$\frac{0}{E}$ \\
$\frac{5}{3}$ \\
$\frac{2}{0}$ \\
$\frac{\pi}{0}$ \\
$\frac{\pi}{0}$
\end{tabular} & 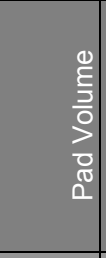 & 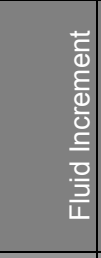 & 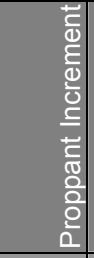 & 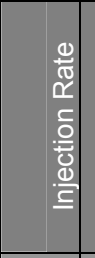 & 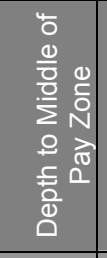 & \begin{tabular}{l|l}
$\frac{0}{3}$ & \\
0 & \\
0 & \\
0 & \\
0 & \\
$\pi$ & \\
$\frac{0}{2}$ & \\
$\frac{1}{0}$ & \\
$z$ &
\end{tabular} & 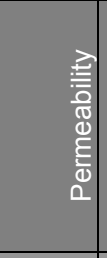 & 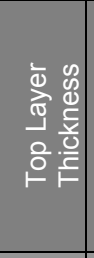 & 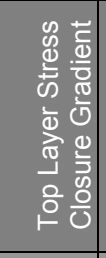 & 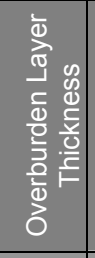 & 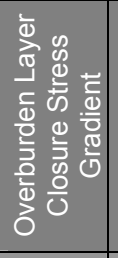 & 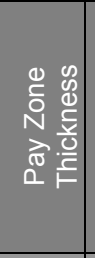 & 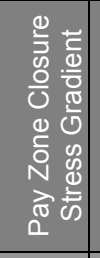 & 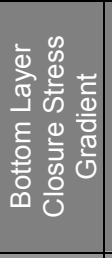 & 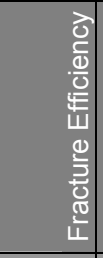 & 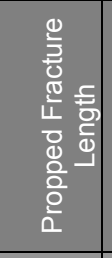 & 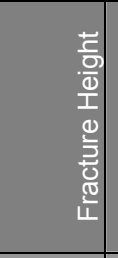 & 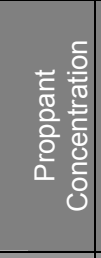 & 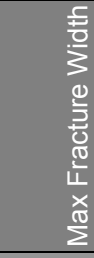 \\
\hline input & input & input & input & input & input & input & input & input & input & input & input & input & input & input & output & output & output & output & output \\
\hline 39,706 & 16,591 & 1,005 & 1.0 & 20 & 8,798 & 3,411 & 0.0916 & 208 & 0.734 & 20 & 0.772 & 80 & 0.692 & 0.721 & 0.51 & 236.59 & 380.51 & 0.75 & 0.35 \\
\hline 151,572 & 48,891 & 4,464 & 1.1 & 40 & 8,173 & 3,204 & 0.0282 & 190 & 0.724 & 40 & 0.740 & 68 & 0.655 & 0.731 & 0.87 & 402.70 & 710.83 & 1.16 & 0.63 \\
\hline 126,338 & 51,255 & 3,264 & 1.0 & 36 & 8,555 & 4,126 & 0.0190 & 180 & 0.722 & 31 & 0.752 & 74 & 0.686 & 0.726 & 0.89 & 351.80 & 651.28 & 0.96 & 0.57 \\
\hline 85,390 & 35,345 & 2,176 & 1.0 & 27 & 6,427 & 2,742 & 0.0483 & 182 & 0.736 & 15 & 0.761 & 105 & 0.694 & 0.741 & 0.71 & 299.84 & 528.11 & 0.93 & 0.50 \\
\hline 144,061 & 51,028 & 4,045 & 1.1 & 15 & 10,169 & 4,857 & 0.0055 & 215 & 0.741 & 20 & 0.770 & 76 & 0.692 & 0.733 & 0.92 & 422.42 & 536.22 & 1.32 & 0.77 \\
\hline 104,749 & 42,977 & 2,686 & 1.1 & 37 & 12,216 & 5,770 & 0.0161 & 221 & 0.721 & 38 & 0.811 & 104 & 0.668 & 0.721 & 0.75 & 472.62 & 546.80 & 0.84 & 0.54 \\
\hline 142,948 & 51,849 & 3,961 & 1.0 & 23 & 14,710 & 5,799 & 0.0677 & 218 & 0.754 & 22 & 0.834 & 69 & 0.701 & 0.755 & 0.72 & 428.08 & 487.32 & 1.28 & 0.75 \\
\hline 142,948 & 51,849 & 3,961 & 1.0 & 35 & 14,710 & 5,799 & 0.0067 & 218 & 0.724 & 22 & 0.800 & 69 & 0.650 & 0.728 & 0.88 & 532.54 & 287.89 & 1.04 & 0.75 \\
\hline 143,371 & 49,805 & 4,068 & 1.1 & 38 & 9,359 & 3,696 & 0.0207 & 188 & 0.758 & 27 & 0.758 & 90 & 0.674 & 0.740 & 0.78 & 484.02 & 585.41 & 1.07 & 0.68 \\
\hline 63,181 & 24,765 & 1,670 & 1.3 & 23 & 14,370 & 6,313 & 0.0176 & 227 & 0.732 & 13 & 0.778 & 107 & 0.658 & 0.720 & 0.53 & 490.78 & 300.04 & 0.92 & 0.52 \\
\hline 47,978 & 17,910 & 1,307 & 1.0 & 20 & 12,705 & 6,009 & 0.0367 & 171 & 0.765 & 16 & 0.848 & 79 & 0.730 & 0.787 & 0.61 & 255.20 & 304.75 & 1.05 & 0.54 \\
\hline 102,656 & 43,619 & 2,567 & 1.5 & 30 & 7,657 & 3,668 & 0.0013 & 197 & 0.733 & 11 & 0.763 & 93 & 0.683 & 0.729 & 0.96 & 318.72 & 522.94 & 1.72 & 0.69 \\
\hline 107,318 & 34,040 & 3,186 & 1.5 & 28 & 7,733 & 3,808 & 0.0200 & 240 & 0.767 & 33 & 0.797 & 127 & 0.685 & 0.798 & 0.77 & 371.19 & 448.02 & 1.94 & 0.85 \\
\hline 72,402 & 29,151 & 1,880 & 1.5 & 26 & 5,244 & 1,743 & 0.0019 & 180 & 0.734 & 30 & 0.734 & 117 & 0.675 & 0.716 & 0.87 & 270.71 & 465.46 & 1.51 & 0.64 \\
\hline 148,715 & 47,951 & 4,381 & 1.0 & 40 & 9,990 & 4,086 & 0.0028 & 211 & 0.793 & 18 & 0.825 & 102 & 0.690 & 0.779 & 0.93 & 623.98 & 500.52 & 0.91 & 0.77 \\
\hline 148,715 & 47,951 & 4,381 & 1.0 & 40 & 9,990 & 4,086 & 0.0028 & 211 & 0.755 & 18 & 0.755 & 102 & 0.650 & 0.720 & 0.94 & 575.57 & 498.28 & 0.99 & 0.79 \\
\hline 150,770 & 53,323 & 4,237 & 1.5 & 40 & 12,960 & 5,159 & 0.0332 & 201 & 0.749 & 13 & 0.763 & 105 & 0.682 & 0.736 & 0.70 & 528.99 & 542.16 & 1.52 & 0.76 \\
\hline 89,086 & 29,864 & 2,575 & 1.5 & 25 & 13,782 & 6,620 & 0.0093 & 201 & 0.717 & 30 & 0.800 & 90 & 0.698 & 0.734 & 0.95 & 378.70 & 470.36 & 1.42 & 0.69 \\
\hline 89,086 & 29,864 & 2,575 & 1.5 & 25 & 13,782 & 6,620 & 0.0020 & 201 & 0.740 & 30 & 0.740 & 90 & 0.670 & 0.724 & 0.91 & 504.64 & 454.11 & 1.10 & 0.64 \\
\hline 145,089 & 48,858 & 4,184 & 1.2 & 36 & 5,927 & 2,066 & 0.0016 & 183 & 0.770 & 36 & 0.781 & 120 & 0.714 & 0.764 & 0.95 & 336.05 & 559.29 & 1.35 & 0.71 \\
\hline 145,089 & 48,858 & 4,184 & 1.2 & 36 & 5,927 & 2,066 & 0.0016 & 183 & 0.741 & 36 & 0.781 & 120 & 0.714 & 0.730 & 0.96 & 314.69 & 586.42 & 1.38 & 0.72 \\
\hline 125,109 & 42,960 & 3,572 & 1.5 & 27 & 7,405 & 3,644 & 0.0052 & 220 & 0.759 & 12 & 0.750 & 133 & 0.681 & 0.727 & 0.91 & 375.52 & 565.17 & 1.69 & 0.78 \\
\hline 143,896 & 51,315 & 4,025 & 1.0 & 40 & 8,236 & 3,597 & 0.0104 & 189 & 0.787 & 33 & 0.794 & 114 & 0.712 & 0.783 & 0.87 & 404.93 & 560.68 & 1.46 & 0.78 \\
\hline 159,816 & 51,809 & 4,696 & 1.0 & 40 & 11,259 & 4,239 & 0.0065 & 234 & 0.753 & 43 & 0.801 & 127 & 0.681 & 0.737 & 0.83 & 605.64 & 582.17 & 0.94 & 0.70 \\
\hline 59,815 & 21,493 & 1,666 & 1.2 & 22 & 8,465 & 3,296 & 0.0122 & 202 & 0.748 & 30 & 0.796 & 104 & 0.661 & 0.730 & 0.90 & 339.28 & 352.30 & 1.25 & 0.67 \\
\hline 42,963 & 13,226 & 1,293 & 1.3 & 27 & 12,001 & 5,508 & 0.0054 & 227 & 0.734 & 19 & 0.736 & 74 & 0.651 & 0.711 & 0.88 & 296.44 & 348.40 & 1.16 & 0.60 \\
\hline 25,349 & 8,418 & 736 & 1.0 & 19 & 5,065 & 1,556 & 0.0063 & 238 & 0.727 & 36 & 0.741 & 128 & 0.670 & 0.711 & 0.91 & 179.08 & 307.50 & 0.92 & 0.50 \\
\hline 134,445 & 53,376 & 3,525 & 1.0 & 35 & 8,112 & 3,803 & 0.0307 & 200 & 0.712 & 19 & 0.736 & 60 & 0.644 & 0.700 & 0.90 & 375.61 & 520.55 & 1.14 & 0.74 \\
\hline 47,689 & 15,023 & 1,420 & 1.0 & 22 & 12,612 & 5,321 & 0.0077 & 179 & 0.745 & 21 & 0.805 & 86 & 0.713 & 0.740 & 0.84 & 286.35 & 411.58 & 0.75 & 0.46 \\
\hline 47,689 & 15,023 & 1,420 & 1.0 & 22 & 12,612 & 5,321 & 0.0077 & 179 & 0.745 & 21 & 0.805 & 86 & 0.660 & 0.740 & 0.79 & 422.45 & 306.38 & 0.74 & 0.59 \\
\hline
\end{tabular}

Table A-3: Data Generated for Eight Stage Treatment Type I, continued 


\begin{tabular}{|c|c|c|c|c|c|c|c|c|c|c|c|c|c|c|c|c|c|c|c|}
\hline \begin{tabular}{l|l|}
$\frac{0}{5}$ & \\
$\frac{3}{0}$ & \\
$\frac{\pi}{\pi}$ & \\
$\frac{\pi}{0}$ &
\end{tabular} & 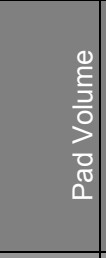 & 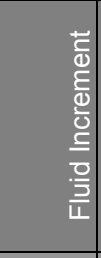 & 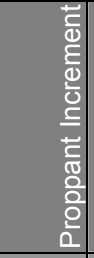 & 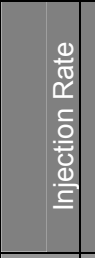 & 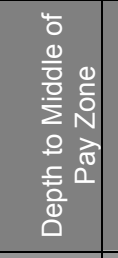 & 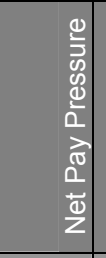 & 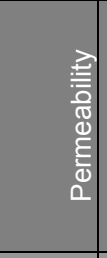 & 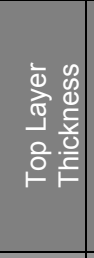 & 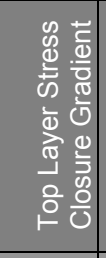 & 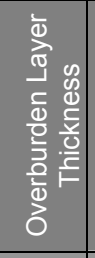 & 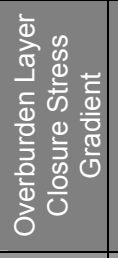 & 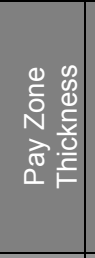 & 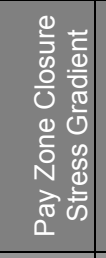 & 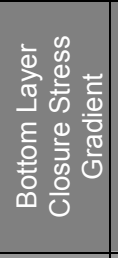 & 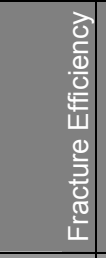 & 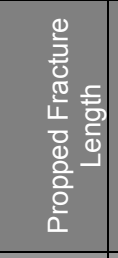 & 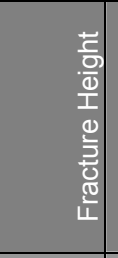 & 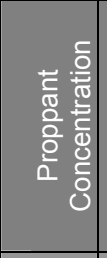 & 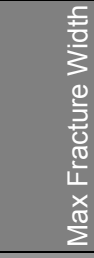 \\
\hline input & input & input & input & input & input & input & input & input & input & input & input & input & input & input & output & output & output & output & output \\
\hline 91,374 & 26,888 & 2,804 & 1.2 & 26 & 11,188 & 4,996 & 0.0720 & 172 & 0.762 & 40 & 0.784 & 129 & 0.707 & 0.758 & 0.51 & 372.84 & 423.39 & 1.41 & 0.67 \\
\hline 91,374 & 26,888 & 2,804 & 1.2 & 26 & 11,188 & 4,996 & 0.0072 & 172 & 0.762 & 40 & 0.784 & 129 & 0.650 & 0.758 & 0.74 & 625.40 & 304.76 & 1.17 & 0.76 \\
\hline 91,230 & 38,545 & 2,291 & 1.2 & 29 & 9,860 & 4,383 & 0.0103 & 174 & 0.741 & 24 & 0.743 & 72 & 0.656 & 0.723 & 0.88 & 399.92 & 450.26 & 1.12 & 0.64 \\
\hline 44,175 & 14,896 & 1,273 & 1.0 & 21 & 5,705 & 2,005 & 0.0115 & 222 & 0.763 & 38 & 0.808 & 122 & 0.688 & 0.732 & 0.83 & 230.43 & 378.70 & 1.90 & 0.58 \\
\hline 120,129 & 34,841 & 3,708 & 1.0 & 29 & 5,108 & 1,840 & 0.0081 & 179 & 0.715 & 37 & 0.774 & 129 & 0.650 & 0.710 & 0.89 & 375.17 & 588.02 & 1.14 & 0.67 \\
\hline 33,043 & 10,294 & 989 & 1.0 & 19 & 8,763 & 4,073 & 0.0210 & 215 & 0.747 & 25 & 0.758 & 55 & 0.690 & 0.735 & 0.84 & 218.79 & 344.49 & 0.81 & 0.49 \\
\hline 61,050 & 22,396 & 1,681 & 1.0 & 24 & 13,483 & 6,649 & 0.0080 & 194 & 0.763 & 35 & 0.793 & 125 & 0.687 & 0.746 & 0.77 & 416.55 & 318.94 & 0.83 & 0.61 \\
\hline 158,130 & 47,266 & 4,820 & 1.0 & 40 & 14,715 & 6,566 & 0.0182 & 231 & 0.711 & 36 & 0.750 & 108 & 0.664 & 0.705 & 0.79 & 519.89 & 618.95 & 1.01 & 0.69 \\
\hline 158,130 & 47,266 & 4,820 & 1.0 & 40 & 14,715 & 6,566 & 0.0070 & 231 & 0.728 & 36 & 0.750 & 108 & 0.650 & 0.710 & 0.81 & 774.88 & 456.12 & 0.92 & 0.77 \\
\hline 142,353 & 47,857 & 4,109 & 1.0 & 38 & 10,399 & 5,171 & 0.0177 & 193 & 0.770 & 24 & 0.799 & 54 & 0.710 & 0.761 & 0.93 & 402.00 & 633.15 & 1.33 & 0.67 \\
\hline 99,299 & 40,857 & 2,541 & 1.1 & 30 & 5,269 & 2,500 & 0.0103 & 206 & 0.722 & 10 & 0.769 & 76 & 0.651 & 0.710 & 0.94 & 309.93 & 618.77 & 1.17 & 0.64 \\
\hline 99,299 & 40,857 & 2,541 & 1.1 & 34 & 11,597 & 5,225 & 0.0103 & 231 & 0.767 & 19 & 0.726 & 92 & 0.695 & 0.748 & 0.85 & 430.02 & 471.80 & 0.90 & 0.62 \\
\hline 35,653 & 12,483 & 1,007 & 1.0 & 21 & 9,152 & 3,776 & 0.0129 & 208 & 0.754 & 19 & 0.771 & 108 & 0.683 & 0.731 & 0.80 & 240.58 & 332.11 & 0.88 & 0.55 \\
\hline 44,699 & 16,204 & 1,239 & 1.0 & 16 & 13,646 & 6,615 & 0.0186 & 201 & 0.754 & 12 & 0.790 & 92 & 0.681 & 0.745 & 0.70 & 372.18 & 304.36 & 0.73 & 0.54 \\
\hline 85,348 & 36,644 & 2,118 & 1.0 & 29 & 11,270 & 4,773 & 0.0099 & 239 & 0.768 & 17 & 0.800 & 72 & 0.700 & 0.745 & 0.87 & 372.42 & 429.99 & 0.87 & 0.61 \\
\hline 97,639 & 28,783 & 2,994 & 1.0 & 18 & 12,690 & 5,664 & 0.0227 & 224 & 0.756 & 15 & 0.752 & 64 & 0.700 & 0.735 & 0.84 & 391.63 & 549.28 & 0.94 & 0.60 \\
\hline 82,735 & 27,150 & 2,417 & 1.5 & 25 & 10,385 & 4,812 & 0.0097 & 173 & 0.740 & 31 & 0.765 & 102 & 0.688 & 0.736 & 0.84 & 338.76 & 490.53 & 1.49 & 0.66 \\
\hline 62,508 & 25,107 & 1,626 & 1.2 & 25 & 14,726 & 7,158 & 0.0112 & 227 & 0.738 & 25 & 0.783 & 88 & 0.658 & 0.720 & 0.79 & 464.29 & 377.99 & 1.38 & 0.76 \\
\hline 62,508 & 25,107 & 1,626 & 1.0 & 27 & 6,902 & 2,965 & 0.0010 & 229 & 0.706 & 22 & 0.746 & 70 & 0.643 & 0.700 & 0.97 & 272.92 & 440.51 & 0.91 & 0.57 \\
\hline 71,773 & 30,485 & 1,795 & 1.0 & 22 & 6,191 & 2,259 & 0.0011 & 201 & 0.787 & 37 & 0.815 & 65 & 0.703 & 0.770 & 0.97 & 311.86 & 451.07 & 0.86 & 0.55 \\
\hline 95,991 & 40,166 & 2,427 & 1.1 & 28 & 10,595 & 5,154 & 0.0137 & 171 & 0.719 & 30 & 0.734 & 109 & 0.652 & 0.707 & 0.82 & 415.39 & 472.16 & 0.89 & 0.60 \\
\hline 37,153 & 11,417 & 1,119 & 1.0 & 19 & 12,473 & 5,368 & 0.0069 & 210 & 0.759 & 11 & 0.769 & 102 & 0.674 & 0.718 & 0.81 & 283.06 & 345.67 & 0.77 & 0.51 \\
\hline 42,002 & 15,364 & 1,158 & 1.1 & 21 & 6,857 & 3,132 & 0.0095 & 224 & 0.715 & 25 & 0.736 & 114 & 0.662 & 0.709 & 0.87 & 229.29 & 382.14 & 0.91 & 0.55 \\
\hline 54,393 & 23,198 & 1,356 & 1.4 & 25 & 11,442 & 5,293 & 0.0088 & 170 & 0.720 & 24 & 0.753 & 65 & 0.670 & 0.711 & 0.90 & 297.48 & 406.98 & 0.88 & 0.50 \\
\hline 83,797 & 34,897 & 2,126 & 1.0 & 25 & 13,022 & 5,704 & 0.0077 & 215 & 0.799 & 32 & 0.869 & 62 & 0.712 & 0.820 & 0.78 & 544.78 & 306.07 & 0.95 & 0.68 \\
\hline 127,080 & 42,404 & 3,682 & 1.0 & 36 & 14,082 & 6,579 & 0.0248 & 194 & 0.741 & 12 & 0.818 & 134 & 0.662 & 0.741 & 0.84 & 552.54 & 356.81 & 1.25 & 0.90 \\
\hline 89,254 & 36,928 & 2,275 & 1.0 & 27 & 10,879 & 4,635 & 0.0092 & 235 & 0.739 & 19 & 0.781 & 113 & 0.652 & 0.733 & 0.79 & 460.43 & 392.55 & 0.85 & 0.65 \\
\hline 89,254 & 36,928 & 2,275 & 1.5 & 23 & 12,745 & 4,525 & 0.0147 & 221 & 0.777 & 12 & 0.794 & 120 & 0.691 & 0.754 & 0.67 & 454.09 & 362.22 & 1.40 & 0.72 \\
\hline 47,391 & 18,008 & 1,278 & 1.2 & 20 & 8,809 & 3,493 & 0.0202 & 204 & 0.757 & 41 & 0.756 & 60 & 0.675 & 0.754 & 0.84 & 270.84 & 376.34 & 0.98 & 0.57 \\
\hline 96,354 & 28,937 & 2,931 & 1.3 & 26 & 6,741 & 2,928 & 0.0146 & 218 & 0.778 & 28 & 0.806 & 91 & 0.708 & 0.750 & 0.86 & 358.96 & 516.12 & 1.46 & 0.70 \\
\hline
\end{tabular}

Table A-3: Data Generated for Eight Stage Treatment Type I, continued 


\begin{tabular}{|c|c|c|c|c|c|c|c|c|c|c|c|c|c|c|c|c|c|c|c|}
\hline \begin{tabular}{l|l}
$\frac{0}{5}$ \\
$\frac{5}{0}$ \\
$\frac{0}{\pi}$ \\
$\frac{\pi}{0}$ \\
$\vdash$
\end{tabular} & 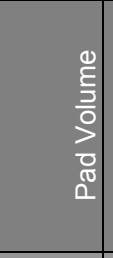 & 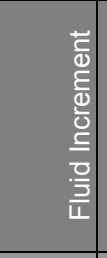 & 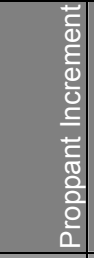 & 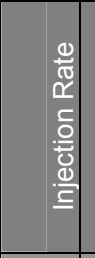 & 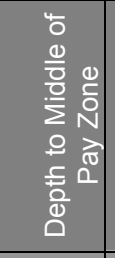 & \begin{tabular}{l|l}
$\frac{0}{3}$ & \\
0 & \\
0 & \\
$\frac{1}{2}$ & \\
$\frac{2}{\pi}$ & \\
0 & \\
$\frac{0}{0}$ & \\
&
\end{tabular} & 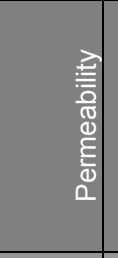 & 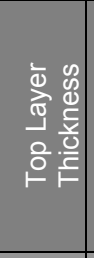 & 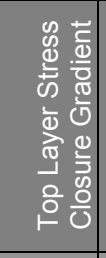 & 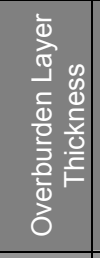 & 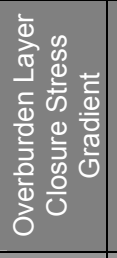 & 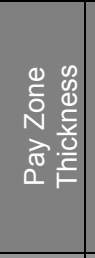 & 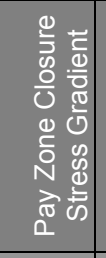 & 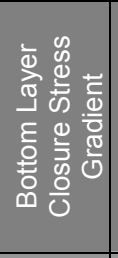 & \begin{tabular}{l|l}
3 \\
0 \\
$\frac{0}{0}$ \\
$\frac{0}{0}$ \\
$\frac{4}{4}$ \\
$\frac{0}{0}$ \\
$\frac{3}{0}$ \\
$\frac{0}{0}$ \\
$\frac{0}{4}$
\end{tabular} & 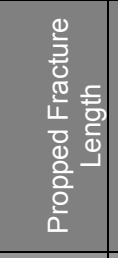 & 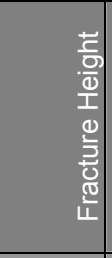 & 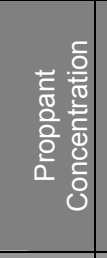 & \\
\hline input & input & input & input & input & input & input & input & input & input & input & input & input & input & input & output & output & output & output & outpu \\
\hline 62,569 & 18,651 & 1,909 & 1.0 & 25 & 10,078 & 4,975 & 0.0122 & 228 & 0.789 & 37 & 0.819 & 126 & 0.646 & 0.755 & 0.72 & 480.64 & 262.83 & 1.00 & \\
\hline 62,569 & 18,651 & 1,909 & 1.2 & 22 & 13,215 & 5,702 & 0.0069 & 197 & 0.747 & 24 & 0.795 & 82 & 0.674 & 0.736 & 0.81 & 457.57 & 391.54 & 0.86 & \\
\hline 98,812 & 35,841 & 2,738 & 1.5 & 30 & 13,586 & 6,101 & 0.0088 & 233 & 0.748 & 13 & 0.768 & 73 & 0.666 & 0.722 & 0.86 & 476.10 & 461.61 & 1.25 & 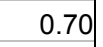 \\
\hline 85,941 & 36,577 & 2,146 & 1.2 & 20 & 9,716 & 3,896 & 0.0206 & 205 & 0.794 & 38 & 0.841 & 96 & 0.720 & 0.784 & 0.83 & 387.93 & 456.63 & 0.91 & \\
\hline 157,458 & 52,417 & 4,567 & 1.0 & 40 & 10,546 & 4,715 & 0.0365 & 188 & 0.755 & 16 & 0.773 & 67 & 0.670 & 0.732 & 0.78 & 569.78 & 569.45 & 0.95 & 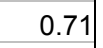 \\
\hline 89,844 & 30,775 & 2,568 & 1.1 & 24 & 8,082 & 3,972 & 0.0054 & 218 & 0.781 & 27 & 0.799 & 65 & 0.686 & 0.757 & 0.92 & 400.40 & 70.99 & 1.10 & \\
\hline 98,592 & 36,237 & 2,711 & 1.4 & 24 & 7,479 & 2,861 & 0.0905 & 234 & 0.746 & 36 & 0.765 & 91 & 0.671 & 0.730 & 0.86 & 369.14 & 508.64 & 1.44 & 0.6 \\
\hline 80,827 & 31,731 & 2,135 & 1.0 & 26 & 13,755 & 6,103 & 0.0047 & 227 & 0.754 & 26 & 0.769 & 114 & 0.659 & 0.728 & 0.79 & 555.37 & 286.58 & 0.91 & \\
\hline 41,149 & 13,016 & 1,223 & 1.0 & 18 & 12,441 & 5,818 & 0.0043 & 189 & 0.782 & 22 & 0.778 & 51 & 0.658 & 0.769 & 0.89 & 421.81 & 283.27 & 0.69 & 0.5 \\
\hline 122,102 & 38,404 & 3,639 & 1.4 & 38 & 9,247 & 3,942 & 0.0044 & 221 & 0.770 & 16 & 0.810 & 95 & 0.680 & 0.750 & 0.93 & 456.11 & 524.92 & 1.43 & 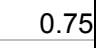 \\
\hline 120,919 & 49,626 & 3,100 & 1.0 & 33 & 10,940 & 5,401 & 0.0074 & 203 & 0.719 & 22 & 0.761 & 65 & 0.659 & 0.700 & 0.93 & 404.76 & 571.26 & 0.90 & \\
\hline 146,848 & 58,004 & 3,863 & 1.4 & 19 & 11,936 & 5,015 & 0.0054 & 177 & 0.795 & 10 & 0.834 & 109 & 0.696 & 0.769 & 0.84 & 604.67 & 454.51 & 1.47 & .8 \\
\hline 69,759 & 26,936 & 1,862 & 1.2 & 23 & 6,236 & 2,219 & 0.0028 & 186 & 0.724 & 15 & 0.760 & 93 & 0.650 & 0.710 & 0.93 & 302.77 & 450.91 & 1.07 & \\
\hline 69,759 & 26,936 & 1,862 & 1.0 & 26 & 14,109 & 6,610 & 0.0090 & 213 & 0.730 & 23 & 0.766 & 111 & 0.669 & 0.709 & 0.78 & 402.41 & 385.91 & 0.77 & 0.5 \\
\hline 90,378 & 27,901 & 2,716 & 1.5 & 29 & 15,021 & 6,930 & 0.0101 & 209 & 0.778 & 23 & 0.793 & 89 & 0.708 & 0.778 & 0.77 & 508.09 & 385.60 & 1.40 & .7 \\
\hline 90,378 & 27,901 & 2,716 & 1.0 & 28 & 10,233 & 4,936 & 0.0254 & 239 & 0.798 & 46 & 0.867 & 70 & 0.733 & 0.800 & 0.81 & 398.55 & 458.00 & 1.09 & 0.6 \\
\hline 83,310 & 26,695 & 2,462 & 1.0 & 30 & 14,595 & 6,433 & 0.0052 & 177 & 0.735 & 27 & 0.784 & 103 & 0.675 & 0.718 & 0.84 & 507.25 & 440.74 & 0.74 & 0.5 \\
\hline 133,416 & 44,950 & 3,846 & 1.8 & 39 & 10,946 & 5,118 & 0.0243 & 179 & 0.766 & 36 & 0.782 & 134 & 0.670 & 0.741 & 0.69 & 579.59 & 452.72 & 1.47 & 0.8 \\
\hline 156,706 & 57,898 & 4,296 & 1.0 & 40 & 14,934 & 6,370 & 0.0752 & 173 & 0.733 & 35 & 0.737 & 126 & 0.669 & 0.735 & 0.45 & 583.06 & 378.57 & 1.93 & 0.8 \\
\hline 34,200 & 12,099 & 961 & 1.0 & 24 & 6,832 & 2,754 & 0.0147 & 209 & 0.739 & 14 & 0.747 & 119 & 0.683 & 0.714 & 0.82 & 202.53 & 345.72 & 0.92 & 0.5 \\
\hline 116,444 & 37,792 & 3,420 & 1.2 & 35 & 5,863 & 2,430 & 0.0155 & 222 & 0.756 & 38 & 0.753 & 57 & 0.663 & 0.731 & 0.93 & 360.39 & 561.71 & 1.32 & 0.7 \\
\hline 158,063 & 58,809 & 4,315 & 1.5 & 40 & 5,634 & 2,791 & 0.0543 & 216 & 0.764 & 37 & 0.804 & 71 & 0.706 & 0.759 & 0.89 & 346.96 & 617.37 & 1.50 & 0.8 \\
\hline 158,063 & 58,809 & 4,315 & 1.5 & 40 & 5,634 & 2,791 & 0.0050 & 216 & 0.720 & 37 & 0.720 & 71 & 0.640 & 0.705 & 0.97 & 376.40 & 618.14 & 1.39 & 0.7 \\
\hline 151,254 & 64,968 & 3,752 & 1.0 & 21 & 9,821 & 3,525 & 0.0211 & 193 & 0.730 & 21 & 0.772 & 90 & 0.663 & 0.716 & 0.82 & 459.61 & 571.54 & 0.96 & 0.6 \\
\hline 110,501 & 43,768 & 2,901 & 1.0 & 36 & 13,988 & 6,129 & 0.0023 & 202 & 0.760 & 25 & 0.787 & 122 & 0.715 & 0.750 & 0.86 & 488.09 & 550.23 & 0.73 & \\
\hline
\end{tabular}

Table A-3: Data Generated for Eight Stage Treatment Type I, continued 


\begin{tabular}{|c|c|c|c|c|c|c|c|c|c|c|c|c|c|c|c|c|c|c|c|}
\hline \begin{tabular}{l|l}
$\frac{0}{5}$ \\
$\frac{5}{0}$ \\
$\frac{0}{\pi}$ \\
$\frac{\pi}{0}$ \\
$\vdash$
\end{tabular} & 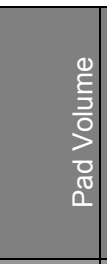 & 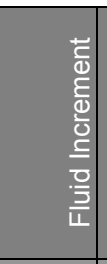 & 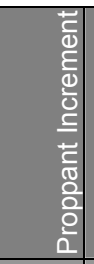 & 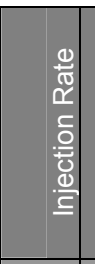 & 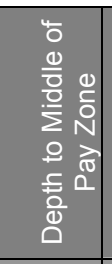 & \begin{tabular}{l|l}
$\frac{0}{3}$ & \\
0 & \\
0 & \\
0 & \\
0 & \\
$\frac{\pi}{0}$ & \\
0 & \\
$\frac{0}{0}$ & \\
$z$ &
\end{tabular} & 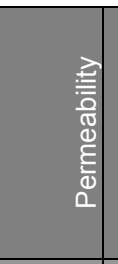 & 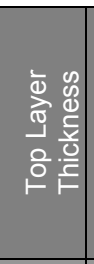 & 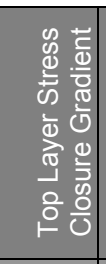 & 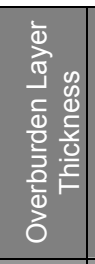 & 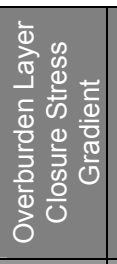 & 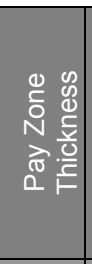 & 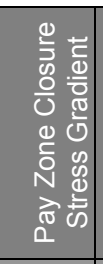 & 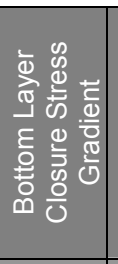 & 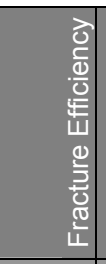 & 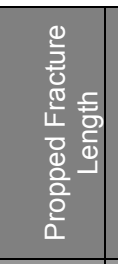 & 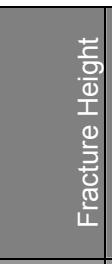 & 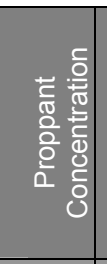 & \\
\hline input & input & input & input & input & input & input & input & input & input & input & input & input & input & input & output & output & output & output & outpl \\
\hline 54,965 & 21,990 & 1,434 & 1.2 & 22 & 6,878 & 2,962 & 0.0377 & 226 & 0.763 & 17 & 0.786 & 132 & 0.728 & 0.755 & 0.61 & 246.59 & 441.48 & 1.07 & \\
\hline 93,749 & 33,085 & 2,638 & 1.1 & 29 & 10,984 & 4,104 & 0.0027 & 222 & 0.731 & 28 & 0.790 & 113 & 0.671 & 0.744 & 0.86 & 409.36 & 430.16 & 1.11 & \\
\hline 116,296 & 43,488 & 3,166 & 1.0 & 32 & 10,072 & 4,418 & 0.0087 & 184 & 0.720 & 22 & 0.780 & 103 & 0.652 & 0.710 & 0.90 & 349.83 & 543.12 & 1.46 & 0.7 \\
\hline 116,296 & 43,488 & 3,166 & 1.3 & 29 & 5,272 & 2,418 & 0.0087 & 184 & 0.754 & 22 & 0.793 & 103 & 0.652 & 0.718 & 0.85 & 355.45 & 487.01 & 1.21 & \\
\hline 135,314 & 45,000 & 3,927 & 1.3 & 35 & 12,394 & 2,337 & 0.0236 & 209 & 0.725 & 31 & 0.770 & 86 & 0.678 & 0.736 & 0.75 & 457.36 & 488.66 & 1.54 & 0.7 \\
\hline 59,262 & 19,551 & 1,727 & 1.0 & 23 & 11,616 & 5,047 & 0.0087 & 186 & 0.758 & 26 & 0.833 & 113 & 0.712 & 0.768 & 0.71 & 379.53 & 428.22 & 0.72 & 0.4 \\
\hline 77,242 & 24,319 & 2,301 & 1.2 & 26 & 10,456 & 4,532 & 0.0938 & 184 & 0.791 & 28 & 0.842 & 67 & 0.736 & 0.805 & 0.60 & 368.39 & 484.95 & 1.04 & 0.2 \\
\hline 77,242 & 24,319 & 2,301 & 1.2 & 26 & 10,456 & 4,532 & 0.0098 & 184 & 0.742 & 28 & 0.822 & 67 & 0.684 & 0.740 & 0.84 & 394.94 & 468.59 & 1.00 & .5 \\
\hline 132,783 & 56,636 & 3,311 & 1.4 & 36 & 9,034 & 3,806 & 0.0068 & 222 & 0.726 & 13 & 0.761 & 111 & 0.659 & 0.726 & 0.86 & 396.67 & 497.24 & 1.58 & 0.7 \\
\hline 31,152 & 11,621 & 849 & 1.0 & 22 & 6,989 & 3,199 & 0.0132 & 170 & 0.756 & 35 & 0.835 & 77 & 0.724 & 0.754 & 0.81 & 218.83 & 368.86 & 0.71 & 0.3 \\
\hline 138,491 & 56,705 & 3,556 & 1.5 & 36 & 11,915 & 5,708 & 0.0121 & 199 & 0.732 & 39 & 0.797 & 95 & 0.700 & 0.741 & 0.83 & 406.87 & 536.71 & 1.65 & 0.7 \\
\hline 133,674 & 44,909 & 3,859 & 1.1 & 31 & 7,792 & 2,970 & 0.0934 & 191 & 0.738 & 40 & 0.774 & 118 & 0.703 & 0.734 & 0.58 & 387.31 & 622.91 & 1.19 & \\
\hline 133,674 & 44,909 & 3,859 & 1.1 & 31 & 7,792 & 2,970 & 0.0060 & 191 & 0.738 & 40 & 0.774 & 118 & 0.670 & 0.734 & 0.85 & 428.06 & 557.70 & 1.20 & 0.6 \\
\hline 102,697 & 36,334 & 2,885 & 1.2 & 35 & 6,899 & 3,146 & 0.0329 & 197 & 0.735 & 36 & 0.760 & 62 & 0.685 & 0.721 & 0.87 & 327.38 & 573.86 & 1.24 & 0.6 \\
\hline 99,620 & 40,480 & 2,571 & 1.5 & 30 & 9,328 & 3,954 & 0.0062 & 213 & 0.744 & 36 & 0.775 & 81 & 0.677 & 0.744 & 0.88 & 360.68 & 535.43 & 1.35 & 0. \\
\hline 153,392 & 65,146 & 3,837 & 1.0 & 40 & 14,852 & 6,850 & 0.0031 & 213 & 0.707 & 18 & 0.735 & 84 & 0.663 & 0.730 & 0.91 & 477.38 & 479.25 & 1.13 & 0.7 \\
\hline 153,392 & 65,146 & 3,837 & 1.0 & 35 & 14,852 & 6,850 & 0.0031 & 213 & 0.715 & 18 & 0.782 & 84 & 0.674 & 0.725 & 0.91 & 457.72 & 461.30 & 1.23 & 0.7 \\
\hline 95,804 & 33,330 & 2,716 & 1.3 & 33 & 9,325 & 4,662 & 0.0057 & 188 & 0.732 & 38 & 0.767 & 130 & 0.694 & 0.738 & 0.86 & 333.61 & 494.23 & 1.44 & 0.6 \\
\hline 159,801 & 59,885 & 4,344 & 1.4 & 40 & 11,026 & 5,239 & 0.0079 & 217 & 0.712 & 17 & 0.766 & 91 & 0.645 & 0.722 & 0.88 & 471.38 & 464.96 & 1.87 & 0.9 \\
\hline 159,801 & 59,885 & 4,344 & 1.0 & 40 & 7,061 & 3,001 & 0.0984 & 201 & 0.727 & 38 & 0.792 & 98 & 0.674 & 0.715 & 0.78 & 424.59 & 651.34 & 1.06 & 0.6 \\
\hline
\end{tabular}

Table A-3: Data Generated for Eight Stage Treatment Type I, continued 


\begin{tabular}{|c|c|c|c|c|c|c|c|c|c|c|c|c|c|c|c|c|c|c|c|}
\hline 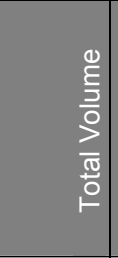 & 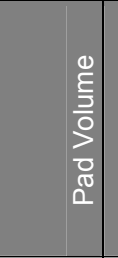 & 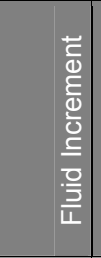 & 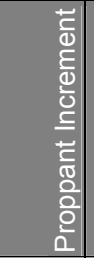 & 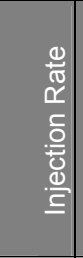 & 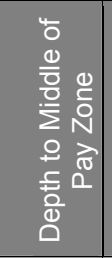 & 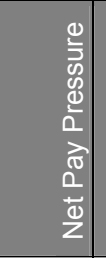 & 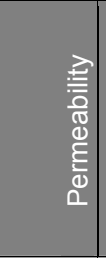 & 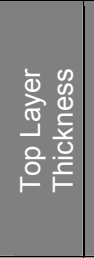 & 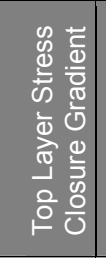 & 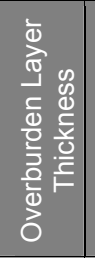 & 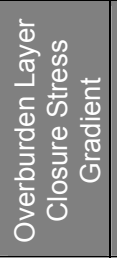 & 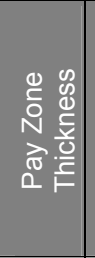 & 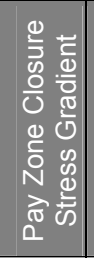 & 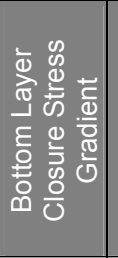 & 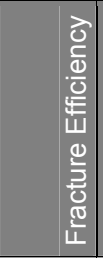 & 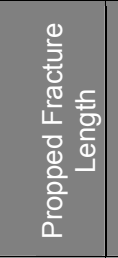 & 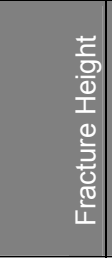 & 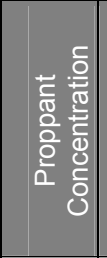 & 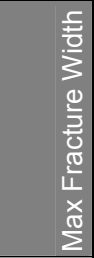 \\
\hline input & input & input & input & input & input & input & input & input & input & input & input & input & input & input & output & output & output & output & output \\
\hline 63,679 & 19,251 & 1,932 & 1.0 & 26 & 5,716 & 1,901 & 0.0920 & 232 & 0.737 & 30 & 0.741 & 128 & 0.659 & 0.724 & 0.63 & 269.75 & 434.16 & 1.08 & 0.63 \\
\hline 46,004 & 17,749 & 1,228 & 1.0 & 22 & 6,907 & 2,544 & 0.0086 & 192 & 0.773 & 16 & 0.803 & 84 & 0.695 & 0.740 & 0.88 & 241.12 & 364.76 & 0.97 & 0.57 \\
\hline 74,291 & 28,315 & 1,999 & 1.0 & 25 & 9,341 & 4,359 & 0.0163 & 200 & 0.727 & 18 & 0.773 & 105 & 0.640 & 0.716 & 0.81 & 371.23 & 404.97| & 1.05 & 0.65 \\
\hline 86,063 & 32,393 & 2,333 & 1.1 & 30 & 10,837 & 5,295 & 0.0215 & 174 & 0.774 & 35 & 0.828 & 105 & 0.713 & 0.757 & 0.77 & 395.30 & 476.09 & 0.81 & 0.57 \\
\hline 93,207 & 29,605 & 2,765 & 1.3 & 35 & 8,042 & 3,983 & 0.0065 & 177 & 0.746 & 13 & 0.836 & 126 & 0.643 & 0.700 & 0.90 & 356.54 & 498.00 & 1.30 & 0.70 \\
\hline 90,379 & 36,043 & 2,362 & 1.6 & 36 & 13,534 & 6,518 & 0.0189 & 190 & 0.712 & 17 & 0.741 & 110 & 0.650 & 0.706 & 0.77 & 422.11 & 447.15 & 1.36 & 0.67 \\
\hline 95,902 & 40,866 & 2,393 & 1.0 & 38 & 6,300 & 2,200 & 0.0081 & 229 & 0.750 & 15 & 0.786 & 82 & 0.694 & 0.733 & 0.90 & 300.12 & 490.88 & 1.02 & 0.63 \\
\hline 95,902 & 40,866 & 2,393 & 1.5 & 40 & 6,733 & 3,207 & 0.0086 & 189 & 0.768 & 28 & 0.800 & 135 & 0.694 & 0.733 & 0.89 & 296.42 & 472.78 & 1.62 & 0.72 \\
\hline 16,783 & 5,892 & 474 & 1.0 & 18 & 8,969 & 3,882 & 0.0154 & 237 & 0.765 & 23 & 0.779 & 114 & 0.688 & 0.749 & 0.74 & 168.72 & 227.32 & 0.71 & 0.45 \\
\hline 143,195 & 50,714 & 4,021 & 1.0 & 40 & 7,328 & 3,255 & 0.0135 & 235 & 0.741 & 28 & 0.740 & 125 & 0.668 & 0.713 & 0.87 & 404.33 & 602.89 & 1.06 & 0.70 \\
\hline 138,075 & 55,006 & 3,612 & 1.5 & 38 & 10,967 & 4,672 & 0.0398 & 196 & 0.772 & 35 & 0.814 & 106 & 0.710 & 0.767 & 0.71 & 466.51 & 563.39 & 1.22 & 0.65 \\
\hline 112,681 & 38,202 & 3,238 & 1.2 & 34 & 14,770 & 6,617 & 0.0073 & 211 & 0.766 & 32 & 0.807 & 58 & 0.693 & 0.740 & 0.91 & 450.46 & 586.68 & 0.96 & 0.58 \\
\hline 64,651 & 26,983 & 1,638 & 1.0 & 26 & 8,862 & 4,222 & 0.0022 & 220 & 0.736 & 19 & 0.732 & 114 & 0.669 & 0.714 & 0.93 & 291.90 & 414.83 & 0.87 & 0.58 \\
\hline 64,651 & 26,983 & 1,638 & 1.1 & 29 & 13,338 & 6,559 & 0.0126 & 182 & 0.740 & 17 & 0.740 & 100 & 0.660 & 0.721 & 0.79 & 395.46 & 333.09 & 0.95 & 0.63 \\
\hline 159,298 & 62,937 & 4,190 & 1.0 & 40 & 12,715 & 5,816 & 0.0509 & 184 & 0.763 & 33 & 0.807 & 122 & 0.695 & 0.754 & 0.59 & 633.63 & 522.44 & 0.87 & 0.66 \\
\hline 41,663 & 16,074 & 1,113 & 1.0 & 23 & 12,272 & 5,959 & 0.0118 & 176 & 0.721 & 30 & 0.783 & 108 & 0.641 & 0.704 & 0.77 & 345.55 & 288.72 & 0.72 & 0.55 \\
\hline 159,298 & 62,937 & 4,190 & 1.0 & 40 & 9,540 & 4,459 & 0.0672 & 188 & 0.749 & 29 & 0.777 & 122 & 0.681 & 0.753 & 0.88 & 461.22 & 509.11 & 1.15 & 0.80 \\
\hline 55,850 & 17,308 & 1,676 & 1.5 & 26 & 5,707 & 1,887 & 0.0124 & 206 & 0.740 & 38 & 0.768 & 107 & 0.646 & 0.733 & 0.85 & 271.87 & 392.26 & 1.52 & 0.70 \\
\hline 78,597 & 26,964 & 2,245 & 1.1 & 25 & 11,453 & 5,033 & 0.0880 & 181 & 0.782 & 40 & 0.795 & 100 & 0.689 & 0.770 & 0.47 & 437.63 & 316.59 & 1.13 & 0.66 \\
\hline 78,597 & 26,964 & 2,245 & 1.0 & 26 & 11,543 & 4,968 & 0.0291 & 221 & 0.748 & 36 & 0.746 & 79 & 0.666 & 0.745 & 0.75 & 417.89 & 427.44| & 0.78 & 0.64 \\
\hline 89,330 & 27,864 & 2,672 & 1.0 & 28 & 5,424 & 1,885 & 0.0190 & 208 & 0.765 & 24 & 0.814 & 101 & 0.672 & 0.756 & 0.84 & 339.81 & 486.98 & 1.27 & 0.69 \\
\hline 80,016 & 32,486 & 2,067 & 1.1 & 30 & 8,815 & 4,159 & 0.0095 & 229 & 0.766 & 11 & 0.765 & 101 & 0.687 & 0.761 & 0.87 & 340.49 & 437.40 & 1.00 & 0.64 \\
\hline 40,603 & 14,235 & 1,146 & 1.0 & 20 & 10,807 & 5,017 & 0.0040 & 192 & 0.740 & 12 & 0.761 & 115 & 0.672 & 0.716 & 0.86 & 275.74 & 334.03 & 0.95 & 0.56 \\
\hline 132,289 & 51,246 & 3,524 & 1.5 & 40 & 11,707 & 5,011 & 0.0115 & 177 & 0.764 & 17 & 0.799 & 94 & 0.704 & 0.767 & 0.85 & 434.47 & 461.12 & 1.57 & 0.82 \\
\hline 122,749 & 37,186 & 3,720 & 1.0 & 34 & 12,278 & 5,331 & 0.0271 & 195 & 0.727 & 32 & 0.727 & 80 & 0.662 & 0.722 & 0.80 & 444.02 & 543.13 & 1.10 & 0.70 \\
\hline 122,749 & 37,186 & 3,720 & 1.5 & 37 & 10,831 & 4,546 & 0.0122 & 175 & 0.760 & 21 & 0.807 & 56 & 0.760 & 0.804 & 0.96 & 397.65 & 445.16 & 2.18 & 0.98 \\
\hline 41,891 & 13,280 & 1,244 & 1.1 & 20 & 7,562 & 3,028 & 0.0139 & 221 & 0.738 & 16 & 0.782 & 66 & 0.650 & 0.710 & 0.88 & 254.35 & 375.07 & 0.92 & 0.54 \\
\hline 155,634 & 45,430 & 4,791 & 1.1 & 40 & 14,804 & 6,770 & 0.0086 & 196 & 0.762 & 29 & 0.830 & 69 & 0.680 & 0.767 & 0.85 & 740.11 & 456.50 & 1.00 & 0.78 \\
\hline 138,732 & 43,383 & 4,146 & 1.4 & 37 & 14,083 & 6,389 & 0.0187 & 201 & 0.732 & 39 & 0.777 & 117 & 0.675 & 0.718 & 0.73 & 588.92 & 569.18 & 1.10 & 0.66 \\
\hline 149,426 & 61,524 & 3,822 & 1.5 & 39 & 9,460 & 3,785 & 0.0075 & 211 & 0.781 & 23 & 0.833 & 55 & 0.704 & 0.780 & 0.93 & 425.42 & 496.47| & 1.62 & 0.83 \\
\hline
\end{tabular}

Table A - 4: Data Generated for Eight Stage Treatment Type II 


\begin{tabular}{|c|c|c|c|c|c|c|c|c|c|c|c|c|c|c|c|c|c|c|c|}
\hline \begin{tabular}{l|}
$\frac{0}{E}$ \\
$\frac{3}{0}$ \\
$\frac{0}{0}$ \\
$\frac{\pi}{0}$ \\
\end{tabular} & 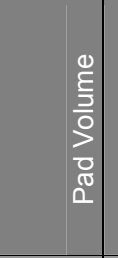 & 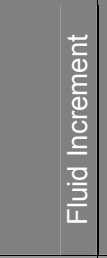 & 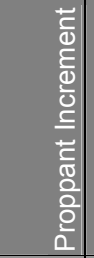 & 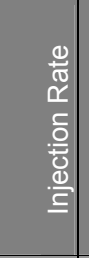 & 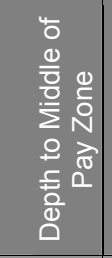 & 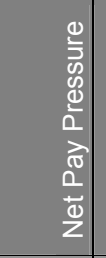 & 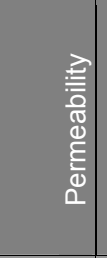 & 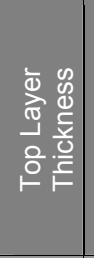 & 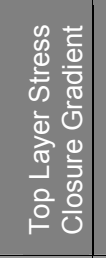 & 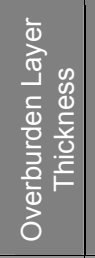 & 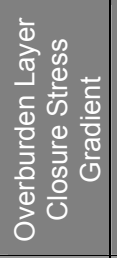 & 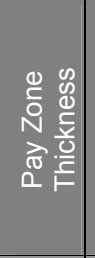 & 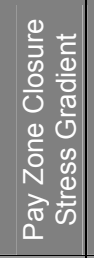 & 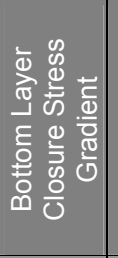 & 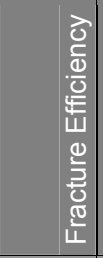 & 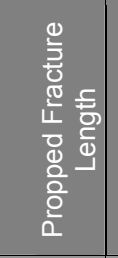 & 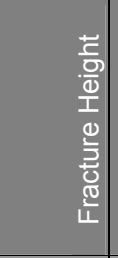 & 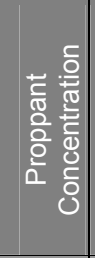 & 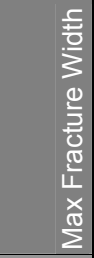 \\
\hline input & input & input & input & input & input & input & input & input & input & input & input & input & input & input & output & output & output & output & output \\
\hline 138,678 & 45,982 & 4,030 & 1.2 & 38 & 13,813 & 6,481 & 0.0561 & 235 & 0.782 & 17 & 0.788 & 126 & 0.705 & 0.775 & 0.51 & 603.97 & 376.33 & 1.37 & 0.77 \\
\hline 138,678 & 45,982 & 4,030 & 1.0 & 38 & 13,813 & 6,481 & 0.0561 & 235 & 0.780 & 17 & 0.812 & 126 & 0.725 & 0.770 & 0.77 & 615.85 & 570.18 & 0.74 & 0.60 \\
\hline 143,276 & 59,155 & 3,657 & 1.2 & 40 & 7,612 & 3,183 & 0.0168 & 226 & 0.747 & 27 & 0.760 & 76 & 0.655 & 0.734 & 0.87 & 423.11 & 554.25 & 1.20 & 0.69 \\
\hline 124,417 & 50,200 & 3,227 & 1.1 & 38 & 11,961 & 5,261 & 0.0913 & 217 & 0.715 & 36 & 0.794 & 111 & 0.666 & 0.722 & 0.45 & 522.88 & 523.96 & 0.25 & 0.50 \\
\hline 124,417 & 50,200 & 3,227 & 1.0 & 38 & 9,227 & 4,034 & 0.0337 & 222 & 0.755 & 38 & 0.760 & 69 & 0.679 & 0.745 & 0.81 & 405.11 & 583.47 & 0.98 & 0.58 \\
\hline 54,454 & 20,158 & 1,491 & 1.0 & 25 & 10,267 & 4,870 & 0.0783 & 224 & 0.793 & 29 & 0.798 & 105 & 0.731 & 0.774 & 0.44 & 311.46 & 403.84 & 0.76 & 0.41 \\
\hline 73,005 & 26,591 & 2,018 & 1.0 & 32 & 14,455 & 7,046 & 0.0115 & 201 & 0.799 & 40 & 0.862 & 83 & 0.727 & 0.789 & 0.70 & 546.61 & 415.01 & 0.70 & 0.53 \\
\hline 73,005 & 26,591 & 2,018 & 1.5 & 29 & 11,124 & 4,642 & 0.0069 & 224 & 0.718 & 25 & 0.752 & 90 & 0.663 & 0.709 & 0.83 & 372.04 & 501.85 & 0.96 & 0.51 \\
\hline 54,454 & 20,158 & 1,491 & 1.0 & $27 \mid$ & 14,298 & 6,803 & 0.0082 & 203 & 0.757 & 15 & 0.817 & 118 & 0.695 & 0.739 & 0.67 & 407.05 & 344.67 & 0.66 & 0.48 \\
\hline 147,206 & 48,441 & 4,294 & 1.0 & 40 & 9,472 & 4,378 & 0.0192 & 238 & 0.703 & 33 & 0.733 & 74 & 0.642 & 0.700 & 0.87 & 410.63 & 535.58 & 1.25 & 0.79 \\
\hline 92,236 & 38,942 & 2,317 & 1.5 & 35 & 9,958 & 4,837 & 0.0386 & 198 & 0.742 & 39 & 0.775 & 120 & 0.688 & 0.731 & 0.65 & 337.97| & 485.20 & 0.93 & 0.47 \\
\hline 92,236 & 38,942 & 2,317 & 1.0 & $27 \mid$ & 14,995 & 7,049 & 0.0141 & 235 & 0.751 & 29 & 0.800 & 122 & 0.698 & 0.743 & 0.55 & 560.35 & 298.94 & 0.82 & 0.54 \\
\hline 121,937 & 37,916 & 3,653 & 1.0 & 37 & 7,749 & 3,673 & 0.0099 & 231 & 0.726 & 21 & 0.754 & 74 & 0.670 & 0.724 & 0.92 & 354.57 & 570.72 & 1.30 & 0.72 \\
\hline 115,429 & 44,021 & 3,105 & 1.0 & 34 & 6,988 & 2,876 & 0.0076 & 195 & 0.783 & 22 & 0.807 & 102 & 0.712 & 0.746 & 0.88 & 379.60 & 591.92 & 0.99 & 0.57 \\
\hline 56,836 & 24,116 & 1,423 & 1.0 & 27 & 13,089 & 5,557 & 0.0026 & 192 & 0.711 & 27 & 0.754 & 92 & 0.642 & 0.706 & 0.84 & 446.75 & 367.02 & 0.68 & 0.50 \\
\hline 37,592 & 13,923 & 1,029 & 1.0 & 20 & 14,280 & 6,606 & 0.0195 & 226 & 0.789 & 40 & 0.822 & 104 & 0.731 & 0.781 & 0.57 & 357.18 & 293.92 & 0.54 & 0.40 \\
\hline 158,466 & 53,708 & 4,555 & 1.5 & 40 & 10,086 & 4,753 & 0.0042 & 172 & 0.748 & 28 & 0.778 & 129 & 0.691 & 0.723 & 0.88 & 487.39 & 658.03 & 1.19 & 0.67 \\
\hline 158,466 & 53,708 & 4,555 & 1.0 & 35 & 10,086 & 4,753 & 0.0042 & 172 & 0.748 & 28 & 0.778 & 129 & 0.660 & 0.730 & 0.84 & 673.23 & 542.27 & 0.81 & 0.68 \\
\hline 59,632 & 18,033 & 1,809 & 1.0 & 26 & 11,219 & 5,407 & 0.0056 & 230 & 0.763 & 36 & 0.806 & 84 & 0.681 & 0.743 & 0.83 & 433.59 & 406.77| & 0.66 & 0.53 \\
\hline 79,217 & 30,064 & 2,137 & 1.1 & 30 & 11,623 & 5,810 & 0.0068 & 190 & 0.789 & 24 & 0.796 & 76 & 0.712 & 0.764 & 0.85 & 445.00 & 446.26 & 0.77 & 0.54 \\
\hline 88,360 & 35,166 & 2,313 & 1.2 & 28 & 13,706 & 6,631 & 0.0097 & 235 & 0.761 & 35 & 0.781 & 111 & 0.690 & 0.735 & 0.72 & 471.06 & 404.43 & 0.90 & 0.58 \\
\hline 81,818 & 29,503 & 2,275 & 1.3 & 25 & 11,299 & 4,905 & 0.0551 & 239 & 0.743 & 14 & 0.801 & 53 & 0.682 & 0.723 & 0.79 & 344.22 & 523.07 & 1.00 & 0.53 \\
\hline 110,542 & 38,403 & 3,136 & 1.0 & 35 & 6,498 & 2,589 & 0.0159 & 173 & 0.726 & 26 & 0.774 & 125 & 0.651 & 0.708 & 0.79 & 391.91 & 570.15 & 1.11 & 0.60 \\
\hline 128,712 & 49,712 & 3,435 & 1.5 & 35 & 7,768 & 3,663 & 0.0026 & 178 & 0.770 & 10 & 0.796 & 110 & 0.681 & 0.741 & 0.92 & 430.53 & 572.50 & 1.27 & 0.69 \\
\hline 91,748 & 33,408 & 2,537 & 1.0 & 28 & 8,724 & 4,074 & 0.0085 & 217 & 0.731 & 35 & 0.771 & 67 & 0.653 & 0.712 & 0.90 & 379.30 & 541.50 & 0.79 & 0.55 \\
\hline 91,748 & 33,408 & 2,537 & 1.2 & 27 & 9,358 & 4,330 & 0.0805 & 227 & 0.770 & 27 & 0.770 & 60 & 0.680 & 0.756 & 0.70 & 385.65 & 503.95 & 1.01 & 0.59 \\
\hline 91,748 & 33,408 & 2,537 & 1.1 & 30 & 5,732 & 2,737 & 0.0073 & 215 & 0.774 & 28 & 0.818 & 117 & 0.709 & 0.743 & 0.88 & 372.57 & 599.30 & 0.99 & 0.57 \\
\hline 106,618 & 34,870 & 3,119 & 1.3 & 35 & 10,995 & 4,947 & 0.0095 & 173 & 0.710 & 13 & 0.752 & 60 & 0.651 & 0.702 & 0.90 & 401.64 & 588.90 & 1.04 & 0.58 \\
\hline 104,289 & 39,812 & 2,803 & 1.0 & 32 & 8,304 & 3,619 & 0.0235 & 193 & 0.787 & 39 & 0.785 & 69 & 0.704 & 0.765 & 0.83 & 377.20 & 546.75 & 1.07 & 0.60 \\
\hline 141,482 & 46,557 & 4,127 & 1.1 & 38 & $13,274 \mid$ & 6,369 & 0.0193 & 234 & 0.768 & 23 & 0.795 & 76 & 0.682 & 0.746 & 0.82 & 714.21 & 425.05 & 0.94 & 0.72 \\
\hline
\end{tabular}

Table A-4: Data Generated for Eight Stage Treatment Type II, continued 


\begin{tabular}{|c|c|c|c|c|c|c|c|c|c|c|c|c|c|c|c|c|c|c|c|}
\hline 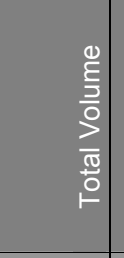 & 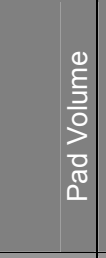 & 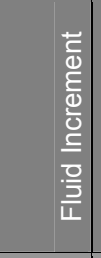 & 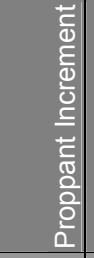 & 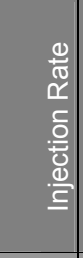 & 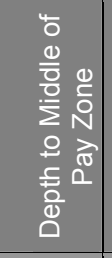 & 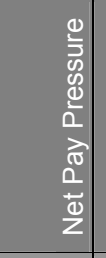 & 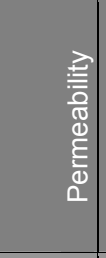 & 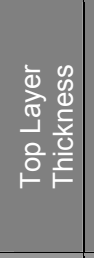 & 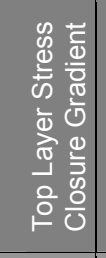 & 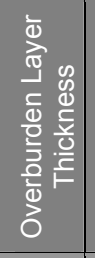 & 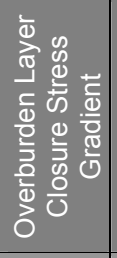 & 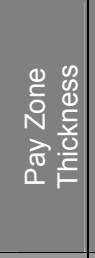 & 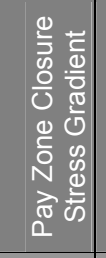 & 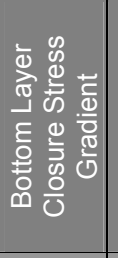 & 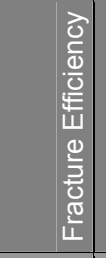 & 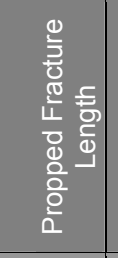 & 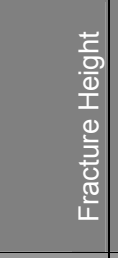 & 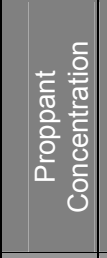 & 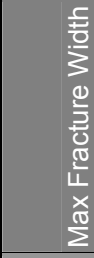 \\
\hline input & input & input & input & input & input & input & input & input & input & input & input & input & input & input & output & output & output & output & output \\
\hline 127,660 & 41,749 & 3,735 & 1.2 & 34 & 13,011 & 6,391 & 0.0273 & 214 & 0.713 & 30 & 0.756 & 103 & 0.651 & 0.702 & 0.69 & 608.30 & 564.67 & 0.81 & 0.57 \\
\hline 70,750 & 22,410 & 2,102 & 1.0 & 22 & 9,273 & 4,310 & 0.0039 & 203 & 0.738 & 32 & 0.771 & 62 & 0.657 & 0.727 & 0.91 & 390.04 & 430.88 & 0.89 & 0.58 \\
\hline 70,750 & 22,410 & 2,102 & 1.2 & 22 & 5,158 & 2,509 & 0.0066 & 219 & 0.746 & 32 & 0.742 & 59 & 0.663 & 0.720 & 0.94 & 306.76 & 557.90 & 0.91 & 0.50 \\
\hline 137,020 & 47,245 & 3,903 & 1.0 & 25 & 10,735 & 4,945 & 0.0714 & 238 & 0.766 & 31 & 0.803 & 77 & 0.700 & 0.744 & 0.67 & 535.73 & 606.61 & 0.78 & 0.55 \\
\hline 140,536 & 42,451 & 4,265 & 1.0 & 35 & 6,096 & 2,176 & 0.0024 & 235 & 0.721 & 13 & 0.788 & 108 & 0.670 & 0.701 & 0.94 & 366.81 & 608.13 & 1.23 & 0.74 \\
\hline 140,536 & 42,451 & 4,265 & 1.5 & 40 & 14,005 & 6,596 & 0.0099 & 209 & 0.722 & 34 & 0.784 & 119 & 0.645 & 0.736 & 0.70 & 765.67 & 411.92 & 1.28 & 0.76 \\
\hline 136,536 & 50,324 & 3,748 & 1.0 & 36 & 13,710 & 6,473 & 0.0072 & 172 & 0.741 & 35 & 0.794 & 130 & 0.684 & 0.732 & 0.84 & 582.24 & 566.45 & 0.81 & 0.57 \\
\hline 153,097 & 62,888 & 3,922 & 1.5 & 19 & 6,552 & 3,227 & 0.0023 & 216 & 0.768 & 16 & 0.768 & 126 & 0.691 & 0.729 & 0.93 & 400.49 & 674.56 & 1.29 & 0.65 \\
\hline 67,375 & 28,188 & 1,704 & 1.0 & 24 & 14,171 & 6,611 & 0.0079 & 236 & 0.745 & 16 & 0.778 & 84 & 0.650 & 0.722 & 0.71 & 570.48 & 267.73 & 0.72 & 0.55 \\
\hline 67,375 & 28,188 & 1,704 & 1.0 & 24 & 14,171 & 6,611 & 0.0079 & 236 & 0.730 & 16 & 0.770 & 84 & 0.685 & 0.715 & 0.80 & 392.71 & 462.02 & 0.60 & 0.43 \\
\hline 67,375 & 28,188 & 1,704 & 1.0 & 28 & 9,864 & 4,003 & 0.0173 & 228 & 0.760 & 26 & 0.816 & 72 & 0.695 & 0.753 & 0.79 & 339.15 & 430.14 & 0.82 & 0.51 \\
\hline 67,375 & 28,188 & 1,704 & 1.0 & 22 & 11,088 & 4,591 & 0.0047 & 217 & 0.721 & 15 & 0.786 & 134 & 0.670 & 0.733 & 0.77 & 350.39 & 402.98 & 0.95 & 0.53 \\
\hline 22,930 & 8,145 & 643 & 1.2 & 15 & 6,502 & 2,865 & 0.0494 & 200 & 0.705 & 25 & 0.768 & 53 & 0.665 & 0.709 & 0.74 & 178.86 & 332.28 & 0.73 & 0.35 \\
\hline 127,168 & 39,055 & 3,831 & 1.0 & 36 & 14,234 & 6,743 & 0.0097 & 187 & 0.735 & 39 & 0.789 & 76 & 0.673 & 0.720 & 0.82 & 568.61 & 648.98 & 0.74 & 0.56 \\
\hline 105,250 & 42,105 & 2,745 & 1.0 & 37 & 13,001 & 6,150 & 0.0053 & 194 & 0.730 & 16 & 0.782 & 80 & 0.666 & 0.718 & 0.87 & 516.62 & 506.02 & 0.67 & 0.55 \\
\hline 120,705 & 37,616 & 3,613 & 1.3 & 40 & 7,167 & 3,208 & 0.0036 & 216 & 0.730 & 18 & 0.768 & 73 & 0.657 & 0.708 & 0.95 & 366.54 & 553.18 & 1.52 & 0.76 \\
\hline 151,397 & 45,473 & 4,605 & 1.0 & 23 & 13,333 & 6,480 & 0.0910 & 233 & 0.773 & 15 & 0.811 & 63 & 0.707 & 0.761 & 0.68 & 539.22 & 555.16 & 0.99 & 0.67 \\
\hline 72,128 & 27,230 & 1,952 & 1.0 & 25 & 5,699 & 2,554 & 0.0036 & 222 & 0.743 & 32 & 0.792 & 56 & 0.677 & 0.730 & 0.95 & 300.55 & 486.75 & 0.86 & 0.53 \\
\hline 72,128 & 27,230 & 1,952 & 1.3 & 27 & 7,533 & 2,871 & 0.0634 & 194 & 0.715 & 17 & 0.796 & 84 & 0.652 & 0.704 & 0.66 & 323.30 & 483.80 & 0.98 & 0.48 \\
\hline 72,128 & 27,230 & 1,952 & 1.0 & 24 & 9,016 & 3,836 & 0.0041 & 200 & 0.730 & 21 & 0.793 & 119 & 0.681 & 0.716 & 0.85 & 351.18 & 514.13 & 0.69 & 0.45 \\
\hline 98,112 & 40,371 & 2,510 & 1.4 & 27 & 14,020 & 6,293 & 0.0084 & 213 & 0.723 & 27 & 0.762 & 98 & 0.651 & 0.715 & 0.72 & 622.92 & 324.59 & 1.04 & 0.63 \\
\hline 35,402 & 14,439 & 911 & 1.3 & 21 & 15,141 & 6,905 & 0.0913 & 223 & 0.743 & 19 & 0.800 & 81 & 0.700 & 0.740 & 0.28 & 283.76 & 318.56 & 0.82 & 0.33 \\
\hline 97,235 & 32,869 & 2,799 & 1.0 & 30 & 6,538 & 2,303 & 0.0069 & 212 & 0.705 & 14 & 0.784 & 128 & 0.672 & 0.708 & 0.86 & 332.94 & 550.14 & 1.00 & 0.59 \\
\hline 51,315 & 20,035 & 1,360 & 1.2 & 20 & 10,149 & 4,465 & 0.0034 & 231 & 0.715 & 26 & 0.782 & 72 & 0.678 & 0.713 & 0.92 & 373.69 & 534.99 & 1.08 & 0.57 \\
\hline 51,315 & 20,035 & 1,360 & 1.0 & 25 & 10,869 & 5,232 & 0.0431 & 186 & 0.785 & 29 & 0.825 & 61 & 0.726 & 0.800 & 0.70 & 281.24 & 323.40 & 0.96 & 0.58 \\
\hline 139,592 & 46,170 & 4,062 & 1.2 & 34 & 10,651 & 5,313 & 0.0047 & 197 & 0.731 & 17 & 0.783 & 66 & 0.702 & 0.721 & 0.93 & 402.85 & 583.90 & 1.28 & 0.70 \\
\hline 49,533 & 18,841 & 1,334 & 1.1 & 24 & 11,292 & 5,603 & 0.0574 & 219 & 0.710 & 22 & 0.788 & 129 & 0.672 & 0.704 & 0.78 & 315.88 & 455.21 & 0.64 & 0.39 \\
\hline 49,533 & 18,841 & 1,334 & 1.0 & 22 & 5,520 & 1,776 & 0.0153 & 202 & 0.733 & 34 & 0.791 & 60 & 0.650 & 0.717 & 0.84 & 282.58 & 430.37 & 0.70 & 0.42 \\
\hline 49,533 & 18,841 & 1,334 & 1.0 & 27 & 7,886 & 2,337 & 0.0073 & 237 & 0.764 & 17 & 0.802 & 76 & 0.715 & 0.746 & 0.85 & 260.27 & 437.73 & 0.75 & 0.43 \\
\hline 25,949 & 7,835 & 788 & 1.0 & 22 & 10,413 & 4,640 & 0.0177 & 171 & $0.727 \mid$ & 27 & 0.796 & 100 & 0.677 & 0.712 & 0.59 & 249.48 & 330.15 & 0.68 & 0.35 \\
\hline
\end{tabular}

Table A-4: Data Generated for Eight Stage Treatment Type II, continued 


\begin{tabular}{|c|c|c|c|c|c|c|c|c|c|c|c|c|c|c|c|c|c|c|c|}
\hline \begin{tabular}{l|}
$\stackrel{0}{E}$ \\
$\frac{5}{0}$ \\
$\frac{0}{0}$ \\
$\frac{\pi}{0}$ \\
$\frac{0}{0}$
\end{tabular} & $\begin{array}{l}\frac{1}{\varepsilon} \\
\frac{1}{3} \\
0 \\
\frac{0}{0} \\
\frac{0}{0} \\
0\end{array}$ & 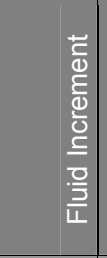 & 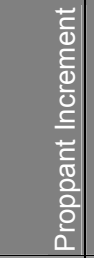 & 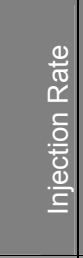 & 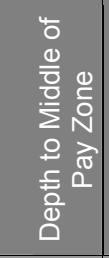 & 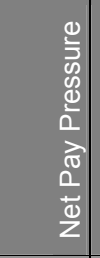 & 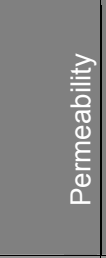 & 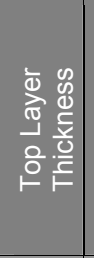 & 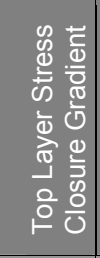 & 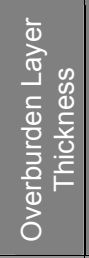 & 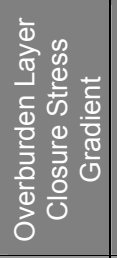 & 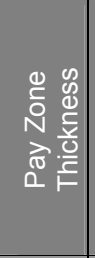 & 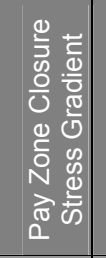 & 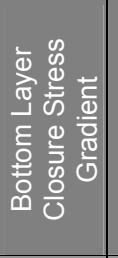 & 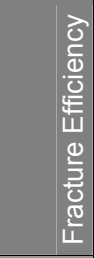 & 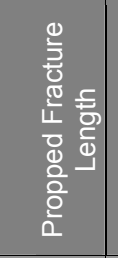 & 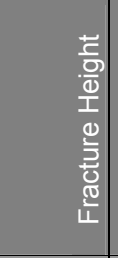 & 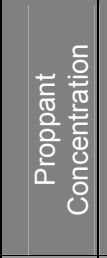 & 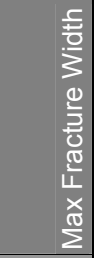 \\
\hline input & input & input & input & input & input & input & input & input & input & input & input & input & input & input & output & output & output & output & output \\
\hline 93,082 & 31,905 & 2,660 & 1.0 & 31 & 8,758 & 4,018 & 0.0054 & 224 & 0.758 & 57 & 0.819 & 105 & 0.707 & 0.788 & 0.86 & 402.16 & 580.01 & 0.82 & 0.50 \\
\hline 97,549 & 39,482 & 2,525 & 1.1 & 35 & 10,608 & 5,252 & 0.0019 & 192 & 0.705 & 34 & 0.762 & 84 & 0.646 & 0.702 & 0.93 & 420.58 & 492.47 & 0.88 & 0.57 \\
\hline 39,915 & 14,788 & 1,092 & 1.0 & 23 & 13,103 & 6,063 & 0.0086 & 173 & 0.758 & 27 & 0.835 & 99 & 0.707 & 0.747 & 0.66 & 349.27 & 377.88 & 0.53 & 0.38 \\
\hline 121,612 & 45,805 & 3,296 & 1.0 & 35 & 14,651 & 6,627 & 0.0147 & 186 & 0.740 & 35 & 0.791 & 104 & 0.666 & 0.726 & 0.65 & 693.96 & 442.04 & 0.69 & 0.61 \\
\hline 44,691 & 16,562 & 1,223 & 1.0 & 25 & 11,677 & 5,478 & 0.0569 & 236 & 0.710 & 27 & 0.769 & 80 & 0.660 & 0.702 & 0.53 & 318.50 & 380.37 & 0.72 & 0.39 \\
\hline 44,691 & 16,562 & 1,223 & 1.0 & 22 & 8,210 & 6,266 & 0.0053 & 173 & 0.755 & 18 & 0.755 & 78 & 0.705 & 0.741 & 0.88 & 268.16 & 487.37 & 0.74 & 0.38 \\
\hline 77,369 & 26,883 & 2,195 & 1.0 & 27 & 10,622 & 4,334 & 0.0050 & 174 & 0.726 & 23 & 0.788 & 98 & 0.658 & 0.718 & 0.82 & 426.34 & 455.68 & 0.89 & 0.55 \\
\hline 43,515 & 15,612 & 1,213 & 1.0 & 17 & 7,302 & 3,080 & 0.0136 & 186 & 0.740 & 16 & 0.738 & 90 & 0.677 & 0.730 & 0.75 & 284.44 & 421.76 & 0.65 & 0.40 \\
\hline 77,369 & 26,883 & 2,195 & 1.0 & 25 & 5,708 & 2,407 & 0.0199 & 201 & 0.779 & 38 & 0.845 & 106 & 0.710 & 0.770 & 0.76 & 339.87 & 510.03 & 0.90 & 0.50 \\
\hline 42,098 & 12,493 & 1,287 & 1.5 & 20 & 7,389 & 3,165 & 0.0754 & 225 & 0.750 & 37 & 0.753 & 133 & 0.667 & 0.746 & 0.36 & 244.62 & 357.79 & 1.31 & 0.45 \\
\hline 42,098 & 12,493 & $1,287 \mid$ & 1.0 & 24 & 15,046 & 6,647 & 0.0750 & 206 & 0.760 & 24 & 0.800 & $87 \mid$ & 0.684 & 0.724 & 0.69 & 380.39 & 337.30 & 0.58 & 0.43 \\
\hline 39,794 & 15,310 & 1,065 & 1.0 & 25 & 9,408 & 4,272 & 0.0052 & 237 & 0.768 & 27 & 0.812 & 56 & 0.693 & 0.743 & 0.85 & 293.31 & 403.63 & 0.58 & 0.40 \\
\hline 102,076 & 35,351 & 2,901 & 1.5 & 36 & 9,828 & 4,688 & 0.0138 & 232 & 0.728 & 14 & 0.803 & 94 & 0.671 & 0.714 & 0.81 & 390.30 & 546.76 & 1.25 & 0.61 \\
\hline 123,820 & 52,370 & 3,107 & 1.0 & 39 & 7,338 & 3,283 & 0.0045 & 201 & 0.746 & 13 & 0.789 & 101 & 0.677 & 0.716 & 0.91 & 377.59 & 582.03 & 1.01 & 0.60 \\
\hline 45,898 & 16,136 & 1,294 & 1.5 & 29 & 5,452 & 2,700 & 0.0043 & 228 & 0.767 & 18 & 0.767 & 98 & 0.709 & 0.776 & 0.90 & 244.21 & 420.78 & 1.12 & 0.50 \\
\hline 68,692 & 29,038 & 1,724 & 1.1 & 25 & 12,656 & 6,164 & 0.0131 & 202 & 0.746 & 29 & 0.777 & 88 & 0.682 & 0.730 & 0.72 & 434.66 & 422.92 & 0.66 & 0.47 \\
\hline 91,813 & 27,917 & 2,778 & 1.0 & 26 & 6,687 & 2,354 & 0.0297 & 228 & 0.757 & 13 & 0.678 & 96 & 0.743 & 0.773 & 0.71 & 386.44 & 534.08 & 0.96 & 0.55 \\
\hline 68,692 & 29,038 & 1,724 & 1.0 & 21 & 10,770 & 5,477 & 0.0079 & 181 & 0.743 & 28 & 0.799 & 97 & 0.689 & 0.730 & 0.79 & 379.84 & 487.19 & 0.67 & 0.45 \\
\hline 149,147 & 55,050 & 4,091 & 1.0 & 35 & 13,126 & 5,649 & 0.0304 & 203 & 0.755 & 11 & 0.817 & 91 & 0.695 & 0.746 & 0.66 & 586.54 & 564.92 & 0.79 & 0.58 \\
\hline 114,965 & 42,156 & 3,166 & 1.1 & 30 & 7,269 & 3,554 & 0.0553 & 202 & 0.729 & 26 & 0.787 & 129 & 0.686 & 0.723 & 0.68 & 369.49 & 596.66 & 1.02 & 0.52 \\
\hline 69,356 & 22,999 & 2,016 & 1.1 & 27 & 7,622 & 2,985 & 0.0178 & 174 & 0.734 & 36 & 0.795 & 125 & 0.700 & 0.725 & 0.73 & 305.45 & 509.15 & 0.92 & 0.47 \\
\hline 49,091 & 18,817 & 1,316 & 1.2 & 22 & 9,835 & 4,386 & 0.0634 & 200 & 0.749 & 18 & 0.792 & 51 & 0.688 & 0.738 & 0.71 & 296.57 & 404.58 & 0.85 & 0.45 \\
\hline 93,789 & 28,640 & 2,833 & 1.1 & 28 & 12,446 & 5,251 & 0.0322 & 191 & 0.744 & 23 & 0.744 & 118 & 0.672 & 0.746 & 0.50 & 525.90 & 413.41 & 0.94 & 0.57 \\
\hline 69,356 & 22,999 & 2,016 & 1.0 & 24 & 10,951 & 4,770 & 0.0439 & 189 & 0.773 & 16 & 0.849 & 70 & 0.731 & 0.783 & 0.69 & 305.79 & 401.44 & 1.05 & 0.59 \\
\hline 92,167 & 37,456 & 2,379 & 1.1 & 30 & 11,889 & 5,818 & 0.0616 & 186 & 0.732 & 15 & 0.782 & 127 & 0.689 & 0.720 & 0.52 & 383.77 & 525.36 & 0.83 & 0.44 \\
\hline 155,619 & 61,119 & 4,109 & 1.0 & 40 & 12,858 & 5,946 & 0.0047 & 226 & 0.744 & 40 & 0.781 & 114 & 0.696 & 0.746 & 0.85 & 519.30 & 542.93 & 0.94 & 0.66 \\
\hline 92,167 & 37,456 & 2,379 & 1.0 & 26 & 7,352 & 3,273 & 0.0179 & 236 & 0.738 & 31 & 0.755 & 65 & 0.700 & 0.740 & 0.88 & 314.93 & 589.51 & 0.82 & 0.51 \\
\hline 152,139 & 49,896 & 4,445 & 1.0 & 38 & 6,497 & 2,727 & 0.0834 & 174 & 0.748 & 28 & 0.818 & 96 & 0.708 & 0.745 & 0.74 & 372.45 & 619.68 & 1.24 & 0.70 \\
\hline 109,039 & 41,433 & 2,939 & 1.1 & 31 & 14,175 & 6,963 & 0.0261 & 238 & 0.777 & 25 & 0.857 & 71 & 0.722 & 0.777 & 0.72 & 483.22 & 483.88 & 0.89 & 0.56 \\
\hline 109,039 & 41,433 & 2,939 & 1.0 & 35 & 8,963 & 4,134 & 0.0056 & 226 & 0.760 & 36 & 0.764 & 106 & 0.673 & 0.758 & 0.86 & 433.27 & 502.59 & 0.87 & 0.65 \\
\hline
\end{tabular}

Table A-4: Data Generated for Eight Stage Treatment Type II, continued 


\begin{tabular}{|c|c|c|c|c|c|c|c|c|c|c|c|c|c|c|c|c|c|c|c|}
\hline \begin{tabular}{l|}
$\frac{0}{E}$ \\
$\frac{3}{0}$ \\
$\frac{0}{0}$ \\
$\frac{\pi}{0}$ \\
\end{tabular} & $\begin{array}{l}0 \\
\frac{0}{5} \\
\frac{3}{0} \\
2 \\
\frac{0}{0} \\
0 \\
0\end{array}$ & 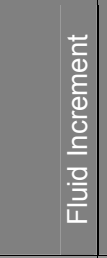 & 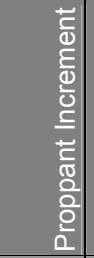 & 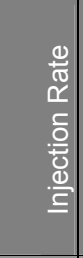 & 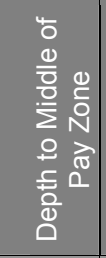 & 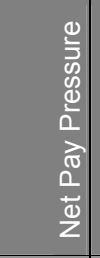 & 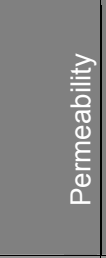 & 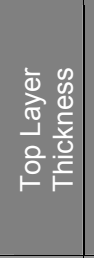 & 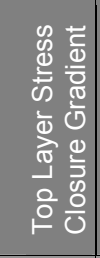 & 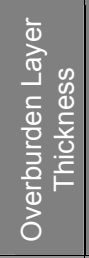 & 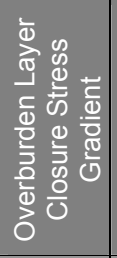 & 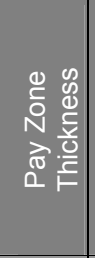 & 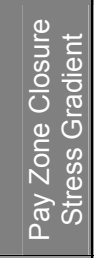 & 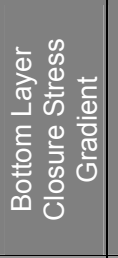 & 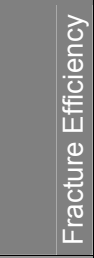 & 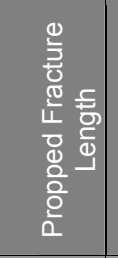 & 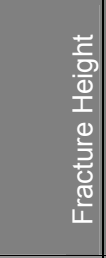 & 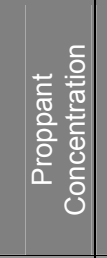 & 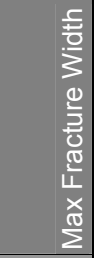 \\
\hline input & input & input & input & input & input & input & input & input & input & input & input & input & input & input & output & output & output & output & output \\
\hline 47,834 & 19,869 & 1,216 & 1.0 & 22 & 7,462 & 3,470 & 0.0182 & 202 & 0.757 & 37 & 0.783 & 59 & 0.698 & 0.748 & 0.84 & 273.81 & 412.45 & 0.69 & 0.44 \\
\hline 150,612 & 58,126 & 4,021 & 1.0 & 36 & 12,819 & 5,643 & 0.0144 & 223 & 0.718 & 11 & 0.784 & 102 & 0.649 & 0.711 & 0.74 & 643.30 & 537.34 & 0.75 & 0.62 \\
\hline 95,175 & 37,670 & 2,500 & 1.2 & 27 & 8,362 & 3,627 & 0.0076 & 189 & 0.755 & 11 & 0.755 & 94 & 0.677 & 0.742 & 0.85 & 383.42 & 522.73 & 0.96 & 0.58 \\
\hline 158,413 & 48,534 & 4,777 & 1.1 & 36 & 13,538 & 6,481 & 0.0302 & 231 & 0.749 & 35 & 0.775 & 75 & 0.684 & 0.730 & 0.82 & 508.02 & 612.85 & 1.08 & 0.67 \\
\hline 65,664 & 20,350 & 1,970 & 1.0 & 22 & 10,847 & 4,874 & 0.0123 & 204 & 0.726 & 25 & 0.796 & 128 & 0.677 & 0.719 & 0.68 & 393.53 & 481.21 & 0.67 & 0.44 \\
\hline 136,859 & 49,791 & 3,786 & 1.0 & 33 & 12,609 & 6,291 & 0.0164 & 206 & 0.708 & 31 & 0.733 & 133 & 0.657 & 0.709 & 0.73 & 493.55 & 519.57 & 1.05 & 0.65 \\
\hline 94,723 & 27,559 & 2,920 & 1.2 & 24 & 9,865 & 4,024 & 0.0446 & 180 & 0.739 & 36 & 0.763 & 114 & 0.686 & 0.736 & 0.56 & 416.77 & 524.30 & 1.03 & 0.52 \\
\hline 38,145 & 14,872 & 1,012 & 1.5 & 20 & 6,766 & 3,085 & 0.0758 & 199 & 0.701 & 35 & 0.769 & 127 & 0.642 & 0.702 & 0.46 & 228.27 & 350.32 & 1.22 & 0.44 \\
\hline 41,528 & 13,474 & 1,220 & 1.0 & 22 & 9,265 & 4,000 & 0.0064 & 206 & 0.799 & 14 & 0.855 & 66 & 0.736 & 0.782 & 0.88 & 296.95 & 412.38 & 0.64 & 0.43 \\
\hline 103,936 & 39,027 & 2,822 & 1.1 & 34 & 5,717 & 2,296 & 0.0846 & 200 & 0.766 & 22 & 0.830 & 116 & 0.716 & 0.775 & 0.64 & 329.02 & 541.53 & 1.12 & 0.57 \\
\hline 126,478 & 49,374 & 3,352 & 1.0 & 39 & 11,937 & 5,169 & 0.0114 & 211 & 0.745 & 40 & 0.812 & 78 & 0.684 & 0.746 & 0.83 & 502.59 & 515.85 & 0.79 & 0.58 \\
\hline 120,086 & 37,040 & 3,611 & 1.2 & 35 & 9,734 & 3,875 & 0.0299 & 218 & 0.750 & 28 & 0.748 & 51 & 0.692 & 0.748 & 0.86 & 380.49 & 670.43 & 1.09 & 0.55 \\
\hline 91,899 & 26,981 & 2,823 & 1.0 & $27 \mid$ & 5,702 & 2,127 & 0.0194 & 199 & 0.718 & 38 & 0.756 & 99 & 0.647 & 0.708 & 0.75 & 395.79 & 535.74 & 0.86 & 0.55 \\
\hline 42,476 & 14,372 & 1,222 & 1.2 & 23 & 9,453 & 4,169 & 0.0685 & 234 & 0.766 & 24 & 0.764 & 106 & 0.719 & 0.760 & 0.48 & 250.83 & 490.29 & 0.96 & 0.41 \\
\hline 55,611 & 19,308 & 1,578 & 1.2 & 28 & 12,930 & 5,587 & 0.0157 & 240 & 0.740 & 23 & 0.783 & 71 & 0.671 & 0.723 & 0.72 & 404.24 & 410.16 & 0.70 & 0.47 \\
\hline 55,611 & 19,308 & 1,578 & 1.5 & 26 & 9,692 & 4,543 & 0.0073 & 213 & 0.788 & 25 & 0.788 & 129 & 0.714 & 0.765 & 0.74 & 326.14 & 429.47 & 1.04 & 0.52 \\
\hline 102,282 & 37,386 & 2,822 & 1.0 & 30 & 14,459 & 6,655 & 0.0255 & 193 & 0.715 & 37 & 0.761 & 124 & 0.662 & 0.705 & 0.52 & 557.43 & 495.74 & 0.81 & 0.51 \\
\hline 65,586 & 25,214 & 1,755 & 1.0 & 28 & 10,596 & 5,427 & 0.0073 & 171 & 0.728 & 36 & 0.796 & 93 & 0.677 & 0.720 & 0.80 & 426.27 & 433.90 & 0.61 & 0.47 \\
\hline 65,586 & 25,214 & 1,755 & 1.3 & 25 & 12,087 & 6,013 & 0.0056 & 210 & 0.733 & 25 & 0.779 & 112 & 0.680 & 0.727 & 0.78 & 391.95 & 453.55 & 0.78 & 0.49 \\
\hline 83,256 & 29,570 & 2,334 & 1.1 & 29 & 8,053 & 3,205 & 0.0117 & 219 & 0.750 & 30 & 0.750 & 75 & 0.662 & 0.737 & 0.84 & 365.06 & 523.34 & 0.86 & 0.56 \\
\hline 33,212 & 9,875 & 1,015 & 1.0 & 29 & 11,470 & 4,850 & 0.0044 & 230 & 0.716 & 16 & 0.767 & 127 & 0.660 & 0.710 & 0.74 & 291.98 & 367.49 & 0.67 & 0.41 \\
\hline 149,723 & 57,602 & 4,005 & 1.0 & 40 & 5,420 & 1,909 & 0.0055 & 206 & 0.763 & 15 & 0.828 & 69 & 0.684 & 0.753 & 0.94 & 371.51 & 538.03 & 1.44 & 0.80 \\
\hline 123,416 & 47,500 & 3,301 & 1.1 & 37 & 8,083 & 3,516 & 0.0187 & 184 & 0.787 & 34 & 0.789 & 109 & 0.700 & 0.768 & 0.83 & 434.29 & 577.43 & 0.93 & 0.63 \\
\hline 69,828 & 21,423 & 2,105 & 1.5 & 26 & 7,717 & 3,748 & 0.0087 & 210 & 0.768 & 32 & 0.811 & 77 & 0.707 & 0.754 & 0.87 & 342.24 & 501.70 & 1.09 & 0.53 \\
\hline 69,828 & 21,423 & 2,105 & 1.0 & $27 \mid$ & 8,289 & 3,347 & 0.0026 & 223 & 0.742 & 33 & 0.784 & 85 & 0.673 & 0.730 & 0.90 & 373.44 & 487.93 & 0.74 & 0.52 \\
\hline 81,374 & 34,176 & 2,052 & 1.4 & 32 & 8,737 & 4,187 & 0.0281 & 178 & 0.718 & 10 & 0.769 & 88 & 0.655 & 0.709 & 0.73 & 330.37 & 464.65 & 1.22 & 0.57 \\
\hline 81,374 & 34,176 & 2,052 & 1.1 & 30 & 10,869 & 5,402 & 0.0010 & 201 & 0.758 & 16 & 0.809 & 119 & 0.679 & 0.744 & 0.90 & 477.35 & 417.33 & 0.72 & 0.56 \\
\hline 145,639 & 43,204 & 4,454 & 1.0 & 40 & 7,899 & 3,392 & 0.0091 & 203 & 0.794 & 28 & 0.794 & 96 & 0.713 & 0.776 & 0.87 & 444.04 & 651.25 & 1.10 & 0.67 \\
\hline 80,444 & 32,311 & 2,093 & 1.0 & 29 & 5,201 & 2,598 & 0.0047 & 170 & 0.741 & 31 & 0.744 & 87 & 0.654 & 0.726 & 0.93 & 329.28 & 510.75 & 0.80 & 0.53 \\
\hline 80,444 & 32,311 & 2,093 & 1.0 & 24 & 10,546 & 4,018 & 0.0053 & 187 & 0.795 & 26 & 0.843 & 58 & 0.720 & 0.790 & 0.82 & 414.25 & 423.09 & 0.94 & 0.57 \\
\hline
\end{tabular}

Table A-4: Data Generated for Eight Stage Treatment Type II, continued 


\begin{tabular}{|c|c|c|c|c|c|c|c|c|c|c|c|c|c|c|c|c|c|c|c|}
\hline 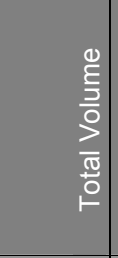 & 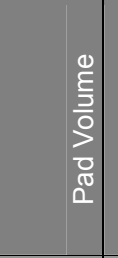 & 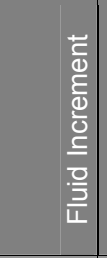 & 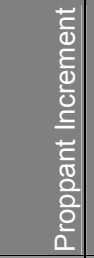 & 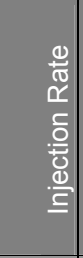 & 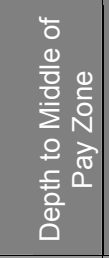 & 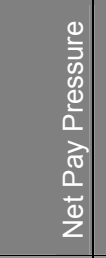 & 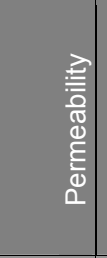 & 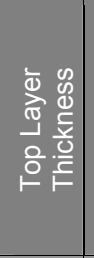 & 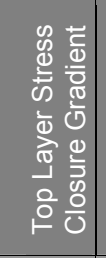 & 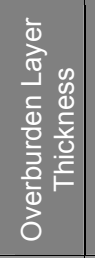 & 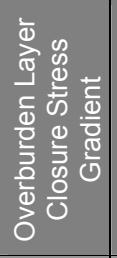 & 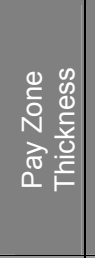 & 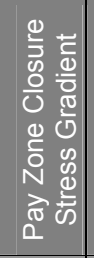 & 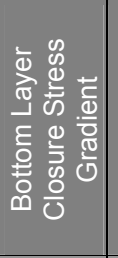 & 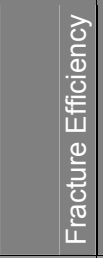 & 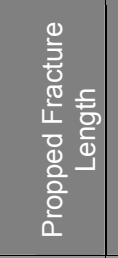 & 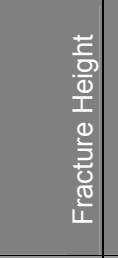 & 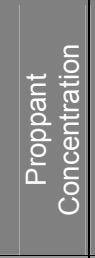 & 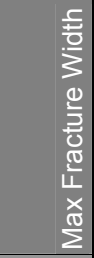 \\
\hline input & input & input & input & input & input & input & input & input & input & input & input & input & input & input & output & output & output & output & output \\
\hline 50,211 & 20,140 & 1,307 & 1.0 & 28 & 11,328 & 4,890 & 0.0080 & 194 & 0.752 & 30 & 0.756 & 105 & 0.675 & 0.738 & 0.71 & 373.64 & 325.62 & 0.68 & 0.48 \\
\hline 52,826 & 18,210 & 1,505 & 1.0 & 23 & 8,358 & 3,190 & 0.0020 & 186 & 0.735 & 25 & 0.800 & 109 & 0.660 & 0.720 & 0.87 & 354.36 & 427.12 & 0.70 & 0.47 \\
\hline 80,504 & 28,978 & 2,240 & 1.0 & 28 & 12,475 & 6,184 & 0.0072 & 172 & 0.755 & 20 & 0.811 & 123 & 0.681 & 0.733 & 0.72 & 504.13 & 349.88 & 1.00 & 0.59 \\
\hline 58,543 & 19,779 & 1,685 & 1.0 & 27 & 5,209 & 1,829 & 0.0146 & 182 & 0.735 & 38 & 0.799 & 99 & 0.655 & 0.736 & 0.88 & 304.30 & 469.97 & 0.76 & 0.47 \\
\hline 124,914 & 50,409 & 3,239 & 1.0 & 32 & 6,980 & 3,073 & 0.0071 & 175 & 0.747 & 17 & 0.742 & 52 & 0.675 & 0.721 & 0.94 & 377.40 & 616.82 & 1.00 & 0.57 \\
\hline 58,543 & 19,779 & 1,685 & 1.0 & 29 & 9,756 & 3,390 & 0.0186 & 207 & 0.742 & 26 & 0.813 & 118 & 0.682 & 0.722 & 0.62 & 329.07 & 433.51 & 0.74 & 0.44 \\
\hline 88,586 & 37,607 & 2,217 & 1.5 & 26 & 7,196 & 3,468 & 0.0014 & 238 & 0.777 & 22 & 0.843 & 91 & 0.697 & 0.768 & 0.95 & 357.75 & 484.58 & 1.14 & 0.59 \\
\hline 88,586 & 37,607 & 2,217 & 1.0 & 30 & 12,667 & 5,359 & 0.0041 & 180 & 0.716 & 15 & 0.713 & 120 & 0.661 & 0.707 & 0.80 & 480.16 & 472.99 & 0.63 & 0.62 \\
\hline 72,751 & 27,560 & 1,965 & 1.2 & 22 & 9,260 & 4,093 & 0.0052 & 176 & 0.748 & 23 & 0.804 & 75 & 0.683 & 0.731 & 0.87 & 378.14 & 490.47 & 0.80 & 0.49 \\
\hline 72,751 & 27,560 & 1,965 & 1.1 & 29 & 11,103 & 4,492 & 0.0175 & 224 & 0.728 & 20 & 0.785 & 63 & 0.649 & 0.717 & 0.78 & 386.37 & 423.12 & 0.90 & 0.56 \\
\hline 72,751 & 27,560 & 1,965 & 1.0 & 25 & 14,203 & 6,358 & 0.0022 & 205 & 0.766 & 13 & 0.766 & 121 & 0.699 & 0.742 & 0.84 & 483.23 & 348.12 & 0.74 & 0.54 \\
\hline 42,518 & 15,003 & 1,196 & 1.0 & 22 & 12,634 & 6,279 & 0.0487 & 208 & 0.770 & 29 & 0.826 & 112 & 0.675 & 0.788 & 0.26 & 364.25 & 272.68 & 0.77 & 0.36 \\
\hline 104,817 & 42,057 & 2,729 & 1.0 & 33 & 6,384 & 2,654 & 0.0091 & 196 & 0.750 & 23 & 0.803 & 78 & 0.660 & 0.742 & 0.88 & 383.62 & 485.49 & 1.05 & 0.63 \\
\hline 40,965 & 16,469 & 1,065 & 1.5 & 25 & 9,938 & 4,469 & 0.0031 & 172 & 0.725 & 28 & 0.785 & 53 & 0.672 & 0.723 & 0.92 & 282.59 & 384.61 & 0.87 & 0.45 \\
\hline 132,165 & 50,812 & 3,537 & 1.0 & 26 & 14,429 & 6,420 & 0.0223 & 177 & 0.764 & 18 & 0.822 & 72 & 0.684 & 0.751 & 0.75 & 749.51 & 386.49 & 0.87 & 0.66 \\
\hline 86,244 & 32,615 & 2,332 & 1.5 & 20 & 12,143 & 5,988 & 0.0097 & 202 & 0.756 & 38 & 0.828 & 53 & 0.705 & 0.747 & 0.89 & 373.15 & 545.50 & 1.11 & 0.57 \\
\hline 119,614 & 42,724 & 3,343 & 1.0 & 26 & 10,655 & 4,331 & 0.0857 & 181 & 0.767 & 10 & 0.794 & 100 & 0.655 & 0.749 & 0.66 & 423.15 & 498.00 & 1.22 & 0.64 \\
\hline 59,099 & 25,195 & 1,474 & 1.0 & 19 & 12,995 & 6,136 & 0.0337 & 221 & 0.759 & 34 & 0.818 & 82 & 0.689 & 0.780 & 0.61 & 381.82 & 424.66 & 0.64 & 0.43 \\
\hline 144,233 & 46,207 & 4,262 & 1.3 & 22 & 14,254 & 6,728 & 0.0618 & 201 & 0.771 & 12 & 0.745 & 54 & 0.736 & 0.735 & 0.65 & 480.94 & 669.01 & 0.85 & 0.54 \\
\hline 102,037 & 43,794 & 2,532 & 1.2 & 36 & 14,780 & 6,395 & 0.0182 & 190 & 0.751 & 22 & 0.797 & 66 & 0.692 & 0.740 & 0.79 & 466.98 & 479.09 & 0.77 & 0.51 \\
\hline 86,016 & 35,617 & 2,191 & 1.0 & 24 & 9,045 & 3,773 & 0.0057 & 214 & 0.733 & 24 & 0.783 & 82 & 0.647 & 0.720 & 0.85 & 421.44 & 454.28 & 0.90 & 0.57 \\
\hline 46,520 & 15,424 & 1,352 & 1.0 & 25 & 14,705 & 6,927 & 0.0336 & 215 & 0.712 & 40 & 0.761 & 96 & 0.656 & 0.708 & 0.42 & 423.02 & 345.26 & 0.59 & 0.44 \\
\hline 46,520 & 15,424 & 1,352 & 1.5 & 20 & 6,063 & 2,383 & 0.0050 & 187 & 0.755 & 27 & 0.828 & 81 & 0.660 & 0.750 & 0.86 & 303.46 & 394.23 & 1.09 & 0.52 \\
\hline 156,719 & 48,984 & 4,684 & 1.0 & 40 & 6,603 & 2,498 & 0.0199 & 198 & 0.751 & 23 & 0.816 & 65 & 0.695 & 0.729 & 0.90 & 392.79 & 639.60 & 1.44 & 0.73 \\
\hline 39,830 & 15,687 & 1,050 & 1.0 & 20 & 8,033 & 3,965 & 0.0093 & 228 & 0.777 & 30 & 0.777 & 104 & 0.699 & 0.769 & 0.75 & 284.47 & 360.17 & 0.73 & 0.43 \\
\hline 140,572 & 41,351 & 4,314 & 1.0 & 38 & 8,750 & 3,922 & 0.0247 & 194 & 0.767 & 26 & 0.801 & 116 & 0.719 & 0.756 & 0.77 & 418.74 & 631.42 & 1.05 & 0.63 \\
\hline 121,642 & 51,158 & 3,064 & 1.0 & 29 & 5,746 & 2,077 & 0.0403 & 227 & 0.739 & 21 & 0.762 & 67 & 0.670 & 0.714 & 0.83 & 360.36 & 584.09 & 0.93 & 0.56 \\
\hline 80,665 & 24,969 & 2,422 & 1.0 & 24 & 9,015 & 4,203 & 0.0137 & 210 & 0.744 & 33 & 0.790 & 109 & 0.704 & 0.744 & 0.78 & 334.57 & 505.42 & 0.92 & 0.54 \\
\hline 80,665 & 24,969 & 2,422 & 1.2 & 27 & 7,785 & 3,744 & 0.0402 & 216 & 0.729 & 18 & 0.726 & 74 & 0.689 & 0.729 & 0.81 & 312.14 & 563.49 & 1.06 & 0.53 \\
\hline 154,167 & 55,952 & 4,270 & 1.5 & $37 \mid$ & 8,755 & 3,484 & 0.0263 & 215 & 0.719 & 26 & 0.762 & 110 & 0.669 & 0.715 & 0.77 & 402.60 & 591.23 & 1.60 & 0.73 \\
\hline
\end{tabular}

Table A-4: Data Generated for Eight Stage Treatment Type II, continued 


\begin{tabular}{|c|c|c|c|c|c|c|c|c|c|c|c|c|c|c|c|c|c|c|c|}
\hline 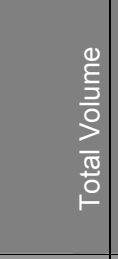 & $\begin{array}{l}\frac{0}{E} \\
\frac{1}{3} \\
0 \\
\frac{0}{0} \\
\frac{0}{\sigma} \\
0\end{array}$ & 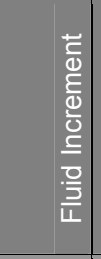 & 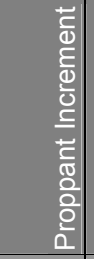 & 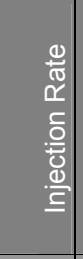 & 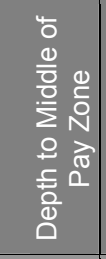 & 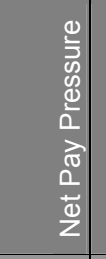 & 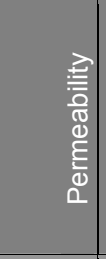 & 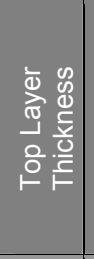 & 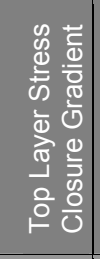 & 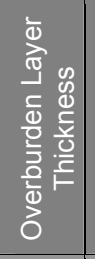 & 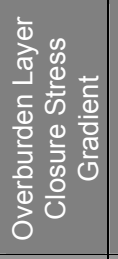 & 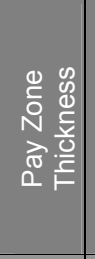 & 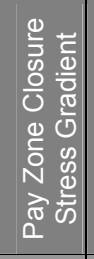 & 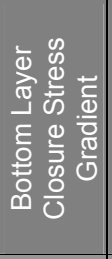 & 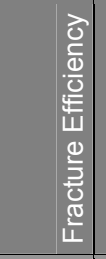 & 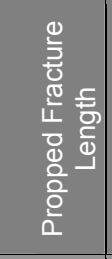 & 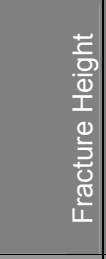 & 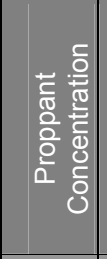 & 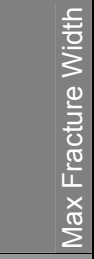 \\
\hline input & input & input & input & input & input & input & input & input & input & input & input & input & input & input & output & output & output & output & output \\
\hline 57,765 & 19,846 & 1,649 & 1.1 & 25 & 10,350 & 4,242 & 0.0189 & 209 & 0.731 & 27 & 0.788 & 97 & 0.673 & 0.724 & 0.69 & 359.15 & 438.85 & 0.74 & 0.45 \\
\hline 127,363 & 50,235 & 3,353 & 1.0 & 33 & 9,014 & 4,274 & 0.0417 & 222 & 0.736 & 36 & 0.761 & 117 & 0.684 & 0.721 & 0.68 & 445.74 & 591.34 & 0.82 & 0.53 \\
\hline 141,600 & 45,664 & 4,171 & 1.2 & 37 & 14,707 & 7,264 & 0.0073 & 237 & 0.725 & 11 & 0.726 & 102 & 0.656 & 0.725 & 0.80 & 644.85 & 434.45 & 1.15 & 0.77 \\
\hline 35,078 & 12,763 & 970 & 1.0 & 18 & 6,261 & 2,950 & 0.0957 & 201 & 0.704 & 36 & 0.736 & 117 & 0.658 & 0.702 & 0.48 & 219.61 & 372.95 & 0.76 & 0.36 \\
\hline 52,007 & 21,146 & 1,342 & 1.3 & 20 & 5,146 & 2,003 & 0.0327 & 232 & 0.723 & 15 & 0.795 & 89 & 0.666 & 0.716 & 0.75 & 244.48 & 421.25 & 1.09 & 0.48 \\
\hline 73,325 & 21,591 & 2,249 & 1.0 & 28 & 9,720 & 4,493 & 0.0081 & 202 & 0.755 & 22 & 0.750 & 62 & 0.684 & 0.750 & 0.89 & 337.12 & 525.65 & 1.00 & 0.56 \\
\hline $94,657 \mid$ & 37,154 & 2,500 & 1.2 & 31 & 14,018 & 6,438 & 0.0223 & 240 & 0.713 & 20 & 0.751 & 116 & 0.646 & 0.710 & 0.57 & 555.40 & 381.84 & 0.91 & 0.58 \\
\hline 70,573 & 23,229 & 2,058 & 1.0 & 25 & 11,967 & 5,514 & 0.0086 & 186 & 0.782 & 39 & 0.780 & 73 & 0.725 & 0.780 & 0.83 & 362.48 & 515.07| & 0.71 & 0.48 \\
\hline 122,772 & 52,504 & 3,055 & 1.0 & 30 & 12,932 & 6,390 & 0.0435 & 226 & 0.774 & 38 & 0.850 & 133 & 0.727 & 0.770 & 0.46 & 528.66 & 526.45 & 0.74 & 0.47 \\
\hline 80,154 & 26,011 & 2,354 & 1.1 & 30 & 11,943 & 5,647 & 0.0535 & 205 & 0.743 & 27 & 0.819 & 62 & 0.702 & 0.746 & 0.73 & 368.46 & 494.78 & 0.91 & 0.49 \\
\hline 26,021 & 8,145 & 777 & 1.0 & 19 & 8,146 & 3,429 & 0.0289 & 191 & 0.758 & 30 & 0.784 & 113 & 0.685 & 0.739 & 0.52 & 231.85 & 320.49 & 0.67 & 0.36 \\
\hline 113,346 & 36,042 & 3,361 & 1.1 & 30 & 7,916 & 3,439 & 0.0075 & 192 & 0.742 & 13 & 0.828 & 87 & 0.679 & 0.741 & 0.90 & 376.94 & 508.12 & 1.29 & 0.75 \\
\hline 65,591 & 22,774 & 1,862 & 1.0 & 26 & 9,610 & 4,445 & 0.0322 & 176 & 0.730 & 34 & 0.780 & 83 & 0.685 & 0.717 & 0.73 & 335.51 & 487.03 & 0.81 & 0.45 \\
\hline 157,130 & 55,704 & 4,410 & 1.0 & 40 & 10,423 & 2,631 & 0.0117 & 171 & 0.712 & 11 & 0.712 & 80 & 0.653 & 0.713 & 0.89 & 457.39 & 688.16 & 0.90 & 0.58 \\
\hline 85,480 & 25,777 & 2,596 & 1.0 & 29 & 7,702 & 3,443 & 0.0272 & 230 & 0.733 & 20 & 0.803 & 131 & 0.685 & 0.728 & 0.56 & 342.82 & 528.55 & 0.92 & 0.49 \\
\hline 79,688 & 31,811 & 2,082 & 1.0 & 26 & 10,858 & 5,086 & 0.0093 & 207 & 0.751 & 23 & 0.820 & 76 & 0.677 & 0.737 & 0.82 & 442.41 & 464.72 & 0.73 & 0.52 \\
\hline 81,656 & 27,304 & 2,363 & 1.0 & 25 & 11,396 & 4,725 & 0.0540 & 237 & 0.720 & 32 & 0.766 & 89 & 0.672 & 0.724 & 0.61 & 367.47 & 454.01 & 0.91 & 0.53 \\
\hline 129,718 & 40,822 & 3,865 & 1.1 & 34 & 7,900 & 3,423 & 0.0204 & 177 & 0.725 & 27 & 0.848 & 55 & 0.675 & 0.718 & 0.90 & 379.45 & 675.87 & 1.07 & 0.58 \\
\hline 91,737 & 29,964 & 2,686 & 1.0 & 27 & 11,673 & 5,835 & 0.0093 & 191 & 0.762 & 30 & 0.781 & 80 & 0.687 & 0.755 & 0.81 & 492.04 & 440.40 & 0.80 & 0.61 \\
\hline 63,266 & 21,085 & 1,834 & 1.0 & 28 & 12,826 & 5,556 & 0.0109 & 176 & 0.750 & 37 & 0.787 & 61 & 0.704 & 0.725 & 0.85 & 348.34 & 523.31 & 0.65 & 0.44 \\
\hline
\end{tabular}

Table A-4: Data Generated for Eight Stage Treatment Type II, continued 


\begin{tabular}{|c|c|c|c|c|c|c|c|c|c|c|c|c|c|c|c|c|c|c|c|}
\hline 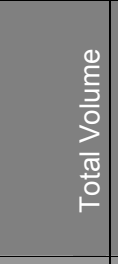 & 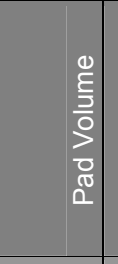 & 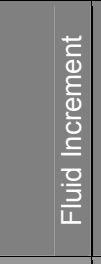 & 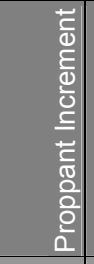 & 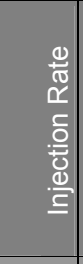 & 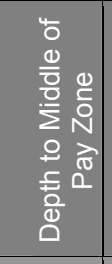 & 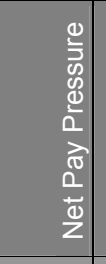 & 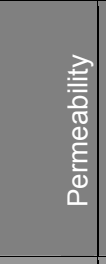 & 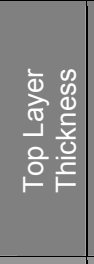 & 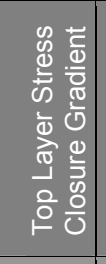 & 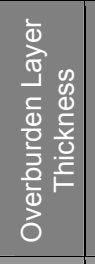 & 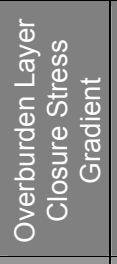 & 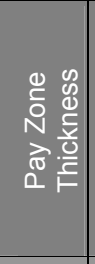 & 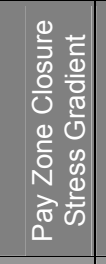 & 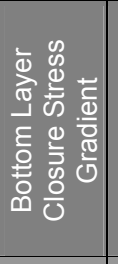 & 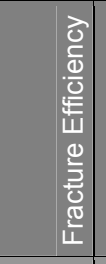 & 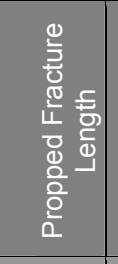 & 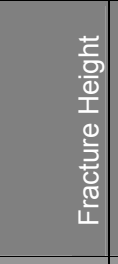 & 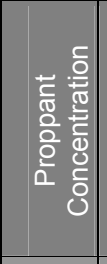 & 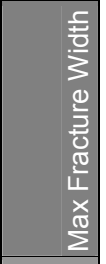 \\
\hline input & input & input & input & input & input & input & input & input & input & input & input & input & input & input & output & output & output & output & output \\
\hline 150,920 & 58,932 & 3,999 & 1.2 & 26 & 11,296 & 5,131 & 0.0050 & 199 & 0.768 & 15 & 0.795 & 122 & 0.691 & 0.750 & 0.82 & 607.37 & 548.52 & 0.90 & 0.65 \\
\hline 120,588 & 48,621 & 3,129 & 1.5 & 35 & 12,317 & 5,564 & 0.0300 & 199 & 0.780 & 30 & 0.842 & 129 & 0.730 & 0.770 & 0.58 & 467.25 & 552.50 & 1.14 & 0.54 \\
\hline 166,561 & 57,026 & 4,762 & 1.0 & 40 & 8,775 & 3,945 & 0.0176 & 228 & 0.726 & 35 & 0.770 & 99 & 0.667 & 0.710 & 0.85 & 474.33 & 652.50 & 0.96 & 0.63 \\
\hline 87,117 & 28,665 & 2,541 & 1.3 & 30 & 8,219 & 3,311 & 0.0085 & 209 & 0.733 & 36 & 0.733 & 89 & 0.670 & 0.722 & 0.87 & 343.01 & 537.45 & 1.13 & 0.59 \\
\hline 173,529 & 56,395 & 5,093 & 1.5 & 39 & 10,588 & 3,578 & 0.0126 & 226 & 0.709 & 19 & 0.782 & 72 & 0.645 & 0.704 & 0.86 & 457.38 & 564.84 & 1.85 & 0.88 \\
\hline 53,308 & 21,036 & 1,403 & 1.0 & 25 & 5,844 & 2,827 & 0.0172 & 227 & 0.724 & 39 & 0.761 & 55 & 0.650 & 0.716 & 0.91 & 274.29 & 424.56 & 0.75 & 0.48 \\
\hline 149,441 & 45,261 & 4,530 & 1.5 & 40 & 7,390 & 3,559 & 0.0085 & 224 & 0.745 & 13 & 0.825 & 78 & 0.688 & 0.732 & 0.92 & 375.32 & 580.88 & 1.96 & 0.87 \\
\hline 143,020 & 49,409 & 4,070 & 1.0 & 38 & 8,345 & 3,986 & 0.0017 & 231 & 0.728 & 38 & 0.795 & 78 & 0.665 & 0.722 & 0.96 & 444.29 & 581.15 & 0.99 & 0.66 \\
\hline 115,293 & 35,510 & 3,469 & 1.0 & 33 & 5,457 & 2,171 & 0.0108 & 218 & 0.751 & 13 & 0.751 & 82 & 0.673 & 0.755 & 0.90 & 370.19 & 619.39| & 0.95 & 0.60 \\
\hline 54,051 & 21,529 & 1,414 & 1.3 & 22 & 14,071 & 6,769 & 0.0043 & 185 & 0.745 & 27 & 0.809 & 71 & 0.646 & 0.742 & 0.84 & 583.49 & 331.47 & 0.59 & 0.51 \\
\hline 158,002 & 48,685 & 4,753 & 1.5 & 39 & 6,273 & 2,446 & 0.0056 & 235 & 0.765 & 34 & 0.798 & 84 & 0.697 & 0.749 & 0.93 & 382.84 & 664.47 & 1.76 & 0.78 \\
\hline 40,412 & 16,744 & 1,029 & 1.0 & 23 & 12,807 & 6,343 & 0.0063 & 192 & 0.723 & 24 & 0.783 & 63 & 0.679 & 0.723 & 0.86 & 299.51 & 375.18 & 0.57 & 0.40 \\
\hline 117,574 & 44,512 & 3,177 & 1.4 & 29 & 7,855 & 3,873 & 0.0096 & 176 & 0.717 & 18 & 0.763 & 120 & 0.667 & 0.715 & 0.89 & 355.02 & 533.61 & 1.47 & 0.70 \\
\hline 60,132 & 23,060 & 1,612 & 1.1 & 27 & 13,049 & 5,813 & 0.0169 & 236 & 0.773 & 36 & 0.816 & 86 & 0.720 & 0.776 & 0.68 & 368.58 & 393.97| & 0.76 & 0.49 \\
\hline 39,445 & 14,333 & 1,092 & 1.0 & 20 & 6,208 & 2,125 & 0.0010 & 236 & 0.788 & 20 & 0.793 & 94 & 0.701 & 0.780 & 0.93 & 278.36 & 386.86 & 0.64 & 0.45 \\
\hline 71,789 & 24,287 & 2,065 & 1.6 & 22 & 6,180 & 2,989 & 0.0485 & 177 & 0.743 & 11 & 0.803 & 69 & 0.666 & 0.756 & 0.79 & 279.86 & 425.32 & 1.74 & 0.72 \\
\hline 154,047 & 49,734 & 4,535 & 1.3 & 17 & 9,749 & 4,856 & 0.0080 & 193 & 0.793 & 17 & 0.864 & 53 & 0.699 & 0.808 & 0.93 & 437.87 & 418.19 & 2.02 & 1.10 \\
\hline 182,032 & 66,949 & 5,004 & 2.0 & 40 & 7,201 & 2,959 & 0.0163 & 220 & 0.745 & 36 & 0.780 & 103 & 0.672 & 0.734 & 0.82 & 430.53 & 609.43 & 2.39 & 0.87 \\
\hline 77,004 & 25,387 & 2,244 & 1.2 & 30 & 5,224 & 2,360 & 0.0032 & 178 & 0.763 & 38 & 0.824 & 94 & 0.701 & 0.741 & 0.93 & 324.61 & 532.59 & 0.98 & 0.53 \\
\hline 67,442 & 19,753 & 2,073 & 1.0 & 26 & 11,440 & 6,258 & 0.0060 & 180 & 0.744 & 26 & 0.805 & 73 & 0.687 & 0.722 & 0.90 & 392.21 & 532.28 & 0.62 & 0.46 \\
\hline 77,959 & 28,224 & 2,162 & 1.5 & 28 & 8,692 & 2,730 & 0.0134 & 196 & 0.711 & 12 & 0.760 & 71 & 0.667 & 0.705 & 0.87 & 290.81 & 458.33 & 1.53 & 0.66 \\
\hline 54,463 & 21,307 & 1,442 & 1.0 & 25 & 15,187 & 7,000 & 0.0056 & 197 & 0.750 & 21 & 0.803 & 56 & 0.679 & 0.723 & 0.89 & 301.92 & 413.81 & 0.72 & 0.50 \\
\hline 60,139 & 24,443 & 1,552 & 1.1 & 26 & 7,297 & 3,112 & 0.0145 & 175 & 0.716 & 36 & 0.782 & 106 & 0.650 & 0.700 & 0.78 & 308.45 & 439.06 & 0.79 & 0.47 \\
\hline 178,023 & 49,882 & 5,571 & 1.5 & 31 & 13,146 & 5,779 & 0.0084 & 222 & 0.746 & 38 & 0.807 & 95 & 0.688 & 0.737 & 0.83 & 607.52 & 621.65 & 1.39 & 0.73 \\
\hline 39,518 & 15,000 & 1,066 & 1.0 & 20 & 5,587 & 1,919 & 0.0167 & 191 & 0.717 & 26 & 0.738 & 73 & 0.645 & 0.710 & 0.81 & 246.12 & 385.75 & 0.70 & 0.41 \\
\hline 82,811 & 25,508 & 2,491 & 1.5 & 30 & 14,369 & 6,819 & 0.0146 & 239 & 0.750 & 27 & 0.787 & 69 & 0.690 & 0.735 & 0.78 & 440.10 & 477.59 & 1.11 & 0.58 \\
\hline 160,396 & 59,530 & 4,385 & 1.4 & 40 & 10,185 & 5,035 & 0.0054 & 206 & 0.751 & 23 & 0.751 & 114 & 0.642 & 0.743 & 0.83 & 690.44 & 455.87 & 1.22 & 0.80 \\
\hline 75,397 & 24,241 & 2,224 & 1.0 & 27 & 12,831 & 6,019 & 0.0163 & 207 & 0.781 & 11 & 0.781 & 59 & 0.721 & 0.786 & 0.82 & 358.00 & 551.99 & 0.70 & 0.47 \\
\hline 72,240 & 27,732 & 1,935 & 1.0 & 26 & 11,246 & 5,385 & 0.0074 & 201 & 0.713 & 20 & 0.760 & 77 & 0.670 & 0.714 & 0.87 & 333.12 & 437.95 & 0.83 & 0.54 \\
\hline 54,130 & 19,583 & 1,502 & 1.0 & 23 & 5,209 & 1,715 & 0.0027 & 221 & 0.731 & 12 & 0.779 & 117 & 0.683 & 0.730 & 0.90 & 263.10 & 462.29 & 0.77 & 0.46 \\
\hline
\end{tabular}

Table A - 5: Data Generated for Eight Stage Treatment Type III 


\begin{tabular}{|c|c|c|c|c|c|c|c|c|c|c|c|c|c|c|c|c|c|c|c|}
\hline 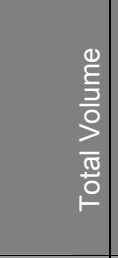 & 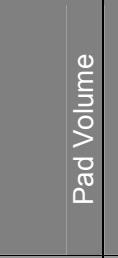 & 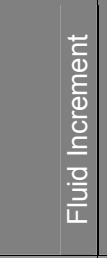 & 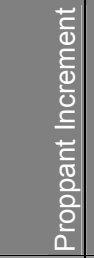 & 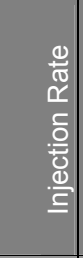 & 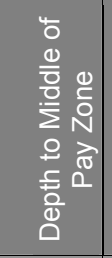 & 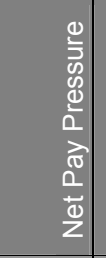 & 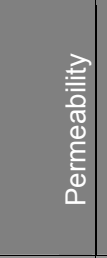 & 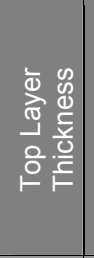 & 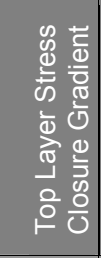 & 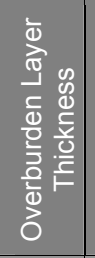 & 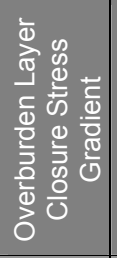 & 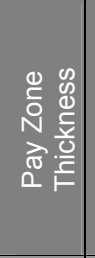 & 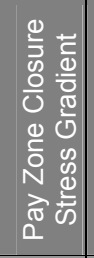 & 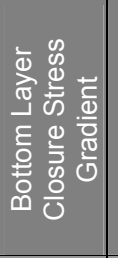 & 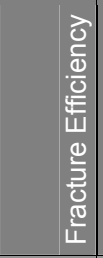 & 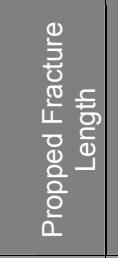 & 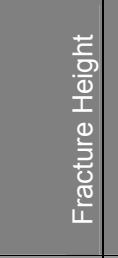 & 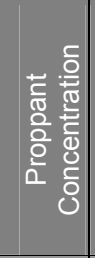 & 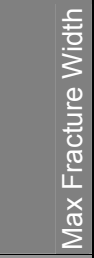 \\
\hline input & input & input & input & input & input & input & input & input & input & input & input & input & input & input & output & output & output & output & output \\
\hline 99,350 & 30,838 & 2,979 & 1.2 & 35 & 12,133 & 5,079 & 0.0072 & 234 & 0.730 & 33 & 0.777 & 86 & 0.644 & 0.726 & 0.81 & 546.77 & 427.37 & 0.96 & 0.68 \\
\hline 103,791 & 41,044 & 2,728 & 1.0 & 31 & 14,140 & 6,475 & 0.0159 & 196 & 0.744 & 27 & 0.740 & 64 & 0.680 & 0.728 & 0.79 & 547.63 & 502.01 & 0.62 & 0.52 \\
\hline $177,597 \mid$ & 69,592 & 4,696 & 1.3 & 40 & 7,277 & 3,396 & 0.0089 & 199 & 0.768 & 22 & 0.837 & 82 & 0.695 & 0.770 & 0.91 & 407.71 & 534.55 & 1.75 & 0.91 \\
\hline 186,757 & 56,300 & 5,672 & 1.1 & 40 & 13,737 & 6,037 & 0.0076 & 174 & 0.759 & 13 & 0.809 & 109 & 0.676 & 0.736 & 0.76 & 815.34 & 465.74 & 0.84 & 0.70 \\
\hline 74,092 & 24,456 & 2,158 & 1.2 & 27 & 7,110 & 3,433 & 0.0096 & 172 & 0.779 & 35 & 0.830 & 52 & 0.712 & 0.770 & 0.92 & 326.23 & 471.23 & 1.06 & 0.58 \\
\hline 130,445 & 37,493 & 4,041 & 1.4 & 34 & 12,983 & 5,847 & 0.0066 & 212 & 0.717 & 35 & 0.783 & 130| & 0.657 & 0.708 & 0.77 & 620.07 & 570.97| & 0.99 & 0.64 \\
\hline 169,498 & 50,147 & 5,189 & 1.3 & 38 & 9,171 & 3,459 & 0.0032 & 219 & 0.751 & 23 & 0.837 & 70 & 0.717 & 0.750 & 0.94 & 393.25 & 604.62 & 1.78 & 0.89 \\
\hline 172,163 & 66,852 & 4,579 & 1.0 & 37 & 11,624 & 5,017 & 0.0441 & 215 & 0.727 & 24 & 0.772 & 92 & 0.679 & 0.716 & 0.71 & 505.19 & 636.56 & 0.89 & 0.58 \\
\hline 78,979 & 30,898 & 2,090 & 1.0 & 25 & 7,775 & 3,026 & 0.0129 & 215 & 0.755 & 39 & 0.797 & 123 & 0.671 & 0.734 & 0.73 & 388.26 & 465.50 & 0.73 & 0.52 \\
\hline 142,588 & 50,393 & 4,008 & 1.1 & 36 & 8,784 & 3,505 & 0.0080 & 203 & 0.749 & 23 & 0.824 & 81 & 0.686 & 0.745 & 0.89 & 432.01 & 550.90 & 1.16 & 0.71 \\
\hline 106,428 & 34,060 & 3,146 & 1.5 & 32 & 10,222 & 4,298 & 0.0101 & 202 & 0.717 & 20 & 0.806 & 127 & 0.644 & 0.758 & 0.75 & 491.57 & 512.33 & 1.18 & 0.64 \\
\hline 174,501 & 64,493 & 4,783 & 1.6 & 40 & 9,916 & 4,629 & 0.0117 & 170 & 0.720 & 34 & 0.720 & 79 & 0.677 & 0.720 & 0.90 & 420.09 & 733.78 & 1.56 & 0.65 \\
\hline 128,272 & 53,339 & 3,258 & 1.0 & 37 & 5,359 & 2,454 & 0.0705 & 185 & 0.713 & 37 & 0.760 & 58 & 0.667 & 0.710 & 0.87 & 320.97 & 600.48 & 1.06 & 0.64 \\
\hline 73,443 & 21,102 & 2,276 & 1.1 & 30 & 5,943 & 2,405 & 0.0448 & 207 & 0.751 & 23 & 0.807 & $107 \mid$ & 0.685 & 0.744 & 0.70 & 323.24 & 501.60 & 0.97 & 0.52 \\
\hline 113,562 & 35,617 & 3,389 & 1.4 & 36 & 11,518 & 2,347 & 0.0034 & 179 & 0.731 & 12 & 0.774 & 113 & 0.672 & 0.724 & 0.86 & 464.11 & 501.02 & 1.28 & 0.71 \\
\hline 184,928 & 56,620 & 5,579 & 1.6 & 37 & 14,329 & 6,660 & 0.0178 & 183 & 0.760 & 26 & 0.839 & 72 & 0.673 & 0.746 & 0.76 & 881.70 & 580.17 & 1.09 & 0.71 \\
\hline 87,803 & 26,176 & 2,679 & 1.2 & 26 & 14,257 & 6,794 & 0.0014 & 193 & 0.750 & 30 & 0.748 & 62 & 0.680 & 0.745 & 0.93 & 502.58 & 495.11 & 0.81 & 0.59 \\
\hline 47,449 & 17,512 & 1,302 & 1.0 & 24 & 6,757 & 2,831 & 0.0030 & 208 & 0.766 & 36 & 0.823 & 129 & 0.696 & 0.741 & 0.86 & 280.91 & 406.82 & 0.72 & 0.46 \\
\hline 50,218 & 21,117 & 1,265 & 1.0 & 22 & 10,314 & 3,143 & 0.0097 & 171 & 0.711 & 17 & 0.780 & 83 & 0.645 & 0.701 & 0.76 & 315.00 & 383.72 & 0.65 & 0.46 \\
\hline 139,169 & 45,204 & 4,085 & 1.5 & 31 & 7,766 & 3,799 & 0.0033 & 237 & 0.720 & 21 & 0.862 & 79 & 0.674 & 0.720 & 0.94 & 360.18 & 547.78 & 1.93 & 0.87 \\
\hline 94,298 & 31,438 & 2,733 & 1.0 & 29 & 5,300 & 2,645 & 0.0470 & 220 & 0.767 & 38 & 0.769 & 62 & 0.651 & 0.759 & 0.85 & 355.83 & 533.33 & 0.90 & 0.59 \\
\hline 128,876 & 52,076 & 3,339 & 1.4 & 34 & 13,201 & 6,039 & 0.0100 & 193 & 0.783 & 23 & 0.865 & 81 & 0.708 & 0.780 & 0.78 & 603.85 & 485.64 & 1.00 & 0.63 \\
\hline 115,072 & 43,506 & 3,112 & 1.1 & 33 & 11,381 & 4,746 & 0.0040 & 202 & 0.749 & 15 & 0.804 & 87 & 0.695 & 0.753 & 0.89 & 409.06 & 458.60 & 1.14 & 0.72 \\
\hline 88,942 & 37,294 & 2,246 & 1.5 & 26 & 7,484 & 3,287 & 0.0135 & 220 & 0.740 & 11 & 0.740 & 115 & 0.640 & 0.728 & 0.77 & 387.77 & 458.24 & 1.19 & 0.62 \\
\hline 37,767 & 11,990 & 1,121 & 1.0 & 20 & 13,097 & 5,660 & 0.0070 & 172 & 0.736 & 31 & 0.781 & 79 & 0.675 & 0.728 & 0.73 & 392.99 & 355.50 & 0.50 & 0.40 \\
\hline 40,772 & 12,593 & 1,225 & 1.0 & 21 & 10,534 & 6,085 & 0.0029 & 223 & 0.723 & 36 & 0.722 & 117 & 0.664 & 0.714 & 0.88 & 327.01 & 404.18 & 0.58 & 0.44 \\
\hline 185,701 & 69,173 & 5,066 & 1.1 & 40 & 8,246 & 3,947 & 0.0028 & 193 & 0.714 & 33 & 0.801 & 118 & 0.689 & 0.738 & 0.93 & 452.29 & 615.21 & 1.26 & 0.75 \\
\hline 185,900 & 75,494 & 4,800 & 1.5 & 40 & 13,218 & 6,022 & 0.0329 & 232 & 0.738 & 24 & 0.731 & 134 & 0.681 & 0.730 & 0.61 & 551.64 & 620.40 & 1.32 & 0.68 \\
\hline 158,850 & 52,850 & 4,609 & 1.3 & 35 & 6,903 & 2,923 & 0.0062 & 181 & 0.734 & 15 & 0.764 & 108 & 0.698 & 0.733 & 0.91 & 390.21 & 725.13 & 1.32 & 0.67 \\
\hline 184,893 & 73,374 & 4,849 & 1.1 & 40 & 6,407 & 2,786 & 0.0065 & 171 & 0.770 & 25 & 0.770 & 74 & 0.710 & 0.773 & 0.94 & 416.00 & 730.35 & 1.10 & 0.62 \\
\hline
\end{tabular}

Table A-5: Data Generated for Eight Stage Treatment Type III, continued 


\begin{tabular}{|c|c|c|c|c|c|c|c|c|c|c|c|c|c|c|c|c|c|c|c|}
\hline \begin{tabular}{l|}
$\stackrel{0}{E}$ \\
$\frac{5}{0}$ \\
$\frac{0}{0}$ \\
$\frac{\pi}{0}$ \\
$\frac{0}{0}$
\end{tabular} & $\begin{array}{l}\frac{1}{\varepsilon} \\
\frac{1}{3} \\
0 \\
\frac{0}{0} \\
\frac{0}{0} \\
0\end{array}$ & 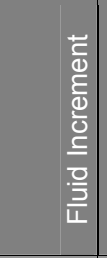 & 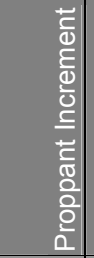 & 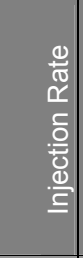 & 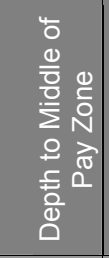 & 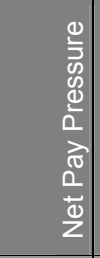 & 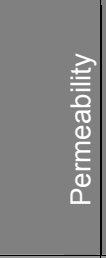 & 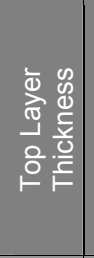 & 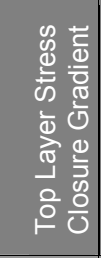 & 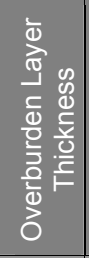 & 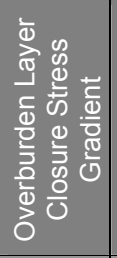 & 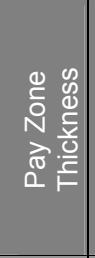 & 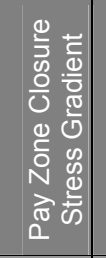 & 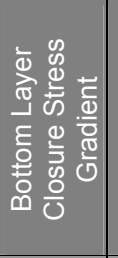 & 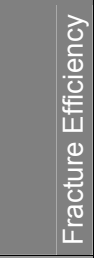 & 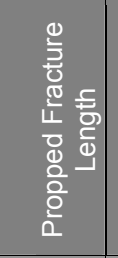 & 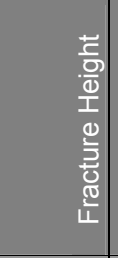 & 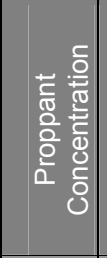 & 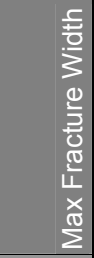 \\
\hline input & input & input & input & input & input & input & input & input & input & input & input & input & input & input & output & output & output & output & output \\
\hline 92,152 & 38,288 & 2,342 & 1.6 & 33 & 11,832 & 5,561 & 0.0046 & 190 & 0.775 & 14 & 0.777 & 80 & 0.720 & 0.765 & 0.88 & 372.60 & 541.02 & 1.16 & 0.56 \\
\hline 83,557 & 32,857 & 2,204 & 1.2 & 30 & 8,906 & 3,463 & 0.0190 & 174 & 0.744 & 18 & 0.800 & 66 & 0.680 & 0.725 & 0.83 & 359.93 & 502.12 & 0.92 & 0.53 \\
\hline 147,152 & 59,630 & 3,805 & 1.0 & 36 & 7,280 & 3,624 & 0.0013 & 220 & 0.728 & 25 & 0.741 & 108 & 0.670 & 0.711 & 0.96 & 396.19 & 643.09 & 0.94 & 0.61 \\
\hline 126,829 & 49,021 & 3,383 & 1.0 & 33 & 12,907 & 5,663 & 0.0149 & 216 & 0.747 & 13 & 0.745 & 60 & 0.645 & 0.735 & 0.79 & 732.82 & 373.55 & 0.77 & 0.70 \\
\hline 134,633 & 50,264 & 3,668 & 1.6 & 36 & 12,555 & 6,086 & 0.0100 & 201 & 0.785 & 22 & 0.845 & 125 & 0.725 & 0.770 & 0.75 & 533.15 & 589.45 & 1.17 & 0.63 \\
\hline 79,118 & 28,969 & 2,180 & 1.0 & 35 & 7,996 & 3,284 & 0.0051 & 184 & 0.734 & 36 & 0.807 & 97 & 0.669 & 0.728 & 0.88 & 381.84 & 506.02 & 0.71 & 0.51 \\
\hline 165,985 & 46,716 & 5,186 & 1.3 & 35 & 12,065 & 5,645 & 0.0207 & 201 & 0.780 & 24 & 0.851 & 51 & 0.679 & 0.757 & 0.86 & 625.02 & 641.49 & 1.05 & 0.70 \\
\hline 97,140 & 40,613 & 2,458 & 1.1 & 28 & 6,014 & 2,914 & 0.0055 & 200 & 0.722 & 30 & 0.721 & $77 \mid$ & 0.664 & 0.728 & 0.93 & 323.92 & 560.60 & 0.93 & 0.54 \\
\hline 55,973 & 22,241 & 1,467 & 1.0 & 28 & 9,111 & 4,416 & 0.0076 & 186 & 0.734 & 31 & 0.790 & 55 & 0.681 & 0.718 & 0.91 & 294.50 & 478.26 & 0.65 & 0.44 \\
\hline 146,112 & 55,154 & 3,955 & 1.5 & 35 & 10,171 & 5,048 & 0.0405 & 190 & 0.736 & 33 & 0.810 & 67 & 0.676 & 0.738 & 0.80 & 449.14 & 528.17 & 1.57 & 0.72 \\
\hline 79,448 & 34,010 & 1,976 & 1.0 & 31 & 5,589 & 1,797 & 0.0095 & 236 & 0.753 & 24 & 0.805 & 58 & 0.695 & 0.743 & 0.92 & 283.01 & 481.42 & 0.91 & 0.55 \\
\hline 87,290 & 32,207 & 2,395 & 1.3 & 29 & 7,133 & 3,382 & 0.0052 & 206 & 0.771 & 37 & 0.828 & 75 & 0.731 & 0.800 & 0.92 & 305.15 & 554.82 & 1.15 & 0.62 \\
\hline 78,961 & 30,087 & 2,125 & 1.0 & $27 \mid$ & 14,968 & 7,261 & 0.0022 & 187 & 0.741 & 18 & 0.846 & 80 & 0.687 & 0.729 & 0.90 & 456.94 & 542.60 & 0.54 & 0.46 \\
\hline 36,207 & 13,801 & 974 & 1.0 & 22 & 11,704 & 5,684 & 0.0283 & 193 & 0.774 & 16 & 0.779 & 115 & 0.714 & 0.766 & 0.49 & 287.57 & 331.03 & 0.64 & 0.38 \\
\hline 72,864 & 27,325 & 1,980 & 1.0 & 25 & 10,544 & 4,935 & 0.0075 & 190 & 0.717 & 18 & 0.747 & 135 & 0.651 & 0.711 & 0.76 & 427.86 & 442.24 & 0.65 & 0.50 \\
\hline 189,449 & 66,239 & 5,357 & 1.3 & 40 & 7,743 & 3,731 & 0.0067 & 239 & 0.728 & 28 & 0.789 & 95 & 0.686 & 0.718 & 0.93 & 403.18 & 656.20 & 1.65 & 0.81 \\
\hline 116,607 & 45,699 & 3,083 & 1.3 & 29 & 13,435 & 6,504 & 0.0165 & 207 & 0.717 & 14 & 0.781 & 63 & 0.662 & 0.710 & 0.83 & 482.17 & 520.33 & 1.00 & 0.58 \\
\hline 103,278 & 35,418 & 2,950 & 1.6 & 33 & 8,022 & 3,142 & 0.0154 & 187 & 0.734 & 23 & 0.747 & 104 & 0.693 & 0.741 & 0.81 & 337.28 & 555.86 & 1.58 & 0.64 \\
\hline 127,489 & 47,640 & 3,472 & 1.0 & 39 & 9,214 & 4,398 & 0.0080 & 172 & 0.789 & 28 & 0.847 & 90 & 0.730 & 0.809 & 0.88 & 378.57 & 443.07 & 1.30 & 0.84 \\
\hline 49,125 & 17,897 & 1,358 & 1.0 & 39 & 15,163 & 6,938 & 0.0073 & 174 & 0.729 & 18 & 0.785 & 71 & 0.678 & 0.717 & 0.82 & 374.83 & 378.13 & 0.55 & 0.42 \\
\hline 93,682 & 31,711 & 2,694 & 1.1 & 22 & 13,552 & 6,053 & 0.0045 & 231 & 0.797 & 18 & 0.800 & 69 & 0.729 & 0.806 & 0.85 & 448.18 & 547.65 & 0.76 & 0.52 \\
\hline 61,888 & 20,416 & 1,803 & 1.0 & 25 & 11,077 & 4,566 & 0.0221 & 200 & 0.705 & 26 & 0.763 & 75 & 0.641 & 0.707 & 0.72 & 384.07 & 406.87 & 0.72 & 0.50 \\
\hline 37,678 & 15,411 & 968 & 1.1 & 20 & 6,225 & 2,721 & 0.0200 & 176 & 0.750 & 17 & 0.826 & 104 & 0.715 & 0.749 & 0.73 & 217.72 & 383.68 & 0.80 & 0.39 \\
\hline 97,258 & 29,179 & 2,960 & 1.2 & 33 & 7,115 & 3,541 & 0.0039 & 208 & 0.775 & 23 & 0.869 & 135 & 0.710 & 0.765 & 0.88 & 401.06 & 564.45 & 0.98 & 0.57 \\
\hline 35,922 & 13,672 & 967 & 1.0 & 22 & 9,127 & 4,539 & 0.0905 & 216 & 0.754 & 18 & 0.750 & 110 & 0.704 & 0.743 & 0.43 & 231.62 & 361.76 & 0.72 & 0.34 \\
\hline 52,240 & 21,634 & 1,331 & 1.2 & 24 & 8,100 & 3,723 & 0.0081 & 178 & 0.710 & 20 & 0.746 & 85 & 0.666 & 0.706 & 0.86 & 269.77 & 426.34 & 0.87 & 0.47 \\
\hline 173,765 & 72,504 & 4,403 & 1.5 & 38 & 7,853 & 3,766 & 0.0038 & 233 & 0.758 & 26 & 0.791 & 73 & 0.690 & 0.728 & 0.95 & 394.33 & 663.11 & 1.58 & 0.72 \\
\hline 40,354 & 16,364 & 1,043 & 1.2 & 20 & 12,893 & 6,420 & 0.0035 & 188 & 0.715 & 37 & 0.809 & 64 & 0.640 & 0.712 & 0.88 & 349.52 & 428.87 & 0.52 & 0.44 \\
\hline 49,905 & 18,084 & 1,384 & 1.0 & 27 & 10,939 & 5,179 & 0.0208 & 189 & 0.794 & 0.744 & 0.822 & 135 & 0.697 & 0.742 & 0.59 & 318.51 & 417.05 & 0.65 & 0.40 \\
\hline 99,867 & 37,752 & 2,701 & 1.3 & 30 & 8,014 & 3,410 & 0.0162 & 232 & 0.749 & 11 & 0.745 & 62 & 0.675 & 0.727 & 0.88 & 363.04 & 603.69 & 1.00 & 0.54 \\
\hline
\end{tabular}

Table A-5: Data Generated for Eight Stage Treatment Type III, continued 


\begin{tabular}{|c|c|c|c|c|c|c|c|c|c|c|c|c|c|c|c|c|c|c|c|}
\hline 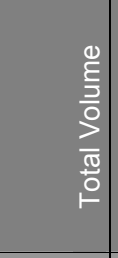 & 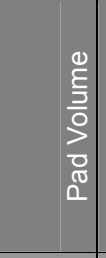 & 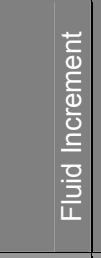 & 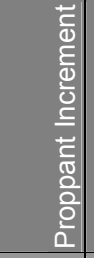 & 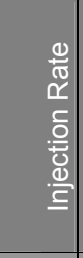 & 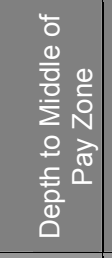 & 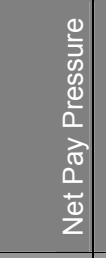 & 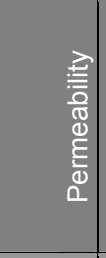 & 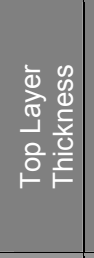 & 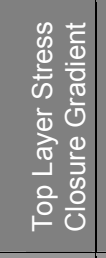 & 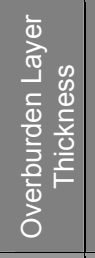 & 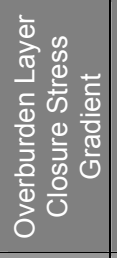 & 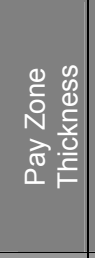 & 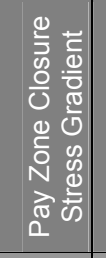 & 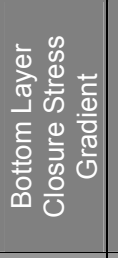 & 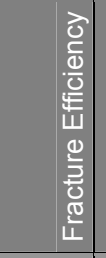 & 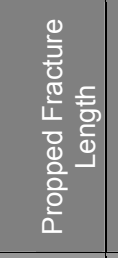 & 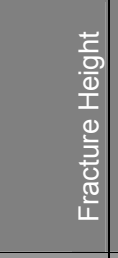 & 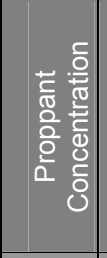 & 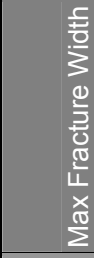 \\
\hline input & input & input & input & input & input & input & input & input & input & input & input & input & input & input & output & output & output & output & output \\
\hline 112,288 & 35,047 & 3,358 & 1.1 & 35 & 11,438 & 5,233 & 0.0307 & 207 & 0.799 & 25 & 0.800 & 68 & 0.735 & 0.780 & 0.92 & 442.60 & 576.16 & 0.91 & 0.61 \\
\hline 162,365 & 46,949 & 5,018 & 1.1 & 39 & 8,138 & 3,391 & 0.0043 & 182 & 0.728 & 28 & 0.784 & 88 & 0.644 & 0.730 & 0.92 & 453.58 & 509.73 & 1.50 & 0.93 \\
\hline 110,880 & 45,489 & 2,843 & 1.0 & 35 & 5,010 & 2,442 & 0.0057 & 205 & 0.711 & 35 & 0.752 & 88 & 0.647 & 0.705 & 0.94 & 331.19 & 567.30 & 0.95 & 0.60 \\
\hline 102,088 & 40,643 & 2,672 & 1.2 & 26 & 8,883 & 3,880 & 0.0035 & 237 & 0.759 & 39 & 0.810 & 117 & 0.646 & 0.741 & 0.83 & 556.00 & 419.80 & 0.86 & 0.65 \\
\hline 68,767 & 28,426 & 1,754 & 1.0 & 29 & 13,799 & 6,875 & 0.0072 & 181 & 0.732 & 33 & 0.784 & 68 & 0.688 & 0.727 & 0.86 & 397.09 & 469.35 & 0.59 & 0.44 \\
\hline 184,863 & 65,931 & 5,171 & 1.5 & 40 & 10,652 & 5,069 & 0.0135 & 234 & 0.722 & 33 & 0.796 & 127 & 0.657 & 0.730 & 0.75 & 679.89 & 589.10 & 1.21 & 0.75 \\
\hline 46,882 & 13,760 & 1,440 & 1.0 & 18 & 7,704 & 4,271 & 0.0177 & 209 & 0.733 & 38 & 0.721 & 73 & 0.653 & 0.714 & 0.79 & 298.85 & 447.41 & 0.67 & 0.44 \\
\hline 153,417 & 60,104 & 4,057 & 1.0 & 39 & 11,141 & 5,441 & 0.0242 & 196 & 0.787 & 31 & 0.844 & 114 & 0.725 & 0.780 & 0.70 & 557.14 & 585.12 & 0.78 & 0.58 \\
\hline 147,378 & 56,166 & 3,966 & 1.2 & 38 & 14,944 & 6,680 & 0.0050 & 199 & 0.754 & 35 & 0.838 & 134 & 0.687 & 0.745 & 0.73 & 779.14 & 348.86 & 1.10 & 0.73 \\
\hline 53,013 & 21,102 & 1,388 & 1.0 & 23 & 12,331 & 5,619 & 0.0089 & 188 & 0.715 & 38 & 0.713 & 75 & 0.662 & 0.706 & 0.82 & 322.14 & 438.38 & 0.62 & 0.44 \\
\hline 159,742 & 45,028 & 4,988 & 1.5 & 35 & 13,478 & 6,361 & 0.0338 & 188 & 0.730 & 34 & 0.831 & 111 & 0.681 & 0.719 & 0.68 & 498.94 & 649.07 & 1.45 & 0.69 \\
\hline 93,700 & 37,102 & 2,461 & 1.2 & 34 & 9,308 & 4,374 & 0.0044 & 198 & 0.747 & 39 & 0.795 & 106 & 0.678 & 0.738 & 0.88 & 414.25 & 502.99 & 0.89 & 0.58 \\
\hline 164,387 & 56,529 & 4,689 & 1.4 & 35 & 11,683 & 5,099 & 0.0083 & 198 & 0.764 & 31 & 0.847 & 118 & 0.699 & 0.756 & 0.77 & 635.34 & 609.48 & 1.06 & 0.66 \\
\hline 41,053 & 13,410 & 1,202 & 1.1 & 20 & 9,365 & 4,619 & 0.0065 & 198 & 0.719 & 27 & 0.791 & 69 & 0.650 & 0.719 & 0.86 & 335.36 & 381.37 & 0.65 & 0.45 \\
\hline 79,452 & 32,868 & 2,025 & 1.0 & 28 & 9,720 & 4,731 & 0.0088 & 209 & 0.790 & 16 & 0.853 & 115 & 0.731 & 0.809 & 0.79 & 341.17 & 395.69 & 0.94 & 0.61 \\
\hline 170,133 & 67,606 & 4,458 & 1.1 & 28 & 5,050 & 2,322 & 0.0297 & 187 & 0.724 & 25 & 0.779 & 60 & 0.677 & 0.724 & 0.92 & 371.26 & 707.03 & 1.17 & 0.69 \\
\hline 43,148 & 12,897 & 1,315 & 1.0 & 24 & 10,995 & 4,815 & 0.0085 & 200 & 0.729 & 39 & 0.769 & 90 & 0.664 & 0.717 & 0.76 & 361.54 & 386.88 & 0.59 & 0.44 \\
\hline 56,838 & 16,294 & 1,763 & 1.1 & 29 & 14,076 & 6,401 & 0.0326 & 218 & 0.768 & 30 & 0.768 & 51 & 0.707 & 0.768 & 0.76 & 332.01 & 484.84 & 0.75 & 0.46 \\
\hline 44,404 & 16,648 & 1,207 & 1.0 & 20 & 7,769 & 3,006 & 0.0026 & 225 & 0.742 & 31 & 0.808 & 52 & 0.698 & 0.736 & 0.94 & 283.17 & 440.11 & 0.61 & 0.39 \\
\hline 176,885 & 52,064 & 5,427 & 1.4 & 32 & 13,200 & 6,411 & 0.0183 & 232 & 0.725 & 22 & 0.770 & 54 & 0.661 & 0.711 & 0.88 & 580.56 & 611.64 & 1.34 & 0.73 \\
\hline 106,367 & 44,369 & 2,696 & 1.5 & 27 & 10,645 & 4,366 & 0.0167 & 216 & 0.733 & 11 & 0.730 & 128 & 0.691 & 0.722 & 0.71 & 364.08 & 573.91 & 1.21 & 0.54 \\
\hline 138,805 & 39,985 & 4,297 & 1.1 & 35 & 14,511 & 6,271 & 0.0453 & 237 & 0.774 & 15 & 0.775 & 129 & 0.705 & 0.760 & 0.41 & 596.61 & 395.17 & 1.26 & 0.65 \\
\hline 73,253 & 20,800 & 2,281 & 1.2 & 25 & 15,098 & 6,626 & 0.0174 & 218 & 0.726 & 12 & 0.788 & 123 & 0.681 & 0.711 & 0.57 & 440.65 & 488.79 & 0.80 & 0.47 \\
\hline 57,389 & 18,595 & 1,687 & 1.2 & 23 & 11,556 & 6,833 & 0.0293 & 233 & 0.756 & 37 & 0.797 & 65 & 0.704 & 0.746 & 0.79 & 351.77 & 445.00 & 0.81 & 0.47 \\
\hline 84,568 & 33,971 & 2,200 & 1.1 & 36 & 12,467 & 6,718 & 0.0058 & 209 & 0.714 & 24 & 0.787 & 111 & 0.665 & 0.711 & 0.86 & 420.23 & 491.67 & 0.74 & 0.53 \\
\hline 148,744 & 57,500 & 3,967 & 1.0 & 40 & 15,013 & 6,649 & 0.0100 & 233 & 0.729 & 29 & 0.753 & 84 & 0.701 & 0.725 & 0.85 & 464.73 & 627.82 & 0.85 & 0.53 \\
\hline 47,068 & 16,121 & 1,346 & 1.0 & 21 & 14,390 & 6,789 & 0.0181 & 173 & 0.728 & 28 & 0.764 & 55 & 0.682 & 0.728 & 0.77 & 308.16 & 343.92 & 0.73 & 0.48 \\
\hline 86,037 & 28,306 & 2,510 & 1.2 & 31 & 9,057 & 4,337 & 0.0151 & 233 & 0.742 & 13 & 0.743 & 78 & 0.693 & 0.730 & 0.86 & 332.32 & 564.46 & 1.00 & 0.54 \\
\hline 50,480 & 14,433 & 1,567 & 1.0 & 22 & 7,307 & 2,825 & 0.0058 & 205 & 0.787 & 13 & 0.787 & 97 & 0.711 & 0.789 & 0.83 & 307.88 & 438.76 & 0.73 & 0.50 \\
\hline 66,187 & 26,775 & 1,714 & 1.2 & 29 & 14,205 & 6,654 & 0.0158 & 210 & 0.745 & 13 & 0.795 & 85 & 0.680 & 0.740 & 0.69 & 431.62 & 386.34 & 0.77 & 0.53 \\
\hline
\end{tabular}

Table A-5: Data Generated for Eight Stage Treatment Type III, continued 


\begin{tabular}{|c|c|c|c|c|c|c|c|c|c|c|c|c|c|c|c|c|c|c|c|}
\hline 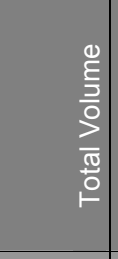 & $\begin{array}{l}\frac{0}{E} \\
\frac{1}{3} \\
0 \\
\frac{0}{0} \\
\frac{0}{\sigma} \\
0\end{array}$ & 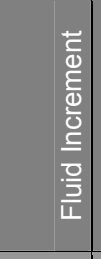 & 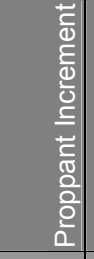 & 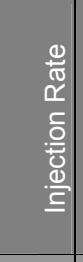 & 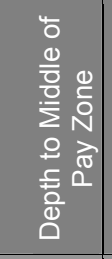 & 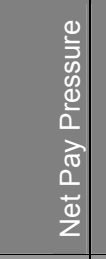 & 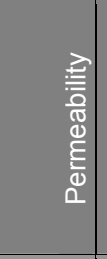 & 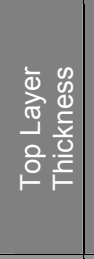 & 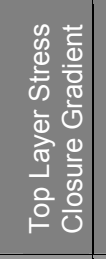 & 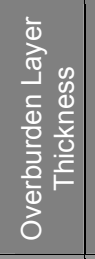 & 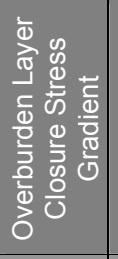 & 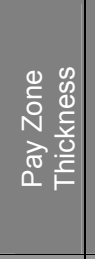 & 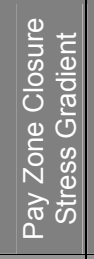 & 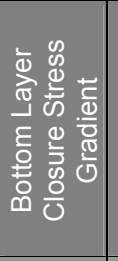 & 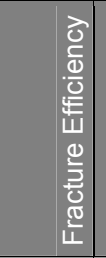 & 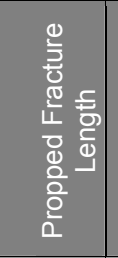 & 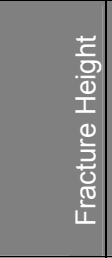 & 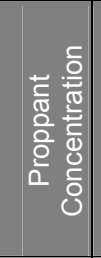 & 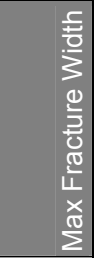 \\
\hline input & input & input & input & input & input & input & input & input & input & input & input & input & input & input & output & output & output & output & output \\
\hline 139,304 & 52,298 & 3,783 & 1.0 & 33 & 9,949 & 4,201 & 0.0181 & 183 & 0.738 & 18 & 0.738 & 129 & 0.651 & 0.721 & 0.70 & 589.35 & 520.98 & 0.77 & 0.63 \\
\hline 70,317 & 21,555 & 2,120 & 1.0 & 25 & 14,518 & 6,850 & 0.0468 & 220 & 0.725 & 23 & 0.781 & 121 & 0.665 & 0.729 & 0.35 & 460.06 & 323.03 & 0.89 & 0.48 \\
\hline 124,558 & 39,693 & 3,690 & 1.3 & 36 & 12,160 & 6,840 & 0.0830 & 199 & 0.750 & 13 & 0.750 & 88 & 0.706 & 0.747 & 0.65 & 390.99 & 613.33 & 1.25 & 0.58 \\
\hline 48,264 & 14,064 & 1,487 & 1.0 & 26 & 10,545 & 5,098 & 0.0170 & 196 & 0.725 & 26 & 0.794 & 61 & 0.678 & 0.724 & 0.85 & 317.36 & 418.20 & 0.70 & 0.46 \\
\hline 99,962 & 37,253 & 2,726 & 1.5 & 29 & 12,920 & 5,756 & 0.0165 & 204 & 0.770 & 11 & 0.770 & 68 & 0.692 & 0.763 & 0.72 & 462.08 & 491.96 & 1.13 & 0.64 \\
\hline 53,957 & 22,096 & 1,385 & 1.0 & 23 & 7,685 & 3,491 & 0.0421 & 211 & 0.725 & 12 & 0.725 & 94 & 0.684 & 0.712 & 0.70 & 263.71 & 451.52 & 0.73 & 0.41 \\
\hline 184,179 & 62,120 & 5,307 & 1.0 & 40 & 11,359 & 5,085 & 0.0251 & 189 & 0.772 & 26 & 0.776 & 128 & 0.677 & 0.774 & 0.58 & 839.38 & 372.56 & 1.07 & 0.76 \\
\hline 167,944 & 58,464 & 4,760 & 1.2 & 38 & 14,099 & 6,073 & 0.0349 & 235 & 0.772 & 18 & 0.854 & 91 & 0.729 & 0.800 & 0.73 & 367.35 & 368.34 & 2.65 & 1.30 \\
\hline 168,516 & 49,092 & 5,192 & 1.5 & 35 & 11,070 & 5,155 & 0.0099 & 209 & 0.767 & 11 & 0.812 & 99 & 0.709 & 0.785 & 0.85 & 417.41 & 436.88 & 2.68 & 1.23 \\
\hline 79,145 & 27,069 & 2,264 & 1.0 & 30 & 5,329 & 1,984 & 0.0083 & 184 & 0.797 & 29 & 0.849 & 51 & 0.715 & 0.800 & 0.93 & 297.26 & 487.33 & 0.98 & 0.61 \\
\hline 173,101 & 52,839 & 5,229 & 1.5 & 32 & 11,226 & 5,370 & 0.0099 & 240 & 0.729 & 31 & 0.744 & 74 & 0.683 & 0.720 & 0.90 & 439.68 & 684.30 & 1.64 & 0.74 \\
\hline 134,063 & 53,813 & 3,489 & 1.4 & 38 & 8,282 & 3,154 & 0.0172 & 205 & 0.713 & 29 & 0.742 & 86 & 0.640 & 0.705 & 0.84 & 395.02 & 519.48 & 1.49 & 0.74 \\
\hline 159,037 & 46,347 & 4,900 & 1.0 & 35 & 12,244 & 5,127 & 0.0065 & 193 & 0.766 & 29 & 0.814 & 106 & 0.711 & 0.754 & 0.82 & 593.44 & 626.86 & 0.83 & 0.61 \\
\hline 48,512 & 20,114 & 1,235 & 1.1 & 22 & 14,378 & 6,330 & 0.0261 & 215 & 0.726 & 35 & 0.780 & 89 & 0.668 & 0.717 & 0.51 & 388.14 & 338.20 & 0.59 & 0.42 \\
\hline 61,026 & 22,422 & 1,678 & 1.1 & 28 & 11,804 & 5,642 & 0.0037 & 232 & 0.740 & 13 & 0.791 & 73 & 0.693 & 0.729 & 0.90 & 319.57 & 450.61 & 0.80 & 0.50 \\
\hline 83,119 & 26,925 & 2,443 & 1.0 & 31 & 12,403 & 5,469 & 0.0501 & 206 & 0.796 & 32 & 0.796 & 94 & 0.733 & 0.789 & 0.68 & 643.16 & 303.77 & 0.78 & 0.63 \\
\hline 71,952 & 21,099 & 2,211 & 1.3 & 28 & 14,899 & 6,535 & 0.0311 & 198 & 0.746 & 32 & 0.809 & 107 & 0.668 & 0.741 & 0.39 & 552.31 & 280.58 & 1.16 & 0.57 \\
\hline 178,436 & 59,039 & 5,191 & 1.2 & 40 & 10,342 & 4,739 & 0.0218 & 193 & 0.731 & 24 & 0.759 & 74 & 0.670 & 0.720 & 0.86 & 443.21 & 598.94 & 1.47 & 0.80 \\
\hline 109,755 & 37,667 & 3,134 & 1.0 & 29 & 13,196 & 6,085 & 0.0053 & 184 & 0.710 & 37 & 0.710 & 122 & 0.653 & 0.709 & 0.80 & 516.20 & 478.43 & 0.79 & 0.62 \\
\hline 185,786 & 60,952 & 5,428 & 1.0 & 40 & 10,607 & 4,959 & 0.0182 & 195 & 0.758 & 11 & 0.794 & 104 & 0.677 & 0.742 & 0.77 & 646.90 & 601.94 & 0.87 & 0.69 \\
\hline 90,647 & 31,381 & 2,577 & 1.2 & 30 & 8,318 & 4,212 & 0.0046 & 186 & 0.779 & 30 & 0.847 & 78 & 0.731 & 0.775 & 0.91 & 357.06 & 504.21 & 1.08 & 0.58 \\
\hline 153,845 & 50,282 & 4,503 & 1.5 & 32 & 7,567 & 6,439 & 0.0294 & 205 & 0.729 & 13 & 0.725 & 63 & 0.664 & 0.725 & 0.91 & 409.28 & 679.17 & 1.52 & 0.67 \\
\hline 81,730 & 29,009 & 2,292 & 1.0 & 25 & 10,627 & 4,758 & 0.0049 & 219 & 0.753 & 27 & 0.835 & 122 & 0.695 & 0.766 & 0.79 & 431.56 & 437.45 & 0.76 & 0.55 \\
\hline 156,355 & 61,286 & 4,133 & 1.4 & 37 & 6,096 & 2,570 & 0.0062 & 173 & 0.740 & 14 & 0.740 & 77 & 0.702 & 0.740 & 0.94 & 365.03 & 589.56 & 1.44 & 0.68 \\
\hline 144,015 & 48,403 & 4,157 & 1.2 & 40 & 13,935 & 6,193 & 0.0149 & 225 & 0.738 & 20 & 0.788 & 91 & 0.671 & 0.719 & 0.75 & 653.60 & 571.25 & 0.84 & 0.61 \\
\hline 55,251 & 22,745 & 1,413 & 1.0 & 21 & 5,694 & 2,804 & 0.0148 & 171 & 0.720 & 29 & 0.750 & 91 & 0.676 & 0.724 & 0.85 & 262.53 & 450.36 & 0.75 & 0.45 \\
\hline 122,734 & 38,609 & 3,658 & 1.3 & 29 & 9,770 & 4,067 & 0.0075 & 228 & 0.784 & 17 & 0.800 & 60 & 0.726 & 0.772 & 0.90 & 395.07 & 275.72 & 1.38 & 0.72 \\
\hline 75,944 & 25,721 & 2,184 & 1.2 & 24 & 10,884 & 5,127 & 0.0133 & 186 & 0.778 & 18 & 0.845 & 97 & 0.703 & 0.794 & 0.84 & 348.15 & 341.57 & 1.38 & 0.79 \\
\hline 165,048 & 67,378 & 4,246 & 1.0 & 34 & 15,122 & 6,937 & 0.0063 & 171 & 0.746 & 14 & 0.820 & 130 & 0.700 & 0.734 & 0.76 & 672.72 & 607.34 & 0.65 & 0.55 \\
\hline 78,923 & 27,258 & 2,246 & 1.5 & 27 & 9,653 & 4,782 & 0.0015 & 219 & 0.726 & 21 & 0.726 & 78 & 0.673 & 0.726 & 0.95 & 329.52 & 549.37 & 1.17 & 0.54 \\
\hline
\end{tabular}

Table A-5: Data Generated for Eight Stage Treatment Type III, continued 


\begin{tabular}{|c|c|c|c|c|c|c|c|c|c|c|c|c|c|c|c|c|c|c|c|}
\hline \begin{tabular}{l|}
$\stackrel{0}{E}$ \\
$\frac{5}{0}$ \\
$\frac{0}{0}$ \\
$\frac{\pi}{0}$ \\
$\frac{0}{0}$
\end{tabular} & $\begin{array}{l}\frac{1}{\varepsilon} \\
\frac{1}{3} \\
0 \\
\frac{0}{0} \\
\frac{0}{0} \\
0\end{array}$ & 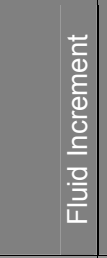 & 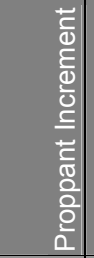 & 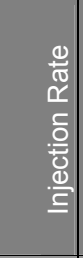 & 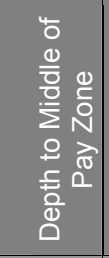 & 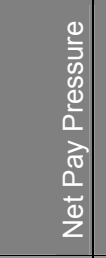 & 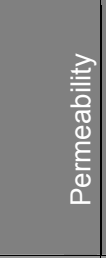 & 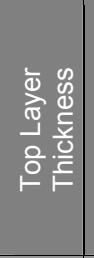 & 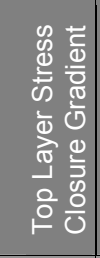 & 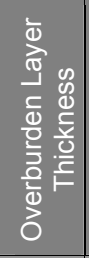 & 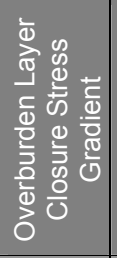 & 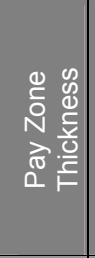 & 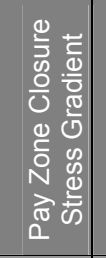 & 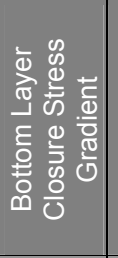 & 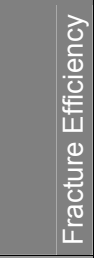 & 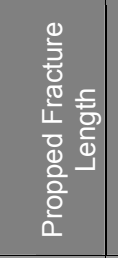 & 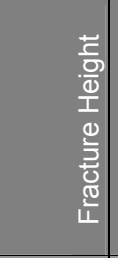 & 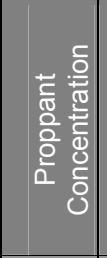 & 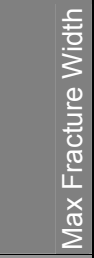 \\
\hline input & input & input & input & input & input & input & input & input & input & input & input & input & input & input & output & output & output & output & output \\
\hline 113,711 & 32,249 & 3,542 & 1.1 & 28 & 9,450 & 4,220 & 0.0386 & 204 & 0.727 & 28 & 0.785 & 116 & 0.677 & 0.718 & 0.65 & 455.17 & 611.67 & 0.90 & 0.52 \\
\hline 53,318 & 16,034 & 1,621 & 1.0 & 24 & 8,105 & 4,304 & 0.0156 & 219 & 0.713 & 37 & 0.767 & 61 & 0.654 & 0.707 & 0.81 & 337.81 & 453.64 & 0.66 & 0.44 \\
\hline 120,942 & 45,710 & 3,271 & 1.1 & $37 \mid$ & 7,177 & 3,432 & 0.0068 & 193 & 0.797 & 40 & 0.847 & 96 & 0.729 & 0.780 & 0.90 & 399.50 & 577.91 & 0.98 & 0.60 \\
\hline 109,061 & 41,378 & 2,943 & 1.0 & 33 & 14,918 & 6,643 & 0.0023 & 213 & 0.748 & 31 & 0.749 & 88 & 0.692 & 0.740 & 0.88 & 537.54 & 475.52 & 0.72 & 0.59 \\
\hline 141,214 & 57,588 & 3,636 & 1.7 & 35 & 13,104 & 6,447 & 0.0559 & 220 & 0.720 & 24 & 0.800 & 128 & 0.670 & 0.714 & 0.89 & 511.59 & 588.00 & 1.29 & 0.64 \\
\hline 66,811 & 19,553 & 2,055 & 1.2 & 30 & 14,535 & 6,445 & 0.0164 & 181 & 0.719 & 28 & 0.796 & 85 & 0.642 & 0.707 & 0.65 & 558.18 & 346.40 & 0.80 & 0.58 \\
\hline 86,034 & 30,044 & 2,434 & 1.0 & 30 & 8,464 & 4,179 & 0.0066 & 170 & 0.739 & 37 & 0.752 & 96 & 0.680 & 0.733 & 0.88 & 350.89 & 523.88 & 0.83 & 0.55 \\
\hline 56,779 & 17,507 & 1,708 & 1.0 & 24 & 10,850 & 4,799 & 0.0166 & 194 & 0.750 & 14 & 0.750 & 73 & 0.693 & 0.741 & 0.78 & 325.09 & 471.31 & 0.70 & 0.47 \\
\hline 94,393 & 33,359 & 2,654 & 1.5 & 28 & 12,896 & 6,078 & .0078 & 236 & 0.742 & 20 & 0.748 & 114 & 0.695 & 0.733 & 0.79 & 386.70 & 498.82 & 1.29 & 0.62 \\
\hline 67,248 & 26,422 & 1,775 & 1.0 & 24 & 11,843 & 5,170 & 0.0591 & 198 & 0.739 & 12 & 0.733 & 103 & 0.687 & 0.730 & 0.48 & 364.64 & 451.24 & 0.67 & 0.41 \\
\hline 182,204 & 58,417 & 5,382 & 1.5 & 40 & 13,730 & 6,688 & 0.0184 & 209 & 0.719 & 21 & 0.777 & 105 & 0.673 & 0.717 & 0.77 & 537.83 & 587.78 & 1.60 & 0.77 \\
\hline 54,164 & 16,304 & 1,646 & 1.1 & 26 & 12,781 & 5,608 & 0.0025 & 192 & 0.742 & 10 & 0.800 & 109 & 0.696 & 0.742 & 0.85 & 344.10 & 512.48 & 0.80 & 0.51 \\
\hline 139,286 & 40,539 & 4,293 & 1.0 & 33 & 8,142 & 3,951 & 0.0265 & 188 & 0.714 & 18 & 0.767 & 126 & 0.662 & 0.710 & 0.77 & 418.32 & 598.82 & 1.07 & 0.66 \\
\hline 182,393 & 65,912 & 5,064 & 1.4 & 28 & 15,000 & 7,446 & 0.0044 & 204 & 0.772 & 19 & 0.841 & 99 & 0.723 & 0.766 & 0.86 & 648.74 & 611.55 & 1.12 & 0.66 \\
\hline 159,377 & 52,415 & 4,651 & 1.1 & 39 & 6,180 & 2,459 & 0.0065 & 217 & 0.774 & 19 & 0.856 & 109 & 0.714 & 0.768 & 0.90 & 391.93 & 609.10 & 1.35 & 0.77 \\
\hline 94,263 & 33,578 & 2,638 & 1.3 & 32 & 13,299 & 5,912 & 0.0588 & 223 & 0.734 & 16 & 0.783 & 101 & 0.696 & 0.726 & 0.55 & 404.81 & 529.41 & 1.00 & 0.49 \\
\hline 76,433 & 25,839 & 2,200 & 1.5 & 26 & 7,119 & 2,598 & 0.0286 & 222 & 0.775 & 26 & 0.847 & 129 & 0.709 & 0.759 & 0.64 & 319.90 & 470.21 & 1.38 & 0.58 \\
\hline 78,638 & 26,805 & 2,254 & 1.1 & 27 & 11,989 & 5,918 & 0.0119 & 214 & 0.777 & 19 & 0.775 & 95 & 0.715 & 0.762 & 0.79 & 388.48 & 472.41 & 0.85 & 0.56 \\
\hline 176,441 & 64,824 & 4,853 & 1.5 & 40 & 8,808 & 3,960 & 0.0705 & 177 & 0.795 & 30 & 0.859 & 79 & 0.732 & 0.784 & 0.73 & 457.12 & 606.26 & 1.65 & 0.73 \\
\hline 123,915 & 49,381 & 3,241 & 1.0 & 30 & 13,118 & 6,262 & 0.0037 & 174 & 0.791 & 16 & 0.782 & 78 & 0.728 & 0.752 & 0.91 & 404.98 & 558.68 & 0.90 & 0.58 \\
\hline 94,343 & 27,439 & 2,909 & 1.3 & 30 & 7,608 & 3,711 & 0.0351 & 172 & 0.741 & 31 & 0.805 & 94 & 0.702 & 0.740 & 0.78 & 343.43 & 545.78 & 1.26 & 0.60 \\
\hline 115,589 & 38,512 & 3,351 & 1.5 & 31 & 12,349 & 5,815 & 0.0031 & 178 & 0.709 & 17 & 0.706 & 113 & 0.648 & 0.702 & 0.87 & 482.91 & 553.92 & 1.18 & 0.66 \\
\hline 51,937 & 19,659 & 1,403 & 1.3 & 24 & 8,958 & 4,366 & 0.0092 & 227 & 0.761 & 22 & 0.761 & 128 & 0.712 & 0.758 & 0.76 & 283.80 & 467.67 & 0.86 & 0.43 \\
\hline 108,183 & 38,021 & 3,051 & 1.2 & 34 & 7,141 & 2,686 & 0.0317 & 187 & 0.728 & 30 & 0.778 & 123 & 0.677 & 0.719 & 0.72 & 365.25 & 571.20 & 1.10 & 0.56 \\
\hline 174,858 & 50,402 & 5,411 & 1.6 & 40 & 7,193 & 3,312 & 0.0055 & 182 & 0.789 & 27 & 0.788 & 130 & 0.727 & 0.764 & 0.89 & 431.87 & 696.55 & 1.80 & 0.78 \\
\hline 37,794 & 14,034 & 1,033 & 1.0 & 19 & 11,458 & 4,772 & 0.0123 & 223 & 0.732 & 27 & 0.765 & 106 & 0.685 & 0.724 & 0.64 & 289.61 & 373.45 & 0.58 & 0.37 \\
\hline 144,053 & 51,831 & 4,010 & 1.5 & 34 & 5,646 & 1,853 & 0.0548 & 239 & 0.714 & 31 & 0.765 & 132 & 0.660 & 0.713 & 0.68 & 376.99 & 614.18 & 1.63 & 0.66 \\
\hline 141,803 & 54,610 & 3,791 & 1.1 & $37 \mid$ & 13,314 & 6,621 & 0.0303 & 219 & 0.768 & 15 & 0.765 & 124 & 0.726 & 0.760 & 0.66 & 453.57 & 647.65 & 0.89 & 0.53 \\
\hline 132,701 & 41,922 & 3,947 & 1.2 & 36 & 8,446 & 3,738 & 0.0147 & 182 & 0.732 & 36 & 0.806 & 105 & 0.693 & 0.731 & 0.83 & 404.16 & 608.38 & 1.21 & 0.62 \\
\hline 87,583 & 28,694 & 2,560 & 1.0 & 25 & 5,086 & 2,051 & 0.0652 & 238 & 0.768 & 23 & 0.841 & 117 & 0.719 & 0.757 & 0.66 & 317.76 & 549.78 & 0.92 & 0.50 \\
\hline
\end{tabular}

Table A-5: Data Generated for Eight Stage Treatment Type III, continued 


\begin{tabular}{|c|c|c|c|c|c|c|c|c|c|c|c|c|c|c|c|c|c|c|c|}
\hline \begin{tabular}{l|}
$\frac{0}{E}$ \\
$\frac{3}{0}$ \\
$\frac{0}{0}$ \\
$\frac{\pi}{0}$ \\
\end{tabular} & $\begin{array}{l}\text { है } \\
\frac{\text { ह }}{3} \\
0 \\
\frac{0}{0} \\
\frac{0}{0} \\
0\end{array}$ & 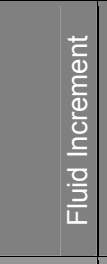 & 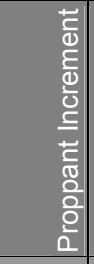 & 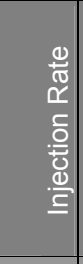 & 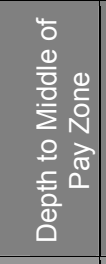 & 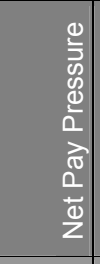 & 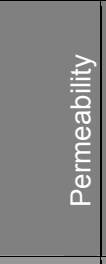 & 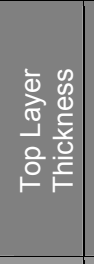 & 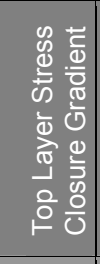 & 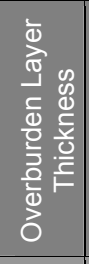 & 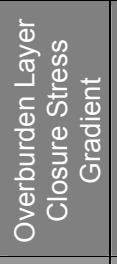 & 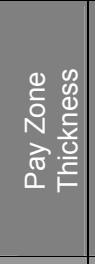 & 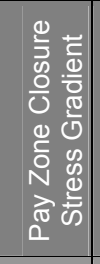 & 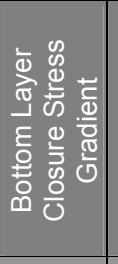 & 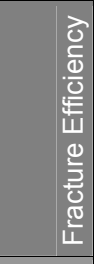 & 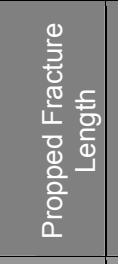 & 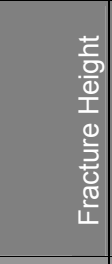 & 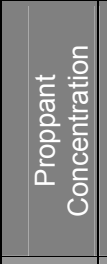 & 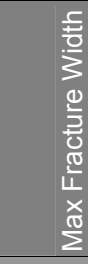 \\
\hline input & input & input & input & input & input & input & input & input & input & input & input & input & input & input & output & output & output & output & output \\
\hline 53,257 & 14,999 & 1,663 & 1.2 & 26 & 9,804 & 3,911 & 0.0441 & 219 & 0.730 & 22 & 0.800 & 88 & 0.678 & 0.724 & 0.62 & 316.64 & 434.65 & 0.91 & 0.46 \\
\hline 80,243 & 26,863 & 2,321 & 1.1 & 28 & 9,021 & 4,477 & 0.0286 & 210 & 0.718 & 11 & 0.768 & 117 & 0.651 & 0.718 & 0.70 & 382.05 & 470.95 & 0.89 & 0.55 \\
\hline 140,348 & 50,259 & 3,917 & 1.0 & 36 & 7,851 & 3,663 & 0.0076 & 225 & 0.724 & 28 & 0.789 & 70 & 0.671 & 0.720 & 0.93 & 388.83 & 561.84 & 1.12 & 0.70 \\
\hline 184,176 & 62,261 & 5,301 & 1.6 & 39 & 14,730 & 7,269 & 0.0038 & 212 & 0.713 & 40 & 0.746 & 88 & 0.669 & 0.703 & 0.91 & 575.36 & 649.32 & 1.42 & 0.69 \\
\hline 53,543 & 19,374 & 1,486 & 1.2 & 21 & 14,349 & 6,481 & 0.0061 & 216 & 0.723 & 19 & 0.784 & 89 & 0.677 & 0.715 & 0.77 & 391.71 & 439.48 & 0.65 & 0.44 \\
\hline 55,673 & 23,041 & 1,419 & 1.0 & 25 & 9,363 & 4,369 & 0.0085 & 220 & 0.756 & 27 & 0.752 & 93 & 0.691 & 0.748 & 0.82 & 304.98 & 442.40 & 0.66 & 0.47 \\
\hline 158,764 & 65,425 & 4,058 & 2.0 & 33 & 9,673 & 4,224 & 0.0123 & 171 & 0.788 & 11 & 0.844 & 65 & 0.730 & 0.774 & 0.88 & 446.68 & 581.53 & 0.98 & 0.64 \\
\hline 43,563 & 15,073 & 1,239 & 1.1 & 18 & 5,256 & 1,744 & 0.0361 & 230 & 0.718 & 20 & 0.718 & 87 & 0.674 & 0.710 & 0.72 & 232.57 & 423.68 & 0.87 & 0.43 \\
\hline 92,892 & 29,650 & 2,750 & 1.1 & 27 & 11,151 & 5,202 & 0.0255 & 220 & 0.726 & 40 & 0.784 & 111 & 0.693 & 0.753 & 0.70 & 332.79 & 420.72 & 1.35 & 0.71 \\
\hline 138,697 & 52,563 & 3,745 & 1.5 & 33 & 8,421 & 3,748 & 0.0156 & 211 & 0.762 & 22 & 0.836 & 83 & 0.729 & 0.827 & 0.87 & 324.96 & 482.96 & 2.24 & 1.01 \\
\hline 71,580 & 29,455 & 1,832 & 1.1 & 31 & 14,796 & 6,461 & 0.0254 & 178 & 0.701 & 21 & 0.743 & 108 & 0.657 & 0.705 & 0.58 & 404.03 & 414.64 & 0.68 & 0.46 \\
\hline 131,268 & 50,470 & 3,513 & 1.4 & 36 & 10,174 & 4,621 & 0.0059 & 228 & 0.728 & 34 & 0.783 & 107 & 0.694 & 0.729 & 0.88 & 378.70 & 576.14 & 1.41 & 0.66 \\
\hline 100,509 & 33,936 & 2,894 & 1.0 & 30 & 10,135 & 4,806 & 0.0326 & 203 & 0.779 & 23 & 0.869 & 92 & 0.683 & 0.718 & 0.76 & 362.61 & 533.21 & 0.94 & 0.55 \\
\hline 47,747 & 18,199 & 1,285 & 1.2 & 25 & 9,481 & 4,710 & 0.0185 & 240 & 0.740 & 20 & 0.767 & 124 & 0.682 & 0.738 & 0.68 & 289.60 & 391.70 & 0.86 & 0.46 \\
\hline 56,877 & 16,091 & 1,773 & 1.0 & 26 & 11,487 & 5,106 & 0.0071 & 198 & 0.713 & 35 & 0.756 & 61 & 0.678 & 0.710 & 0.88 & 314.32 & 447.94 & 0.79 & 0.49 \\
\hline 115,159 & 34,332 & 3,514 & 1.2 & 24 & 13,800 & 5,999 & 0.0181 & 229 & 0.734 & 31 & 0.734 & 119 & 0.674 & 0.734 & 0.63 & 513.05 & 497.11 & 1.04 & 0.64 \\
\hline $188,167 \mid$ & 54,259 & 5,822 & 1.2 & 40 & 7,627 & 3,360 & 0.0120 & 229 & 0.709 & 16 & 0.708 & 99 & 0.652 & 0.709 & 0.89 & 448.02 & 773.76 & 1.26 & 0.66 \\
\hline 75,043 & 28,827 & 2,009 & 1.1 & 29 & 7,996 & 3,929 & 0.0481 & 219 & 0.780 & 18 & 0.836 & 86 & 0.738 & 0.777 & 0.79 & 283.27 & 463.25 & 1.06 & 0.56 \\
\hline 91,399 & 33,013 & 2,539 & 1.2 & 30 & 14,596 & 6,736 & 0.0300 & 216 & 0.736 & 18 & 0.760 & 125 & 0.663 & 0.726 & 0.44 & 577.55 & 337.01 & 0.98 & 0.56 \\
\hline 77,793 & 27,146 & 2,202 & 1.2 & $27 \mid$ & 10,522 & 4,492 & 0.0109 & 233 & 0.725 & 36 & 0.790 & 85 & 0.674 & 0.724 & 0.81 & 392.50 & 534.50 & 0.86 & 0.51 \\
\hline 131,627 & 39,039 & 4,026 & 1.1 & 38 & 13,120 & 6,053 & 0.0889 & 182 & 0.730 & 29 & 0.729 & 84 & 0.672 & 0.734 & 0.62 & 449.79 & 608.92 & 1.01 & 0.59 \\
\hline 88,605 & 34,296 & 2,361 & 1.1 & 25 & 8,174 & 3,812 & 0.0335 & 226 & 0.741 & 33 & 0.740 & 117 & 0.680 & 0.740 & 0.68 & 349.55 & 518.87 & 0.90 & 0.52 \\
\hline
\end{tabular}

Table A-5: Data Generated for Eight Stage Treatment Type III, continued 


\section{CURRICULUM VITAE}

Andrei Popa was born November 30, 1970, in Campina, Romania. He is the son of Sergiu Popa and Stela Popa.

He received his B.S. degree in Petroleum and Natural Gas Engineering from University of Ploiesti, Romania, in 1995. He achieved a Master of Science in Petroleum and Natural Gas Engineering in 1998 and his Doctor of Philosophy degree in Petroleum and Natural Gas Engineering in 2004, both from West Virginia University.

Andrei is a member of the Honor Society of Petroleum Engineers (Pi Epsilon Tau) and member of the Society of Petroleum Engineers (SPE). He has served as technical reviewer for different publications and currently serves as member of the Board of Directors for the San Joaquin Valley SPE Section. He has authored/coauthored more than a dozen technical papers on the subject of Artificial Intelligence applications in the energy industry for SPE, IEEE, Elsevier, and others.

Prior to coming to the United States, he was a Petroleum Engineer for PETROMAR, the offshore division of Romanian Oil Company, and then a Reservoir Engineer for the Institute of Research and Technology in Campina, Romania. During this time, he worked on various projects involving reservoir simulation, forecasting, and economic evaluation for several oil and gas fields in Romania.

While working towards his $\mathrm{PhD}$, Andrei was also employed as a Research Associate at West Virginia University. His projects included pioneer research in the application of Artificial Intelligence to the oil and natural gas industry.

Currently he is a Petroleum Engineer with ChevronTexaco where he is responsible for the production and operation of over 1,500 wells in the Kern River field in Kern County, California. $\mathrm{He}$ is also involved in steamflood and workover optimization and continues to find new and innovative ways to apply his expertise in Artificial Intelligence for increased oil production opportunities.

$\mathrm{He}$ is married to Carrie Goddard Popa, and they currently reside in Bakersfield, California.

He plans to continue his research and application of Artificial Intelligence techniques to topics related to Petroleum and Natural Gas Engineering. 